\title{
Förderung der Lerneffizienz \\ beim Einsatz von \\ Unternehmensplanspielen
}

\author{
Eine empirische Erhebung \\ in der kaufmännischen Fortbildung \\ berufstätiger Erwachsener
}

Dissertation

zur Erlangung des wirtschaftswissenschaftlichen Doktorgrades der Wirtschaftswissenschaftlichen Fakultt

der Universität Göttingen

vorgelegt am 18. Oktober 1999

von Susanne Hartung

aus Gehrden, Ldkr. Hannover

Göttingen 1999 
Erstgutachter:

Zweitgutachter:

Tag der mündlichen Prüfung: 06. Dezember 1999
Prof. Dr. Dr. h.c. Jürgen Bloech

Prof. Dr. Dr. h.c. Frank Achtenhagen 


\section{Inhaltsverzeichnis}

Abbildungsverzeichnis $\quad$ ix

Tabellenverzeichnis $\quad$ xiii

1 Problemstellung 1

1.1 Bildungstheoretischer Kontext . . . . . . . . . . . . 1

1.2 Einordnung und Ziel der Arbeit ............. 5

1.3 Aufbau der Arbeit ................. 7

2 Berufliches Lernen und Handeln 11

2.1 Vorbemerkungen .................... 11

2.2 Modelle der kognitiven Struktur . . . . . . . . . . . . 12

2.2.1 Arten von Wissensrepräsentationen . . . . . . . . 12

2.2.2 Zwei Ansätze der Wissensrepräsentation . . . . . . . 16

2.3 Zusammenhang zwischen Kognition, Emotion und Motivation . . 21

2.3.1 Grundlegende Überlegungen . . . . . . . . . . . . . 21

2.3.2 Darstellung des Modells ............. 25

2.4 Wirtschaftspädagogischer Hintergrund . . . . . . . . . 29

2.4.1 Schlüsselqualifikationen als Lernziele beruflicher Bildung • 29

2.4.2 Das Konzept der Handlungsorientierung . . . . . . . . 33

2.4.3 Planung von Lernzielen in der beruflichen Bildung . . . . . 35 
2.5 Komplexe Lehr-Lern-Arrangements . . . . . . . . . . . . . . 39

2.5.1 Defizite traditionellen Unterrichts und Auswege . . . . . 39

2.5 .2 Exkurs: Komplexität . . . . . . . . . . . . . . . . 40

2.5.3 Gestaltung komplexer Lehr-Lern-Arrangements . . . . . 42

3 Unternehmensplanspiele in der Aus- und Weiterbildung 47

3.1 Vorbemerkungen . . . . . . . . . . . . . . . 47

3.2 Grundlagen zu Unternehmensplanspielen . . . . . . . . . . 48

3.2 .1 Begriffsabgrenzung . . . . . . . . . . . . . . 48

3.2.2 Einsatzmöglichkeit von Unternehmensplanspielen . . . . 52

3.2.3 Klassifikation von Unternehmensplanspielen . . . . . . . 55

3.3 Planung im Unternehmensplanspiel . . . . . . . . . . . . 60

3.3.1 Grundlagen der Unternehmensplanung . . . . . . . . . 60

3.3 .2 Absatzplanung . . . . . . . . . . . . 63

3.3.3 Produktions- und Beschaffungsplanung . . . . . . . . 65

3.3.4 Investitions- und Finanzplanung . . . . . . . . . . 68

3.4 Lernziele beim Einsatz von Unternehmensplanspielen . . . . . . 73

3.4.1 Lernhandeln im Unternehmensplanspiel . . . . . . . . . 73

3.4.2 Klassifikation der Lernziele . . . . . . . . . . . . 76

3.5 Besonderheiten beim Einsatz von Unternehmensplanspielen . . . . 79

3.5.1 Allgemeine Überlegungen . . . . . . . . . . . . . . 79

3.5.2 Organisatorische und methodische Besonderheiten . . . . 80

3.6 Forschungsstand zum Einsatz von Unternehmensplanspielen . . . 84

3.6.1 Einfluss der Modell-Komplexität. . . . . . . . . . . . 84

3.6.2 Umgang mit komplexen Simulationsmodellen . . . . . . 89

3.6.3 Weitere Forschungsergebnisse . . . . . . . . . . . 92 
4 Konzeption und Evaluation der Maßnahme $\quad 97$

4.1 Vorbemerkungen ..................... 97

4.2 Analyse der Rahmenbedingungen . . . . . . . . . . . . 98

4.3 Überlegungen zur Konzeption . . . . . . . . . . . . . . . 102

4.4 Lernziele der Maßnahme . . . . . . . . . . . . . . . 104

4.5 Überlegungen zur Evaluation . . . . . . . . . . . . . 109

4.5.1 Funktionen und Arten von Evaluation . . . . . . . . 109

4.5.2 Evaluation in der beruflichen Weiterbildung ....... 111

4.5.3 Zum Planspiel-Einsatz .............. 113

4.6 Aspekte der Leistungsbeurteilung . . . . . . . . . . . . . 117

4.6.1 Begriffsklärungen ................. 117

4.6.2 Funktionen von Leistungsbeurteilungen . . . . . . . . . . 118

4.6.3 Bezugsnormen der Leistungsbeurteilung . . . . . . . 120

4.7 Gütekriterien von Erhebungsinstrumenten . . . . . . . . . . 122

4.7.1 Allgemeine Überlegungen . . . . . . . . . . . . . 122

4.7.2 Objektivität .................... 125

4.7 .3 Reliabilität .................... 126

4.7.4 Validität ................... 127

4.7 .5 Weitere Kriterien . . . . . . . . . . . 128

4.8 Konzeption der Maßnahme . . . . . . . . . . . . 130

4.8.1 Gestaltung der Evaluation ............. 130

4.8.2 Gestaltung des Planspiel-Einsatzes ........... 132

4.8.3 Design der Erhebung . . . . . . . . . . . 133

4.8.4 Hypothesen der Erhebung ............. 135

4.9 Versuchsplan und Ablauf der Erhebung . . . . . . . . . . . 138

4.9.1 Entwicklung des Versuchsplans ............ 138

4.9.2 Ablauf der Erhebung ................ 139 
$5 \quad$ Erhebungsinstrumente des Prä- und Posttests 143

5.1 Vorbemerkungen ...................... 143

5.2 Fragebogen zum Faktenwissen . . . . . . . . . . . 144

5.2.1 Inhaltliche Konstruktion ............... 144

5.2 .2 Beurteilung des Lernerfolgs . . . . . . . . . . . 145

5.2.3 Gütekriterien des Fragebogens . . . . . . . . . . . 146

5.3 Netzwerk-Erhebung zum Zusammenhangswissen . . . . . . . . . 146

5.3.1 Inhaltliche Konstruktion . . . . . . . . . . . 146

5.3 .2 Beurteilung des Lernerfolgs . . . . . . . . . . . 149

5.3.3 Gütekriterien der Netzwerk-Erhebung . . . . . . . . 150

5.4 Fallstudie zur Transferleistung . . . . . . . . . . . 153

5.4.1 Inhaltliche Konstruktion . . . . . . . . . . . . 153

5.4 .2 Beurteilung des Lernerfolgs . . . . . . . . . . 157

5.4.3 Gütekriterien der Fallstudie . . . . . . . . . 159

5.5 Zusammenhang der drei Instrumente . . . . . . . . . 160

6 Die Planspiel-Phase und ihre Erhebungsinstrumente 163

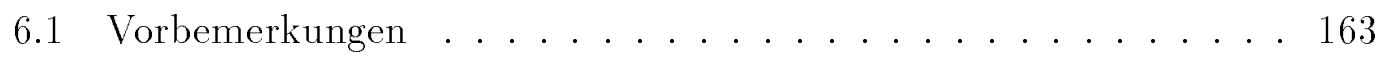

6.2 Das Unternehmensplanspiel EpUS . . . . . . . . . . . . 164

6.2.1 Planspiel-Modell ................. 164

6.2.2 Planspiel-Unterlagen . . . . . . . . . . . . . 168

6.3 Die experimentelle Phase . . . . . . . . . . . . 172

6.3 .1 Ausgangslage . . . . . . . . . . . . . 172

6.3.2 Stufenweise Komplexitäts-Steigerung . . . . . . . 173

6.3.3 Lernziele der Komplexitäts-Stufen . . . . . . . . . . 175 
6.4 Die Testphase . . . . . . . . . . . . . . . . 181

6.4.1 Inhaltliche Konstruktion ............... 181

6.4 .2 Ausgangslage .................. 181

6.4.3 Bewertung des Lernerfolgs ............. 186

6.4.4 Gütekriterien der Testphase ........... 187

6.5 Der Fragebogen zur Entscheidung . . . . . . . . . . . 192

6.5.1 Inhaltliche Konstruktion . . . . . . . . . . . 192

6.5.2 Gütekriterien des Fragebogens ............. 194

6.6 Der Fragebogen zum situativen Erleben . . . . . . . . . . . 195

6.6.1 Inhaltliche Konstruktion .............. 195

6.6.2 Gütekriterien des Fragebogens ........... 197

$\begin{array}{lll}7 & \text { Auswertungsmethoden } & 199\end{array}$

7.1 Vorbemerkungen . . . . . . . . . . . . . 199

7.2 Statistische Tests . . . . . . . . . . . . . 199

7.3 Fisher's exakter Test .................. 200

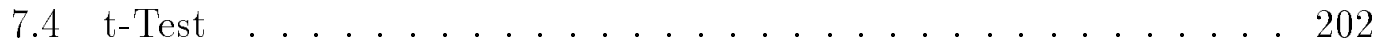

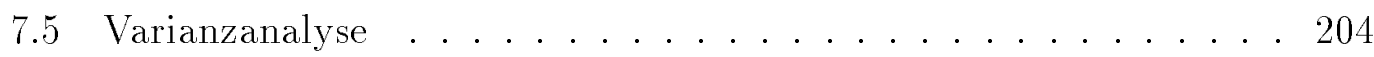

7.6 Korrelationsanalyse .................. 206

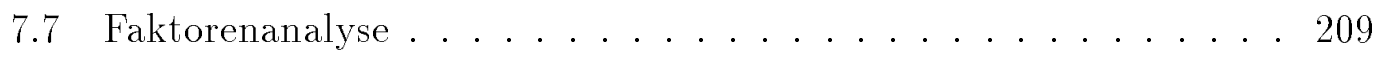

8 Ergebnisse der Planspiel-Phase $\quad 211$

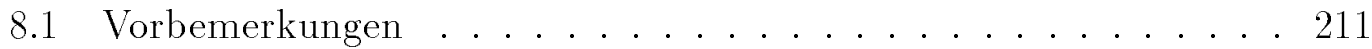

8.2 Entwicklung einer optimalen Entscheidung in der Testphase . . . 212

8.2.1 Unternehmensplanung . . . . . . . . . . 212

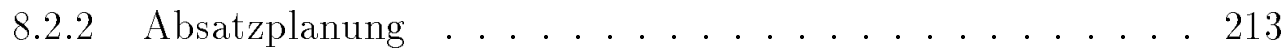

8.2.3 Produktions- und Beschaffungsplanung . . . . . . . . 215

8.2.4 Kostenrechnung . . . . . . . . . . . . 218

8.2.5 Finanzplanung ................ 221 
8.3 Vergleich der experimentellen Bedingungen . . . . . . . . 223

8.3.1 Produktions- und Beschaffungsplanung . . . . . . . . 223

8.3.2 Absatzplanung ................... 230

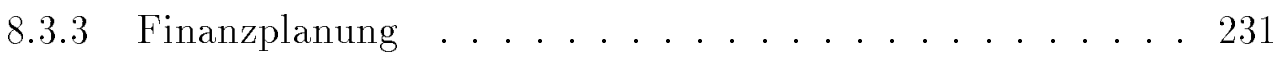

8.3.4 Unternehmenserfolg. . . . . . . . . . . . 235

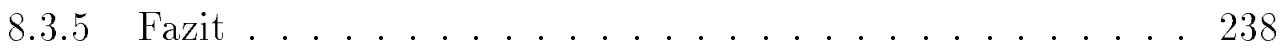

8.4 Vollständiges Design . . . . . . . . . . . . . 238

8.4.1 Einsatz des Fragebogens zur Entscheidung . . . . . . 238

8.4.2 Fragebogen zum situativen Erleben ......... 243

9 Ergebnisse des Prä- und Posttests 253

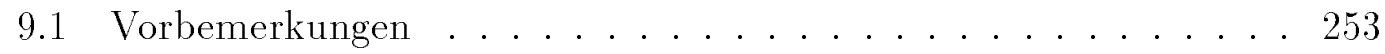

9.2 Betrachtung der gesamten Stichprobe . . . . . . . . . . 254

9.2.1 Multiple-Choice-Fragebogen ... . . . . . . . . 254

9.2.2 Netzwerk-Erhebung . . . . . . . . . . . 255

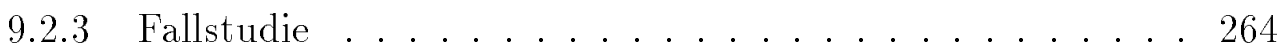

9.3 Vergleich der experimentellen Bedingungen . . . . . . . . 268

9.3.1 Netzwerk-Erhebung................ 268

9.3 .2 Fallstudie .................. 270

9.4 Vollständiges Untersuchungsdesign . . . . . . . . . . . 274

9.4.1 Multiple-Choice-Fragebogen ............. 274

9.4 .2 Netzwerk-Erhebung. . . . . . . . . . . 274

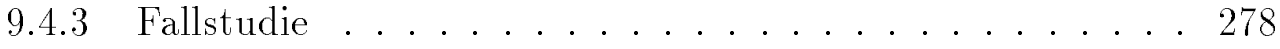

10 Zusammenfassung $\quad 283$

$\begin{array}{lr}\text { Literaturverzeichnis } & \mathbf{2 8 9}\end{array}$ 
A Anhang 1

A.1 Zeitplan der Erhebung ................. 1

A.2 Einteilung der Gruppen ................ 2

A.3 Konstruktion der Fallstudie ............... 3

A.4 Komplexitäts-Steigerung in der experimentellen Phase . . . . . 4

A.5 Instruktion zum Prätest ............... 5

A.6 Instruktion zum Posttest . . . . . . . . . . . . . 6

A.7 Fallstudie ................... 7

A.8 Lösungsblatt zur Fallstudie. . . . . . . . . . . . . . . . 11

A.9 Multiple-Choice-Fragebogen, Version A . . . . . . . . . 12

A.10 Multiple-Choice-Fragebogen, Version B . . . . . . . . . 15

A.11 Lösungsblatt zum Fragebogen . . . . . . . . . . . . . . 18

A.12 Netzwerk-Erhebung . . . . . . . . . . . . . . . . . 19

A.13 Legende zur Darstellung der Netzwerke . . . . . . . . . . . . . . . 21

A.14 Entscheidungsblätter der modularen Bedingung . . . . . . . 22

A.15 Entscheidungsblätter der herkömmlichen Bedingung . . . . . . . 33

A.16 Entscheidungsblätter in der Testphase . . . . . . . . . 36

A.17 Fragebogen zur Entscheidung . . . . . . . . . . 40

A.18 Fragebogen zum situativen Erleben . . . . . . . . . . . . 41

A.19 Ausgangslage der experimentellen Phase . . . . . . . . . . . . 42

A.20 Ausgangslage der Testphase . . . . . . . . . . . . 52

A.21 Finanzplanung in EpUS . . . . . . . . . . . . 62

A.22 Korrelations-Koeffizienten der Fallstudie . . . . . . . . . . . 64

A.23 Auswertungsergebnisse des Prä-Post-Vergleichs . . . . . . . . . 65

A.24 Faktorenanalyse der Ergebnisse der Testphase . . . . . . . . . . 69

A.25 Auswertungsergebnisse Testphase . . . . . . . . . . . . . 71

A.26 Auswertungsergebnisse Fragebogen zum situativen Erleben . . . 75 
A.27 Korrelationsanalyse der Items des Fragebogens zum situativen Er-

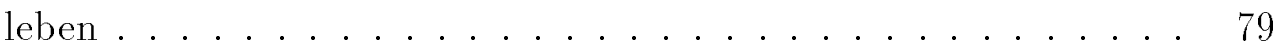




\section{Abbildungsverzeichnis}

1.1 Einordnung der beruflichen Weiterbildung . . . . . . . . 3

2.1 Kognitive Struktur - Kognitive Strukturierungen _ . . . . . . 13

2.2 Arten von Wissen . . . . . . . . . . . . . . . 15

2.3 TOTE-Schema $\ldots \ldots \ldots \ldots \ldots \ldots \ldots \ldots$

2.4 Erweitertes kognitives Motivationsmodell . . . . . . . . 26

2.5 Struktur des psychophysischen Systems . . . . . . . . . . 27

2.6 Vom Lehrinhalt zum Lehrziel _ . . . . . . . . . . . . 36

2.7 Taxonomie der kognitiven Lernziele nach BLoom $\ldots \ldots \ldots$. . 37

3.1 Phasen einer Spielrunde im Planspiel . . . . . . . . . . . 50

3.2 Teilpläne der Unternehmensplanung . . . . . . . . . . . . . . 62

3.3 Finanzierungsarten $\ldots \ldots \ldots \ldots \ldots \ldots \ldots$

3.4 Qualitätsmerkmale der Handlung im Planspiel . . . . . . . . . 75

4.1 Einflussgrößen des Lernens . . . . . . . . . . . . . . . . . 103

4.2 Hierarchisierung der kognitiven Lernziele . . . . . . . . . 105

4.3 Untersuchungsdesign . . . . . . . . . . . . . . . . . . . 134

4.4 Versuchsplan . . . . . . . . . . . . . . . . . . . 139

4.5 Ablauf der Untersuchung . . . . . . . . . . . . . . . . . 141

5.1 Zusammenhang zwischen Fragebogen, Netzwerk und Fallstudie . . 162 
6.1 Ermittlung der tatsächlichen Produktionsmenge im Planspiel EpUS 167

6.2 Verlauf der Nachfrage im Planspiel EpUS . . . . . . . . . 168

6.3 Zusammenhänge im Planspiel EpUS (1) . . . . . . . . . . . 170

6.4 Zusammenhänge im Planspiel EpUS (2) . . . . . . . . . . . 171

6.5 Stufenweise Erhöhung der Komplexität . . . . . . . . . . . . . 174

6.6 Ausgangslage der Testphase: Marktforschung 1. . . . . . . . 183

6.7 Ausgangslage der Testphase: Marktforschung 2 . . . . . . . . . 184

6.8 Ausgangslage der Testphase: Relative Preis-Abweichungen . . . . 185

7.1 Zweifaktorielle Varianzanalyse . . . . . . . . . . . 205

7.2 Interpretation des Korrelations-Koeffizienten . . . . . . . . . 207

8.1 Optimale Entscheidung: Absatzprognosen . . . . . . . . . 215

8.2 Testphase: Beschaffungs- und Produktionsplanung, herkömmliche Bedingung . . . . . . . . . . . . . . . . 224

8.3 Testphase: Beschaffungs- und Produktionsplanung, modulare Be-

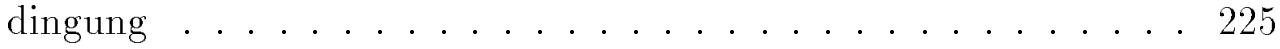

8.4 Testphase: Investitionsplanung und Herstellungskosten, herkömmliche Bedingung . . . . . . . . . . . . . . . 228

8.5 Testphase: Investitionsplanung und Herstellungskosten, modulare Bedingung . . . . . . . . . . . . . . 229

8.6 Testphase: Absatzplanung, herkömmliche Bedingung . . . . . . . 232

8.7 Testphase: Absatzplanung, modulare Bedingung . . . . . . . 233

8.8 Testphase: Kassenbestand . . . . . . . . . . . . . . 234

8.9 Testphase: Erzielte Gewinne . . . . . . . . . . . 236

8.10 Testphase: Kumulierte Gewinne . . . . . . . . . . . . . 237

8.11 Testphase mit vollständigem Design: Rohstoffbeschaffung . . . . . 241

8.12 Testphase mit vollständigem Design: Bestandsveränderungen . . . 242

8.13 Ergebnisse des Fragebogens zum situativen Erleben (Item 1-3) . . 244

8.14 Ergebnisse des Fragebogens zum situativen Erleben (Item 4-6) . . 245 
8.15 Ergebnisse des Fragebogens zum situativen Erleben (Item 7-9) . . 247

8.16 Ergebnisse des Fragebogens zum situativen Erleben (Item 10-12) 248

8.17 Ergebnisse des Fragebogens zum situativen Erleben (Item 13) . . 250

8.18 Vergleich der experimentellen Bedingungen: Einsatz des Fragebogens zum situativen Erleben . . . . . . . . . . . . 251

9.1 Prä-Post-Vergleich: Anzahl richtiger Antworten im Fragebogen . . 255

9.2 Prä-Post-Vergleich: Größe der Netzwerke . . . . . . . . . . . . . 256

9.3 Prä-Post-Vergleich: Größe und Ordnung der Netzwerke . . . . . . 257

9.4 Prä-Post-Vergleich: Modales Netzwerk des Prätests . . . . . . . 260

9.5 Prä-Post-Vergleich: Modales Netzwerk des Posttests . . . . . . . . 261

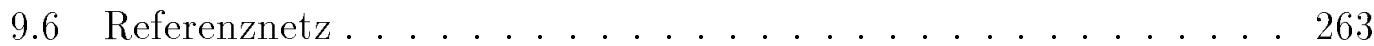

9.7 Prä-Post-Vergleich: Gewinne in der Fallstudie . . . . . . . . . . 264

9.8 Prä-Post-Vergleich: Absatzmengen in der Fallstudie . . . . . . . 265

9.9 Prä-Post-Vergleich mit Planspiel-Durchführung: Netzwerk . . . 269

9.10 Prä-Post-Vergleich mit Planspiel-Durchführung: Fallstudie (1) . 272

9.11 Prä-Post-Vergleich mit Planspiel-Durchführung: Fallstudie (2) . 273

9.12 Vollständiges Design: Netzwerk-Erhebung (1) . . . . . . . . 275

9.13 Vollständiges Design: Netzwerk-Erhebung (2) _ . . . . . . . 276

9.14 Vollständiges Design: Netzwerk-Erhebung (3) . . . . . . . . 277

9.15 Vollständiges Design: Fallstudie (1) . . . . . . . . . . 279

9.16 Vollständiges Design: Fallstudie, Einfluss des Fragebogens zum situativen Erleben ............... . 280

9.17 Vollständiges Design: Fallstudie (3) . . . . . . . . . . . 281 
Abbildungsverzeichnis 


\section{Tabellenverzeichnis}

3.1 Klassifikation von Planspielen . . . . . . . . . . . 56

6.1 Korrelations-Koeffizienten der Testphase . . . . . . . . . . . 190

8.1 Subziele und Mittel zur Gewinn-Maximierung im Planspiel EpUS 213

8.2 Optimale Entscheidung: Marketing-Instrumente . . . . . . . . . 214

8.3 Auswirkungen unterschiedlicher Kapazitätsauslastungen im Planspiel EpUS . . . . . . . . . . . . . . . . . 217

8.4 Optimale Entscheidung: Vorkalkulation der Selbstkosten . . . . 220

8.5 Optimale Entscheidung: Liquiditätsplanung . . . . . . . . 222 


\section{Problemstellung}

\subsection{Bildungstheoretischer Kontext}

In den letzten Jahren ist ein Strukturwandel des Beschäftigungssystems festzustellen, der sich in verschiedenen Bereichen zeigt. Diese Veränderungen werden häufig unter dem Begriff der Megatrends zusammengefasst: ${ }^{1}$ Hierzu zählen die zunehmende Internationalisierung und Globalisierung der Wirtschaft, aber gleichzeitig auch die damit verbundenen Auswirkungen auf die Umwelt, die das Wirtschafts-Wachstum begrenzen. Der zunehmende Einsatz neuer Technologien ermöglicht die Automatisierung von Routine-Tätigkeiten und eine flexiblere Steuerung der Produktionssysteme, hat jedoch insbesondere in Zusammenhang mit der demographischen Entwicklung einen negativen Einfluss auf das Beschäftigungssystem.

Die Unternehmen sind einem erhöhten Rationalisierungsdruck ausgeliefert und reagieren mit der Umstrukturierung von Organisationseinheiten und dem Abbau von Hierarchien. ${ }^{2}$ Aber auch die Strategien der Unternehmungen auf den Strukturwandel verändern sich. In diesem Zuge kommt der 'lernenden Unternehmung' eine große Bedeutung zu. ${ }^{3}$ Der Erfolg unternehmerischer Aktivitäten hängt mehr denn je von den Qualifikationen der im Unternehmen tätigen Führungskräfte ab. Das sich verändernde Arbeitsumfeld stellt zunehmend höhere Anforderungen an ihre Flexibilität und beruflichen Kompetenzen, ${ }^{4}$ wozu auch moderne Management-Methoden wie Lean Management und Benchmarking zählen. ${ }^{5}$

\footnotetext{
1 Vgl. Buttler (Tätigkeitslandschaft 1992).

Vgl. McKinsey Company Inc. \& Rommel (Unternehmenskonzept 1993).

Vgl. KRuse (Unternehmen 1995); Savage (Management 1997), S. 189-230.

Vgl. Staudt, Kröll \& Hören (Potentialorientierung 1993).

Vgl. Füser (Management 1999).
} 
Im kaufmännischen Bereich gewinnen langfristig Funktionen an Bedeutung, in denen sich Eigenständigkeit, Dispositions-, Koordinations-, Organisations- und Führungsaufgaben mit fachlichen Kompetenzen und sachkundigem Umgang mit DV-gestützten Informationssystemen verbinden. ${ }^{6}$ Eine gezielte Förderung solcher Schlüsselqualifikationen durch die Weiterbildung gehört deshalb zu den wichtigsten Aufgaben der Unternehmensführung ${ }^{7}$ und wird weiterhin an Bedeutung gewinnen. ${ }^{8}$ Daher ist sie auch als öffentliche Aufgabe zu verstehen. ${ }^{9}$

Berufliche Bildung hat im Zuge der skizzierten Veränderungen eine zweifache Funktion: ${ }^{10}$ Erstens müssen die Arbeitnehmenden ausreichend und effektiv auf die Anforderungen im Berufsleben vorbereitet werden, ${ }^{11}$ zweitens muss die berufliche Aus- und Weiterbildung die Persönlichkeitsentwicklung ermöglichen. ${ }^{12}$ Eine Anpassung der Weiterbildung an den Wandel in der Arbeitswelt hätte jedoch nur kurzfristig 'Reparatur-Charakter' und kann längerfristige Entwicklungen nicht berücksichtigen. Hier muss vielmehr den zukünftigen Anforderungen vorgegriffen werden, wenn berufliche Bildung ihr Ziel erreichen soll. ${ }^{13}$

Zur beruflichen Bildung im Sinne des Berufsbildungsgesetzes (BBiG) zählen die berufliche Erstausbildung, die in Deutschland im Rahmen des Dualen Systems durchgeführt wird, ${ }^{14}$ und die berufliche Weiterbildung als "Fortsetzung oder Wiederaufnahme organisierten Lernens nach Abschluss einer unterschiedlich ausgedehnten Ausbildungsphase"15. Das Ende der Ausbildungsphase und damit der Beginn möglicher Weiterbildung ist in der Regel durch den Eintritt in die volle Erwerbstätigkeit gekennzeichnet. ${ }^{16}$ Mehr als ein Drittel der Beschäftigten neh-

6 Vgl. Tessaring (Qualifikationsentwicklungen 1996).

7 Vgl. KorndörfER (Unternehmensführungslehre 1995), S. 271-274.

8 Vgl. Enquête-Kommission (Bildungspolitik 1991), S. 84 sowie Deutscher Bundestag (Bildungspolitik 1990); auch Jagenlauf, Schulz \& Wolgast (Weiterbildung 1995) und ARNoLD (Schlüsselqualifikationen 1992).

9 Vgl. Buttler (Weiterbildung 1995), S. 61-63; auch Amt für Berufs- und WeiterbilDUNG (Weiterbildung 1995).

$10 \mathrm{Vgl}$ z. B. die Darstellung der European Cooperation in the Field of scientific and technical Research (COST) bei Achtenhagen, Nijhof \& Raffe (Feasibility Study 1995).

11 Vgl. auch Bundesinstitut Für Berufsbildung (Fabrikstrukturen 1990), S. 158.

12 Vgl. auch Bojanowski, Brater \& Dedering (Qualifizierung 1991), S. 102.

13 Vgl. Heid (Bildung 1998); auch Bundesinstitut für Berufsbildung (Lernen 1994).

14 Vgl. Tessaring (Berufsausbildung 1993).

15 Deutscher Bildungsrat (Empfehlungen 1970), S. 197.

16 Vgl. auch Bundesinstitut Für Berufsbildung (Weiterbildung 1994). 
men an Maßnahmen der beruflichen Weiterbildung teil. ${ }^{17}$ Von der beruflichen Bildung lässt sich die Erwachsenenbildung abgrenzen. Hierzu zählen die politische und gewerkschaftliche Bildungsarbeit, die insbesondere an Volkshochschulen und Bildungseinrichtungen der Gewerkschaften durchgeführt wird. ${ }^{18}$ Sie hat im Allgemeinen eher nichtberuflichen Charakter, kann jedoch auch im Sinne einer beruflichen Weiterbildung - z. B. bei Mitgliedern des Betriebsrats - aufgefasst werden. Abbildung 1.1 gibt einen Überblick über das Gesamtsystem der Bildung.

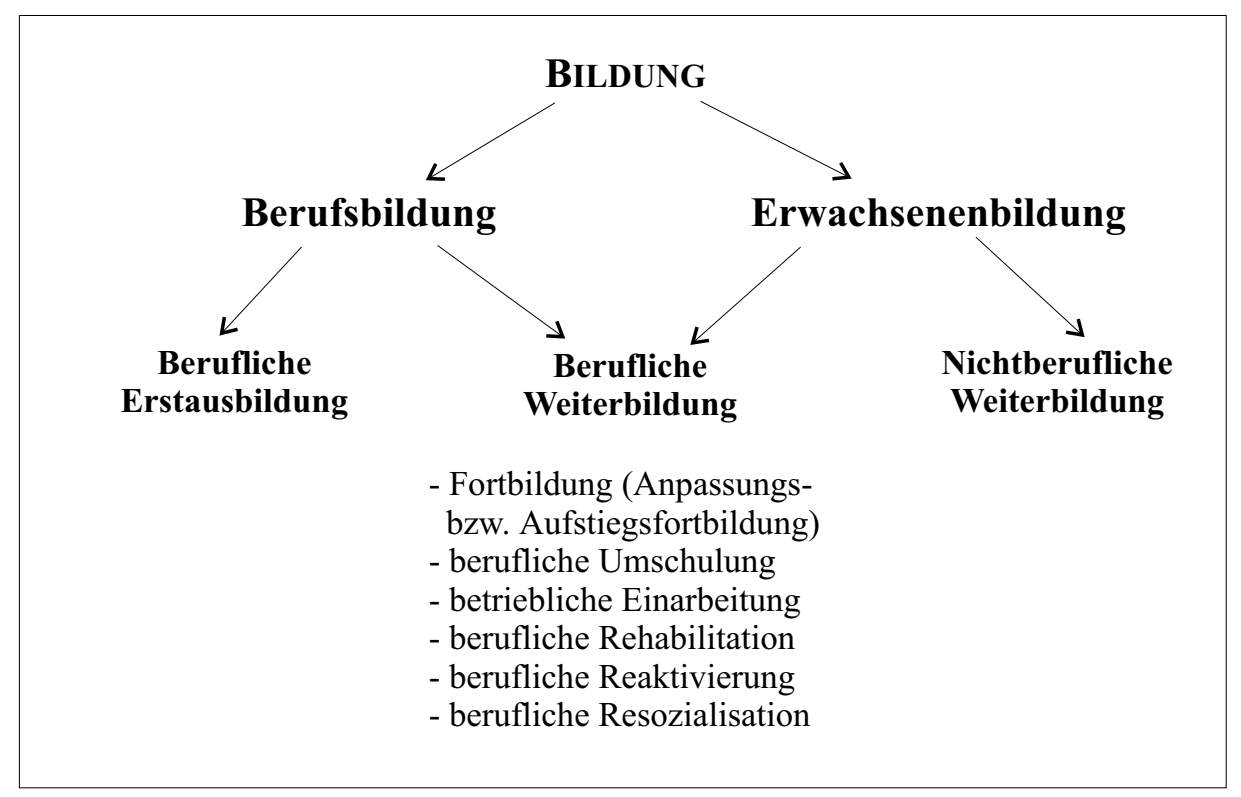

Abbildung 1.1: Einordnung der beruflichen Weiterbildung in das Gesamtsystem der Bildung ${ }^{19}$

Die traditionelle berufliche Aus- und Weiterbildung ist durch die fehlende Einbettung des Lernens in authentische Kontexte gekennzeichnet. ${ }^{20}$ Dadurch wird die Vermittlung von 'trägem Wissen' ${ }^{21}$ gefördert, das zwar im Lernkontext vorhanden und reproduzierbar ist, sich im entsprechenden Umfeld, also im beruflichen

\footnotetext{
17 Vgl. Kuwan, Gnahs \& Seusing (Weiterbildungsstatistik 1991), S. 284.

18 Vgl. z. B. Faulstich, Bayer \& Krohn (Zukunftskonzepte 1998).

19 In Anlehnung an Weyerich (Gestaltung 1995), S. 10.

$20 \mathrm{Vgl}$. Bransford et al. (Learning skills 1989).

21 Vgl. RenkL (Wissen 1994).
} 
Alltag, nur als schwer einsetzbar erweist. ${ }^{22}$ Als Mängel werden im Einzelnen genannt: ${ }^{23}$

- Es wurden eher abstrakte und anwendungsunspezifische Inhalte vermittelt, die in ihrer Struktur nicht den beruflichen Anforderungssituationen entsprachen.

- Die Lerninhalte wurden zerfasert dargestellt, d. h. eine Integration verschiedener Aspekte wurde vernachlässigt. Gerade die Zusammenhänge zwischen den Inhalten sind jedoch für die berufliche Praxis von großer Relevanz.

- Weiterhin fehlten kooperative Lernformen, durch die die im Berufsleben geforderte Teamfähigkeit vermittelt werden könnte.

Lernen im Rahmen der Handlungsorientierung wird als Lernhandeln betrachtet ${ }^{24}$ und es werden so genannte 'komplexe Lehr-Lern-Arrangements' entwickelt, ${ }^{25} \mathrm{um}$ die Defizite der traditionellen beruflichen Bildung zu beseitigen. Dabei steht die Entwicklung zum selbstständigen Handeln im Vordergrund. ${ }^{26}$

Zur Überprüfung, ob die Anforderungen durch entsprechende Bildungsmaßnahmen erreicht werden, wird Qualitätssicherung auch in der beruflichen Weiterbildung gefordert. ${ }^{27}$ Dabei ist nicht nur die Effektivität, also eine Analyse des Input-Output-Verhältnisses zu betrachten, sondern ein mehrdimensionales Qualitätskonzept unter Einbezug von Output-Qualitäten, d. h. eine Überprüfung der Effizienz von Weiterbildungsmaßnahmen vorzunehmen. ${ }^{28}$ Weiterhin ist eine Betrachtung von außerfachlichen Bildungseffekten fachlicher Qualifizierungsprozesse von Bedeutung. ${ }^{29}$ Insbesondere die Entwicklung der Informationstechnologie lässt die Grenzen zwischen den Berufen hinsichtlich der zu vermittelnden Inhalte eher fließend werden. Dies ist insbesondere in den meisten handwerklichen Bereichen sowie bei den akademischen Berufen von Bedeutung. ${ }^{30}$

\footnotetext{
22 Vgl. auch Deutscher Industrie- und Handelstag (Wettbewerb 1993), S. 108.

23 Vgl. Mandl, Gruber \& Renkl (Wissen 1993) sowie Reetz (Wissen 1996).

24 Vgl. Achtenhagen et al. (Lernhandeln 1992), S. 30-33.

$25 \mathrm{Vgl}$. Achtenhagen \& John (Lehr-Lern-Arrangements 1992).

26 Vgl. auch Bundesinstitut Für BerufsBildung (Entwicklung 1991).

27 Vgl. Feuchthofen \& Severing (Qualitätsmanagement 1995), S. XI-XVII.

28 Vgl. Arnold \& Krämer-StürzL (Qualität 1995).

29 Vgl. Arnold (Schlüsselqualifikationen 1996).

30 Vgl. Lipsmeier (Wert 1998).
} 
Aus den dargestellten Entwicklungen ergeben sich Folgerungen für die Evaluation solcher Maßnahmen: ${ }^{31}$ Alle Beteiligten am Forschungsprozess - Lehrende, Lernende und Forschende - müssen mit ihren Zielen und Anforderungen als gleichberechtigte Partner angesehen werden. Die Vielzahl der nur qualitativ zu erfassenden Variablen erfordert zudem eine angemessene Umsicht bei der Evaluation von Weiterbildung: ${ }^{32}$ Es sollte versucht werden, die wesentlichen Elemente, die in einer komplexen Struktur die spezifische Qualität einer Maßnahme ausmachen, herauszuarbeiten und entsprechend zu verbessern. ${ }^{33}$ Die bisherigen Forschungen im Bereich der Berufsbildung sind in erster Linie als quantitative Bildungsforschung konzipiert, ${ }^{34}$ qualitative Forschungen werden eher als ergänzende Komponenten behandelt. ${ }^{35}$ Es ist jedoch eine Entwicklung festzustellen, nach der qualitative Forschung verstärkt in einem spiralförmig organisierten Forschungsprozess durchgeführt wird, d.h. die Forschung wird während des Prozesses kontinuierlich verbessert und verläuft nicht mehr linear. ${ }^{36}$

\subsection{Einordnung und Ziel der Arbeit}

Die vorliegende Arbeit entstand aus dem von der Deutschen Forschungsgemeinschaft 1994-1997 geförderten Forschungsprojekt 'Lehren und Lernen mit Hilfe computergestützter Simulationen zur Unternehmensführung, insbesondere zur Investitions- und Produktionsplanung ${ }^{37}$ heraus. ${ }^{38}$ Im Rahmen des Projekts wurde ein Unternehmensplanspiel an der Verwaltungs- und Wirtschaftsakademie Göttingen eingesetzt und der Lernerfolg mit verschiedenen Instrumenten erhoben. Diese

31 Vgl. Achtenhagen et al. (Lernhandeln 1992), S. 30.

32 Vgl. Wesseler (Evaluation 1994), S. 677.

33 Vgl. Joint Committee \& Sanders (Handbuch 1999), S. 83-86.

34 Vgl. z.B. Achtenhagen (Erträge 1991).

35 Vgl. Deutsche Forschungsgemeinschaft (Berufsbildungsforschung 1990), S. 25.

36 Vgl. Arnold \& Müller (Berufsbildung 1992), S. 100-102; auch Arnold (Forschung 1995).

37 Das Projekt wurde im Rahmen des Schwerpunktprogramms 'Lehr-Lern-Prozesse in der kaufmännischen Erstausbildung' (Az.Bl 133/4) gefördert.

38 Vgl. Bloech et al. (Einsatz 1996); Blozch, Kauer \& Orth (Unternehmensplanspiele 1996); Bloech et al. (Einsatz 1998); Blozch, Hartung \& Orth (Unternehmensplanspiele 1998); Bloech, Hartung \& Orth (Einsatz 1999). 
Konzeption folgte der Forderung, dass pädagogische Forschung das Lernen als Prozess untersuchen und im Feld durchgeführt werden sollte. ${ }^{39}$

Die Ausbildung an der Verwaltungs- und Wirtschaftsakademie ist als Aufstiegsfortbildung einzuordnen. ${ }^{40}$ Sie wird nebenberuflich durchgeführt und dient dazu, durch Erweiterung der Kenntnisse und Fertigkeiten im Beruf weiterzukommen, und setzt eine abgeschlossene Berufsausbildung und Berufserfahrung voraus. ${ }^{41}$

Basierend auf den Ergebnissen und Erfahrungen des DFG-Projekts wird ein weitergehendes Konzept zur Förderung der Lerneffizienz beim Einsatz eines Unternehmensplanspiels entwickelt. Dabei wurden drei wissenschaftliche Blickrichtungen kombiniert:

1. Der erste Ansatz ist die System- und Entscheidungsorientierung der Betriebswirtschaftslehre: Die unternehmerische Realität wird als System verstanden und kann in Unternehmensplanspielen abgebildet werden. Dabei erfolgt eine Orientierung an der Handlung im Sinne einer planvollen Entscheidung auf Grund einer gegebenen betrieblichen Situation.

2. In der Wirtschaftspädagogik wurden mehrdimensionale Lehr-Lern-Arrangements entwickelt, um die Defizite der traditionellen beruflichen Bildung zu überwinden und die Teilnehmenden für die Anforderungen des Berufslebens auszubilden. Zu ihnen zählen auch Unternehmensplanspiele und ihre instruktionale Unterstützung.

3. Weiterhin werden zwei Ansätze der Psychologie bei der Konzeption der Arbeit herangezogen: Das zu Grunde liegenden Menschenbild ist das epistemologische Subjektmodell, ${ }^{42}$ wobei insbesondere die Eigenschaft der Reflexivität des Menschen für die Gestaltung der Fortbildungs-Maßnahme bedeutsam ist. Weiterhin wird zur Erklärung des Zusammenhangs zwischen Kognition, Emotion und Motivation beim Lernen ein Modell herangezogen, das auf diesem Menschenbild basiert. ${ }^{43}$

\footnotetext{
39 Vgl. Deutsche Forschungsgemeinschaft (Berufsbildungsforschung 1990).

40 Vgl. dazu Abbildung 1.1.

41 Vgl. Bundesverband Deutscher VWA (Rahmen-Studienordnung 1994), §4 (2).

42 Vgl. Groeben \& Scheele (Argumente 1977).

43 Vgl. Becker, Oldenbürger \& Piehl (Motivation 1987).
} 
Ziel der Arbeit ist die Untersuchung der lerneffizienten Gestaltung des Einsatzes eines Unternehmensplanspiels in der beruflichen Fortbildung. ${ }^{44}$ Dabei liegt ein Schwerpunkt auf der Analyse des Einflusses der Entscheidungs-Komplexität auf den Lernerfolg. Hierzu wird die Komplexität der Anforderungssituation systematisch variiert. Es wird ein Design entwickelt, das neben einem Vergleich des Wissens vor und nach dem Planspiel-Einsatz auch die Leistungen während der Planspiel-Phase betrachtet. Im Prä-Post-Design werden drei verschiedene Instrumente eingesetzt, die das betriebswirtschaftliche Faktenwissen, das Wissen um betriebliche Zusammenhänge sowie eine Transferleistung erheben. Dabei wird auf bereits eingesetzte und weiterentwickelte Instrumente aus dem DFG-Projekt zurückgegriffen. Der Einsatz des Unternehmensplanspiels wird ebenfalls wie in den bisherigen Untersuchungen durchgeführt. Für die Forschungsarbeit werden jedoch zusätzliche Maßnahmen zur Förderung der Reflexivität der Teilnehmenden entwickelt und eingesetzt, von denen ein förderlicher Einfluss auf den Lernerfolg erwartet wird. Insgesamt wird davon ausgegangen, dass sich die beiden Durchführungsformen des Unternehmensplanspiels unterschiedlich auf den Lernerfolg im Planspiel sowie im Prä- und Posttest auswirken. Bei der Analyse des Datenmaterials wird entsprechend die Art des Einflusses auf die verschiedenen Bereiche des Lernens betrachtet.

\subsection{Aufbau der Arbeit}

Im folgenden Kapitel werden die theoretischen Grundlagen von beruflichem Lernen und Handeln, die für die Konzeption und Durchführung der FortbildungsMaßnahme von Bedeutung sind, dargestellt. Um einen Lernerfolg messen zu können, muss das Wissen der Teilnehmenden erhoben werden. Dazu werden kognitionspsychologische Modelle der Repräsentation von Wissen herangezogen. Zwei Ansätze, die dafür besonders geeignet erscheinen, werden dargestellt. Neben den Kognitionen sind aber auch die Emotion und Motivation der Teilnehmenden im Unterricht von Bedeutung. Die dargestellten Ansätze berücksichtigen diese jedoch nur unzureichend, so dass zur Erklärung des Zusammenhangs zwischen Kognition, Emotion und Motivation ein umfassenderes Modell herangezogen wird.

44 Systematische Evaluations-Studien zum Einsatz von Unternehmensplanspielen sind bisher eher von zweitrangigem Interesse; vgl. Geilhardt \& Mühlbradt (Konzepte 1995), S. 14. 
Nachfolgend wird der wirtschaftspädagogische Hintergrund der Arbeit dargestellt. Auf Grund der Veränderungen im Berufsleben werden Schlüsselqualifikationen als Lernziele beruflicher Bildung gefordert, die im Konzept der Handlungsorientierung in entsprechende Unterrichts-Konzepte umgesetzt werden. Dabei wird auch auf die Planung der Lernziele eingegangen. Die allgemeinen Kriterien von komplexen Lehr-Lern-Arrangements, die zur Überwindung der Defizite traditionellen Unterrichts und zur Vermittlung übergreifender Qualifikationen entwickelt werden, werden nach einem Exkurs zur Klärung des Begriffs der Komplexität, dargestellt.

Das dritte Kapitel beschäftigt sich mit dem Einsatz von Unternehmensplanspielen in der beruflichen Aus- und Weiterbildung. Nach einer Begriffsabgrenzung wird eine Klassifikation von Planspielen vorgenommen. Nachfolgend wird auf die Planung im Unternehmensplanspiel eingegangen, wobei auf Grundlagen der Betriebswirtschaftslehre zurückgegriffen wird. Ein weiterer Abschnitt beschäftigt sich mit den Lernzielen, die mit dem Einsatz eines Unternehmensplanspiels verfolgt werden können. Dabei wird die Handlung der Teilnehmenden näher betrachtet. Abschließend wird auf Forschungsergebnisse zum Einsatz von Unternehmensplanspielen in der beruflichen Aus- und Weiterbildung eingegangen.

In den folgenden drei Kapiteln wird die empirische Erhebung dargestellt. Dabei wird im vierten Kapitel zunächst auf die Konzeption und die Evaluation der Maf3nahme eingegangen. Zur angemessenen Gestaltung der Fortbildungs-Maßnahme werden die Rahmenbedingungen analysiert und die Lernziele präzisiert. Nachfolgend werden methodologische Überlegungen zur Evaluation und zum PlanspielEinsatz angestellt. Da der Lernerfolg des Planspiel-Einsatzes auch gemessen und beurteilt werden soll, werden verschiedene Aspekte der Leistungsbeurteilung näher betrachtet. Die zu konstruierenden Erhebungsinstrumente müssen den klassischen Gütekriterien der Objektivität, der Reliabilität und der Validität sowie weiteren Kriterien genügen, damit die gewonnenen Daten eine Aussagekraft haben. Der nachfolgende Abschnitt beschäftigt sich mit der Umsetzung der bisherigen Überlegungen in ein konkretes Konzept. Dabei wird auf die Gestaltung der Evaluation und des Planspiel-Einsatzes eingegangen. Abschließend wird der Versuchsplan sowie der Ablauf der Erhebung dargestellt. Es ist ein mehrfaktorielles, vollständig gekreuztes Design vorgesehen, wobei die Komplexität der Anforderungs-Situation im Planspiel und die Maßnahmen zur Förderung der 
Lerneffizienz jeweils in zwei Formen variiert werden. Es werden Hypothesen aufgestellt, die bei der Auswertung der Ergebnisse überprüft werden. Darüber hinaus werden jedoch auch Hypothesen generiert, die neue Erkenntnisse zum Einsatz von Unternehmensplanspielen in der beruflichen Weiterbildung berufstätiger Erwachsener bringen sollen. ${ }^{45}$

Das fünfte Kapitel beschäftigt sich mit der Konstruktion der Erhebungsinstrumente, die im Prä- und Posttest eingesetzt wurden. Dabei wird jeweils auf die inhaltliche Gestaltung, die Operationalisierung der Lernziele sowie die Gütekriterien der Tests eingegangen. Zum Einsatz kam ein Instrumentarium zur Erfassung unterschiedlicher Aspekte des Unterrichts. Mit einem Fragebogen wurde das betriebswirtschaftliche Faktenwissen vor und nach dem Unterricht erhoben. Zur Erhebung des betriebswirtschaftlichen Zusammenhangswissens - ebenfalls im Präund Posttest - diente die Netzwerk-Technik, die auf der Basis der Heidelberger Struktur-Lege-Technik entwickelt wurde. ${ }^{46}$ Weiterhin ist eine Betrachtung von Effekten des Lernens über den Bereich des Qualifizierungsprozesses hinaus von Bedeutung. ${ }^{47}$ Entsprechend wurde zur Messung der Transferleistung sowie des methodischen Arbeitens eine betriebswirtschaftliche Fallstudie konstruiert und eingesetzt.

Im sechsten Kapitel erfolgt eine Beschreibung der Planspiel-Phase und der eingesetzten Erhebungsinstrumente. Zunächst wird das eingesetzte Planspiel dargestellt und nachfolgend auf die experimentelle Phase sowie die Testphase eingegangen. Bei der Betrachtung der experimentellen Phase wird neben der inhaltlichen Gestaltung auch die Operationalisierung der mit dem Planspiel-Einsatz verfolgten Lernziele beschrieben. Des Weiteren wird die inhaltliche Konstruktion der Testphase, in der das Unternehmensplanspiel als Erhebungsinstrument eingesetzt wird, sowie die Bewertung des Lernerfolgs erörtert. Sie wird ebenfalls hinsichtlich der Gütekriterien beurteilt. Nachfolgend wird auf die zusätzlichen Maßnahmen zur Förderung der Lerneffizienz eingegangen.

Die zur Auswertung der erhobenen Daten herangezogenen statistischen Methoden werden im siebten Kapitel beschrieben. Im achten Kapitel werden die Ergebnisse der Planspiel-Phase dargestellt, wobei zunächst eine optimale Entscheidung auf

$45 \mathrm{Vgl}$. Joint Committee \& Sanders (Handbuch 1999), S. 61-65.

$46 \mathrm{Vgl}$. ScheEle (Struktur-Lege-Verfahren 1992).

$47 \mathrm{Vgl}$. ARnold (Schlüsselqualifikationen 1996). 
Grund der in der Testphase präsentierten Ausgangslage des Planspiels entwickelt wird. Sie dient als Grundlage für die Beurteilung der Lernerfolge der Teilnehmenden. Im neunten Kapitel wird auf die Ergebnisse der Instrumente des Prä-PostDesigns eingegangen. Im abschließenden zehnten Kapitel werden die empirischen Ergebnisse interpretiert, das eingesetzte Instrumentarium bewertet und ein Ausblick auf zukünftige Forschungen zum Einsatz von Unternehmensplanspielen in der kaufmännischen Weiterbildung vorgenommen. 


\section{Berufliches Lernen und Handeln}

\section{$2.1 \quad$ Vorbemerkungen}

Dieses Kapitel beschäftigt sich mit den theoretischen Grundlagen beruflichen Lernens und Handelns, die für die Konzeption und Evaluation einer Maßnahme der beruflichen Weiterbildung von Bedeutung sind. Dabei wird zunächst auf ver-

schiedene Arten von Wissensrepräsentationen eingegangen und nachfolgend zwei Ansätze der Informationsverarbeitung dargestellt, gegeneinander abgegrenzt und kritisch betrachtet, um bei der Planung des Unterrichts auf die unterschiedlichen Wissenformate zurückgreifen zu können (Abschnitt 2.2).

Solche eher an der Arbeitsweise eines Computers angelehnten Modelle der Informationsverarbeitung eröffnen zwar die Möglichkeit, Einblicke in die kognitiven Prozesse eines Menschen zu gewinnen. Zur Beschreibung von komplexeren Denkprozessen, wie sie in Unternehmensplanspielen erforderlich sind, greifen sie jedoch angesichts der fehlenden Berücksichtigung von Umwelteinflüssen sowie von Emotion und Motivation zu kurz. Als logische Konsequenz wird der Mensch im epistemologischen Subjektmodell umfassend als handelndes Subjekt mit den Merkmalen der Intentionalität, Reflexivität, potentiellen Rationalität und sprachlichen Kommunikationsfähigkeit beschrieben (Abschnitt 2.3). Nachfolgend wird auf die Subjektiven Theorien von Menschen eingegangen, die auch zur Erklärung seiner Handlungen herangezogenen werden können. Der Abschnitt wird fortgesetzt mit der Beschreibung eines auf dem Forschungsprogramm 'Subjektive Theorien' basierenden Modells über den Zusammenhang von Kognition, Emotion und Motivation, das als Ausgangspunkt dieser Arbeit dient. 
Aufbauend auf diesen Überlegungen werden die wirtschaftspädagogischen Grundlagen beruflichen Handelns und Lernens betrachtet (Abschnitt 2.4). Auf Grund der veränderten Anforderungen im Berufsleben werden Schlüsselqualifikationen als Lernziele der beruflichen Bildung gefordert. ${ }^{1}$ Zur Vermittlung von Schlüsselqualifikationen sind außerdem Metakognitionen von Bedeutung, da ohne das Bewusstsein über den eigenen Lernprozess der Lernerfolg nicht gewährleistet ist. Dabei liegt der eigentliche Wissenserwerb, also der Aufbau der kognitiven Struktur, jenseits des Bewusstseins der Lernenden. Im Bereich der Berufs- und Wirtschaftspädagogik hat das Konzept der Handlungsorientierung die Forschung in den letzten Jahren maßggeblich beeinflusst. Nachfolgend werden die Grundsätze eines handlungsorientierten Unterrichts sowie die Definition des Lernhandelns dargestellt. Lernziele müssen genaustens geplant werden, wobei sie zunächst operationalisiert und dann mit Hilfe einer Taxonomie dimensioniert und hierarchisiert werden.

Um die Defizite traditionellen Unterrichts zu überwinden und Lernziele wie Schlüsselqualifikationen unter Berücksichtigung des Einflusses von Emotion und Motivation auf die Lernprozesse verfolgen zu können, werden im Rahmen der Handlungsorientierung komplexe Lehr-Lern-Arrangements konstruiert (Abschnitt 2.5). In einem Exkurs wird der Begriff der Komplexität näher betrachtet.

Abschließend wird auf das Lernen im und am Modell als zentrale Eigenschaft komplexer Lehr-Lern-Arrangements eingegangen.

\subsection{Modelle der kognitiven Struktur}

\subsubsection{Arten von Wissensrepräsentationen}

Im Bereich der Kognitionswissenschaft haben die Theorien über die Art der mentalen Repräsentation von Wissen eine zentrale Bedeutung, die jedoch nicht ausschließlich neuronal, sondern auch sozio-kulturell zu betrachten ist. ${ }^{2}$ Im Paradigma des Informationsverarbeitungsansatzes werden Kognitionen betrachtet, ${ }^{3}$ die

\footnotetext{
1 Vgl. Kapitel 1.

2 Vgl. Gardner (Denken 1989), S. 399-401.

3 Vgl. Groeben et al. (Forschungsprogramm 1988), S. 17.
} 
vergleichweise einfache Phänomene beinhalten wie Begriffe oder Konzepte sowie deren mehrstelligen Beziehungen untereinander. ${ }^{4}$ Grundsätzlich wird davon ausgegangen, dass semantisches Wissen in einer bestimmten Form organisiert ist bzw. strukturiert vorliegt. ${ }^{5}$ Dabei muss jedoch eine Begriffsabgrenzung vorgenommen werden (Abbildung 2.1): ${ }^{6}$ Die Realität wird in der kognitiven Struktur abgebildet. Diese internen Repräsentationen von Objekten der Anschauung, der Vorstellung und des Denkens umfassen Perzepte, Konzepte und Merkmale sowie ihre gewichteten Beziehungen untereinander. Es kann davon ausgegangen werden, dass die Ausbildung dieser Strukturen individuell unterschiedlich geschieht und in Interaktion mit der aktuellen Situation steht. Kognitive Strukturen sind dem Forschenden jedoch nicht direkt zugänglich und können nicht einfach erfasst oder erhoben werden. Für einen empirischen Zugang muss dieses innere Modell der Wirklichkeit wiederum abgebildet werden und durch geeignete Instrumente möglichst verlustfrei erhoben werden. Das Ergebnis dieses Vorgangs kann als kognitive Strukturierung bezeichnet werden. Dieses äußere Modell kann weiterhin mit einer formalen Struktur verglichen werden und es können Hypothesen über die kognitive Strukturierungen untersucht werden.

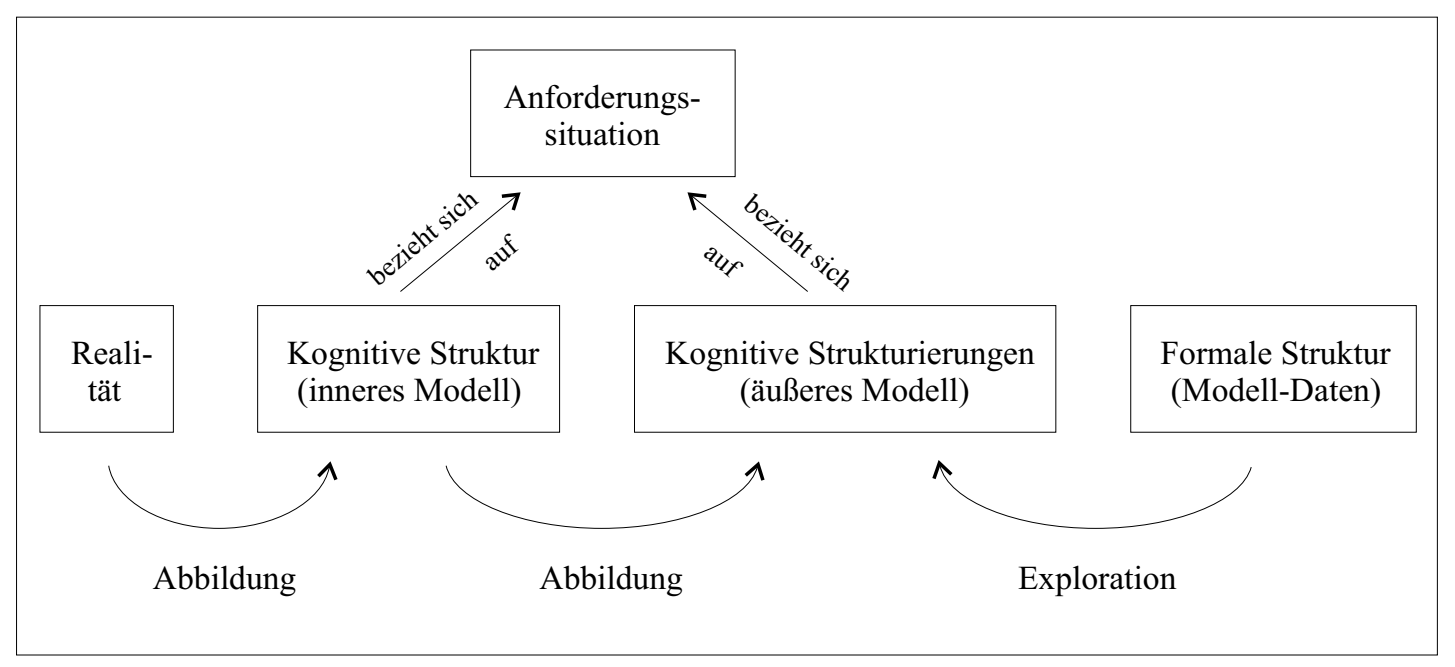

Abbildung 2.1: Zusammenhang zwischen den Begriffen 'Kognitive Struktur' und 'Kognitive Strukturierungen'

\footnotetext{
4 Vgl. Oldenbürger (Erhebung 1986), S. 19.

Vgl. Tergan (Modelle 1986), S. 4.

Vgl. Oldenbürger (Erhebung 1986), S. 19-29.

In Anlehnung an Oldenbürger (Erhebung 1986), S. 27.
} 
Dieser Interpretation liegen verschiedene Theorien zu Grunde: ${ }^{8}$ Grundsätzlich beziehen sich die Vorgänge auf eine konkrete Anforderungssituation. Hinter dem inneren Modell liegt ein psychologisches Modell über die inhaltlichen und formalen Aspekte der individuellen Wissensrepräsentation, die im Kontext des jeweiligen Grundansatzes entwickelt werden. Aber auch die Annahmen über die kognitiven Strukturierungen basieren auf einer entsprechenden Theorie. Dabei können die Modelle nach einer analogen, propositionalen bzw. regelbasierten Wissensrepräsentation unterschieden werden. ${ }^{9}$ Eine andere Klassifikation teilt sie in analoge Repräsentationen, semantische Raum-Modelle und Produktionssysteme ein. ${ }^{10}$ Diese drei Grundtypen von Repräsentationssystemen werden auch als 'klassische' Modelle bezeichnet. ${ }^{11}$ Davon abzugrenzen sind die so genannten 'konnektionistischen' Modelle, die sich vorrangig auf elementare Wahrnehmungsphänomene beziehen, wobei vor allem weitgehend unbewusst verlaufende kognitive Operationen wie die Buchstaben- und Worterkennung betrachtet werden. ${ }^{12}$

Zu den analogen Repräsentationsmodellen, ${ }^{13}$ gehören die so genannten Mentalen Modelle. ${ }^{14}$ Sie sind hierarchisch aufgebaute Wissensnetze und bilden die Realität ab, ohne dabei jedoch Emotionen und Motivationen einzubeziehen. ${ }^{15}$

Zu den propositionalen Modellen zählen u. a. die Netzwerkansätze: ${ }^{16}$ Nach diesem Modell werden Informationen als Propositionen im Gedächtnis gespeichert. Propositionen bestehen aus Argumenten, die Konstante oder Variable sein können, die durch Prädikate verbunden werden. Argumente können über die Verknüpfungen 'und' sowie 'oder' verbunden werden. Da nicht alle Informationen auf diese einfache Art und Weise gespeichert werden können, werden zusätzlich Quantoren eingeführt, die die Formulierung von All- und Existenz-Aussagen ermöglichen. Weiterhin gibt es Funktionen, die auf ein Argument angewendet werden und als Ergebnis wiederum ein Element liefern, das verknüpft werden kann. Die ent-

\footnotetext{
8 Vgl. Tergan (Grundlagen 1989a) , S. 159-161.

9 Vgl. Opwis \& LüEr. (Modelle 1994).

10 Vgl. Tergan (Modelle 1986), S.9.

11 Vgl. Tergan (Grundlagen 1989a), S. 160-163.

12 Vgl. MeClelland, Rumelhart \& PDP Research Group (Explorations 1988) und McClelland, Rumelhart \& PDP Research Group (Explorations 1987).

13 Vgl. Mandl \& Spada (Wissenspsychologie 1988), S. 99-119.

14 Ausführliche Darstellung z. B. bei Fürstenau (Problemlösen 1994), S. 38-50.

15 Vgl. Fuhrer (Mehrfachhandeln 1985), S. 121-136; Minsky (Mentopolis 1990), S. 300-308.

16 Vgl. z. B. Rumelhart \& Norman (Netz 1978) und Palm (Modellvorstellungen 1988).
} 
standenen Propositionen sind untereinander über die verschiedenen Argumente vernetzt und bilden das individuelle Wissen in einem Netzwerk ab. In der Literatur wird die Darstellung in Netzwerk-Form teilweise mit einer Listendarstellung gleichgesetzt. ${ }^{17}$ Bei Relationen, die mehr als zweistellig sind, treten in der Listenform jedoch Informationsverluste auf.

Für die regelbasierte Wissensrepräsentation werden zwei Ansätze dargestellt, die grundlegend für weitere Überlegungen sind: Der Ansatz von DörnER 1976/1987 kommt aus dem Bereich der Problemlöseforschung. Er unterscheidet zwischen epistemischem und heuristischem Wissen und mündet in psychologischintegrative Modellvorstellungen über die Repräsentation von semantischem Wissen. Der Ansatz von Anderson 1983/1988 geht von einem Computermodell des Gehirns aus und grenzt deklaratives gegenüber prozeduralem Wissen ab. Abildung 2.2 zeigt die Begriffsabgrenzung zwischen beiden Ansätzen sowie die Beziehungen zwischen den Begriffen.

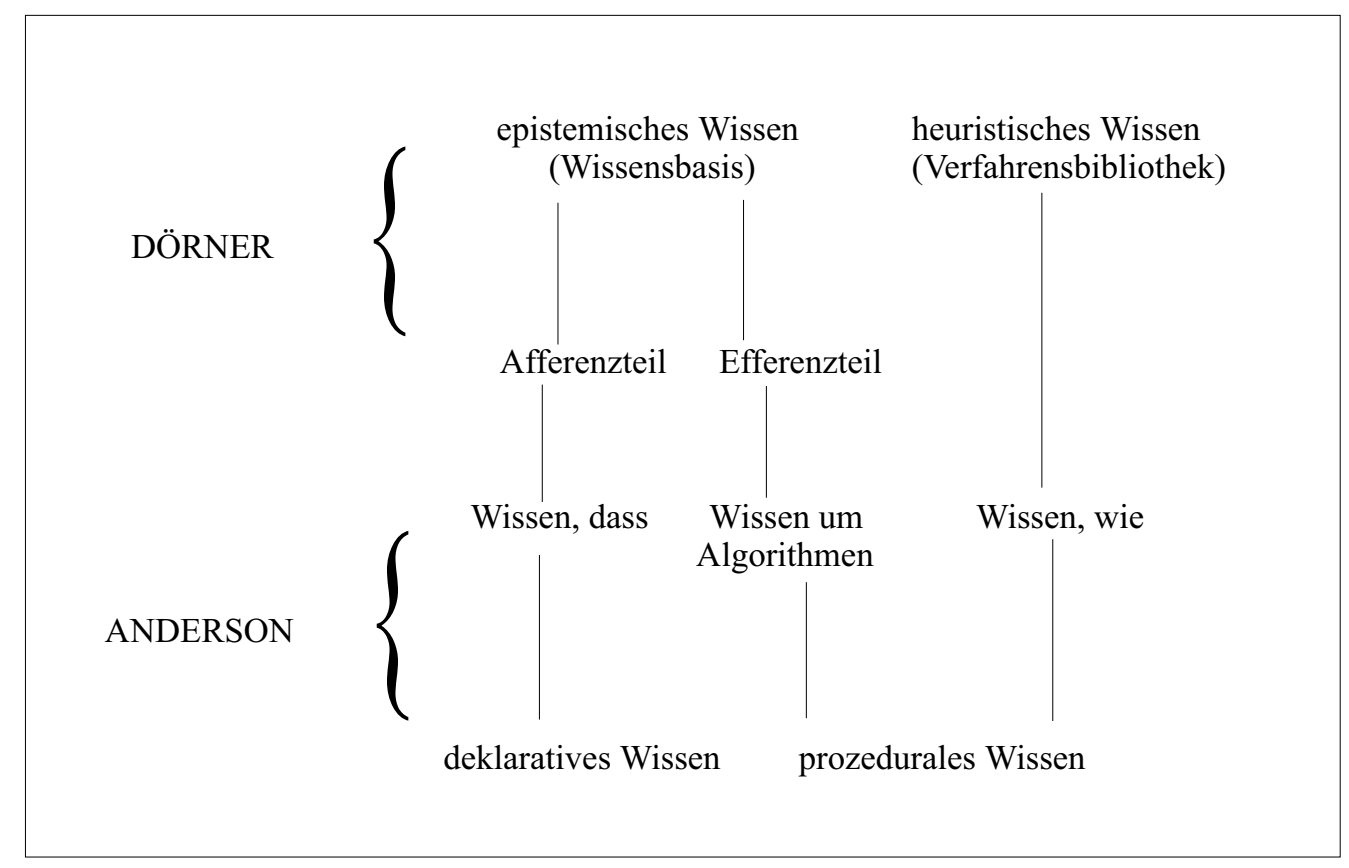

Abbildung 2.2: Arten von Wissen ${ }^{18}$

\footnotetext{
17 Vgl. Tergan (Modelle 1986), S. 37; auch Bonato (Wissensstrukturierung 1990).

18 In Anlehnung an SEmBiLl (Problemlösefähigkeit 1992), S. 86.
} 
Beide Modelle teilen die interne Wissensrepräsentation, also die kognitive Struktur, in zwei Bereiche auf, wobei zwischen einem grundlegenden Wissensbereich und einem darauf aufbauenden Wissenbereich unterschieden wird. An der Lösung von Probleme sind beide Bereiche beteiligt. Ein Problem wird dabei als Situation beschrieben, die beim Übergang von einem (unerwünschten) Ist-Zustand in einen (erwünschten) Zielzustand auftritt und in der die zur Erreichung des Ziels erforderlichen Handlungen gar nicht oder zumindest zum Teil nicht bekannt sind. ${ }^{19}$ Je nach Bekanntheitsgrad der auszuführenden Operationen bzw. der Kriterien des Ist-/Soll-Zustands können Probleme wie folgt unterschieden werden: ${ }^{20}$

Analytische Probleme liegen vor, wenn das Problem geschlossen definiert ist, d.h. sowohl die Ist-/Soll-Kriterien bekannt sind als auch die Operatoren. Diese Probleme treten vorwiegend in der mathematischen Beweisführung auf.

Synthetische Probleme sind durch einen geringen Bekanntheitsgrad der Operationen gekennzeichnet, jedoch sind die Ist-/Soll-Kriterien bekannt. Solche Probleme treten z. B. in Form von Textaufgaben auf, in denen Methoden des Fachgebiets angewendet werden müssen.

Dialektische Probleme weisen einen geringen Bekanntheitsgrad der Kriterien des Ist- sowie des Soll-Zustands auf, hier sind jedoch die durchzuführenden Operationen bekannt.

Dialektische, synthetische Probleme sind durch einen geringen Bekanntheitsgrad sowohl der Ist-/Soll-Kriterien als auch der Operationen gekennzeichnet. Sie kommen häufig im Berufsleben vor und der Umgang mit ihnen fällt auf Grund der mangelnden Informationen besonders schwer.

\subsubsection{Zwei Ansätze der Wissensrepräsentation}

\section{Der Ansatz von DöRNER}

In dem Ansatz von DörNER wird das semantische Wissen nach epistemischem und heuristischem Wissen unterschieden. Ersteres wird weiterhin in einen

\footnotetext{
19 Genauer bei Funke (Problemlösen 1986) und die dort angegebene Literatur.

20 Vgl. SELL (Problemlösungsverhalten 1989), S. 17-18.
} 
Afferenz- und einen Efferenzteil unterteilt. Dabei bezeichnet der Afferenzteil das Gedächtnis für Sachverhalte, der Efferenzteil das Gedächtnis für Handlungen. Zur Lösung von einfachen Aufgaben genügt das epistemische Wissen, da gemäß der Definition im Efferenzteil der Wissensbasis das Wissen um Algorithmen bereits enthalten ist. Zur Bewältigung von Problemen ist jedoch heuristisches Wissen erforderlich, da nicht alle Handlungen zur Zielerreichung bekannt sind und erst erworben werden müssen ${ }^{21}$ wobei das epistemische Wissen in diese Überlegungen einbezogen wird. Der Vorgang folgt dem TOTE-Schema, das in Abbildung 2.3 dargestellt ist.

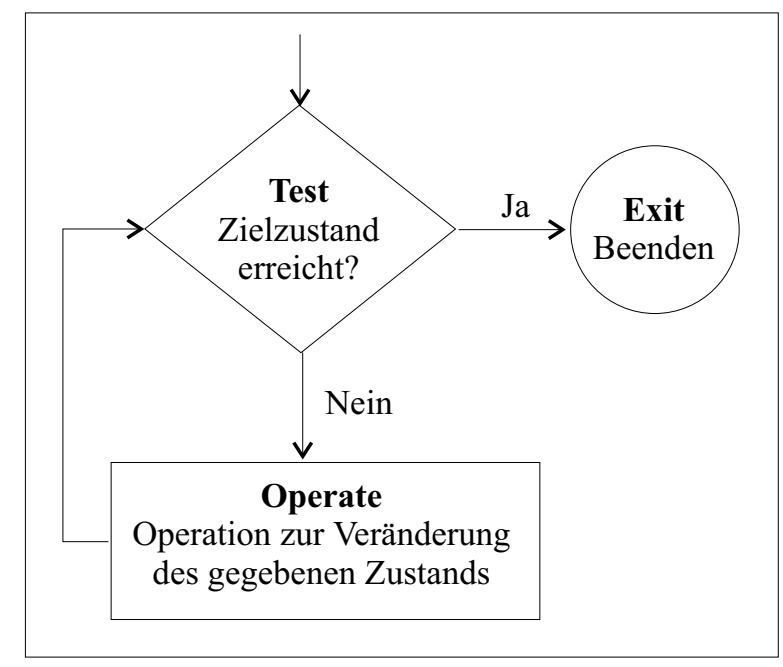

Abbildung 2.3: TOTE-Schema $a^{22}$

Die bei der Problemlösung erforderlichen, aber noch unbekannten Handlungen werden in einem bestimmten Problemraum gesucht, der aus relevanten WissensZuständen, Situationen und Sachverhalten der Realität besteht. ${ }^{23}$ Dieses Absuchen geschieht zum einen durch eine Suchraum-Einengung, deren Kernstück die Ziel-Mittel-Analyse des General Problem Solver ${ }^{24}$ ist. Zum anderen wird bei der Suchraum-Erweiterung durch ein systematisches Trial-and-Error-Vorgehen eine unter Umständen langwierige Handlung anhand von Ausschluss-Kriterien

21 Vgl. Dörner (Problemlösen 1987), S. 27.

22 In Anlehnung an Dörner (Problemlösen 1987), S.40 nach Miller, Galanter \& PriBRAm (Strategie 1973), S. 34.

23 Vgl. Eisenstadt \& Kareev (Aspekte 1978).

24 Vgl. Newell \& Simon (Problem Solving 1972). Er basiert auf dem Ansatz des Prädikatenkalküls; vgl. MandL \& SPADA (Wissenspsychologie 1988), S. 57. 
verkürzt. Dieses geschieht durch drei rekursiv miteinander verknüpfte Methoden: ${ }^{25}$

1. In der Transformation wird eine Unterschiedsanalyse zwischen Start- und Zielpunkt durchgeführt.

2. Die Reduktion ist eine Operatorsuche zur Beseitigung des gefundenen Unterschieds.

3. In der Operator-Anwendung wird der Operator auf Anwendbarkeit geprüft und und bei positivem Ergebnis angewendet.

Allerdings gilt diese Vorgehensweise nur für die Suche innerhalb geschlossener Systeme und ist immer auch situationsgeleitet. ${ }^{26}$

\section{Der Ansatz von ANDERSON}

In der ACT*-Theorie ${ }^{27}$ nach ANDERSON 1983/1988 besteht das deklarative Wissen aus Fakten, die in einem propositionalen Netzwerk repräsentiert sind, sowie zeitlichen Reihen und räumlichen Vorstellungen. Diese Repräsentation wird von ANDERSON als 'mixed' und 'tangled hierarchies' bezeichnet, $d . h$. verschiedene Hierarchien können durch gleiche Knotenpunkte verbunden sein. ${ }^{28}$ Das prozedurale Wissen beinhaltet Wenn-Dann-Sätze, so genannte Produktionen, wobei die Wenn-Teile dieser Sätze die Bedingungen darstellen und im deklarativen Wissen gespeichert sind. Der Dann-Teil stellt bei Erfüllung der Bedingung den Aktionsteil dar.

Das Zusammenwirken der beiden Wissensbereiche ist dabei in dieser Theorie gegenüber der von DöRNER stärker ausgearbeitet: Es wird davon ausgegangen, dass zwischen den beiden Wissensbereichen eine Instanz exisistiert, die als Interpreter bezeichnet wird und das Kontrollwissen beinhaltet. Die Menge der anwendbaren Produktionen wird durch Auswertung des Wenn-Teils einschränkt. Aus dieser Menge werden eine oder mehrere Produktionen ausgewählt und ausgeführt. ${ }^{29}$

25 Vgl. Dörner (Problemlösen 1987), S. 74-76.

26 Vgl. Fuhrer (Mehrfachhandeln 1985), S. 81-83.

27 ACT = Adaptive Control of Thought. Der Stern bezeichnet "the final major reformulation"; Anderson (Architecture 1983), S. 18.

28 Vgl. Anderson (Architecture 1983), S. 78-79.

29 Vgl. Mandl \& Spada (Wissenspsychologie 1988), S. 77. 
Die Ausbildung von prozeduralem Wissen beim Problemlösen geschieht in einem dreiphasigen Wissenserwerb: ${ }^{30}$

1. Im deklarativen Stadium wird Wissen über den Ablauf der Handlungen erworben. Dieses Wissen liegt entweder in Form eines Rezepts vor, das genau festlegt, wie bei der Lösung des Problems vorgegangen werden muss, oder in Form einer Analogie, die interpretativ angewendet wird. ${ }^{31}$

2. In der Phase der Wissenskompilation wird deklaratives Wissen in prozedurales Wissen überführt. In dieser Phase laufen zwei Prozesse parallel ab: ${ }^{32}$ Während bei der Komposition aus einer festen Folge von Produktionen mit gleicher Zielsetzung eine Makro-Produktion gebildet wird, bewirkt die Prozeduralisation die Speicherung des notwendigen deklarativen Wissens als Teil der zugehörigen Produktionen, so dass es nicht in einem zusätzlichen Vorgang abgerufen werden muss.

3. Die Phase der Wissensoptimierung ${ }^{33}$ besteht aus drei Vorgängen: ${ }^{34}$ Die Generalisation strebt eine Anwendung der Produktionen in anderen Bereichen an. Die Diskrimination versucht umgekehrt, allgemeine Produktionen auf einen Anwendungsbereich einzuschränken. Die Verstärkung dient als Korrektur-Instanz. Im letzten Prozess werden die Produktionen hinsichtlich ihrer erfolgreichen Anwendung bewertet und entweder eliminiert oder verstärkt.

Hierzu ist grundsätzlich kritisch anzumerken, dass auf die Bedeutung der im deklarativen Bereich gespeicherten zeitlichen Folgen oder räumlichen Vorstellungen in der Phase der Prozeduralisierung nicht mehr explizit eingegangen wird. ${ }^{35}$

30 Vgl. Anderson (Psychologie 1988), S. 219-220.

31 Vgl. Anderson (Architecture 1983), S. 217.

32 Vgl. Anderson (Architecture 1983), S. 235.

33 In Anlehnung an Rumelhart \& Norman (Accretion 1978) nennt Anderson diese Phase tuning, was ins Deutsche mit 'Einstimmen' übersetzt wurde; vgl. Anderson (Psychologie 1988), S. 220. Der Begriff erscheint in diesem Zusammenhang jedoch irreführend; vgl. SEMBILL (Problemlösefähigkeit 1992), S. 91.

34 Vgl. Anderson (Architecture 1983), S. 398.

35 Vgl. Hesse (Review 1985), S. 234. Zur möglichen Kodierung von Zeitbezügen in begrifflichen Wissensstrukturen vgl. Meer \& Kolbe (Kodierung 1997). 


\section{Grenzen dieser Ansätze}

Beiden vorgestellten Ansätzen liegt die Operatordoktrin zu Grunde, nach der das Denken definiert wird als Verketten einer Menge elementarer Operatoren. Die Theorie über das Absuchen der Problemräume bleibt jedoch hinter den notwendigen Handlungen zur Lösung eines Problems zurück, denn problemlösendes Denken ist mehr als die probeweise Verkettung von Handlungseinheiten. ${ }^{36}$ Das Problem der Intentionalität einer Handlung, die sie erst in einen Gesamtzusammenhang einordnet und beurteilbar macht, ${ }^{37}$ wird in der dargestellten ACT*-Theorie durch einen höheren Auflösungsgrad einzelner Kognitionen umgangen. Somit bleibt das Problem der Intentionalität weiterhin ein Problem für Erklärungsmuster der Alltagspsychologie. ${ }^{38}$

Auch gehen beide Modelle von einem genau definierten Zustand aus, von dem aus das Lernen startet. Diese Annahme ist jedoch nicht haltbar, da die kognitiven Strukturen verschiedener Lernenden nicht als homogen angenommen werden können. Es wird auch nicht darauf eingegangen, wie dieser Zustand überhaupt erreicht wird, d.h. wie die Wissensbasis, die für die Vorgänge beim Problemlösen heranzuziehen ist, aufgebaut wird. Insgesamt beziehen sich diese Termini zwar schon auf größere Aggregate einzelner Konzepte oder Kognitionen, ihre Struktur bleibt allerdings offen. Zur Erklärung von Lernen greifen sie zu kurz, da Handlungswissen bezüglich Maßnahmen oder auch Wissen, wie Wissen generiert wird, nicht in die Systematik einbezogen wird. ${ }^{39}$

Weiterhin beziehen sich beide Modelle ausschließlich auf die Kognitionen und vernachlässigen die Emotionen und Motivationen, die beim Lernen jedoch eine Rolle spielen. ${ }^{40}$ Im folgenden Abschnitt wird ein umfassenderes Modell über den Zusammenhang zwischen Kognition, Emotion und Motivation dargestellt, deren Annahmen als Grundlage für die Konzeption des Unterrichts in der vorliegenden Arbeit dient.

\footnotetext{
36 Vgl. Dörner (Prozesse 1983).

37 Vgl. Abschnitt 2.3.1.

38 Vgl. Gerjets \& Westermann (Theorien 1997).

39 Vgl. Groeben et al. (Forschungsprogramm 1988), S. 18.

40 Vgl. Abschnitt 2.3; auch Dörner (Prozesse 1983), der diese Kritikpunkte anbringt, ohne jedoch näher auf sie einzugehen.
} 


\subsection{Zusammenhang zwischen Kognition, Emo- tion und Motivation}

\subsubsection{Grundlegende Überlegungen}

\section{Epistemologisches Subjektmodell}

Durch die eher an der Informationsverarbeitung orientierten Ansätze wurden zwar genauere Einblicke in die kognitiven Prozesse gewonnen. Zur Erklärung von komplexeren Denkprozessen wie der Lösung von Problemen sind sie jedoch eher ungeeignet, weil sie die Aspekte der Irrationalität und Subjektivität unberücksichtigt lassen. Bei der Konstruktion dieser Modelle bleiben die Umwelteinflüsse, affektive Aspekte des Erlebens sowie Auswirkungen kultureller und historischer Faktoren ausgegrenzt. Dies führt zu einer verzerrten Sicht des Menschen, der im Gegensatz zum Computer kein mechanisches, sondern ein biologisches System ist. ${ }^{41}$

Die entsprechend konsequente Formulierung eines Menschenbildes, das diese Missstände versucht zu beseitigen, führt zur Definition des epistemologischen Subjektmodells. ${ }^{42}$ Hier wird der Mensch parallel zum Bild des Wissenschaftlers als 'man scientist' Mensch in individuell unterschiedlichen Ausprägungen durch die Merkmale der Intentionalität, Reflexivität, potentiellen Rationalität und sprachlichen Kommunikationsfähigkeit gekennzeichnet. ${ }^{44}$

\section{Definition und Beschreibung von Subjektiven Theorien}

Die Merkmale des epistemologischen Subjektmodells beschreiben nicht nur den Menschen, sondern werden im Rahmen des Forschungsprogramms 'Subjektive Theorien' ${ }^{\prime 5}$ (wertend) als positive Zieldimensionen konstruktiver Entwick-

41 Vgl. Gardner (Denken 1989), S. 401-405.

42 Vgl. Groeben et al. (Forschungsprogramm 1988), S.16; ausführlich bei Groeben \& ScheELE (Argumente 1977).

43 Nach Kelly (Psychology 1955).

44 Vgl. z. B. bereits die Ausführungen zur Notwendigkeit in der Pädagogik von der Reflexivität des Menschen auszugehen bei Henz (Lehrbuch 1964), S. 129-134.

45 Vgl. Groeben et al. (Forschungsprogramm 1988). 
lungsmöglichkeit gefordert. Entsprechend muss auch der Forschungs-Gegenstand neu definiert werden. Insbesondere erscheinen die Merkmale der Zielsetzung und der Konstruktivität des Menschen als wichtig. ${ }^{46}$

Dabei sind die Erkenntnis-Interessen durchaus unterschiedlich: Während es im Bereich der objektiven Theorien um die Erklärung von Phänomenen geht, haben Subjekte einen wesentlich breiteres Feld an Bedürfnissen zu befriedigen. ${ }^{47}$ Die vom Menschen gebildeten Theorien werden hier parallel zu den 'objektiven' wissenschaftlichen Theorien als Subjektive Theorien bezeichnet. ${ }^{48}$ Subjektive Theorien sind zeitlich relativ überdauernde mentale Strukturen. ${ }^{49}$ Sie werden definiert als

- "Kognitionen der Selbst- und Weltsicht,

- die im Dialog-Konsens aktualisier- und rekonstruierbar sind

- als komplexes Aggregat mit (zumindest impliziter) Argumentationsstruktur,

- das auch die zu objektiven (wissenschaftlichen) Theorien parallelen Funktionen

- der Erklärung, Prognose, Technologie erfüllt,

- deren Akzeptierbarkeit als 'objektive' Erkenntnis zu prüfen ist" ${ }^{\text {"50 }}$.

Die beiden kursiv gesetzten Merkmale dieser Definition grenzen die enge gegenüber der weiten Variante des Konstrukts ein: ${ }^{51}$ Sie ist zum einen als enger zu bezeichnen, weil sie mehr Merkmale enthält als die weite Definition. Sie ist aber auch aus einem inhaltlichen Grund enger: Stärker als in den bisherigen Forschungsansätzen tritt durch das zweite Merkmal die Kommunikation zwischen Forschenden und Erkenntnis-Subjekten in den Vordergrund und beinhaltet hierdurch ein Innovationspotential für die Psychologie. Das letzte Merkmal bezieht sich auf die Möglichkeit des Vergleichs von Subjektiven Theorien und den objektiven wissenschaftlichen Theorien zu einem Gegenstandsbereich. ${ }^{52}$ Im Bereich der

\footnotetext{
46 Vgl. im Folgenden Groeben et al. (Forschungsprogramm 1988), S. 22-24.

47 Vgl. Hofer, Pekrun \& Zielinski (Psychologie 1986), S. 227.

48 Dabei wird die bei den Autoren des Forschungsprogramms 'Subjektive Theorien' übliche Großschreibung zur Abgrenzung gegenüber anderen Begriffsdefinitionen beibehalten.

49 Vgl. Dann (Subjektive Theorien 1983), S. 80.

50 Groeben et al. (Forschungsprogramm 1988), S. 22.

51 Vgl. Groeben et al. (Forschungsprogramm 1988), S. 22-24.

52 Vgl. Abbildung 2.1.
} 
qualitativen Forschung stellt die Erforschung der Subjektiven Theorien derzeit das am weitesten entwickelte Gebiet dar. ${ }^{53}$

Die Unterschiede der objektiven zu den Subjektiven Theorien sind nicht in jedem Fall eindeutig trennende, sondern eventuell nur akzentuierende. Subjektive Theorien erfüllen wie objektive Theorien vor allem die Funktionen der Beschreibung, der Erklärung sowie der Rechtfertigung. Sie sind also nicht nur als Merkmal einer Person zu verstehen, sondern immer auch in ihrer Funktion in Bezug auf individuelles Handeln, das in einen Kontext eingebunden ist, zu betrachten. Auch hier wird von der Erforschung Subjektiver Theorien ein Fortschritt für die Erklärung von Zielkriterien objektiver Theorien erwartet. Dies gilt insbesondere in solchen Fällen, in denen unrealistische Zielvorstellungen auch für objektive Theorien überprüft werden. ${ }^{54}$

Ein scheinbar bisher ungelöstes Problem in der (psychologischen) Forschung ist die Frage, ob der zu erforschende Gegenstand erst durch die Forschungsmethode konstituiert wird oder ob bereits das Vorverständnis über den Gegenstand den Forschenden in seiner Wahl der Methode einschränkt. ${ }^{55}$ Nimmt man in jedem Fall die gegenseitige Abhängigkeit von Gegenstand und Methodik ernst, dann ergeben sich daraus Konsequenzen für die Art der Erkenntnisgewinnung in der Forschung: ${ }^{56}$ Die Eignung einer Methode zur Erfassung eines bestimmten Forschungs-Gegenstands muss am (theoriegeleiteten) Vorverständnis über den Gegenstand gemessen werden. Dies bedeutet jedoch, dass sich im Gegensatz zum bisher herrschenden Verständnis methodologischer Ziel- und Bewertungskriterien - mit dem Anspruch auf Genauigkeit und Objektivität - die Auswahl und Anwendung der Methoden, die Regeln für die Forschungsprozeduren sowie die Auswertung der Ergebnisse an der Stimmigkeit zu diesem Vorverständnis orientieren sollten. Die herkömmlichen Maßstäbe wissenschaftlicher Tätigkeit werden dabei im Rahmen des Forschungsprogramms 'Subjektive Theorien' diesem Vorverständnis nicht übergeordnet. Im Fall einer Unvereinbarkeit mit den Zieldimensionen des Gegenstands-Vorverständnisses sollten sie entsprechend keinen ausschlaggebenden Einfluss auf die Wahl der Methode haben.

\footnotetext{
$\overline{53}$ Vgl. König (Forschung 1995), S. 12.

54 Vgl. Groeben et al. (Forschungsprogramm 1988), S. 47-55.

55 Vgl. Groeben (Handeln 1986), S. 49-54, ausführlich dazu Gigerenzer (Messung 1981).

56 Vgl. Groeben et al. (Forschungsprogramm 1988), S. 11-12.
} 
Die traditionelle empirische Forschung kann als asymmetrisch-hierarchisch beschrieben werden: Die Teilnehmenden wurden über die Absichten und Hintergründe der Forschung selten umfassend informiert. Auf Grund der MenschenbildAnnahmen des Forschungsprogramms 'Subjektive Theorien' ergibt sich ein verändertes Verhältnis zwischen den Forschenden und den Erkenntnis-Subjekten: ${ }^{57}$ Die Forschenden sollten die Untersuchungs-Bedingungen so gestalten, dass die Fähigkeit des Erkenntnis-Subjekts zur Reflexivität und Rationalität optimale Möglichkeit zur Umsetzung erhält. Dazu gehört auch eine umfassende Aufklärung über den Untersuchungszweck und -ablauf. ${ }^{58}$

\section{Definition Handlung}

Subjektive Theorien können dabei zur Erklärung von Handlungen herangezogen werden. Sie werden vom Menschen entsprechend dem epistemologischen Subjektmodell konstruktiv geplant und als Mittel zur Erreichung von meist selbstgewählten Zielen eingesetzt. Sie folgen Motiven und Interessen und sind daher nur auf der Grundlage eines Erfahrungs- und Wissenssystems denkbar. Menschen bilden Hypothesen, sie entwickeln Konzepte und kognitive Schemata. ${ }^{59}$ Diese internen Prozesse und Strukturen steuern ihr Handeln. ${ }^{60}$

Handlungen können als absichtsvolle und sinnhafte Verhaltensweisen beschrieben werden: ${ }^{61}$ Sie lassen sich entsprechend ihrem Grad an Abstraktion zu einer übergeordneten Handlung zusammenfassen bzw. umgekehrt in Teilhandlungen zerlegen. Diese Teilhandlungen sind jedoch erst umfassend zu verstehen, wenn die Zusammenhänge zu den übergeordneten Handlungen bekannt sind, da diese den Rahmen bilden und die Absicht der Handlung erkennen lassen. Mit dem Ebenenwechsel verändern sich allerdings auch die Intentionen und Bedeutungen der Handlungen. Entsprechend ist die Beschreibung einer Handlung abhängig von der Situation, den Beobachtenden, den Handelnden sowie von dem Sinn, den ihr die handelnden Personen vor dem Hintergrund der Situation zuschreibt. Handlungen

57 Vgl. Groeben et al. (Forschungsprogramm 1988), S. 24-29.

58 Was nicht heißen soll, dass in den meisten bisher durchgeführten Untersuchungen die Teilnehmenden nicht über den Ablauf informiert wurden. Jedoch ist auch die Aufklärung über das Ziel der Forschung erforderlich.

$59 \mathrm{Vgl}$. Groeben (Handlungsperspektive 1981).

60 Vgl. Abschnitt 2.2.

61 Vgl. Groeben et al. (Forschungsprogramm 1988), S. 12-17. 
existieren im Gegensatz zu Verhaltensweisen nicht als solche, sondern immer nur relativ zu Beschreibungen, in die durch unterschiedliche Bezugnahmen interpretative Momente eingehen. ${ }^{62}$

Im Gegensatz zu Verhaltensweisen können Handlungen nicht im physikalischen Sinn der Zeit zerteilt werden, weil sie immer auch mit Ereignissen zusammenhängen, die in der Vergangenheit oder Zukunft liegen. Auch sind die wesentlichen Bestimmungsmerkmale einer Handlung nicht in dem 'äußeren Aspekt des Handelns ${ }^{963}$, also im beobachtbaren Verhalten, zu finden, sondern immer erst in der Zuschreibung von Bedeutungen, dem Konstruieren von Sinn und dem Verfolgen von Absichten. Diese 'Innenaspekte' des Handelns sind entsprechend nur interpretativ zu erschließen bzw. mit entsprechenden Methoden zu rekonstruieren. ${ }^{64}$ Ursprünglich bewusste und geplante Handlungsweisen können durch permanente Wiederholung zu Routinen im Sinne einer Automatisierung werden und damit an bewusster Zugänglichkeit verlieren. ${ }^{65}$

\subsubsection{Darstellung des Modells}

In verschiedenen Ansätzen werden Emotion und Motivation nur eine untergeordnete Rolle im Verhältnis zu Kognition beim Lernen eingeräumt: Bei HEIMAnN, OTTo \& Schulz 1979 sind Emotionen im Bereich der Intentionalität als eine von drei Lernziel-Dimensionen zu finden, Motivation wird als stabiles Merkmal im Rahmen der anthropogenen Voraussetzungen eingeordnet. SPIES \& HESSE 1986 erweiterten die ACT*-Theorie von ANDERSON 1983 durch Emotionen, betrachten diese aber als Phänomene. Bei Hofer, PEkrun \& Zielinski 1986 sind die Motive und Gefühle der Lernenden als Ziele des Unterrichts zu verstehen.

Aufbauend auf dem Forschungsprogramm 'Subjektive Theorien' wurde von Becker, Oldenbürger \& Piehl 1987 durch Erweiterung der Subjektiven Theorien um Emotionen und Motivationen und Einbeziehen des Normen- und Zielbezugs sowie der Validität ein umfassenderes Modell konzipiert. Grundsätzlich wird hier von einer Bewusstseinsfähigkeit des Menschen in Bezug auf Kogni-

\footnotetext{
62 Vgl. Heid (Schlüsselqualifikationen 1995).

63 Vgl. Wright (Erklären 1974), S. 85-91.

64 Vgl. Oldenbürger (Erhebung 1986), S. 25.

65 Vgl. Groeben et al. (Forschungsprogramm 1988), S. 37-41.
} 
tion, Emotion und Motivation ausgegangen. Die drei Begriffe werden wie folgt definiert: ${ }^{66}$

Kognition ist die zusammenfassende Bezeichnung für Inhalte und Prozesse der Wahrnehmungs- und Erkenntsnistätigkeit. ${ }^{67}$

Emotion bezieht sich auf die Qualität des Zustands des psychophysischen Systems und dessen Abbildung im Erleben. ${ }^{68}$

Motivation ist der Prozess der Zielbildung und Regulation menschlicher Aktivitäten. Nach dem Modell von Heckhausen \& Rheinberg 1980 wird Motivation aus einem Zusammenspiel von handlungsbezogenen Erwartungen und Wertigkeiten erklärt (vgl. Abbildung 2.4).

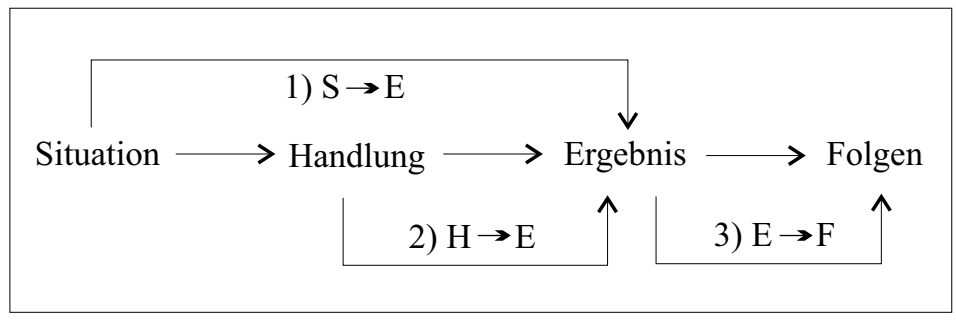

Abbildung 2.4: Erweitertes kognitives Motivationsmodell ${ }^{69}$

1) $S \rightarrow E:$ Situations-Ergebnis-Erwartung

2) $H \rightarrow E:$ Handlungs-Ergebnis-Erwartung

3) $E \rightarrow F:$ Ergebnis-Folge-Erwartung

Motivation entsteht, wenn die folgenden Erwartungen vorhanden sind: ${ }^{70}$

Situations-Ergebnis-Erwartung: Die Umstände der Situation erzeugen ohne eigenes Handeln keinen Erfolg.

Handlungs-Ergebnis-Erwartung: Eigenes Handeln führt zu einem Ergebnis.

Ergebnis-Folge-Erwartung: Erfolg zieht weitere Konsequenzen nach sich, die wichtig und erstrebenswert erscheinen.

66 Vgl. Becker, Oldenbürger \& Piehl (Motivation 1987), S.433-435.

67 Vgl. Abschnitt 2.2.

68 Zur Emotionsgenese vgl. Becker, Oldenbürger \& Piehl (Motivation 1987), S. 436-439 und die dort angegebene Literatur; auch Scheele (Emotionen 1990). An dieser Stelle soll nicht näher darauf eingegangen werden.

69 Quelle: Heckhausen \& Rheinberg (Lernmotivation 1980), S. 16.

70 Vgl. Heckhausen \& Rheinberg (Lernmotivation 1980), S. 19. 
Dabei wird Erfolg hier immer als subjektiv positiv bewertetes Ergebnis definiert. Der Zusammenhang zwischen Kognition, Emotion und Motivation wird von den Autoren als 'System von Systemen' verstanden. Ein System ist eine Menge von Elementen, zwischen denen Beziehungen bestehen. Es hat demnach eine spezifische Struktur. ${ }^{71}$ Die drei Systeme Kognition, Emotion und Motivation setzen sich gegenseitig voraus und bedingen einander. In Systemen, die aus mehr als zwei Elementen bestehen, kommen jedoch zu diesen einfachen, direkten wechselseitigen Wirkungen noch die Wirkungen einer Komponente auf die Wechselwirkung zwischen zwei anderen Elementen hinzu. Diese Moderator-Effekte sind in Abbildung 2.5 durch die drei im Zentrum sich kreuzenden Pfeile dargestellt. Weiterhin sind die Wirkungen zwischen Umgebung, Person und innerpsychischer Welt (bzw. Welten) durch entsprechende Pfeile verdeutlicht. ${ }^{72}$

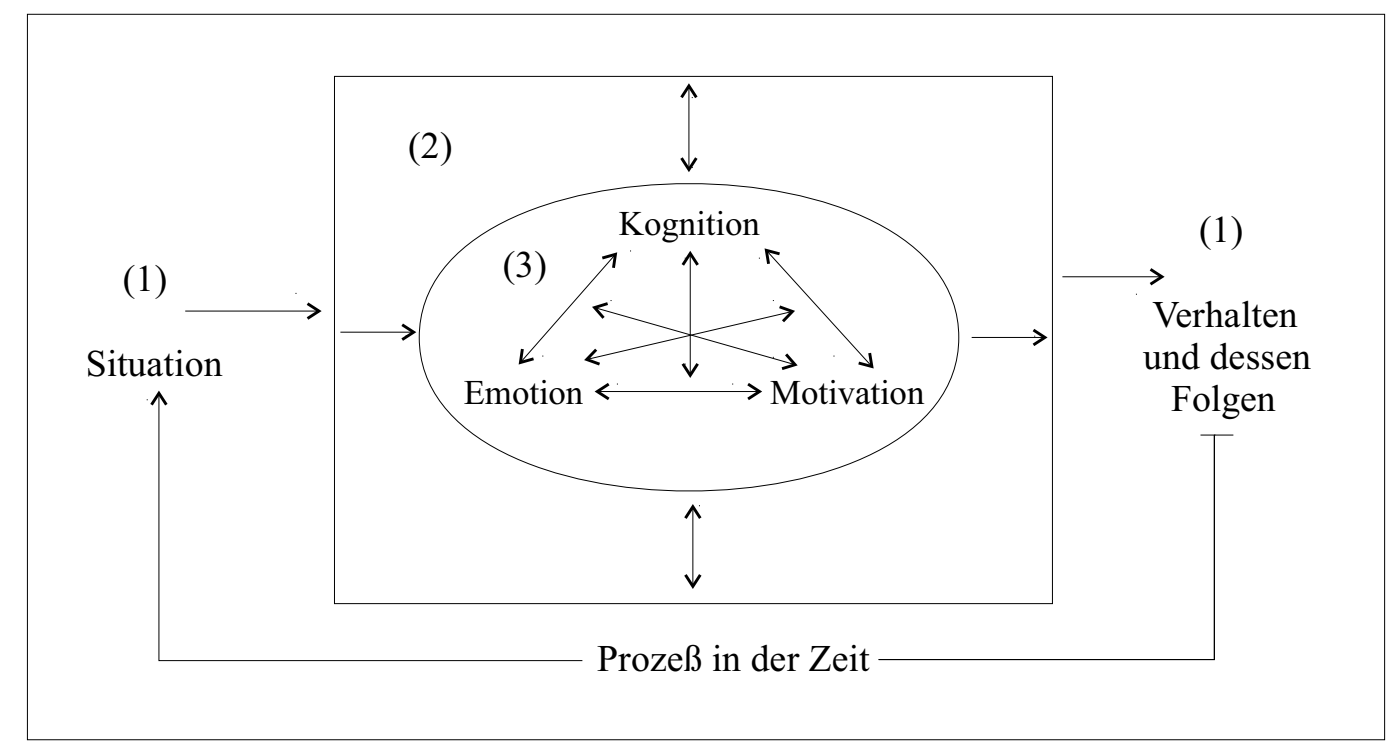

Abbildung 2.5: Struktur des psychophysischen Systems

im Person-Umwelt-Bezug ${ }^{73}$

(1) physikalisch-soziale Außenwelt (Umgebung)

(2) psychophysischer Organismus (Person)

(3) innerpsychische Welt(en)

71 Zum Begriff des Systems vgl. Abschnitt 3.2.1.

72 Vgl. Becker, Oldenbürger \& Piehl (Motivation 1987), S. 433-435.

73 In Anlehnung an Becker, Oldenbürger \& Pienl (Motivation 1987), S. 433. 
In der Abbildung ist der Zusammenhang für die Person-Umwelt-Beziehung dargestellt. Die Komponenten der Situationswahrnehmung, der internen Informationsverarbeitung sowie der Handlungsplanung und -regulation lassen sich jedoch auch jeweils in Form von Prozessen und Teilprozessen darstellen. ${ }^{74}$ Die Ergebnisse eines solchen Prozesses führen nicht nur zu einer Änderung der Systemzustände, sondern schlagen sich auch in Änderungen der internen Struktur der Systeme und Teilsysteme nieder. In den unterschiedlichen, situationsbezogenen und kurzfristigen Prozessen dominieren jeweils bestimmte Teilsysteme, die sich zu verschiedenen Zeitpunkten voneinander unterscheiden können. ${ }^{75}$

Innerhalb dieses Prozesses wirken die aktuellen Sinneseindrücke mit den vorhandenen Strukturen sowie mit Motivation und Emotion wechselseitig aufeinander ein und beeinflussen so die Wahrnehmung. Dadurch erhalten bestimmte Inhalte der Situationswahrnehmung ihren Aufforderungscharakter und ihre Akzentuierung. Andererseits beeinflussen die Inhalte der Wahrnehmung in diesem Prozess die emotionale und motivationale Befindlichkeit. ${ }^{76}$

Dabei ist das Selbstkonzept entscheidend für diese Vorgänge: ${ }^{77}$ Die Situationswahrnehmung wird nicht nur von den momentanen emotionalen und motivationalen Zustände bestimmt, sondern auch durch bisherige Erfahrungen und daraus abgeleiteten Gewohnheiten, Strategien und Stile. Dies gilt auch für die Ausführung von Handlungen. Im Laufe des Lebens hat der Mensch Rollenerwartungen an die eigenen Handlungen erfahren und zum Teil übernommen bzw. selber ausgebildet. Diese beeinflussen die Zielfindung und den Handlungsablauf. Um eine Handlung durchführen zu können, muss also eine Vorstellung über das eigene Handlungsrepertoire und deren Durchführbarkeit existieren.

Erfolgszuversichtlichkeit oder Misserfolgsängstlichkeit sind Eigenschaften, die im Laufe des Lebens als Folge von Erziehungseinwirkungen und anderen Umwelteinflüssen entstanden sind. In der Lernsituation neigen erfolgszuversichtliche Ler-

74 Vgl. z. B. die Darstellung bei Schunck (Subjektive Theorien 1993), S. 24-26.

75 Vgl. Becker, Oldenbürger \& Piehl (Motivation 1987), S. 434.

76 Vgl. auch SEmbill (Problemlösefähigkeit 1992), S. 106.

77 Vgl. Becker, Oldenbürger \& Piehl (Motivation 1987), S. 436; auch Diehl-Becker (Personal-Effectiveness-Trainings 1990). Zur Erhebung von Selbstkonzepten vgl. MuMmENDEY (Fragebogen-Methode 1995). Zur so genannten 'Erlernten Hilflosigkeit' vgl. PETERson, Maier \& Seligman (Helplessness 1993); auch Stiensmeier-Pelster \& SchlanGEN (Hilflosigkeit 1996). 
nende dazu, sich mit anspruchsvolleren Aufgaben zu befassen. Sie sind auch eher bereit, sich aktiv an der Lösung von Aufgaben zu beteiligen. Misserfolgsängstliche Personen neigen demgegenüber dazu, Anforderungen auszuweichen. Für sie ist im Wesentlichen einer eher vermeidendes Verhalten kennzeichnend. Erfolgszuversichtliche Personen pflegen auf gelegentliche Misserfolge mit verstärkter Anstrengung zu reagieren, während Misserfolgsängstliche relativ schnell zu Resignation neigen. ${ }^{78}$ Diese Aspekte müssen entsprechend bei der Gestaltung von Unterricht berücksichtigt werden.

Im Bereich des beruflichen Handelns spielen die Subjektiven Theorien der Arbeitnehmerinnen und Arbeitnehmer eine große Rolle, da sich - wie eben ausgeführt auf ihnen das Handeln begründet. Hier ist neben dem Fachwissen als Wissenbasis die Reflexivität über die Vorgänge sehr wichtig. Um im Beruf erfolgreich zu sein, muss ein Bewusstsein über die eigene Handlungsfähigkeit existieren. Im Folgenden soll sich eingehender mit Lernzielen der betriebswirtschaftlicher beruflicher Bildung beschäftigt werden.

\subsection{Wirtschaftspädagogischer Hintergrund}

\subsubsection{Schlïsselqualifikationen als Lernziele beruflicher Bildung}

Die Debatte um die Schlüsselqualifikationen wird aktuell in drei theoretischen Kontexten geführt: ${ }^{79}$

1. In der Arbeitsmarkt-Theorie geht es um die Formulierung von Qualifikationen, die benötigt werden, um den ständig wechselnden Anforderungen am Arbeitsplatz zu begegnen. ${ }^{80}$

2. Im Rahmen der Kompetenz-Theorie werden Fähigkeiten zur situationsgerechten Erzeugung bzw. Aktualisierung von konkreten Handlungen be-

\footnotetext{
78 Vgl. Schmiel (Förderung 1988), S. 18-20.

79 Vgl. BREYde (Schlüsselqualifikationen 1996).

80 Vgl. Mertens (Schlüsselqualifikationen 1974), der den Begriff geprägt hat.
} 
schrieben. Dadurch wird auch das enge fachliche Verständnis beruflicher Bildung überwunden. ${ }^{81}$

3. Die Bildungs- und Curriculum-Theorie beschäftigt sich mit Zielgrößen beruflicher Bildung und der Darstellung möglicher Konsequenzen. ${ }^{82}$

Der Begriff der Schlüsselqualifikationen wird jedoch unscharf verwendet: ${ }^{83}$ Es mangelt an Inhaltlichkeit und Verbindlichkeit der Schlüsselqualifikationen und es fehlt eine konsistente theoretische Einbettung des Konstrukts. Weiterhin ungeklärt bleibt die Vermittelbarkeit bzw. Lernbarkeit dieser komplexen Fähigkeiten unter verschiedenen Bedingungen beruflicher Bildung sowie ihre angemessene Überprüfung.

Grundlage jeder Schlüsselqualifikation ist das Fachwissen. ${ }^{84}$ Dieses Wissen hat zwei Funktionen: ${ }^{85}$ Zum einen ist es Erschließungswissen für neue InformationsHorizonte, zum anderen ist es Generierungswissen als Basis für Prozesse des Denkens und lebenslangen Lernens.

Schlüsselqualifikationen können nicht losgelöst vom beruflichen Kontext vermittelt werden, da sonst ein Transfer in Situationen des Arbeitslebens nicht gewährleistet wäre. Sie müssen vielmehr mit verschiedenen berufsrelevanten Gegenständen verknüpft und im Berufsfeld angewandt werden. ${ }^{86}$ Dabei müssen diese Fähigkeiten den Handelnden jedoch bewusst sein, weil sie sonst nicht umgesetzt werden. ${ }^{87}$

Das Ziel beruflichen Lernens im Sinne einer handlungsorientierten Konzeption ${ }^{88}$ liegt weitergehend in der Fähigkeit und Bereitschaft der Lernenden, ihre zukünftige Lebenssituation verantwortlich und kompetent zu gestalten. Dies erfordert ein permanentes Abwägen zwischen den eigenen Bedürfnissen und Interessen einerseits und den Ansprüchen des sozialen Umfelds andererseits. Berufliche Bildung

\footnotetext{
81 Vgl. Arnold (Weiterbildung 1994).

82 Vgl. ReEtz (Konzept 1989).

83 Vgl. Laur-ERnst (Schlüsselqualifikationen 1996), S. 19-21.

$84 \mathrm{Vgl}$. auch Abschnitt 2.2.

85 Vgl. DuBs (Schlüsselqualifikationen 1996), S. 51.

86 Vgl. Bunk, Kaiser \& ZedLer (Schlüsselqualifikationen 1991), S. 367.

$87 \mathrm{Vgl}$. Goetze (Schlüsselqualifikationen 1996), S.64-65; auch Abschnitt 2.3.

88 Vgl. Abschnitt 2.4.2.
} 
zielt somit nicht allein auf Kompetenzen, sondern auch auf die Entwicklung der Persönlichkeit als Voraussetzung für verantwortliches Handeln und Entscheiden. ${ }^{89}$

Um diesen Forderungen gerecht $\mathrm{zu}$ werden, ist das Lernen mit Situationen anzureichern, in denen die Lernenden an komplexen Aufgaben selbständig ihren Lernprozess gestalten können. ${ }^{90}$ Die Teilnehmenden bekommen so die Möglichkeit, Fähigkeiten zu erwerben, die eher grundlegend, breit und dauerhaft angelegt sind. Dabei ist die Ganzheitlichkeit der Kompetenzentwicklung im Hinblick auf Qualifizierungsprozesse wichtig. ${ }^{91}$

Allerdings muss klar sein, dass diese Schlüsselqualifikationen auch immer inhaltsspezifisch und an den Kontext gebunden bleiben und nicht universell und unbedingt transferfähig sind. ${ }^{92}$ Trotz empirischer Bestätigung der Bereichsspezifität von Schlüsselqualifikationen ist die Frage der Verallgemeinerung von Fähigkeiten über die Situation des Erwerbs hinaus nicht endgültig wissenschaftlich beantwortet. 93

Schlüsselqualifikationen sind weder hierarchisch geordnet, noch existieren sie unabhängig voneinander, sondern sind als Dimensionen in einem Raum zu begreifen. ${ }^{94}$ Jede Schlüsselqualifikation basiert auf der Person als Träger der Kompetenz. Dabei sind ihre Wahrnehmung, ihre Kognition, ihre Emotion und Motivation bei der Beschreibung der Kompetenz zu berücksichtigen. Schlüsselqualifikationen stellen also nicht direkt beobachtbare Persönlichkeitsmerkmale dar. Als Lernziel beruflicher Bildung ist eine Beschreibung der beobachtbaren Handlung notwendig. Entsprechend sollte für jede Schlüsselqualifikation ein eigenes Handlungsmodell aufgestellt werden. ${ }^{95}$

Zur Vermittlung von Schlüsselqualifikationen sind die Metakognitionen der Teilnehmenden von besonderer Bedeutung, da sie den Lernerfolg maßgeblich beein-

89 Vgl. Tramm (Entwicklungsperspektiven 1991); auch Halfpap (Lernen 1991).

90 Vgl. BECK (Schlüsselqualifikationen 1995), S. 54.

91 Vgl. Achtenhagen (Operationalisierung 1996), S. 107-113; vgl. dazu auch die Ausführungen in Kapitel 1.

92 Vgl. Kaiser (Schlüsselqualifikationen 1992), S. 82.

93 Vgl. Laur-Ernst (Schlüsselqualifikationen 1996), S. 21.

94 Vgl. die Übersichten in ReEtz \& Reitmann (Schlüsselqualifikationen 1990) bzw. Dörig (Konzept 1994).

95 Vgl. dazu bereits Klauer (Methodik 1974), S. 66-69, sowie die Ausführungen in den Abschnitten 2.2 und 2.3 . 
flussen. Nur etwa ein Zehntel des eigenen Lernens wird in seinen Ergebnissen und Wirkungen bewusst wahrgenommen; der Lernprozess als solcher liegt jenseits des Bewusstseins. Wenn aber das Bewusstsein derart beschränkt ist, können fördernde oder behindernde Gewohnheiten nicht durch weiteres Lernen ergänzt oder verändert werden. Diese Vernachlässigung jedoch ist auch verantwortlich für das Problem der Nachhaltigkeit des Gelernten bzw. der Sicherung der Transferfähigkeit. ${ }^{96}$

Der Begriff der Metakognitionen bezeichnet dabei kein präzise definiertes Konstrukt, sondern grob umrissene Konzepte, deren gemeinsame Basis in der Beschreibung von Verhaltensweisen besteht, die zur Bewältigung von Aufgaben und Problemen dienen können: ${ }^{97}$ Metakognitionen werden als Sequenz einzelner Lerntechniken verstanden, die von den Lernenden zur Erreichung eines bestimmten Ziels eingesetzt werden. Sie dienen dazu, den eigenen Lernprozess zu planen, zu steuern und $\mathrm{zu}$ kontrollieren. ${ }^{98}$

Neben den Metakognitionen ist auch das Bewusstsein der Lernenden über die eigenen Gedankengänge sowie der Lernmethoden wichtig, da diese zur Steuerung der kognitiven Prozesse angewendet werden. Die Förderung dieses Bewusstseins muss entsprechend in den Lernziel-Katalog eines Unterrichts aufgenommen werden, damit die eigene Steuerung des Lernens gefördert und die Art des Lernens an die Bedingungen der Lernsituation vorgenommen werden kann. Dass dies von großer Bedeutung ist, zeigen die Forschungen zum 'Lernen Lernen'. ${ }^{99}$ In einer Maßnahme der beruflichen Weiterbildung sollten entsprechend alle Aspekte gleichzeitig angesprochen werden. ${ }^{100}$ Als Konsequenz aus diesen Forderungen ist im Bereich der Berufs- und Wirtschaftspädagogik das Konzept der Handlungsorientierung zu nennen, das im folgenden Abschnitt dargestellt werden soll.

\footnotetext{
96 Vgl. Wesseler (Evaluation 1994), S. 680-683.

97 Vgl. Wild, Schiefele \& Winteler (Verfahren 1992), S. 1.

98 Vgl. auch WiLd \& Schiefele (Fragebogenverfahren 1993), S. 315.

99 Vgl. Weinert (Lernen 1994), S. 186.

100 Zur Klassifikation von Metakognitionen vgl. BAUMERT (Lernstrategien 1993); auch EHRHaRd (Metakognition 1995), S.8-27 sowie Wuttke (Motivation 1999), S. 107-146. Konkrete Hinweise zur Förderung im Unterricht vgl. z. B. Metzig \& Schuster (Lernen 1993).
} 


\subsubsection{Das Konzept der Handlungsorientierung}

Das disziplinäre Themenspektrum der Berufs- und Wirtschaftspädagogik konzentrierte sich zunächst auf didaktisch-methodische und curriculare Fragen des Lernens an berufsbildenden Schulen und auf kultur-philosophisch fundierte normative Theorien beruflicher Bildung. Heutzutage ist diese Disziplin als empirische Sozialwissenschaft einzuordnen und setzt sich verstärkt mit Theorien über die Entwicklung der beruflichen Aus- und Weiterbildung im Kontext ökonomischer Modernisierungsprozesse auseinander. ${ }^{101}$ Im Bereich der Wirtschaftspädagogik beschäftigt sich die Forschung in den letzten Jahren insbesondere mit dem Konzept der Handlungsorientierung. ${ }^{102}$

Im Allgemeinen ist die Ausbildung von Handlungsfähigkeit im Berufsleben Ziel eines auf diesen Grundsätzen basierenden Unterrichts. Handlungsorientierter Unterricht liegt - in negativer Abgrenzung - jedoch noch nicht vor, wenn die Lernenden nur eine praktische Tätigkeit ausführen, ${ }^{103}$ sondern ist durch Ganzheitlichkeit, Aktivität der Lernenden, Orientierung an den Lernprozessen sowie Anstoß zur Reflexion gekennzeichnet. ${ }^{104}$ Im Einzelnen umfasst die Konzeption folgende grundlegende Ideen: ${ }^{105}$

1. Grundlage der Überlegungen ist das epistemologische Subjektmodell ${ }^{106}$ Weiterhin wird von einem interaktionistisches Verhältnis zwischen Individuum und Umwelt ausgegangen. ${ }^{107}$

2. Handlungen umfassen neben ihrem operativen Moment, d. h. dem beobachtbaren Verhalten, immer auch eine spezifische psychische Struktur. ${ }^{108}$

3. Der Ablauf von Handlungen wird als Regelkreis angenommen: ${ }^{109}$ Auf Grund einer (selbstgestellten) Aufgabe wird zunächst eine Zielsetzung formuliert.

\footnotetext{
101 Vgl. Georg (Perspektiven 1998).

102 Vgl. grundlegend dazu Söltenfuss \& Halfpap (Ausbildung 1987).

103 Vgl. Tramm (Entwicklungsperspektiven 1991), S. 252.

104 Vgl. Beck (Handlungsorientierung 1996), S. 60.

$105 \mathrm{Vgl}$. Achtenhagen et al. (Lernhandeln 1992), S. 31-33; auch PÄTzold (Handlungsorientierung 1992). Zur Abgrenzung verschiedener Konzepte von handlungsorientiertem Unterricht vgl. BECK (Handlungsorientierung 1996), S. 17-52.

106 Vgl. Abschnitt 2.3.1.

107 Vgl. Abschnitt 2.3.

108 Vgl. Abschnitt 2.3.1.

109 Vgl. Abschnitt 2.2.
} 
Nachfolgend wird basierend auf dem grundlegenden Erfahrungs- und Wissenssystem die Handlung geplant. Hierbei werden Alternativen hinsichtlich ihrer Durchführbarkeit sowie Zielerreichung bewertet. Nach der Ausführung der optimalen Handlung wird das Ergebnis kontrolliert und die Zielerreichung beurteilt. Entsprechend wird die vorangegangene Auswahl der optimalen Alternative bewertet und die Handlung bei Erfolg für ähnliche Aufgaben im Gedächtnis gespeichert bzw. bei Misserfolg eliminiert. Grundlage jeder Handlung ist die subjektive Repräsentation eines Handlungsfeldes. Handeln enthält Wissen und setzt Wissen voraus. ${ }^{110}$

4. Lernen wird als Ausweitung und Ausdifferenzierung der individuellen Erkenntnis-, Urteils- und Handlungs-Möglichkeiten definiert. Die Lernenden setzen sich dabei aktiv mit der Umwelt auseinander: ${ }^{111}$ Lernen knüpft zum einen an bestehende Strukturen an, zum anderen ist der Auf- und Ausbau kognitiver Strukturen ein konstruktiver Prozess. ${ }^{112}$ Der Wissenserwerb gliedert sich dabei in zwei Teilprozesse: ${ }^{113}$

- In einer ersten Phase werden Informationen gespeichert. Dies ist der eigentliche Veränderungsprozess, der jedoch nicht (unmittelbar) im Verhalten des Lernenden sichtbar wird.

- In der aktuellen Situation werden die gespeicherten Informationen dann abgerufen bzw. aktiviert und beeinflussen so das beobachtbare Handeln und Verhalten.

5. Handlungsorientierung erfordert immer auch eine Situationsbewertung, eine Schwerpunktbildung innerhalb der Situation sowie das Bilden von Prioritäten.

Lernen unter den Prämissen der Handlungsorientierung ist das Auseinandersetzen mit Problemstellungen, die Handlungen und Denken sowie die Reflexion über Zusammenhänge, Werte und Konsequenzen des Handelns beinhalten. Dabei ist besonders das methodische Prinzip der Anschauung, d. h. eine anschauliche Darstellung der einzelnen Elemente des zu vermittelnden Modells, von Bedeutung. ${ }^{114}$

\footnotetext{
110 Vgl. Abschnitt 2.3.1.

111 Vgl. AEBLi (Grundlagen 1987), S. 20-21.

112 Vgl. Aebli (Denken 1981), S. 128.

113 Vgl. Prenzel \& Schiefele (Konzepte 1993), S. 120-121.

114 Vgl. DuBs (Anfänger-Unterricht 1992), S. 261-262.
} 
Lernen wird zum Lernhandeln. ${ }^{115}$ Dieser Begriff beinhaltet den Auf- und Ausbau der individuellen Handlungs- und Erkenntnissysteme und wird in den formalen Rahmen der Handlungstheorie eingeordnet. ${ }^{116}$

Lernhandeln wird definiert als ein "bewusster, aktiver, relativ selbstgesteuerter und zielgerichteter Prozess des Planens, Entscheidens, Ausführens und Beurteilens in Interaktion mit der Umwelt und der bisherigen eigenen Lernerfahrung zum Aufbau von relativ stabiler beruflicher Handlungskompetenz im fachlichen, sozialen und persönlichen Bereich als notwendige Voraussetzung späterer beruflicher Tätigkeit"117. "Lernhandeln unterscheidet sich von anderen Tätigkeitsarten durch seine finale Ausrichtung am Wissens- und Kompetenzerwerb zum Zwecke der Erweiterung künftiger Orientierungs- und Handlungsmöglichkeiten.“118

Zur Planung einer Bildungsmaßnahme müssen die Lernziele entsprechend genau geplant werden, um dieses Lernhandeln im Unterricht zur Erreichung der Ziele auch umzusetzen. ${ }^{119}$ Zur genaueren Beschreibung von Lernzielen dient eine Taxonomie, bei der die verfolgten Lernziele in eine Systematik eingeordnet werden.

\subsubsection{Planung von Lernzielen in der beruflichen Bildung}

\section{Begriffsdefinition}

Im Folgenden werden die theoretischen Grundlagen der Methoden der Operationalisierung, Dimensionierung und Hierarchisierung, die zur Planung der Lernziele der vorliegenden Arbeit herangezogen werden, dargestellt. ${ }^{120}$

Der Begriff des Lernziels kann wie folgt definiert werden: ${ }^{121}$ Die Elemente der Aufgabenmenge bestehen aus Aufgaben, die durch eindeutige Beschreibung der

115 Vgl. Achtenhagen et al. (Lernhandeln 1992), S. 82-83.

116 Vgl. Kaiser (Lernen 1987), S. 14.

117 Diepold (Modellversuch 1991), S. 19.

118 Tramm (Konzeption 1992), S. 131.

119 Vgl. z. B. ReBmann (Komplexität 1993) zur Diskrepanz zwischen Zielen der Lehrpläne berufsbildender Schulen und der mangelnden Komplexität von Lehrbüchern für den Wirtschaftslehreunterricht.

120 Vgl. einführend dazu Jank \& Meyer (Modelle 1994), S. 298-310.

121 Vgl. Klauer (Teaching 1988), S. 14-17. Dabei hat sich in den letzten Jahren der Begriff des Lernziels gegenüber dem Begriff des Lehrziels durch die stärkere Orientierung am Lernen denn am Lehren durchgesetzt. 
'Leistung'122 im Sinne einer zu erwartenden Handlung in Bezug auf einen festgelegten Inhalt und eine konkrete Situation gekennzeichnet sind. Die Ausprägung einer Kompetenz ist die Wahrscheinlichkeit, mit der die Lernenden eine bestimmte Aufgabe aus einer Aufgabenmenge lösen sollen. Das Lernziel wiederum ist die geforderte Ausprägung dieser Kompetenz. Abbildung 2.6 fasst die Definition zusammen.

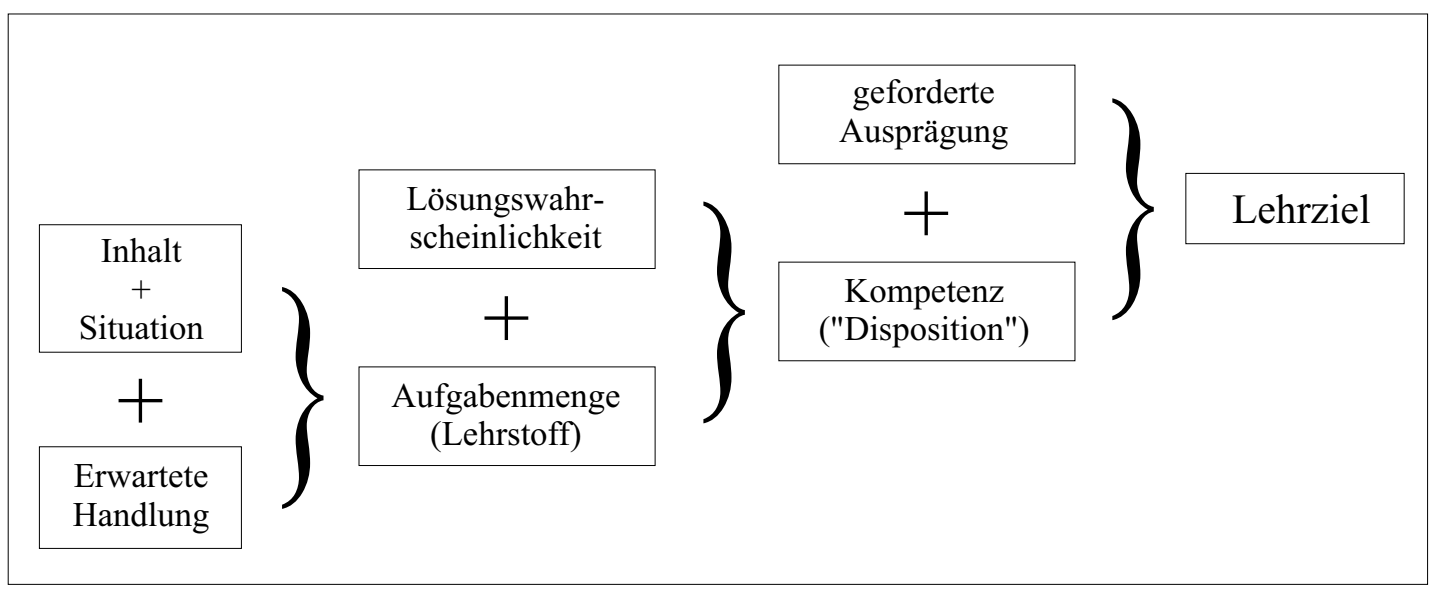

Abbildung 2.6: Vom Lehrinhalt zum Lehrziel ${ }^{123}$

Die allgemeine Vorgehensweise bei der Planung von Lernzielen für einen Unterricht entspricht der Folgenden: ${ }^{124}$

1. Operationalisierung: Das Lernziel muss in einem ersten Schritt in Unterziele aufgeteilt werden, die nachfolgend in eindeutig beobachtbare Verhaltensweisen der Lernenden umgesetzt werden. Für diese müssen zum einen genau bestimmte Situationen vorgegeben werden, in denen das Verhalten beobachtet werden soll. Zum anderen müssen Messmethoden konstruiert werden, mit deren Hilfe der zu erreichende Lernerfolg möglichst eindeutig gemessen werden kann. ${ }^{125}$

122 Zum Begriff vgl. Abschnitt 4.6.

123 In Anlehnung an Klauer (Teaching 1988), S. 16.

124 Die Techniken zur Lernzielananlyse sind zwar auf der methodologischen Grundlage des Behaviorismus entwickelt worden und dem empirisch-analytischen Forschungsparadigma verpflichtet. Sie bringen aber wertvolle Hinweise zur systematischen Unterrichtsplanung; vgl. Jank \& Meyer (Modelle 1994), S. 298-310.

125 Vgl. dazu auch Klauer (Teaching 1988), S.17-18. 
2. Dimensionierung: In einem zweiten Schritt werden die operationalisierten Lernziele nach formalen Kriterien bestimmten Dimensionen zugeordnet, damit sie anhand vorliegender Kriteriensysteme beurteilt werden können.

3. Hierarchisierung: Im dritten Schritt werden die Lernziele innerhalb der Dimensionen in eine Rangfolge gebracht.

An dieser Stelle soll das seit seiner Entwicklung gebräuchlichste Dimensionierungskonzept und Hierarchisierungsinstrument zur Klassifizierung des Anforderungsniveaus von Lernzielen dargestellt werden: die Taxonomie von Lernzielen im kognitiven Bereich nach BLoom et al. 1976.

\section{Taxonomie der kognitiven Lernziele}

Eine Taxonomie stellt eine Klassifikation des Verhaltens dar, das die beabsichtigten Ergebnisse einer Bildungsmaßnahme repräsentiert. ${ }^{126}$ Der Ansatz differenziert die kognitiven Lernziele in sechs Hauptkategorien von Komplexitätsstufen (vgl. Abbildung 2.7). ${ }^{127}$

$\left.\begin{array}{l}\text { 6. Beurteilung } \\ \text { 5. Synthese } \\ \text { 4. Analyse } \\ \text { 3. Anwendung } \\ \text { 2. Verständnis }\end{array}\right\}$ und Fertigkeiten
intellektuelle Fähigkeiten
und

\section{Kenntnisse}

Abbildung 2.7: Taxonomie der kognitiven Lernziele nach BLoom et al. 1976

1. Kenntnisse: Diese Stufe wird gleichgesetzt mit der Erinnerung von Besonderheiten und Allgemeinheiten, d.h. der Fähigkeit, gelehrte Methoden und Prozesse, Muster, Strukturen und Festlegungen wiederzugeben. Demzufolge zielt eine Messung des Lernerfolgs darauf ab, inwieweit sich die Lernenden das angeeignete Material bewusstmachen können.

126 Vgl. BLoom et al. (Taxonomie 1976), S. 26.

127 Vgl. Bloom et al. (Taxonomie 1976), S. 217-223. Auf eine Darstellung der Unterkategorien wird hier verzichtet. 
Die zweite bis sechste Stufe werden unter dem Begriff der intellektuellen Fähigkeiten und Fertigkeiten zusammengefasst:

2. Verständnis: Die zweite Stufe ist dadurch gekennzeichnet, dass die Lernenden um die Inhalte eines Textes oder Gesprächs wissen. Sie sollen in der Lage sein, mit dem Material oder einer Idee umzugehen, ohne es mit anderen Elementen in Beziehung setzen zu müssen. Somit umfasst das Verständnis das Übersetzen, Interpretieren und Extrapolieren von Sachverhalten innerhalb des Gebiets.

3. Anwendung: Die dritte Stufe der Taxonomie beinhaltet den Gebrauch von Abstraktionen in besonderen und in konkreten Situationen. Darunter werden Ideen, Regeln über Prozeduren oder verallgemeinerte Methoden verstanden.

4. Analyse: Die vierte Stufe erfasst die Fähigkeit, Sachverhalte und Gegenstände so zu zerlegen, dass die Beziehungen der Elemente untereinander sichtbar werden. Dabei steht die Frage nach der Struktur der konkreten Sachverhalte und Gegenstände im Vordergrund sowie die Überlegung, welche Wirkungen von ihnen ausgehen.

5. Synthese: Aufbauend auf den vorherigen Stufen umfasst diese Stufe durch Zusammenführung der gewonnenen Erkenntnisse eine einheitliche Kommunikation oder einen Plan für eine Tätigkeit und bezieht sich auf die Ableitung einer Reihe abstrakter Beziehungen.

6. Beurteilung: Die höchste Stufe der Taxonomie nimmt Bezug auf die Fähigkeit des Individuums, Werturteile über Sachverhalte und Methoden abzugeben. Diese werden dahingehend beurteilt, inwiefern sie (selbstgesteckten) Kriterien genügen.

Eine solche Taxonomie dient der genaueren Beschreibung eines Lernziels hinsichtlich des Anforderungsniveaus an die Lernenden, nachdem das Lernziel festgelegt wurden. So kann zum Beispiel untersucht werden, ob zur Vermittlung von Lernzielen der oberen Stufen ausreichend Grundlagen auf den ersten vier Stufen gelegt wurden. Umgekehrt erlaubt eine solche Vorgehensweise auch die Überprüfung, ob der Unterricht Lernziele enthält, die über die ersten drei Stufen hinaus gehen. ${ }^{128}$

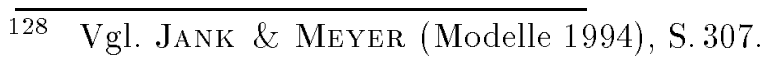


Allerdings existiert eine Taxonomie immer erst in Beziehung zu einer spezifischen Lerngruppe, ${ }^{129}$ orientiert sich also an dem Vorwissen der Lernenden sowie an ihren bisherigen Lernerfahrungen.

\subsection{Komplexe Lehr-Lern-Arrangements}

\subsubsection{Defizite traditionellen Unterrichts und Auswege}

Das Ziel der Erziehung zum selbstständigen, reflektierenden und verantwortungsbewussten (beruflichen) Handeln stellt Ansprüche an die Lernorganisation und an das entsprechende praktisch-pädagogische Handeln. ${ }^{130}$ Es gibt kein Handeln ohne Verhalten oder Tun, ${ }^{131}$ aber Verhalten, das nicht schon als Handeln verstanden werden kann. Jedem Verhalten und jeder Handlung liegen jedoch notwendigerweise Verhaltensdispositionen zu Grunde, die nicht unmittelbar beobachtet werden können. Hierzu zählen Wollen und Können, die bei der interpretativen Erschließung von Handlung einbezogen werden sollen und auf die durch pädagogische Maßnahmen eingewirkt wird. ${ }^{132}$

Die traditionellen Unterrichtsprinzipien waren durch Elementarisierung des Lernens, einem Vorgehen vom Isolierten zum Komplexen sowie vom Konkreten zum Abstrakten gekennzeichnet und verlangten Anschaulichkeit im Unterricht. ${ }^{133}$ Das vermittelte Wissen war jedoch häufig abstraktes Begriffswissen, das arm an Bedeutungen ist. Die Lerninhalte wurden unverbunden im Curriculum nach verschiedenen Fächern aufgeteilt. ${ }^{134}$ Diese Prinzipien behinderten das Verständnis komplexer Probleme, stattdessen förderten sie so genanntes 'träges Wissen'. ${ }^{135}$

\footnotetext{
129 Vgl. Achtenhagen (Didaktik 1984), S. 128.

130 Vgl. Schmiel \& Sommer (Legitimationsproblematik 1996).

131 Zur Begrifflichkeit vgl. Groeben (Handeln 1986).

132 Vgl. Heid (Erfordernis 1996).

133 Vgl. z. B. DuBs (Lehr-Lern-Arrangements 1996).

134 Vgl. ReEtz (Wissen 1996), S. 175-178.

135 Vgl. ausführlicher dazu z. B. RENkL (Wissen 1994).
} 
Neuere Ansätze mit konstruktivistischem Ursprung wie Cognitive Apprenticeship $^{136}$, Situated Learning ${ }^{137}$ oder Anchored Instruction ${ }^{138}$ basieren auf folgenden Grundannahmen: ${ }^{139}$

- Das Wissen wird durch die Lernenden konstruiert und nicht durch die Lehrenden vorgegeben.

- Lernen ist immer in eine Situation eingebettet.

- Handeln und Denken sollen in sinnvollen Kontexten stattfinden, damit das Verständnis für die Lerngegenstände gefördert wird.

In die Didaktik der Wirtschaftsfächer wurden diese Ideen durch die Konzeption des Forschungsprojekts 'Lernen, Denken, Handeln in komplexen ökonomischen Situationen' ${ }^{\prime 40}$ eingeführt. Die entsprechenden Unterrichtsmethoden werden als komplexe oder mehrdimensionale Lehr-Lern-Arrangements bezeichnet, die bisher hauptsächlich für das Lernen an berufsbildenden Schulen konstruiert werden. Dabei wird von Lehr-Lern-Arrangements gesprochen, weil neue Lehr-Lern-Verfahren auch neue Lehr-Lern-Techniken erfordern. ${ }^{141}$ Das Attribut der Komplexität bzw. der Mehrdimensionalität bezieht sich zum einen auf die Zielausrichtung des Unterrichts, zum anderen auf die inhaltliche Gestaltung. ${ }^{142}$

\subsubsection{Exkurs: Komplexität}

Nicht nur im Bereich der Problemlöseforschung wird der Begriff der 'Komplexität' in zunehmendem Maße benutzt. Komplexität ist ein Schlagwort, ist Alltagserfahrung, ist ein wissenschaftliches Problem und ein Problem des Managements. ${ }^{143}$

\footnotetext{
136 Vgl. Collins, Brown \& Newman (Cognitive apprenticeship 1989).

137 Vgl. Lave \& Wenger (Situated Learning 1991).

138 Vgl. Cognition and Technology Group at Vanderbilt (Instruction 1993) sowie BransFord et al. (Anchored instruction 1990).

139 Vgl. Gerstenmaier \& Mandl (Wissenserwerb 1995). Zur Übersicht zu den verschiedenen Ansätzen vgl. MandL \& Reinmann-Rothmeier (Unterrichten 1995). Zur Kritik am Radikalen Konstruktivismus vgl. NüsE et al. (Erfindung/en 1991), Groeben (Kritik 1995). Auf die nähere Darstellung der verschiedenen Konzepte wird in dieser Arbeit verzichtet.

$140 \mathrm{Vgl}$. Achtenhagen (Lernen 1992).

141 Vgl. Achtenhagen \& John (Lehr-Lern-Arrangements 1992), S. 5.

142 Die Begriffe 'komplex' und 'mehrdimensional' werden in der Literatur in diesem Zusammenhang weitgehend synonym verwendet. Im Folgenden wird daher von komplexen LehrLern-Arrangements die Rede sein.

143 Vgl. Malik (Strategie 1989), S. 184-186.
} 
Daher soll an dieser Stelle eine kurze Definition des Begriffs vorgenommen werden, der den weiteren Betrachtungen zu Grunde liegen wird.

Nach DörnER 1987 sind die beiden Merkmale der Komplexität eines Sachverhalts zum einen die Anzahl der Komponenten, die den Sachverhalt beschreiben, und zum anderen die Vielfalt der Verknüpfungen zwischen den Komponenten. ${ }^{144}$ Dies entspricht im Wesentlichen der Definition von Komplexität in der Systemtheorie. Dieser Ansatz wird jedoch nur unzureichend in der Forschung zu komplexen Problemen genutzt. Häufig wird von komplexen Problemen gesprochen, ohne die beiden anderen Merkmale von Systemen zu berücksichtigen. ${ }^{145}$ Die so genannten 3 C's der Systemtheorie sind complexity, connectivity und catastrophe: ${ }^{146}$

Komplexität kann in statischer und dynamischer Ausprägung auftreten: ${ }^{147}$ Unter statischer Komplexität werden Eigenschaften wie die hierarchische Struktur eines Systems, die Verbindungsmuster der Variablen, die Verschiedenheit der Komponenten sowie die Stärke der Interaktionen zwischen ihnen verstanden. Dabei sind die Interaktionen zwischen den Subsystemen vermutlich eher schwach, aber nicht vernachlässigbar. ${ }^{148}$ Die dynamische Komplexität beschreibt den Zusammenhang zwischen Input und Output: Der Output stellt bei dynamisch komplexen Systemen keine einfache Funktion des Inputs dar. Insbesondere Zeitverzögerungen können in die Zusammenhänge eingreifen. Statik bzw. Dynamik und Komplexität haben jedoch nicht die gleichen Ausprägungen, sind also nicht als gegensätzliche Merkmale zu begreifen. Als Beispiel für ein statisch komplexes System kann eine mechanische Uhr gelten, die in ihrem Aufbau komplex ist, in ihrer Dynamik jedoch statisch. Ein Beispiel für ein dynamisch nicht-komplexes System ist das 'Apfelmännchen' aus der Chaostheorie, ${ }^{149}$ ein Unternehmensplanspielkann als dynamisch komplexes System verstanden werden. ${ }^{150}$

Die Art und Weise der Vernetzung der Komponenten eines Systems führt zur Konnektivität. Sie bezieht sich auf die mathematische Dimensionalität des Systems und auf die Möglichkeit der Hierarchisierung seiner Komponenten. Weiterhin

\footnotetext{
144 Vgl. Dörner (Problemlösen 1987), S. 18.

145 Vgl. Funke (Problemlösen 1986), S. 26.

146 Vgl. Casti (Connectivity 1979).

147 Vgl. Funke (Problemlösen 1986), S. 26-32; auch Casti (Connectivity 1979), S. 43-44.

148 Vgl. Casti (Connectivity 1979), S. 41.

149 Vgl. Mandelbrot (Geometrie 1987).

150 Vgl. Abschnitt 3.2.3.
} 
kann die Länge der Kette von verbundenen Elementen als Merkmal herangezogen werden..$^{151}$

Als drittes Merkmal gilt die Katastrophe bzw. - positiv formuliert - die Stabilität eines Systems. Diese Eigenschaft beinhaltet auf der einen Seite die Reaktion auf Änderungen in der Umwelt, um zu überleben. Auf der anderen Seite muss ein System ein vergleichbares dynamisches Verhalten über einem gewissen Zeitraum beibehalten. Von struktureller Stabilität wird gesprochen, wenn sich ein System im Wesentlichen so verhält wie seine Umgebung, also nicht überreagiert und dadurch die Stabilität des Gesamtsystems, in das es eingebettet ist, gefährdet. Ein System ist jedoch nicht unbedingt stabil, wenn es im Ausgangszustand verharrt. Bei der Evolutions-Stabilität ist durchaus eine Entwicklung wünschenswert, so z. B. in einem Unternehmen mit Gewinnmaximierungs-Absichten. ${ }^{152}$

\subsubsection{Gestaltung komplexer Lehr-Lern-Arrangements}

An die Konstruktion von komplexen Lehr-Lern-Arrangements im Rahmen eines handlungsorientierten Unterrichts werden verschiedene Anforderungen gestellt: ${ }^{153}$

Die Gestaltung orientiert sich grundsätzlich an den Lernzielen, die neben den Schlüsselqualifikationen auch Metakognitionen umfassen und die Bedeutung von Emotion und Motivation auf die Lernprozesse berücksichtigen. Allerdings ist die Vermittlung des grundlegenden Faktenwissens und und ihrer Strukturen nicht zu vernachlässigen, da ohne sie ein Aufbau höherer Kognitionen wie Schlüsselqualifikationen nicht möglich ist. Die explizite Berücksichtigung des Vorwissens der Lernenden verhindert eine Über- oder Unterforderung.

Die Problemstellungen, mit denen die Lernenden konfrontiert werden, sollten bereits zu Beginn des Unterrichts eine komplexe Ziel- und Inhaltsstruktur haben und

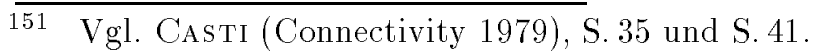

152 Vgl. Funke (Problemlösen 1986), S. 37-40; auch Casti (Connectivity 1979), S.45-46.

153 An dieser Stelle werden die Anforderungen zusammenfassend dargestellt. Vgl. AchtenHagen (Kriterien 1998); Klauser (Problem-based learning 1998), S. 250 sowie Dubs (Schlüsselqualifikationen 1996), S. 53-56, Dubs (Lehr-Lern-Arrangements 1996), S.164165 und BECK (Handlungsorientierung 1996). Zu Grenzen beim Einsatz komplexer LehrLern-Arrangements, speziell von Unternehmensplanspielen, vgl. Abschnitt 3.5. 
die Erarbeitung allgemeiner Regeln und Schlussfolgerungen ermöglichen. Hierbei sollten unterschiedliche Arten von Probleme zur Lösung vorgegeben werden. ${ }^{154}$

Ein weiteres Merkmal der Problemstellung ist die Wissenschaftsorientierung. Sie orientiert sich an den dem Gegenstandsbereich zu Grunde liegenden Theorien und sollte sinnvolle Problemstellungen aus der zukünftigen Berufswelt der Lernenden enthalten. Sie müssen subjektiv relevant sein, wobei sich die Bewertung der Relevanz nach den Erfahrungsbereichen der Lernenden richtet. Dabei sollten auch Aufgaben gestellt werden, die solchen aus dem Arbeitsleben entsprechen. Hierbei muss jedoch immer die Möglichkeiten zur Fehler-Korrektur gegeben sein.

Die Problemstellungen sind zwar auf der einen Seite in einen Kontext einzubetten, wobei der Bezug zu den Lernzielen zu behalten ist. Auf der anderen Seite sollte aber auch ein Wechsel der Kontexte möglich sein. Dabei müssen sowohl die Bedeutung als auch der Sinn der verwendeten Begriffe vermittelt werden, um einen Transfer der gelernten Inhalte zu fördern.

Das Lernen sollte aus kombinierter Einzel- und Gruppenarbeit bestehen, wobei die institutionellen Rahmenbedingungen so gut wie möglich auszunutzen sind. Die Lernenden sollten zu selbstständigem Lernhandeln angeregt werden. Die Lernprozesse dürfen jedoch nicht ungeleitet bleiben. Auch ist zu berücksichtigen, dass die Lernziele nicht in jedem Fall identisch mit den Handlungszielen der Lernenden sind.

Entsprechend ist die Rolle der Lehrenden neu zu definieren: Sie sind nicht mehr ausschließlich Wissensvermittler, sondern unterstützen beratend die Lernprozesse. Dies hat auch Auswirkungen auf ihre Aus- und Weiterbildung. ${ }^{155}$

Der Einsatz komplexer Lehr-Lern-Arrangements hat auch einen Einfluss auf die Messung des Lernerfolgs. Die Instrumente müssen sich an der Komplexität der Lernumgebung orientieren und ihr gerecht werden. Dabei sollte berücksichtigt werden, dass offene Lernumgebungen auch zufällige Lernergebnisse produzieren können.

Der Perspektivwechsel zwischen Handlung und System spielt in einem komplexen Lehr-Lern-Arrangement eine große Rolle. Hierdurch wird ein Lernen im Modell

\footnotetext{
154 Vgl. Abschnitt 2.2.

155 Vgl. z. B. Young (Education 1998).
} 
sowie ein Lernen am Modell ermöglicht, auf das im nächsten Abschnitt näher eingegangen wird.

\section{Lernen im und am Modell}

Der Vorteil der Abbildung eines komplexen Realitätsbereichs im Modell gegenüber einem Lernen anhand der Wirklichkeit selber liegt in der Möglichkeit, Erkenntnisse über bestimmte Sachverhalte zu fördern. Modelle haben dabei grundsätzlich folgende Funktionen: ${ }^{156}$

Ersatzfunktion: Lerninhalte, die im Original nicht direkt zugänglich sind, werden im Modell durch bestimmte Elemente abgebildet und so sichtbar gemacht.

Strukturierungsfunktion: Bestimmte Strukturen oder Funktionen werden im Modell hervorgehoben.

Trainings-/Steuerungsfunktion: Ein bestimmtes Verhalten, insbesondere das Steuern des Modells, wird am Modell geübt.

Ideologiekritische Funktion: Die bestehende Realität wird anhand des konstruierten Modells hinterfragt.

Bei der Aufzählung der Funktionen von Modellen wird bereits deutlich, dass zwischen einem Lernen im Modell und einem Lernen am Modell unterschieden werden kann. Dabei zielt das Lernen im Modell auf den Erwerb der verknüpften Lernziele und -inhalte, während das Lernen am Modell in einem metakognitiven Zugriff die Modelleigenschaften und die der Modellierung zu Grunde liegenden Entscheidungen thematisiert. ${ }^{157}$

Die ersten zwei Funktionen von Modellen beschäftigen sich mit dem Lernen im Modell. Hierbei sollen Fähigkeiten entwickelt werden, zu denen das Analysieren und Abstrahieren von Realitätsbereichen, die Beschaffung und Bewertung von Informationen über den Realitätsbereich, das Transformieren der Informationen in Modelle sowie methodische Fähigkeiten zählen. ${ }^{158}$

\footnotetext{
$1 \overline{56}$ Vgl. Buddensiek (Simulationsspiele 1979), S. 125-126.

157 Zur analytischen Unterscheidung zweier Ebenen des Lernhandelns in der Übungsfirma vgl. TRAmM (Konzeption 1992), S. 203-216.

158 Vgl. Böhret \& Wordelmann (Planspiel 1975), S.43-45.
} 
Den Teilnehmenden muss jedoch bei der Einführung in das Lehr-Lern-Arrangement klargemacht werden, dass das Modell die Wirklichkeit reduziert abbildet, sie aber nicht umfassend darstellen kann. Sie sollen im Modell und nicht in der Wirklichkeit lernen. Durch die Reduzierung und Akzentuierung der Modellierung werden bestimmte Mechanismen des Fachgebiets für die Teilnehmenden transparenter und somit wiederum in der Realität anwendbar. ${ }^{159}$

Geschieht dies nicht, so besteht die Gefahr, dass die Teilnehmenden das Modell für die Realität halten. Dies führt u. U. dazu, dass bei der Reflexion über eigene Erfahrungen und über die Erfahrungen mit dem Modell Unstimmigkeiten auftreten, ${ }^{160}$ die die Motivation der Teilnehmenden sinken lässt und damit einen negativen Einfluss auf den Lernerfolg hat. ${ }^{161}$

Die beiden letzten Funktionen eines Modells dagegen behandeln das Lernen am Modell. Nach der sozial-kognitiven Lerntheorie von BANDURA 1979, auf der das Lernen am Modell basiert, hat der Mensch nicht nur die Auswirkungen seines eigenen Handelns als Grundlage für zukünftige Handlungen, sondern kann Modelle in jeglicher Form beobachten und aus ihnen lernen. ${ }^{162}$ Durch die Übernahme einer bestimmten Rolle in einem komplexen Lehr-Lern-Arrangement, ihren Handlungen und die den entsprechenden Rückmeldungen erhalten die Teilnehmenden die Möglichkeit, die modellierten Zusammenhänge zu erkennen und daraus zu lernen. ${ }^{163}$ Im Rahmen der betriebswirtschaftlichen Aus- und Weiterbildung können Unternehmensplanspiele als komplexe Lehr-Lern-Arrangements im Rahmen eines handlungsorientierten Unterrichts-Konzepts zum Einsatz kommen. Damit beschäftigt sich das folgende Kapitel.

\footnotetext{
159 Vgl. Buddensiek (Simulationsspiele 1979), S. 123.

160 Vgl. Achtenhagen et al. (Lernhandeln 1992), S. 129.

161 Vgl. Kaiser \& Kaminski (Methodik 1994), S. 176-178 sowie Hesse, Spies \& Lüer (Einfluß 1983), S. 404-405.

$162 \mathrm{Vgl}$. Bandura (Lerntheorie 1979). Die aufgestellte Theorie beschäftigt sich zwar hauptsächlich mit dem Beobachtungslernen bei Kindern, kann jedoch genauso auf den Umgang mit Modellen bezogen werden.

163 Vgl. Schunck (Subjektive Theorien 1993), S. 65-66.
} 


\section{Unternehmensplanspiele in der Aus- und Weiterbildung}

\section{$3.1 \quad$ Vorbemerkungen}

Unternehmensplanspiele und ihre instruktionale Unterstützung können in der Aus- und Weiterbildung als komplexe Lehr-Lern-Arrangements eingesetzt werden. In Abschnitt 3.2 werden die Charakteristika von Unternehmensplanspielen dargestellt. Bevor jedoch auf den Einsatz und die Klassifikation von Unternehmensplanspielen eingegangen wird, sollen zum besseren Verständnis einige Begriffsabgrenzungen vorgenommen werden. Da Unternehmensplanspiele zur Vermittlung von Kenntnissen der Unternehmensplanung herangezogen werden können, wird in Abschnitt 3.3 die allgemeine Theorie der Unternehmensplanung im Hinblick auf eine mögliche Umsetzung in einem Unternehmensplanspiel erläutert.

Abschnitt 3.4 beschäftigt sich mit den Lernzielen des Einsatzes von Unternehmensplanspielen. Dabei wird die Handlung im Planspiel betrachtet und eine Klassifikation der möglichen Lernziele vorgenommen. Nachfolgend wird auf Besonderheiten beim Einsatz von Unternehmensplanspielen eingegangen (Abschnitt 3.5), wobei sowohl allgemeine Überlegungen angestellt als auch organisatorische und methodische Besonderheiten aufgezeigt werden.

Der Forschungsstand zum Einsatz von Unternehmensplanspielen wird abschließend Abschnitt 3.6 erörtert. Hier werden 11. a. Forschungsergebnisse zum Einfluss der Komplexität auf den Lernerfolg dargestellt. Dabei interessieren für die vorlie- 
gende Arbeit insbesondere die Designs der Forschungen. Eine kritische Betrachtung der Erhebungsinstrumente sowie der eingesetzten Planspiele kann auf Grund mangelnder Publikation nur zum Teil durchgeführt werden.

\subsection{Grundlagen zu Unternehmensplanspielen}

\subsubsection{Begriffsabgrenzung}

Unter Simulation wird eine Methode verstanden, mit der Entwicklungen von Systemzuständen im Zeitverlauf beobachtet und analysiert werden können. Diese Veränderungen werden durch unterschiedliche Inputs innerhalb dynamischer Modelle, den Simulationsmodellen, erreicht. ${ }^{1}$ Dabei werden nur die Attribute des Realitätsausschnitts erfasst, die für den Modellbildenden oder -verwendenden von Bedeutung sind. Modelle erfüllen ihre Ersatzfunktion immer für bestimmte Erkenntnis- oder Aktionssubjekte innerhalb bestimmter Zeitspannen der Originalrepräsentation und zu bestimmten Zwecken und Zielen, denen die Modellbildung unterliegt. Das heißt, sie sind immer in einen Kontext eingebettet. ${ }^{2}$

Die Entwicklung von Simulationen geht auf Erfahrungen im militärischen Bereich zurück. Sie wurden zunächst als Strategie- und 'Sandkastenspiele' bekannt. Der Vorteil von Simulationen liegt hier auf der Hand: Handlungsalternativen können in hypothetischen Szenarien erarbeitet werden und die eigenen Aktionen sowie die Reaktionen des Gegners bleiben ohne tatsächliche Konsequenzen. ${ }^{3}$

Auf Grund der immer komplexer werdenden Zusammenhänge wurden auch im Bereich der Betriebswirtschaft - und seit den 50er Jahren auch in der betriebswirtschaftlichen Aus- und Weiterbildung - verstärkt Simulationen eingesetzt, um Reaktionen des Unternehmens auf mögliche Veränderungen der Umwelt abzubilden. ${ }^{4}$ Dabei orientiert sich die Darstellung an einem systemorientierten Ansatz.

1 Vgl. Böhret \& Wordelmann (Planspiel 1975), S. 22.

2 Vgl. Stachowiak (Erkenntnisstufen 1980), S. 18; zu den Funktionen vgl. Abschnitt 2.5.3.

3 Zu einer Darstellung der geschichtlichen Entwicklung von Planspielen vgl. z. B. RoHN (Ursprung 1995); RoHN (Führungsentscheidungen 1964), S.19-27.

4 Vgl. Bleicher (Unternehmungsspiele 1962), S.11-14; Rohn (Führungsentscheidungen 1964), S. $27-31$. 
Systeme bestehen aus Elementen als nicht weiter zerlegbare Einheiten und deren Beziehungen untereinander. ${ }^{5}$

Der Begriff des Simulationsspiels beinhaltet den spielerischen Umgang mit der Abbildung des Realitätsausschnitts. ${ }^{6}$ Simulationsspiele für didaktische Zwecke sind eine Unterrichtsmethode, in denen die Teilnehmenden eine Rolle übernehmen, bestimmte Interessen verfolgen und versuchen, diese innerhalb des Modells durchzusetzen. ${ }^{7}$

Im Gegensatz zu reinen Simulationen, die ohne Eingriff ablaufen, haben die Teilnehmenden in Planspielen die Möglichkeit, während des Verlaufs einzugreifen. Entscheidungen, die in der Realität u. U. mit großen Risiken behaftet sind, können so simuliert werden. Dabei sind die Auswirkungen der Entscheidungen durchaus erfahrbar, ziehen aber keine realen Konsequenzen nach sich. Die Teilnehmenden eines Planspiels erleben jedoch die Verantwortlichkeit für die getroffenen Entscheidungen und können die Auswirkungen sowohl richtiger als auch falscher Entscheidungen nachvollziehen. ${ }^{8}$

Seinen besonderen Charakter erhält das Planspiel durch entgegengesetzte Interessen, Zielkonflikte und den Entscheidungsdruck sowie die zeitliche Gliederung. Dabei besteht die Möglichkeit, das Verhalten der Teilnehmenden anhand der Simulationsergebnisse systematisch festzuhalten und nach verschiedenen Kriterien zu bewerten. ${ }^{9}$ Die Teilnehmenden bilden den Aktions- und Steuerungsbereich, die Spielleitung - bzw. der programmierte Algorithmus beim Einsatz eines computergestützten Planspiels - repräsentiert den Reaktions- oder Simulationsbereich des Planspiels. ${ }^{10}$ Die Phasen des Ablaufs einer Spielrunde im Planspiel werden in Abbildung 3.1 dargestellt.

5 Vgl. Bossel (Simulation 1989), S. 16; grundlegend zur Systemtheorie vgl. Casti (Connectivity 1979); Zur Klassifizierung von Systemen vgl. z. B. Biethahn, Mucksch \& RuF (Informationsmanagement 1996), S.88-93.

6 Vgl. Buddensiek (Simulationsspiele 1979), S. 14-15.

7 Vgl. Buddensiek (Entscheidungstraining 1992), S. 18.

8 Vgl. Rohn (Führungsentscheidungen 1964), S.9 sowie BECK (Schlüsselqualifikationen 1995), S. 63-64.

9 Vgl. Geilhardt \& Mühlbradt (Konzepte 1995), S. 49; auch Abschnitt 3.4.

10 Vgl. Bleicher (Unternehmungsspiele 1962), S. 21-22 sowie EBERT (Planspiel 1992), S. 30. 


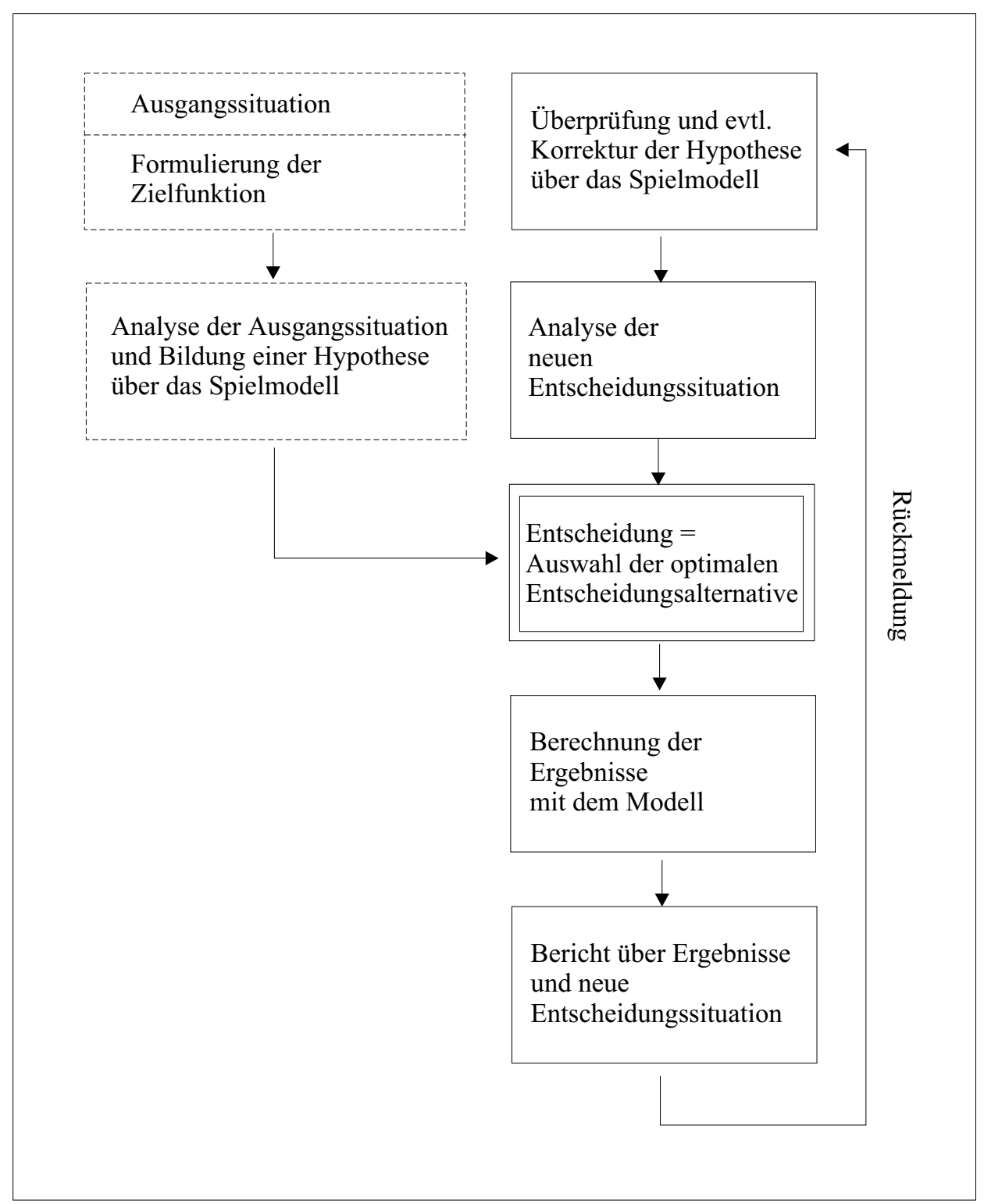

Abbildung 3.1: Phasen einer Spielrunde im Planspiel ${ }^{11}$

$\overline{11}$ Quelle: Koller (Simulation 1969) , S. 101. 
Die Ausgangssituation des Planspiels ist im Modell enthalten. Die verfolgten Ziele werden entweder von den Teilnehmenden aufgestellt oder von der Spielleitung vorgegeben. Die Teilnehmenden analysieren zunächst die präsentierte Ausgangssituation und stellen Hypothesen über das Spielmodell auf. Nachfolgend überlegen sie, welche Handlungsalternativen in der gegebenen Situation existieren, und wählen die zur Zielerreichung optimale Handlung aus. Die Spielleitung - bzw. das Computerprogramm - übernimmt die Aufgabe der Simulation, indem sie die Ergebnisse mit dem Modell berechnet und den Teilnehmenden über die Ergebnisse sowie die neue Entscheidungssituation berichtet. Durch die Rückmeldung sind die Teilnehmenden aufgefordert, ihre aufgestellten Hypothesen zu überprüfen und gegebenenfalls zu korrigieren. Weiterhin wird die neue Situation analysiert und die Handlungsalternativen beurteilt. Nun wiederholt sich der Vorgang von Entscheidung und nachfolgender Simulation entsprechend der Anzahl der zu spielenden Perioden. ${ }^{12}$

Im Begriff des Planspiels oder der Simulation ist jedoch noch nicht festgelegt, welche konkreten Inhalte im Simulationsmodell abgebildet werden. Bildet eine Simulation unternehmerische Zusammenhänge ab, wird von einer Unternehmenssimulation gesprochen. Unternehmens- oder Unternehmungsplanspiele sind entsprechend Planspiele, die eine Unternehmung oder Teile dieser im Modell abbilden. Sie basieren auf dem Regelkreisprinzip und entsprechen somit der systemkybernetischen Betrachtungsweise der Betriebswirtschaft. ${ }^{13}$ Dabei werden die theoretischen Grundlagen als formaler Rahmen genutzt und auf betriebswirtschaftliche Problemsituationen angewandt. ${ }^{14}$

Unternehmensplanspiele dienen dazu, Abhängigkeiten zwischen den einzelnen Teilen einer Unternehmung darzustellen, die Wirkungen verschiedener Entscheidungen auf das Unternehmen als Ganzes zu verdeutlichen bzw. einzelne Unternehmensbereiche detaillierter darzustellen. ${ }^{15}$

12 Vgl. Bleicher (Unternehmungsspiele 1962), S. 71-79 sowie Rohn (Führungsentscheidungen 1964), S. 82-107.

13 Vgl. Ulrich \& Probst (Anleitung 1988) und Probst \& Gomez (Denken 1989); ausfïhrliche Darstellung z. B. bei WiLms (Entscheidungsverhalten 1995), S 112-130.

14 Vgl. Ulrich (Unternehmen 1970), S. 137-138.

15 Vgl. Rohn (Führungsentscheidungen 1964), S. 11. 


\subsubsection{Einsatzmöglichkeit von Unternehmensplanspielen}

Zur Erforschung des Verhaltens beim Lösen komplexer Probleme werden Planspiele auch in psychologischen Untersuchungen eingesetzt. Hier wird jedoch eher auf die Abbildung von Systemen aus der Alltagswelt zurückgegriffen, deren Parameter sich im Zeitverlauf nicht ändern. ${ }^{16}$

Planspiele im Allgemeinen sind inzwischen sowohl im Bereich der Hochschulen als auch in der betrieblichen und schulischen Aus- und Weiterbildung eine weit verbreitete Lehrmethode: ${ }^{17}$ Unternehmensplanspiele bieten im Bereich des wirtschaftswissenschaftlichen Hochschulstudiums eine zusätzliche Form des Lernens neben den herkömmlichen Vorlesungen und Seminaren. ${ }^{18}$ Weiterhin gewinnen sie in den berufsbildenden Schulen sowie zur Lehrerfortbildung ${ }^{19}$ und in überbetrieblichen Planspiel-Seminaren an Bedeutung. ${ }^{20} \mathrm{Im}$ Folgenden werden die Ergebnisse von drei Untersuchungen zum Einsatz von Planspielen an wirtschaftswissenschaftlichen Fakultäten, an berufsbildenden Schulen des Bereichs Wirtschaft und Verwaltung und in der betrieblichen Ausbildung sowie an Institutionen der kaufmännischen Weiterbildung dargestellt.

Eine empirische Erhebung, die in Deutschland in wirtschaftswissenschaftlichen Fakultäten durchgeführt wurde $(\mathrm{n}=268)$, befasste sich mit verschiedenen Fragen zum Einsatz von Unternehmensplanspielen im Hochschulstudium. Dabei wurde untersucht, welche Gründe für oder gegen den Einsatz von Unternehmensplanspielen sprechen und welche Erfahrungen mit ihnen gemacht wurden. Knapp zwei Drittel der befragten Lehrstühle gaben an, keine Unternehmensplanspiele einzusetzen. Etwa ein Drittel dagegen führte sie in unterschiedlicher Art durch, wobei sie hauptsächlich semesterbegleitend in wöchentlichem Abstand im Hauptstudium eingesetzt wurden. ${ }^{21}$

16 Vgl. z. B. die Übersicht in Funke (Problemlösen 1986) und Dörner et al. (Lohhausen 1983) bzw. Abschnitt 3.6.3.

17 Vgl. Rohn (Management-Lernen 1991); auch FassheBer (Planspiele 1995), S.611 und Cohen \& Rhenman (Rolle 1974). Zu den damit verfolgten Lernzielen vgl. Abschnitt 3.4 .2 .

18 Vgl. Bloech (Modellierung 1989), S. 105-107.

19 Vgl. z. B. Kaiser \& Kaminski (Methodik 1994), S. 164; auch Klippert (Planspiele 1992) und BACH (Planspiele 1983).

20 Vgl. Rohn (Einsatzgebiete 1995).

21 Vgl. Bronner \& Kollmannsperger (Planspieleinsatz 1997). 
Die zweite Erhebung mittels eines Fragebogens beschäftigte sich mit der Sicht des Planspiel-Einsatzes von Lehrenden berufsbildender Schulen $(\mathrm{n}=349)$ sowie Ausbildenden Hamburger Unternehmen $(\mathrm{n}=90) .{ }^{22}$ Der Fragebogen bestand aus verschiedenen Aussagen, die auf einer zum Teil zwei-, zum Teil vierstufigen Rating-Skala zu bewerten waren, sowie offenen Fragen. Mögliche Lernerfolge eines PlanspielEinsatzes sowie die Vorzüge von Simulationen gegenüber anderen, nicht bezeichneten Unterrichtsmethoden werden von den Befragten positiv eingeschätzt. Dabei lassen sich die Befragten in zwei Gruppen teilen: Eine Gruppe stimmt voll mit den vorgegebenen Aussagen überein, die andere ist eher skeptisch, urteilt aber insgesamt positiv. $23 \%$ der Lehrenden und $28 \%$ der Ausbildenden setzten zum Zeitpunkt der Befragung Planspiele in der Ausbildung ein. Als Gründe gegen den Einsatz wird als Rahmenbedingung hauptsächlich die mangelnde Verfügbarkeit passender Planspiele genannt. Dabei nennen die Ausbildenden wesentlich weniger Gründe gegen einen Einsatz. Unter veränderten organisatorischen Bedingungen wären insbesondere die Lehrenden verstärkt bereit, Planspiele einzusetzen. Von den Ausbildenden werden keine der genannten Bedingungen als förderlich für einen Planspiel-Einsatz eingeschätzt.

Eine weitere Frage in dieser Erhebung beschäftigte sich mit den negativen Erfahrungen, die mit einem Planspiel gemacht wurden. Hier wurden wiederum insbesondere von den Lehrenden die zeitliche Belastung vor und während des Einsatzes, die Überforderung der Lernenden sowie Probleme mit der Kontrolle des Lernerfolgs genannt. Hinsichtlich positiver Erfahrungen im Umgang mit Planspielen lässt sich die Stichprobe in zwei Gruppen teilen: $44 \%$ der Befragten haben sehr positive Erfahrungen mit dem Einsatz gemacht, wobei die Aspekte der sozialen Beziehungen zwischen Lernenden und Lehrenden sowie das Fachwissen und die Vermittlung der Lerninhalte eher schlechter beurteilt werden. Mehr als die Hälfte haben eher schlechte Erfahrungen mit Planspielen gemacht, wiederum im Bereich der sozialen Beziehungen zwischen Lernenden und Lehrenden sowie der Vermittlung der Lerninhalte, zusätzlich bei der Problemlösefähigkeit. Planspiele werden aber auch förderlich hinsichtlich des Interesses und der Motivation von knapp zwei Dritteln der Befragten eingeschätzt. Die bisherigen Ergebnisse erklären nicht den vergleichsweise seltenen Einsatz von Planspielen. Eine offene

22 Vgl. Rebmann (Planspieleinsatz 1998). Die Stichprobe reduzierte sich jedoch bei den einzelnen Bereichen zum Teil erheblich. 
Frage nach dem grundsätzlichen Zweck eines Planspiel-Einsatzes zeigt, dass gut drei Viertel der Ausbildenden und gut die Hälfte der Lehrenden Planspiele als Ergänzung einsetzen würden. Knapp die Hälfte der Ausbildenden und Lehrenden würde Planspiele weiterhin als Präsentations-Medium benutzen. Ebenfalls knapp die Hälfte der Lehrenden, aber nur noch $16 \%$ der Ausbildenden würden Planspiele zur Einführung in neue Stoffgebiete einsetzen. Insgesamt scheint hinsichtlich einer positiven Bewertung des didaktischen Nutzens von Planspielen Nachholbedarf zu bestehen.

Die dritte Umfrage zum Einsatz von Planspielen an Institutionen der kaufmännischen Weiterbildung $(n=36)$ kam zum Teil zu vergleichbaren Ergebnissen. Knapp die Hälfte der befragten Institutionen setzten nach ihren Angaben Planspiele ein. In fast allen Institutionen wurden andere komplexe Lehr-Lern-Arrangements zur Gestaltung der Maßnahmen herangezogen, und zwar unabhängig davon, ob Planspiele eingesetzt wurden oder nicht. ${ }^{23}$ Ein wichtiger Aspekt dieser Untersuchung war der Zusammenhang zwischen dem Einsatz komplexer Lehr-Lern-Arrangements - wie Planspielen - und der Qualitätskontrolle der eigenen Lehre. So wurden die Institutionen u. a. danach befragt, ob sie den Qualitätsstandards des Arbeitsförderungsgesetzes (AFG) entsprechen. ${ }^{24}$ Den Standards entsprachen 87,5\% der Institutionen, die Planspiele einsetzen, und $75 \%$ der Institutionen, die Planspiele nicht einsetzen. Entsprechend waren auch relativ mehr der Institutionen durch das Bundesland anerkannt, die Planspiele einsetzen, als Institutionen, die keine Planspiele einsetzen (31\% gegenüber $25 \%$ ). Eine Zertifizierung nach DIN ISO $9000 \mathrm{ff}^{25}$ hatte nur eine einzige Institution, die allerdings keine Planspiele einsetzte. Einige Institutionen streben nach eigenen Angaben eine Zertifizierung nach DIN ISO 9000 ff. an, wobei es sich wiederum verstärkt um Institutionen handelt, die Planspiele einsetzen (31\% gegenüber 10\%). Interne Qualitätskontrollen werden jeweils von drei Vierteln der Institutionen durchgeführt. Dies wurde jedoch in dem eingesetzten Fragebogen nicht weiter spezifiziert.

\footnotetext{
23 Vgl. Pietzsch (Einsatz 1997), S. 74-80.

24 Dies bedeutet eine Förderung einzelner Maßnahmen durch das Arbeitsamt; vgl. BARToNZiEmann (Qualitätskriterien 1995). Dabei werden die Regelungen des AFG derzeit in wesentlichen Teilen durch das Arbeitsförderungs-Reformgesetz (AFRG) abgelöst, das seit dem 1. Januar 1997 stufenweise in Kraft tritt.

25 Vgl. z. B. KöHLER (DIN ISO 9000 ff. 1995).
} 
In der Weiterbildung spielt die Qualitätssicherung eine große Rolle, da insbesondere durch Lohn- und Gehaltsfortzahlungen den Unternehmen Kosten bei der Weiterbildung ihrer Mitarbeiterinnen und Mitarbeiter entstehen. Insofern ist die Sicherung der Effektivität einer Bildungsmaßnahme für die Verantwortlichen von Bedeutung. ${ }^{26} \mathrm{Um}$ den Ansprüchen gerecht zu werden, ist es wichtig, neuere Methoden - wie zum Beispiel Unternehmensplanspiele - einzusetzen.

\subsubsection{Klassifikation von Unternehmensplanspielen}

In diesem Abschnitt werden verschiedene Aspekte dargestellt, nach denen Unternehmensplanspiele klassifiziert werden können. Dabei wird auch auf den möglichen Einsatz in der betriebswirtschaftlichen Aus- und Weiterbildung eingegangen. Tabelle 3.1 fasst alle Merkmale zusammen.

Bezüglich der Wirklichkeitsnähe des Modells kann zwischen der Abbildung einer speziellen Unternehmung, eines bestimmten Produkts bzw. einer Branche unterschieden werden: Während Standard-Planspiele stärker von der Realität einer spezifischen Unternehmung abstrahieren und übergeordnete Prinzipien abbilden, gibt es auch firmenspezifische Planspiele. ${ }^{27}$

Weiterhin üblich ist die Differenzierung nach speziellen Planspielen, die im Modell eine spezifische Branche abbilden, und allgemeinen Planspielen, die auf einem branchen-unabhängigen Modell basieren. ${ }^{28}$ Weiterhin kann nicht nur eine spezielle Branche abgebildet werden, sondern auch ein bestimmtes Produkt oder eine bestimmte Produktpalette, so dass sich ein konkretes bzw. ein abstraktes Modell ergibt. ${ }^{29}$ In der innerbetrieblichen Aus- und Weiterbildung kommen eher spezifischere Modelle zum Einsatz, während in überbetrieblichen Bildungsmaßnahmen sowie an Berufsschulen oder Hochschulen eher die weniger spezifischen Modelle

26 Vgl. Woortmann (Qualität 1995); auch Bundesinstitut für Berufsbildung (Qualitätsaspekte 1993).

27 Vgl. Högsdal (Planspiele 1996), S. 15; auch Rohn (Methodik 1980), S. 21.

28 Vgl. Bleicher (Entscheidungsprozesse 1974), S. 19-20. Zur Konzeption eines Unternehmensplanspiels für den mittelständischen Textileinzelhandel vgl. ScHoEL (Konzeption 1998).

29 Vgl. Rohn (Führungsentscheidungen 1964), S. 50-51. 
eingesetzt werden, da hier die Teilnehmenden unterschiedliche Erfahrungen mitbringen. ${ }^{30}$

\begin{tabular}{|l|l|}
\hline \multicolumn{2}{|c|}{ Klassifikation von Unternehmensplanspielen } \\
\hline Merkmal & \multicolumn{1}{c|}{ Ausprägung } \\
\hline Wirklichkeitsnähe: & $\begin{array}{l}\text { allgemeines - spezielles Modell } \\
\text { branchen-, produkt-, firmen-(un)spezifisch }\end{array}$ \\
\hline Zeitaspekt: & $\begin{array}{l}\text { simulierte Zeiträume (Tage, ..., Jahre) } \\
\text { periodische - kontinuierliche Simulation }\end{array}$ \\
\hline Umfang des Entscheidungsrahmens: & Gesamt-Planspiel - Teil-Planspiel \\
\hline Freiheitsgrad der Entscheidungen: & freies - starres Planspiel \\
\hline Abhängigkeit Entscheidungen: & deterministisches - stochastisches Planspiel \\
\hline Aktion der Teilnehmenden: & Kleingruppen - Einzel-Entscheidung \\
\hline Interaktion der Teilnehmenden: & $\begin{array}{l}\text { interaktives - isoliertes Planspiel } \\
\text { offenes/geschlossenes interaktives Planspiel } \\
\text { kompetetives - nicht-kompetetives Planspiel }\end{array}$ \\
\hline Abbildung der Marktform: & Monopol - Konkurrenz \\
\hline Komplexität des Spielmodells: & einfaches - kompliziertes/komplexes Modell \\
\hline Variation der Komplexität: & herkömmliches - modulares Planspiel \\
\hline technische Anforderungen: & $\begin{array}{l}\text { manuelles - computergestïtztes Planspiel } \\
\text { (kein) Einsatz von Planungshilfen }\end{array}$ \\
\hline Art der Durchführung: & Fern-Planspiel - Vor-Ort-Planspiel \\
\hline
\end{tabular}

Tabelle 3.1: Klassifikation von Planspielen

Bezüglich des Zeitaspekts kann ebenfalls nach verschiedenen Planspiel-Typen unterschieden werden: Zum einen ist der Umfang der simulierten Zeiträume im Modell entscheidend. Durch die Simulation können die Effekte im Zeitraffer dargestellt werden. Zum anderen sind periodische oder kontinuierliche Simulationen möglich. Entweder springt das Modell in die nächste Periode nach Eingabe einer Entscheidung oder es wird ein kontinuierlicher Zeitverlauf vorgegeben, der sich am realen Zeitverlauf orientiert. ${ }^{31}$

30 Vgl. Ebert (Planspiel 1992), S. 31.

31 Vgl. Högsdal (Planspiele 1996), S. 15. 
Nach dem Umfang des Entscheidungsrahmens bzw. den Ebenen des Entscheidungsprozesses kann zwischen Gesamt- und Teil-Planspielen unterschieden werden. Dabei treffen die Teilnehmenden ihre Entscheidungen entweder für das gesamte simulierte Unternehmen oder nur für einen Teilbereich. ${ }^{32}$ Der Vorteil von Gesamt-Planspielen liegt in der Möglichkeit, Interdependenzen zwischen allen betrieblichen Funktionsbereichen abzubilden. Teil-Planspiele hingegen sind geeignet, um einen bestimmten Funktionsbereich genauer zu modellieren und seine Eigenarten herauszustellen. ${ }^{33}$

Hinsichtlich des Freiheitsgrads des Entscheidungsbereichs wird zwischen freien oder starren Planspielen unterschieden. Die Entscheidungen der Teilnehmenden unterliegen in einem starren Spiel festen Restriktionen, d.h. ihr Handlungsraum ist begrenzt, während dieser in freien Spielen nicht vorstrukturiert ist. ${ }^{34}$ Unternehmensplanspiele sind eher starr, indem sie ein bestimmtes Unternehmen durch ein Simulationsmodell mit festen Regeln abbilden. Sie haben den Vorteil der einfacheren Durchführung und leichteren Auswertbarkeit und sind so für den Einsatz in der Aus- und Weiterbildung besser geeignet als freie Planspiele. ${ }^{35}$

Bezüglich der Abhängigkeit der Entscheidungen wird zwischen deterministischen und stochastischen Planspielen unterschieden. Erstere sind durch eindeutig definierte Zusammenhänge zwischen den Variablen gekennzeichnet. Letztere enthalten stochastische Elemente, die nicht durch die Entscheidungen der Teilnehmenden beeinflussbar sind. Möglich sind auch teil-determinierte Modelle, in denen nur ein Teil der Variablen stochastisch modelliert ist. ${ }^{36}$ Diese zufälligen Elemente der Simulation können die Vorhersage der Auswirkung der getroffenen Entscheidungen stark beeinflussen und das Modell für die Teilnehmenden undurchschaubar machen. Die Informationen, die den Teilnehmenden gegeben werden, sollten jedoch hinreichend sicher sein. Ein gewisser Unsicherheitsfaktor kann im Planspiel

32 Vgl. z. B. zur Simulation von Produktionssystemen Bloech, Goertzen \& Maurer (Unternehmenssimulation 1992) sowie BloEch (Lehr-Lern-Effekte 1999).

33 Vgl. Ebert (Planspiel 1992), S. 30; auch Bleicher (Entscheidungsprozesse 1974), S. 2021; RoHn (Führungsentscheidungen 1964), S. 50. Zur Konzeption eines Unternehmensplanspiels mit Instrumenten zur Entscheidungsvorbereitung für das Marketing vgl. PREHM (Marketing-Unternehmensspiel 1995).

34 Vgl. Christopher (Games 1988).

35 Vgl. Ebert (Planspiel 1992), S.31; Bleicher (Entscheidungsprozesse 1974), S. 24.

36 Vgl. Rohn (Führungsentscheidungen 1964), S. 51 sowie Bleicher (Entscheidungsprozesse 1974), S. 23-24. 
abgebildet werden, darf jedoch nicht dazu führen, dass die Teilnehmenden sich selbst als wirkungslos erfahren. ${ }^{37}$

In einem interaktiven Planspiel spielen mehrere Teilnehmende gegeneinander, d. h. die Entscheidungen der anderen Teilnehmenden haben einen Einfluss auf die eigenen Ergebnisse. Ein Beispiel für interaktive Planspiele sind Unternehmensplanspiele, die eine Konkurrenz-Situation abbilden und bei denen die verschiedenen Unternehmen durch verschiedene Teilnehmende geführt werden. Bei offenen interaktiven Planspielen findet ein Informationsaustausch zwischen den Teilnehmenden statt, während dies bei geschlossenen interaktiven Planspielen nicht der Fall ist. ${ }^{38}$ Die Entscheidungen können dabei in Kleingruppen oder einzeln als individuelle Entscheidung getroffen werden. ${ }^{39}$

Interaktive Planspiele in der Aus- und Weiterbildung haben den Nachteil, dass die Simulationsergebnisse nur relativ zu bewerten sind. Ist eine Gruppe besonders stark oder hat durch Zufall gleich zu Beginn des Planspiels ein besonders gutes Ergebnis erzielt, haben die anderen Teilnehmenden u. U. wesentlich größere Schwierigkeiten, mit ihren Unternehmen ebenfalls gute Ergebnisse zu erzielen. Da aber eine Zusammensetzung der Gruppen mit gleich starken Teilnehmenden auf Grund fehlender verlässlicher Größen zur Vorhersage der Entscheidungsgüte in einem Planspiel nur schwer möglich ist, empfiehlt sich der Einsatz eines interaktiven Planspiels für evaluatorische Zwecke nicht unbedingt. Weiterhin sind große Anforderungen an die Spielleitung hinsichtlich der Motivation der einzelnen Teilnehmenden zu stellen. Für den Bereich der Aus- und Weiterbildung erscheinen nicht-interaktive oder isolierte Planspiele eher empfehlenswert, da sich die Entscheidungen der Teilnehmenden untereinander nicht beeinflussen. ${ }^{40}$

RoHN spricht in diesem Zusammenhang von kompetetiven bzw. nichtkompetetiven Planspielen, ${ }^{41}$ was jedoch keine Aussage über die KonkurrenzSituation der simulierten Unternehmung beinhaltet: Im Planspiel-Modell kann entweder ein Monopol-Unternehmen abgebildet werden oder die KonkurrenzUnternehmen werden durch das Modell simuliert. Insofern kann es auch nicht-

37 Vgl. die Ausführungen in Abschnitt 2.3.

38 Vgl. Rohn (Führungsentscheidungen 1964), S. 52-53 sowie BlEICHER (Entscheidungsprozesse 1974), S. 26.

39 Vgl. Högsdal (Planspiele 1996), S. 15; auch Rohn (Führungsentscheidungen 1964), S. 52.

40 Vgl. Ebert (Planspiel 1992), S. 32; auch Fassheber (Planspiele 1995).

41 Vgl. Rohn (Führungsentscheidungen 1964), S. 51-52. 
interaktive Planspiele geben, in denen das zu führende Unternehmen als Polypolist agiert. ${ }^{42}$

Hinsichtlich der Komplexität des Spielmodells wird zwischen einfachen und komplexen Planspielen unterschieden. Als mögliche Unterscheidung wird die Anzahl der Entscheidungen, die getroffen werden, oder die Anzahl der Produkte und Märkte in einem Unternehmensplanspiel vorgeschlagen. Weiterhin wird hier zwischen Komplexität und Kompliziertheit eines Planspiels unterschieden. Dabei wird der Vernetzungsgrad der Entscheidungen untereinander als Kriterium herangezogen. ${ }^{43}$ Das Ausmaß der Komplexität ist in diesem Zusammenhang jedoch abhängig von dem Handlungsrepertoire, das den Teilnehmenden zur Verfügung steht, und sollte nicht auf Grund externer Merkmale bestimmt werden. ${ }^{44}$ Wird die Komplexität des Modells im Zeitverlauf variiert, wird von einem modularen im Gegensatz zum herkömmlichen Planspiel gesprochen. ${ }^{45}$

Weiterhin wird zwischen computergestützten Planspielen, bei denen die Auswertung mit Hilfe eines Computerprogramms vorgenommen wird, und manuellen Planspielen, bei denen die Spielleitung die Auswertung ohne solche Software durchführen kann, unterschieden. Im Bereich der Aus- und Weiterbildung bietet sich der Einsatz von computergestützten Planspielen an, da diese eine bessere Handhabung bieten sowie eine objektivere Auswertung ermöglichen. ${ }^{46}$ Hier gibt es auf Grund der technischen Entwicklung mittlerweile die Möglichkeit, dass die Teilnehmenden selbst die Dateneingabe am PC vornehmen. Manche Planspiele bieten darüber hinaus die Möglichkeit, durch integrierte Planungshilfen die Entscheidung zu unterstützen. ${ }^{47}$ Der Einsatz des Computers im Rahmen des Planspiels kann jedoch auch von den eigentlichen Lernzielen ablenken, da die Teilnehmenden mehr mit dem Umgang mit der Hard- und Software beschäftigt sind als mit dem eigentlichen Unternehmensplanspiel. ${ }^{48}$

\footnotetext{
42 Vgl. Bleicher (Entscheidungsprozesse 1974), S. 26.

43 Vgl. Högsdal (Planspiele 1996), S. 15.

44 Vgl. Kastner (Denken 1995), S. 30-31. Zur Begriffsdefinition vgl. Abschnitt 2.5.2.

45 Vgl. Högsdal (Planspiele 1996), S. 15; auch Gerlach (Weiterbildung 1996). Vgl. hierzu auch die Ausführungen zum Einfluss der Komplexität des Planspiel-Modells auf den Lernerfolg in Abschnitt 3.6.1.

46 Vgl. EBert (Planspiel 1992), S. 34; auch Rohn (Führungsentscheidungen 1964), S. 53.

47 Vgl. Högsdal (Planspiele 1996), S. 15.

48 Vgl. Heidack (Lerninstrument 1992), S. 118; kontrovers dazu vgl. Schunck (Subjektive Theorien 1993), S.67.
} 
Ein weiteres Kriterium ist die Art der Durchführung des Unternehmensplanspiels. Sind alle Parteien an einem Ort, so wird das Planspiel als Vor-Ort-Planspiel bezeichnet. Bei Fern-Planspielen sind Teilnehmende und Spielleitung räumlich voneinander getrennt, was insbesondere in der Aus- und Weiterbildung Probleme hinsichtlich der Betreuung während der Entscheidungsphasen bringen kann. ${ }^{49}$

Mit dem Einsatz von Unternehmensplanspielen in der Aus- und Weiterbildung werden verschiedene Ziele verfolgt. Sie können ebenfalls als Kriterien zur Unterscheidung herangezogen werden. ${ }^{50}$ Bevor diese Lernziele genauer dargestellt werden, soll im nächsten Abschnitt auf die Möglichkeiten der Abbildung der Unternehmensplanung in Planspiel-Modellen sowie ihre Umsetzung eingegangen werden.

\subsection{Planung im Unternehmensplanspiel}

\subsubsection{Grundlagen der Unternehmensplanung}

Planung ist die "gedankliche Vorwegnahme zukünftigen Handelns"51, also das Abwägen zwischen alternativen Handlungsmöglichkeiten zur Erreichung von gesetzten Zielen. ${ }^{52}$ Damit die Führung eines Unternehmens auf Dauer erfolgreich sein kann, wird planvolles Wirtschaften vorausgesetzt. Eine wichtige Grundlage betrieblicher Planungs- und Entscheidungsprozesse bildet die Informationsbeschaffung. ${ }^{53}$ Weiterhin spielen die Erwartungen an die Handlungsfolgen, die die Auswahl der Alternativen beschränken, eine Rolle. ${ }^{54}$

Zur Identifikation von Chancen und Risiken des Unternehmens ist eine Analyse und Prognose der Umwelt vorzunehmen. Dabei beeinflussen Entwicklungen der globalen Umwelt das Unternehmen nur indirekt, indem sie die Rahmenbedingungen für die Unternehmung und die unternehmenspezifische Umwelt festlegen. ${ }^{55}$

\footnotetext{
49 Vgl. Rohn (Einsatzgebiete 1995) sowie Ebert (Planspiel 1992), S. 34.

50 Vgl. Rohn (Methodik 1980), S. 21.

51 WöHE (Einführung 1996), S. 140.

52 Vgl. Adam (Planung 1996), S. 1-3.

53 Vgl. Korndörfer (Unternehmensführungslehre 1995), S. 104-106.

54 Vgl. auch das Erwartung-mal-Wert-Modell in Abschnitt 2.3.2.

55 Vgl. Götze \& Rudolph (Planung 1994), S.6.
} 
Ausschlaggebend für das Risiko bzw. die Unsicherheit unternehmerischen Handelns ist die Dynamik der Entwicklung. Das Unternehmen selbst hat nur wenig Möglichkeiten, Einfluss auf die globale Umwelt zu nehmen. ${ }^{56}$

Demgegenüber wirkt die unternehmensspezifische Umwelt, zu denen die Kunden, die Konkurrenten, die Lieferanten, die Anteilseigner und Kapitalgeber, Gewerkschaften und staatliche Institutionen zählen, direkt auf das Unternehmen ein. Hier kann das Unternehmen durch verschiedene Handlungen Einfluss nehmen. Eine besondere Stellung nehmen dabei die Marktbedingungen und die KonkurrenzSituation des Unternehmens ein. Sie gelten als grundlegende Einflussfaktoren auf die Aufgaben der Unternehmensplanung. ${ }^{57}$

Mit zunehmender Dynamik der Umwelt steigen auch die Anforderungen an die Planung der Unternehmung: ${ }^{58}$.Je komplexer sich die Problemstellungen darstellen, umso mehr muss das Management Systeme entwickeln, die einen Beitrag zur Reduzierung der Komplexität sowie zur Strukturierung und Lösung des Problems leisten können. Dabei kann auf die Grundlagen der Systemtheorie zur Betrachtung der Zusammenhänge zurückgegriffen werden. ${ }^{59}$

Als Unternehmensziel kann die Maximierung verschiedener Kennzahlen wie der Gewinn oder Rentabilitäten dienen, die Erhaltung der Liquidität einer Unternehmung, der Sicherheit sowie ihre Unabhängigkeit. ${ }^{60}$ Ausgangspunkt aller unternehmerischen Planungen ist nach dem GuTENBERG'schen Ausgleichsgesetz der Planung der Bereich, in dem ein Engpass auftritt. ${ }^{61}$ Ist dieser Bereich identifiziert, werden alle anderen Zielgrößen der verschiedenen Unternehmensbereiche simultan geplant. ${ }^{62}$

Die taktische Planung als mittelfristige Planung stellt das Bindeglied zwischen der strategischen und operativen Planung dar: ${ }^{63}$ Sie ist detaillierter als die stra-

56 Vgl. Hammer (Unternehmensplanung 1998), S. 39.

57 Vgl. Götze \& Rudolph (Planung 1994), S. 8.

58 Vgl. Hammer (Unternehmensplanung 1998), S. 16-18.

59 Vgl. BaEtge (Systemtheorie 1974).

60 Vgl. Perridon \& Steiner (Finanzwirtschaft 1995), S. 10-15.

61 Vgl. Gutenberg (Grundlagen 1983), S. 164-165.

62 Zu verschiedenen Aspekten der Unternehmensplanung und Controlling vgl. BogAsCHEWSKY \& GöTzE (Unternehmensplanung 1998).

63 Vgl. Adam (Planung 1996), S. 314-318; auch CARL \& KiEsEL (Unternehmensführung 1996), S. 26-27. Zu verschiedenen Aspekten der strategischen Planung vgl. BLoEcH et al. (Planung 1994). 
tegische Planung und sollte kontinuierlich die Entwicklung der Umwelt und des Unternehmens analysieren.

Die mittel- und langfristig angelegten Ziele sind in der operativen Planung in konkrete Maßnahmen umzusetzen. Sie zielt dabei auf sachlich und zeitlich genau abgegrenzte Teilbereiche der Unternehmung und ist auf Kontrollierbarkeit angelegt. Hier werden die in der strategischen Planung identifizierten Grobziele in ein System von Feinzielen überführt, das sich z. B. an den betrieblichen Funktionsbereichen orientiert. ${ }^{64}$ Die Teilpläne werden im erfolgs-, leistungs- oder finanzwirtschaftlichen Bereich aufgestellt (Abbildung 3.2). Dabei kann die Erstellung aller Pläne simultan erfolgen oder sich an einem vorgegebenen Plan orientieren. ${ }^{65}$

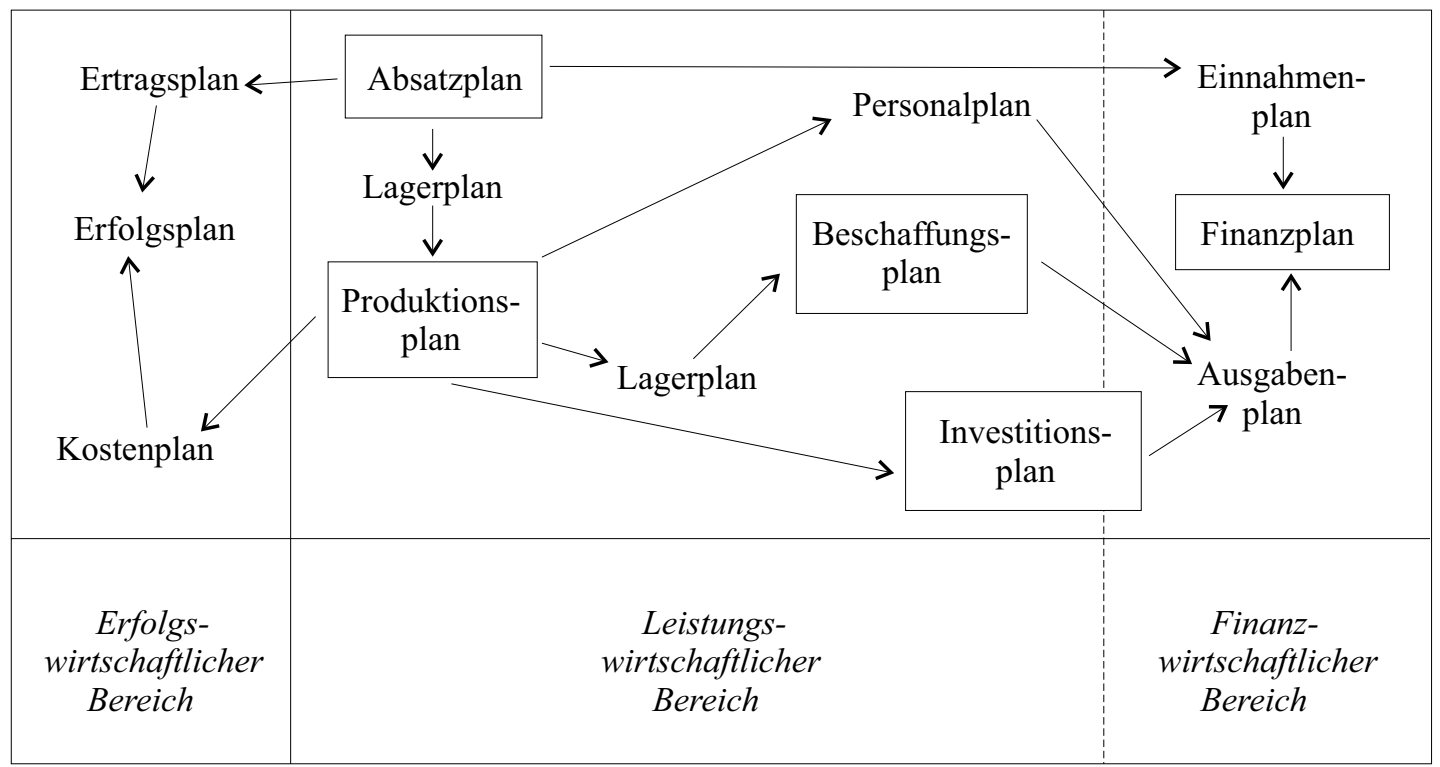

Abbildung 3.2: Teilpläne der Unternehmensplanung ${ }^{66}$

Im Modell eines Unternehmensplanspiels werden eventuell einige Funktionsbereiche einer Unternehmung ausgeblendet, um den Fokus auf einen Bereich zu lenken. ${ }^{67} \mathrm{Im}$ Folgenden soll jedoch von einem generellen Unternehmensplanspiel ausgegangen werden, in dem alle Funktionsbereiche abbildet werden.

\footnotetext{
64 Vgl. Hammer (Unternehmensplanung 1998), S. 59-62; auch WöHE (Einführung 1996), S. $153-155$.

65 Vgl. Carl \& Kiesel (Unternehmensführung 1996), S. 28-30; auch Korndörfer (Unternehmensführungslehre 1995), S. 111-113.

66 In Anlehnung an Olfert (Finanzierung 1994), S. 106.

67 Vgl. Abschnitt 3.2.3.
} 
In vielen Fällen ist eine Orientierung der Unternehmensplanung am Absatzbereich sinnvoll. ${ }^{68}$ Dabei kann sich im Rahmen der langfristigen strategischen Planung ${ }^{69}$ z. B. am konjunkturellen Verlauf orientiert werden: Jedes Produkt durchläuft nach dem Lebenszyklus-Konzept die Phasen der Einführung am Markt, des Wachstums und der Reife, der Sättigung, des Verfalls sowie des Absterbens. ${ }^{70}$ Das Einbeziehen eines konjunkturellen Verlaufs im Planspiel-Modell stellt eine zusätzliche Möglichkeit der realitätsnäheren Abbildung dar.

Im Folgenden werden die Absatzplanung, die Produktions- und Beschaffungsplanung sowie die Investitions- und Finanzplanung genauer betrachtet. Ertragsund Kostenplan, die zum Erfolgsplan führen, benötigen Informationen des betrieblichen Rechnungswesens. Die Lagerpläne, die zwischen Absatz- und Produktionsplan sowie zwischen Produktions- und Beschaffungsplan erstellt werden, sollen in die jeweils vorgeschalteten Pläne integriert werden. Ebenso kann die grundsätzliche Planung des Personalbedarfs im Rahmen des Produktionsplans geschehen. Darüber hinaus sind jedoch Aspekte wie die Aus- und Weiterbildung der Mitarbeiterinnen und Mitarbeiter sowie die Disposition der Führungskräfte eigenständig zu planen. ${ }^{71}$ Die Pläne der Einnahmen- und Ausgaben werden als Basis des Finanzplans erstellt.

\subsubsection{Absatzplanung}

Die Absatzplanung setzt sich aus drei Bereichen zusammen: ${ }^{72}$ Die Marktforschung untersucht die Verhältnisse am Markt und bringt Erkenntnisse über die Nachfrage, die Konkurrenz und die Absatzwege. Die Analyse des eigenen Absatzes ermittelt die Struktur der Abnehmenden und eventuelle Abhängigkeiten zwischen den eigenen Produkten. Hier können die mengen- und wertmäßjigen Umsatzstatistiken zu Kennzahlen verarbeitet werden. Mit Hilfe der ersten beiden Schritte

68 Vgl. Hopfenbeck (Managementlehre 1992), S.582-583; auch CarL \& Kiesel (Unternehmensführung 1996), S. 30.

69 Zur Klassifikation von Planung in zeitlicher Hinsicht vgl. BLoEcH et al. (Einführung 1998), S. 118-119.

70 Vgl. Meffert (Marketing 1991), S. 370-371.

71 Vgl. Hammer (Unternehmensplanung 1998), S. 66.

72 Vgl. Wöнe (Einführung 1996), S.608-631; auch HAMmer (Unternehmensplanung 1998), S. 62-63. 
werden Prognosen über den Absatz aufgestellt, der aus Schätzungen der zukünftigen Marktlage und der Berechnung des zukünftigen Absatzes besteht.

Ein wichtiger Teilbereich der Absatzplanung betrifft die Festlegung des absatzpolitischen Instrumentariums: ${ }^{73}$

- Die Produktpolitik bezieht sich auf die Gestaltung der Absatzleistungen und umfasst Entscheidungen, die die Neuentwicklung, die Differenzierung, die Änderungen und die Eliminierung der Produkte betreffen. Der Kundendienst spielt dabei als Zusatzleistung eine wesentliche Rolle.

- Die Kontrahierungspolitik umfasst die Gesamtheit der vertraglichen Vereinbarungen über das Leistungsangebot, insbesondere über die Preispolitik.

- Die Distributionspolitik bezieht sich auf Entscheidungen, die im Zusammenhang mit dem Weg des Produkts zu den Endverbrauchern stehen.

- Die Kommunikationspolitik schließlich beinhaltet die Instrumente der Werbung, der Verkaufsförderung sowie der Öffentlichkeitsarbeit.

Alle vier Bereiche zusammen werden unter dem Begriff Marketing-Mix zusammengefasst. ${ }^{74} \mathrm{Ihm}$ kommt die Aufgabe zu, durch die Umsetzung der MarketingStrategien in operative Maßnahmen die Zielerreichung im Absatzbereich zu sichern: ${ }^{75}$

- Betriebsgerichtete Ziele betreffen die Anpassung des Markts an den Produktionsprozess, die Vollbeschäftigung oder die Verwirklichung einer optimalen Kostensituation.

- Als marktgerichtete Ziele gelten die Maximierung der Absatzmengen oder des Umsatzes, die Erhaltung eines gewissen Kundenstamms, das Erreichen einer Vorzugsstellung im Wettbewerb bzw. der Gewinn von Marktanteilen sowie die Beeinträchtigung bis zur Ausschaltung von Konkurrenten.

Diese Ziele können neben der Gestaltung der Verkaufspreise (Kontrahierungspolitik) mittels der Werbung (Kommunikationspolitik) erreicht werden. Durch sie werden die den Konsum steuernden Einstellungen und die daraus resultierenden Entscheidungsprozesse für oder wider den Kauf eines Produkts beeinflusst. Dabei

\footnotetext{
73 Vgl. Meffert (Marketing 1991), S. 114-120.

74 Vgl. auch die Übersicht bei WöHE (Einführung 1996), S. 635.

75 Vgl. Meffert (Marketing 1991), S. 263.
} 
wird zum einen versucht, die Bedürfnisbildung der Konsumierenden durch eine Strukturierung ihrer Bedürfnisse zu verändern. Zum anderen wird der besondere Nutzen des Produkts zur Befriedigung der Bedürfnisse herausgestellt. ${ }^{76}$

Neben der inhaltlichen und medialen Ausgestaltung der Werbung ist die Entscheidung über die Höhe des Werbe-Etats besonders wichtig. Grundsätzlich kann er sowohl heuristisch als auch analytisch festgelegt werden: ${ }^{77}$ Die heuristische Festlegung orientiert sich an Größen wie dem Umsatz, dem Gewinn, den verfügbaren finanziellen Mitteln sowie den Werbeaufwendungen der KonkurrenzUnternehmen, soweit die Zahlen verfügbar sind. Im Gegensatz dazu versuchen analytische Ansätze, Werbebudgets mit zugehörigen Ziel-Erreichungs-Funktionen zu verknüpfen. Dabei stellt die Informationsbeschaffung bezüglich der Auswirkung der Werbung auf das Verhalten der Konsumierenden einen Unsicherheitsfaktor dar.

Im Rahmen der Produktpolitik wird mit dem Kundendienst das Ziel verfolgt, sich durch das Angebot entgeltlicher oder unentgeltlicher Zusatzleistungen gegenüber der Konkurrenz abzugrenzen. Die Bedeutung des Kundendienstes liegt in der Herausbildung von Präferenzen bei den Konsumierenden, die auch einen preispolitischen Spielraum schaffen. ${ }^{78}$

Die Abbildung des Absatzbereichs in einem Unternehmensplanspiel wird an die Komplexität der Realität nicht heranreichen. Je nach Zielsetzung des PlanspielEinsatzes werden die einzelnen Politiken unterschiedlich stark hervorgehoben. Entscheidend für die Absatzplanung ist auch die Marktform, wobei die Anzahl der Anbieter und Nachfrager bzw. die Beziehungen zwischen ihnen die Handlungen beeinflussen. $^{79}$

\subsubsection{Produktions- und Beschaffungsplanung}

Unter Produktion wird der gelenkte Einsatz von Produktionsfaktoren - also Gütern und Dienstleistungen - zum Rohstoffabbau bzw. zur Herstellung von Er-

\footnotetext{
76 Vgl. WöнE (Einführung 1996), S. 697-699.

77 Vgl. Nieschlag, Dichtl \& Hörschgen (Marketing 1997), S. 586-593.

78 Vgl. MefFert (Marketing 1991), S. 411-415.

79 Vgl. WöнE (Einführung 1996), S.634.
} 
zeugnissen und zur Erbringung von Dienstleistungen verstanden. ${ }^{80}$ Im engeren Sinne umfasst der Begriff die betriebliche Leistungserstellung, im Gegensatz zur Leistungsverwertung, die dem Absatzbereich zugeordnet wird. ${ }^{81}$ Von der Modellierung des Produktionsbereichs im Unternehmensplanspiel hängt im Wesentlichen die gesamte Gestaltung des Planspiel-Modells ab. Dabei ist auch die Abbildung eines Unternehmens der Dienstleistungsbranche wie einer Bank oder einer Versicherung denkbar. Im Folgenden soll jedoch von einem Unternehmen ausgegangen werden, das Güter herstellt.

Unter dem Ziel der Gewinnmaximierung wird im Bereich der Produktion eine Minimierung der Kosten anzustreben sein, da die Erlöse dem Absatzbereich zuzuordnen sind, die bei isolierter Betrachtung des Produktionsbereichs als konstant anzunehmen sind. Teilaufgaben der Produktionsplanung sind die Bestimmung des Produktionsprogramms, die Festlegung der Produktionsprozesse sowie die Bereitstellung der erforderlichen Produktionsfaktoren. ${ }^{82}$

Die Planung des Produktionsprogramms kann hinsichtlich des zeitlichen Rahmens unterteilt werden: ${ }^{83}$

- Im Rahmen der strategischen Programmplanung werden die Erfolgspotentiale verschiedener Produktfelder identifiziert und das zur Erreichung des Unternehmensziels am besten geeignete ausgewählt.

- Die taktische Programmplanung bezieht sich auf die Frage nach der Einführung neuer Produkte, der Modifikation sowie der Elimination bereits bestehender Produkte.

- Die operative Programmplanung hat die Aufgabe, die durch die strategische Planung vorgegebenen Restriktionen optimal zu nutzen. Dazu gehört unter anderem die zeitliche Abstimmung zwischen den Produktions- und den Absatzmengen.

Die zweite Komponente der Produktionsplanung ist die Durchführungsplanung, die von der Struktur der zu Grunde liegenden Produktionsprozesse abhängt. Dabei kann der Produktionsprozess nach den Eigenschaften der Produkte, der Struk-

\footnotetext{
80 Vgl. Bloech \& Ihde (Logistiklexikon 1997), S. 812.

81 Vgl. Wöнe (Einführung 1996), S. 463.

82 Vgl. Bloech \& Ihde (Logistiklexikon 1997), S. 823.

83 Vgl. WöHe (Einführung 1996), S. 533-539; auch BLOECH et al. (Einführung 1998), S.118131.
} 
tur der Produktion sowie nach den Eigenschaften des Produktionsprogramms typisiert werden. Innerhalb dieser Typisierungen gibt es verschiedene Merkmale mit unterschiedlichen Ausprägungen: ${ }^{84}$ So kann z. B. zwischen materiellen und immateriellen oder standardisierten und individuell gestalteten Produkten unterschieden werden. Im Bereich der Struktur der Produktion kann zwischen Fließ-, Gruppen-, Werkstatt- und Baustellen-Fertigung differenziert werden. Weiterhin wird die Planung u. a. durch die Zahl der Arbeitsgänge - einstufig versus mehrstufig - sowie die Verbundenheit der Produktion mehrerer Güter determiniert.

Die Abbildung des Produktionsprozesses beeinflusst die Komplexität des Planspiel-Modells, wobei alle Variationen von einer einstufigen Fertigung eines einzelnen Produkts ${ }^{85}$ bis hin zu einer mehrstufigen Produktion mit verschiedenen Zwischenprodukten und der Berücksichtigung von Qualitätsaspekten abgebildet werden können. ${ }^{86}$ In einigen Modellen können die Einstellungen durch die Spielleitung variiert werden. ${ }^{87}$

Sind Produktionsprogramm und -prozess durch die Planung festgelegt, kann auf die Bereitstellung des Materials eingegangen werden. Dabei kann auch diese Planung wiederum einen Einfluss auf die Planung des Produktionsprogramms haben, z. B. wenn ein Engpass bei einem bestimmten Rohstoff auftritt. Unter dem bereitzustellenden Material werden die drei Produktionsfaktoren Betriebsmittel, Arbeitskräfte sowie Werkstoffe verstanden. ${ }^{88}$ Die Beschaffung der Arbeitskräfte und Betriebsmittel kann jedoch bereits im Rahmen der Planung des Produktionsprogramms vorgenommen werden.

Unter dem enger gefassten Begriff der Materialwirtschaft wird dann die Beschaffung der Werkstoffe verstanden. ${ }^{89}$ Darunter fallen die Roh-, Hilfs- und Betriebsstoffe sowie Zulieferteile und Handelswaren. ${ }^{90}$ Es muss eine Ermittlung der Be-

84 Vgl. BLohm et al. (Produktionswirtschaft 1997), S. 243.

85 So z. B. in den Planspielen JEANSFABRIK, vgl. Preiss (Planspiel 1994), und EpUS, vgl. Bloech \& Rüscher (Modellbeschreibung 1995).

86 Z. B. die Planspiele SIM-LOG, vgl. Bloеch \& Rüscher (Unternehmenssimulation 1991), PENTA, vgl. Bloech \& Rüscher (Unternehmenssimulation 1992), und MARKUS, vgl. Prenm (Marketing-Unternehmensspiel 1995).

87 Vgl. z. B. in den Planspielen PUMA, vgl. Bloech \& Rüscher (Marketing-Simulation 1992), und MEdUSa, vgl. OrTh (Entwicklung 1998).

88 Vgl. Gutenberg (Grundlagen 1983), S. 171.

89 Vgl. WöHe (Einführung 1996), S. 539-555.

90 Vgl. Bloech \& Ihde (Logistiklexikon 1997), S. 706, 2.Sp. 
darfsmengen sowie eine Festlegung der Bestellmengen und -zeitpunkte vorgenommen werden. Weiterhin müssen eventuell optimale Sicherheitsmengen bei den Lagerbeständen bestimmt werden. Hierzu stehen eine Vielzahl von Berechnungsverfahren zur Verfügung. ${ }^{91}$

\subsubsection{Investitions- und Finanzplanung}

Die Planung von Investitionen ist für Unternehmen von großer Bedeutung, da durch sie häufig finanzielle Mittel für einen längeren Zeitraum gebunden werden und so den Unternehmenserfolg beeinflussen. ${ }^{92}$ Nach der Art der Investitionsobjekte lassen sich Finanz- und Sach-Investitionen unterscheiden. Die Entscheidungen über Investitionen bzw. Desinvestitionen im Sachbereich hängen eng mit der Produktionsplanung zusammen. Hier können Ersatz- oder ErweiterungsInvestitionen vorgenommen werden. Erstere lassen sich weiter nach identischem Ersatz und Rationalisierungs-Investitionen differenzieren. ${ }^{93}$

Grundsätzlich sind Investitionsobjekte durch Aus- und Einzahlungen gekennzeichnet, ${ }^{94}$ wobei zwischen verschiedenen Arten unterschieden werden kann: ${ }^{95}$

- Erstens können sie als Auszahlungen im Bereich des Absatzes und der Beschaffung auf Gütermärkten sowie für die disponierenden oder produzierenden Tätigkeiten, die diese Markthandlungen begleiten, auftreten.

- Zweitens können sie als Nachfragehandlungen auf Finanzmärkten zur Schaffung zukünftiger Einzahlungen dienen.

- Schließlich können durch Vertragsgestaltung und organisatorische Maßnahmen Institutionen gebildet werden, um Unsicherheiten zukünftiger Einnahmen oder Ausgaben zu verringern. Hierzu zählen die Gründung von Subunternehmen oder Zusammenschlüsse von kleineren Unternehmen.

Zur Beurteilung der Vorteilhaftigkeit alternativer Objekte können verschiedene Verfahren der Investitionsrechnung herangezogen werden. ${ }^{96}$ In einem Unterneh-

91 Vgl. BloEch et al. (Einführung 1998), S. 167-228.

92 Vgl. Götze \& Bloech (Investitionsrechnung 1995), S.1.

93 Vgl. Perridon \& Steiner (Finanzwirtschaft 1995), S. 29-30.

94 Vgl. Schneider (Investition 1992), S. 34.

95 Vgl. Schneider (Investition 1992), S. 10-11.

96 Vgl. Götze \& BloEch (Investitionsrechnung 1995). 
mensplanspiel kann die Investitionsplanung z. B. anhand des Kapazitätsbedarfs der Produktion dargestellt werden. Dabei kann der Fokus auf den Vermögensendwert als Zielgröße der Investitionsrechnung gelenkt werden. ${ }^{97}$

Finanzierungen sind unternehmerische Handlungen, die Geldmittel für Investitionen bereitstellen. Dabei kann im Hinblick auf die Kapitalherkunft zwischen Innen- und Außenfinanzierung unterschieden werden: ${ }^{98}$

- Die Außenfinanzierung umfasst eine Geldaufnahme auf Finanzmärkten,

- während die Innenfinanzierung ein zeitliches Vorverlegen von Einnahmen bzw. ein zeitliches Hinausschieben von Ausgaben bedeutet, ohne dass dabei das Unternehmen als Nachfrager auf Finanzmärkten auftritt und ohne dass geplante leistungswirtschaftliche Tätigkeiten korrigiert werden.

- Weiterhin ist auch hier eine Institutionenbildung denkbar, um Unsicherheiten hinsichtlich der zukünftigen Zahlungen der Innen- oder Außenfinanzierung zu verringern. Hierzu zählen u. a. Verträge mit Kreditnehmern über eine Verlängerung der Zahlungsfrist oder Bürgschaften.

Ein Unternehmen finanziert sich von innen insbesondere durch den Rückfluss finanzieller Mittel aus dem Umsatzprozess, so z. B. aus Gewinnen. Dabei können rechtliche Vorschriften diese Selbstfinanzierung vorschreiben. ${ }^{99}$ Die Finanzierung aus gesetzlichen bzw. freiwilligen Rücklagen oder Rückstellungen für ungewisse, zukünftige Verpflichtungen zählt zur Innenfinanzierung, indem Gewinne einbehalten wurden. Unter die Innenfinanzierung fallen auch Finanzierungen durch Nutzung der Spielräume bei Ansatz und Bewertung z. B. des Umlaufvermögens. ${ }^{100}$

Von außen kann sich ein Unternehmen durch eine Erhöhung der Einlagen der Gesellschafter oder durch eine Beteiligung an anderen Kapitalgesellschaften finanzieren. Weiterhin fließen durch die Aufnahme von Krediten bei Bankinstituten finanzielle Mittel in das Unternehmen. ${ }^{101}$

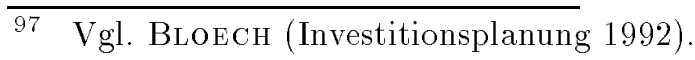

98 Vgl. DrukarczyK (Finanzierung 1996), S. 3-14; auch Schneider (Investition 1992), S. 11-13.

99 So sind z. B. gesetzliche Rücklagen in einer Aktiengesellschaft vorgeschrieben; vgl. 1150 Abs. 1 und 2 AktG.

100 Sog. 'Stille Rücklagen'; vgl. WöHe (Einführung 1996), S. 1068-1071.

101 Vgl. Perridon \& Steiner (Finanzwirtschaft 1995), S. 319-323. 
Nach der Rechtsstellung der Kapitalgeber wird zwischen Eigen- und Fremdfinanzierung unterschieden: ${ }^{102}$

- Die Eigenfinanzierung bedeutet eine Finanzierung aus eigenen Mitteln,

- während die Fremdfinanzierung eine Finanzierung durch fremde Mittel umfasst.

Finanzielle Mittel können in Form von Beteiligungen oder Einlagen von den Anteilseignern dem Unternehmen zugeführt werden. Unter die 'stille Selbstfinanzierung', d.h. eine Finanzierung, die nicht als solche direkt sichtbar ist, fällt eine Finanzierung aus Abschreibungen, wenn z. B. Geldmittel aus dem Umsatzprozess über die Gewinne zurück in das Unternehmen geflossen sind. Die Kosten der Abschreibungen wurden also in die Verkaufspreise der Produkte eingerechnet und durch die Umsatzerlöse erwirtschaftet. Eigenfinanzierung hat den Vorteil, dass keine Zinszahlungen anfallen. Eine Verzinsung des Eigenkapitals wird jedoch in Form von kalkulatorischen Zinsen als Kosten bei der Preiskalkulation berücksichtigt.

Zur Fremdfinanzierung zählt die Aufnahme von Kredite sowie die Bildung von Rückstellungen für besondere Vorkommnisse bzw. Rücklagen aus einbehaltenen Gewinnen. Der Nachteil einer Fremdfinanzierung besteht in den Zinszahlungen im Falle einer Kreditfinanzierung auf die Liquiditätslage der Unternehmung. Zinsaufwendungen müssen weiterhin durch den Verkauf der Produkte erwirtschaftet werden, erhöhen also die Preisuntergrenze. ${ }^{103}$

Eine Finanzierung durch Vermögensumschichtung bzw. Kapitalfreisetzung ist eine Innenfinanzierung, indem nicht mehr benötigte Vermögensteile verkauft werden oder durch Rationalisierungsmaßnahmen Kapazitäten frei werden, die zur Herstellung zusätzlicher Mengen genutzt werden können. Eine Trennung zwischen zwischen Eigen- und Fremdfinanzierung ist in diesem Fall häufig nicht eindeutig. ${ }^{104}$

102 Vgl. Perridon \& Steiner. (Finanzwirtschaft 1995), S. 428-433.

103 Vgl. Drukarczyk (Finanzierung 1996), S. 24.

104 Vgl. Perridon \& Steiner (Finanzwirtschaft 1995), S. 435-436. 
Die Fremdfinanzierung bietet gegenüber der Eigenfinanzierung einige Vorteile: ${ }^{105}$ Ein Vorteil liegt in der relativen Risikolosigkeit von Krediten gegenüber z. B. Beteiligungen. Die Verhältnisse der Stimmrechte der Anteilseigner verändern sich außerdem nur bei der Aufnahme eines relativ großen Kredits.

Ein weiterer Vorteil der Fremdfinanzierung lässt sich mit dem Leverage-Effekt erklären: Mit zunehmender Verschuldung, also einem relativ großen Anteil Fremdkapital am Gesamtkapital, erhöht sich die Rendite des Eigenkapitals, solange eine positive Differenz zwischen der Rendite der zusätzlich durchgeführten Investitionen und deren Fremdkapital-Kosten besteht. Letztendlich sind auch steuerliche Gesichtspunkte für die Bevorzugung einer Fremdkapital-Finanzierung ausschlaggebend, da die Zinsen vom zu versteuernden Gewinn abzugsfähig sind. Abbildung 3.3 fasst die Finanzierungsarten zusammen.

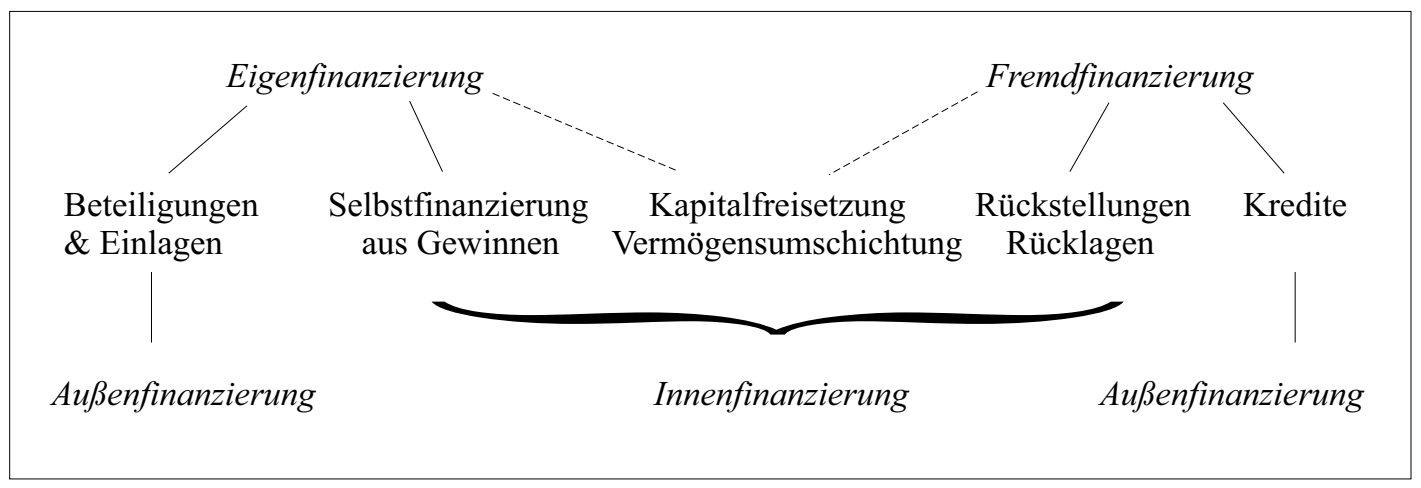

Abbildung 3.3: Finanzierungsarten ${ }^{106}$

Grundsätzliches Ziel der Finanzplanung ist die Aufrechterhaltung der Liquidität des Unternehmens. ${ }^{107}$ Unter Liquidität wird die Fähigkeit einer Unternehmung verstanden, zu jeder Zeit den bestehenden Zahlungsverpflichtungen termingerecht und betragsgenau nachzukommen. Dabei können verschiedene Liquiditätsquellen identifiziert werden: ${ }^{108}$

105 Vgl. Perridon \& Steiner (Finanzwirtschaft 1995), S. 478-484; auch Spremann (Investition 1996), S. 275-277.

106 In Anlehnung an Perridon \& Steiner (Finanzwirtschaft 1995), S. 320.

107 Vgl. Spremann (Investition 1996), S. 222.

108 Vgl. DrukarczYK (Finanzierung 1996), S. 32. 


\section{Originäre Liquiditätsquellen:}

- Die güterwirtschaftliche Liquidität bezieht sich auf vorhandene Vermögensgegenstände,

- während sich die zukünftige Liquidität auf zukünftige finanzielle Überschüsse bezieht.

\section{Abgeleitete Liquiditätsquellen:}

- Die verliehene Liquidität geht von einer Beleihung vorhandener Vermögensgegenstände aus,

- die antizipierte Liquidität von der Beleihung zukünftiger finanzieller Überschüsse.

Dabei ist die Eignung von Vermögensgegenständen und Rechten zur Beschaffung von Geld an der Veräußerungsfähigkeit am Markt, der Beleihbarkeit bei Kreditinstituten sowie der Möglichkeit der Geldwerdung im Rahmen des Unternehmensprozesses während der Selbstliquidationsperiode zu messen. Das Problem der 'optimalen Finanzierung' besteht in der unsicheren Datengrundlage, auf der die Planung aufbaut. 109

In einem Unternehmensplanspiel ist ebenfalls eine Finanzplanung durchzuführen, die sich an der Liquidität orientieren kann. Im Planspiel-Modell können jedoch auch Maßnahmen zur Verhinderung einer Illiquidität eingebaut sein, um einen Konkurs des Modell-Unternehmens abzuwenden. ${ }^{110}$ Die Planung betrifft hier eher die Vermeidung hoher Zinsbelastungen bzw. das rentabilitätsmaximierende Anlegen freier Mittel. ${ }^{111}$

Grundsätzlich sind die Möglichkeiten der Finanzierung in einem Unternehmensplanspiel von der abgebildeten Rechtsform der Unternehmung abhängig. So kann die Höhe der Rücklagen einer Aktiengesellschaft in den Parametern festgelegt werden. Einen weiteren Parameter des Modells stellen die Abschreibungen dar. Eine Finanzierung aus Gewinnen wiederum hängt von den Entscheidungen der Teilnehmenden zur Umsatz-Maximierung bzw. Aufwands-Minimierung ab. Die Höhe der Kreditfinanzierung kann ebenfalls durch die Entscheidungen beeinflusst werden.

109 Vgl. DrukarczyK (Finanzierung 1996), S. 21-22.

110 Vgl. z. B. Bloech \& Rüscher (Modellbeschreibung 1995), S. 49-50.

111 Vgl. Olfert (Finanzierung 1994), S. 32. 


\subsection{Lernziele beim Einsatz von Unternehmens- planspielen}

\subsubsection{Lernhandeln im Unternehmensplanspiel}

Mit dem Einsatz komplexer Lehr-Lern-Arrangements, ${ }^{112}$ zu denen Unternehmensplanspiele zählen, können unterschiedliche Lernziele vermittelt werden. ${ }^{113}$ Dabei ist die Handlung der Teilnehmenden im Planspiel ein zentraler Aspekt, da sie einen Einfluss auf den Lernerfolg hat, aber auch selbst als Lernziel gesehen werden kann. Zur genaueren Bestimmung soll die Handlung im Planspiel betrachtet werden. ${ }^{114}$

Als Merkmale einer allgemeinen komplexen Handlungssituation nennt DörNER die Komplexität, die Dynamik, die Intransparenz der Situation sowie die nicht ausreichenden Kenntnisse der Handelnden. ${ }^{115}$ Diese Merkmale lassen sich auch auf die Handlungssituation im Planspiel übertragen: Die Komplexität der Situation ist zwar objektiv bestimmbar, ${ }^{116}$ ihre Wahrnehmung in einer konkreten Situation ist jedoch subjektiv und kann zwischen den Teilnehmenden erheblich variieren. ${ }^{117}$ Eine wesentliche Rolle im Planspiel spielt die Dynamik der Situation, die einen Zeitdruck erzeugt und die Handelnden dazu veranlasst, genauere Überlegungen nicht durchzuführen. Auch die Unkenntnis und falsche Hypothesen über den Zustand des Systems und die Struktur des Modells führen zu einer falscher Einschätzung der aktuellen Situation.

Im Planspiel gibt es für die Teilnehmenden sichtbare und nicht-sichtbare sowie beeinflussbare und nicht-beeinflussbare Variablen, ${ }^{118}$ die auf die Intransparenz der Situation einwirken. Auf sichtbare Variablen wird z. B. in der Darstellung der Unternehmens-Ergebnisse eingegangen. Sie sind zum Teil als EntscheidungsVariablen von den Teilnehmenden zu beeinflussen. Parameter sind als sichtbare

112 Vgl. Abschnitt 2.5.

113 Vgl. Böhret \& Wordelmann (Planspiel 1975), S. 151-152; zu kognitiven Lernzielen und deren Taxonomie vgl. Abschnitt 2.4.

114 Zur Definition des Begriffs der Handlung vgl. Abschnitt 2.3.1.

115 Vgl. Dörner (Logik 1989), S. 58-66.

116 Vgl. Abschnitt 2.5.2.

117 Vgl. Abschnitt 2.3; auch Abschnitt 2.5.

118 Vgl. Dörner et al. (Lohhausen 1983), S. 28-29. 
Variablen jedoch nicht-beeinflussbar. Weiterhin gibt es nicht-sichtbare Variablen, die auch nicht durch Entscheidungen der Teilnehmenden zu beeinflussen sind. Hierzu zählen z. B. die Variablen der Preis-Absatz-Funktion.

Die Handlung im Planspiel hat zwei Ebenen der Aktivität: ${ }^{119}$ Wenn Planspiele in Gruppen gespielt werden, kann sie als zielgerichtetes und zweckrationales Entscheidungshandeln innerhalb der Gruppe verstanden werden. Sie ist somit eine externe Handlung. Zum anderen beinhaltet sie als interne Handlung der einzelnen Teilnehmenden die spielsteuernden Gedanken- und Gefühlsprozesse, die Gewinnung von Erkenntnissen im Bewusstsein der Teilnehmenden sowie das kommunikative Handeln. ${ }^{120}$

Abbildung 3.4 zeigt die Qualitätsmerkmale einer Handlung. Dabei stellt die durchgezogene Linie eine idealtypische Handlung dar, bei der alle Merkmale gleich wichtig sind und die in der Realität so nicht anzutreffen sein wird. ${ }^{121}$ Entsprechend muss für jede Handlung in der aktuellen Situation ein individuelles Muster erstellt werden. Die Anforderungen an eine (ebenfalls idealtypische) Handlung im Planspiel ist in der Abbildung durch eine gestrichelte Linie dargestellt.

Zur Beschreibung der Qualität einer Handlung im Planspiel können folgende Merkmale herangezogen werden: ${ }^{122}$

Subjektbezogenheit: Dieses Merkmal bezieht sich auf die Beschränkung der Handlungsqualität durch physische und psychische Eigenschaften des Handlungssubjekts. Im Planspiel sind physische Eigenschaften wie Körpergröße oder Sportlichkeit der Teilnehmenden nicht relevant. Dagegen spielen psychische Einflüsse eine große Rolle, zum einen in Bezug auf die Lernmotivation, zum anderen hinsichtlich der Interaktionen während der Gruppenarbeit. ${ }^{123}$

\footnotetext{
119 Zur Definition einer Handlung vgl. Abschnitt 2.3.1.

120 Vgl. Geuting (Planspiel 1992), S. 88-89.

121 Vgl. Bachmann (Grundlagen 1988), S. 45.

122 Vgl. Flothow (Förderung 1992), S. 32-50.

123 Vgl. Abschnitt 2.3.
} 


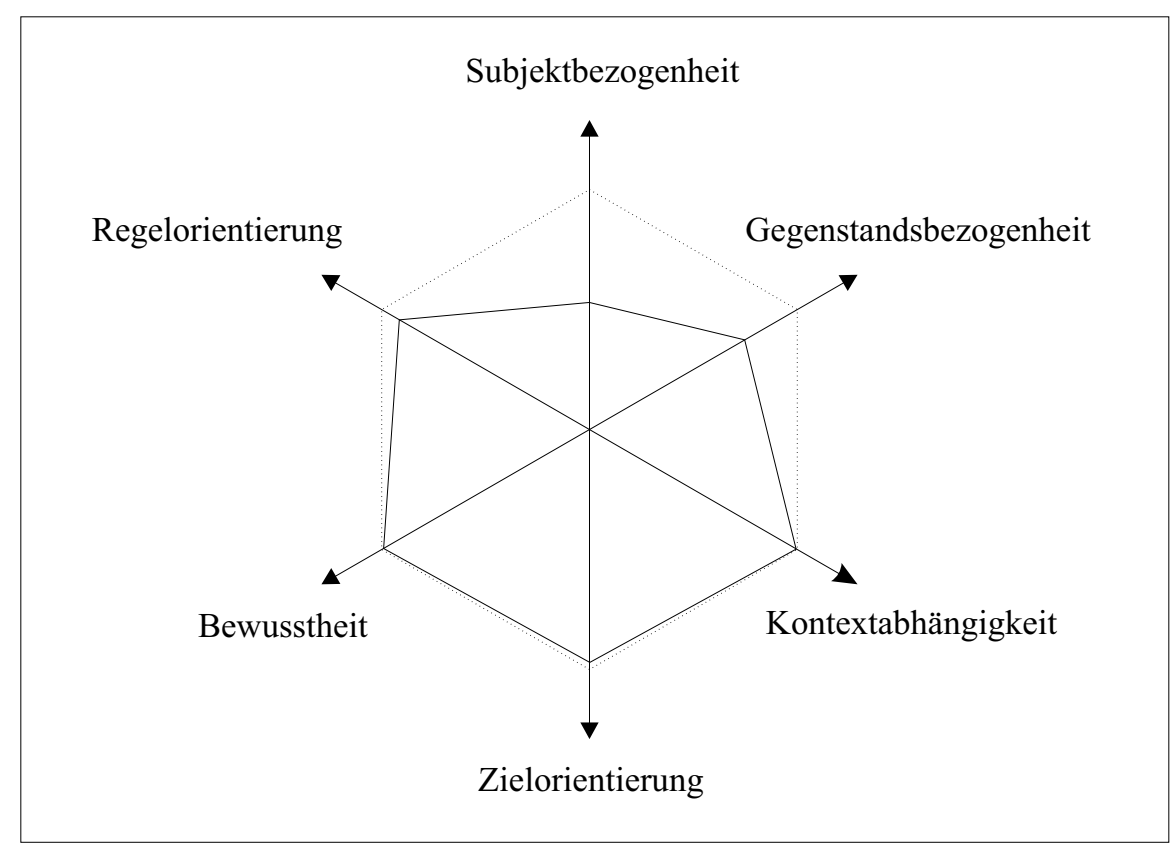

Abbildung 3.4: Qualitätsmerkmale einer Handlung ${ }^{125}$

gestrichelte Linie = idealtypische Handlung

durchgezogene Linie $=$ Handlung im Planspiel

Gegenstandsbezogenheit: Handeln ist immer auf einen Gegenstand bezogen, der jedoch nicht unbedingt physischer Natur sein muss. Wichtig ist hierbei, dass der Lehrgegenstand von den Teilnehmenden als Lerngegenstand angenommen wird. Dies ist im Planspiel insofern wichtig, als dass die Teilnehmenden die simulierte Situation ernst nehmen müssen, damit sie sich aufgefordert fühlen zu handeln. ${ }^{126}$

Kontextabhängigkeit: Ein wichtiges Merkmal ist die Kontextabhängigkeit. Die Handlung im Planspiel ist abhängig von der bisherigen Entwicklung der simulierten Unternehmung sowie der aktuellen Situation. Dabei ist dieser Faktor durchaus interpretativ zu verstehen, d. h. die Teilnehmenden müssen die vorliegenden Informationen erst deuten, um sie in sinnvolle Handlungen umzusetzen. Dieses Merkmal ist im Planspiel entsprechend wichtig, da bei Nichtbeachtung des Kontextes mitunter Entscheidungen getroffen werden, die nicht zu den gewünschten Ergebnissen führen.

\footnotetext{
125 Modifiziert für die Handlung im Planspiel nach Flothow (Förderung 1992), S. 36.

126 Vgl. Sembill (Problemlösefähigkeit 1992), S. 79.
} 
Zielorientierung: Im Planspiel dient die Handlung als Mittel zur Erreichung von Zielen. Dabei wird sich an den von den Teilnehmenden gesetzten oder durch die Lehrenden vorgegebenen Zielen orientiert.

Selbstkontrolle: Reflex- oder Instinkthandlungen führen in einem Planspiel nicht zum gewünschten Erfolg. Es muss eine Selbstkontrolle der Handlung stattfinden, da ohne die Kontrolle der eigenen Handlung und deren Auswirkungen ein Lernen kaum möglich ist. ${ }^{127}$ Diese Kontrolle wird durch die Mehrperiodizität des Planspiels gewährleistet. Auf der einen Seite werden die Handlung in wechselnden Kontexten wiederholt, auf der anderen Seite bekommen die Teilnehmenden mehrfach eine Rückmeldung über die Ergebnisse. $^{128}$

Regelorientierung: Schließlich ist die Regelorientierung ein Merkmal der Handlungsqualität. Sie beinhaltet ein aktives Auseinandersetzen mit den Normen und Werten, die im Kontext der Handlung gelten. Grundsätzlich sind Planspiele eher regelbasiert, jedoch kann durch die Modellierung eine mehr oder weniger starke Einschränkung der Regeln vorgenommen werden. In der Betriebswirtschaftslehre spielen normative Fragen jedoch immer eine Rolle, so dass dieser Punkt auch ein Ziel beim Einsatz von Planspielen in der Ausund Weiterbildung sein sollte. ${ }^{129}$

Die Lernziele, die mit dem Einsatz von Unternehmensplanspielen verfolgt werden, werden im folgenden Abschnitt näher betrachtet.

\subsubsection{Klassifikation der Lernziele}

Die Lernziele, die mit dem Einsatz eines Unternehmensplanspiels verfolgt werden, können nach verschiedenen Kriterien klassifiziert werden. In diesem Abschnitt sollen hierfür die für den kaufmännischen Bereich abgeleiteten Schlüsselqualifikationen herangezogen werden, die Systemdenken und Selbstständigkeit sowie Kooperations- und Kommunikationsfähigkeit umfassen. ${ }^{130}$ Anschliefend werden

\footnotetext{
127 Vgl. Kapitel 2.

$128 \mathrm{Vgl}$. Abschnitt 3.2.

129 Vgl. DuBs (Anfänger-Unterricht 1992), S. 262.

130 Vgl. Windecker (Kompetenzen 1991), S. 101-106.
} 
weitere Überlegungen bezüglich der Lernziele beim Einsatz von Unternehmensplanspielen in der Aus- und Weiterbildung angestellt. Grundsätzliches Ziel eines Planspiel-Einsatzes ist das Lernen in größeren Zusammenhängen, d.h. die Lernziele dürfen nicht zu stark zerfasert werden. ${ }^{131}$

\section{Systemdenken und Selbstständigkeit}

Diese beiden Schlüsselqualifikationen sind insbesondere im Unternehmensplanspiel nicht voneinander zu trennen: Durch eine Aufgabenstellung, die vergleichbar mit dem zu erlernenden Handeln ist und an die vorhandene Handlungskompetenz der Lernenden anknüpft, wird eine selbstständige Bewältigung der Aufgaben gewährleistet. ${ }^{132}$

Unternehmensplanspiele fördern ganzheitliches und vorausschauendes Denken. ${ }^{133}$ Durch den Einsatz von Unternehmensplanspielen sollen allgemeine ökonomische Kenntnisse zum besseren Verständnis der wirtschaftlichen Umwelt vermittelt werden. Weitere Ziele sind das Erkennen verschiedener Zusammenhänge aus der Sicht eines Unternehmens: Dabei werden sowohl überbetrieblich die Abhängigkeiten zwischen volks- und betriebswirtschaftlichen Elementen, als auch innerbetrieblich die Abhängigkeiten zwischen den verschiedenen unternehmerischen Funktionsbereichen betrachtet. ${ }^{134}$

Der Einsatz von Unternehmensplanspielen beinhaltet aber auch den Umgang mit Systemen. Ein lineares Kausaldenken würde im Planspiel nicht zum gewünschten Erfolg führen. Die Handlungen in dem System 'Planspiel' führen zu Kenntnissen darüber, was ein System im Allgemeinen ist und worin im besonderen Fall die konkrete Struktur des vorliegenden Systems besteht. ${ }^{135}$

Unternehmensplanspiele dienen der aktiven Umsetzung des Wissens in Handlungen. Durch die Modellierung einer konkreten Entscheidungssituation sind die Teilnehmenden zur aktiven Mitwirkung aufgefordert. ${ }^{136}$ Hierdurch soll die Fähigkeit

\footnotetext{
131 Vgl. Rohn (Methodik 1980), S. 16.

132 Vgl. Abschnitt 2.4.2; auch WEYERICH (Gestaltung 1995).

133 Vgl. BEck (Schlüsselqualifikationen 1995), S. 63-64.

134 Vgl. Ebert (Planspiel 1992), S. 37-38.

135 Vgl. Geuting (Planspiel 1992), S. 185; auch Abschnitt 2.5.3.

136 Vgl. EBert (Planspiel 1992), S.27.
} 
der Teilnehmenden gefördert werden, in einer komplexen Situation mit Risiken behaftete Entscheidungen zu treffen. Dazu gehören das Formulieren von Zielen und Strategien zur Zielerreichung, das Umsetzen von Strategien in Maßnahmen sowie die Früherkennung, Analyse und Beurteilung von kritischen Situationen. Weiterhin sollten die Folgen der Entscheidungen für die Teilnehmenden mit der Zeit vorhersehbarer werden und die Entscheidungssituation insgesamt transparenter werden. ${ }^{137}$ Das Fällen von Entscheidungen in einem Unternehmensplanspiel erfordert methodisches Arbeiten sowie die nutzbringende Anwendung betriebswirtschaftlicher Methoden. ${ }^{138}$ Dabei wird deutlich, dass die Selbstständigkeit in diesen Lernzielen implizit enthalten ist.

Hierbei kann allerdings ein Ziel-Konflikt entstehen: Während die Erreichung der genannten Lernziele ein methodisches Arbeiten der Teilnehmenden fordert, sind sie u. U. an einer schnellen Entscheidung interessiert und übersehen zentrale Zusammenhänge. ${ }^{139}$ Entsprechend wichtig sind neben den eher entscheidungsorientierten Zielen die eher verhaltensbezogenen, affektiven Lernziele eines PlanspielEinsatzes.

\section{Kooperations- und Kommunikationsfähigkeit}

Neben den fachlichen Zielen werden im Zuge der komplexer werdenden betrieblichen Aufgaben sowie der Entwicklung der Informationstechnologie teamorientiertes Denken und Handeln als zentrale Lernziele beruflicher Bildung gesehen. ${ }^{140}$ Hierzu gehört auch die Förderung des Verständnisses für die Auswirkungen gruppendynamischer Prozesse auf die Entscheidungsergebnisse ${ }^{141}$ sowie die explizite Berücksichtigung emotionaler und motivationaler Aspekte. ${ }^{142}$ Dies lässt sich unter dem Aspekt der Förderung der Reflexivität in der Phase des Einsatzes eines Unternehmensplanspiels zusammenfassen.

137 Vgl. Högsdal (Planspiele 1996), S. 14; BECK (Schlüisselqualifikationen 1995), S. 63-64 und Ebert (Planspiel 1992), S. 37-38.

138 Vgl. BECK (Schlüsselqualifikationen 1995), S.63-64; HöGsdaL (Planspiele 1996), S. 14.

139 Vgl. Rohn (Methodik 1980), S. 32-46.

$140 \mathrm{Vgl}$. BudDENSIEK (Entscheidungstraining 1992).

141 Vgl. Högsdal (Planspiele 1996), S. 14. Zum Aspekt der Gruppendynamik in Planspielen vgl. auch Boos (Entscheidungsfindung 1996) sowie DaniEL (Planspiel 1996).

142 Vgl. auch Abschnitt 2.3. 


\subsection{Besonderheiten beim Einsatz von Unter- nehmensplanspielen}

\subsubsection{Allgemeine Überlegungen}

Der Einsatz eines Planspiels muss sorgsam vor- und nachbereitet werden, da komplexe Lehr-Lern-Arrangements ${ }^{143}$ grundsätzlich keine 'Selbstläufer' sind. ${ }^{144}$ Die Lernziele können nicht durch den alleinigen Einsatz eines Unternehmensplanspiels erreicht werden. Es können aber Voraussetzungen geschaffen werden, die durch den nachfolgenden Einsatz weiterer komplexer Lehr-Lern-Arrangements gesichert und vertieft werden. ${ }^{145}$

Es gibt eine Reihe von Gründen, die von Praktikern als Argumente für den Einsatz von Unternehmensplanspielen genannt werden: In einer Befragung an deutschen Hochschulen $(n=168)$ wurden Gründe wie 'Vernetztes Denken fördern' und 'Treffen von Entscheidungen einüben' genannt. Interessanterweise gaben zwei Drittel der Befragten an, Unternehmensplanspiele zur 'Motivation für Betriebswirtschaftslehre' einzusetzen. Aber auch zur Vermittlung von betriebswirtschaftlichen Grund- und Fachkenntnissen sowie zum Training von Führungsverhalten eignen sich Unternehmensplanspiele nach Angaben der Lehrstühle. ${ }^{146} \mathrm{Ob}$ diese angestrebten Lernziele jedoch auch erreicht werden, wird nur selten systematisch überprüft. ${ }^{147}$ Die Reaktionen der Teilnehmenden auf den Einsatz von Unternehmensplanspielen sind überwiegend positiv, sodass die Lehrstühle auch weiterhin Planspiele einsetzen würden. ${ }^{148}$

In einer empirischen Erhebung an Weiterbildungs-Institutionen setzten 16 regelmäßig Planspiele ein. Mehr als die Hälfte dieser Institutionen gaben die veränderten Anforderungen an die Arbeitnehmerinnen und Arbeitnehmer als Grund für den Einsatz an. Besonders wichtig erschienen ihnen in diesem Zusammenhang die Förderung des Verständnisses komplexer Zusammenhänge, die Vermittlung

\footnotetext{
143 Vgl. Abschnitt 2.5.

144 Vgl. Keys \& Wolfe (Role 1990), S. 316.

145 Vgl. Achtenhagen (Einsatz 1992), S. 7.

146 Vgl. Bronner \& Kollmannsperger (Planspieleinsatz 1997), S. 410-411.

147 Vgl. dazu auch Wolfe (Teaching Effectiveness 1985), S. 311.

148 Vgl. Bronner \& Kollmannsperger (Planspieleinsatz 1997), S. 416-418.
} 
kaufmännischen Wissens, die Erhöhung der Lernmotivation der Teilnehmenden, die Förderung der Kommunikations- und Konfliktfähigkeit, die Förderung der Teamfähigkeit, die Möglichkeit des Handelns und Entscheidens in realistischen Situationen ohne negative Folgen sowie das Training von Führungsverhalten. ${ }^{149}$ Dabei wird deutlich, dass die Institutionen von deren Wirksamkeit überzeugt sind: Alle gaben an, dass sie Planspiele für lerneffektiv halten.

Die in der Literatur vorgefundenen sowie in den Befragungen genannten Lernziele sind häufig nur unzureichend operationalisiert. Um den hohen Anforderungen an die Lernziele eines Planspiels gerecht zu werden, muss der Einsatz dahingehend überprüft werden, welche Kompetenzen in welchen Phasen schwerpunktmäßig vermittelt werden können. Dabei muss auch auf die Dimensionen der Leistungen eingegangen werden. ${ }^{150}$

Im Bereich der fachlichen Fähigkeiten bedeutet dies zwar einen erheblichen Aufwand, ist inhaltlich aber durchaus zu bewältigen. Die Beurteilung der Lernphasen übergreifenden sozialen Kompetenzen stellen den Beurteilenden jedoch vor große Probleme, da diese nicht ohne Weiteres zu beobachten oder zu erheben sind. Für Forschungszwecke mag sich der Aufwand lohnen, im täglichen Einsatz geraten die Lehrenden jedoch schnell an die Grenzen der verfügbaren Ressourcen insbesondere hinsichtlich des zeitlichen Rahmens. Nur wenn die Unterrichts-Sequenz entsprechend lang und für eine abschließende Beurteilung wichtig genug ist, scheint sich der Aufwand zu lohnen. ${ }^{151}$

\subsubsection{Organisatorische und methodische Besonderheiten}

Ein Problem, das sich häufig beim Einsatz eines Unternehmensplanspiels in der Aus- oder Weiterbildung ergibt, ist die fehlende Einbindung in den bestehenden

149 Vgl. Pietzsch (Einsatz 1997), S. 67-73.

$150 \mathrm{Vgl}$. Abschnitt 2.4.

151 Vgl. Kramer (Probleme 1995), S. 157-180. Kramer analysierte die Lernbüro-Phase im Fach Wirtschaftspraxis an der Berufsfachschule Wirtschaft und erstellte ein spezifisches Kontroll-Schema auf Grund abgeleiteter Leistungskriterien. Seine grundsätzlichen Überlegungen lassen sich auch auf andere komplexe Lehr-Lern-Arrangements wie Unternehmensplanspiele übertragen. 
Lehrplan. Unternehmensplanspiele werden meist auf freiwilliger Basis und außerhalb der sonstigen Lehre in Block- oder Sonderveranstaltungen angeboten. ${ }^{152}$

Ein Grund, ganz auf den Einsatz eines Planspiels zu verzichten, ist die Tatsache, dass die Begriffe in den zu Grunde liegenden Modellen ungenau verwendet werden und zum Teil nicht mit den Curricula abgestimmt sind. Die Modelle sind weiterhin selten flexibel im Hinblick auf ihre Komplexität, ${ }^{153}$ so dass der Einsatz eines Planspiels nur für eine bestimmte Lerngruppe sinnvoll ist und nicht in verschiedenen Gruppen eingesetzt werden kann. ${ }^{154}$ Schließlich ist die geringe Ausstattung der Ausbildungsinstutionen mit Hard- und Software ein Grund, der den Einsatz von Planspielen verhindert. ${ }^{155}$

Diese Aussagen werden durch die Befragung der Lehrstühle gestützt: An den meisten Lehrstühlen ist die Teilnahme an Planspielen freiwillig. Grund sind hierfür die begrenzten Kapazitäten bzw. der fächerübergreifende Einsatz. ${ }^{156}$ Als Gründe für einen Verzicht auf den Einsatz von Planspielen gaben mehr als ein Drittel der Lehrstühle den hohen Zeitaufwand oder organisatorische Probleme an. Weitere Gründe waren, dass keine geeigneten Planspiele vorhanden sind bzw. die verfügbaren Planspiele zu hohen Anschaffungskosten haben. Es wurde auch angeführt, die herkömmlichen Methoden seien ausreichend bzw. es würden andere - wie z. B. Fallstudien - eingesetzt. ${ }^{157}$

Ein teilweise anderes Bild zeichnet die Befragung der Weiterbildungs-Institutionen: Hier wurde nur von einem Viertel der Institutionen als Grund für den Verzicht auf den Einsatz von Planspielen angegeben, die Lerninhalte könnten auf herkömmliche Weise schneller gelehrt werden. Sie schrieben den herkömmlichen Unterrichtsmethoden im Vergleich zu Planspielen nicht mehr Effektivität zu und waren auch nicht der Meinung, dass Kosten und Nutzen des Einsatzes von Planspielen in einem ungünstigen Verhältnis stünden. Noch weniger relevant für ihre Entscheidung war die Tatsache, dass Planspiele auf Modellen basieren und deshalb unrealistisch sind. Wichtig war ihren Angaben zu Folge jedoch, dass ihnen keine für ihre Zwecke geeigneten Planspiele bekannt sind oder die Entwick-

\footnotetext{
152 Vgl. Heidack (Lerninstrument 1992), S. 56-57.

153 Vgl. dazu auch Abschnitt 3.6.1.

154 Vgl. Amann \& Streb (Planspiel 1994); Gerlach (Weiterbildung 1996).

155 Vgl. Achtenhagen \& Preiss (Planspieleinsatz 1991), S. 26.

156 Vgl. Bronner \& Kollmannsperger (Planspieleinsatz 1997), S. 412-413.

157 Vgl. Bronner \& Kollmannsperger (Planspieleinsatz 1997), S. 408-410.
} 
lung bzw. der Erwerb eines Planspiels zu teuer ist. Weiterhin wurden als wichtige Gründe angegeben, dass die Durchführung eines Planspiels zu lange dauert und die Lehrenden mit dem Einsatz von Planspielen nicht vertraut sind. ${ }^{158}$

Neben den mit einem Planspiel-Einsatz verbundenen organisatorischen Gründen sind auch methodische zu beachten: Eine Entscheidung im Planspiel treffen zu können, bedeutet noch nicht, sie in der Realität auch gegen den Willen anderer durchzusetzen. ${ }^{159}$ Das vom Alltag entfernte Spielhandeln erweist sich zwar in einer realen Situation als produktive Erkenntnisquelle. Ein Unterricht, der sich lediglich auf simuliertes Handeln beschränkt, greift jedoch zu kurz. Um nicht nur eine 'Spielkompetenz' im Planspiel zu vermitteln, sollte die Eigenverantwortlichkeit in einer Ernstsituation angeregt und gefördert werden. Sonst besteht die Gefahr, dass sich Planspiele in 'bloßem Aktionismus' erschöpfen. ${ }^{160}$

Problematisch im Hinblick auf Unternehmensplanspiele ist auch die Reduzierung des Zielekatalogs auf die Maximierung des Gewinns, der Rentabilitäten oder sonstiger betriebswirtschaftlicher Kennzahlen. Aspekte wie Motivation der Mitarbeiterinnen und Mitarbeiter oder der Ökologie dürfen heutzutage nicht außen vor gelassen werden. ${ }^{161}$

Weiterhin ist der Einsatz des Computers zu hinterfragen. Grundsätzlich wird dadurch zwar der Einfluss der Lehrenden auf die Lernbedingungen verringert, ${ }^{162}$ durch die technischen Möglichkeiten besteht aber die Gefahr, dass die Zusammenhänge immer komplexer modelliert werden, im Simulationsmodell 'verschwinden' und somit für die Teilnehmenden nicht mehr nachvollziehbar sind. Dies ist jedoch kontraproduktiv, weil das Ziel des Planspiel-Einsatzes gerade die Reduzierung und Verdeutlichung solcher Zusammenhänge ist. ${ }^{163}$

Auch im Hinblick auf die Rolle der Spielleitung bzw. der Lehrenden konnten bisher keine eindeutigen Handlungsempfehlungen gegeben werden. Verschiede-

\footnotetext{
158 Vgl. Pietzsch (Einsatz 1997), S. 67-73. Bei der Befragung der Institutionen wurden die einzelnen Gründe in dem Fragebogen zur Bewertung vorgegeben, während in der Befragung der Lehrstühle offene Fragen zum Verzicht auf den Einsatz von Planspielen gestellt wurden. Das dürfte zum Teil die Unterschiede zwischen den Ergebnissen erklären.

159 Vgl. BuddensieK (Entscheidungstraining 1992), S. 13-15.

160 Vgl. Buddensiek (Simulationsspiele 1979), S. 173 sowie Geuting (Planspiel 1992), S. 89.

161 Vgl. Kaiser \& Kaminski (Methodik 1994), S. 180.

162 Vgl. Wagner \& Krause (Einsatz 1990), S. 10.

163 Vgl. Kaiser \& Kaminski (Methodik 1994), S. 181.
} 
ne Untersuchungen kommen zu dem Schluss, dass die Lehrenden sich keinesfalls passiv verhalten dürfen. Darüber hinaus sind jedoch kaum Forschungsergebnisse bekannt. Gerade aber der Einfluss der Unterstützung durch die Lehrenden, also die Einbettung des Planspiel-Einsatzes in ein Gesamtkonzept, bedarf einer genaueren Betrachtung. ${ }^{164}$

Eine empirische Untersuchung von 39 Unternehmensplanspielen kommt zu dem Schluss, dass hinsichtlich der Komplexität und dem Abstraktionsgrad des Modells Variationen in zwei Arten durchzuführen sind: Zum einen kann ein hoher Abstraktionsgrad zu Beginn eines Einsatzes gewählt werden, der mit einer geringen Komplexität bis hin zur Reduzierung auf die Abbildung bestimmter Betriebsbereiche einhergeht. Zum anderen könnten die Entscheidungen im Planspiel sich zunächst auf den operativen Bereich konzentrieren, um z. B. die Anwendung von betriebswirtschaftlichen Verfahren zu erlernen. Im Verlauf des Planspiels würden sie sich auf mehr strategisch und längerfristig wirksame Entscheidungen verlagern. Beide Variationen sind durch die Einführung von 'schlafenden Variablen' erreichbar, die bei Bedarf hinzugeschaltet werden können und so eine größere Flexibilität des Simulations-Modells erzeugen. ${ }^{165}$

Die alleinige Hinzunahme weiterer Entscheidungs-Variablen innerhalb des Planspiels zur Erhöhung der Komplexität der Entscheidungssituation scheint - nach Auffassung der Teilnehmenden einer empirischen Untersuchung - jedoch kein hinreichendes Mittel zu sein, um Probleme wie zunehmende Unterforderung zu überwinden. Auf Grund der nachlassenden Motivation zum Ende des Unterrichts hin sollte neben dem Lernen im Modell stärkeres Gewicht auf das Lernen am Modell gelegt werden. Die im Planspiel gemachten Erfahrungen müssen im weiterführenden Unterricht systematisiert werden und an konkreten Erfahrungen in der betrieblichen Arbeitswelt gespiegelt und kritisch hinterfragt werden. ${ }^{166}$

Diese Aspekte müssen bei der Konzeption eines Planspiel-Einsatzes zur Sicherung des Lernerfolgs berücksichtig werden. Der nächste Abschnitt beschäftigt sich mit Ergebnissen verschiedener empirischer Untersuchungen zum Einsatz von Unternehmensplanspielen in der Aus- und Weiterbildung. Dabei wird zunächst auf

164 Vgl. Keys \& Wolfe (Role 1990), S. 317; erste Ansätze hierzu vgl. Abschnitt 3.6.3.

165 Vgl. FaBel (Fortentwicklungsperspektiven 1993).

$166 \mathrm{Vgl}$. Schunck (Subjektive Theorien 1993), S.114; zum Lernen im und am Modell vgl. Abschnitt 2.5.3. 
mögliche Einflüsse der Komplexität des Planspiel-Modells auf den Lernerfolg eingegangen. Nachfolgend werden weitere Forschungsergebnisse aufgezeigt, die für die vorliegende Arbeit von Bedeutung sind.

\subsection{Forschungsstand zum Einsatz von Unter- nehmensplanspielen}

\subsubsection{Forschungsergebnisse zum Einfluss der Komplexität des Planspiel-Modells auf den Lernerfolg}

Wie bereits in Abschnitt 2.5 ausgeführt wurde, sollten die Problemstellungen, mit denen die Lernenden konfrontiert werden, bereits zu Beginn des Unterrichts eine komplexe Ziel- und Inhaltsstruktur haben. Mit dem Einfluss der Komplexität des Planspiel-Modells auf den Lernerfolg beschäftigten sich einige Untersuchungen, deren Ergebnisse im Folgenden dargestellt werden. ${ }^{167}$

In einer 1966 durchgeführten Studie wurden zwei Experimentalgruppen (jeweils $n=45)$ einer Kontrollgruppe $(n=51)$ gegenüber gestellt. Die Untersuchung fand an Studierenden der University of Maryland (USA) statt. Die Komplexität des Modells des eingesetzten Unternehmensplanspiels MANSYM wurde in den Experimentalgruppen auf Grund der Anzahl der zu treffenden Entscheidungen sowie der Anzahl der Produkte und Rohstoffe definiert:

- In der Variante mit niedriger Komplexität mussten acht Entscheidungen in jeder Runde getroffen werden. Es wurde ein Produkt aus einem Rohstoff hergestellt.

- Die hohe Komplexität der zweiten Variante spiegelte sich in 18 Entscheidungsgrößen wider. Es wurden drei Produkte aus jeweils einem Rohstoff hergestellt.

Die untersuchten Kriterien waren das Wissen über Fakten, das Wissen über Techniken sowie die Anwendung des Wissens in einer speziellen Situation. A1le drei Arten des Wissens wurden mit einer schriftlichen Lernkontrolle nach dem

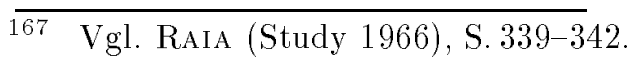


Planspiel-Einsatz erhoben. Sie bestand aus einem Richtig-Falsch-Fragebogen inklusive Erläuterungen sowie zwei Tests, die Methoden - wie z. B. die Break-EvenAnalyse - abfragten. Zum anderen wurde vor und nach dem Unterricht eine Fallbearbeitung eingesetzt. Weiterhin wurden die Einstellungen der Teilnehmenden zum Planspiel sowie ihr Interesse und ihre Motivation im Unterricht mit Fragebögen erhoben. ${ }^{168}$

Die Ergebnisse dieser Studie brachten keine eindeutigen Ergebnisse hinsichtlich der untersuchten Merkmale: Der Planspiel-Einsatz führte gegenüber dem herkömmlichen Unterricht zwar zu einem höheren Lernerfolg beim Wissen über Fakten und Techniken sowie bei der Anwendung des Wissens in speziellen Situationen. Hinsichtlich der Fallbearbeitung wurde jedoch ein eher niedrigerer Lernerfolg gemessen. Zwischen den Experimentalgruppen ergaben sich in allen Instrumenten vergleichbare Ergebnisse. Das heißt, die Komplexität des Planspiels, wie sie in dieser Studie variiert wurde, zeigte in den eingesetzten Instrumenten keinen Einfluss auf den Lernerfolg. ${ }^{169}$

Insgesamt lässt sich jedoch festhalten, dass der Einsatz des Planspiels von den Teilnehmenden begrüßt wurde und ihr Interesse und ihre Motivation im Unterricht im Vergleich zur Kontrollgruppe höher lag. Interessant an dieser Studie ist der Einsatz einer Fallbearbeitung zur Messung des Lernerfolgs, die über einen einfachen Fragebogen hinausgeht. Die Auswertung wurde jedoch auf eine nicht näher erläuterte Maßzahl beschränkt. ${ }^{170}$

An der Universität Tübingen wurde im Rahmen des wirtschaftswissenschaftlichen Hauptstudiums eine Untersuchung durchgeführt $(n=59)$, die den Zusammenhang zwischen dem Wissenszuwachs, der Komplexität des Planspiel-Modells und dem Vorwissen erhob. Die Komplexität des volkswirtschaftlichen Planspiels MACRO wurde in drei Stufen durch die Einführung weiterer Entscheidungsgrößen erhöht. Dadurch ergaben sich verstärkt Zielkonflikte und eine erhöhte Anzahl an Beziehungen zwischen den Variablen. Die Bewertung des Vorwissens bzw. des Wissenszuwachses wurde mit Hilfe eines Wissenstests erhoben, der allgemeines volkswirtschaftliches Hochschulwissen erfasste. ${ }^{171}$

\footnotetext{
168 Vgl. Raia (Study 1966), S. 343-345.

169 Vgl. Raia (Study 1966), S. 345-347.

170 Vgl. Raia (Study 1966), S. 348-349.

171 Vgl. Ripper et al. (Ökonomieplanspiele 1993), S. 114-116.
} 
Der Lernerfolg wurde durch eine Kennzahl gemessen, die sich aus den erreichten Punkten im Vor- und Nachtest ergab. Für die weiteren varianzanalytischen Auswertungen wurde jedoch nur diese Zahl herangezogen. Als Ergebnis der Studie kann festgehalten werden: Personen mit hohem Vorwissen profitieren hinsichtlich des erfassten Wissens mehr von einem Planspiel mittlerer und hoher Komplexität als Personen mit niedrigem Vorwissen. Letztere wiederum erreichen einen höheren Lernerfolg beim Einsatz eines Planspiels mit niedriger Komplexität. Dieser Zusammenhang besteht auch bei der erhobenen Akzeptanz des Planspiel-Einsatzes: Die Motivation ist in dem komplexen Planspiel für Personen mit höherem Vorwissen größer als bei denen mit niedrigerem Vorwissen. ${ }^{172}$

An der Universität München wurde eine Studie zum Einfluss der Instruktionen während des Planspiel-Einsatzes sowie die Art der Durchführung des Planspiels auf den Lernerfolg untersucht $(n=60)$. Dabei wurden die Schülerinnen und Schüler einer Berufsschule in ein 2x2-faktorielles Design aufgeteilt: ${ }^{173}$

- Ein Faktor war die Art des Lernkontextes:

- Im uniformen Lernkontext wurden die Teilnehmenden in den drei aufeinander folgenden Planspiel-Perioden jeweils mit einer vergleichbaren Ausgangssituation konfrontiert,

- während sich die Situationen in der multiplen Umgebung hinsichtlich der abgebildeten Unternehmens-Situation voneinander unterschieden.

- Der zweite Faktor umfasste die Anleitung zur Problemlösung:

- In der geleiteten Bedingung wurde ein Schema zum Lösen von Probleme vorgegeben und anhand einer Standardsituation im Planspiel erklärt und geübt.

- Ungeleitet bedeutet entsprechend, dass die Teilnehmenden keine Anleitung erhielten.

Die Versuche wurden im Labor durchgeführt und die Teilnehmenden trafen die Entscheidungen im Planspiel einzeln. Zur Messung des Vorwissens wurde ein Lückentest mit betriebswirtschaftlichen Items, zur Messung des Lernerfolgs ein Sachwissenstest mit 15 Items aus dem Umgang mit dem Planspiel eingesetzt. Als 'Steuerungsleistung' im Planspiel wurden die erzielten Gewinne herangezogen.

172 Vgl. Ripper et al. (Ökonomieplanspiele 1993), S. 116-121.

173 Vgl. STark et al. (Förderung 1995) und STARK et al. (Lernen 1995). 
Weitere Aspekte waren zum einen die Qualität der Mentalen Modelle, die über eine Fallbearbeitung am PC erhoben wurde, ${ }^{174}$ zum anderen die Motivation, die mit einem Fragebogen erhoben wurde. ${ }^{175}$

Hinsichtlich des Lernerfolgs ergaben sich in beiden Studien keine eindeutigen Ergebnisse. Insgesamt lässt sich aber festhalten, dass es sinnvoll ist, bei einer Variation der Lernkontexte im Planspiel eine Anleitung vorzugeben. Bei gleichbleibenden Kontexte führt die Anleitung ebenfalls zu einem besseren Ergebnis. Besonders negativ auf den Lernerfolg wirkte sich das Fehlen einer Anleitung in dem multiplen Lernkontext aus. Die Unterstützung der Teilnehmenden bei der Problemlösung hat offensichtlich einen Einfluss auf den Lernerfolg. ${ }^{176}$ Hinsichtlich der Motivation zeigt sich der uniforme Lernkontext mit Anleitung als besonders ungünstig. Hier scheinen die Teilnehmenden von der gleichförmigen Art des Planspiels eher gelangweilt. ${ }^{17 \tau}$

An der Verwaltungs- und Wirtschaftsakademie Göttingen wurden zwei Erhebungen zum Einsatz des Unternehmensplanspiels EpUS durchgeführt ( $n=65$ bzw. $\mathrm{n}=70) .{ }^{178}$ Dabei wurden zwei verschiedene Planspiel-Durchführungen experimentell gegenübergestellt: ${ }^{179}$

- In der modularen Durchführung wurde die Komplexität der Anforderungssituation sukzessive gesteigert,

- während sie in der herkömmlichen Durchführung über den gesamten Verlauf des Planspiels konstant blieb.

Der Lernerfolg wurde in einem Prä-Post-Design mit Hilfe eines Fragebogens zum Faktenwissen, eines Leerfeld-Schemas bzw. in der zweiten Erhebung mit einer Netzwerk-Erhebung zum Zusammenhangswissen sowie einer Fallstudie zur Transferleistung erhoben. Zusätzlich wurden die Leistungen in einer 'Testphase' bewertet, in der das Planspiel selbst als Erhebungsinstrument eingesetzt wurde. Der

174 Vgl. STaRK et al. (Förderung 1995), S. 19.

175 Vgl. Stark et al. (Lernen 1995), S. 14.

176 Vgl. Stark et al. (Förderung 1995), S. 20-27 bzw. Stark et al. (Lernen 1995), S. 14-17.

177 Vgl. STARK et al. (Lernen 1995), S. 19-20.

178 Zu einem Vergleich dieser Erhebungen und der vorher dargestellten Münchner Studie vgl. Klauser (Gestaltung 1998) und Klauser (Befunde 1998).

179 Vgl. Bloech, Hartung \& Orth (Einsatz 1999). 
Unterricht erstreckte sich jeweils über ein gesamtes Semester. ${ }^{180}$ Bezüglich des Faktenwissens wurde von einem eher geringen Zuwachs ausgegangen, was durch die beiden Erhebungen auch bestätigt wurde. Beim Zusammenhangswissen wurde in beiden Erhebungen ein großer Zuwachs festgestellt. Bezüglich der Transferleistung war ebenfalls von einem Lernerfolg durch den Einsatz des Planspiels ausgegangen, der durch beide Erhebungen bestätigt wurde. Die Art der PlanspielDurchführung hatte bei beiden Instrumenten entgegen den Erwartungen keinen Einfluss auf den Lernerfolg. In der Transferleistung wurden der erzielte Gewinn, der Auslastungsgrad der Maschinen sowie die Bestandsveränderungen zur Beurteilung herangezogen. Im Posttest bringen die Teilnehmenden wesentlich bessere Leistungen als im Prätest. Die Planspiel-Durchführung brachte jedoch keine signifikanten Unterschiede. In der Testphase des Planspiels zeigte sich hinsichtlich der betrachteten Zielgröße des Gewinns eine Überlegenheit der modularen gegenüber der herkömmlichen Durchführung. ${ }^{181}$ Eine genauere Betrachtung der Lernprozesse kann durch Heranziehen unterschiedlicher Kennzahlen geschehen, wobei sich in der zweiten Erhebung zum Teil große Unterschiede in der Qualität der Entscheidung im Planspiel zeigten. ${ }^{182}$

Für den englisch-sprachigen Raum kommt eine Meta-Analyse verschiedener Studien zu dem Ergebnis, dass sich die durchgeführten Untersuchungen hauptsächlich auf den Bereich der strategischen Planspiele konzentrieren. Sie stellen fest, dass im Hinblick auf die Komplexität des Planspiel-Modells bisher keine hinreichende Klassifikation vorliegt, so dass die Untersuchungsergebnisse kaum vergleichbar sind. ${ }^{183}$ Dies lässt sich sicherlich auch auf den deutsch-sprachigen Raum übertragen und hat sich in den vergangenen knapp zehn Jahren kaum verändert. Grundsätzlich ist zu vermuten, dass es für jede und jeden Teilnehmenden eine 'optimale' Komplexität gibt, die zur Maxierung des individuellen Lernerfolgs führt. ${ }^{184}$

\footnotetext{
$\overline{180 \text { Vgl. Bloech et al. (Einsatz 1996) }}$, S. 33-39; auch Bloech, Kauer \& Orth (Unternehmensplanspiele 1996).

181 Vgl. Bloech et al. (Einsatz 1998), S. 25-32; auch Bloech, Hartung \& Orth (Förderung 1999) sowie Bloech, Hartung \& Orth (Unternehmensplanspiele 1998).

182 Erste Ansätze dazu in Blozch et al. (Einsatz 1998), S. 33-43.

183 Vgl. Keys \& Wolfe (Role 1990), S. 316.

184 Vgl. Hauschild (Komplexität 1990).
} 
Eine Möglichkeit zur Reduzierung der Komplexität in einer Entscheidungssituation - speziell in einem computersimulierten dynamischen System - ist die Hilfestellung durch adaptive Hinweise während der Bearbeitung am Computer, die teilweise eine positive Auswirkung auf den Lernerfolg im Sinne einer Erhöhung der Fähigkeit zur Systemsteuerung hat. ${ }^{185}$ Hier spielen jedoch die konkrete Ausgestaltung der Hilfen sowie die räumlichen Bedingungen des Einsatzes eine große Rolle.

\subsubsection{Umgang mit komplexen Simulationsmodellen}

In verschiedenen Untersuchungen wurde mit dem Einsatz prozessorientierter komplexer Simulationsmodelle der Umgang mit komplexen, dynamischen Situationen erforscht. Diese Systeme unterscheiden sich von Planspielen insofern, als dass sie "komplizierte, vernetzte und eigendynamische 'Modellwelten' simulieren, mit denen der Akteur interaktiv umgehen kann ... [und] den 'Weg' der Entscheidungen und Handlungen und deren Konsequenzen mit in die Analyse“186 einbezieht. Zu diesen gehören die im Folgenden vorgestellten Forschungen.

Die Vorhersagbarkeit des Lernerfolgs beim Umgang mit komplexen Systemen durch Ergebnisse in einem Intelligenztest wurde in einem Experiment untersucht, in dem die Teilnehmenden in Einzelversuchen ein komplexes Kleinsystem, das Planspiel SCHNEIDERWERKSTATT zu steuern hatten $(n=70)$. Dieses Planspiel hat 11 direkt beeinflussbare und insgesamt 24 Variablen. ${ }^{187}$ Die Teilnehmenden wurden in zwei experimentelle Bedingungen geteilt: Die Gruppe der 'transparenten' Bedingung bekam eine Abbildung mit den Verknüpfungen der Variablen des Planspiel-Modells, der anderen Gruppe wurden nur die Systemvariablen und ihre Eingriffsmöglichkeiten genannt. Als Maßzahlen zur Beurteilung der Lernerfolge wurde die Höhe verschiedener Variablen herangezogen. Es stellte sich heraus, dass die Leistung während der Systemsteuerung nicht mit den Leistungen in dem Intelligenz-Test korrelierten. Das heißt, dieser Intelligenz-Test fragt offensichtlich nicht solche Leistungen ab, die zur Steuerung des komplexen Systems erforder-

\footnotetext{
185 Vgl. ausführlicher dazu LeUTnER (Lehrsysteme 1992), S. 121-156.

186 Reither (Schwierigkeiten 1991), S. 31.

187 Vgl. Putz-Osterloh \& Lüer (Vorhersagbarkeit 1981), S. 313-315. Zur Unterscheidung zwischen beeinflussbaren und nicht-beeinflussbaren Variablen im Planspiel vgl. Abschnitt 3.4.1.
} 
lich sind. Unter der experimentellen Bedingung der 'Transparenz' konnten jedoch die Teilnehmenden mit guten Leistungen im Intelligenz-Test die Informationen über die Zusammenhänge der Variablen besser in entsprechende Handlungen bei der Systemsteuerung umsetzen als solche, die im Test schlecht abgeschnitten hatten. ${ }^{188}$

Einen wichtigen Aspekt beim Lösen komplexer Probleme stellt die Motivation dar, die im Verlauf des Planspiels erheblich variieren kann. In einer Studie wurden die Teilnehmenden $(n=60)$ mit dem komplexen System MORI konfrontiert, das aus 13 Variablen bestand, wovon sieben beeinflussbar waren. Dabei wurde die Stärke der persönlichen Betroffenheit zum einen durch die Gefährlichkeit der Situation, zum anderen durch die persönliche Verantwortung im Planspiel experimentell variiert. ${ }^{189}$

Die Stärke der persönlichen Betroffenheit scheint die Güte der Entscheidungen zu beeinflussen. Dabei sind die Teilnehmenden in der Bedingung der mit starker Betroffenheit eher in der Lage, das System zu steuern, als diejenigen in der Bedingung mit geringerer Betroffenheit. Die Steuerungsleistung wurde wiederum über Maßzahlen im Planspiel operationalisiert. Ein weiterer Faktor, der die Systemsteuerung beeinflusst, ist die Erfolgs- bzw. Misserfolgsorientierung der Teilnehmenden: ${ }^{190}$ Erfolgsorientierte neigten dazu, ihre Bearbeitungszeit besser aufzuteilen, insbesondere bei persönlicher Betroffenheit. Außerdem beschäftigten sie sich stärker mit tieferliegenden Strukturen der Problemsituation und wählten Maßnahmen aus, die längerfristig einen größeren Erfolg brachten. Insgesamt ließ sich feststellen, dass Erfolgsorientierung und persönliche Betroffenheit die Leistung im Planspiel positiv beeinflussten. ${ }^{191}$

Zur Untersuchung, inwieweit sich 'Experten', in diesem Fall sieben Professoren der Wirtschaftswissenschaften, von 'Novizen', d.h. Studierenden unterschiedlicher Fachbereiche $(n=30)$, bei der Bearbeitung eines intransparenten, komplexen Problems unterscheiden, kamen die Planspiele SCHNEIDERWERKSTATT und MOROSYSTEM mit jeweils 50 Variablen zum Einsatz. Den Teilnehmenden wurden vor der Bearbeitung mehrere konkrete Zielvariablen vorgegeben, an denen ih-

\footnotetext{
188 Vgl. Putz-Osterloh \& Lüer (Vorhersagbarkeit 1981), S. 330.

189 Vgl. Hesse, Spies \& LüEr (Einfluß 1983), S. 407-410.

190 Vgl. Abschnitt 2.3.

191 Vgl. Hesse, Spies \& Lüer (Einfluß 1983), S. 419-423.
} 
re Leistung bewertet wurde. Beide Planspiele wurden in Einzelsitzungen gespielt, die Äußerungen der Teilnehmenden wurden auf Tonband aufgenommen. ${ }^{192}$

Zum erfolgreichen Steuern von Systemen können in diesen beiden Fällen zunächst nur allgemeine Heuristiken herangezogen werden, weil die Zusammenhänge zwischen den Variablen den Teilnehmenden unbekannt sind und erst im Laufe der Bearbeitung herausgefunden werden müssen. In der Studie zeigen sich die Experten überlegen: Sie betrachten häufiger die Beziehungen zwischen den Variablen und erkennen dabei mehr richtige Beziehungen. Sie bilden weiterhin wesentlich häufiger richtige Hypothesen über die Zusammenhänge zwischen den Variablen und planen ihr Vorgehen systematischer.

Zusammenfassend lässt sich bei dieser Studie feststellen, dass Expertenwissen auch bei schlecht-definierten Problemen eine Voraussetzung für besseres Problemlösen ist. ${ }^{193}$ Dabei sind jedoch die geringen Stichproben-Größen sowie die Durchführung als Laborversuche kritisch anzumerken. ${ }^{194}$

Eine neuere Studie betrachtete kognitive, emotionale und motivationale Determinanten des Handelns bei der Steuerung des Systems SCHOKO-FIN. STARKER \& DöRNER stellen abschließend fest, dass zwar das Verhalten der Teilnehmenden erkennbaren Gesetzmäßigkeiten unterliegt, einfache Indizes zur Prognose der Problemlöse-Güte jedoch nicht geeignet sind. Es erscheint ihnen notwendig, Merkmale der ablaufenden kognitiven Prozesse mit Merkmalen des emotionalmotivationalen Zustands zu verbinden. ${ }^{195}$

192 Vgl. Putz-Osterloh (Experten 1987), S. 63-67. Dabei bleibt offen, ob die Teilnehmenden explizit aufgefordert wurden, ihre Überlegungen im Laufe der Bearbeitung auszusprechen. Die Bemerkung "Bei den Studenten war jeweils einer Person das Tonband aufgefallen." (S.77) und der nachfolgende Hinweis auf die entsprechende Reduzierung der Stichprobe um diese Probanden deuten eher daraufhin, dass diese Aufnahmen ohne das Wissen der Teilnehmenden gemacht wurden. Es ist zu hinterfragen, inwieweit die gefundenen Unterschiede bezüglich der verbalisierten Lösungen nicht auch auf eine unterschiedliche Redezeit zurückzuführen sind.

193 Vgl. Putz-Osterloh (Experten 1987), S. 77-82. Zu vergleichbaren Ergebnissen kommen verschiedene Studien über den Umgang mit komplexen Systemen unter intransparenten Bedingungen; vgl. Dörner et al. (Lohhausen 1983) und Dörner (Logik 1989); auch REITHER (Schwierigkeiten 1991).

194 Ausführlicher zur Kritik vgl. Funke (Problemlösen 1986) und Funke (Probleme 1990).

195 Vgl. Starker \& Dörner (Determinanten 1997). 


\subsubsection{Weitere Forschungsergebnisse}

An der Graduate School of Business Administration der Harvard University, USA wurde mit Studierenden im ersten Studienjahr $(n=90)$ ein Vergleich zwischen dem Einsatz eines Unternehmensplanspiels und herkömmlichem Unterricht durchgeführt. Dabei wurde die Planspiel-Phase durch mehrfache Diskussionsrunden unterstützt. Vor und nach der Unterrichtseinheit wurde zur Kontrolle des Lernerfolgs eine Fallbearbeitung eingesetzt, deren Auswertung sich auf folgende qualitative Kriterien stützte: ${ }^{196}$

- Die heutigen Entscheidungen wirken sich auf die nächsten Ergebnisse aus.

- Die Ziele und Pläne werden in entsprechend sinnvolle Entscheidungen umgesetzt, die auch situationsbezogen sind.

- Die Entscheidungen in den einzelnen Betriebsbereichen hängen voneinander ab und sind in sich stimmig.

Die Ergebnisse brachten bei allen drei Kriterien eine Überlegenheit des PlanspielUnterrichts gegenüber dem herkömmlichen Unterricht. Es wurde festgestellt, dass das eingesetzte Planspiel offensichtlich kompliziert und interessant genug war, um einen positiven Einfluss auf die Motivation zu haben. Weiterhin konnte den Teilnehmenden die Erfahrung ermöglicht werden, Entscheidungen unter Unsicherheit zu treffen und die Bedeutung eines planvollen Vorgehens zu erkennen. Interessant ist bei dieser Studie, dass neben quantitativen Maßzahlen, die direkt aus dem Planspiel gewonnen werden, auch eine Fallbearbeitung, die qualitativ ausgewertet wurde, zur Bewertung des Lernerfolgs herangezogen wurde. ${ }^{197}$

An der TU Hamburg-Harburg wurde ein fertigungs-orientiertes Planspiel eingesetzt. Die Stichprobe bestand aus Studierenden verschiedener Fachbereiche sowie Mitarbeiterinnen und Mitarbeitern der Arbeitsvorbereitung eines Industrieunternehmens. Sie wurden in jeweils drei Gruppen geteilt, wobei sie sich nach der Einführung in das Planspiel eine Strategie überlegen und diese im Verlauf des Planspiels durch geeignete Entscheidungen verfolgen sollten. Die Strategien wurden bei der nachfolgenden Auswertung nach der Fristigkeit eingeteilt. Die Gruppen mit der kurzfristigen Strategie 'Kapazitätsauslastung' erzielten in dieser

196 Vgl. McKenney (Evaluation 1962), S. 278-280.

197 Vgl. McKenney (Evaluation 1962), S. 282-285. 
Kennzahl die besten Ergebnisse. Sie schnitten jedoch auch bei den betrachteten Größen 'Rohgewinn', 'Termintreue' und 'Umsatzrentabilität' am besten ab. Dies steht im Gegensatz zu dem gesetzten Lernziel für den Planspiel-Einsatz, nach dem die Bedeutung einer langfristigen Planung mit entsprechenden strategischen Zielgrößen vermittelt werden sollte. ${ }^{198}$

Bei dieser Studie ist jedoch zu vermuten, dass im Planspiel die Kapazitätsauslastung in engem Zusammenhang mit dem Gewinn steht: Die Termintreue wird durch eine hohe Kapazitätsauslastung bei vollen Lagern gewährleistet. Der Umsatz hängt allein von der Termintreue ab, da der Marketing-Bereich nicht in der Entscheidung der Teilnehmenden lag und ceteris paribus für alle Gruppen gleich war. Insofern sind Ziele wie die Arbeitsplatz-Erhaltung oder die Gewinnmaximierung zwar grundsätzlich längerfristig angelegt, in dem Planspiel-Modell aber nicht direkt über die Entscheidungsgrößen beeinflussbar. Interessant ist bei dieser Untersuchung die Betrachtung verschiedener Kennzahlen im zeitlichen Verlauf des Planspiels. Bei der Formulierung der verfolgten Lernziele muss jedoch überprüft werden, ob das Planspiel geeignet ist, diese abzubilden und zu vermitteln.

Am wirtschaftswissenschaftlichen Fachbereich der Universität Göttingen wurde an Studierenden mittlerer Semester eine Untersuchung zum Diskussionsprozess und zur Effektivität der Gruppenarbeit beim Lösen komplexer ökonomischer Probleme durchgeführt $(\mathrm{n}=60)$. Dabei wurde als Ziel die Maximierung des Eigenkapitals im Unternehmensplanspiel OPEX zum Ende des Einsatzes vorgegeben. Die Hälfte der Stichprobe erhielt Richtlinien, anhand derer sie ihre Diskussionen während der Entscheidungsfindung führen sollten. Im Wesentlichen beinhalteten diese, dass die Entscheidungen nicht durch Konsensbildung im Sinne von durchschnittlichen Werten der individuellen Entscheidungen getroffen werden sollen. Die Gruppenprozesse wurden durch Befragung der Teilnehmenden sowie durch Inhaltsanalyse der Protokolle der Gruppendiskussion erhoben. So wurde versucht, die getroffenen Entscheidungen nachzuvollziehen. Dabei stellte sich heraus, dass die Mehrzahl der Entscheidungen auf einfache Zusammenhänge zu reduzieren waren. Eine Steigerung der 'Gruppeneffektivität' durch die Ausgabe der Diskussionsrichtlinien konnte nicht nachgewiesen werden. ${ }^{199}$

198 Vgl. Schmager \& Wirth (Planspiel 1990).

199 Vgl. Stumpf (Diskussionsprozeß 1992), S. 198-203. 
Eine weitere Erhebung an Studierenden $(n=26)$ und zehn Graduierten des wirtschaftswissenschaftlichen Faachbereichs der Universität Göttingen mit dem Planspiel OPEX differenzierte zwischen deklarativem Strukturwissen und prozeduralem Handlungswissen: ${ }^{200}$ Zum einen wurden Fragen zu möglichen Zusammenhängen im Planspiel im Sinne einer Wirkungsanalyse - wie einer Preiserhöhung auf die Absatzmenge - in Form eines Interviews gestellt. Zum anderen sollten die Teilnehmenden die Entscheidungsgrößen des Planspiels schriftlich in eine Rangfolge nach ihrem Einfluss auf die Zielgröße bringen. Als Zielgröße im Planspiel wurde auch in dieser Erhebung die Höhe des Eigenkapitals am Ende des Planspiel-Einsatzes gewählt. Dabei ist die Gestaltung der Interview-Instruktion bzw. des Fragebogens zu hinterfragen: Den Teilnehmenden wurde in beiden Aufgaben ausdrücklich gesagt, es gäbe keine richtige Antwort und sie sollten spontan antworten. Zur Bewertung der Leistung wurde jedoch jeweils die Anzahl der richtigen Antworten herangezogen. Dadurch sollte zum einen verhindert werden, dass sich die Teilnehmenden "unter Leistungsdruck gesetzt fühlten und die erfragten Modellbeziehungen sehr genau analysieren, bevor sie eine Antwort abgeben“"201, zum anderen sollte es zu einem "intuitiven Vorgehen führen"202. Beides wirft die Frage nach einem möglichen Einfluss auf die erhaltenen Antworten auf. ${ }^{203}$

Als unabhängige Variable wurde in dieser Erhebung die Instruktion der Teilnehmenden variiert. Während eine Hälfte der Teilnehmenden neben den Informationen über das Planspiel-Modell keine weiteren Informationen bekam, wurde der anderen Hälfte eine Darstellung der Zusammenhänge der Variablen des Planspiels in Form eines Netzwerks vorgelegt. Weiterhin bekamen sie ausführlichere Erläuterungen zu den einzelnen Kennzahlen der Unternehmens-Ergebnisse. ${ }^{204}$ Die Auswertung der Daten erfolgte durch Zusammenführen der Antworten und Ergebnisse mit einer Sensitivitäts-Analyse der Zusammenhänge im Planspiel sowie der Entwicklung einer optimalen Entwicklung der Zielgröße. Hierzu wurden Verfahren des Operations Research herangezogen. Hinsichtlich der Problemlösegüte waren die Teilnehmenden ohne zusätzliche Erläuterungen besser als diejenigen mit Erläuterungen. In der Wirkungsanalyse verschiedener Maßnahmen im Planspiel

\footnotetext{
$200 \mathrm{Vgl}$. Abschnitt 2.2.

201 Petzing (Untersuchung 1993), S. 109.

202 Petzing (Untersuchung 1993), S. 111.

203 Vgl. dazu auch Abschnitt 2.3.1.

204 Vgl. Petzing (Untersuchung 1993), S. 97-108.
} 
erbrachten umgekehrt die Teilnehmenden mit zusätzlichen Erläuterungen bessere Ergebnisse als diejenigen ohne. Bei der Einschätzung bezüglich der Rangfolge der Planspiel-Variablen konnten keine Unterschiede zwischen den Gruppen festgestellt werden. Insgesamt wurden die zusätzlichen Informationen überwiegend von den Teilnehmenden benutzt und als hilfreich bei der Entscheidungsfindung bewertet. ${ }^{205}$

Ein Grund für das teilweise Fehlen von Nachweisen der Effekte in beiden Erhebungen kann in der Wahl der zur Beurteilung herangezogenen Kriterien liegen. Zur Bewertung der Gruppeneffektivität oder der Problemlösegüte scheint eine einzige Kennzahl, die am Ende des Planspiel-Einsatzes ermittelt wird, nicht hinreichend zu sein, um Auswirkungen aufzuzeigen. Dazu bedarf es einer Reihe von Daten, die den Lernerfolg im Prozess zeigen können, bzw. zusätzliche Tests, die den Lernerfolg in unterschiedlichen Bereichen aufzeigen.

Die Vermittlung von Zusammenhangswissen durch den Einsatz des Unternehmensplanspiels B-P-A in der betrieblichen Ausbildung von Industriekaufleuten wurde mit Hilfe einer multi-relationalen Netzwerk-Erhebung ${ }^{206}$ im Prä-Post-Design erhoben $(n=61)$. Nach dem Planspiel-Einsatz benutzten die Auszubildenden wesentlich stärker die vorgegebenen Begriffe und Relationen als vor dem Einsatz. Die Abbildungsleistung, also die Anzahl der Begriffspaare in den individuell konstruierten Netzwerken, die sich auch im Referenznetz wiederfinden, war im Posttest höher als im Prätest, allerdings insgesamt nicht sehr hoch (12,6\% bzw. 18\%). Weiterhin hat die Größe der individuellen Netzwerke zugenommen. Es konnte gezeigt werden, dass der Einsatz des Planspiels geeignet ist, durch die inhaltliche Auseinandersetzung mit den im Planspiel abgebildeten Zusammenhängen das Wissen im Bereich Betriebswirtschaftslehre zu erweitern. ${ }^{207}$

Mit den Subjektiven Theorien ${ }^{208}$ zu einem planspiel-gestützten Betriebswirtschaftslehre-Unterricht beschäftigte sich eine Untersuchung, die an einer Berufsfachschule durchgeführt wurde. Dabei wurde das Unternehmensplanspiel JEANSFABRIK $^{209}$ eingesetzt und die Beurteilung des Unterrichts durch die Lernen-

\footnotetext{
205 Vgl. Petzing (Untersuchung 1993), S. 113-137.

206 Zu diesem Erhebungsinstrument vgl. Abschnitt 5.3.

207 Vgl. Getsch (Möglichkeiten 1990), S. 243-245. Allerdings wurde keine Kontrolle durchgeführt, ob andere Unterrichtsformen ähnliche Effekte bringen.

208 Zur Begriffsdefinition vgl. Abschnitt 2.3.1.

209 Vgl. Preiss (Planspiel 1994).
} 
den mit Hilfe verschiedener Erhebungsinstrumenten vor, während und nach dem Planspiel-Einsatz erhoben. Die Hypothesen hinsichtlich existierender Zusammenhänge zwischen kognitiven, emotionalen und motivationalen Prozessen wurde durch die Ergebnisse im Wesentlichen bestätigt. ${ }^{210}$

Zum Teil wurde von den Lernenden allerdings eine zunehmende Unterforderung im Verlauf des Planspiels geäußert, was auf die nur geringfügige Steigerung der Komplexität des Planspiel-Modells zurückgeführt werden kann und negative Auswirkung auf die Emotionen und Motivation im Unterricht hatte. Auferdem wurde ein fehlender Bezug des Modells zu Handlungen im beruflichen Alltag bemängelt. Als Resultat wird für einen Planspiel-Einsatz u. a. empfohlen, den Unterricht stärker an den Lernprozessen zu orientieren sowie die Handlungen der Lernenden im Sinne eigenständiger Zielbildung, Planung, Durchführung und Reflexion zu unterstützen. ${ }^{211}$

Zur Erfassung der Vorwissensstrukturen und ihrer Veränderungen wurde an einer Berufsfachschule ein Instrumentarium bestehend aus einer multi-relationalen Netzwerk-Erhebung zum Zusammenhangswissen sowie einer Methode zur Erhebung betriebswirtschaftlichen Begriffswissens eingesetzt. In der Experimentalgruppe $(n=26)$ wurde ebenfalls das Unternehmensplanspiel JEANSFABRIK eingesetzt, während die Kontrollgruppe $(n=26)$ 'herkömmlich' unterrichtet wurde. Im Ergebnis konnten jedoch keine statistisch bedeutsamen Unterschiede zwischen den experimentellen Gruppen festgestellt werden. ${ }^{212}$ Interessant ist die im Ausblick der Studie aufgeworfene Frage, “inwiefern sich das hier ermittelte ökonomische Zusammenhangswissen auch tatsächlich im ökonomischen Handeln von Schülern niederschlägt“213. Mit dieser Fragestellung beschäftigte sich die vorliegende empirische Arbeit, die in den folgenden Kapiteln dargestellt wird.

\footnotetext{
210 Vgl. dazu auch Abschnitt 2.3.

211 Vgl. Schunck (Subjektive Theorien 1993), S. 193-196.

212 Vgl. WeBer (Vorwissen 1994).

213 WeBER (Vorwissen 1994), S. 202.
} 


\section{Konzeption und Evaluation der Maßnahme}

\section{$4.1 \quad$ Vorbemerkungen}

Auf der Basis der vorherigen theoretischen Überlegungen wird in diesem Kapitel die Konzeption einer Fortbildungs-Maßnahme, in der ein Unternehmensplanspiel eingesetzt werden soll, vorgestellt. In Abschnitt 4.2 werden die Rahmenbedingungen analysiert, die die Konzeption der Veranstaltung beeinflussen. Es wird die Institution genauer betrachtet und die inhaltlichen Vorgaben durch die Studienpläne und -verordnungen beschrieben. Abschnitt 4.3 beschäftigt sich mit grundlegenden Überlegungen zur Gestaltung der Maßnahme. Dabei steht die Förderung der Fähigkeit der Teilnehmenden, in komplexen Situationen des Berufslebens erfolgreich handeln zu können, im Vordergrund. Dies ist auf Grund der veränderten Bedingungen im Arbeitsleben notwendig. ${ }^{1}$ Unter Rückgriff auf das Forschungsprogramm 'Subjektive Theorien' wird die Wichtigkeit der Förderung der Reflexivität der Teilnehmenden unterstrichen. Dabei werden nicht nur kognitive Aspekte einbezogen, sondern auch Emotionen und Motivationen.

Im Abschnitt 4.4 werden auf der Grundlage der bisherigen Betrachtungen die Lernziele sowie die Inhalte in dem für die Maßnahme vorgesehenen Fach mittels der Taxonomie der kognitiven Lernziele eingeordnet und weitergehend auf die Entwicklung von möglichen Testinstrumenten eingegangen. Anschließend geht Abschnitt 4.5 auf allgemeine Merkmale von Evaluationen ein, wobei verschie-

1 Vgl. Kapitel 1. 
dene Arten und Funktionen gegeneinander abgegrenzt werden und auf spezielle Aspekte der Evaluation in der beruflichen Weiterbildung eingegangen wird.

In Abschnitt 4.6 wird eine kritische Auseinandersetzung mit dem Begriff der Leistung und ihrer Beurteilung vorgenommen. Dabei werden die Funktionen der Leistungsbeurteilung betrachtet sowie Konsequenzen daraus für die Gestaltung von Prüfungen gezogen. Weiterhin wird auf die verschiedenen Bezugsnormen bei der Leistungsbeurteilung eingegangen, die unterschiedlichen Informationsgehalt haben und in ihrer motivationalen Auswirkung auf die Lernenden differieren.

Abschnitt 4.7 beschäftigt sich mit den Gütekriterien für pädagogische Tests. Dabei wird aufbauend auf einem allgemeinen Modell der Forschung die Notwendigkeit der verschiedenen Kriterien abgeleitet und nachfolgend genauer auf die einzelnen Gütekriterien eingegangen. Abschnitt 4.8 stellt die konkrete Umsetzung der Überlegungen in eine Untersuchung dar. Dabei wird auf die durchzuführende Evaluation, die Gestaltung des Planspiel-Einsatzes, die Erhebung im Prä-PostDesign sowie die Hypothesen der Erhebung eingegangen. Der Versuchsplan und der Ablauf der Erhebung werden in Abschnitt 4.9 dargestellt.

\subsection{Analyse der Rahmenbedingungen}

Zur angemessenen Konzeption einer Bildungsmaßnahme müssen verschiedene Rahmenbedingungen überprüft werden. Zu diesen Bedingungen gehören zum einen die anthropogenen Voraussetzungen, die die individuellen Eigenschaften der Lernenden inklusive ihrer Emotionen, Motivationen und ihres Vorwissens umfassen, zum anderen die sozio-kulturellen Voraussetzungen, zu denen die institutionellen Rahmenbedingungen sowie die zu Grunde liegenden Normen und rechtlichen Bestimmungen zählen. ${ }^{2}$

Die Maßnahme wird an der Verwaltungs- und Wirtschaftsakademie in Göttingen durchgeführt. Sie ist eine Institution der nebenberuflichen Fortbildung für Personen, die im öffentlichen Dienst oder in der Wirtschaft tätig sind. Die Teilnehmenden schließen das 6-semestrige Studium mit einem Verwaltungs- oder Wirtschafts-

2 Vgl. grundlegend zur Theorie der Unterrichtsplanung aus unterschiedlichen Blickwinkeln z. B. Posch, Schneider \& Mann (Unterrichtsplanung 1977); Heimann, Оtto \& Schulz (Unterricht 1979) sowie KLAFKi (Unterrichtsplanung 1980). 
diplom ab. Die Ausbildung erfolgt in Form von Vorlesungen und Übungen, die an zwei Abenden in der Woche mit jeweils zwei Doppelstunden stattfinden. Als Voraussetzung für die Zulassung zum Studium müssen die Teilnehmenden eine abgeschlossene Berufsausbildung und eine mindestens einjährige Berufstätigkeit vorweisen. ${ }^{3}$ Insofern ist das Studium an dieser Akademie als Aufstiegsfortbildung einzustufen, die das Ziel hat, den Teilnehmenden durch die Erweiterung ihrer Kenntnisse und Fähigkeiten ein Weiterkommen im Beruf zu ermöglichen. ${ }^{4}$

Auf Grund der Möglichkeit, an jeder Stelle des Berufslebens an dieser Fortbildung teilzunehmen, sowie der nicht vorhandenen Beschränkung der Teilnahme auf bestimmte Berufsgruppen muss die Stichprobe als heterogen hinsichtlich des Alters, der Berufszugehörigkeit sowie der Berufserfahrung eingestuft werden. ${ }^{5}$ Dabei entspricht der Wunsch, an solchen Fortbildungen teilzunehmen, durchaus dem allgemeinen Trend in der Berufswelt. Stellt man den Ausbildungsberuf dem aktuellen Erwerbsberuf gegenüber, zeigt sich in erheblichem Umfang ein Berufswechsel. ${ }^{6}$ Entsprechend heterogen sind auch die bisherigen Lernerfahrungen sowie das Vorwissen der Teilnehmenden einzustufen, soweit dies nicht im bisherigen Studium erworben wurde. Insgesamt ist im Bereich der Weiterbildung von Erwachsenen hinsichtlich der Gestaltung der Lernprozesse ein größeres Augenmerk auf die Selbststeuerung zu legen. ${ }^{7}$

Die Anzahl der Teilnehmenden pro Semester beträgt ca. 100, jedoch nehmen nicht alle durch die Umstände des nebenberuflichen Studiums regelmäßig an den Veranstaltungen teil. ${ }^{8}$ Die Verwaltungs- und Wirtschaftsakademie nutzt einen Hörsaal der Georg-August-Universität Göttingen mit ca. 250 Sitzplätzen. Für die gesamte Maßnahme stehen 14 Doppelstunden zur Verfügung.

3 Vgl. Bundesverband Deutscher. VWA (Rahmen-Studienordnung 1994), 4 (2).

4 Vgl. Kuwan, Gnahs \& Seusing (Weiterbildungsstatistik 1991), S.197; ausführlicher in Kapitel 1.

5 Dies zeigte sich z. B. in den Untersuchungen, die im Rahmen des DFG-Forschungsprojekts ebenfalls an dieser Akademie durchgeführt wurden; vgl. BLOECH et al. (Einsatz 1996), S. 39-40. Dort wurde jedoch in drei verschiedenen Erhebungen kein Einfluss des Alters, der Berufszugehörigkeit oder der Berufserfahrung auf den Lernerfolg festgestellt, so dass dieser Aspekt in der vorliegenden Arbeit nicht näher betrachtet wird.

6 Vgl. Beitr AB 195 (Weiterbildung 1996), S. 243-250.

7 Vgl. Reinmann-Rothmeier \& Mandl (Lernen 1994), S. 22-46.

8 Zur Lebens- und Studiensituation von Teilnehmenden an einer Berufsakademie vgl. WiNTER (Studiensituation 1995); auch Stentzel (Lernschwierigkeiten 1986). 
Mit dem Studium an der Verwaltungs- und Wirtschaftsakademie werden nach der Rahmen-Studienordnung folgende Ziele verfolgt: ${ }^{9}$

- Die Anwendung vertiefter Kenntnisse u. a. auf den Gebieten der Betriebsund der Volkswirtschaftslehre,

- das Erkennen, Strukturieren und Lösen von Entscheidungsproblemen,

- die Wahrnehmung von Führungsaufgaben,

- die Einordnung der Aufgaben und Entscheidungen in volkswirtschaftliche und gesamtgesellschaftliche Zusammenhänge.

Auf den Einsatz handlungsorientierter Unterrichtsmethoden - wie z. B. Unternehmensplanspiele oder Fallstudien - wird weder in der Rahmen-Studienordnung noch im Studienplan explizit eingegangen. Für die Vermittlung der genannten Kompetenzen sind diese jedoch besonders geeignet. Insbesondere der Umgang mit Entscheidungsproblemen sowie die Wahrnehmung von Führungsaufgaben kann durch sie im spielerischen Umgang gelernt werden. ${ }^{10}$ Insofern wird bereits an dieser Stelle grundsätzlich der Einsatz eines Unternehmensplanspiels vorgeschlagen.

Das Studium ist in vier Bereiche aufgeteilt, die in jedem Semester mit anderen Schwerpunkten unterrichtet werden: Rechtswissenschaft, Volkswirtschaftslehre, Betriebswirtschaftslehre sowie je nach Studiengang Betriebswirtschaftslehre der öffentlichen Verwaltung bzw. der Versicherung. Die fachliche Vorbildung durch das Studium umfasst in den ersten zwei Semestern im Bereich der Betriebswirtschaftslehre die folgenden Veranstaltungen: ${ }^{11}$

\section{Semester:}

- Allgemeine Betriebswirtschaftslehre: Liquiditätsplanung und unternehmerische Zielsetzungen

- Industriebetriebslehre: Zielsysteme, Kennzahlen und -systeme, Grundzüge der Produktionswirtschaft, Materialwirtschaft und Logistik, Organisation der Fertigung

- Wirtschaftsmathematik: Grundlagen für Finanz- und Investitionsplanungen, Umsatz- und Gewinnfunktionen

\footnotetext{
9 Vgl. Bundesverband Deutscher VWA (Rahmen-Studienordnung 1994), §2 (2).

10 Vgl. Abschnitt 3.4.

11 Vgl. VWA Göttingen (Vorlesungs-Verzeichnis 1997), dabei wurden die Inhalte herausgegriffen, die für die zu konzipierende Maßnahme von Bedeutung sind.
} 
- Einführung in das Marketing: absatzwirtschaftliches Instrumentarium, Aufgaben und Methoden der Marktforschung

\section{Semester:}

- Finanzierungen: Kapitalbedarf und -deckung, verschiedene Arten der Finanzierungen

- Organisationslehre und -theorie: Organisationstypen der Fertigung

- Kosten- und kurzfristige Erfolgsrechnung: Gesamt- und Umsatzkostenverfahren, Deckungsbeitragsrechnung, Kontrollrechnungen

Die Maßnahme wird für das 3. Semester im Fach Unternehmensführung und Unternehmensforschung konzipiert, für das nach dem Studienplan folgende Inhalte vorgesehen sind: ${ }^{12}$

- Management und Managementlehre

- Führungsprobleme und Führungsmethoden

- Optimalplanungen für Produktion, Logistik und Preispolitik

- Optimale Produktionspläne

- Zuweisungsprobleme und ihre Optimierung

- Strukturen von Transport- und Rundreiseproblemen

- Graphische Optimierung

- Projektplanungen und Methoden der Netzplantechnik

- Bestimmung optimaler Industriestandorte

Ein Teil dieser Inhalte kann durch den Einsatz eines Unternehmensplanspiels abgedeckt werden, ${ }^{13}$ ein anderer Teil muss über eine begleitende Lehre vermittelt werden. Parallel dazu werden die Studierenden in folgenden Bereichen der Betriebswirtschaftslehre unterrichtet: ${ }^{14}$

\section{Semester:}

- Handels- und Steuerbilanzen: Jahresabschluss, Analyse und -kritik

- Grundzüge des Controlling: Arten und Instrumente des Controlling

- Marketing: Produktpolitik, Distributionspolitik, Preispolitik

\footnotetext{
12 Vgl. VWA Göttingen (Vorlesungs-Verzeichnis 1997).

13 Vgl. Abschnitt 3.4 zu Lernzielen in Unternehmensplanspielen allgemein bzw. Abschnitt 4.4 zu den Lernzielen der geplanten Maßnahme.

14 Hier wurden wiederum nur einige Inhalte herausgenommen.
} 


\section{$4.3 \quad$ Überlegungen zur Konzeption}

Im Zuge der Veränderungen der Bedingungen am Arbeitsplatz ist es für die Mitarbeiterinnen und Mitarbeiter unerlässlich, in komplexen Situationen erfolgreich handeln zu können. ${ }^{15}$ Das zentrale Ziel der zu konzipierenden Maßnahme umfasst entsprechend die Förderung der Fähigkeit der Lernenden, in einer komplexen ökonomischen Situation Ziele setzen zu können und Entscheidungen so zu treffen, dass die gesetzten Ziele auch erreicht werden.

Erfolgreiches Lernhandeln kann sich jedoch nur dann in unbestimmten und komplexen Situationen bewähren, wenn den Teilnehmenden ihre Fähigkeiten auch bewusst sind. Die Reflexivität über die eigenen Kognitionen ist entscheidend für den Erfolg von Handlungen. ${ }^{16}$ Weiterhin muss das notwendige und hinreichende Wissen für die Lerngruppe identifiziert und in ein entsprechendes Konzept umgesetzt werden. Dabei erfolgt ein Rückgriff auf Ergebnisse der aktuellen Curriculumund Lehr-Lern-Forschung. ${ }^{17}$

Neben den Kognitionen sind auch die Emotionen und Motivationen sowie die sozialen Interaktionen in der Lerngruppe von Bedeutung. Dabei kommt der Lernmotivation eine wichtige Rolle zu: ${ }^{18}$ Eine beeinträchtigte oder ganz fehlende Motivation zum Lernen führt zu Unsicherheit in der aktuellen Lehr-Lern-Situation und zu Zurückhaltung bei der aktiven Mitarbeit. Häufig strahlt dies auch auf andere Teilnehmende aus und beeinflusst das Lernklima in nachteiliger Weise. Die Folgen sind Frustration und 'Abschalten'. Die Konsequenz aus langanhaltender Demotivation wäre das Abbrechen der Bildungsmaßnahme. Ziel jeden Unterrichts muss entsprechend neben der Vermittlung kognitiver Fähigkeiten auch die Unterstützung der emotionalen und motivationalen Prozesse sein, damit die Lernergebnisse objektiv und subjektiv befriedigend sind, was wiederum für die persönliche Entwicklung der Lernenden förderlich ist. ${ }^{19}$

\footnotetext{
15 Vgl. Kapitel 1; auch Abschnitt 2.4.1.

16 Vgl. Sembilt (Problemlösefähigkeit 1992), S. 111; auch Abschnitt 2.3.

17 Vgl. Arnold (Schlüsselqualifikationen 1996).

18 Vgl. Schmiel (Förderung 1988), S. 1.

19 Vgl. Schunck (Subjektive Theorien 1993), S. 122; auch Abschnitt 2.3.
} 
Die Konzeption der Maßnahme lehnt sich an die Theorie der Handlungsorientierung an, ${ }^{20}$ Zum Einsatz soll ein komplexes Lehr-Lern-Arrangement kommen, das zur Vermittlung der genannten Qualifikationen geeignet ist. ${ }^{21}$ Dabei sind verschiedene Einflussgrößen auf das Lernen zu berücksichtigen (vgl. Abbildung 4.1):

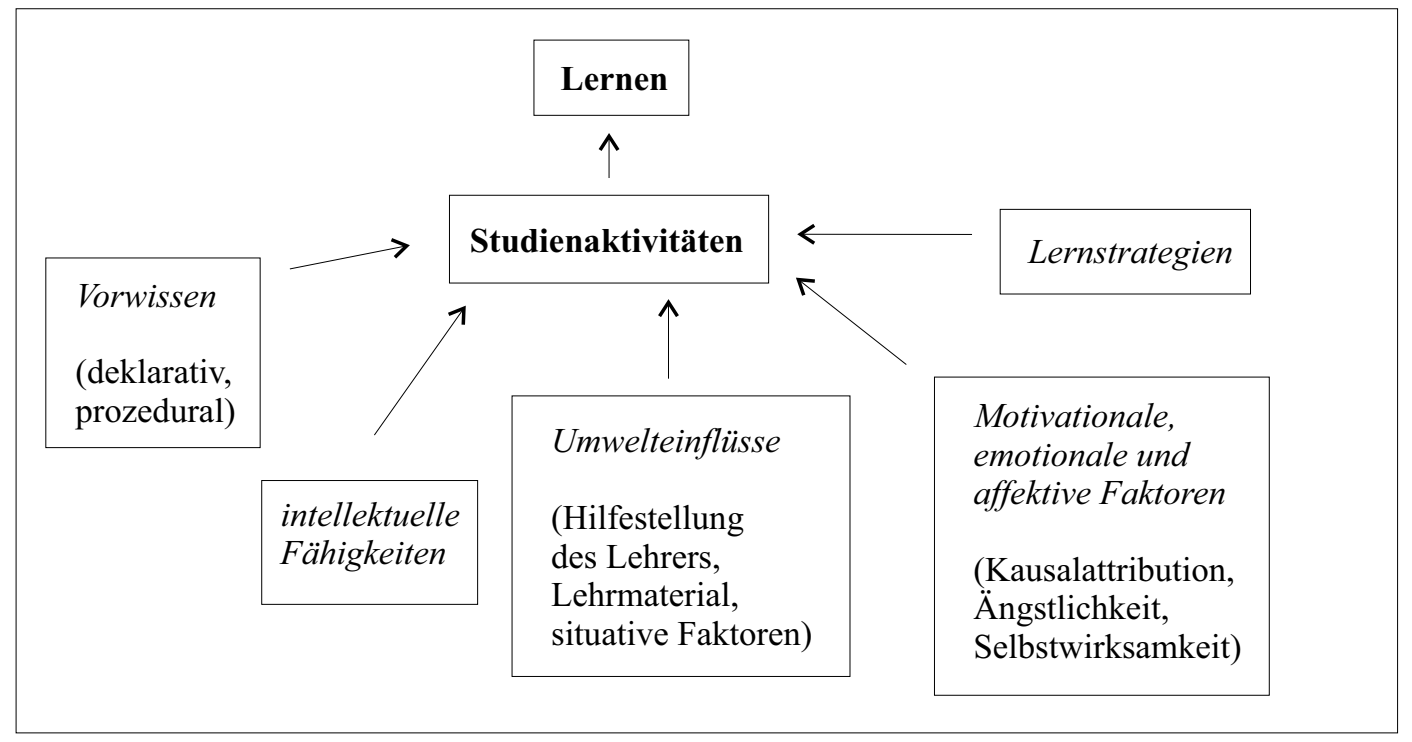

Abbildung 4.1: Einflussgrößen des Lernens ${ }^{22}$

Zum einen muss das Vorwissen bei der Konzeption berücksichtigt werden, da umfassende Kenntnisse bereits zu Beginn der Maßnahme zu Langeweile führt, während zu geringe Vorkenntnisse insbesondere in komplexen Situationen demotivierend wirken können. ${ }^{23}$ Eine nicht-beeinflussbare Größe stellen die intellektuellen Fähigkeiten der Teilnehmenden dar. Das Vorwissen sollte entsprechend zu Beginn der Maßnahme erhoben werden. Es kann jedoch auch auf Erfahrungen der bisher durchgeführten Maßnahmen zurückgegriffen werden, die auch einen Rückschluss auf die intellektuellen Fähigkeiten der Teilnehmenden zulassen. ${ }^{24}$ Weiterhin sind die Umwelteinflüsse, die aus dem Lernmaterial, der Rolle der Lehrkraft sowie aus verschiedenen situativen Faktoren bestehen, zu kontrollieren, um ihre positive Wirkung auf das das Lernhandeln der Teilnehmenden zu nutzen. Wichtig

\footnotetext{
20 Vgl. Abschnitt 2.4.2.

21 Vgl. Abschnitt 2.5.

22 In Anlehnung an KLAUER (Teaching 1988), S. 354.

23 Ausführlicher dazu RenkL (Vorwissen 1996).

24 Vgl. Bloech et al. (Einsatz 1996) und BLoEch et al. (Einsatz 1998).
} 
sind auch die motivationalen, emotionalen und affektiven Faktoren, deren Einfluss auf den Lernerfolg ebenfalls zu kontrollieren bzw. sogar zu nutzen ist. Schließlich sind die Lernstrategien bei der Vermittlung von höheren Qualifikationen - wie Schlüsselqualifikationen - in die Betrachtung einzubeziehen. ${ }^{25}$

Die Effekte der Maßnahme müssen systematisch kontrolliert werden. Eine solche Wirkungsanalyse erfordert die Operationalisierung der angestrebten Qualifikationen sowie eine Messung der Transferwirkung. ${ }^{26}$ Im Berufsalltag entstehen beim Transfer von Gelerntem oftmals Probleme. Insbesondere psychologische Barrieren und das soziale Umfeld verursachen, dass das Gelernte nicht oder nur zum Teil am Arbeitsplatz Anwendung findet. ${ }^{27}$

\subsection{Lernziele der Maßnahme}

\section{Kognitive Lernziele}

Zunächst ist festzuhalten, dass die Lernziele in der Studienverordnung der Verwaltungs- und Wirtschaftsakademie sowie die Inhalte im Studienplan - wie in Abschnitt 4.2 dargestellt - wenig präzisiert sind, um als Grundlage für die weitergehende Planung herangezogen zu werden. Deshalb werden zunächst die Lernziele, die grundsätzlich mit dem Einsatz von Unternehmensplanspielen verfolgt werden, ${ }^{28}$ mit Hilfe der Taxonomie der kognitiven Lernziele nach BLoom et al. 1976 eingeordnet. ${ }^{29}$ Dabei wird überprüft, ob die genannten Ziele des Studiums, speziell die Inhalte des Fachs 'Unternehmensführung und Unternehmensforschung', in dem das Unternehmensplanspiel eingesetzt werden soll, abgedeckt werden. Die Vorgehensweise lehnt sich an die theoretischen Grundlagen zur Hierarchisierung der kognitiven Lernziele an. ${ }^{30}$ Weiterhin ist auch zu prüfen, welche kognitiven Wissensbereiche abgedeckt werden. ${ }^{31}$ Abbildung 4.2 fasst die Hierarchisierung

\footnotetext{
$25 \quad$ Vgl. Abschnitt 2.4.1.

26 Vgl. Bunk, Kaiser \& ZedLer (Schlüsselqualifikationen 1991), S. 372.

27 Vgl. LemkE (Transfermanagement 1995), S. 1-2. Zum Transfer wissenschaftlichen Wissens in die Praxis vgl. auch Dewe (Wissensverwendung 1988).

28 Vgl. Abschnitt 3.4.

29 Vgl. Abschnitt 2.4.3.

30 Vgl. Abschnitt 2.4.

31 Vgl. Abschnitt 2.2.
} 
der kognitiven Lernziele sowie die mögliche Konstruktion von Erhebungsinstrumenten zusammen. ${ }^{32}$

\begin{tabular}{|c|c|c|}
\hline Stufe: & kognitives Lernziel: & Messung des Lernerfolgs: \\
\hline $\begin{array}{l}\text { 6. Beurteilung } \\
\text { 5. Synthese }\end{array}$ & $\begin{array}{l}\text { aktive Umsetzung des } \\
\text { Wissens in Handlungen }\end{array}$ & $\begin{array}{l}\text { komplexe Aufgabenstellung } \\
\text { ohne eindeutige Lösung, } \\
\text { mehrere Entscheidungen in Folge }\end{array}$ \\
\hline 4. Analyse & $\begin{array}{l}\text { Erkennen von Zusammen- } \\
\text { hängen und Abhängigkeiten }\end{array}$ & $\begin{array}{l}\text { grafische Darstellung, } \\
\text { offene Struktur }\end{array}$ \\
\hline 3. Anwendung & $\begin{array}{l}\text { methodisches Arbeiten und } \\
\text { Anwendung betriebswirt- } \\
\text { schaftlicher Methoden }\end{array}$ & $\begin{array}{l}\text { komplexe Aufgabenstellung } \\
\text { ohne eindeutige Lösung, } \\
\text { ein Erhebungszeitpunkt (Transfer) }\end{array}$ \\
\hline 2. Verständnis & Umgang mit Systemen & (kein eigenes Instrument) \\
\hline 1. Kenntnisse & $\begin{array}{l}\text { allgemeine ökonomische } \\
\text { Kenntnisse }\end{array}$ & $\begin{array}{l}\text { offener oder geschlossener } \\
\text { Fragebogen }\end{array}$ \\
\hline
\end{tabular}

Abbildung 4.2: Hierarchisierung der kognitiven Lernziele und ihre mögliche Umsetzung in Erhebungsinstrumente

Auf der ersten Stufe der Taxonomie von BLOOM werden allgemeine ökonomischen Kenntnisse eingeordnet. Die Teilnehmenden sollen betriebswirtschaftliche Begriffe richtig verwenden können und verschiedene Methoden der Unternehmensrechnung und Strukturen eines Unternehmens wiedergeben können. Diese Kenntnisse sind notwendig, um die in der Studienordnung geforderte 'Anwendung vertiefter Kenntnisse auf den Gebieten der Betriebs- und Volkswirtschaftslehre' umzusetzen. Nach der Systematik von Dörner 1976/1987 sind sie dem Afferenzteil des epistemischen Wissens bzw. nach ANDERSON 1983/1988 dem deklarativen Wissen zuzuordnen. Die Messung dieser Kenntnisse kann mittels eines offenen oder geschlossenen Fragebogens erfolgen. Er sollte den Stand des Wissens sowohl vor als auch nach dem Einsatz des Unternehmensplanspiels erfassen, um den Lernerfolg dokumentieren zu können.

32 Vgl. Echterhoff (Veränderungsmessung 1978); auch Abschnitt 2.2. Auf die genaue Konstruktion der Erhebungsinstrumente sowie die Darstellung der Messung des Lernerfolgs wird in Kapitel 5 und 6.2 eingegangen. 
Der Einsatz eines Unternehmensplanspiels fördert weiterhin den Umgang mit Systemen, ${ }^{33}$ was zum einen das Wissen über ein System im Allgemeinen und zum anderen konkretes Wissen über das vorliegende System sowie das Erkennen der Kontextabhängigkeit des Systems beinhaltet. Dieses Lernziel ist der zweiten Stufe der Taxonomie zuzuordnen. Es wird in der Studienordnung nicht explizit genannt. Jedoch kann davon ausgegangen werden, dass zur Bewältigung von Entscheidungsproblemen Kenntnisse über die Systeme, in denen die Entscheidungen gefällt werden, von Bedeutung sind. Die Teilnehmenden sollen in der Lage sein, sich in einem Unternehmen als komplexes System zurechtzufinden, einen gegebenen Sachverhalt zu interpretieren und mit ihm umzugehen. In der vorliegenden Untersuchung wird hierfür kein eigenes Messinstrument, da dieses Lernziel als Teilziel höherer Lernziele gesehen werden kann. ${ }^{34}$

Weitere Lernziele sind das methodische Arbeiten und die Anwendung betriebswirtschaftlicher Methoden. Diese werden auf der dritten Stufe der Taxonomie eingeordnet. Hier werden auch im Studienplan verschiedene Methoden - wie die grafische Optimierung oder die Optimalplanungen für Produktions, Logistik und Preispolitik - genannt, die von den Teilnehmenden beherrscht werden sollen. Zur methodischen Arbeit wird zusätzlich zum Basiswissen - je nach Systematik - heuristisches bzw. prozedurales Wissen benötigt. Die Erfassung könnte mit Hilfe einer einfachen Aufgabenstellung in Form einer Textaufgabe vorgenommen werden. Da jedoch für den kaufmännischen Bereich die Transferleistung eine große Rolle spielt, ${ }^{35}$ wird eine komplexe Aufgabenstellung ohne eindeutige Lösung konzipiert, in der die Teilnehmenden sowohl ihre Fähigkeiten zum methodischen Arbeiten als auch eine Transferleistung zeigen können. Das zu entwickelnde Instrument sollte ebenfalls zur Feststellung des Lernerfolgs vor und nach der Planspiel-Phase eingesetzt werden.

Auf der vierten Stufe der Taxonomie lässt sich das Lernziel des Erkennens überund innerbetrieblicher Zusammenhänge und Abhängigkeiten aus der Sicht eines Unternehmens einordnen. In der Studienordnung wird von 'volkswirtschaftlichen

33 Zum Begriff des Systems vgl. Abschnitt 3.2.1.

34 Allerdings wäre es auch denkbar, das spezifische Wissen über das System zu erheben und mit der Steuerleistung des Systems zusammenzuführen; vgl. z. B. Putz-Osterloh \& LÜER (Vorhersagbarkeit 1981) sowie die Darstellung weiterer Forschungsergebnisse in Abschnitt 3.6.3.

35 Vgl. Abschnitt 2.4.1. 
und gesamtwirtschaflichen Zusammenhängen' gesprochen. Hier bleibt allerdings die Blickrichtung offen, d. h. ob die Teilnehmenden in der Lage sein sollen, aus der Sicht der Geschäftsführung, der Management- oder der Sachbearbeitungs-Ebene Interdependenzen zu erkennen. Auf Grund der Entwicklungen in der Arbeitswelt $^{36}$ sowie der Ausrichtung des Studiums als Aufstiegsfortbildung kann jedoch von davon ausgegangen werden, dass die Teilnehmenden mehr Zusammenhänge erkennen sollen, als es zur bloßen Erfüllung ihrer Funktionen am Arbeitsplatz notwendig ist. Zum Erwerb dieser Fähigkeit ist epistemisches Wissen des Efferenzteils nach DöRNER bzw. prozedurales Wissen nach ANDERSON erforderlich. Eine Kontrolle des Lernerfolgs sollte mit einer grafischen Darstellung der Zusammenhänge geschehen, wobei die Begriffe vorgegeben werden, die Struktur jedoch offen bleibt. Auch hier erscheint es sinnvoll, das Instrument vor und nach dem Unternehmensplanspiel einzusetzen, um eine Verbesserung der Fähigkeiten erfassen zu können.

Auf den beiden höchsten Stufen der Taxonomie kann die aktive Umsetzung des Wissens in Handlungen eingeordnet werden, die auf den Fähigkeiten der ersten vier Stufen aufbaut. Hierzu gehört das Lernziel des Studiums des 'Erkennens, Strukturierens und Lösens von Entscheidungsproblemen', aber auch das 'Wahrnehmen von Führungsaufgaben'. Implizit ist hier die Selbstständigkeit und Verantwortlichkeit des Handelns als Lernziel enthalten. Dieses Lernziel darf in der beruflichen Bildung nicht vernachlässigt werden, da eine Beschränkung auf die Problemlösung nur zu einer 'Halbbildung' führen würde. ${ }^{37}$ An dieser Stelle lässt sich das Lernziel der zweiten Stufe einbinden, das den Umgang mit Systemen umfasst. Zur Bewältigung solch einer Anforderung ist nach DöRNER heuristisches bzw. ANDERSON prozedurales Wissen erforderlich. Der Lernerfolg soll sich ebenfalls in einer komplexen Aufgabenstellung ohne eindeutige Lösung zeigen. Jedoch erscheint hier ein zeitlicher Verlauf sinnvoller als ein Einsatz vor bzw. nach der Planspiel-Phase. Das heißt, die Teilnehmenden sollten mehrere Entscheidungen in einem komplexen System in zeitlicher Folge treffen, um der zufälligen Erzeugung einer erfolgreichen Entscheidung vorzubeugen. ${ }^{38}$

\footnotetext{
36 Vgl. Kapitel 1.

37 Vgl. PÄtzold (Schlüsselqualifikationen 1996).

38 Vgl. Abschnitt 3.5.
} 


\section{Soziale und affektive Lernziele}

Neben den kognitiven Lernzielen der Maßnahme müssen auch die sozialen und affektiven Lernziele geplant werden. Hier lassen sich die Kooperations- und die Kommunikationsfähigkeit zuordnen, die ebenfalls für den kaufmännischen Bereich zunehmend eine Rolle spielen. ${ }^{39}$ Hierzu gehört zum einen die Förderung des Verständnisses für die Auswirkung gruppendynamischer Prozesse auf die Entscheidungsergebnisse. ${ }^{40}$ Zum anderen ist eine explizite Berücksichtigung der emotionalen und motivationalen Aspekte während des Lernprozesses zur Sicherung des Lernerfolgs erforderlich. Das heißt, die Reflexivität der Teilnehmenden soll durch entsprechende Maßnahmen gefördert werden.

Das Unternehmensplanspiel wird in Gruppenarbeit durchgeführt, um einen Anreiz zur Kooperation und Kommunikation zwischen den Teilnehmenden zu schaffen. Insbesondere in der nebenberuflichen Fortbildung, in der sich die Teilnehmenden an zwei Abenden in der Woche für vier Unterrichtsstunden sehen und in der die meisten Veranstaltungen in Form von Vorlesungen durchgeführt werden, dient dies der Anregung zum Austausch zwischen den Teilnehmenden, nicht nur während der Planspiel-Phase, sondern über den Unterricht hinaus. In der konkreten Situation des Planspiels könnte die Reflexivität über die gemeinsam zu treffende Entscheidung mittels eines Instruments angeregt werden, das die Teilnehmenden auffordert, ihre Vorgehensweise zu dokumentieren und dadurch zu kontrollieren. Dies entspricht der Metakognition monitoring. ${ }^{41}$ Weiterhin soll die individuelle Wahrnehmung der aktuellen Situation im Planspiel durch die Teilnehmenden selbst kontrolliert werden. Hierzu wird ein Instrument konstruiert, das einen geringen zeitlichen Aufwand für die Teilnehmenden bedeutet, jedoch in der Lage ist, die Wahrnehmungen für weitere Auswertungen festzuhalten.

Beide Instrumente sollten während der gesamten Planspiel-Phase eingesetzt werden. Es wird jedoch kein Lernerfolg im eigentlichen Sinne postuliert. Der Effekt des Einsatzes soll sich in einer Erhöhung des Lernerfolgs im kognitiven Bereich zeigen. Im nächsten Abschnitt werden grundsätzliche Aspekte der Evaluation sowie die konkrete Umsetzung in der vorliegenden Arbeit dargestellt.

\footnotetext{
39 Vgl. Beck (Schlüsselqualifikationen 1995), S. 73.

40 Vgl. dazu auch Mande, Gruber \& Renkl (Prozesse 1992).

41 Vgl. Friedrich (Analyse 1995), S. 118-121.
} 


\section{5 Überlegungen zur Evaluation}

\subsubsection{Funktionen und Arten von Evaluation}

Der Begriff der Evaluation wird uneinheitlich verwendet. Evaluation ist im Allgemeinen durch eine Bewertung von Handlungsalternativen als Planungs- und Entscheidungshilfe gekennzeichnet. Sie ist ziel- und zweckorientiert und sollte den aktuellen Stand wissenschaftlicher Techniken und Forschungsmethoden berücksichtigen. ${ }^{42}$ Ein Problem dabei ist jedoch, dass Evaluation niemals nur Beobachtung ist, sondern immer auch ein Eingriff. ${ }^{43}$ Dabei lassen sich verschiedene Funktionen verfolgen: ${ }^{44}$

- Die Steuerungs- und Optimierungs-Funktion umfasst die Erhebung von Informationen über den Verlauf und über Zwischenergebnisse des aktuellen Lehr-Lern-Prozesses.

- Zur Bewertungs- und Beurteilungs-Funktion gehören das adäquate Beschreiben der Endergebnisse sowie das Einordnen, Gewichten und Bewerten der Ergebnisse nach Qualitätskriterien.

- Die Kontroll- und Disziplinierungs-Funktion beschränkt sich auf personenneutrale Aspekte einer Maßnahme. Weiterhin bezieht sie sich auf die Aspekte der Beurteilung der Lehrenden oder der Einsatzmöglichkeiten der Teilnehmenden nach der Maßnahme.

- Die Entscheidungs-Funktion beschreibt die Nutzung der Befunde als Entscheidungshilfe oder -begründung.

- Die Dokumentations- und Legitimation-Funktion beinhaltet die Beschreibung und Veröffentlichung der gefundenen Resultate zur Rechtfertigung und Begründung gegenüber den Beteiligten.

- Die Erkenntnis-Funktion bezieht sich auf die Entwicklung übertragbarer Erfahrungen und Strategien bei der Einbettung in theoretische Rahmenüberlegungen.

42 Vgl. Wottawa \& Thierau (Lehrbuch 1998), S.16.

43 Verschiedene Untersuchungen belegen, dass schon die Ankündigung einer Evaluation das Verhalten der Betroffenen verändert. Vgl. Wesseler (Evaluation 1994), S. 684; auch Joint Committee \& Sanders (Handbuch 1999), S. 83-86.

44 Vgl. Will, Winteler \& Krapp (Evaluation 1987), S. 20-25. 
Eine mögliche Definition von Evaluation für die Pädagogik umfasst die Bereiche der Zielorientierung im Sinne einer Verbesserung einer Maßnahme, der Systematik insbesondere bei der Datengewinnung, der Kontinuität eines Konzepts, der Bewertung und Interpretation der erhobenen Daten sowie der Objekt-Orientierung, d. h. den Bezug auf die jeweilige Maßnahme. ${ }^{45}$ Evaluation lässt sich nach verschiedenen Gesichtspunkten einteilen, wobei diese Unterteilung nicht immer eindeutig ist. ${ }^{46}$

Eine Kontext- oder Ziel-Evaluation untersucht z. B. die Rahmenbedingungen oder die mögliche Zielsetzung einer Maßnahme. Eine Input-Evaluation beschäftigt sich neben den materiellen und finanziellen Ressourcen auch mit den Eingangsqualifikationen der Teilnehmenden, den Kompetenzen der Lehrenden, den übergreifenden Zielvorgaben und Planungen sowie den gegebenen Curricula. Die didaktischen Medien, die eingesetzten Methoden und Arbeitsformen, die Informationsund Kommunikationsstrukturen zwischen den Interaktionspartnern und mögliche Interventionen von außen werden in einer Prozess-Evaluation untersucht.

Eine Ergebnis-, Produkt- oder Output-Evaluation untersucht alle kurzfristig beobachtbaren Resultate, unabhängig davon, ob sie als Ziele beabsichtigt waren oder sich als Nebeneffekte ergaben. Die Wirkungs- oder Program-ImpactEvaluation betrachtet die längerfristigen Folgen der Bildungsmaßnahme. Speziell die Transfer-Evaluation beschäftigt sich mit der wichtigen Frage der Übertragbarkeit des Gelernten in den Berufsalltag. Dabei ist auch eine Kombination der einzelnen Aspekte denkbar und durchaus sinnvoll.

Bezüglich der Modelle der Untersuchung kann zwischen folgenden Arten unterschieden werden: Eine Evaluation kann intern durch die Mitarbeiterinnen und Mitarbeiter der Bildungsinstitution durchgeführt werden oder extern an andere Personen vergeben werden. Die Unterscheidung zwischen Selbst- und FremdEvaluation bezieht sich auf die Betroffenen, die die Evaluation selbst durchführen oder jemandem übergeben, der nicht direkt betroffen ist. Dabei kann eine FremdEvaluation durchaus intern durch geführt werden.

\footnotetext{
45 Vgl. Grüner (Evaluation 1993); Will, Winteler \& Krapp (Evaluation 1987), S. 14.

46 Vgl. Will, Winteler \& Krapp (Evaluation 1987), S. 18-27; Windecker (Kompetenzen 1991), S.187-189; Wesseler (Evaluation 1994), S.671-679; Wottawa \& Thierau (Lehrbuch 1998), S. 31-35.
} 
Die formative Evaluation begleitet die Maßnahme und dient der Steuerung des Verlaufs im Sinne einer Prozess-Evaluation. Die während der Bildungsmaßnahme gewonnenen Ergebnisse werden direkt in Veränderungen umgesetzt. Die Versuchspersonen nehmen aktiv handelnd als Subjekte am Evaluationsprozess teil. Die summative Evaluation wird dagegen nach Ende der Maßnahme im Sinne einer Feststellung der Ergebnisse durchgeführt. Die Versuchspersonen sind hier Objekte, die ihre Ergebnisse in standardisierter Form liefern. Allerdings ist eine strenge Unterscheidung zwischen formativer und summativer Evaluation in der Praxis oft nicht möglich, da ein formatives Vorgehen kaum ohne die Erhebung 'harter' Fakten vollzogen werden kann und umgekehrt die summative Evaluation kaum auf die subjektiven Elemente der formativen Evaluation verzichten kann.

\subsubsection{Evaluation in der beruflichen Weiterbildung}

\section{Grundlagen zur Evaluation in der beruflichen Weiterbildung}

Evaluationen in der beruflichen Weiterbildung in Deutschland werden unter allen genannten Aspekten durchgeführt. ${ }^{47}$ Dabei finden sich hier alle Elemente der qualitativen Forschung. ${ }^{48}$ Es kann jedoch nicht davon gesprochen werden, dass dadurch eine eigene Forschungstradition entstanden ist. ${ }^{49}$

Eine besondere Schwierigkeit liegt hier in der Heterogenität der Lerngruppe. Die Verwendung eines einzigen Instruments kann zu ganz unterschiedlichen Reaktionen und Deutungen bei den Teilnehmenden führen, so dass die Vergleichbarkeit zwischen einzelnen Gruppen schwierig wird. ${ }^{50}$ Die Erhebungsverfahren sollten sich deshalb mit einer gewissen Redundanz ergänzen und eine Rückkoppelung ermöglichen. Dies erfordert allerdings ein hohes Maß an Ressourcen für die Evaluation, sowohl beim Einsatz der Instrumente als auch in der Auswertung der erhobenen Daten. ${ }^{51}$

\footnotetext{
$\overline{47 \text { Vgl. Seyfried (Evaluation 1998) }}$, S. 16.

48 Vgl. König (Erwachsenenbildung 1995).

49 Vgl. Born (Geschichte 1991); auch Tietgens (Reflexionen 1992).

50 Vgl. z. B. BLoech et al. (Einsatz 1998), S. 25-32 zu einem Vergleich zweier Erhebungen an der Verwaltungs- und Wirtschaftsakademie Göttingen.

51 Vgl. Wesseler (Evaluation 1994), S. 682.
} 


\section{Überlegungen zur Evaluation der Maßnahme}

Um die Lerninhalte gemäß des Studienplans und die gesetzten Zielen in einer Bildungsmaßnahme vermitteln zu können, ist eine Problemsituation zu schaffen, in der die Lernenden aufgefordert sind, durch Handlungen den aktuellen Zustand in einen gewünschten zu überführen. Dabei wird eine Komplexionsstrategie verfolgt, unter der Folgendes verstanden wird: ${ }^{52}$ Anhand der vorgegebenen Inhalte wird eine komplexe, für Handeln und Lernen motivierend wirkende Lernumgebung so konstruiert, dass Lernprozesse über die Handlungen der Lernenden initiiert werden. Genauer gesagt geschieht dies über die kognitiven Regulationen der Handlungen und über die Wahrnehmungen und Erklärungen der Handlungsfolgen. Dieser Vorgang der Reflexion muss durch eine entsprechende Modellierung der Unterrichtssituation unterstützt werden. ${ }^{53}$

Zur Erreichung der formulierten Lernziele sind strategisch angelegte Unternehmensplanspiele besonders geeignet. Sie stellen ein Verfahren zur Simulation der Auswirkungen wirtschaftlichen Handelns in zeitlich gerafften Abläufen dar. Die Teilnehmenden übernehmen in Kleingruppen die Rolle der Unternehmensleitung und sammeln so Erfahrungen in der gemeinsamen Findung, der Festlegung und der Korrektur von Entscheidungen. Insbesondere die Periodengliederung sowie die Rückmeldung über die Simulations-Ergebnisse an die Teilnehmenden sind von Bedeutung für den Lernerfolg. Im Unternehmensplanspiel wird ein unternehmerisches Verhalten geübt, wobei die wesentlichen funktionalen Zusammenhänge innerhalb des Unternehmens sowie die Interdependenzen zwischen dem abgebildeten Unternehmen und der simulierten Umwelt wie Banken oder Kunden entsprechend zu modellieren sind. ${ }^{54}$

Gerade in der beruflichen Weiterbildung ist die Berücksichtigung psychischer Prozesse bei der Gestaltung von Maßnahmen besonders wichtig. Hier sollte bezüglich der Lernprozesse ein Übergang von der Fremd- zur Selbstregulation vorgenommen werden. ${ }^{55}$ Ausschlaggebend ist die Eingliederung der Teilnehmenden in das Gesamtsystem der Handlungsbereiche. Entscheidend ist dabei auch, dass den Teilnehmenden zu Beginn des Planspiel-Einsatzes die Ziele der Maßnahme erläutert

\footnotetext{
52 Vgl. Preiss (Komplexität 1992), S. 60.

53 Vgl. auch Abschnitt 2.4.2.

54 Vgl. EBert (Planspiel 1992), S. 25-26; auch Abschnitt 3.4.

55 Vgl. Weyerich (Gestaltung 1995), S. 93.
} 
werden. Für sie steht häufig jedoch erst einmal die Beherrschung des Unternehmensplanspiels als solches, d.h. die optimale Steuerung des Systems, im Vordergrund. Sie versuchen durch eine Input-Output-Analyse die Systemstruktur zu erkennen und daraus sinnvolle Entscheidungen abzuleiten. ${ }^{56}$ Entsprechend muss bei der Gestaltung der zu konzipierenden Maßnahme versucht werden, zwischen den übergreifenden Lernzielen der Maßnahme und den Wünschen der Teilnehmenden nach optimaler Steuerung des Planspiels eine Balance zu finden, so dass die gesamte Weiterbildungs-Maßnahme den Teilnehmenden gerecht wird, die Lernziele der Lehrenden erreicht werden und die Forschungen durchgeführt werden können.

Grundsätzlich sollte die Erhebung des Lernerfolgs auf Grund der bisherigen Überlegungen aus einem Prä-Post-Design bestehen, um sowohl das Wissen der Teilnehmenden vor dem Planspiel-Einsatz zu erfassen als auch die Lerneffekte kontrollieren zu können. Hierbei müssen Instrumente eingesetzt werden, die in der Lage sind, die verschiedenen Aspekte der zu vermittelnden Qualifikationen zu erheben. $^{57}$

\subsubsection{Zum Planspiel-Einsatz}

Der Erfolg eines Planspiel-Einsatzes wird maßgeblich von der Gestaltung der auf die Lerngruppe abgestimmten und angemessenen Lernumgebung beeinflusst. Die Komplexität der Anforderungssituation ist den Voraussetzungen der Lerngruppen anzupassen, ${ }^{58}$ um Motivationsverluste durch Über- bzw. Unterforderung zu vermeiden. ${ }^{59}$ Die überwiegend an Universitäten durchgeführten Untersuchungen zum Einfluss der Modellkomplexität eines Planspiels auf den Lernerfolg kommen jedoch zu unterschiedlichen Ergebnissen. Dies ist zum einen auf die unterschied-

\footnotetext{
$\overline{56}$ Vgl. Funke (Problemlösen 1986) S. 16.

57 Vgl. Abschnitt 4.4.

58 Vgl. Achtenhagen (Überlegungen 1990), S. 120; auch Posch, Schneider \& Mann (Unterrichtsplanung 1977), S. 35. Dies wurde bereits 1895 bei der Entwicklung des Kriegsspiels STRATEGOS berücksichtigt, das aus einer Version für Anfänger und einer für Fortgeschrittene besteht; vgl. Rohn (Führungsentscheidungen 1964), S. 22.

59 Vgl. auch Schunck (Subjektive Theorien 1993), S. 195.
} 
liche Definition der Komplexität, zum anderen auf die unterschiedliche Messung des Lernerfolgs zurückzuführen. ${ }^{60}$

Insbesondere in der beruflichen Weiterbildung ist die Modellierung des Unternehmens im Unternehmensplanspiel sorgfältig durchzuführen, damit keine Unstimmigkeiten beim Lernen im und am Modell auftreten können. Die modellierte Realität muss Ernstcharakter für die Teilnehmenden besitzen, nicht für die oder den Modellierenden bzw. Lehrenden, um einen entsprechenden Effekt zu haben. ${ }^{61}$ Der Spielcharakter sollte sich im Sinne einer Revidierbarkeit der Entscheidungen und dem Fehlen von Konsequenzen - im Gegensatz zur realen berufliche Situation - zeigen. Weiterhin ist die Betroffenheit der Teilnehmenden in der PlanspielSituation für den Lernerfolg von besonderer Bedeutung: ${ }^{62}$ Zum einen ist sie als emotionale Bewertung eine zentrale Komponente der Identitätsbildung, von der abhängt, ob etwas als subjektiv bedeutsam erlebt wird oder nicht. Zum anderen bezieht sie sich in dieser Wechselwirkung auf die Unterrichtsinhalte, so dass die Teilnehmenden sich aufgefordert fühlen zu handeln, sie ihr geplantes Handeln nachvollziehen können und es ihnen ernst genug ist, sich damit auseinanderzusetzen.

Auf Grund der heterogenen Lernerfahrungen und Vorbildung der Teilnehmenden ist der Einsatz eines Gesamt-Planspiels einem Teil-Planspiel vorzuziehen. ${ }^{63}$ Dabei sollte auf die Abbildung einer speziellen Branche zu Gunsten der Möglichkeit einer abstrakteren Darstellung der Zusammenhänge im Unternehmen verzichtet werden. ${ }^{64}$ Das Unternehmensplanspiel sollte weiterhin keine stochastischen Elemente enthalten, sondern auf einem deterministischen Simulationsmodell basieren, damit die Unsicherheit der Teilnehmenden beim Einsatz nicht noch erhöht wird. Weiterhin sollte das Unternehmensplanspiel in Gruppenarbeit durchgeführt werden, da dies einen zusätzlich motivierenden Effekt hat, der sich positiv auf den

60 Zur Darstellung der Forschungsergebnisse in diesem Bereich vgl. Abschnitt 3.6.1; zur Definition von Komplexität vgl. Abschnitt 2.5.2. Aus diesen Überlegungen heraus entstand z. B. auch das Unternehmensplanspiel MEdUSa (Modular Einstellbare UnternehmensSimulation), bei dem die Modellkomplexität durch Hinzuschalten bzw. Ausblenden einzelner Betriebsbereiche variabel und systematisch den Anforderungen der Lerngruppe angepasst werden kann; vgl. OrTh (Entwicklung 1998). Zu diesem Aspekt vgl. auch HameL (IntensivSeminar 1996) sowie Hahne (Schlüsselqualifikationen 1989).

61 Vgl. Ebert (Planspiel 1992), S. 27; auch Abschnitt 2.5.

62 Vgl. Sembill (Problemlösefähigkeit 1992), S. 78-79; auch Abschnitt 2.3.

63 Vgl. zur Klassifikation von Unternehmensplanspielen Abschnitt 3.2.3.

64 Vgl. speziell zu diesem Aspekt auch Heinecke \& Oelsnitz (Planspiele 1994). 
Lernerfolg auswirken kann. Neben den fachlichen Lernzielen dient dies auch der Förderung der sozialen Kompetenz der Teilnehmenden, ${ }^{65}$ insbesondere da der überwiegende Teil der Lehre an der Verwaltungs- und Wirtschaftsakademie in Form von Vorlesungen durchgeführt wird.

Beim Einsatz eines interaktiven Planspiels, in dem die simulierten und durch die Teilnehmenden geführten Unternehmen miteinander konkurrieren, könnte das Problem auftreten, dass die konkurrierenden Gruppen unterschiedlich stark sind und eine Gruppe das Spielgeschehen dominiert. Sinnvoll erscheint vielmehr ein Unternehmensplanspiel, in dem zwar eine Konkurrenz-Situation abgebildet wird, aber nur jeweils eins der simulierten Unternehmen von den Teilnehmenden geleitet wird und die Konkurrenz-Unternehmen durch das Modell simuliert werden. Solch eine nicht-interaktive Durchführung gewährleistet die Vergleichbarkeit der Ergebnisse der Gruppen untereinander, weil die Entscheidungen der KonkurrenzUnternehmen nicht von den Entscheidungen der Teilnehmenden abhängen. ${ }^{66}$

Auf Grund der institutionellen Rahmenbedingungen sollte das Unternehmensplanspiel vor Ort eingesetzt werden, d.h. die Teilnehmenden treffen ihre Entscheidungen in den Unterrichtsstunden, die im wöchentlichen Abstand stattfinden. Die Simulation wird außerhalb der Maßnahme von der Spielleitung auf einem PC durchgeführt und die Ergebnisse in der nächsten Stunde den Teilnehmenden zurückgemeldet. Dies hat allerdings den Nachteil, dass der Lernprozess unterbrochen wird.

Um den Lernerfolg durch den Einsatz des Unternehmensplanspiels zu gewährleisten, sind zusätzlich stützende Maßnahmen erforderlich. ${ }^{67}$ Auf der Basis der Überlegungen in Abschnitt 2.3 wird eine Erhöhung der Reflexivität in zwei Bereichen angestrebt. ${ }^{68}$ Dabei sollen die Teilnehmenden durch geeignete Instrumente aufgefordert werden, sich ihre Subjektiven Theorien zum Gegenstandsbereich bewusst zu machen.

- Der kognitiv-prozessuale Bereich ist an die konkreten Inhalte der Maßnahme gebunden und baut auf dem vorhandenen Wissen der Teilnehmenden auf.

\footnotetext{
65 Vgl. Abschnitt 4.4.

66 Vgl. Fassheber (Planspiele 1995), S.613-614; auch Abschnitt 3.2.3.

67 Vgl. Kaiser \& Kaminski (Methodik 1994), S. 180-181.

68 Vgl. auch Abschnitt 4.4 .
} 
- Der zweite Aspekt bezieht sich auf den emotionalen, motivationalen und sozialen Bereich des Lernprozesses, der Auswirkungen auf die Ausbildung der kognitiven Strukturen der Teilnehmenden hat.

Weiterhin ist festzuhalten, dass einige Inhalte nicht durch den Einsatz des Unternehmensplanspiels allein zu vermitteln sind. Ergänzend werden entsprechende Inhalte in einer begleitenden Lehre in Form einer Vorlesung vermittelt. Grundsätzlich sollen hier die Subjektiven Theorien der Teilnehmenden mit den objektiven, wissenschaftlichen Theorien zum Erkenntnisobjekt 'Unternehmen' konfrontiert werden. So wird vor der experimentellen Phase das Modell des Unternehmensplanspiels erläutert und den Teilnehmenden eine grundlegende Einführung in die Wirkungszusammenhänge im Planspiel gegeben. Dadurch soll erreicht werden, dass sich die Teilnehmenden vor der ersten zu treffenden Entscheidung mit dem Planspiel-Modell vertraut machen. Weiterhin wird im Verlauf des Planspiels auf Aspekte der Unternehmensplanung in verschiedenen Bereichen sowie der Kostenrechnung eingegangen. ${ }^{69}$

Die Inhalte des planspiel-begleitenden Unterrichts wurden auf die Gebiete hin, die in der Unternehmens-Simulation zum Tragen kommen, ausgewählt. Das betrifft insbesondere das Niveau und die Auflösung des Sachgebiets, was auch durch die zeitlichen Vorgaben mitbestimmt wird. Für die inhaltliche Bestimmung des Sachgebiets wird sich zunächst im Wesentlichen am Unternehmensplanspiel selbst angelehnt. Allerdings erfolgt eine Ergänzung um weitere Bereiche aus dem Gebiet der Unternehmensplanung, insbesondere im Bereich der Produktion und Investition. Ein Grund war die Vermittlung von Wissen, das über das PlanspielModell hinausgeht, ein weiterer die vorgegebenen Inhalte in der Studienordnung der Verwaltungs- und Wirtschaftsakademie für das 3. Semester. ${ }^{70}$ Die Loslösung von der konkreten Anwendung im Planspiel bei der Vermittlung der betriebswirtschaftlichen Methoden erhöht auch ihre Anwendung in anderen Kontexten.

Bei der Beurteilung der Leistungen im Planspiel können verschiedene Aspekte betrachtet werden, auf die im Folgenden zunächst allgemein eingegangen werden soll. Diese theoretische Überlegungen dienen als Grundlage der Auswertung der erhaltenen Daten und werden im Ergebnisteil zur Darstellung benötigt.

\begin{tabular}{ll}
\hline 69 & Vgl. Abschnitt 3.3. \\
70 & Vgl. Abschnitt 4.2.
\end{tabular} 


\subsection{Aspekte der Leistungsbeurteilung}

\subsubsection{Begriffsklärungen}

Leistung wird allgemein definiert als Erfüllung bestimmter sozialkultureller Normen, wobei gesellschaftliche Berechtigungen an den Grad der Erfüllung geknüpft sind, die sich beispielsweise in Beruf, Einkommen und sozialem Prestige äußern. ${ }^{71}$ Das Prinzip der Leistung wird somit zum 'Prinzip sozialer Verteilungsgerechtigkeit'. ${ }^{22}$ Leistung kann - konkreter auf das Subjekt bezogen - definiert werden als Vollzug oder Ergebnis von Tätigkeiten und Handlungen einschließlich der kognitiven Lernprozesse. ${ }^{73}$ Diese Definition hat zwei Komponenten: ${ }^{74}$ Zum einen werden Maßstäbe für die Handlungen und Handlungsergebnisse mit einer verbindlichen Selbstverpflichtung auf Einhaltung derselben festgelegt. Zum anderen erfolgt eine Selbstbewertung nach erfolgter Tätigkeit, die Konsequenzen nach sich zieht.

In der Pädagogik wird die Leistung, die beim Lernen gezeigt werden soll, als Aneignung von Fertigkeiten, Fähigkeiten, Wissen, Erkenntnissen und Einstellungen definiert. Damit sind aber nicht nur die kognitiven Prozesse, sondern auch die emotionalen, psychomotorischen und sozialen Aspekte von Lernen angesprochen. $^{75}$ Leistung existiert jedoch nicht als solche, sondern immer erst in der Zuschreibung bestimmter Merkmale zum menschlichen Handeln. Handlungen oder Handlungsergebnisse werden von Handelnden zunächst als solche nicht unbedingt als Leistung empfunden. Erst durch eine Beurteilung werden sie zu einer Leistung. Entsprechend kann Leistung niemals abstrakt gefordert werden. Eine Leistungsbeurteilung kann immer nur inhalts-spezifisch und an bestimmte Arbeitsaufgaben gebunden vorgenommen werden. ${ }^{76}$

Die Begriffe der Bewertung, der Beurteilung, der Diagnose sowie der Evaluation werden häufig synonym verwendet, lassen sich jedoch auf Grund ihrer Ausprägungen unterscheiden: ${ }^{77}$ Die Bewertung bezeichnet das Zuschreiben eines Werts oder

\footnotetext{
71 Vgl. Klafki (Leistung 1983), S. 491.

72 Vgl. Heid (Schlüsselqualifikationen 1995), S. 212.

73 Vgl. Klafki (Leistung 1983), S. 491-492.

74 Vgl. Heckhausen (Motivation 1989), S. 231.

75 Vgl. Klafki (Leistung 1983), S. 492.

76 Vgl. HEID (Schlüsselqualifikationen 1995), S. 216; auch Abbildung 2.6.

77 Vgl. Kleber (Diagnostik 1992), S. 77-78.
} 
einer Bedeutung zu einer Leistung. Die Beurteilung ist begrifflich eng mit speziellen Prüfungen, also der Abgabe eines Urteils über eine Leistung verknüpft. Im pädagogischen Zusammenhang ist der Begriff unmittelbar auf Personen bezogen. Bei der Diagnose handelt es sich im engeren Sinn um eine Bewertung auf Grund von präzisen, begründeten Fragestellungen sowie kontrollierter, theoriegeleiteter Datenerhebungsprozesse. Evaluation wiederum ist die ziel- und zweckorientierte Bewertung von Handlungsalternativen zur Hilfe bei Planungen und Entscheidungen. $^{78}$

\subsubsection{Funktionen von Leistungsbeurteilungen}

Die Beurteilung von Leistungen erfüllt im gesellschaftlichen Zusammenleben verschiedene Funktionen: ${ }^{79}$

1. Die Herrschafts- und Sozialisations-Funktion umfasst die Setzung und Kontrolle von Leistungskriterien für das soziale Zusammenleben.

2. Die Steuerungs- und Allokationsfunktion beinhaltet Aspekte des Berichtens über Erfolg und Misserfolg sowie der Berechtigung zum Zwecke der Zuweisung oder Verteilung auf mögliche Positionen des Beschäftigungs- und Bildungssystems.

3. Die pädagogische und didaktische Funktion orientiert sich an den Lernenden: Zum einen soll durch individuelle Leistungsdiagnose und -prognose der erzielte Lernerfolg offengelegt werden, um die Lernenden zu fördern. Hierzu gehört auch die Förderung der Fähigkeit zur Selbsteinschätzung. Zum anderen wird eine Verbesserung der Maßnahme durch didaktische Steuerung des Lernens sowie eine Motivierung der Lernenden zur Intensivierung ihrer Lernaktivitäten angestrebt. Diese Funktion dient auch als Orientierung für die Lehrenden hinsichtlich der Planung weiterer Maßnahmen. Dies wird u. a. angestrebt durch eine Standardisierung von Ausbildungsgängen. ${ }^{80}$

$78 \mathrm{Vgl}$. Abschnitt 4.

79 Vgl. Walter (Prüfungen 1996), S. 27-44 und die dort angegebene Literatur.

80 Z. B. werden Qualitätsstandards in der beruflichen Bildung entwickelt; vgl. BundesinstiTUt Für Berufsbildung (Qualitätsaspekte 1993) sowie Faulstich (Qualitätskriterien 1991). 
Die ersten beiden Funktionen sind auf Grund der leistungsorientierten Gesellschaftsstrukturen von besonderer Bedeutung. Im Zuge der veränderten Rahmenbedingungen ergeben sich auch neue Ansprüche an einen berufspädagogischen Leistungsbegriff. Der didaktische Positionswandel und die Neuorientierung der beruflichen Bildung zeigen sich in den Diskussionen um Ganzheitlichkeit, Schlüsselqualifikationen und einem Lernen unter den Prämissen der Handlungsorientierung. Entsprechend sind Entwicklungstendenzen bezüglich einer Weiterentwicklung des Leistungsbegriffs in der Berufsbildung festzumachen: ${ }^{81}$ Insbesondere die Auffassung der zeitraum- statt zeitpunktbezogenen Beurteilung spiegelt sich im 'dynamischen Leistungsbegriff' wider, der sowohl eine Produkt- als auch eine Prozesskomponente umfasst. ${ }^{82}$ Dieser Leistungsbegriff wird mit der Forderung verbunden, dass die Beurteilung als didaktische Hilfe aufgefasst werden muss, um die Teilnehmenden zunehmend zu Selbständigkeit und Selbstbeurteilung zu befähigen. Die Bewertung ist demnach nicht mehr nur Endkontrolle, sondern vor allem Rückmeldung im Lernprozess.

Entsprechend ergeben sich Konsequenzen für die Gestaltung von Prüfungen und Beurteilungen: ${ }^{83}$ Die Kontrolle des Lernerfolgs erfolgt individuell und ist den jeweiligen Lernprozessen angepasst. Eine Leistungsbeurteilung sollte sich an der Unterrichtplanung und am Verlauf der Maßnahme orientieren. Es sollten Möglichkeiten für die Teilnehmenden geschaffen werden, die Fähigkeiten für die Realität zu erproben, d. h. auch Empfehlungen zum weiteren Lernen beinhalten. Leistungsbeurteilungen sollten sich inhaltlich und methodisch auf die einzelnen Lernenden beziehen. Die Aufgabenstellungen sollten offen und komplex sein und die Lernenden zum Handeln auffordern. Dabei müssen die Lösungen Gestaltungsmöglichkeiten lassen. Gruppenprüfungen sollten gleiches Gewicht wie Einzelprüfungen haben. Die Beurteilung kann auch durch die Lernenden selbst vorgenommen werden, nicht nur durch Beobachtung und Tests. ${ }^{84}$

Erste Ansätze für die Umsetzung eines veränderten Verständnisses von Leistung bringen jedoch auch Probleme mit sich: ${ }^{85}$ Bei einer gruppenorientierten Leistungsbeurteilung sind zum einen die fachpraktischen und -theoretischen Lernziele zu

81 Vgl. Kapitel 1 und 2.

82 Vgl. Klafki (Leistung 1983), S. 493-494.

83 Vgl. Walter (Prüfungen 1996), S. 255-258.

84 Vgl. Heid (Schlüsselqualifikationen 1995), S. 219.

85 Vgl. ausführlicher bei WALTER (Prüfungen 1996), S. 184-210. 
bestimmen, zum anderen die funktionalen und extrafunktionalen Qualifikationen. Erstere betreffen die fachbezogenen Schlüsselqualifikationen, letztere das Planen, Durchführen, Kontrollieren sowie Präsentieren eines konkreten Projekts. Bei der Bewertung sind Inhalts- und Prozessdimensionen ganzheitlich zu berücksichtigen. Für eine umfassende Beurteilung ist eine Mischung aus Gruppen- und Einzelbewertung sowie Fremd- und Eigenkontrolle vorzunehmen. Eine Verlaufsbeobachtung ist zwar zur Beurteilung der Lernprozesse notwendig, aber auch eine Kontrolle des Wissensstands vor bzw. nach dem Lernprozess sollte durchgeführt werden. Ein Problem stellt dabei die Gruppenarbeit dar, denn die Gruppenprozesse beeinflussen das Lernergebnis in erheblichem und nicht vollständig zu kontrollierendem Umfang. Weiterhin ist es schwierig, übergeordnete Qualifikationen wie Schlüsselqualifikationen zu operationalisieren. ${ }^{86}$

Die Umsetzung auf handlungsorientierten Grundsätzen rückt die pädagogische Funktion der Leistungsbeurteilung in den Vordergrund. Insbesondere in der beruflichen Aus- und Weiterbildung spielt jedoch die Allokationsfunktion für die Teilnehmenden, aber auch für die Bildungsinstitution eine wichtige Rolle. ${ }^{87}$ Weiterhin gibt es verschiedene Bezugsmöglichkeiten bei der Beurteilung der individuellen Leistungen. Mit ihnen beschäftigt sich der nächste Abschnitt.

\subsubsection{Bezugsnormen der Leistungsbeurteilung}

Bezugsnormen stellen den Rahmen zur Orientierung bei der Bewertung von Lernerfolgen dar. Sie sind jedoch keine Soll-Normen - wie etwa gesellschaftliche Normen - sondern Werte, an denen etwas beurteilend gemessen werden soll. Auf diese Werte wird die Leistung bezogen und insofern wirken sie als Maßstab für die Beurteilung normierend. ${ }^{88}$ Im Folgenden werden drei verschiedenen Arten der Bezugsnorm dargestellt, wobei auch auf Auswirkungen auf die Lernmotivation eingegangen wird. ${ }^{89}$

\footnotetext{
86 Vgl. Abschnitt 2.4.

$87 \mathrm{Zu}$ verschiedenen Anstätzen der Leistungsbeurteilung im Rahmen der Handlungsorientierung vgl. BECK (Handlungsorientierung 1996), S. 133-140. Vgl. Klauer (Notengebung 1987), S. 182.

89 Vgl. dazu Schlag (Leistungsmotivation 1995), S. 112-117; auch KLAUER (Notengebung 1987) und Kleber (Diagnostik 1992), S. 143-151 sowie Rheinberg (Leistungsvergleiche 1987) zur motivationalen Auswirkung der Bezugsnorm.
} 
Die soziale Bezugsnorm ist eine Realnorm für eine bestimmte Bezugsgruppe. Dabei werden häufig die Leistungen der Lerngruppe herangezogen. Es kann aber auch ein ganzer Jahrgang einer Bildungseinrichtung, eines Landes oder Staats sein. Zur Beurteilung einer Einzelleistung wird der Gruppen-Durchschnitt herangezogen. Das heißt, diese Bezugsnorm kann erst auf Grund empirischer Erhebungen angewandt werden. Mit ihr lässt sich feststellen, ob die individuelle Leistung über oder unter dem Durchschnitt der Bezugsgruppe liegt. Die motivationale Auswirkung dieser Bezugsnorm ist abhängig von der Leistungsstärke der Lernenden: Bei überdurchschnittlicher Leistung wirkt sie eher positiv motivierend, während sie sich auf unterdurchschnittlich Beurteilte negativ auswirkt. Eine Darstellung der Leistungen im Vergleich kann durch standardisierte z-Werte vorgenommen werden, der aussagt, um welchen Anteil der Gesamt-Varianz $S$ der Mittelwert $x$ einer bestimmten Gruppe vom Mittelwert $M$ der gesamten Stichprobe entfernt ist: $:^{90}$

$$
z=\frac{x-M}{S}
$$

Die sachliche Bezugsnorm ist eine Idealnorm, weil sie sich an den Lernzielen des Unterrichts orientiert. Sie wird entsprechend auch curriculare Norm oder Lernzielnorm genannt. Zur Beurteilung ist eine Operationalisierung der Lernziele erforderlich, wobei genaue Leistungskriterien definiert werden müssen. ${ }^{91}$ An ihnen werden die einzelnen Leistungen gemessen und festgestellt, ob das Lernziel eher übertroffen oder verfehlt wurde. Die motivationale Auswirkung ist positiv, wenn Erreichbarkeit und Herausforderung im angemessenen Umfang gegeben sind. Trifft dies nicht zu, werden die Lernenden also im Unterricht über- oder unterfordert, hat das einen negativen Einfluss auf die Lernmotivation. Eine Darstellung kann durch prozentuale Abweichungen vom zu erreichenden Kriterium vorgenommen werden, wie z. B. der Anteil der individuell richtig gelösten Aufgaben an der Anzahl der gestellten Aufgaben.

Die individuelle Bezugsnorm ist wie die soziale Bezugsnorm eine Realnorm, jedoch wird sich hier an den persönlichen Leistungen orientiert. Sie ist also eine

90 Vgl. Hays (Statistic 1973), S. 251-252; auch Lienert \& RaAtz (Testaufbau 1998), S. 331 und S. 562.

91 Vgl. dazu Abschnitt 2.4. 
Realnorm für jede/n einzelne/n Lernende/n und trifft Aussagen über die Verbesserung oder Verschlechterung im Vergleich. Um die Effekte des Lernzuwachses messen zu können, ist eine Erhebung der Leistungen zu verschiedenen Zeitpunkten mit vergleichbaren Tests erforderlich. Diese Bezugsnorm bietet Möglichkeiten der individuellen Förderung der Lernenden sowie eine Selbstbewertung, die zur Steigerung der Lernerfolge beiträgt. Sie hat unter den drei Bezugsnormen die stärkste positive Auswirkung auf die Lernmotivation, da auf das Anspruchsniveau der Lernenden Bezug genommen wird. ${ }^{92}$ Zur Ermittlung des Lernerfolgs erscheint es sinnvoll, den gleichen Test zweifach einzusetzen und die Differenz zwischen beiden Testwerten zu ermitteln.

Zusammenfassend lässt sich festhalten, dass die dargestellten Bezugsnormen unterschiedliche Informationen über den Lernerfolg liefern. Entsprechend sind alle drei Normen pädagogisch sinnvoll und sollten gleichzeitig angewandt werden. ${ }^{93}$ Die individuelle Bezugsnorm ist zwar am stärksten förderlich für die Lernmotivation, insbesondere für die Allokations-Funktion aber ist die soziale Bezugsnorm entscheidend. ${ }^{94}$

Die Leistungsbewertung von führt zu Problemen hinsichtlich der Vergleichbarkeit zwischen verschiedenen Messinstrumenten. Daher sollen in der vorliegenden Untersuchung Bewertungsschemata entwickelt werden, die Intervallskalen darstellen und metrische Eigenschaft haben, so dass Vergleiche und statistische Auswertungen möglich sind. ${ }^{95}$

\subsection{Gütekriterien von Erhebungsinstrumenten}

\subsubsection{Allgemeine Überlegungen}

Im Allgemeinen enthält das Modell der Forschung verschiedene Komponenten, die untereinander in Zusammenhang stehen: ${ }^{96}$ Die Forschenden möchten ein be-

\footnotetext{
92 Vgl. Heckhausen (Motivation 1989), S. 272-275.

93 Vgl. KLauer (Notengebung 1987), S. 188-190.

94 Vgl. Rheinberg (Leistungsvergleiche 1987), S. 101-104.

95 Vgl. Lienert \& Eye (Statistik 1994), S. 17-20.

96 Vgl. Birkhan ((Un)brauchbarkeit 1992), S. 235-236; auch Vorwort zu Feger \& BredenKAMP (Datenerhebung 1983).
} 
obachtetes Phänomen im Verhalten und Handeln von Menschen erklären. Dabei unterliegen ihre Vorgehensweisen den erkenntnis- und wissenschaftstheoretischen Regeln ihrer Disziplin. Die Daten werden mit Hilfe von Messinstrumenten, bei den Erkenntnis-Subjekten, also den Personen, deren Handlungen zu erklären sind, durch die Forschenden, erhoben. Wichtig ist hierbei, dass eine Wechselbeziehung zwischen dem zu erfassenden Gegenstand und dem Instrument, das zur Erfassung eingesetzt wird, besteht. Das heißt, die Messung ist immer Modell bildend und niemals in der Lage, die Realität umfassend abzubilden. ${ }^{97}$ Entsprechend ist das Messinstrument auf Grund der Theorien des Bereichs zu konstruieren. Im Bereich der Pädagogik sind dies die Theorien der Wahrnehmung, der kognitiven Präsentation, der Informationsverarbeitung usw., die versuchen, die 'unsichtbaren' psychischen Eigenschaften des Menschen mit Hilfe von Tests für die Forschenden sichtbar zu machen. Aus den erhobenen Daten müssen nun Rückschlüsse gezogen werden, um Einsichten zu gewinnen. Diese Erklärungen sollten dem Schema der deduktiv-nomologischen Erklärung unterliegen. ${ }^{98}$ Das Schema kann als Prototyp der Subsumtion zu erklärender Sachverhalte unter Gesetzmäßigkeiten gelten. Dabei trat in den Sozialwissenschaften das Problem auf, dass die Anforderung an die Gesetze, deterministische All-Aussagen zu sein, im Allgemeinen nur selten erfüllt ist. Wenn weiterhin für den Sachverhalt eine Begründung verlangt wird, muss auch für die Erkenntnis, auf die jeweils die zu begründende Auffassung zurückgeführt wird, eine Begründung verlangt werden. ALBERT bezeichnete diese Situation als Münchhausen-Trilemma. ${ }^{99}$ Sie ist theoretisch mit Hilfe von drei Alternativen zu lösen:

1. Ein infiniter Regress ist praktisch aber nicht durchführbar, da auf der Suche nach Begründungen zeitlich immer weiter zurückgegangen werden muss.

2. Ein logischer Zirkel in der Deduktion kann zu keiner weitergehenden Begründung führen, weil er auf bereits benutzte Aussagen zurückgreift.

97 Vgl. Gigerenzer (Messung 1981), S. 86-87.

98 Vgl. Hempel \& Oppenheim (Studies 1948).

99 Vgl. Albert (Traktat 1991), S.13-18. Der als 'Lügenbaron' bezeichnete Karl Friedrich Hieronymus Freiherr von Münchhausen (1720-1797) aus Bodenwerder erzählte zu Lebzeiten abenteuerliche Geschichten, deren Phänomene nicht zu erklären sind. Insbesondere die Geschichte, in der er sich an seinem eigenen Schopf aus dem Sumpf zieht und dadurch rettet, ist paradox, also unerklärlich und kann als Metapher für die Situation in der Wissenschaft gesehen werden. 
3. Da ersten beiden Möglichkeiten unpraktikabel erscheinen, besteht die Neigung, einen Abbruch des Verfahrens an einer bestimmten Stelle vorzunehmen. In diesem Fall könnte die oder der Begründende von 'Intuition' oder 'Erfahrung' sprechen oder sich auf ein Dogma zurückziehen, um den Begründungsvorgang abbrechen zu können. Dies würde jedoch das Prinzip der vollständigen Begründung ad absurdum führen. An dieser Stelle liefert auch die Verwendung induktiver Verfahren - im Gegensatz zum bisherigen deduktiven Vorgehen - zur logischen Begründung keine weiteren Erkenntnisse.

Da es offensichtlich keine rationale Lösung dieser Situation gibt, ist zu fragen, ob das Trilemma an sich nicht vermeidbar ist. ${ }^{100}$ Tritt nun an die Stelle der vollständigen Begründung einer Aussage die Idee der kritischen Prüfung, so wird zwar darauf verzichtet, eine selbst produzierte Gewissheit zu erreichen. Im Gegenzug wird aber die Aussicht gewonnen, durch versuchsweise Konstruktion prüfbarer Theorien und ihrer kritischen Diskussion an Hand relevanter Gesichtspunkte der Wahrheit näher zu kommen, ohne sie jedoch jemals vollständig zu erreichen. ${ }^{101}$ Die Liberalisierung führte schließlich zum Konzept einer schwachen Kausal-Erklärung: ${ }^{102}$

Ein Umstand wird als ursächlich für ein Ereignis angesehen, wenn der Umstand zeitlich vor dem Ereignis stattgefunden hat und das Auftreten des Ereignisses hierdurch wahrscheinlicher wurde.

Um eine Erklärung des Verhaltens und Handelns aus den erhobenen Daten ableiten zu können, müssen die Daten im Explanans, also die Gesetze und die Randbedingungen, verlässlich sein. An dieser Stelle kommen die Gütekriterien der 'Tests', also der Informationserhebung und Interpretation in einem diagnostischen Prozess, ins Spiel, da sich aus unzuverlässig erhobenen Daten keine verlässlichen Folgerungen ziehen lassen. Bei der Konstruktion von Erhebungsinstrumenten zur empirischen Forschung werden entsprechend verschiedene Kriterien zur Beurteilung ihrer Güte herangezogen. Dabei gibt es neben den drei Haupt-Gütekriterien der Objektivität, Reliabilität und Validität weitere Kriterien wie Fairness, Ver-

\footnotetext{
100 Vgl. Albert (Traktat 1991), S. 35-44.

101 Vgl. dazu auch Popper (Logik 1989).

102 Vgl. der Darstellung bei Groeben (Handeln 1986), S. 202-208 sowie 283-297.
} 
gleichbarkeit, Ökonomie und Nützlichkeit. Ein Test sollte nach Möglichkeit alle diese Forderungen erfüllen. ${ }^{103}$

\subsubsection{Objektivität}

Das Kriterium der Objektivität bezeichnet den Grad der Unabhängigkeit der Ergebnisse von der Person der/des Untersuchenden. Dabei werden verschiedene Aspekte betrachtet: ${ }^{104}$

Unter der Durchführungs-Objektivität wird der Grad der Unabhängigkeit der Testergebnisse durch zufällige oder systematische Verhaltensvariationen des Untersuchenden im Verlauf der Untersuchung verstanden, die zu Verhaltensänderungen bei den Teilnehmenden führen und deren Ergebnis beeinflussen. Zur Maximierung dieser Art der Objektivität sollten die sozialen Interaktionen zwischen den Untersuchenden und den Teilnehmenden so gering wie möglich gehalten werden. Das bedeutet die Vorgabe genauer Instruktionen sowie eine Standardisierung der Untersuchungssituation. Überprüft werden kann die Durchführungs-Objektivität, indem mehrere Untersuchende eine Stichprobe testen und nachfolgend die Ergebnisse korreliert werden. Ist der Wert des Korrelations-Koeffizienten hoch, so sind die Ergebnisse eher unabhängig von der durchführenden Person.

Die Auswertungs-Objektivität bezieht sich auf den Grad der Unabhängigkeit von der auswertenden Person. Bei Tests, in denen die Teilnehmenden nur über den Wahrheitsgehalt einer Aussage im Sinne von richtig oder falsch zu entscheiden haben, ist diese Objektivität praktisch vollkommen gegeben. Sie ist entsprechend geringer bei Möglichkeiten der freien Beantwortung. Hier kann eine Orientierung an vorgegebenen Kriterien wie operationalisierten Lernzielen sinnvoll sein, d. h. eine numerische oder kategoriale Auswertung des Testverhaltens nach vorgegebenen Regeln. ${ }^{105}$ Um diese Art der Objektivität zu bewerten, ist eine Auswertung der Ergebnisse einer Stichprobe durch mehrere Personen und nachfolgende Kor-

103 Vgl. Kleber (Diagnostik 1992), S. 174.

104 Vgl. Lienert \& RaAtz (Testaufbau 1998), S. 7-9; Kleber (Diagnostik 1992), S. 189-192.

105 Z. B. wiesen die Urteile verschiedener Lehrkräfte bei der freien Beurteilung von schriftlichen Prüfungen wie Aufsätzen eine starke Streuung auf; vgl. Ingenkamp (Lehrbuch 1995), S. $107-108$. 
relation der Ergebnisse vorzunehmen. Auch hier ist die Objektivität umso höher, je größer der erhaltene Koeffizient ist.

Als drittes Kriterium beeinflusst die Interpretation der Ergebnisse die Objektivität. Die Interpretations-Objektivität ist gegeben bei normierten Tests mit feststehenden Antworten und Testskalen, also bei Tests, die keine Interpretation erfordern. Bei offenen Bewertungen oder indirekten Messungen, die noch interpretiert werden müssen, ist sie jedoch nicht trivial. Hier ist eine Lenkung erforderlich, d.h. es sollten Schemata zur Interpretation der Ergebnisse vorgegeben werden. Die Überprüfung dieser Objektivität geschieht durch die Ermittlung des Kontingenz-Koeffizienten zwischen den Ergebnissen der Interpretation mehrerer Personen der gleichen Rohdaten einer Stichprobe. Auch hier kann von einem hohen Koeffizienten auf eine hohe Objektivität geschlossen werden.

Zusammengefasst lässt sich sagen, dass eine Beurteilung mittels eines Tests dann objektiv ist, wenn sie in der Durchführung, Auswertung und Interpretation dem Objekt entspricht und die Subjektivität der Forschenden vermeidet.

\subsubsection{Reliabilität}

Unter dem Gütekriterium der Reliabilität wird der Grad der Genauigkeit verstanden, mit dem ein bestimmtes Merkmal gemessen wird. Dabei wird nicht bewertet, ob dieses Merkmal mit dem Test zu messen ist oder nicht. Dies entspricht dem Aspekt der Validität. Das Gütekriterium der Zuverlässigkeit wird ebenfalls unter verschiedenen Aspekten konkretisiert: ${ }^{106}$

Die Paralleltest-Reliabilität bezieht sich auf die Messung eines Merkmals durch zwei verschiedene, aber einander streng vergleichbare Tests, sog. Paralleltests, die einer Stichprobe vorgelegt werden und deren Ergebnisse korreliert werden. Ein hoher Korrelations-Koeffizient deutet auf eine große Übereinstimmung der beiden Tests hin, d. h. beide Tests messen in etwa das gleiche Merkmal.

Durch Testwiederholung kann die Retest-Reliabilität gemessen werden. Hier wird einer Stichprobe ein Test zweimal zur Bearbeitung vorgelegt und die Ergebnisse

106 Vgl. Lienert \& RaATZ (Testaufbau 1998), S. 9-10, S. 173-220; auch Kleber (Diagnostik 1992), S. 184-189. 
werden korreliert. Der Korrelations-Koeffizient kann jedoch auch einen Erinnerungseffekt messen, d.h. die Messergebnisse werden durch die Erinnerung der Teilnehmenden an den ersten Test beim zweiten Durchgang verfälscht. Die Ergebnisse ermöglichen weiterhin Rückschlüsse auf die zeitliche Stabilität des Merkmals. Dabei sind aktuelle Persönlichkeitsmerkmale wie Emotionen, Bedürfnisse oder bestimmte Interessen eher instabil, während habituelle Merkmale wie Fähigkeiten und Fertigkeiten eine höhere Konstanz aufweisen.

Ein weiterer Aspekt ist die innere Konsistenz eines Tests. Sie kann durch Testhalbierung gemessen werden, $\mathrm{d}$.h. die Items eines Tests werden in zwei gleichwertige Teile geteilt ('split half'). Nach der Bearbeitung des gesamten Tests durch eine Stichprobe werden die Testergebnisse gemäß der Halbierung ebenfalls geteilt und miteinander korreliert. Der Koeffizient gibt Auskunft darüber, inwieweit beide Testhälften das gleiche Merkmal messen.

\subsubsection{Validität}

Die Validität bezieht sich auf den Grad der Genauigkeit, mit dem der Test das misst, was er messen soll. Das heißt, hier wird die Gültigkeit der Ergebnisse bezüglich des zu messenden Merkmals überprüft. Dabei wird hinsichtlich des Inhalts, der Konstrukte sowie externer Kriterien unterschieden: ${ }^{107}$

Die Inhalts-Validität bezieht sich auf eine optimale Messung eines bestimmten Merkmals durch den Test. Die Testergebnisse sollten also einen Schluss auf das gleiche Verhalten außerhalb der Testsituation zulassen. Für pädagogische Tests gilt, dass die zu messenden Merkmale entsprechend operationalisiert werden müssen. Hierbei kann auf Experten-Urteile bezüglich der geforderten Merkmale als externes Kriterium zurückgegriffen werden.

Ein Test soll weiterhin die zu Grunde liegende Theorie in den gemessenen Merkmalen abbilden. Hier wird von Konstrukt-Validität gesprochen. Das heißt, auf Grund der gefundenen Ergebnisse kann ein Rückschluss auf eine Verhaltensdisposition, also eine Fähigkeit, eine Eigenschaft oder eine Einstellung usw. geschlossen werden. Besteht ein Test aus mehreren Items, so sollten alle Items gemeinsam

107 Vgl. Lienert \& RaAtz (Testaufbau 1998), S. 10-11, S. 220-271; auch KLeBer (Diagnostik 1992), S. 176-184. 
ein Merkmal prognostizieren. Dies kann durch eine Faktoranalyse überprüft werden. ${ }^{108}$ Unter diesem Aspekt ist auch das Konzept der ökologischen Validität zu fassen: Wird eine diagnostische Aussage zu einer Fragestellung und einer Situation bzw. über eine Person in einer konkreten Situation gemacht, so muss ihre Gültigkeit ebenfalls an den Bedingungen dieser Situation gemessen werden.

Ein Schluss auf der Grundlage einer nachgewiesenen Übereinstimmung mit einem bestimmten Kriterium auf ein Verhalten außerhalb der Testsituation wird unter dem Begriff der Kriteriums-Validität gefasst. Hier misst ein externes Kriterium z. B. die Beurteilung eines Vorgesetzen - das Merkmal valide, wenn die Ergebnisse wiederholbar sind. Zur Überprüfung wird die Korrelation zwischen dem externen Kriterium und den Testwerten herangezogen. Dabei kann der zeitliche Aspekt in die Betrachtung einbezogen werden: Die Übereinstimmungs-Gültigkeit bezieht sich auf den Zusammenhang mit einem aktuell relevanten Kriterium, während die Vorhersage-Gültigkeit etwas über den Zusammenhang mit einem zukünftig relevanten Kriterium aussagt. Die Höhe des Validitäts-Koeffizienten hängt von drei Faktoren ab: Der Reliabilität des Tests, der Reliabilität des Kriteriums sowie der gemeinsamen Erfassung des Merkmals durch Test und Kriterium. ${ }^{109}$

Je größer die gemeinsame Erfassung des Merkmals durch Test und Kriterium ist, umso größer ist die kriteriumsbezogene Validität des Tests. Umgekehrt ist die Validität umso geringer, je geringer die Reliabilität von Test und Kriterium ausfällt. Insgesamt lässt sich sagen, dass die Validität einer empirischen Erhebung steigt, wenn mehrere Tests eingesetzt werden, die verschiedene Aspekte einer übergeordneten Fähigkeit erfassen. ${ }^{110}$

\subsubsection{Weitere Kriterien}

Neben den Hauptgütekriterien gibt es einige weitere Kriterien zur Beurteilung der Güte eines Tests, die jedoch zum Teil keine Maßzahlen vorweisen können: ${ }^{111}$ Die Normierung eines Tests bezieht sich auf die Standardisierung der Testergebnisse mittels einer Messskala. Ohne eine solche Normierung ist kein Forschungsinstru-

\footnotetext{
$108 \mathrm{Vgl}$. Abschnitt 7.7.

109 Im letzten Fall wird auch von der Zulänglichkeit eines Tests gesprochen.

110 Vgl. z. B. die Untersuchung von Hollmann (Validität 1991).

111 Vgl. Lienert \& RaAtz (Testaufbau 1998), S. 12-13.
} 
ment zum Vergleich von Gruppen geeignet. Existieren mehrere Paralleltests oder Tests mit vergleichbarer Validität, so kann von einer Vergleichbarkeit der Tests bezüglich des zu messenden Merkmals gesprochen werden. Insbesondere in der Evaluation von Bildungsmaßnahmen ist die Ökonomie eines Tests von Bedeutung. Ein Test ist ökonomisch, wenn er die zur Verfügung stehenden Ressourcen effizient ausnutzt. Dazu gehören eine kurze Durchführungszeit, ein geringer Materialverbrauch und eine einfache Handhabung. Weiterhin sollten die Durchführung des Tests als Gruppentest und eine schnelle und bequeme Auswertung möglich sein.

Fast trivial ist der Aspekt der Nützlichkeit eines Tests: ${ }^{112}$ Es sollte zum einen ein praktisches Bedürfnis für die Messung eines Merkmals bestehen. ${ }^{113}$ Zum anderen sollten nicht bereits mehrere Tests zur Erfassung dieses Merkmals vorhanden sein. Die Nützlichkeit von schriftlichen Lernkontrollen ermöglicht jedoch keine Aussagen über das Lern- und Arbeitsverhalten der Lernenden. Sie bilden lediglich kognitive Strukturen ab, wobei sie keine abgesicherten Aussagen darüber ermöglichen, in welchem Maße den Lernenden eine Umsetzung der kognitiven Strukturen in Relation zu ihrer tatsächlichen Leistungsfähigkeit gelingt.

Ein weiterer Aspekt bei der Beurteilung der Güte eines Tests ist die Fairness, die in der klassischen Testtheorie nicht explizit genannt wird: ${ }^{114}$ Erhebungsverfahren zur Leistungsbeurteilung sind fair, wenn sie den Gegenstand des Unterrichts erfassen, in der Lage sind, den Lernprozess der Lernenden widerzuspiegeln und die anderen gemessenen Merkmale gesondert ausweisen. Bewertungen sind fair, wenn sie die individuellen Lernprozesse und die individuellen Lebens- und Lernsituationen der Lernenden berücksichtigen. Fairness ist also zu realisieren, indem Tests gruppenspezifisch normiert und mehrere Tests zu mehreren Zeitpunkten zur bessereren Prognose von Merkmalen durchgeführt werden.

Im Folgenden wird die Konzeption der Evaluation dargestellt, die die Lernerfolge beim Einsatz eines Unternehmensplanspiels im dritten Semester an der Vewaltungs- und Wirtschaftsakademie Göttingen untersuchen soll. Dabei wird

112 Vgl. Lienert \& RaAtz (Testaufbau 1998), S. 13.

113 Dabei sollte dieses Merkmal als erstes überprüft werden, denn "Wen interessiert schon die Validität eines überflüssigen Tests!“, Birkhan ((Un)brauchbarkeit 1992), S. 233.

114 Vgl. KLeber (Diagnostik 1992), S. 174-176. 
auch auf die konkrete Gestaltung des Planspiel-Einsatzes eingegangen sowie das grundlegende Design hergeleitet.

\subsection{Konzeption der Maßnahme}

\subsubsection{Gestaltung der Evaluation}

Die Evaluation der Fortbildungs-Maßnahme wird im Rahmen dieser Untersuchung als interne Fremd-Evaluation, d. h. innerhalb der Institution durch Außenstehende durchgeführt. Sie hat in Bezug auf die Lernfortschritte der Teilnehmenden summativen Charakter, wird also im Sinne einer Ergebnis-Evaluation nach Abschluss der Veranstaltungen auf Grund der erhobenen Daten mit Hilfe von statistischen Auswertungsmethoden durchgeführt. Bezogen auf das eingesetzte didaktische Material hat die Evaluation formativen Charakter. Vorrangiges Ziel dieser Bewertung, die während der Bildungsmaßnahme durchgeführt wird, ist die Optimierung des Planspiel-Einsatzes im Sinne einer Prozess-Evaluation. ${ }^{115}$

Bei der Beurteilung von Lernerfolgen gibt es grundsätzlich verschiedene Kriteriumsvariablen. Dabei kann zwischen primären, direkt zu erhebenden und sekundären, nur indirekt zu erhebenden Variablen unterschieden werden: ${ }^{116}$ Bei sofort lernbaren Inhalten, die kurzfristig zu vermitteln sind, ist ein experimentelles Design zur Erfassung der Primär-Variablen angebracht. Beim Lernen komplexerer Inhalte, das sich in einem mittel- bis langfristigen Prozess vollzieht, sind quasi-experimentelle Designs vorzuziehen. Zu den Sekundär-Variablen gehören die Aktivitäten der Lernenden, die direkt zu beobachten bzw. mittels Fragebögen zu erheben sind, sowie motivationale, emotionale und affektive Faktoren, die hauptsächlich über Fragebögen erfasst werden. Dabei sind jedoch die Anteile der Begabung und Anstrengung, die von den Teilnehmenden erbracht werden, messtheoretisch und -methodisch schwierig zu erfassen. ${ }^{117}$

\footnotetext{
115 Zur Klassifikation vgl. Abschnitt 4.5.1.

116 Vgl. KLAUER (Teaching 1988), S. 353.

117 Vgl. HEID (Schlüsselqualifikationen 1995), S. 221.
} 
Idealerweise sollte bei der Evaluation eines auf die Vermittlung von Schlüsselqualifikationen ausgerichteten Unterrichts wie folgt vorgegangen werden: ${ }^{118}$ Zunächst ist das Sachwissen zu erheben und eine inhaltsbezogene Problemlöseaufgabe zu stellen. Nachfolgend ist die Fähigkeit der Steuerung einer simulierter Unternehmen im Team sowie die Entwicklung von kognitiver Strukturiertheit mit geeigneten Instrumenten zu erheben. Ein wichtiger Aspekt ist die Sicherung des Transfers der erworbenen Fähigkeiten. Weiterhin ist eine Förderung der Reflexivität bezüglich Emotionen und Motivationen erforderlich. Einzelkenntnisse - wie Fakten- und Zusammenhangswissen - sind notwendig, aber nicht hinreichend für erfolgreiches Handeln im Beruf. Die verschiedenen Dimensionen sind dabei nicht additiv, sondern ganzheitlich-integrativ zu verstehen.

Entsprechend und auf Grund der Überlegungen der vorangegangenen Abschnitten sind folgende Erhebungsinstrumente zur Evaluation zu konstruieren, wobei die Reihenfolge Evaluation-Konstruktion-Implementation-Evaluation einzuhalten ist. ${ }^{119}$ Dies ist in der vorliegenden Untersuchung gewährleistet, da auf den Erfahrungen des DFG-Projekts aufgebaut wurde. ${ }^{120}$

- Zur Erhebung allgemeiner ökonomischer Kenntnisse wird ein Fragebogen konzipiert.

- Die Untersuchung der Entwicklung von kognitiver Strukturiertheit erfolgt mittels der Netzwerk-Technik.

- Zur Erfassung des methodischen Arbeitens sowie der Anwendung betriebswirtschafliche Methoden wird eine Fallstudie eingesetzt, die auch die Möglichkeit bietet, die Transferleistung der während des Planspiel-Einsatzes erworbenen Fähigkeiten zu erheben.

Diese Instrumente werden sowohl vor als auch nach der Planspiel-Phase eingesetzt, um den Lernfortschritt erfassen zu können. ${ }^{121}$ Aspekte des 'training-on-test' werden aufgrund des großen Zeitraums zwischen den beiden Testzeitpunkten als zu vernachlässigen angesehen. Der Einsatz des Unternehmensplanspiels wird zur Evaluation im Wesentlichen wie folgt konstruiert:

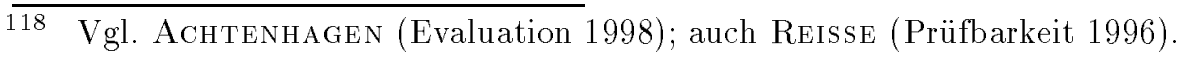

119 Vgl. Achtenhagen (Evaluation 1998).

$120 \mathrm{Vgl}$. Kapitel 1.

121 Vgl. Cook \& Campbell (Quasi-Experimentation 1979), S. 99-103.
} 
- Die Fähigkeit der Steuerung eines simulierten Unternehmens wird mittels des eingesetzten Unternehmensplanspiels in einer eigenen Phase im Anschluss an die eigentliche Lernphase erhoben.

- Zur Erhebung des Problemlösens im Team wird ein Fragebogen eingesetzt. Er ist jedoch nicht nur Erhebungsinstrument, sondern auch Treatment. Mit ihm wird das Ziel der Erhöhung der Reflexivität der Teilnehmenden über den Prozess der Entscheidungsfindung verfolgt.

- Zur Erfassung der individuellen Emotionen und Motivationen in der Entscheidungssituation wird ebenfalls ein Fragebogen konstruiert, der gleichzeitig als Treatment zur Erhöhung des Bewusstseins dient.

\subsubsection{Gestaltung des Planspiel-Einsatzes}

Der Umgang mit einem Unternehmensplanspiel als komplexes System ist für die Teilnehmenden insbesondere zu Beginn der Maßnahme schwierig. Im Allgemeinen gibt es verschiedene Möglichkeiten zur Reduzierung von Komplexität: ${ }^{122}$ Zunächst können durch Abstraktion bestimmte Merkmale aus der Betrachtung ausgeklammert werden. Weiterhin können durch Komplexbildung einzelne Komponenten zu einer Gesamtheit zusammengefasst werden, die nachfolgend als (unzerlegbare) Einheit betrachtet werden. Schließlich bietet die Reduktion durch Zurückführen einer Menge von Einzelmerkmalen auf ein Grundmerkmal, dessen Auswirkungen oder Symptome die Einzelmerkmale sind, eine Möglichkeit zur Verringerung der Komplexität.

Die entsprechende Umsetzung in ein Planspiel geschieht durch einen modularen Aufbau. ${ }^{123}$ Das heißt, die Komplexität der Ausgangssituation wird für die Teilnehmenden reduziert, so dass sie sich auf die wesentlichen Dinge beschränken und sich im System zurecht finden können. Um eine Unterforderung im Laufe des Unternehmensplanspiels zu umgehen, sollte jedoch die Komplexität sukzessive gesteigert werden. Dabei muss der individuelle Lernfortschritt der Kleingruppe berücksichtigt werden. Hier greift dann auch der motivationale Aspekt der individuellen Bezugsnorm. ${ }^{124}$

\footnotetext{
$1 \overline{22}$ Vgl. Dörner (Problemlösen 1987) , S. 18-19.

123 Zum Begriff des modularen Planspiels vgl. Abschnitt 3.2.3.

$124 \mathrm{Vgl}$. Abschnitt 4.6.3.
} 
Um den Effekt dieser Durchführung auf den Lernerfolg im Vergleich zu einer herkömmlichen Version des Planspiels mit konstanter Komplexität überprüfen zu können, sollten die Teilnehmenden in zwei experimentelle Gruppen aufgeteilt werden. Beiden Durchführungsformen sollte die gleiche Ausgangslage im Planspiel zu Grunde liegen, um einen Einfluss der modellierten Unternehmenssituation zu vermeiden. Weiterhin werden hierdurch die erzielten Ergebnisse der beiden experimentellen Gruppen bereits in dieser Phase des Planspiels vergleichbar. Eine Kontrollgruppe ist in dieser Untersuchung nicht erforderlich, da die Fragestellung auf der Konzeption einer Bildungsmaßnahme mit Einsatz eines Unternehmensplanspiels liegt.

In der Testphase soll das gleiche Planspiel mit einer neuen Ausgangslage allen Teilnehmenden in voller Komplexität präsentiert werden. Diese Phase dient der Überprüfung des Lernerfolgs in einer komplexen Situation. Der Vorteil des Einsatzes eines Planspiels als diagnostisches Mittel gegenüber eher eindimensional konstruierten diagnostischen Verfahren wie Fragebögen liegt in der Möglichkeit, die Kompetenzen wesentlich realitätsnäher zu erheben.

Problematisch ist jedoch die Standardisierung der Ergebnisse, da jede Runde im Planspiel einen anderen Verlauf nehmen kann. Hier bietet der Einsatz eines nicht-interaktiven Planspiels große Vorteile hinsichtlich der Vergleichbarkeit der Ergebnisse. Neben der Bewertung der Simulationsergebnisse werden weitere Instrumente eingesetzt, die nähere Aufschlüsse über die Gruppenarbeit geben. ${ }^{125}$

\subsubsection{Design der Erhebung}

Der Lernerfolg des Einsatzes des Unternehmensplanspiels wird weiterhin in einem Prä-Post-Design kontrolliert. Dabei werden zu beiden Zeitpunkten die gleichen Instrumente eingesetzt, um eine bessere Überprüfung und eine Vergleichbarkeit der Leistungen zu gewährleisten. ${ }^{126}$ In diesen Tests werden mehrere Erhebungsinstrumente eingesetzt, die eine breite Erfassung des spezifischen Vorwissens im Prätest sowie des Lernerfolgs im Prä-Post-Vergleich ermöglichen. ${ }^{127}$

125 Vgl. Fassheber (Planspiele 1995), S. 611-616.

126 Vgl. Cook \& Campbell (Quasi-Experimentation 1979), S. 99-103.

127 Zur inhaltlichen Gestaltung der Erhebungsinstrumente und Beurteilung hinsichtlich der Gütekriterien vgl. Kapitel 5 sowie 6.2 . 
Zur Erhebung allgemeiner ökonomischer Kenntnisse wird ein Fragebogen erstellt, der auf Grund der relativ großen Stichprobe, des geringen zeitlichen Umfangs sowie der eher untergeordneten Rolle in der Evaluation als Multiple-ChoiceFragebogen konzipiert wird.

Die Anwendung betriebswirtschaftlicher Methoden und die Transferleistung wird mit einer Fallstudie erhoben, die eine komplexe ökonomische Problemsituation darstellt und den hohen Anforderungen einer Planspiel-Situation entspricht. Sie muss zur Auswertung exakt konstruiert werden, damit eine Vergleichbarkeit der Ergebnisse gewährleistet ist.

Das betriebswirtschaftliche Zusammenhangswissen wird mit einer NetzwerkTechnik erhoben, die den Teilnehmenden bei der Konstruktion genügend Freiräume lässt, jedoch auch gut ausgewertet werden kann.

Die Aufteilung der Erhebung in einen Prä- und Posttest sowie eine Testphase mit mehreren Erhebungszeitpunkten (vgl. Abbildung 4.3) hat den Vorteil, im Prä-Post-Vergleich die individuellen Fortschritte der Teilnehmenden kontrollieren zu können und gleichzeitig in der Testphase die Leistungen der Gruppen zu erheben. In weitergehenden Auswertungen können die Prä-Post-Ergebnisse mit denen der Testphase zusammengeführt werden, um genauere Aufschlüsse über den Einfluss der Gruppenarbeit auf die individuellen Lernerfolge zu bekommen. Weiterhin kann der Effekt der eingesetzten Instrumente während der PlanspielPhase kontrolliert werden.

\begin{tabular}{|c|c|c|c|}
\hline \multirow[b]{2}{*}{ 苑 } & Experimentelle Phase & \multicolumn{2}{|l|}{ Testphase } \\
\hline & Herkömmliches Planspiel & $\begin{array}{l}\text { Herkömmliches Planspiel } \\
\text { (neue Ausgangssituation) }\end{array}$ & $\begin{array}{l}\tilde{y} \\
\stackrel{\Xi}{0} \\
\delta \\
0\end{array}$ \\
\hline & 5 Perioden & 3 Perioden & \\
\hline
\end{tabular}

Abbildung 4.3: Untersuchungsdesign 


\subsubsection{Hypothesen der Erhebung}

In dieser Untersuchung soll zum einen hypothesengeleitet vorgegangen werden. Die Überlegungen orientieren sich dabei an den theoretischen Grundlagen, die in den vorangegangenen Kapiteln dargelegt wurden. Zum anderen sollen jedoch auch Hypothesen generiert werden, die neue Aufschlüsse über den Lernerfolg beim Einsatz von Unternehmensplanspielen in der beruflichen Weiterbildung geben können. ${ }^{128}$

Hypothese 1 - Faktenwissen

Bezüglich des betriebswirtschaftlichen Faktenwissens wird von eher geringen Lernerfolgen ausgegangen, da ein Planspiel nicht vorrangig zur Vermittlung von Faktenwissen eingesetzt wird. ${ }^{129}$ Zur Vermittlung von Qualifikationen, die mit dem Einsatz eines Planspiels angestrebt werden und über das reine Faktenwissen hinausgehen, ist jedoch eine breite Wissensbasis erforderlich, auf dem prozedurales bzw. heuristisches Wissen aufgebaut werden kann. ${ }^{130}$ Um den Stand des Wissens vor dem Einsatz des Unternehmensplanspiels festzustellen, soll dieser Fragebogen im Prätest eingesetzt werden. Zur Kontrolle wird er jedoch auch im Posttest herangezogen.

\section{Hypothese 2 - Zusammenhangswissen}

Im Bereich des Zusammenhangswissens wird von einem großen Lernerfolg ausgegangen, da in einem Unternehmensplanspiel isoliertes Denken nicht zu erfolgreichen Entscheidungen führt, sondern eine gleichzeitige Berücksichtigung verschiedener Einflussfaktoren notwendig ist. ${ }^{131}$ Dies würde sowohl die Ergebnisse bestätigen, die im Rahmen des DFG-Forschungsprojekts gewonnen wurden, ${ }^{132}$ als auch verschiedene empirische Untersuchungen, die eine Vernetzung des betriebswirtschaftlichen Wissens durch den Einsatz von Unternehmensplanspielen belegen. ${ }^{133}$

\section{Hypothese 3 - Methodenanwendung und Transferleistung}

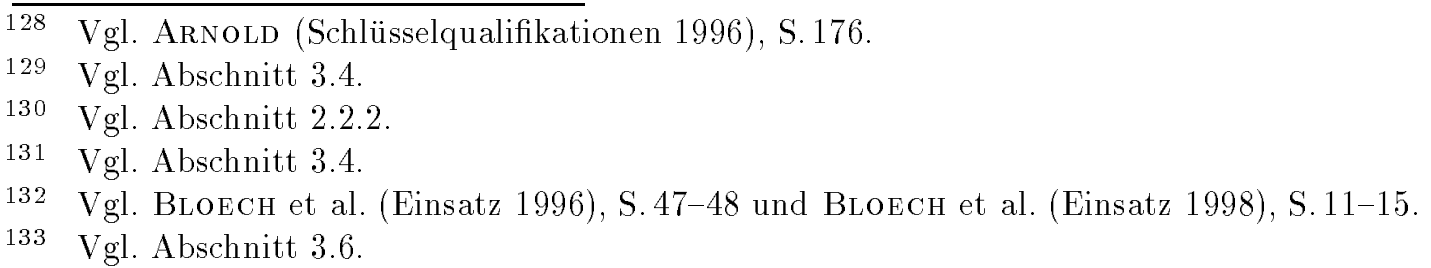


Im Hinblick auf die Transferleistung wird erwartet, dass die Teilnehmenden nach dem Planspiel-Einsatz bessere Ergebnisse als vorher erzielen. Diese Erwartung stützt sich auf die Annahme, dass bei der Entscheidungsfindung im Planspiel die vermittelten betriebswirtschaftlichen Methoden zur Anwendung kommen und sich so festigen (vgl. Hypothese 4). Durch die Variation der Anforderungssituation sind die Teilnehmenden aufgefordert, die Methoden strukturell zu durchschauen und in wechselnden Kontexten anzuwenden. Dadurch sollte ihnen auch die Transfer gelingen. ${ }^{134}$ Auch hier werden die Erwartungen von vorherigen Forschungsergebnisse gestützt. ${ }^{135}$

\section{Hypothese 4-Aktive Umsetzung des Wissens in Handlungen}

An die Leistung der Teilnehmenden in der gesamten Planspiel-Phase werden folgende Erwartungen gestellt: Zum einen soll durch die wiederholte Entscheidung eine Verringerung der Diskrepanz zwischen Absicht und Ausführungsqualität deutlich werden. Die Teilnehmenden sollen eine sachverständige Situationsbeurteilung entwickeln. Ihre Entscheidungen sollen schneller und subjektiv leichter getroffen werden, 'Notfallreaktionen' seltener werden. Sie sollen weiterhin Rückgriff auf Erfahrungen aus den vorherigen Perioden nehmen und die Zielsetzung zunehmend an selbst gesetzten Strategien orientieren. Dabei sollen sie sich mehr und mehr auf übergeordnete Ziele beziehen und eine 'breitere Sicht' als Entscheidungsgrundlage haben. ${ }^{136}$

\section{Hypothese 5 - Einfluss der Komplexität des Planspiel-Modells}

In der Testphase des Planspiels wird von den in der experimentellen Phase in modularer Durchführung spielenden Gruppen eine bessere Umsetzung des Wissens in Handlungen, hier in erfolgreiche Entscheidungen im Unternehmensplanspiel, erwartet als von denen der herkömmlichen Durchführung. Die theoretische Überlegung ist, dass die Teilnehmenden durch die sukzessive Steigerung der Komplexität mehr Zeit haben, sich mit dem aktuellen Funktionsbereich der Unternehmung zu

134 Vgl. dazu Abschnitt 2.4.

135 Vgl. dazu auch die Ergebnisse im DFG-Projekt; BLoEcH et al. (Einsatz 1996), S. 49-50 bzw. S. 53-54 und BloEch et al. (Einsatz 1998), S. 18-20 bzw. S. 29-30.

136 Allerdings wird auf die Erhebung von Strategien verzichtet. In den Untersuchungen des DFG-Projekts wurde dies mit einer offenen Frage versucht, die jedoch nur von wenigen Teilnehmenden beantwortet wurde. In dieser Erhebung wird durch den Fragebogen zur Entscheidung ein zusätzliches Treatment in den Planspiel-Einsatz gebracht, wodurch die Kontrolle der Effekte ermöglicht wird. 
beschäftigen und nicht durch die hohe Komplexität überfordert werden und eher raten, denn rational die Entscheidungen treffen. ${ }^{137}$

Aber auch hinsichtlich der Ergebnisse im Prä- und Posttest wird erwartet, dass die Teilnehmenden der modularen Bedingung bessere Ergebnisse erzielen. Im Bereich des Zusammenhangswissens wird davon ausgegangen, dass das Wissen über die betriebswirtschaftlichen Zusammenhänge durch die modulare Durchführung des Planspiels sukzessive aufgebaut wird. Aber auch hinsichtlich der Transferleistung sollen die Teilnehmenden von dem modularen Aufbau des Planspiels profitieren, weil sie die Gelegenheit erhalten, die Methoden nacheinander in den einzelnen Funktionsbereichen anzuwenden. ${ }^{138}$ Auch diese Erwartungen werden von Untersuchungs-Ergebnissen des DFG-Projekts gestützt. ${ }^{139}$

\section{Hypothese 6 - Aktive Umsetzung des Wissens in Handlungen}

Die Entscheidungen sollen im Planspiel zunehmend strukturierter getroffen werden. Die Teilnehmenden können ihre Entscheidungen in der Testphase besser treffen als noch während der experimentellen Phase, in der sie Gelegenheit hatten, den Planspiel-Verlauf zu durchdenken und die Zusammenhänge zu reflektieren. Dies wird durch den Einsatz des Fragebogens zur Entscheidung zusätzlich gefördert, d. h. die Entscheidungen in den Gruppen, in denen der Fragebogen eingesetzt wird, erzielen bessere Ergebnisse in der Testphase gegenüber den Gruppen, in denen der Fragebogen nicht eingesetzt wird.

\section{Hypothese 7 - Kontrolle der Emotionen und Motivationen}

Die Kontrolle der emotionalen, motivationalen und sozialen Aspekte der Anforderungssituation im Planspiel spiegelt sich in den Einschätzungen der Teilnehmenden wider. Die Teilnehmenden, die den Fragebogen zum situativen Erleben ausfüllen sollten, sind in der Planspiel-Situation zufriedener und erzielen bessere Ergebnisse. ${ }^{140}$

\footnotetext{
137 Vgl. Abschnitt 4.8.

138 Vgl. auch Kapitel 2.

139 Vgl. Bloech et al. (Einsatz 1996), S. 48 und Bloech et al. (Einsatz 1998), S. 16-18.

140 Vgl. Abschnitt 2.3.
} 
Hypothesen-Generierung

Zusätzlich zu den allgemeinen Aspekten des Planspiel-Einsatzes werden auch die Effekte der unterschiedlichen Maßnahmen auf die Förderung der Selbstständigkeit und Handlungsfähigkeit durch entsprechende Gruppenbildung und nachfolgende Auswertung kontrolliert. ${ }^{141}$

\subsection{Versuchsplan und Ablauf der Erhebung}

\subsubsection{Entwicklung des Versuchsplans}

Um die genannten Hypothesen überprüfen zu können, muss der Versuchsplan entsprechend gestaltet werden. ${ }^{142}$ Die während der Planspiel-Phasen einzusetzenden Instrumente sollten den Teilnehmenden die Möglichkeit zur Reflexion über die eigenen Emotionen und Motivationen in der aktuellen Gruppensituation sowie über die Handlungen im Planspiel bieten. Die Untersuchung wird im laufenden Semester, nicht in einer künstlich geschaffenen Situation durchgeführt, so dass von einem Feldexperiment gesprochen werden kann. Dabei müssen die Prädiktor-Variablen, die unabhängig von der Durchführung des Versuchs sind, und die abhängigen Kriteriums-Variablen bestimmt werden. Die unabhängigen Variablen werden auch Faktoren genannt und sind in der vorliegenden Untersuchung die Planspiel-Durchführung in der experimentellen Phase sowie der Einsatz der zusätzlichen Instrumente während der Planspiel-Phasen. Sie treten jeweils in zwei Stufen auf. Das heißt, das Design ist 3-faktoriell und die Anordnung ist 2x2x2. Der Versuchsplan sollte vollständig gekreuzt sein, um alle Effekte kontrollieren zu können. Das heißt, es kommen alle möglichen Kombinationen der drei Faktoren vor, es gibt also acht verschiedene experimentelle Gruppen. Dies ist auf Grund der Stichproben-Größe auch möglich. Abbildung 4.4 stellt den Versuchsplan dar.

141 Zur Vorgehensweise vgl. z. B. Oldenbürger (Datenanalyse 1996).

142 Vgl. dazu Hager (Grundlagen 1987); auch Cook \& Campbell (Quasi-Experimentation 1979). 


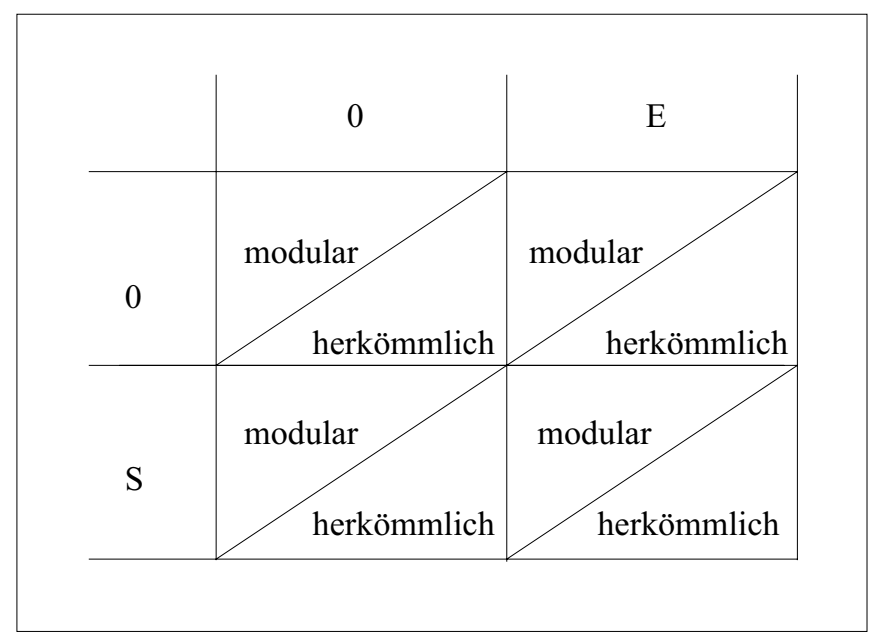

Abbildung 4.4: Versuchsplan

$\mathrm{E}=$ Einsatz des Fragebogens zur Entscheidung

$\mathrm{S}=$ Einsatz des Fragebogens zum situativen Erleben

$0=$ kein Einsatz eines der Fragebögen

\subsubsection{Ablauf der Erhebung}

In der ersten Doppelstunde des Wintersemesters 1997/98 fand eine Vorbesprechung statt. ${ }^{143}$ Hier wurden die Lernziele der Maßnahme sowie insbesondere der Ablauf und die Art der Erhebung und die abschließende Benotung im Rahmen der Veranstaltung besprochen. Nachfolgend wurden einige Inhalte wiederholt, die in den ersten zwei Semestern gelehrt wurden und für den Einsatz des Unternehmensplanspiels wichtig waren.

Der Prätest fand in der zweiten Doppelstunde statt und erhob das spezifische Vorwissen der Teilnehmenden mit Hilfe verschiedener Instrumente. Er diente der Kontrolle des Faktenwissens, des Zusammenhangswissens sowie der Anwendung dieses Wissens in einer komplexen Anforderungssituation.

Die Teilnehmenden wurden nachfolgend für die Planspiel-Phase in Gruppen zu zwei bis drei Personen aufgeteilt. Die Einteilung in die Gruppen erfolgte nach den Wünschen der Teilnehmenden, um negative Einflüsse auf die Motivation durch eine willkürliche Gruppen-Zusammensetzung zu vermeiden. Die Zuordnung in die

$1 \overline{43}$ Der Zeitplan der Erhebung befindet sich im Anhang A.1. 
beiden experimentellen Gruppen sowie der Einsatz der zusätzlichen Fragebögen wurde nach dem spezifischen Vorwissen im Prätest vorgenommen, so dass in etwa eine Gleichverteilung gewährleistet ist. ${ }^{144}$

Für die Planspiel-Phase standen zehn Doppelstunden zur Verfügung, wobei dreimal jeweils zwei Doppelstunden an einem Abend lagen. ${ }^{145}$ Nach dem Prätest waren vier Stunden für eine Einführung in das Planspiel-Modell und die erste Entscheidung vorgesehen, was den Vorteil der zeitlichen Nähe hatte. Zwischen den Abenden lagen sonst mindestens eine Woche. Da die Auswertung der Entscheidungen und das Ausdrucken der Ergebnisse für die Teilnehmenden nicht in der 15-minütigen Pause zwischen zwei Doppelstunden vorgenommen werden konnte, blieben noch sieben Abende, an denen das Planspiel eingesetzt werden konnte. Da das Unternehmensplanspiel auch als Testinstrument eingesetzt werden sollte, in dem die Teilnehmenden mindestens zwei Entscheidungen in Folge treffen sollten, wurde die experimentelle Phase des Planspiels auf insgesamt fünf Doppelstunden festgesetzt, in der das Unternehmensplanspiel in den beiden experimentellen Gruppen in Folge gespielt wurde. ${ }^{146}$

Für die Testphase blieben zwei Doppelstunden, in der die Teilnehmenden auf einer neuen Ausgangslage aufsetzten und das Unternehmensplanspiel mit voller Komplexität spielten. Erfahrungsgemäß treffen die Teilnehmenden die Entscheidungen im Verlauf des Semesters zunehmend schneller, ${ }^{147}$ so dass in der letzten Doppelstunde eine Entscheidung für zwei Perioden getroffen werden sollte. Die Teilnehmenden trafen die erste Entscheidung auf Grund der vorliegenden Planspiel-Ergebnisse und die zweite auf Grund der erwarteten Ergebnisse. Hierdurch kann zusätzlich untersucht werden, inwiefern sie in der Lage sind, die Wirkung ihrer Entscheidungen einzuschätzen. ${ }^{148}$

Während der gesamten Planspiel-Phase wurden die zusätzlichen Fragebögen gekreuzt eingesetzt. ${ }^{149}$ Den Gruppen mit einem oder beiden Fragebögen entstand jedoch kein zeitlicher Nachteil gegenüber den Gruppen, in denen keine Fra-

\footnotetext{
144 Vgl. Anhang A.2.

$145 \mathrm{Vgl}$. Anhang A.1.

146 Vgl. Abschnitt 6.3.

147 Diese Beobachtung wurde in den beiden Erhebungen im Rahmen des DFG-Projekts im Wintersemester 1995/96 sowie im Wintersemester 1996/97 gemacht.

148 Vgl. Abschnitt 6.4.3

149 Vgl. Abschnitt 4.9 .
} 
gebögen eingesetzt wurden, da allen Gruppen genügend Zeit zur Entscheidung zur Verfügung gestellt wurde. Waren alle Gruppen innerhalb der vorgesehenen Zeit mit ihrer Entscheidung fertig, wurde der Rest der Doppelstunde verwendet, um gemeinsam Fragen zu klären und Kenntnisse über verschiedene betriebswirtschaftliche Methoden zu vertiefen. An den beiden Abenden, an denen zwei Doppelstunden zur Verfügung standen, wurden in der ersten Doppelstunde die Entscheidungen im Planspiel getroffen und in der zweiten Doppelstunde Lehre in Form einer Vorlesung gehalten. Hier wurden weitergehende Fragestellungen behandelt, die im Rahmen des Planspiel-Einsatzes nicht zu vermitteln waren.

Nach der Testphase fand der Posttest statt, in dem die gleichen Erhebungsinstrumente wie im Prätest eingesetzt wurden, um einen Vergleich des Wissens zu ermöglichen. Aspekte des 'training-on-test' wurden aufgrund des großen Zeitraums zwischen den beiden Tests von vier Monaten als zu vernachlässigen angesehen. Im Posttest wurde wiederum das Faktenwissen, das Zusammenhangswissen sowie die Leistung in der Transfersituation getestet. Abbildung 4.5 stellt den Ablauf der Untersuchung zusammenfassend dar.

\begin{tabular}{|l|c|c|c|l|}
\hline \multicolumn{1}{|c|}{ Prätest } & \multicolumn{2}{|c|}{ Experimentelle Phase } & Testphase & \multicolumn{1}{c|}{ Posttest } \\
\hline \multirow{2}{*}{$\begin{array}{l}\text { - Multiple-Choice- } \\
\text { Fragebogen } \\
\text { - Netzwerk }\end{array}$} & modular & herkömmlich & neue Situation & - Multiple-Choice- \\
\cline { 2 - 4 } - Fallstudie & sukzessive & $\begin{array}{c}\text { volle } \\
\text { Komplexität }\end{array}$ & $\begin{array}{c}\text { volle } \\
\text { Komplexität } \\
\text { Steigerung }\end{array}$ & $\begin{array}{l}\text { Ketzwerk } \\
\text { - Fallstudie }\end{array}$ \\
\cline { 2 - 5 } individuell & \multicolumn{2}{|c|}{ gekreuzter Einsatz der zusätzlichen Fragebögen } & individuell \\
\hline \multicolumn{3}{|c|}{ Gruppenarbeit } \\
\hline
\end{tabular}

Abbildung 4.5: Ablauf der Untersuchung 
4 Konzeption und Evaluation der Maßnahme 


\section{Erhebungsinstrumente des Prä- und Posttests}

\section{$5.1 \quad$ Vorbemerkungen}

In diesem Kapitel wird die Konstruktion der im Prä- und Posttest der Untersuchung eingesetzten Erhebungsinstrumente dargestellt. ${ }^{1}$ Zur Erfassung des betriebswirtschaftlichen Faktenwissens wird ein Fragebogen konzipiert. Die Netzwerk-Erhebung soll die kognitiven Strukturen der Teilnehmenden bezüglich der Zusammenhänge in einer Unternehmung möglichst genau abbilden. Schlieflich wird zur Beurteilung der Transferleistung eine Fallstudie herangezogen.

Bei der Darstellung der Instrumente wird auf die mit dem Einsatz verfolgten Zielsetzung, auf die Möglichkeiten der Auswertung sowie die Beschreibung des Lernerfolgs eingegangen. Weiterhin wird soweit möglich eine Analyse der Gütekriterien der Instrumente vorgenommen. ${ }^{2}$

\footnotetext{
1 Nach Auffassung von Sievers gibt es grundsätzlich nur zwei Möglichkeiten, über Ereignisse, die sich im Inneren des Menschen abspielen, Erkenntnisse zu gewinnen: die Beobachtung und die Befragung. Dabei haben die Forschenden in den Naturwissenschaften nur die Möglichkeit der Beobachtung, denn "die Befragung z. B. des gestirnten Himmels überläßt man üblicherweise den Dichtern und Philosophen“; Sievers (Forschungsmethoden 1990), S.iii.

2 Zur allgemeinen Darstellung der Gütekriterien vgl. Abschnitt 4.7.
} 


\subsection{Fragebogen zum Faktenwissen}

\subsubsection{Inhaltliche Konstruktion}

Die Zielsetzung bei der Leistungsbeurteilung, die mit diesem Fragebogen verfolgt wird, ist die Messung allgemeiner ökonomischer Kenntnisse. Das entspricht einem Lernziel auf der ersten Stufe der Taxonomie nach BLoom et al. $1976 .^{3}$ Inhaltlich sollte das Instrument so gestaltet werden, dass das im bisherigen Studium, aber auch das während des Planspiel-Einsatzes erworbene Wissen zur Lösung herangezogen werden muss. Dies soll ermöglichen, sowohl das Wissen zu Beginn des Semesters als auch den Lernzuwachs einschätzen zu können. Dabei sollten die Inhalte jedoch nicht zu sehr auf das Planspiel bezogen sein, sondern aus der allgemeinen Betriebswirtschaftslehre kommen.

Auf Grund des Einsatzes mehrerer Instrumente im Prä- bzw. Posttest sowie der Kürze der Zeit ${ }^{4}$ wird ein geschlossener Fragebogen mit Multiple-Choice-Items konstruiert. ${ }^{5}$ Es werden drei Antwort-Alternativen vorgegeben, von denen nur eine richtig ist. ${ }^{6}$ Dadurch wird unter Umständen zwar das Raten gefördert, auf der anderen Seite hat dies jedoch einen motivierenden Effekt, da der Faktor Unsicherheit in Bezug auf die Richtigkeit der Antwort reduziert wird. Weiterhin verringert sich auf diese Weise der Schwierigkeitsgrad des Fragebogens. An die Konstruktion von Fragebogen-Items werden folgende Anforderungen gestellt: ${ }^{7}$

Verständlichkeit: Hierzu gehören die Merkmale der Einfachheit in der Wortwahl, der Gliederung und Ordnung innerhalb der Satzstellung, der Kürze und Prägnanz der Aussagen sowie das Einbeziehen zusätzlicher stimulierender Satzteile.

Eindeutigkeit: Es sind Subjekt, Objekt und Prädikat der Frage zu bestimmen und Ergänzungen bezüglich des Umstands zu nennen. Dabei muss auf mehr-

\footnotetext{
3 Vgl. Abschnitt 4.4

4 Vgl. Abschnitt 4.2.

5 Zu Vor- und Nachteilen von offenen bzw. geschlossenen Fragen vgl. Sievers (Forschungsmethoden 1990), S. 134-141.

6 Der Fragebogen befindet sich im Anhang A.9.

7 Vgl. Sievers (Forschungsmethoden 1990), S. 121-131.
} 
fache Bedeutungen der Wörter geachtet werden. Eventuell sind Hervorhebungen zur Verdeutlichung der Aussage vorzunehmen.

Vollständigkeit: Alle wesentlichen Teile der Aussage müssen vorhanden sein. ${ }^{8}$

Kohärenz: Insbesondere bei vorgegebenen Antworten ist dieser Aspekt wichtig. Die Antwort muss sich direkt auf die Frage beziehen.

Wahrheit von Präsupposition: Hierbei muss auf die Erfüllung der Voraussetzung zur Beantwortung der Frage geachtet werden. Dies spielt in diesem Fall eine eher untergeordnete Rolle, sondern hat eher im Bereich von Persönlichkeitsmerkmalen eine Bedeutung. ${ }^{9}$

Die Aussagen und die Antworten wurden aus verschiedenen Lehrbüchern zur Allgemeinen Betriebswirtschaftslehre entnommen und ggf. umformuliert. Der Paralleltest wurde hauptsächlich durch Negierung der Aussagen erreicht.

\subsubsection{Beurteilung des Lernerfolgs}

Zur Auswertung werden die richtigen Antworten ausgezählt. Der Lernerfolg zeigt sich an einer größeren Anzahl richtiger Antworten im Posttest gegenüber dem Prätest. Die Betrachtung der Anzahl der richtigen Antworten berücksichtigt jedoch nicht, dass Fragen eventuell übersprungen und nicht falsch beantwortet wurden. Bei der Beurteilung des Lernerfolgs wird eine fehlende Antwort im Lösungsblatt als falsch kodiert. Bei der Berechnung von Korrelationen wird sie jedoch als 'missing data' kodiert. Dabei kann allerdings nicht kontrolliert werden, welche Gründe für den Abbruch der Bearbeitung vorlagen und ob richtige Lösungen eventuell durch bloßes Raten entstanden.

\footnotetext{
8 Vgl. Abschnitt 4.7.

9 Zum Beispiel kann jemand eine Erfahrung nicht bewerten, wenn sie oder ihm die Voraussetzungen zum Erwerb dieser Erfahrung fehlen.
} 


\subsubsection{Gütekriterien des Fragebogens}

Die Objektivität ist bei einem Multiple-Choice-Fragebogen bezüglich der Durchführung sowie der Auswertung gegeben. ${ }^{10}$ Aber auch hinsichtlich der Interpretation der Ergebnisse gibt es nur wenig Spielraum.

Die inhaltliche Validität ist durch die Konstruktion der Items gegeben. Bezüglich der Konstrukt-Validität lässt sich sagen, dass eine richtig beantwortete Frage im Prinzip zeigt, dass das Wissen über diesen Sachverhalt vorhanden ist. Hier ist jedoch keine Kontrolle möglich, ob das Ergebnis zufällig geraten wurde. Die Kriteriums-Validität ist gegeben, da eine richtige Beantwortung der Frage betriebswirtschaftliches Wissen widerspiegelt. Als externes Kriterium zur Beurteilung des Inhalts der Fragen dient die Darstellung der theoretischen Grundlagen in den Lehrbüchern der Allgemeinen Betriebswirtschaftslehre.

Der Fragebogen wurde im Rahmen des Forschungsprojekts, in das diese Erhebung eingebettet ist, kontinuierlich kontrolliert und weiterentwickelt. Zur Bestimmung der Zusammenhänge zwischen der Beantwortung der einzelnen Fragen wurde eine Faktorenanalyse durchgeführt. Dabei zeigten sich ähnliche Ergebnisse in beiden Paralleltests. Die Korrelationen zwischen beiden Versionen lagen jedoch nur im Bereich zwischen 0.3 und -0.3 , sind also insgesamt nicht aussagekräftig. ${ }^{11}$

\subsection{Netzwerk-Erhebung zum Zusammenhangs- wissen}

\subsubsection{Inhaltliche Konstruktion}

Mit diesem Instrument soll das Erkennen von betriebswirtschaftlichen Zusammenhängen und Abhängigkeiten getestet werden. ${ }^{12}$ Die Vernetztheit eines Systems - wie z. B. eines Unternehmens - kann durch die grafische Darstellung als Pfeildiagramm oder als Inzidenz-Matrix geschehen. Hierbei werden mindestens

\footnotetext{
10 Vgl. Lienert \& RaAtz (Testaufbau 1998), S.8.

11 Dabei liegt der Umfang der Stichprobe in beiden Fragebogen-Versionen bei n=34 im Prätest und bei n=24 im Posttest. Auf eine Darstellung der Ergebnisse wird verzichtet.

12 Vgl. dem Lernziel auf Stufe 4; Abschnitt 4.4.
} 
zwei Elemente eines Systems miteinander verbunden, wobei die Verbindung eine Bezeichnung erhalten kann. ${ }^{13}$

Die Erfassung der Repräsentation der kognitiven Strukturen der Teilnehmenden geschieht durch eine Netzwerk-Erhebung. ${ }^{14}$ Sie wurde aus der Heidelberger Struktur-Lege-Technik ${ }^{15}$ im Wesentlichen von H.-A. OLdenBürger im Rahmen der Ideen des Forschungsprogramms 'Subjektive Theorien' weiterentwickelt. ${ }^{16}$ Messobjekt, also die Person, die den Test bearbeiten soll, und Messinstrument wirken hier wechselseitig aufeinander ein. Das bedeutet aber, dass das erhaltene Messdatum ein Ergebnis dieser Wechselwirkung ist, nicht nur eine bloße Reaktion auf den Test. Ein Instrument wie die Netzwerk-Technik nutzt diese Wechselwirkung, indem sie - im Gegensatz zu geschlossenen Fragen - zum längeren und genaueren Nachdenken über den Gegenstandsbereich auffordert und das Ergebnis dokumentiert. Auf der anderen Seite hat diese Art der Erhebung gegenüber Fragebögen den Vorteil, die Strukturiertheit der Subjektiven Theorien direkt in dem Netz abbilden zu können. ${ }^{17}$

Sie besteht in der vorliegenden Erhebung aus einer Liste von Konzepten, die betriebswirtschaftliche Begriffe darstellen, und gerichteten, benannten Relationen zur Verbindung der Konzepte. ${ }^{18}$ Dabei werden keine Strukturvorgaben gemacht. Netzwerke eignen sich auch zur Analyse komplexer Problemsituationen, in denen analytisches Denken alleine nicht ausreicht, um die Vernetztheit der Elemente hinreichend darzustellen. ${ }^{19}$

Das Basislexikon der Konzepte sollte dem Sprachgebrauch der Teilnehmenden entsprechen, um Missverständnissen und Fehlinterpretationen vorzubeugen. Hierzu wurde im Sommersemester 1996 am Seminarzentrum Göttingen eine Vorerhebung durchgeführt. Diese Institution wurde gewählt, weil sie bezüglich der Ausbildung mit dem Studium an der Verwaltungs- und Wirtschaftsakademie ver-

13 Vgl. Funke (Problemlösen 1986), S. 32-37; auch die Ausführungen zu propositionalen Modellen der Wissensrepräsentation in Abschnitt 2.2.1.

14 Vgl. Oldenbürger (Erhebung 1986).

15 Vgl. Scheele (Struktur-Lege-Verfahren 1992).

16 Eine Veröffentlichung dazu ist in Vorbereitung.

17 Vgl. BirkHan ((Un)brauchbarkeit 1992), S. 257-261.

18 Vgl. zur Erhebung von Zusammenhangswissen mit der Netzwerk-Technik auch GETsCH (Möglichkeiten 1990) und WEBER (Vorwissen 1994).

19 Vgl. WiLms (Entscheidungsverhalten 1995), S. 218-221. 
gleichbar ist. Weiterhin sind die Teilnehmenden ebenfalls Erwachsene mit Berufserfahrung. ${ }^{20}$ Die Teilnehmenden wurden in dieser Erhebung aufgefordert, ein Netzwerk zu Zusammenhängen in einem Unternehmen zu zeichnen. Dazu wurde ihnen ausschließlich die Art der Konstruktion vorgegeben, jedoch keine Begriffe oder Sichtweisen. Zweck dieser Vorerhebung war zum einen die Erfassung möglicher Schwierigkeiten bei der Konstruktion eines Netzwerks. Zum anderen sollte die Menge der benutzten Konzepte und Relationen ermittelt werden. Es stellte sich heraus, dass die Teilnehmenden die Zusammenhänge aus vielen unterschiedlichen Blickrichtungen darstellten.

Weiterhin wurde eine inhaltsanalytische Auswertung der Entscheidungsberichte zum Planspiel EpUS vorgenommen. In drei Erhebungen im Rahmen des DFGForschungsprojekts, ${ }^{21}$ die an der Verwaltungs- und Wirtschaftsakademie durchgeführt wurden, sollten die Teilnehmenden über die mit ihren Entscheidungen in der Planspiel-Phase verfolgten Ziele zu berichten. Durch diese Auswertung wurden die Konzepte und Relationen erfasst, die von den Teilnehmenden selbst genannt wurden, sie entstammen also dem Wortgebrauch der Zielgruppe. Parallel dazu wurde das Spielerhandbuch zu EpUS analysiert, um die in den Entscheidungsberichten gefundene Menge an Konzepten und Relationen zu ergänzen.

Nachfolgend wurde eine Abstimmung der Mengen an Konzepten und Relationen der Vorerhebung sowie der Inhaltsanalysen vorgenommen und diese zu einer Menge mit 35 Konzepten und fünf Relationen aggregiert. Diese Anzahl wurde wegen der Übersichtlichkeit und der begrenzten Zeit zur Konstruktion gewählt. ${ }^{22}$ Die Instruktion wurde inhaltsfern und relativ einfach in schriftlicher Form dargestellt. Hier konnte auf die Erfahrungen der Vorerhebung zurückgegriffen werden, in der die Instruktionen den Teilnehmenden als Gruppe mündlich gegeben wurden.

Die Konstruktion der Netzwerke selbst wurde nicht am Computer vorgenommen. Es gibt zwar verschiedene Programme, die eine Erfassung von Netzwerken direkt

\footnotetext{
20 Die Vorerhebung konnte nicht an der Akademie selbst durchgeführt werden, da die Teilnehmenden des vierten Semesters im vorherigen Semester bereits an dem Planspiel-Unterricht im Rahmen des DFG-Projekts teilgenommen hatten und bei den Teilnehmenden des zweiten Semesters im folgenden Semester das Planspiel eingesetzt werden sollte.

$21 \mathrm{Vgl}$. Bloech et al. (Einsatz 1996) und Blozch et al. (Einsatz 1998).

22 Vgl. Scheele \& Groeben (Dialog-Konsens-Methoden 1988), S. 64.
} 
am PC ermöglichen, ${ }^{23}$ die den Vorteil haben, dass keine zusätzliche Datenerfassung notwendig ist. Teilweise sind auch Auswertungsmöglichkeiten hinsichtlich verschiedener Kennzahlen zu den Netzwerken implementiert und es werden Protokoll-Dateien erstellt.

In dieser Erhebung bekamen die Teilnehmenden ein leeres DIN-A3-Papier und wurden durch die Instruktionen aufgefordert, ihr Netzwerk zu zeichnen. ${ }^{24}$ Die Vorteile der Konstruktion auf dem Papier, die für diese Erhebung ausschlaggebend waren, sind die kurze Einarbeitungszeit in das Instrument im Gegensatz zur notwendigen Einarbeitungszeit in die Bedienung eines PCs und die entsprechende Software. Die räumlichen Gegebenheiten wären für einen Computer-Einsatz auch nicht geeignet. Weiterhin ist auf dem Papier eine freiere Gestaltung der Netzwerke möglich. Die Teilnehmenden verlieren nicht so leicht den Überblick, wie das u. U. auf einem Bildschirm der Fall sein könnte, wenn das Netzwerk auf Grund seiner Größe nicht mehr ganz dargestellt werden kann. Während der Konstruktion der Netzwerke war die Möglichkeit der Erweiterung der Liste der Konzepte und Relationen jederzeit gegeben.

\subsubsection{Beurteilung des Lernerfolgs}

Die mit Hilfe der Netzwerk-Technik erhobenen Subjektiven Theorien der Teilnehmenden können auf verschiedenen Ebenen betrachtet werden: ${ }^{25}$

- Die benutzten Begriffe, die Konzepte bilden die erste Komplexitätsstufe.

- Die zweite Stufe bilden die Propositionen, also die durch eine Relation verbundenen Begriffspaare.

- Auf der dritten Stufe stehen n-Tupel von Propositionen, also Teilnetze, die einen Satz der Theorie darstellen.

- Die vierte Komplexitätsstufe betrifft die Gesamtheit der Fundamentalsätze der Theorie, das Gesamtnetz.

23 Z. B. GAMMA der Firma UNICON Management Systeme GmbH; vgl. Hub (Denken 1994), S. 75, 'Netzwerk Elaborations Technik (NET)'; vgl. EckERT (Kognition 1997), oder 'The Concept Mapping Software Tool (COMASOTO)'; vgl. Weber \& Schumann (Software 1998).

24 Das Instrument befindet sich im Anhang A.12.

25 Vgl. Birkhan ((Un)brauchbarkeit 1992), S. 271. 
Die Auswertung erfolgt zunächst über die Kennzahlen der Größe, der Ordnung sowie der Dichte der Netzwerke. Sie entsprechen der Anzahl der gebildeten Propositionen (= 'Kanten'), der benutzen Konzepte (= 'Punkte') sowie dem Verhältnis zwischen der Anzahl der Kanten und der Anzahl der Kanten eines Referenznetzes. ${ }^{26}$ Weiterhin wird ein Vergleich der Modalen Netzwerke verschiedener Gruppen vorgenommen. Ein Modales Netzwerk entsteht durch Aggregation verschiedener Netzwerke einer Gruppe und kann als Mittelwert der Netzwerk-Erhebung interpretiert werden.

Der Lernerfolg zeigt sich in diesem Erhebungsinstrument zum einen in einer größeren Ordnung sowie Größe der Netzwerke im Posttest. Dabei sollten die Teilnehmenden sich nicht auf ein bestimmtes Konzept konzentrieren, sondern die Zusammenhänge möglichst genau darstellen. Entsprechend wird eine Annäherung an das Referenznetz angestrebt.

\subsubsection{Gütekriterien der Netzwerk-Erhebung}

Grundsätzlich sind Subjektive Theorien, die mit Verfahren wie der NetzwerkTechnik erhoben werden können, keine Zufallsvariablen. Sie sind nicht in Zahlen abbildbar, sondern Reflexionen des Erkenntnis-Subjekts über einen bestimmten Gegenstands-Bereich. Das heißt, der Raum, in dem sich die Messung vollzieht, ist nicht nur durch Objekt und Merkmal begrenzt, also zweidimensional, sondern dreidimensional, indem das Individuum hinzukommt, ${ }^{27}$ und stellt eine Unsicherheitsgröße bei der Datenerhebung dar. Entsprechend ist bei der Betrachtung der Instrumente, die Subjektive Theorien erheben, bei den Wechselwirkungen zwischen Erkenntnis-Subjekt, Rekonstruktions-Verfahren und Erkenntnis-Objekt anzusetzen.

Die Gütekriterien der klassischen Testtheorie müssen für diese Erhebungsinstrumente weitergefasst werden. Dabei sind insbesondere die Stellen im Prozess der Datenerhebung zu identifizieren, die die Erfüllung der Güterkriterien stören könnten. ${ }^{28}$

26 Vgl. Wilbers (Netzwerke 1997), S. 63-65.

27 Vgl. Gigerenzer (Messung 1981), S. 91.

28 Vgl. dazu Cook \& Campbell (Quasi-Experimentation 1979), S. 55. 
Die Durchführungs-Objektivität ist im vorliegenden Fall gewährleistet, da den Teilnehmenden eine ausführliche Instruktion vorgelegt wurde, die jedoch keinen Einfluss auf das Ergebnis hat, da sie aus einem anderen Inhaltsbereich stammt. Zur Auswertung sind die Schemata genau vorgegeben. Eine Interpretation wird anhand festgelegter Kriterien vorgenommen.

Bei der Konstruktion des Instruments wurde sich an das eingesetzte Unternehmensplanspiel sowie an Literatur der Allgemeinen Betriebswirtschaftslehre angelehnt. Beides kann als Expertenurteil für die Inhalts-Validität angesehen werden. Weiterhin ist davon auszugehen, dass die im Netzwerk rekonstruierten Subjektiven Theorien zu Zusammenhängen in einer Unternehmung handlungsleitend für die Entscheidungen im Planspiel sind. Entsprechend kann von einer Inhaltsvalidität im weiteren Sinne gesprochen werden. ${ }^{29}$ Durch die Art des Instruments wird in der Güte der Netzwerke die Vernetzung des Wissens verdeutlicht (KonstruktValidität). ${ }^{30}$

Bei der Bestimmung der Reliabilität der Netzwerk-Erhebung gibt es verschiedene methodische Schwierigkeiten: Zum einen ist eine Konstruktion einer Parallelform des Tests nicht möglich, da es zu den Begriffen keine Synonyme, nur ähnliche Begriffe - wie z. B. 'Gewinn' und 'Jahresüberschuss' - gibt. Eine Halbierung der Items ist ebenfalls nicht möglich, da die Struktur der Netze verändert würde, was wiederum die Aussage über die Güte der beiden Testhälften beeinflussen würde.

Bezüglich der Beurteilung der Stabilität des erhobenen Merkmals kann ein Stabilitäts-Koeffizient nur verwendet werden, wenn ein Mittelwert über alle Personen durch Summieren der Rohwerte gebildet werden kann. ${ }^{31}$ In diesem Fall wird z. B. die Anzahl der gebildeten Propositionen betrachtet. Ein Mittelwert wäre ein Modales Netzwerk, d.h. ein Netzwerk, das aus den am häufigsten gebildeten Propositionen einer Stichprobe besteht. Stabilität ist aber nicht der Abstand eines individuellen Netzwerks zu dem Modalen Netzwerk, sondern die Wiederholbarkeit der Messung des Merkmals. Bei der Erhebung durch entsprechende Verfahren wird im Rahmen des Forschungsprogramms 'Subjektive Theorien' jedoch davon

29 Vgl. Birkhan ((Un)brauchbarkeit 1992), S. 280-281.

30 Dies hat auch einen Einfluss auf die Reliabilität des Instruments, denn "eine in Gummibegriffe und -relationen gefaßte Rekonstruktion ist ein Indiz für geringe Reliabilität“, Birkhan ((Un)brauchbarkeit 1992), S. 267.

31 Vgl. Lienert \& RaATZ (Testaufbau 1998), S. 181. 
ausgegangen, dass sich allein durch die Rekonstruktion die Subjektiven Theorien bereits verändern.

Im Rahmen der kriteriumsorientierten Testtheorie kann als absolute Norm ein Referenznetz, als kritischer Wert die Grundstruktur des Referenznetzes herangezogen werden. ${ }^{32}$ Hier besteht jedoch das Problem, dass mit diesem Modell wiederum nur eine Messung an der Anzahl der Propositionen, nicht jedoch eine inhaltliche Bewertung der Stabilität erfolgen kann. Die Lösung sind ÜbereinstimmungsKoeffizienten und Distanz-Maße: ${ }^{33}$ Die von KoHnE 1992 durchgeführte Untersuchung entspricht im Wesentlichen dem Einsatz eines Netzwerks zur Erfassung betriebswirtschaftlichen Zusammenhangswissens in dieser Untersuchung. Da seine Untersuchung ausschließlich auf die Retest-Reliabilität der Netzwerk-Erhebung ausgerichtet war, zwischen den Erhebungszeitpunkten also kein Treatment stattfand, sind die Daten wesentlich aussagekräftiger als die der vorliegenden Arbeit, bei der ein Lernerfolg auch in der Netzwerk-Erhebung postuliert wird. Die dortigen Ergebnisse werden im Folgenden dargestellt. Es wird davon ausgegangen, dass sich die Aussagen auch für diese Arbeit übernehmen lassen.

Die Stichprobe bestand aus 20 Studierenden des ersten Semesters an der Wirtschaftswissenschaftlichen Fakultät der Universität Göttingen. Die Anzahl der Propositionen zwischen beiden Erhebungszeitpunkten nahm ohne Treatment um mehr als ein Drittel zu. Die Übereinstimmung in den Netzen einer Person liegt höher als die Übereinstimmung der Netze von unterschiedlichen Personen. Auf der Ebene der Konzept-Kombinationen, also einer Auswertung ohne Berücksichtigung der Relationen, liegt die Übereinstimmung im Schnitt bei $25 \%$. Die Effektstärke, die eine alternative Berechnung des Korrelations-Koeffizienten für die Stabilität erster Art darstellt, liegt hier bei 0.7392 auf der Ebene der Propositionen bzw. bei 0.8575 auf der Ebene der Konzept-Kombinationen, zeigt also ein reliables Ergebnis. Insofern kann die Netzwerk-Erhebung auf der Ebene der Gemeinsamkeiten zwischen den Teilnehmenden bei vorhandener Validität als diagnostisches Instrument gesehen werden.

Die Ähnlichkeitsmaße als Stabilitäts-Koeffizient zweiter Art lagen bei 0.1547 für Propositionen bzw. bei 0.2682 für die Konzept-Kombinationen. Ein anderes Maß

32 Vgl. Klauer (Tests 1987), S.107-109.

33 Vgl. Kohne (Reliabilitätsuntersuchungen 1992), S. 62-76. 
kommt auf Werte von 0.1240 bzw. 0.2149. Beide Maße lassen auf eine geringe Reliabilität schließen. Ihnen liegt jedoch eine sehr konservative Ansicht von Stabilität zu Grunde, die sich nur bedingt auf die Art der Erhebung von kognitiven Strukturierungen mittels Netzwerken übertragen lässt. Zur Bewertung der Stabilität auf individueller Ebene sind die Netzwerke jedoch nicht geeignet, da sich die Subjektiven Theorien der Personen im Zeitverlauf verändern. Bei der Berechnung der Kennzahlen für die Stabilität dritter Art tritt das Problem auf, dass die Netzwerke des Retests aus wesentlich mehr Propositionen bestehen als die des ersten Zeitpunkts. Die Werte der Koeffizienten liegen nur knapp über 0.5. Dieser Wert stellt bei der Beurteilung eines Tests die Grenze dar, ab der von Reliabilität gesprochen werden kann. ${ }^{34}$

Die Bewertung der Reliabilität ist in diesem Fall nicht eindeutig. Ein Test ist grundsätzlich als reliabel zu bezeichnen, wenn er in der Lage ist, die Subjektiven Theorien zu einem bestimmten Gegenstands-Bereich möglichst genau zu rekonstruieren. Entsprechend ist die Validität einzuschätzen: Je genauer die rekonstruierten Subjektiven Theorien einer Person den von den Forschenden intendierten Inhaltsbereich treffen, desto höher ist die Validität. ${ }^{35}$ Davon ist jedoch auf Grund der Konstruktion des Instruments sowie den Umständen der Erhebung - wie eben dargelegt - auszugehen.

\subsection{Fallstudie zur Transferleistung}

\subsubsection{Inhaltliche Konstruktion}

Mit diesem Instrument soll das methodische Arbeiten und die Anwendung betriebswirtschaftlicher Methoden in einer Transfer-Situation gezeigt werden. ${ }^{36}$ Die inhaltliche Gestaltung sollte sich dabei eng genug am eingesetzten Unternehmensplanspiel und den damit vermittelten Inhalten und Kompetenzen orientieren, gleichzeitig aber weit genug davon entfernt sein, um eine Transferleistung

34 Vgl. Wiersma \& Jurs (Measurement 1990), S. 256.

35 Vgl. BirkHan ((Un)brauchbarkeit 1992), S. 267-268.

36 Vgl. Abschnitt 4.4 . 
messen zu können. Als geeignete Aufgabenform erscheint eine komplexe Aufgabenstellung ohne eindeutige Lösung. Hier wird eine Fallstudie konstruiert.

Mit der Methode der Fallstudie wird eher die Anwendung als die Vermittlung von Wissen angestrebt. Deshalb beziehen sich die Lernziele des Einsatzes einer Fallstudie primär auf die Analyse von Problemen und Fakten des individuellen Entscheidungsprozesses. ${ }^{37}$ In einer Fallstudie wird exemplarisch ein praxisrelevanter Ausschnitt der Realität modelliert, mit einer problemhaltigen Handlungsabfolge verbunden und den Teilnehmenden ein selbstständiges Lösen ermöglicht. ${ }^{38}$ Dabei gibt es verschiedene Varianten, die in der Ausbildung eingesetzt werden: ${ }^{39}$

- Die Case-Study-Methode ist durch eine umfassende Darstellung des Falls inklusive aller benötigten Informationen gekennzeichnet. Ziel ist die Erkennung der zu Grunde liegenden Problemstellung.

- Bei der Case-Problem-Methode werden das Problem genannt und die relevanten Informationen vorgegeben. Ziel ist die Entwicklung von Lösungsalternativen sowie die Erörterung der Entscheidungen.

- Im Gegensatz dazu wird bei der Case-Incident-Methode der Fall nur lückenhaft dargestellt. Dadurch wird eine Nähe zur Praxis erreicht. Ziel ist das Sammeln und Aufbereiten der benötigten Informationen, was jedoch zeitaufwendig ist.

- Bei der Stated-Problem-Methode wird die Problemstellung inklusive der Lösung des Falls vorgegeben. Ziel ist die Analyse der den Entscheidungen zu Grunde liegenden Strukturen. Im Gegensatz zu den ersten drei Methoden, bei denen die Lernenden die Lösungsvarianten des Problems ermitteln und anschließend eine Entscheidung treffen, liegt der Schwerpunkt bei dieser Methode auf der Kritik der vorgegebenen Lösungen.

Die Fallstudie als Forschungsmethode hat einige Vorteile: ${ }^{40}$ Zum einen bietet sie eine Vielfalt an Auswertungsmöglichkeiten, da mehrere Methoden bei der Lösung herangezogen werden müssen. Zum anderen können unterschiedliche Sichtweisen

$\overline{37}$ Vgl. Kaiser (Grundlagen 1983), S. 25.

38 Vgl. BECK (Schlüsselqualifikationen 1995), S. 62-63; auch KAIsER (Entscheidungstraining 1976), S. 56.

39 Vgl. Kaiser (Grundlagen 1983), S. 20-23.

40 Vgl. Kaiser (Grundlagen 1983), S. 32. 
verdeutlicht und Bezug auf eine konkrete Situation genommen werden. Weiterhin besteht die Möglichkeit, die Bandbreite der alternativen Lösungen aufzuzeigen.

Allerdings sind auch der Fallstudie Grenzen gesetzt: ${ }^{41}$ Zum einen wird hier nur eine Vorübung zur Durchsetzung des eigenen Willens gegeben. In der konkreten Situation gegenüber Geschäftspartnern oder im Kollegenkreis, also außerhalb des geschützten Raums einer Bildungs-Institution, ist die Durchsetzung des eigenen Willens und der eigenen Entscheidung wesentlich schwieriger. Zum anderen eignen sich Fallstudien zwar zur Bearbeitung einzelner zeitlich eng begrenzter Problemsituationen, nicht jedoch für längerfristige, dynamische Zeitabläufe oder Prozesse. Insbesondere die Annahme bei der Case-study-Methode, dass alle Informationen gegeben sind, ist unrealistisch. Allerdings kann sich dadurch stärker auf die Problemfindung und -lösung konzentriert werden. Weiterhin werden in Fallstudien qualitative Dimensionen von Handlungs- und Entscheidungssituationen abgebildet, sie geben jedoch keine Auskunft über die Häufigkeit des Auftretens des modellierten Problems. Grundlage der Konstruktion einer Fallstudie ist das exemplarische Prinzip, ${ }^{42}$ d.h. der Fall soll ein Beispiel für die Wirklichkeit darstellen. Es sind verschiedene Prinzipien und Kriterien zu berücksichtigen: ${ }^{43}$

Situative Repräsentation: Der Fall sollte einen Ausschnitt der Wirklichkeit abbilden, der für den angestrebten bzw. ausgeübten Beruf von Bedeutung ist. Dies bezieht sich auf die Verwendung der Begriffe, die präsentierten Materialien sowie die erforderliche Handlungsfolge.

Wissenschaftliche Repräsentation: Bei der Fall-Konstruktion muss weiterhin eine Beziehung zur Systematik der zu Grunde liegenden Wissenschaft erkennbar sein. Er darf in keinem Widerspruch zu wissenschaftlichen Erkenntnissen stehen. Im vorliegenden Fall sind die Forderungen der systemorientierten Betriebswirtschaftslehre zu berücksichtigen. ${ }^{44}$

Subjektive Bedeutsamkeit: Die präsentierte Situation muss für die Lernenden eine Bedeutsamkeit im Hinblick auf gegenwärtige und zukünftige Berufs- und Lebens-Situationen haben. Dieses Merkmal steht in engem Zu-

41 Vgl. BuddensieK (Entscheidungstraining 1983), S. 131-135.

42 Vgl. Reetz \& Sievers (Begründung 1983).

43 Vgl. ReEtz (Einsatz 1988), S. 41-42; auch ReETz (Fälle 1988), S. 149-151 und John (Fallstudien 1992).

44 Vgl. dazu ULRich (Unternehmen 1970). 
sammenhang mit dem ersten Merkmal. Der Fall sollte so konstruiert sein, dass er bei den Lernenden ein Bedürfnis nach Lösung des Problems weckt.

Fasslichkeit: Dieses Kriterium ist ebenfalls zu interpretieren und betrifft die Voraussetzungen der Lernenden. Hier wird eine Reduzierung der Komplexität verlangt, so dass der Fall für die Lernenden überschaubar bleibt. Die Akzentuierung richtet sich dabei nach der Maßgabe der Lernziele.

Die Fallstudie, die für die vorliegende Arbeit konstruiert werden soll, dient als Erhebungsinstrument. ${ }^{45}$ Sie wird im Prä- und Posttest eingesetzt, in dem den Teilnehmenden nur ein sehr begrenzter Zeitraum von etwa 45 Minuten zur Bearbeitung zur Verfügung steht. Aus diesem Grund fällt die Entscheidung auf die Case-Problem-Methode. Dabei sollte der Fall auf einem computerbasierten System basieren, so dass die Auswertung durch eine Simulation der Entscheidungen der Teilnehmenden standardisiert wird. Die Wahl fiel auf das ebenfalls am Institut für betriebswirtschaftliche Produktions- und Investitionsforschung entwickelte Unternehmensplanspiel SIM-LOG, ${ }^{46}$ das auf einem generellen Unternehmensmodell basiert und alle betrieblichen Funktionsbereiche abbildet. ${ }^{47}$ Dieses Planspiel bietet die Möglichkeit der Darstellung einer Konkurrenz-Situation, wobei die Konkurrenten durch das Modell simuliert werden. Dadurch ist gewährleistet, dass die Ergebnisse in der Fallstudie untereinander vergleichbar sind. Die Transferweite wird durch die Verwendung von SIM-LOG erhöht: ${ }^{48}$

- Im Gegensatz zum Planspiel EpUS, das in den Planspiel-Phasen eingesetzt wurde, wird in der Fallstudie ein Produkt aus vier Rohstoffen hergestellt, die alternativ bei zwei verschiedenen Lieferanten bestellt werden können.

- Hinzu kommt die Auswahl des Transport-Mittels der Rohstoffe vom Lieferanten zum modellierten Unternehmen.

- Im Marketing-Bereich stehen neben dem Verkaufspreis der Aufwand für Werbung zur Beeinflussung der Nachfrage zur Entscheidung.

- Weiterhin haben die Teilnehmenden über die Investition bzw. Desinvestition der eingesetzten Maschinen zu entscheiden.

Die Fallstudie befindet sich im Anhang A.7.

Vgl. Bloech \& Rüscher (Unternehmenssimulation 1991).

Vgl. Abschnitt 3.2.3.

Dabei wurde das Unternehmensmodell der Vollversion von SIM-LOG in einigen Bereichen reduziert; vgl. Anhang A.3. 
- Der Bereich der Finanzierung wird in der Fallstudie ausgeblendet, da er in der Planspiel-Phase nicht im Mittelpunkt der Lernziele stand und insgesamt für die Bearbeitung nur wenig Zeit zur Verfügung steht.

Die Präsentation des Falls wird im Gegensatz zur Präsentation der Ausgangslage in der Planspiel-Phase wesentlich konkreter an eine hypothetische berufliche Anforderungsssituation angelehnt. Das bedeutet, den Teilnehmenden werden Briefe und Grafiken vorgelegt, wie sie im Berufsleben üblich sind. Die Teilnehmenden agieren als Mitarbeiter/in in einem Unternehmen einer bestimmten Branche, das ein konkretes Produkt herstellt. Zusätzlich zu den Entscheidungen in nummerischer Form sollen die Teilnehmenden ihre Entscheidung begründen. Dazu wird auf dem Entscheidungsblatt eine offene Frage zu den verfolgten Zielen und Absichten gestellt. ${ }^{49}$

\subsubsection{Beurteilung des Lernerfolgs}

Ziel des Einsatzes der betriebswirtschaftlichen Fallstudie ist die Erhebung der Fähigkeit der Teilnehmenden, eine Entscheidung in einer neuartigen Anforderungssituation zu treffen. Neu an dieser Situation war für die Teilnehmenden zum einen die Darstellung der Daten als Fließtext sowie das Zahlengefüge. Zum anderen entschieden sie im Gegensatz zur Planspiel-Phase allein. Neben der Entscheidung in Zahlenform wurden auch die von ihnen verfolgten Ziele, die Strategien sowie die zur Zielerreichung eingesetzten Mittel durch eine offene Frage erhoben.

Zur Auswertung werden die Simulationsergebnisse herangezogen. Den Teilnehmenden wird in der Instruktion zur Fallstudie der erzielte Gewinn als Zielgröße vorgegeben. Zur Beurteilung des Lernerfolgs werden jedoch weitere Kennzahlen, die eine Zielgröße darstellen, herangezogen. ${ }^{50}$ Der Lernerfolg zeigt sich entsprechend in einer besseren Zielerreichung.

Die vorhandene Produktions-Kapazität reicht für eine Produktionsmenge von 2.500 Stück aus, die im vergangenen Jahr abgesetzt werden konnte. Das Ge-

49 Das Lösungsblatt zur Fallstudie befindet sich im Anhang A.8.

50 Dabei werden die Überlegungen zur Planung im Unternehmensplanspiel in Abschnitt 3.3 herangezogen. 
samtvolumen des Markts lag bei 10.000 Stück, der Marktanteil bei $25 \%$. Für das zu planende Jahr geht eine pessimistische Prognose von einer Halbierung der gesamten Nachfrage aus. Entsprechend sollte die Produktionsmenge nicht erhöht werden. Als Untergrenze mit entsprechender Reduzierung der Kapazitäten kann das halbierte Absatzpotentials des vergangenen Jahres genommen werden. Die Produktionsmenge sollte entsprechend bei $x_{p} \in[1.250 ; 2.500]$ liegen.

Die Bestellmengen der vier Rohstoff-Arten ergeben sich entsprechend der Stückliste durch Multiplikation der Produktionsmenge $x_{p}$ mit der benötigten Anzahl des Rohstoffs je Stück. Dabei ist eine Just-in-time-Lieferung ${ }^{51}$ der Rohstoffe zu Grunde gelegt, d.h. Lagerbestände oder Qualitätsaspekte sind bei der Entscheidungsfindung nicht zu berücksichtigen. Die so erhaltenen Bestellmengen müssen nun auf die beiden Lieferanten aufgeteilt werden. Neben den Rabatt-Grenzen sind auch fixe Verwaltungskosten je Lieferant zu berücksichtigen. ${ }^{52}$ Weiterhin ist die Wahl des Transport-Mittels in der Fallstudie abgebildet. Es stehen zwei Alternativen zur Auswahl, zwischen denen allein unter Kostenaspekten gewählt werden sollte.

Weiterhin werden verschiedene Kennzahlen, die durch das Planspiel-Modell berechnet werden, zur Bewertung herangezogen. Hierzu gehören der Grad der Maschinenauslastung sowie die Differenz zwischen der tatsächlichen und der geplanten Produktionsmenge. Diese Lernziele stellen die Anwendung des Wissens dar. ${ }^{53}$ Die Gestaltung der Marketing-Instrumente Verkaufspreis und Werbung wird an der Absatzmenge festgemacht, wobei die Differenz zwischen der tatsächlichen Produktionsmenge und der Summe aus Absatzmenge und einem eventuellen Export berechnet wird. Diese Kennzahl stellt die so genannten Bestandsveränderungen der Produkte dar. ${ }^{54}$

Im Investitionsbereich stehen zwei Maschinentypen zur Auswahl, zwischen denen bei einer eventuellen Erweiterungsinvestition eine Entscheidung getroffen werden muss. Dabei kann auf das statische Modell der Kostenvergleichsrechnung zurückgegriffen werden. ${ }^{55}$

\footnotetext{
51 Vgl. dazu WöHE (Einführung 1996), S. 540-541.

52 Vgl. dazu Bloech et al. (Einführung 1998), S. 194-198.

53 Vgl. Abschnitt 2.4.3.

54 Vgl. Schmolke \& Deitermann (Rechnungswesen 1999), S. 54-55.

55 Vgl. Götze \& Bloech (Investitionsrechnung 1995), S. 53-60.
} 


\subsubsection{Gütekriterien der Fallstudie}

Die Durchführungs-, Auswertungs- und Interpretations-Objektivität können als gegeben vorausgesetzt werden, weil alle entsprechenden Schritte formalisiert und standardisiert wurden. Alle Teilnehmenden bekamen eine Instruktion zum Präbzw. Posttest allgemein sowie zur Fallstudie. ${ }^{56}$ Die Ergebnisse werden nicht subjektiv interpretiert, sondern die Entscheidungen werden mit dem Unternehmensplanspiel simuliert und anhand von Kriterien objektiv beurteilt. Die Ergebnisse auf jeder Stufe können deshalb als unabhängig von der durchführenden, der auswertenden sowie der interpretierenden Person gesehen werden. ${ }^{57}$

Die Inhalts- und Konstrukt-Validität ist insofern gesichert, als die Modellierung der Entscheidungs-Situation durch Experten auf ihre Relevanz beurteilt wurde. Eine Abbildung der Theorie in den getesteten Merkmalen erfolgt durch die Abbildung einer realitätsnahen Situation.

Zur Überprüfung, ob die zur Bewertung herangezogenen Maßzahlen das gleiche Merkmal messen, können Korrelations-Koeffizienten berechnet werden. Dabei ergaben sich zum Teil unterschiedliche Ergebnisse. Der größte Koeffizient wurde im Prättest zwischen der Absatzmenge und den Bestandsveränderungen gemessen. Im Posttest waren starke Zusammenhänge zwischen der geplanten und tatsächlichen Produktionsmenge sowie den Bestandsveränderungen festzustellen. ${ }^{58}$ Dies ist bei der Beurteilung der Ergebnisse zu berücksichtigen.

Die Identifizierung eines externen Merkmals zur Beurteilung der KriteriumsValidität im Bereich der Transferfähigkeit gestaltet sich schwierig, da keine Daten z. B. über den Erfolg im Beruf oder den Aufstieg nach Beendigung des Studiums vorliegen. Bezüglich des methodischen Arbeitens könnten Ergebnisse der Testphase herangezogen werden, die jedoch in Gruppenarbeit erstellt wurden und insofern nur bedingt Aussagekraft über die individuelle Leistungsfähigkeit haben.

Zur Beurteilung der Reliabilität der Fallstudie ist die Konstruktion eines Paralleltests nur schwer möglich. Zwar kann eine weitere beliebige Fallstudie nach obigen Kriterien erstellt werden, sie würde aber eine andere Konjunktur-Situation abbil-

56 Vgl. Anhang A.5 und A.6.

57 Vgl. Lienert \& RaAtz (Testaufbau 1998), S.9.

58 Die Ergebnisse befinden sich im Anhang A.22. 
den und somit eine andere Bearbeitung erfordern. Ändern sich nur die Zahlen in der Fallstudie, nicht aber die konjunkturelle Situation und die Zusammenhänge zwischen den Variablen, so müssten die Einstellungen in der Simulation neu programmiert werden, was einen erheblichen Aufwand darstellt. Ein 'split-half' der Fallstudie ist nicht sinnvoll, da mit jeder Maßzahl eine andere Fähigkeit des methodischen Arbeitens getestet wird. ${ }^{59}$

Insofern wäre nur eine Überprüfung der Retest-Reliabilität sinnvoll. Ein Retest mit der gleichen Stichprobe erweist sich jedoch als schwierig. Da keine Rückmeldung der Ergebnisse an die Teilnehmenden vorgenommen wird, ist ein Einfluss hierdurch zwar ausgeschlossen. Trotzdem bleibt zu vermuten, dass auf Grund der Spezifität der Anforderung in der Fallstudie eine Erinnerung an das erste Mal eine zweite, zeitlich nahe Bearbeitung beeinflussen würde. Ein Retest der Fallstudie kann also nicht mit dem Retest eines Fragebogens verglichen werden. Die Ergebnisse des Prä- und Post-Tests sind prinzipiell zwar Ergebnisse einer Testwiederholung. Da aber im Verlauf des Semesters das Planspiel eingesetzt wurde mit dem Ziel, die Transferfähigkeit und das methodische Arbeiten zu verbessern, lassen sich die Zahlen nicht zur Beurteilung heranziehen.

\subsection{Zusammenhang der drei Instrumente zum betriebswirtschaftlichen Wissen}

Die drei Instrumente, die zur Erhebung eingesetzt werden, sollen unterschiedliche Arten des betriebswirtschaftlichen Wissens erheben. Entsprechend kann von einer heterogenen Testbatterie gesprochen werden, die ebenfalls hinsichtlich der Gütekriterien beurteilt werden kann. ${ }^{60}$

An dieser Stelle soll eine Analyse der Korrelationen zwischen den Ergebnissen der Instrumente vorgenommen werden, von der auf eine Abhängigkeit bzw. Unabhängigkeit der gemessenen Merkmale geschlossen werden kann.

\footnotetext{
59 Vgl. die Überlegungen bei der Netzwerk-Erhebung in Abschnitt 5.3.3 bzw. zur Testphase in Abschnitt 6.4.4.

60 Vgl. Lienert \& RaAtz (Testaufbau 1998), S. 318-332.
} 
Für den Fragebogen zum Faktenwissen wurde die Anzahl der richtigen Antworten als Maßzahl genommen. Bei der Netzwerk-Erhebung kann die Größe der Netze herangezogen werden. Als Kennzahl für die Entscheidungsgüte in der Fallstudie kann der erzielte Gewinn gelten. Es ergeben sich folgende KorrelationsKoeffizienten:

\begin{tabular}{c|c|c|c} 
& Fragebogen & Netzwerk & Fallstudie \\
\hline Fragebogen & - & -0.2250 & -0.0838 \\
\hline Netzwerk & -0.0627 & - & 0.0249 \\
\hline Fallstudie & -0.0987 & -0.1346 & -
\end{tabular}

Dabei sind in der unteren linken Dreiecks-Matrix die Koeffizienten für den Prätest, in der oberen rechten Dreiecks-Matrix die Koeffizienten für den Posttest angegeben. Die zugehörigen Punktwolken sind in Abbildung 5.1 dargestellt. Es kann auf eine Unabhängigkeit zwischen den durch die Instrumente gemessenen Merkmale geschlossen werden. 

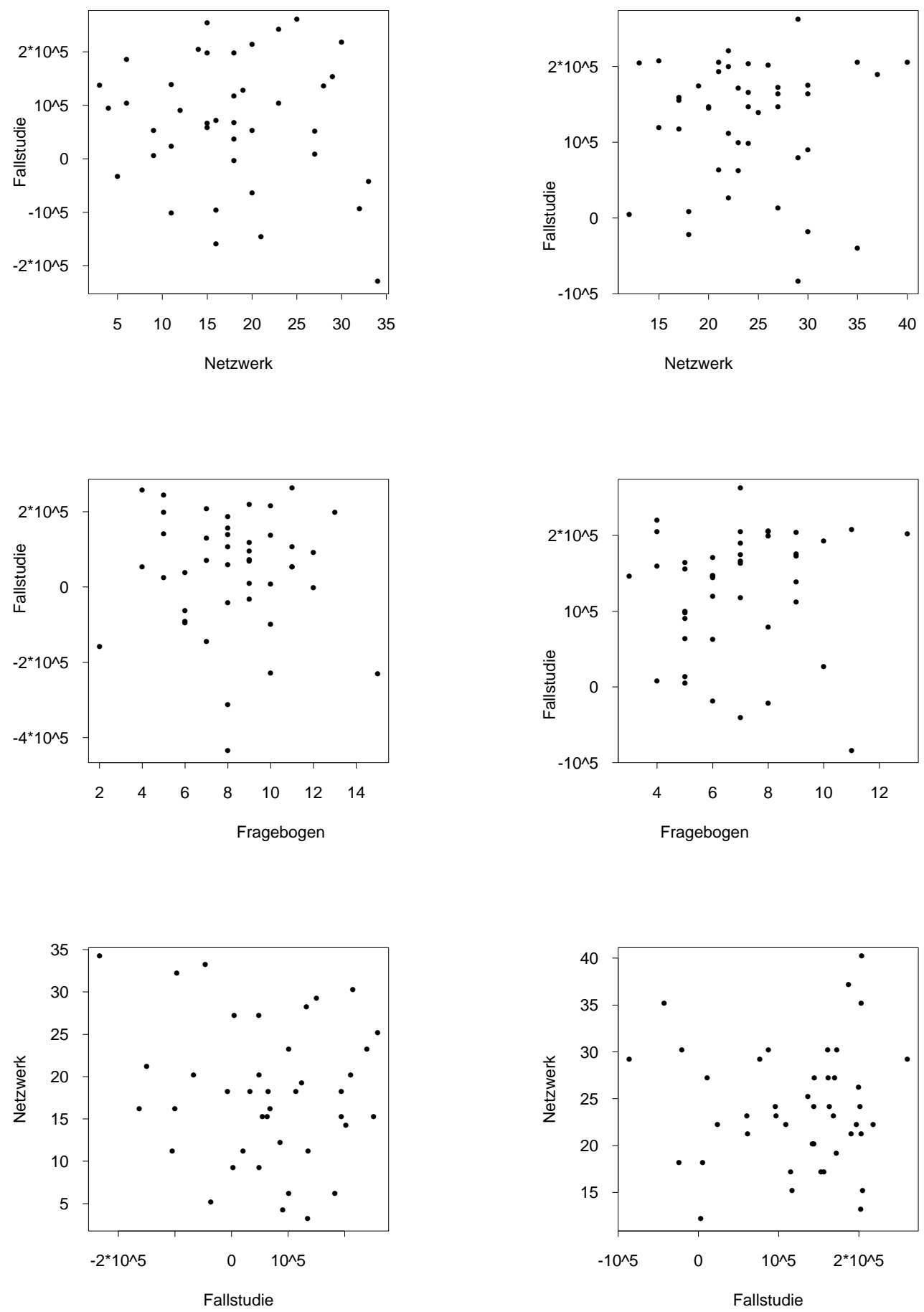

Prätest

Posttest

Abbildung 5.1: Zusammenhang zwischen den mit Fragebogen, Netzwerk und Fallstudie gemessenen Merkmalen 


\section{Die Planspiel-Phase und ihre Erhebungsinstrumente}

\subsection{Vorbemerkungen}

In diesem Kapitel wird die Planspiel-Phase dargestellt. Zunächst wird in Abschnitt 6.2 das eingesetzte Unternehmensplanspiel vorgestellt, das im Hinblick auf die formulierten Lernziele unter den Rahmenbedingungen an der Verwaltungsund Wirtschaftsakademie geeignet ist. Die experimentelle Phase wird in Abschnitt 6.3 dargestellt, wobei zunächst auf die präsentierte Ausgangslage eingegangen wird. In dieser Phase werden zwei verschiedene Bedingungen gegenübergestellt: In der herkömmlichen Durchführung spielen die Teilnehmenden das Unternehmensplanspiel in voller Komplexität, während in der modularen Durchführung des Planspiels die Komplexität der Anforderungssituation stufenweise gesteigert wird. Diese Steigerung erfolgte auf der Grundlage der individuellen Leistungen der Planspiel-Gruppen, wobei für jede Stufe Lernziele entwickelt werden.

Die nachfolgende Testphase wird in Abschnitt 6.4 beschrieben. Sie dient der Überprüfung der Anwendung des Wissens in einer komplexen Situation. Hier spielen alle Teilnehmenden das Unternehmensplanspiel in der herkömmlichen Durchführung mit voller Komplexität gespielt. Neben der Darstellung der Ausgangslage werden auch die Gütekriterien erörtert. Nachfolgend werden die beiden zusätzlichen Instrumente zur Förderung der Reflexivität der Teilnehmenden im Planspiel dargestellt: In Abschnitt 6.5 wird die Konstruktion des Fragebogens zur Entscheidung, in Abschnitt 6.6 die des Fragebogens zum situativen Erleben näher betrachtet und eine Beurteilung hinsichtlich der Gütekriterien vorgenommen. 


\subsection{Das Unternehmensplanspiel EpUS}

\subsubsection{Planspiel-Modell}

Auf Grund der Überlegungen in den vorangegangen Abschnitten fiel die Entscheidung auf das Planspiel EpUS ${ }^{1}$, das am Institut für betriebswirtschaftliche Produktions- und Investitionsforschung, Abteilung für Unternehmensplanung, der Universität Göttingen entwickelt und seit längerem in der universitären Lehre eingesetzt wurde. 'EpUS' bedeutet EinPlatz-UnternehmensSimulation, d. h. es handelt sich um ein nicht-interaktives Planspiel, in dem jedoch eine KonkurrenzSituation abgebildet wird. Die Teilnehmenden führen ein Unternehmen und spielen gegen vier fiktive Konkurrenz-Unternehmen. Dabei sind die Aktionen der Konkurrenten nicht von den Entscheidungen der Teilnehmenden abhängig, sondern im Vorfeld auf Grund der abgebildeten Konjunktur-Entwicklung eingestellt worden. So wird die Vergleichbarkeit der Ergebnisse der Gruppen untereinander gewährleistet. Die Komplexität des zu Grunde liegenden Simulationsmodell, das im Folgenden näher erläutert wird, ist dem Wissenstand der Lerngruppe angemessen. ${ }^{2}$ In voller Komplexität entscheiden die Teilnehmenden über 24 Variablen.

Das simulierte Unternehmen stellt eine Aktiengesellschaft der Industrie dar. Der Produktionsbereich besteht aus einer einstufigen Fertigung, in der zwei Produkte aus zwei Rohstoffen hergestellt werden. Für die Fertigung steht eine Universalmaschine sowie nicht näher spezifizierte Arbeitskräfte zur Verfügung. Zur Bedienung einer Maschine sind zwei Arbeitskräfte notwendig. Dieses Verhältnis ändert sich im Verlauf des Planspiels nicht. Die Kapazität der Maschinen bleibt ebenfalls konstant, sodass eine Verringerung oder Erhöhung der Produktionsmengen kurzfristig durch Leerzeiten der Maschinen bzw. den Zukauf von Fertigprodukten über den Beschaffungsmarkt, längerfristig durch Investition bzw. Desinvestition der Maschinen über den Investitionsgütermarkt erreicht werden kann. ${ }^{3}$

Die zur Bedienung der Maschinen notwendigen Arbeitskräfte werden über den Arbeitsmarkt eingestellt bzw. in diesen entlassen. Dabei entstehen dem Unternehmen Einstellungs- bzw. Entlassungskosten. Weiterhin fallen für die eingestellten

1 Vgl. Bloech \& Rüscher (Modellbeschreibung 1995).

2 Vgl. Abschnitt 4.2.

3 Vgl. Gutenberg (Grundlagen 1983), S. 421-431. 
Arbeitskräfte Lohnzahlungen sowie lohnabhängige Sozialkosten an. Die benötig-

ten Rohstoffe werden auf dem Beschaffungsmarkt zu variablen Preisen eingekauft.

Bei allen Beschaffungsvorgängen (Maschinen, Arbeitskräfte, Rohstoffe) vergeht eine Periode, so dass die Teilnehmenden die Zeitverzögerung bei ihren Entscheidungen berücksichtigen müssen. Übersteigt die zur Herstellung der geplanten Produktionsmengen erforderliche Maschinenkapazität die vorhandene, werden die Produktionsmengen durch das Modell proportional reduziert, so dass die Maschinen voll ausgelastet werden. Die restlichen, nicht produzierten Mengen werden automatisch zu höheren Preisen auf einem anonymen Weltmarkt als Handelsware zugekauft. Stehen für die geplanten Produktionsmengen keine ausreichenden Rohstoffmengen am Lager zur Verfügung, so wird die Differenz zwischen der benötigten und vorhandenen Mengen durch eine automatische Notbeschaffung vom Modell vorgenommen. Ebenso werden bei einer zu geringen Zahl an Arbeitskräften kurzfristig, also nur für die aktuelle Periode, Zeitarbeitskräfte zu einem höheren Lohn eingestellt. Abbildung 6.1 fasst die Ermittlung der tatsächlichen Produktionsmengen zusammen. ${ }^{4}$

Die beiden Produkte werden auf zwei verschiedenen Absatzmärkten angeboten, auf denen auch vier fiktiven Konkurrenten agieren. Die Märkte unterscheiden sich hinsichtlich des Nachfrage-Potentials sowie der Reaktionen auf die Gestaltung der Marketing-Instrumente, so dass sich vier verschiedene Produkt-MarktKombinationen ergeben. Die Teilnehmenden entscheiden im Bereich des Marketing über die Höhe der Verkaufspreise, der Werbeausgaben sowie der Ausgaben für Kundendienst je Produkt und Markt.

4 Zur Darstellung von Flussdiagrammen vgl. z. B. Scheele \& Groeben (Dialog-KonsensMethoden 1988), S. 122-141. 
Einen besonderen didaktischen Wert des Planspiels stellt die Abbildung eines konjunkturellen Verlaufs dar. Dazu werden die Parameter der einzelnen PreisAbsatz-Funktionen sowie die Nachfrage-Potentiale der Märkten variiert. Den Teilnehmenden stehen allerdings nur allgemeine Prognosen über den zukünftigen Konjunktur-Verlauf zur Verfügung. Die Stagnation bzw. der Rückgang der Nachfrage tritt jeweils für die Produkte und Märkte zu unterschiedlichen Zeitpunkten auf. Der Konjunkturverlauf ist in Abbildung 6.2 dargestellt.

Alle Ein- und Auszahlungen erfolgen über die Kasse. Ein negativer Kassenbestand wird durch die Aufnahme eines Kontokorrentkredits bei der Bank ausgeglichen. Dieser kurzfristige Kredit wird jede Periode inklusive der fälligen Zinsen automatisch durch das Simulations-Modell, also ohne Entscheidung der Teilnehmenden, zurückgezahlt. Wird dadurch der Kassenbestand wiederum negativ, wird ein neuer Kontokorrentkredit aufgenommen. Weiterhin haben die Teilnehmenden die Möglichkeit, über die Aufnahme bzw. die Tilgung eines langfristigen Kredits sowie über eine einperiodige Terminanlage für Einzahlungs-Überschüsse zu entscheiden. Die Höhe des langfristigen Kredits ist durch eine Obergrenze im Modell vorgegeben, die den Teilnehmenden zusammen mit den Zinssätzen in der Parameter-Liste mitgeteilt wird. Auf die Unterlagen, die den Teilnehmenden in jeder Periode zur Verfügung gestellt werden, wird im Folgenden eingegangen.

5 In Anlehnung an Bloech \& Rüscher (Modellbeschreibung 1995), S. 29. PiMj= Produkt i auf Marktj, Angaben in Stück für den gesamten Markt. 


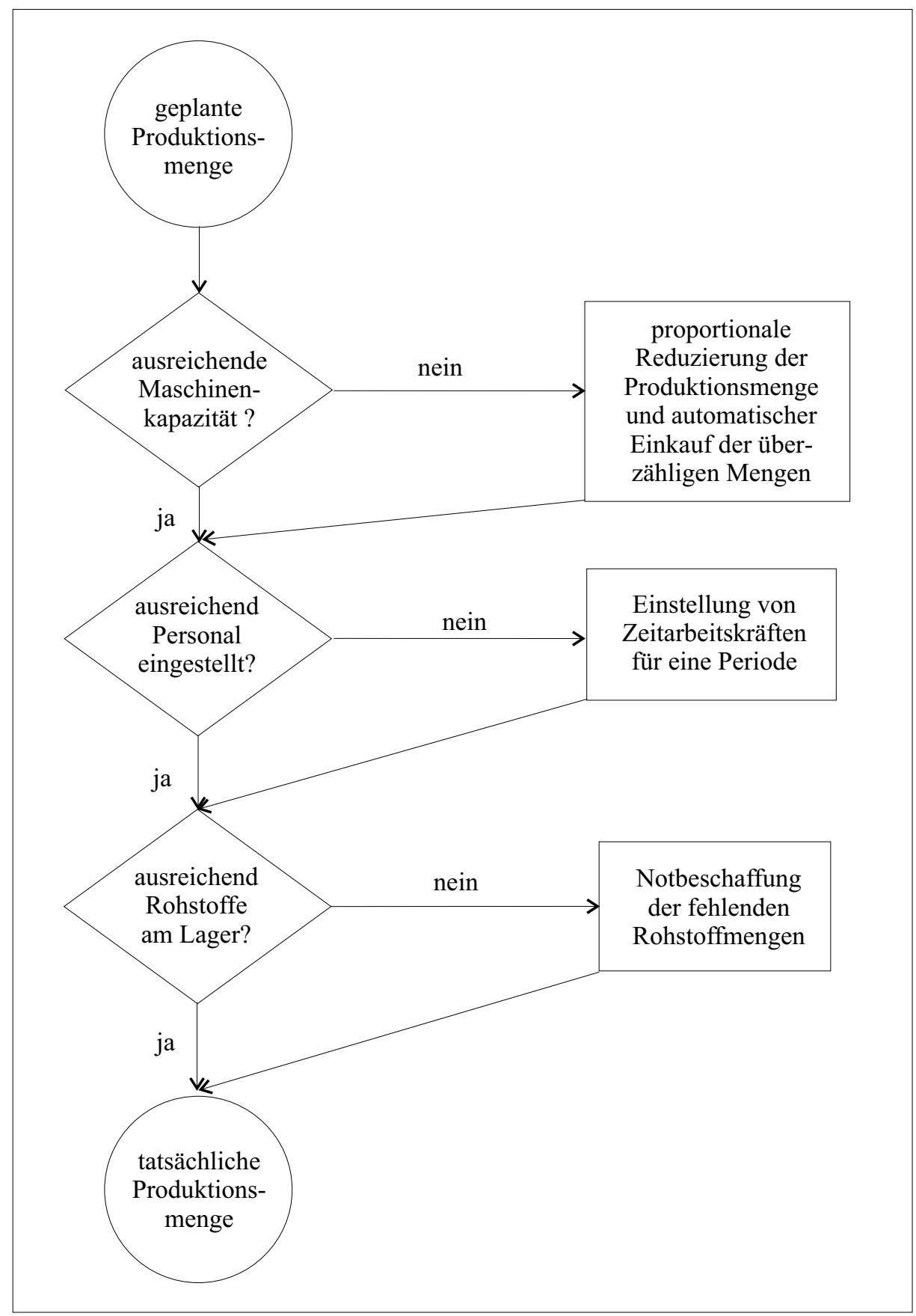

Abbildung 6.1: Ermittlung der tatsächlichen Produktionsmenge im Planspiel EpUS 


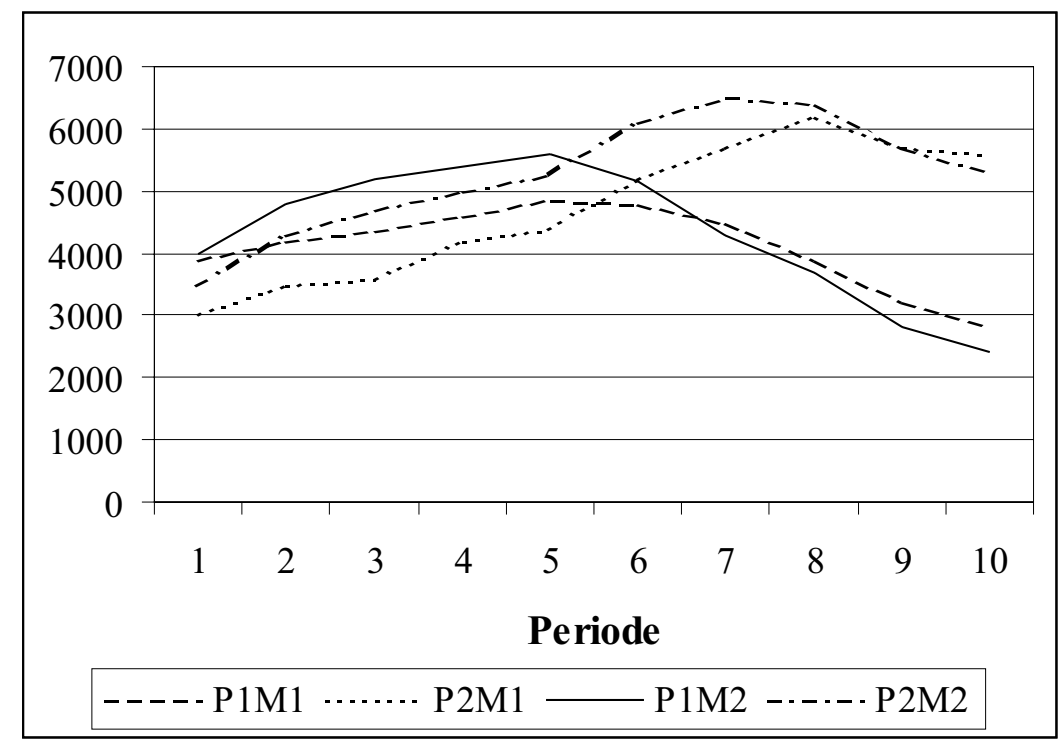

Abbildung 6.2: Verlauf der Nachfrage im Planspiel Ep US ${ }^{5}$

\subsubsection{Planspiel-Unterlagen}

In jeder Periode erhalten die Teilnehmenden die aktuellen Unternehmens-Ergebnisse in Form von Ausdrucken. ${ }^{6}$ Sie beinhalten folgende Komponenten:

1. In den Bestandsrechnungen werden die Lagerbestände mengen- und wertmäßige für beide Rohstoffe und beide Produkte angegeben. Weiterhin wird der Bestand und der Wert der vorhandenen Maschinen, die verfügbaren Leistungseinheiten und die Anzahl des Personals dargestellt.

2. Weiterhin wird der Auslastungsgrad der Maschinen durch die Produktion der aktuellen Periode berechnet und eine Kalkulation der Herstellungskosten der Produkte durchgeführt.

3. Der Absatzbericht beinhaltet Angaben zu den Absatzmengen, den Verkaufspreisen und dem entsprechend erzielten Umsatz und dem Marktanteil, weiterhin der Überhangnachfrage, die sich bei einer größeren Nachfrage- als Lagermenge ergibt, der Kundendienst-Ausgaben pro Stück sowie der wirksamen Werbung für beide Produkte auf beiden Märkten.

6 Die Unternehmens-Ergebnisse für die Ausgangslage der experimentellen Phase befinden sich im Anhang A.19 bzw. für die Testphase im Anhang A.20. 
4. Die Finanzrechnung stellt die Ein- und Auszahlungen gegenüber und ermittelt den Kassenbestand.

5. Die Gewinn- und Verlustrechnung wird im Gesamtkostenverfahren ${ }^{7}$ durchgeführt, wobei auch der kumulierte Gewinn als Summe der Jahresüberschüsse vor Steuern bzw. Jahresfehlbeträge berechnet.

6. Die Bilanz wird nach den gesetzlichen Grundlagen einer Aktiengesellschaft, die im Unternehmensmodell abgebildet wird, dargestellt. ${ }^{8}$

7. Je nach Entscheidung der Teilnehmenden wird eine (kostenpflichtige) Marktforschung durchgeführt, die die Verkaufspreise, die kumulierten Ausgaben für Werbung und Kundendienst, die Absatzmengen, den Gesamtumsatz, den Jahresüberschuss bzw. -fehlbetrag sowie den kumulierten Gewinn aller fünf Unternehmen angibt.

8. Die Parameter-Liste enthält Angaben zu den für alle Teilnehmenden gleichen Beschaffungspreise für Rohstoffe und Fertigprodukte, Angaben der Löhne und Sozialkosten des Personals, der Beschaffungspreise und Leistungsmerkmale der Maschinen, der Marktpotentiale, verschiedener Fixkosten, der Zinssätze des Finanzbereichs sowie die Stückliste der vergangenen Periode.

Der kumulierte Gewinn stellt die vorgegebene Zielgröße im Planspiel dar. Dabei gibt es verschiedene Ziele, die durch das Planspiel-Modell vorgegeben sind und die bestimmte Sachverhalte wie die optimale Auslastung der vorhandenen Kapazität oder die Beschaffung der benötigten Rohstoffmengen abbilden. Andere Ziele wie die Erhöhung der Marktanteile oder das Erreichen eines hohen Umsatzes müssen von den Teilnehmenden formuliert und durch entsprechende Entscheidungen umgesetzt werden.

Die folgenden beiden Abbildungen 6.3 und 6.4 zeigt die Zusammenhänge zwischen den Variablen im Planspiel EpUS auf. Dabei wurden die Relationen unbenannt gelassen. In den Abbildungen sind die Entscheidungsgrößen unterstrichen, die Parameter, die die Dynamik simulieren sollen, sind kursiv dargestellt, die Funktionsbereiche sind durch fett gedruckte Bezeichnungen gekennzeichnet. Die Zusammenhänge in der Abbildung 6.4 beziehen sich hauptsächlich auf die Betrachtung der Gewinn- und Verlustrechnung. Zusammenhänge, die sich eher auf

7 Vgl. Freidank (Kostenrechnung 1994), S. 172-176.

8 Vgl. WöHE (Einführung 1996), S. 1049-1053. 
die Bilanzierung beziehen und die Forderungen und Verbindlichkeiten sowie den Kassenbestand einbeziehen, sind aus Gründen der Übersichtlichkeit in Abbildung 6.3 dargestellt:

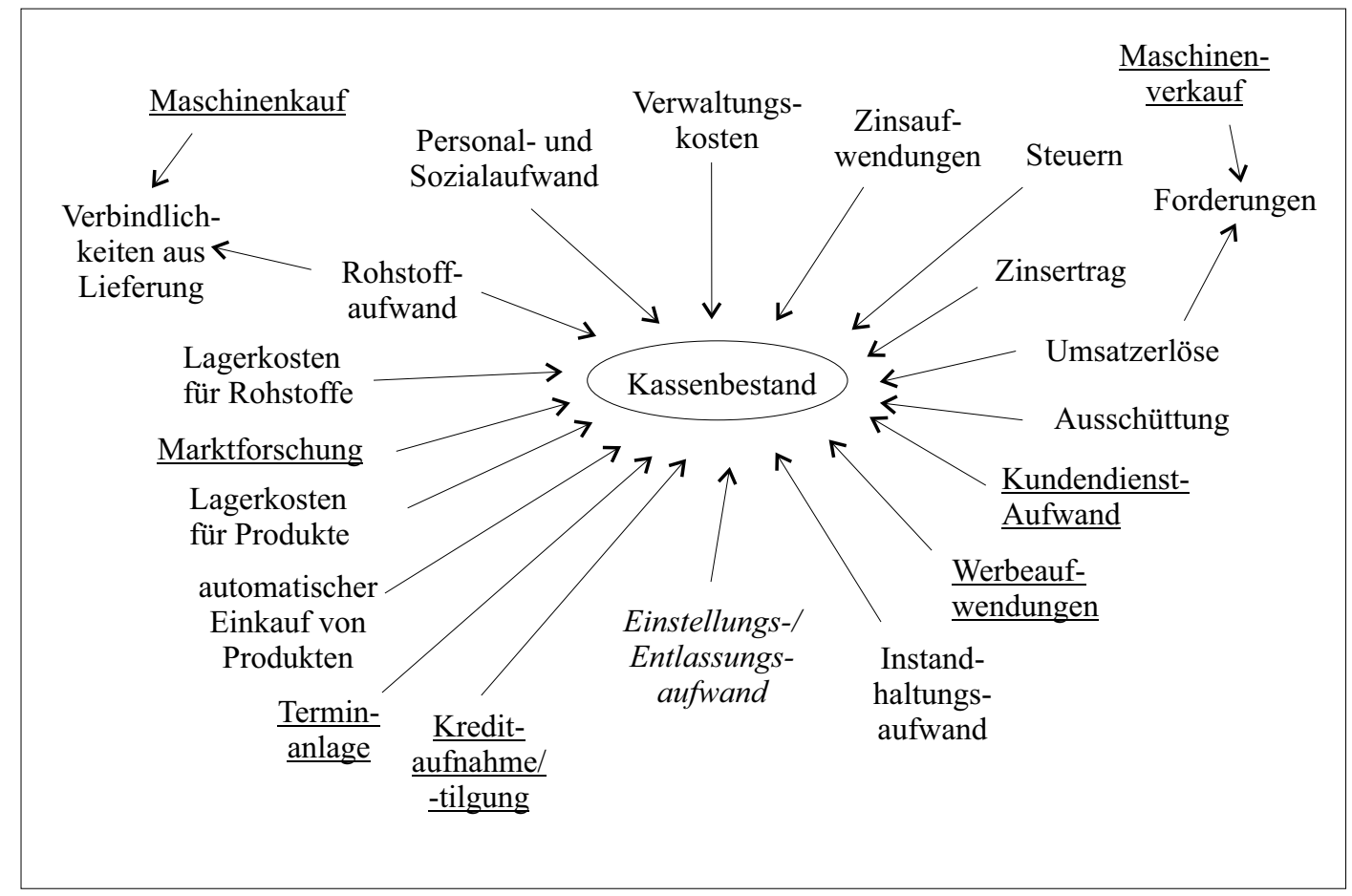

Abbildung 6.3: Zusammenhänge im Planspiel EpUS (1) 


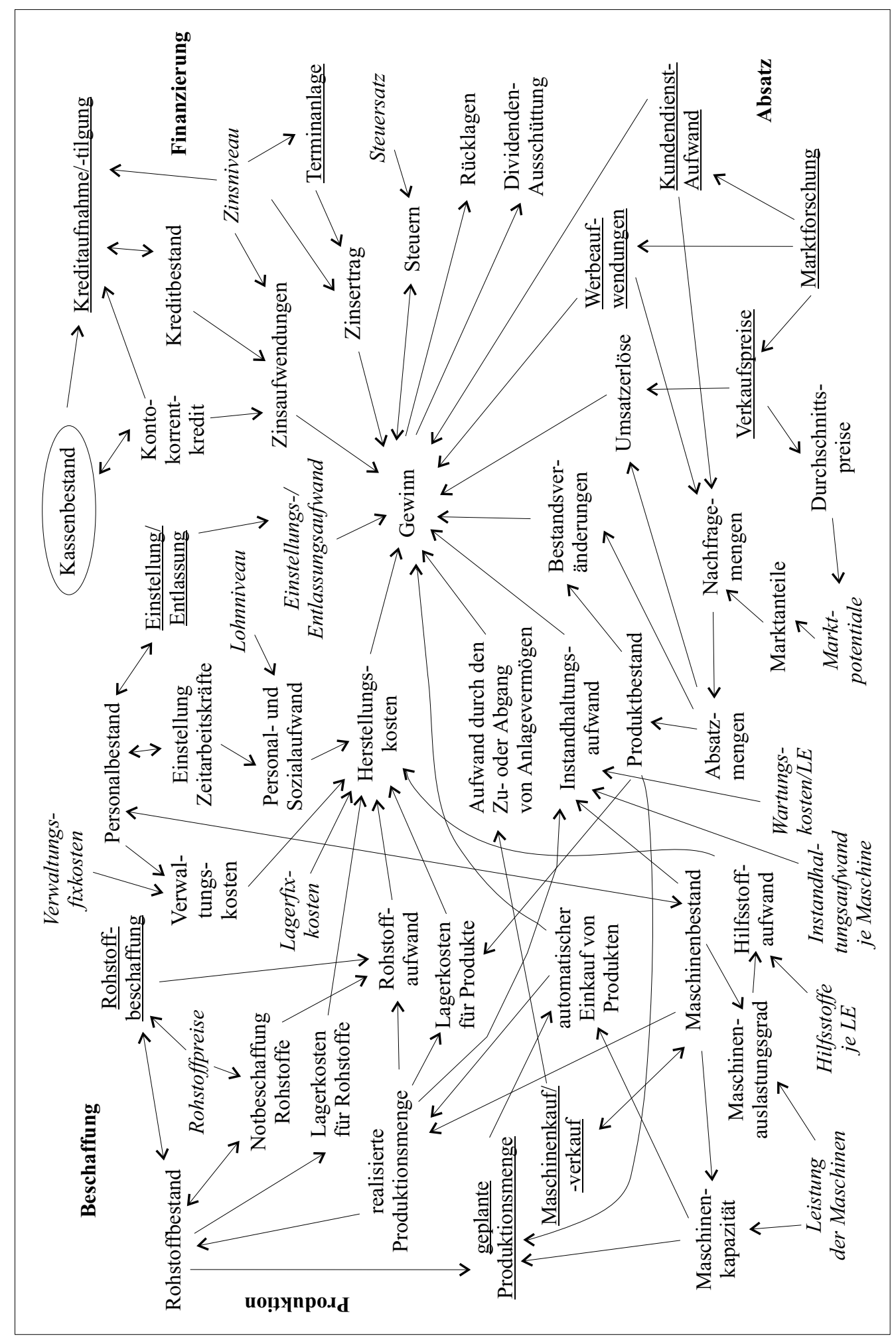

Abbildung 6.4: Zusammenhänge im Planspiel EpUS (2) 


\subsection{Die experimentelle Phase}

\subsubsection{Ausgangslage}

Das Unternehmensplanspiel wird in der experimentellen Phase in zwei verschiedenen Durchführungen eingesetzt, um den Einfluss der Komplexität des PlanspielModells auf die Lernprozesse zu kontrollieren. ${ }^{9}$ Die präsentierte Ausgangslage der simulierten Unternehmung ist jedoch in beiden Durchführungen gleich. Die Komplexität der Anforderungssituation in der herkömmlichen Durchführung bleibt im Verlauf des Planspiels konstant. Sie entspricht der höchsten Komplexitätsstufe der zweiten, der modularen Durchführungsform.

Damit die Teilnehmenden eine Entscheidung in einem bereits existierenden und produzierenden Unternehmen treffen können, werden die ersten drei Perioden vorgespielt, so dass Rohstoffe, Maschinen und sogar ein geringer Bestand an Produkten zur Fertigung zur Verfügung standen. Den Teilnehmenden werden die Unternehmens-Ergebnisse der dritten Periode vorgelegt und sie entscheiden für die vierte Periode.

In der dritten Periode wurde durch die Voreinstellung ein Jahresüberschuss erwirtschaftet, der über denen der Konkurrenz-Unternehmen liegt. Die für das von den Teilnehmenden zu führende Unternehmen gesetzten Verkaufspreise der Produkte sowie die Ausgaben für Werbung und Kundendienst liegen auf beiden Märkten im Durchschnitt. Die Rohstoffe wurden in der dritten Periode fast vollständig verbraucht und entsprechend nachgekauft. Diese Vorgehensweise sollte den Teilnehmenden verdeutlichen, dass es im Planspiel nicht notwendig ist, einen Sicherheitsbestand an Rohstoffen zu halten.

Die Planung der Produktionsmenge in der dritten Periode wurde nicht ganz richtig durchgeführt. Die Menge lag über der Kapazitätsgrenze, d.h. die Maschinen wurden zwar voll ausgelastet, es wurden aber die fehlenden Produkte automatisch auf dem Beschaffungsmarkt eingekauft. Dies soll zum einen zeigen, dass es möglich ist, die Maschinen voll auszulasten, da auf die Darstellung von AusschussProduktion im Modell nicht eingegangen wird. Zum anderen soll den Teilneh-

9 Vgl. Abschnitt 4.8 . 
menden aber auch der automatische Einkauf von Produkten gezeigt werden. ${ }^{10}$ Die Nachfrage ist auf den beiden Absatzmärkten steigend (vgl. Abbildung 6.2). Ebenso steigen die Einkaufspreise für Rohstoffe und Maschinen sowie die Löhne für die Arbeitskräfte. Auch die Fixkosten steigen im Laufe der nächsten Perioden an.

In der herkömmlichen Durchführung des Planspiels entscheiden die Teilnehmenden über die gesamten fünf zu spielenden Perioden hinweg über alle Variablen. Die im Folgenden dargestellte Komplexitäts-Steigerung in der modularen Bedingung wird anhand verschiedener Kriterien durchgeführt, die auch bei der herkömmlichen Bedingung zur Bewertung herangezogen werden können.

\subsubsection{Stufenweise Komplexitäts-Steigerung}

In der modularen Durchführung wird ausgehend von einem geringen Entscheidungsumfang im zeitlichen Verlauf des Planspiels die Komplexität der Anforderungssituation durch Hinzunahme weiterer Entscheidungsbereiche in fünf Stufen erhöht. Dadurch vergrößert sich zum einen die Anzahl der EntscheidungsVariablen, zum anderen aber auch die Anzahl der Zusammenhänge zwischen ihnen. ${ }^{11}$ Die Reihenfolge der Hinzuschaltung orientiert sich dabei am Modell der Unternehmensplanung: ${ }^{12}$

1. Stufe: Die Produktionsmengen werden den Teilnehmenden vorgegeben. Für die Herstellung dieser Mengen müssen sie durch den Kauf oder Verkauf von Maschinen sowie die Disposition des Personals eine ausreichende Produktionskapazität bereitstellen. Darüber hinaus sind die erforderlichen Rohstoffmengen zu beschaffen.

2. Stufe: Zu den Entscheidungen der ersten Stufe kommen zusätzlich die Produktionsmengen.

10 Auf diese Aspekte wurde im Rahmen der Einführung in das Planspiel-Modell in der dritten Unterrichts-Doppelstunde eingegangen; vgl. Abschnitt 4.9.2.

11 Vgl. Abschnitt 2.5.2 zum Begriff der Komplexität. Zur Komplexitäts-Steigerung vgl. auch Hahne (Schlüsselqualifikationen 1989).

12 Vgl. Abschnitt 3.3. 
3. Stufe: Um die geplanten Produktionsmengen absetzen zu können, sind die Marketing-Instrumente entsprechend zu gestalten. Dazu gehört auf dieser Stufe zunächst die Festlegung der Verkaufspreise für die Produkte.

4. Stufe: Auf dieser Stufe kommen die Entscheidungen über die Aufwendungen für Werbung und Kundendienst hinzu.

5. Stufe: Schließlich ist im Finanzierungs-Bereich über die Aufnahme bzw. Tilgung von Krediten sowie über eine kurzfristige Terminanlage für Einzahlungs- ̈̈berschüsse zu entscheiden.

Abbildung 6.5 zeigt die stufenweise Erhöhung der Komplexität in der modularen Durchführung. Die Steigerung von der ersten auf die zweite Stufe geschieht in jedem Fall, um Motivationsverlusten vorzubeugen. Bei Erreichung der gesetzten Lernziele werden einige Gruppen jedoch gleich auf die dritte Stufe hochgesetzt, bekommen also neben der Produktionsmenge auch die Verkaufspreise der Produkte zur Entscheidung. Die erste Entscheidung ist quasi eine Probe-Entscheidung. Alle nachfolgenden Entscheidungen werden - unabhängig von der Stufe - in Folge getroffen. In der letzten Runde werden alle Gruppen mindestens auf die 4. Stufe erhöht, so dass sie vor der Testphase mindestens einmal über alle MarketingInstrumente entscheiden.

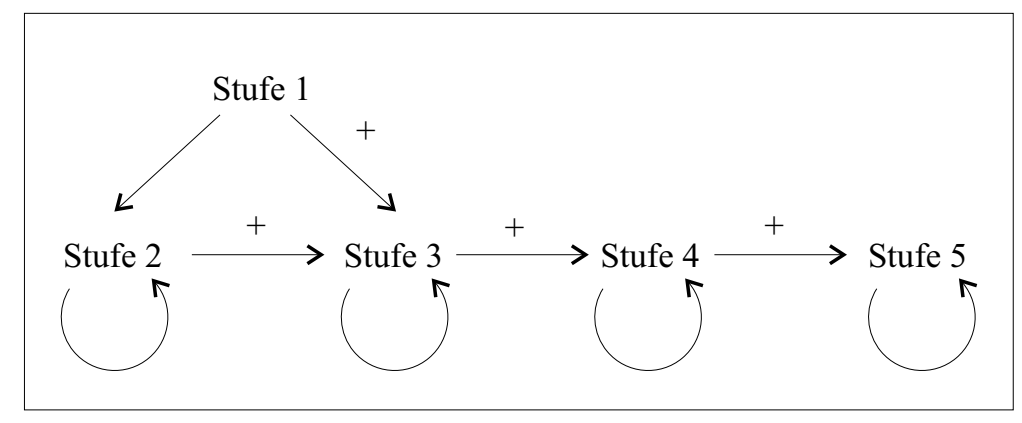

Abbildung 6.5: Stufenweise Erhöhung der Komplexität in der modularen Durchführung $^{14}$

Insgesamt orientiert sich die Steigerung der Komplexität an den Lernfortschritten der Teilnehmenden. Dafür werden auf jeder Stufe Lernziele formuliert, bei deren

14 Dabei bedeutet das Plus-Zeichen die Erreichung der Lernziele der Stufe und entsprechend eine Höherstufung der Gruppe. 
Erreichen die Komplexität für die jeweilige Gruppe erhöht wird. Die übergeordneten Lernziele des Planspiel-Einsatzes beziehen sich auf die aktive Umsetzung des betriebswirtschaftlichen Wissens in Handlungen. Hierzu gehört das Lernziel des Erkennens, Strukturierens und Lösens von Entscheidungsproblemen, aber auch das Wahrnehmen von Führungsaufgaben. ${ }^{15}$ Dies soll durch den modularen Aufbau gefördert werden. Im Folgenden werden die Lernziele, die mit den einzelnen Stufen verfolgt werden, dargestellt.

\subsubsection{Lernziele der Komplexitäts-Stufen}

Die folgenden Überlegungen basieren auf der theoretischen Darstellung der Unternehmensplanung in Abschnitt 3.3. ${ }^{16}$

\section{Die Lernziele der ersten Stufe}

Aufsetzend auf der Ausgangslage wird den Teilnehmenden die Produktionsmengen von $x_{1}=1.900$ und $x_{2}=1.840$ für die nächste Periode vorgegeben. Da die Teilnehmenden für die Entscheidung über Rohstoffe, Maschinen und Personal im Verhältnis zur Entscheidung in der herkömmlichen Durchführung in voller Komplexität wesentlich weniger Zeit brauchen würden, wird ihnen zwei Szenarien mit unterschiedlicher Erwartung bezüglich der Nachfrage vorgelegt. Die entsprechenden Produktionsmengen sind für die übernächste Periode genau angegeben. Die Teilnehmenden sollen nun über die Beschaffung der entsprechenden Rohstoffmengen sowie über den Kauf oder Verkauf von Maschinen und die Einstellung bzw. Entlassung des Personals in beiden Szenarien entscheiden.

Das erste Kriterium zur Beurteilung des Lernerfolgs bezieht sich auf die Ermittlung der Beschaffungsmenge für die beötigten Rohstoffe $r_{1}$ und $r_{2}$ der folgenden Periode. Die Produktion der aktuellen Periode wird mit dem Lagerbestand durchgeführt. Es gelten die Formeln 6.1. Dabei sind $B_{1}$ und $B_{2}$ die Bedarfe der Rohstoffe für die nächste Periode. $L_{1}$ und $L_{2}$ stellen den jeweiligen Lagerbestand

15 Vgl. Abschnitt 4.4.

16 Zu den Erläuterungen der einzelnen Entscheidungen vgl. auch die Entscheidungsblätter im Anhang A.14. 
der Rohstoffe 1 und 2 am Ende der Periode dar, der sich nach der gleichen Formel berechnet. Nach der ersten Entscheidung beträgt $L_{1}=372$ und $L_{2}=580$.

$$
r_{1}=\underbrace{3 x_{1}+5 x_{2}}_{B_{1}}-L_{1} \text { und } r_{2}=\underbrace{6 x_{1}+4 x_{2}}_{B_{2}}-L_{2}
$$

Das zweite Kriterium bezieht sich auf die Beschaffung von Produktionsmitteln. Die zusätzlich benötigten bzw. zu viel vorhandenen Maschinen $B_{M}$ ermitteln sich aus der Differenz von benötigten und vorhandenen Leistungseinheiten (LE) geteilt durch die Anzahl LE je Maschine. Ein positiver Wert bedeutet den Kauf, ein negativer Wert den Verkauf der entsprechenden Anzahl von Maschinen. Da je Maschine zwei Arbeitskräfte benötigt werden, muss der Personalbestand $B_{P}$ ebenfalls aufgestockt oder verringert werden. Dabei muss eventuell gerundet werden. Das Kriterium ist erfüllt, wenn beide Gleichungen 6.2 gelten.

$$
B_{M}=\frac{\overbrace{8 x_{1}+10 x_{2}}^{\text {Bedarfan } L E}-\overbrace{28 \cdot 1200}^{\text {vorhandene LE }}}{\underbrace{1200}_{\text {LE } \text { je Maschine }}} \text { und } B_{P}=2 \cdot B_{M}
$$

Diese beiden Lernziele sind nach der Taxonomie von BLoom et al. 1976 auf der dritten Stufe der Anwendung vorhandenen Wissens einzuordnen. ${ }^{17}$ Alle notwendigen Informationen liegen den Teilnehmenden in der Aufgabenstellung bzw. den Unternehmens-Ergebnissen sowie der Stückliste in den Parametern der Ausgangslage vor. Die Methoden der Ermittlung des Rohstoff-Bedarfs sowie der Ermittlung der Leistungseinheiten der Maschinen im Rahmen des Planspiel-Modells wurden in der Einführung in das Unternehmensplanspiel zu Beginn des Semesters dargestellt. Dabei kann auf dem Wissen aus dem zweiten Semester aus der Industriebetriebslehre aufgebaut werden. ${ }^{18}$ Die Lösung der beiden Szenarien auf Grund der eben dargestellten Kriterien ergibt sich wie folgt:

Szenario 1: Es wird mit einer positiven Entwicklung der Nachfrage gerechnet. Vorgesehen sind Produktionsmengen für die übernächste Periode von $x_{1}=2.500$ und $x_{2}=2.200$.

\footnotetext{
17 Vgl. Abschnitt 2.4.

18 Vgl. Abschnitt 4.2.
} 
Bei der Bewertung des ersten Kriteriums (6.1) bleibt unberücksichtigt, ob der Lagerbestands bei den Rohstoffen am Ende der letzten Periode von den Teilnehmenden eingeplant wurde oder nicht. Dadurch ergeben sich folgende beide Intervalle:

$$
r_{1} \in[18.125 ; 18.500] \text { und } r_{2} \in[23.220 ; 23.800]
$$

Das zweite Kriterium bezieht sich auf die Anzahl der zu beschaffenden Maschinen und die Einstellung der entsprechenden Arbeitskräfte (vgl. Formel 6.2). Es ergibt sich folgende Lösung, wobei nicht gerundet werden muss:

$$
B_{M}=7 \text { und entsprechend } B_{P}=14
$$

Szenario 2: Das zweite Szenario geht von einer eher pessimistischen Erwartung bezüglich der Nachfrage-Entwicklung aus. Hier sind Produktionsmengen von jeweils 2.000 Stück vorgegeben.

Kriterium 6.1 führt entsprechend - je nach Berücksichtigung des Lagerbestands - zu Beschaffungsmengen bei den Rohstoffen von

$$
r_{1} \in[15.628 ; 16.000] \text { und } r_{2} \in[19.420 ; 20.000] \text {. }
$$

Kriterium 6.2 ist erfüllt, wenn gilt:

$$
B_{M}=2 \text { und entsprechend } B_{P}=4
$$

Eine Höherstufung auf Stufe 3 wird bei denjenigen Gruppen vorgenommen, die beide Kriterien in beiden Szenarien erfüllt haben. Bei den anderen Gruppen wird die Komplexität nur um eine Stufe erhöht.

\section{Die Lernziele der zweiten Stufe}

Auf der zweiten Stufe setzen die Teilnehmenden wiederum auf der Ausgangslage auf, planen aber zusätzlich die Produktionsmengen für die nächste Periode $(t=4)$. Zur Kontrolle sollen sie die Produktionsmengen der fünften Periode angeben. So kann überprüft werden, ob die Beschaffung von Rohstoffen sowie die Investition in Maschinen und Arbeitskräfte richtig vorgenommen wird.

Die bisherigen zwei Kriterien beziehen sich auf die Anwendung von Wissens, in diesem Fall von betriebswirtschaftlichen Methoden zur Ermittlung von Bedarfen mittels linearer Zusammenhänge. Hierzu sind alle Informationen verfügbar 
und eine Transparenz der Entscheidungssituation ist gegeben. Auf der zweiten Stufe kommt jedoch die Planung der Produktionsmengen auf Grund der Rohstoffbestände und der vorhandenen Kapazität der Maschinen hinzu. Dazu sind Fähigkeiten der Analyse und Synthese erforderlich, da den Teilnehmenden nur unsichere Prognosen über den Verlauf der Nachfrage vorliegen.

Der Lagerbestand $L_{i}$ plus die Beschaffung $r_{i}$ des Rohstoffs $i$ am Ende der vierten Periode müssen größer oder gleich dem Bedarf $B_{i}(5)$ der fünften Periode sein. Der Bedarf $B_{i}(5)$ berechnet sich auf Grund der geplanten Produktionsmengen $x_{i}$ für die fünfte Periode. Der Bedarf berechnet sich nach Formel 6.1. Das dritte Kriterium lautet:

$$
L_{i}(4)+r_{i}(4) \geq B_{i}(5) \quad \text { für } \quad i=1,2
$$

Das vierte Kriterium bezieht sich auf die Berücksichtigung der Kapazität der Maschinen in der aktuellen Periode. Zur Bewertung kann der automatische Einkauf autom $_{i}$ des Produkts $i$ herangezogen werden. ${ }^{19}$

$$
\text { autom }_{i}=0 \quad \text { für } \quad i=1,2
$$

Das fünfte und sechste Kriterium ist erfüllt, wenn die vorhandene Kapazität der Maschinen in der vierten bzw. fünften Periode voll ausgelastet wird. Dabei wird ein Toleranz-Bereich von fünf Prozentpunkten eingeräumt, um geringfügige Rechenfehler oder Unsicherheiten in der Planung nicht zu überbewerten:

$$
\begin{aligned}
& \text { Maschinenauslastung }(4) \in[95 \% ; 100 \%] \\
& \text { Maschinenauslastung }(5) \in[95 \% ; 100 \%]
\end{aligned}
$$

Eine Höherstufung auf Stufe 3 wird vorgenommen, wenn die Kriterien überwiegend, d. h. mindestens vier der sechs Kriterien erfüllt sind, sonst bleibt die Gruppe eine weitere Runde auf der zweiten Stufe. Nach der dritten Runde werden jedoch alle Gruppen auf die dritte Stufe hochgestuft, um die Lernmotivation nicht zu belasten.

19 Vgl. Abbildung 6.1. 


\section{Die Lernziele der dritten Stufe}

Zusätzlich zu den Kriterien der ersten beiden Stufen kommt auf dieser Stufe die Bewertung der Entscheidung über die Verkaufspreise der Produkte hinzu. Die Marketing-Instrumente der Werbung und des Kundendiensts werden für alle Gruppen voreingestellt, so dass eine Vergleichbarkeit gewährleistet wird. Die Auswirkung der Preis-Festsetzung wird an der Höhe der Marktanteile beurteilt, die den Teilnehmenden in den Unternehmens-Ergebnissen mitgeteilt werden. Die Unsicherheit und die Intransparenz dieser Entscheidungssituation nimmt hier wiederum gegenüber den vorherigen Stufen zu, da sich die Ermittlung der tatsächlichen Absatzmengen am Durchschnittspreis auf dem jeweiligen Markt orientiert. Dabei haben auch die voreingestellten Entscheidungen der KonkurrenzUnternehmen eine Auswirkung. Weiterhin beeinflusst das Nachfragepotential als Parameter die Absatzmengen.

Das siebte Kriterium betrachtet die Marktanteile der beiden Produkte auf beiden Märkten. In der Ausgangslage $(t=3)$ lagen die Marktanteile bei 20-25\%. Das Lernziel wird entsprechend vorsichtig formuliert.

$$
M A_{i j} \geq 15 \% \text { mit } \text { Produkt }_{i}=1,2 \text { und } \text { Markt }_{j}=1,2
$$

Hier erfolgt eine Höherstufung der Gruppe, wenn mindestens fünf der sieben Kriterien erfüllt sind, da weiterhin die Lernziele der ersten Stufe erreicht sein sollen. Es ist aber auch vorgesehen, die Gruppen eine weitere Runde auf der dritten Stufe zu lassen. Nach der vierten Runde jedoch werden alle Gruppen auf die dritten Stufe hochgestuft, damit sie vor der Testphase zumindest einmal über die Marketing-Instrumente entscheiden.

\section{Die Lernziele der vierten Stufe}

Auf der vierten Stufe wird der Marketing-Bereich weiter ausgebaut und es kommt die Planung der Aufwendungen für Werbung und Kundendienst hinzu. Die Lernziele orientieren sich wiederum am Marktanteil, hier aber auch an den Kundendienst-Ausgaben pro Stück, die vom Modell berechnet werden.

Das achte Kriterium besagt, dass die Summe der Marktanteile über alle Produkte und Märkte gegenüber der Ausgangslage nicht sinken soll. Auch dieses Lernziel ist 
vorsichtig formuliert, weil die Wirkung der drei Marketing-Instrumente gleichzeitig berücksichtigt und geplant werden muss. Die Aufwendungen für Werbung und Kundendienst wirken zum Teil jedoch erst zeitverzögert, was die Einschätzbarkeit verringert.

$$
\sum_{i, j} M A_{i j} \geq 77,36 \% \quad \text { mit } \text { Produkt }_{i}=1,2 \text { und } \text { Markt }_{j}=1,2
$$

Das neunte Kriterium bezieht sich auf die Kundendienst-Ausgaben pro Stück. Sie sollen nicht geringer als in der Vorperiode sein, um die Nachfrage zu halten. Dieses Lernziel ist besonders schwer zu erreichen, da die Kundendienst-Ausgaben mehrere Perioden nachwirken und den Teilnehmenden nicht alle zur Berechnung des Werts benötigten Informationen vorliegen.

$$
K D_{i j}(6) \geq K D_{i j}(5) \quad \text { mit } \text { Produkt }_{i}=1,2 \text { und } \text { Markt }_{j}=1,2
$$

Die nächste Stufe wird erreicht, wenn mindestens fünf der neun Kriterien erfüllt sind. Eine Hochstufung auf die fünfte Stufe erfolgt nicht automatisch in der letzten Runde, sodass nur ein Teil der Gruppen über die Finanzplanung entscheiden. Es wird jedoch davon ausgegangen, dass es für eine eher schwächere Gruppe sinnvoller ist, sich eine weitere Runde auf die Planung der Marketing-Instrumente und auf die Beschaffung und Produktion zu konzentrieren, da der Finanzbereich eher eine untergeordnete Rolle in den Lernzielen spielt.

\section{Die Lernziele der fünften Stufe}

Für die Gruppen, die die fünfte und damit die Stufe mit voller Komplexität erreichten, kommt die Finanzplanung hinzu. Als zehntes Kriterium wird der Kassenbestand zur Bewertung herangezogen. Wird bei der Berechnung des Kassenbestands ein negativer Wert ermittelt, so wird dieser im Planspiel-Modell zwar durch einen Kontokorrentkredit ausgeglichen, so dass grundsätzlich keine Illiquidität des Unternehmen auftreten kann. Es entstehen jedoch kurzfristig hohe Zinsaufwendungen, die durch eine mittelfristige Kreditplanung vermieden werden können. ${ }^{20}$

$$
\text { Kasse } \geq 0
$$

$\overline{20}$ Zur Finanzplanung vgl. Abschnitt 3.3. 
Die zehn Kriterien dienen zum einen der individuellen Steigerung der Komplexität während der experimentellen Phase. Sie können jedoch auch zur Bewertung der Leistungen in der Testphase herangezogen werden, auf die im folgenden Abschnitt eingegangen wird.

\subsection{Die Testphase}

\subsubsection{Inhaltliche Konstruktion}

In der Testphase soll sich die Fähigkeit der Teilnehmenden zeigen, in einer komplexen Anforderungssituation Entscheidungen treffen zu können. Dazu muss die Situation und Prognosen analysiert, Alternativen bewertet und sich in der Gruppe für eine Alternative entschieden werden. Es wird das gleiche Unternehmensplanspiel wie in der experimentellen Phase eingesetzt, ${ }^{21}$ dieses Mal als Erhebungsinstrument. Insofern sind den Teilnehmenden zwar die Zusammenhänge des Planspiels sowie die Unterlagen bereits bekannt, sie setzen jedoch auf einer neuen Ausgangslage auf. ${ }^{22}$ Die Testphase hat neben dem Aspekt der Erhebung der Anwendung des Wissens auch einen Lerneffekt, ist also gleichzeitig als Lernphase im Planspiel zu betrachten.

Zur Bewertung wird neben dem Unternehmensgewinn die gesamte Planung des Unternehmens herangezogen. Insbesondere die zeitlichen Effekte der einzelnen Entscheidungen bedürfen einer näheren Betrachtung, da sich Fehler in der Planung erst zeitlich verzögert auf den Gewinn auswirken. Dabei wird auf die in Abschnitt 6.3.3 für die Steigerung der Komplexität in der modularen Durchführung dargestellten Lernziele zurückgegriffen. Im Folgenden soll jedoch zunächst die Ausgangslage, die den Teilnehmenden präsentiert wird, näher betrachtet werden.

\subsubsection{Ausgangslage}

Die Ausgangssituation in der Testphase ist die 8. Periode im Planspiel-Verlauf, die vorgespielt wurde und für alle Teilnehmenden gleich ist. Sie ist bei Produkt 2

21 Vgl. Abschnitt 6.2.

22 Vgl. Anhang A.20. 
durch Marktsättigung gekennzeichnet, Produkt 1 befindet sich bereits in der Degenerationsphase. ${ }^{23}$

Im Produktionsbereich wird eine Rationalisierungs-Maßnahme durchgeführt, die zu Beginn der 9. Periode wirksam wird und eine Verringerung der benötigten Leistungseinheiten je Produkt beinhaltet. Das bedeutet, dass mehr Produkte auf den vorhandenen Maschinen hergestellt werden können. Dies verringert zwar die Herstellungskosten, verschärft jedoch die Situation bezüglich der eher zu hohen Kapazität. Ein weiteres Problem ist der vorhandene Rohstoffbestand, der nach der alten Leistung der Maschinen beschafft wurde, so dass die Rohstoffe für eine volle Maschinenauslastung nicht ausreichen würden. Schließlich trat in der 8. Periode ein Nachfrage-Überhang auf einem Markt auf, d. h. ein Teil der Nachfrage, die auf Grund der Setzung der Marketing-Instrumente entstand, konnte nicht durch die vorhandenen Lagerbestände an Produkten befriedigt werden.

Das von den Teilnehmenden zu führende Unternehmen $\mathrm{U1}^{24}$ hat in der Ausgangslage zwar den höchsten kumulierten Gewinn erreicht, jedoch den geringsten Jahresüberschuss erwirtschaftet. Im Vergleich mit den Konkurrenz-Unternehmen liegt es mit den Produktpreisen, den Absatzmengen sowie den Umsätzen jedoch im Durchschnitt (vgl. Abbildung 6.6, S.183). Für Werbung und Kundendienst gaben die fünf Unternehmen ebenfalls unterschiedlich viel aus. Das Modellunternehmen liegt auch hier im Durchschnitt (vgl. Abbildung 6.7, S. 184).

Insgesamt scheinen die vier Konkurrenz-Unternehmen verschiedene Strategien zu verfolgen und lassen sich dadurch gegeneinander abgrenzen: Während das Unternehmen U2 die höchsten Preise bei beiden Produkten und auf beiden Märkten verlangt, gibt es im Vergleich am wenigsten für Werbung und Kundendienst aus. Entsprechend niedrig ist hier der Umsatz. Der Gewinn jedoch liegt in der 8. Periode im Vergleich zu allen anderen Unternehmen mit Unternehmen U3 an der Spitze. Vergleichbar in der Höhe ist der Gewinn des Unternehmens U3. Die Preise liegen deutlich unter denen von U2, entsprechend höher ist der Umsatz. Dies wird jedoch durch die höheren Aufwendungen für Werbung und Kundendienst ausgeglichen.

23 Vgl. Abbildung 6.2; zum Konjunkturverlauf allgemein vgl. HopfenBECK (Managementlehre 1992), S. 590-595, sowie WöHE (Einführung 1996), S. 644-648.

24 Im Folgenden als 'Modellunternehmen' bezeichnet. 


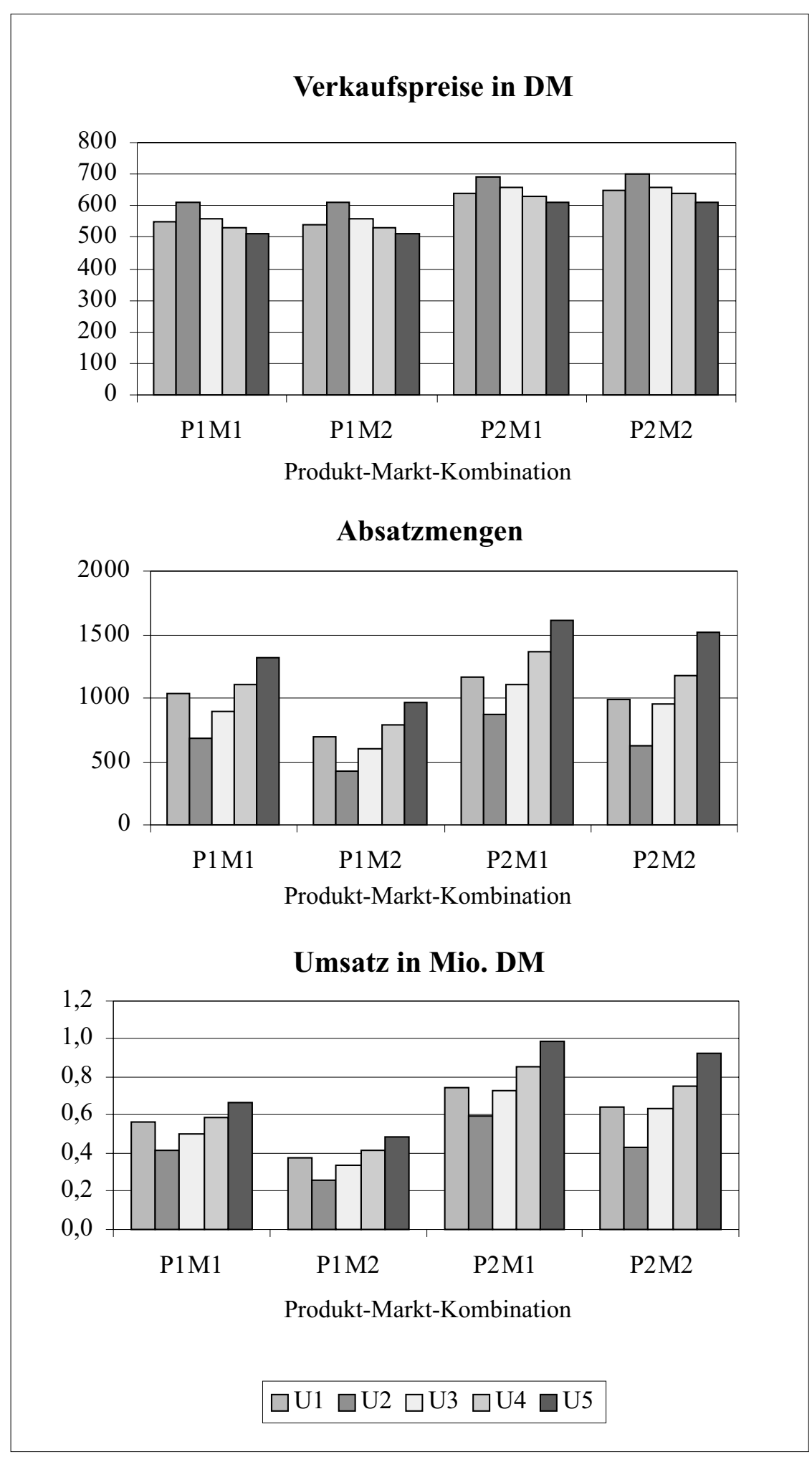

Abbildung 6.6: Ausgangslage der Testphase: Verkaufspreise, Absatzmengen und Umsätze 


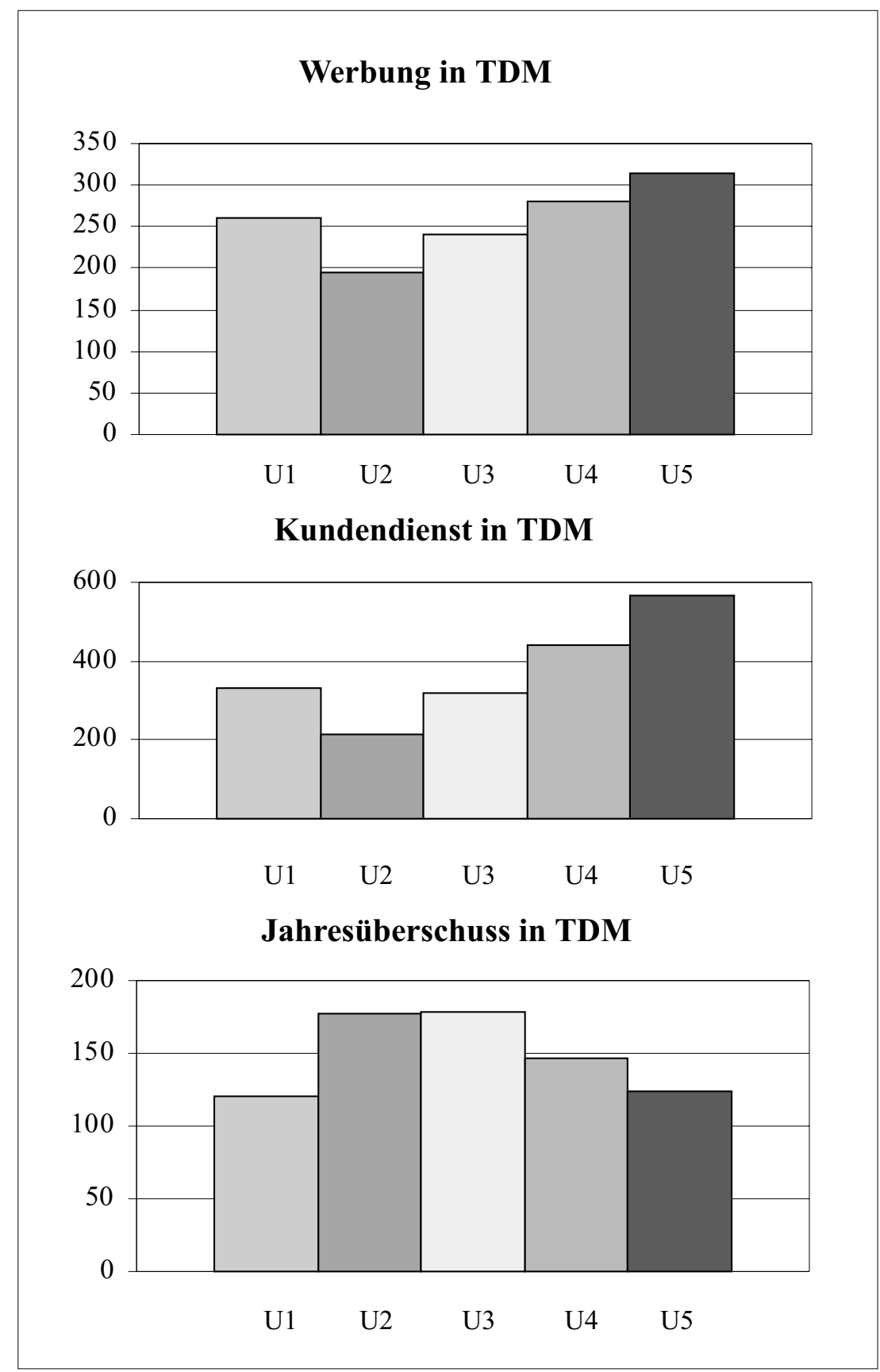

Abbildung 6.7: Ausgangslage der Testphase: Aufwendungen für Werbung und Kundendienst 
Die Unternehmen U4 und U5 verfolgen eher eine Niedrig-Preis-Strategie. Für Werbung und Kundendienst geben sie im Gegensatz zu U2 und U3 wesentlich mehr aus. Dabei übertrifft U5 alle anderen Unternehmen beim Kundendienst sehr deutlich, im Bereich der Werbung weniger. Entsprechend sind die Umsätze der beiden Unternehmen. Bedingt durch die hohen Aufwendungen fällt der Gewinn kleiner aus. Abbildung 6.8 zeigt die Abweichungen der jeweiligen Verkaufspreise vom Durchschnittspreis für die fünf Unternehmen. ${ }^{25}$

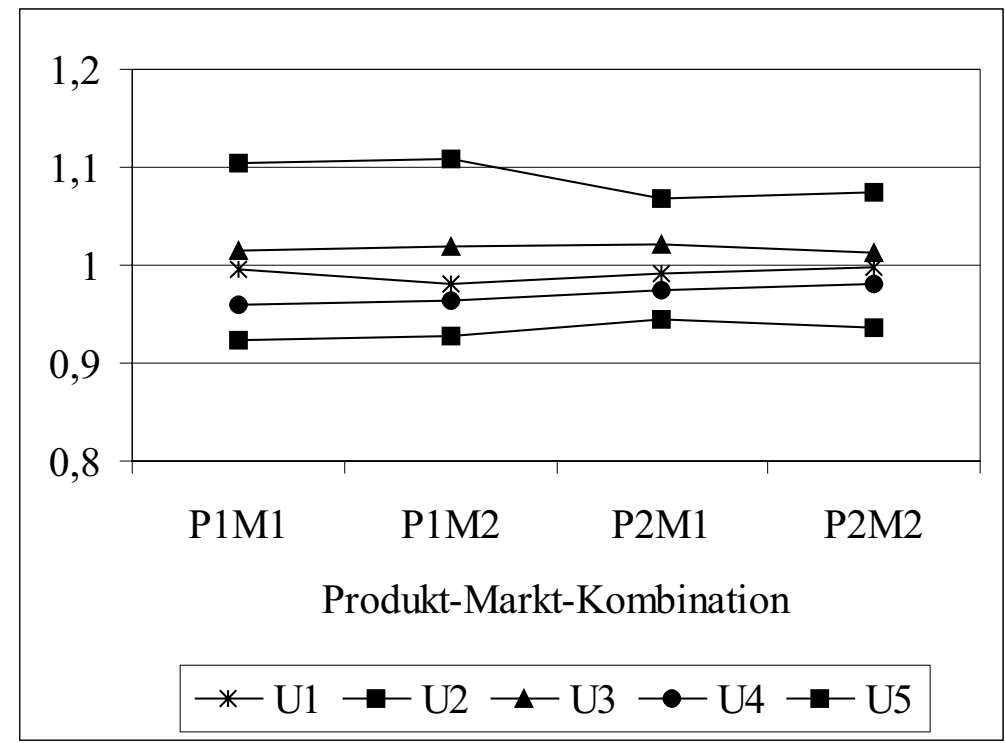

Abbildung 6.8: Ausgangslage der Testphase: Relative Abweichung vom Durchschnittspreis

Zusammenfassend lässt sich feststellen, dass in dieser Situation mehrere Ziele aufeinander treffen und sich nicht gleichzeitig realisieren lassen. Insgesamt verschärft sich durch den Verlauf der Konjunktur die Konkurrenz-Situation, dadurch nehmen die Gewinnchancen ab. Auf die genaue Darstellung des Planspiels als Erhebungsinstrument wird in Abschnitt 6.4 eingegangen.

25 Die Verbindungslinien zwischen den einzelnen Prozentzahlen dienen dabei der Übersichtlichkeit, zeigen also keinen stetigen Verlauf. 


\subsubsection{Bewertung des Lernerfolgs}

Mit der Testphase des Planspiels wurde die Zielsetzung verfolgt, die aktive Umsetzung des Wissens in Handlungen zu messen. ${ }^{26}$ Grundsätzlich gibt es verschiedene Maße zur Beurteilung der Güte der getroffenen Entscheidungen in komplexen Systemen: ${ }^{27}$

Endwerte ausgewählter Systemvariablen sind erst im Vergleich mit anderen Variablen zu bewerten, da sie kontextabhängig sind und als absolute Werte keine Aussagekraft haben. Hier wird kein Verlauf sichtbar, der jedoch gerade in Planspielen eine didaktische Bedeutung hat.

Aggregierte Maße bilden zwar den Systemzustand umfassender ab als Endwerte, sind jedoch eher intransparent und wenig objektiv.

Prozessorientierte Maße - wie z. B. die Veränderung einzelner Variablen im Zeitablauf - sind relativ sinnvoll zu bestimmen. Hierbei ist aber eine präzise Zieldefinition erforderlich, um die Aussagekraft zu erhöhen.

Abweichungsmaße als Vergleich mit Idealwerten bieten die beste Grundlage zur Beurteilung der Entscheidungen. Allerdings sind sie in Planspielen nur schwer oder gar nicht zu bestimmen, weil auf Grund der Dynamik jede Entscheidung auf einer anderen Situation basiert, für die eine optimale Entscheidung hergeleitet werden müsste. ${ }^{28}$

Expertenurteile bieten als weitere Möglichkeit die Verhaltensbeobachtung durch Außenstehende. Sie beziehen sich nicht auf Ergebnisse der Simulation, sondern auf Merkmale der Handlungen, die von außen beobachtbar sind. Hierbei können Probleme bei der Objektivität hinsichtlich der Operationalisierung der Merkmale auftreten.

In der vorliegenden Erhebung sollen verschiedene Aspekte betrachtet werden. Zum einen wird der Versuch unternommen, auf Grund der präsentierten Aus-

26 Zur inhaltlichen Konstruktion vgl. Abschnitt 6.4.1.

27 Vgl. Hasselmann (Problemstellungen 1993), S. 47-49.

28 Dabei könnten z. B. Methoden des Operations Research herangezogen werden; vgl. PetZING (Untersuchung 1993). 
gangslage eine optimale Entscheidung abzuleiten, die dann zur Beurteilung der ersten Entscheidung der Teilnehmenden in der Testphase herangezogen wird. ${ }^{29}$

Weiterhin orientiert sich die Bewertung an den für die modulare Durchführung des Unternehmensplanspiels in der experimentellen Phase formulierten Lernzielen. Hierbei kann auch der zeitliche Verlauf berücksichtigt werden, da drei Entscheidungen in Folge getroffen wurden.

\subsubsection{Gütekriterien der Testphase}

Die Durchführungs-Objektivität ist in der vorliegenden Untersuchung gegeben, da keine Beeinflussung der Entscheidungen durch die Durchführenden vorgenomen wurde. Alle Teilnehmenden bekamen die gleichen Unterlagen. Während der Planspiel-Phasen wurde nur auf Fragen zum Planspiel-Modell und nicht zur konkreten Entscheidung geantwortet. Die Durchführungs-Objektivität komplexer Systeme wie Unternehmensplanspiele muss aber auch hinsichtlich der Formulierung der Aufgabenstellung sowie der Schnittstelle zwischen Programm und Benutzenden betrachtet werden: Letzteres ist in diesem Fall gewährleistet, da die Bearbeitung nicht am Computer, sondern auf dem Papier stattfand. Die Aufgabenstellung war ebenfalls für alle Teilnehmenden gleich. Sie wurde in Form einer Vorlesung als Einführung ins Planspiel-Modell in der zweiten Unterrichtsstunde vorgenommen. Dabei wurde den Teilnehmenden als Unternehmensziel für die experimentelle Phase und die Testphase eine Maximierung des Gewinns vorgegeben. Die Instruktionen auf den Entscheidungsblättern waren zwar für die beiden experimentellen Gruppen unterschiedlich, die Informationen über die konjunkturelle Entwicklung imd das Planspiel-Modell waren jedoch gleich. ${ }^{30}$

Die Entscheidungen wurden durch die Spielleitung simuliert, dabei erfolgte eine Kontrolle der Dateneingabe anhand der Unternehmens-Ergebnisse. Die erfassten Entscheidungen sowie die Ergebnisse wurden gespeichert. Nachfolgend wurden betriebswirtschaftliche Kennzahlen identifiziert, die auch in der Realität zur Beurteilung eines Unternehmens herangezogen werden. Dabei wurde sichergestellt, dass diese Maße auch im Planspiel durch entsprechende Eingrif-

\footnotetext{
29 Vgl. Abschnitt 8.2.

30 Die Entscheidungsblätter befinden sich im Anhang A.14 und A.15.
} 
fe der Teilnehmenden zu beeinflussen sind. Hierdurch sind Auswertungs- und Interpretations-Objektivität gesichert. Diese Vorgehensweise hat aber auch Einfluss auf die Inhalts-Validität: Sie kann als gewährleistet angesehen werden, da die zu messenden Merkmale im Rahmen des Forschungsprojekts durch die Mitarbeiterinnen und Mitarbeiter als Experten entwickelt und operationalisiert wurden, wobei auf Grundlagen der Allgemeinen Betriebswirtschaftslehre zurückgegriffen wurde.

Die Identifizierung eines externen Merkmals mit zeitlichem Abstand zur Beurteilung der Kriteriums-Validität ist äußerst schwierig. Bisherige Untersuchungen im Bereich Experten-Novizen-Forschung mit komplexen Systemen ${ }^{31}$ zeigen zum Teil zwar bessere Leistungen der so genannten Experten. Die Frage ist jedoch, was die Experten von den Novizen unterscheidet. Weiterhin waren die Aufgabenstellungen zum Teil unpräzise formuliert und interpretierfähig, was jedoch in den Studien nicht erhoben wurde. Die Ergebnisse sind insgesamt uneinheitlich, dabei fallen insbesondere methodische Fehler hinsichtlich der Größe der Stichproben sowie des Einflusses der Versuchsleitung bei der Durchführung auf. ${ }^{32}$

In einem Teil dieser Studien wurden Untersuchungen mit Intelligenz-Tests durchgeführt. Sie bringen jedoch nur wenig Aufschluss über die Fähigkeit des Umgangs mit komplexen Systemen, sind also als Maß für die Konstrukt-Validität nur bedingt zu nehmen. Eine weitere Möglichkeit sind Persönlichkeits-Tests, die zum Teil Zusammenhänge zeigen. Die Bearbeitung unterschiedlicher komplexer Systeme schließlich, die durch eine geringe Anzahl Variablen gekennzeichnet sind, bringen eher geringe Korrelationen zwischen den herangezogenen Maßzahlen. Dies liegt zum einen an den Maßzahlen selbst, zum anderen aber auch an den Systemen. Zur Bearbeitung ist hier kein bereichsspezifisches Wissen notwendig, so dass eher von einer Steuerleistung des Systems gesprochen werden muss. Die Frage bleibt, mit welcher Leistung im Berufsleben oder Alltag diese Steuerleistung korrespondiert. ${ }^{33}$

In der vorliegenden Erhebung wurden zur Beurteilung der Validität zwei Faktorenanalysen gerechnet: Bei der ersten wurden alle erfassten Kennzahlen der Testphase korreliert und orthogonal zu Faktoren zusammengefasst. Es ergaben

31 Vgl. die Arbeiten der Gruppe um Dörner und Putz-Osterloh; vgl. Abschnitt 3.6.3.

32 Vgl. Funke (Probleme 1990) und Hasselmann (Problemstellungen 1993), S. 57-62.

33 Vgl. Hasselmann (Problemstellungen 1993), S. 62-74. 
sich neun Faktoren. Eine zweite Faktorenanalyse mit den Daten der gesamten Planspiel-Phase, also von acht Planspiel-Entscheidungen, brachte zehn Faktoren. Die Faktoren beider Analysen unterscheiden sich nur unwesentlich. ${ }^{34}$

Die Bestimmung eines externen Merkmals ist auf Grund der Gruppenarbeit in der Planspiel-Phase schwierig. Sicherlich könnten Maßzahlen der Netzwerk-Erhebung oder der Fallstudie zur Bewertung herangezogen werden. Eine Korrelation würde aber verfälschte Ergebnisse bringen, da in der Planspiel-Phase mehrere Teilnehmende in einer Gruppe spielten und so gleiche Ergebnisse erzielten.

Eine Test-Halbierung sowie Konsistenz-Analysen setzen (stochastisch) unabhängige Variablen voraus. Dies ist im Planspiel jedoch nicht gegeben, so dass eine Überprüfung der Reliabilität der Testphase nicht erfolgen kann. Ein Paralleltest ist ebenfalls nur schwer zu konstruieren: Es ist zwar theoretisch möglich, einer Simulation, die nur aus Zahlen und Funktionen besteht, zwei verschiedene Labels zu geben, d.h. z. B. den Produkten und Rohstoffen andere Namen zu geben. Allerdings beeinflusst auch die präsentierte Situation ihre Entscheidungen und damit auch die Ergebnisse. ${ }^{35}$

Insofern ist in einem Planspiel grundsätzlich zur Beurteilung der Reliabilität nur ein Retest durchführbar. Dies wurde in verschiedenen Untersuchungen durchgeführt. ${ }^{36}$ Die erhaltenen Korrelationen waren unterschiedlich hoch. Ein Grund hierfür sind die verschiedene Maßzahlen, die zur Bewertung herangezogen wurden. ${ }^{37}$ Weitere Kennzeichen dieser Systeme sind die konstanten funktionalen Zusammenhänge zwischen den Variablen, eine geringere Anzahl von Entscheidungsvariablen sowie wesentlich mehr Durchgänge der Simulation, die direkt am Computer oder Taschenrechner durchgeführt wurde. Die gefundenen Ergebnisse bezüglich der Retest-Reliabilität sind entsprechend nicht auf das eingesetzte Unternehmensplanspiel EpUS übertragbar, da sich hier die funktionalen Zusammenhänge und Parameter im Zeitverlauf ändern, um eine konjunkturelle Situation abzubilden. ${ }^{38}$ Weiterhin wurden nur wenige Perioden gespielt und die Entscheidungen nicht direkt am Computer durchgeführt. Ein wichtiges Merkmal ist hier

34 Die Tabellen mit den sortierten rotierten Faktorladungen befinden sich im Anhang A.24.

35 Vgl. dazu Hesse, SpIes \& Lüer (Einfluß 1983), insbesondere S. 419-423.

36 Vgl. die Darstellung in Abschnitt 3.6.3.

37 Vgl. Hasselmann (Problemstellungen 1993), S. 49-57; Hösch (Evaluation 1995), S. 35-40.

38 Vgl. Abschnitt 6.4.1. 
auch das notwendige Vorhandensein von betriebswirtschaftlichem Fachwissen zur Erzielung eines Erfolgs im Planspiel.

Zur Beurteilung der Retest-Reliabilität können in der vorliegenden Untersuchung die beiden ersten Entscheidungen in der experimentellen Phase genommen werden, die von den Gruppen unter herkömmlicher Bedingung getroffen wurden. Alle Gruppen wurden nach Rückmeldung der Ergebnisse der ersten Entscheidung am zweiten Planspiel-Abend aufgefordert, ihre Entscheidung zu wiederholen. Dieses kann als Retest interpretiert werden, allerdings mit der Einschränkung, dass ein Lernerfolg durchaus erwünscht ist: Fielen die rückgemeldeten Ergebnisse entsprechend den Erwartungen der Gruppen-Mitglieder aus, könnte die Tendenz bestehen, die Entscheidung zu wiederholen, was hohe Korrelations-Koeffizienten ergeben würde und auf eine hohe Reliabilität schließen ließe. Fielen die Ergebnisse jedoch nicht wie erwartet aus, würde das Verhalten sicherlich verändert werden und die Ergebnisse auf Grund einer zahlenmäßig anderen Entscheidung entsprechend gering reliabel ausfallen. Tabelle 6.1 zeigt die Korrelations-Koeffizienten.

\begin{tabular}{|l|c|}
\hline \multicolumn{1}{|c|}{ Kennzahl } & $\begin{array}{c}\text { Korr.-Koeff. } \\
\text { Produkt } 1 \text { und } 2 \\
\text { Rohstoff } 1 \text { und } 2\end{array}$ \\
\hline Rohstoffmengen & 0.086 und 0.039 \\
Produktionsmengen & 0.042 und 0.450 \\
\hline Rohstofflager-Differenz & 0.008 und -0.102 \\
Produktlager-Differenz & 0.482 und 0.693 \\
\hline automatischer Einkauf & 0.233 und -0.251 \\
\hline Herstellungskosten & 0.402 und 0.449 \\
\hline Marktanteile Markt 1 & 0.416 und 0.629 \\
Marktanteile Markt 2 & 0.398 und 0.700 \\
\hline Absatzmengen Markt 1 & 0.575 und 0.560 \\
Absatzmengen Markt 2 & 0.328 und 0.761 \\
\hline Kasse & 0.119 \\
\hline Gewinn & 0.703 \\
\hline
\end{tabular}

Tabelle 6.1: Korrelations-Koeffizienten der Testphase 
Bei den Entscheidungen über die Rohstoff- und die Produktionsmengen lässt sich eine starke Veränderung feststellen. Entsprechend sind auch die KorrelationsKoeffizienten bei den Differenzen zwischen Lager-Abgang in die Produktion bzw. durch Verkauf und Lager-Zugang durch Einkauf bzw. Produktion gering. Beim automatischen Einkauf an Produkten, der anfällt, wenn die vorhandene Maschinenkapazität nicht zur Produktion der geplanten Mengen ausreicht, ${ }^{39}$ ist der Koeffizient ebenfalls gering. Hier wurden von den Teilnehmenden offensichtlich Fehler in der Planung mit der zweiten Entscheidung korrigiert.

Da die Herstellungskosten nicht nur von Entscheidungen der Teilnehmenden, sondern auch von Parametern abhängen, die konstant blieben, da die gleiche Ausgangslage zu Grunde lag, ist der Korrelations-Koeffizient hier höher.

Im Marketing-Bereich ist zum Teil Stabilität bei den Entscheidungen über die Verkaufspreise sowie bei den Ausgaben für Werbung und Kundendienst festzustellen. ${ }^{40}$ Diese Stabilität spiegelt sich in zum Teil hohen Koeffizienten wider. Auch hier spielen die konstanten Einstellungen der Parameter eine Rolle.

Beim Kassenbestand sind zu beiden Zeitpunkten ähnlich hohe Varianzen, aber sehr unterschiedliche Mittelwerte festzustellen. Entsprechend gering ist der Korrelations-Koeffizient.

Trotz unterschiedlicher Entscheidungen in manchen Bereichen ist bezüglich der Zielgröße des Gewinns das Ergebnis reliabel. Hier liegt der Koeffizient bei 0.703, d.h. diese Maßzahl kann zur Beurteilung verschiedener Gruppen auf jeden Fall herangezogen werden.

Zusammenfassend lässt sich feststellen, dass eine Aussage über die RetestReliabilität des eingesetzten Planspiels nur bedingt möglich ist, da ein Lernerfolg nicht ausgeschlossen werden kann. Bessere Aussagen könnten sicherlich gemacht werden, wenn zu mehreren Zeitpunkten mit einer größeren Stichprobe Entscheidungen auf Grund der gleichen Ausgangslage getroffen würden.

\footnotetext{
39 Vgl. Abschnitt 6.2.

40 Dabei wurde das Verhalten der Nachfrage auf Markt 2 offensichtlich von den Teilnehmenden besser eingeschätzt als bei Markt1, denn hier sind die Ergebnisse stabiler. Auf eine Darstellung dieser Korrelations-Koeffizienten wird auf Grund des Umfangs jedoch verzichtet.
} 


\subsection{Der Fragebogen zur Entscheidung}

\subsubsection{Inhaltliche Konstruktion}

Dieses Instrument dient der Protokollierung der herangezogenen Begründungen und gesetzten Ziele der Entscheidungen in der Planspiel-Phase. Dies soll ein Nachvollziehen des Entscheidungs-Prozesses ermöglichen, da allein die numerischen Simulations-Ergebnisse keine detaillierten Aufschlüsse über die Subjektiven Theorien der Teilnehmenden sowie die ablaufenden Lernprozesse geben können. Das mit dem Instrument verfolgte Lernziel umfasst ein Heranführen an einen Entscheidungsprozess, der sich an Strategien orientiert und durch die Teilnehmenden selbst kontrolliert und strukturiert wird.

Die inhaltliche Gestaltung orientiert sich am Modell des Planungsprozesses in einer Unternehmung. Dabei können sechs Stufen identifiziert werden, ${ }^{41}$ die jedoch nicht chronologisch abgearbeitet werden, sondern in einem iterativen Verfahren mehrfach durchlaufen werden. ${ }^{42}$

1. Zielformulierung: Alle nachfolgenden Planungsaktivitäten richten sich weitgehend nach Zielinhalt und -intensität. Das Ziel dient auch als Basis für einen späteren Soll-Ist-Vergleich nach Realisierung der Entscheidung.

2. Prognose: Hier werden Vorstellungen über die zukünftigen Entwicklungen wichtiger Bestimmungsfaktoren - wie z. B. der Nachfrage - und deren Prämissen erlangt.

3. Alternativplanung: Auf Grund der Zielformulierung und der Prognosen werden alternative Handlungsmöglichkeiten entwickelt.

4. Eventualplanung: Die einzelnen Handlungsmöglichkeiten werden hinsichtlich ihrer Güte, Angemessenheit und Aktualität der ihnen zu Grunde liegenden Informationen aufbereitet.

5. Planungsrechnung und 6. Entscheidung: Auf Grund der Planungsrechnung wird sich für eine Alternative entschieden, die in der Lage ist, das vorgegebene Ziel unter den identifizierten Prämissen optimal zu erreichen.

41 Vgl. Korndörfer (Unternehmensführungslehre 1995), S. 104.

42 Vgl. CarL \& KiesEL (Unternehmensführung 1996), S. 22-23. 
7. Sollvorgabe: Durch die Entscheidung wird aus einer Alternative ein verbindlicher Soll-Plan, der nach der Durchführung der Maßnahme einen Soll-IstVergleich ermöglicht.

Eine Möglichkeit, die Subjektiven Theorien über die konkrete Handlungssituation zu erfassen, ist die Methode des Lauten Denkens. Hier werden die Teilnehmenden aufgefordert, ihre Überlegungen während der Handlung zu verbalisieren. ${ }^{43}$ Eine Schwierigkeit, die beim Einsatz dieser Methode auftritt, ist eine Tendenz der Handelnden zur Rechtfertigung statt bloßer Erläuterungen zum Thema. ${ }^{44}$ Dies würde in der vorliegenden Untersuchung zwar nicht unbedingt nachteilig wirken: Sie ist jedoch auf Grund der Anlage als Feldstudie sowie der räumlichen Gegebenheiten und der großen Anzahl Teilnehmender nicht durchführbar. ${ }^{45}$

Eine offene Form der Befragung hat zwar eine geringe Reaktivität, d.h. die Teilnehmenden re-agieren nicht nur auf die Fragestellung, sondern strukturieren selbstständig den Entscheidungsprozess und bilden Hypothesen über den Gegenstand. Die Auswertungs-Objektivität fällt jedoch geringer aus, da eine Inhaltsanalyse erforderlich ist. Weiterhin sind die Ergebnisse nur schwer miteinander vergleichbar. Eine strukturierte Fragestellung hat den Vorteil der besseren Auswertbarkeit und Vergleichbarkeit der Ergebnisse, erzeugt aber eine hohe Reaktivität und hält die Teilnehmenden eher davon ab, selbst Hypothesen über das Planspiel-Modell zu bilden.

Am sinnvollsten erscheint daher eine halboffene Form der Befragung, die zwar eine Leitung durch entsprechende Fragen vorsieht, aber kein direktes 'Abarbeiten' der Fragestellungen ermöglicht, um die Reaktivität in Grenzen zu halten. Es wird ein 'Brückenschlag' zwischen den numerischen Ergebnissen der Simulation und dem Handlungswissen der Teilnehmenden versucht. Die Fragen sollen durch ihre Struktur bzw. Reihenfolge die Teilnehmenden auffordern und anleiten, bei ihrer Entscheidungsfindung ebenfalls in einer entsprechenden Reihenfolge vorzugehen, sich die herangezogenen Begründungs-Zusammenhänge bewusst zu machen und explizit darzustellen. ${ }^{46}$

\footnotetext{
43 Vgl. Weidle \& Wagner (Methode 1982).

$44 \mathrm{Vgl}$. Knoblich \& Rhenius (Reaktivität 1992).

45 Vgl. Abschnitt 4.2.

46 Der Fragebogen befindet sich im Anhang A.17.
} 
Für die vorliegende Untersuchung wurden entsprechend ein Fragebogen formuliert, der in seiner Einleitung die Intention des Einsatzes verdeutlicht und dabei auf die Entscheidungsunterstützung sowie die Offenheit der Antworten hinweist. Im zweiten Teil werden - angelehnt an die Stufen des Modells des Planungsprozesses in einer Unternehmung - Fragen gestellt, an denen sich die Teilnehmenden während der Entscheidungsfindung orientieren können und sollen.

Je Planspiel-Gruppe soll nur ein Fragebogen ausgeteilt werden, so dass die Teilnehmenden gemeinsam antworten müssen. Dies erscheint sinnvoll, da auch die Entscheidungsfindung innerhalb der gesamten Gruppe geschehen soll. Hinsichtlich der Art der Darstellung werden keine Vorgaben gemacht. Es sind sowohl Zeichnungen und Rechnungen als auch stichpunktartige Aussagen und Erläuterungen zulässig. Dadurch sollen Probleme bei der inhaltlichen Interpretation in der Auswertung umgangen werden.

\subsubsection{Gütekriterien des Fragebogens}

Bezüglich der Durchführungs-Objektivität ist kein Einfluss zu erwarten, da die Fragebögen an die Teilnehmenden nur verteilt wurden und die Bearbeitung mittels einer Instruktion angeregt wurde.

Die Auswertungs-und Interpretations-Objektivität sind durch entsprechende Formalisierung der Schritte bei der Inhaltsanalyse zu sichern.

Das Instrument ist gleichzeitig auch Treatment, d.h. ein Einfluss auf die Entscheidungsfindung ist geplant und erwünscht: ${ }^{47}$ Hierbei könnte jedoch das Problem entstehen, dass die Teilnehmenden nicht unbedingt willens und in der Lage sind, die Vorgehensweise bei jeder Entscheidungsfindung aufzuschreiben. ${ }^{48}$ Der Fragebogen ist jedoch in der Lage, die Dokumentation die Entscheidungsfindung anzuleiten (Inhalts-Validität). Es kann anhand der Ergebnisse ein Rückschluss auf das Verhalten der Teilnehmenden gezogen werden (Konstrukt-Validität). Die Validierung durch ein externes Kriterium geschieht durch Vergleich der Anga-

47 Vgl. Abschnitt 4.3.

48 Diese Erfahrung wurde in dem DFG-Forschungsprojekt mit einer offenen Frage zur Entscheidung in der Planspiel-Phase gemacht. Die wenigsten Teilnehmenden antworteten derart, dass Rückschlüsse auf die Vorgehensweise bei der Entscheidungsfindung gemacht werden konnten. 
ben im Fragebogen mit den getroffenen Entscheidungen im Planspiel. Dabei wird auch auf eine mögliche Zielhierarchisierung eingegangen. ${ }^{49}$

Die Reliabilität des Fragebogens ist sicherlich nicht sehr hoch, da sie von der Bereitschaft der Teilnehmenden in der aktuellen Situation abhängt. In der Instruktion wird deshalb auf die Unterstützung der Entscheidungsfindung hingewiesen, zu deren Zweck der Fragebogen eingesetzt wird. In der Einführung der Veranstaltung wurde auf die Bedeutung der zusätzlichen Fragebögen für die parallel laufende Untersuchung insgesamt hingewiesen.

\subsection{Der Fragebogen zum situativen Erleben}

\subsubsection{Inhaltliche Konstruktion}

Ein weiterer Aspekt ist die Kontrolle der Emotionen und Motivationen in der aktuellen Anforderungssituation im Planspiel sowie die Gruppenarbeit. Es wird ein geschlossener Fragebogen konstruiert, der das subjektive Erleben der aktuellen Unterrichts-Situation in der Planspiel-Phase erfassen soll. ${ }^{50}$

Dazu werden Aussagen formuliert und mit Ratingskalen den Teilnehmenden zur Bewertung vorgelegt. Dies spart zum einen Zeit bei der Bearbeitung und erleichtert zum anderen die Auswertung. Die Ratingskalen funktionieren hierbei als Medien zur 'Anzeige' von latenten Merkmalsausprägungen. Die Messung erfolgt - im Vergleich zu deskriptiven Beschreibungen von Beobachtungen - diagnostisch, da die Teilnehmenden auf die vorgelegten Aussagen reagieren und damit Aufschluss über ihre interne Struktur geben. ${ }^{51}$

Zunächst sollen die Teilnehmenden ihre aktuelle Stimmung am Abend bewerten. Diese Aussage wird allgemein gehalten, um die Intimsphäre nicht zu verletzen. Sie soll die Teilnehmenden jedoch auffordern, ihre Stimmung zu reflektieren, da die diffuse Stimmungslage einen Einfluss auf den Lernerfolg haben kann. ${ }^{52}$

$\overline{49}$ Vgl. Möвus (Modellierung 1988) , S. 428-430.

50 Vgl. Anhang A.18.

51 Vgl. BeCK (Grundlagen 1987), S. 38-43.

52 Vgl. Abele (Einfluß 1996); ausführlich Abele (Stimmung 1995). 
Ein wichtiger Aspekt ist die Lust der Teilnehmenden zum Planspiel, die von Abend zu Abend wechseln kann, sowie das allgemeine Interesse am Unterricht. Hierauf hat auch die Qualität der Unterrichtsmaterialien einen Einfluss. ${ }^{53}$

Weiterhin sollen sie ihr Gefühl hinsichtlich der Sicherheit sowie einer Über- bzw. Unterforderung in der Planspiel-Situation beurteilen. Hier ist von einer Beeinflussung der Lernmotivation auszugehen, die wiederum die Mitarbeit im Unterricht beeinträchtigt. 54

Verschiedene Aspekte der Gruppenarbeit werden ebenfalls in die Bewertung einbezogen. ${ }^{55}$ Dabei wir nachgefragt, ob die oder der Teilnehmende grundsätzlich gerne in Gruppen arbeitet. Eine Abneigung gegen Gruppenarbeit würde den Lernerfolg eher negativ beeinflussen. Weiterhin wird auf die Diskussion in der Gruppe, das Gruppenklima sowie den Verlauf der Gruppenarbeit eingegangen. Hierbei ist die subjektive Einschätzung der Gruppenarbeit wichtig, nicht eine eventuelle objektive Beurteilung durch Beobachtung, da der Einfluss auf die kognitiven Aktivitäten erhoben werden sollte und nicht die Art und Weise der Gruppenarbeit. Ein weiterer Aspekt ist, ob die Teilnehmenden ihre Beiträge in die Diskussion einbringen konnten und ob ihre eigenen Beiträge in der Gruppe berücksichtigt wurden.

Die letzte Aussage bezieht sich auf das individuelle Gefühl der Sicherheit bezüglich der getroffenen Entscheidung. Dies dient der Kontrolle, ob die Teilnehmenden sich im zeitlichen Verlauf des Planspiels zunehmend sicherer in ihren Entscheidungen waren. Den Teilnehmenden wird auf dem Fragebogen auferdem Platz für weitere Bemerkungen gelassen.

Auch dieser Fragebogen ist als Erhebungsinstrument und zugleich als Treatment zu verstehen. ${ }^{56}$ Er wird einzeln an die Teilnehmenden ausgegeben, so dass die individuellen Wahrnehmungen kontrolliert werden und mit dem Lernerfolg in den anderen Instrumenten zusammengeführt werden können.

\footnotetext{
53 Vgl. Schunck (Subjektive Theorien 1993), S. 153-166.

54 Vgl. Schmiel (Förderung 1988).

55 Vgl. dazu Boos (Entscheidungsfindung 1996).

56 Vgl. Abschnitt 4.3.
} 


\subsubsection{Gütekriterien des Fragebogens}

Mit diesem Fragebogen werden emotionale und motivationale Aspekte erhoben, also relativ instabile Merkmale, die abhängig sind von der aktuellen Situation. Ein Fragebogen mit selbstbezogenen Aussagen, zu denen die Teilnehmenden Stellung nehmen sollen, ist zur Erfassung dieser Aspekte gut geeignet. Selbst-Rating ermöglicht die Kontrolle der Subjektivität in hohem Maße und stellt in der Diagnostik eine unverzichtbare Komponente dar. ${ }^{57}$

Ein grundlegendes methodisches Problem des Ratingverfahrens besteht darin, dass die Hauptgütekriterien mit zunehmender Komplexität der Kriterien nicht gewährleistet sind. Zur Sicherung der Validität werden die Skalen durch disjunkte Wörter im Sinne von Nein-Ja benannt. Weiterhin wird der Zeitbezug der Aussagen durch entsprechende Benennung - wie 'heute' oder 'immer' - verdeutlicht. 58 Die Skalen bestehen aus fünf Punkten, um eine hohe Trennschärfe zu erreichen. ${ }^{59}$

Die Items werden anhand der Kriterien zur Formulierung von Fragen erstellt. ${ }^{60}$ Ein Raten ist in diesem Fragebogen sinnlos, da kein Lernerfolg gemessen wird. Es könnte jedoch die Tendenz zur Mitte im Sinne von 'Ich weiß nicht' entstehen. Ein eigenes Feld wird nicht vorgegeben, da davon ausgegangen werden kann, dass die Teilnehmenden in der Lage sind, die Fragen zu beantworten bzw. die Beantwortung eher auslassen. Dabei besteht allerdings das Problem, dass einige Teilnehmende die Antwort so geben, wie es ihrer Meinung nach erwünscht ist, eventuell aber nicht ihrer Ansicht entspricht. ${ }^{61}$ Hier wurde kein externes Kriterium zur Kontrolle erfasst. Insgesamt ist es bei einer solchen Art von Fragebogen schwierig, ein valides Außenkriterium zur Ermittlung der externen Validität bereitzustellen. ${ }^{62}$ Da die Erhebung in anonymisierter Form durchgeführt wurde, bleibt zu hoffen, dass die Teilnehmenden ehrlich sind.

Die Durchführungs-Objektivität ist gegeben, da die Fragebögen mit den Planspiel-Unterlagen verteilt werden und eine ausreichende Instruktion vorhanden ist.

57 Vgl. Mummendey (Fragebogen-Methode 1995), S. 38.

58 Vgl. KLEBER (Diagnostik 1992), S. 249-252.

59 Vgl. Sievers (Forschungsmethoden 1990), S. 190.

60 Vgl. Sievers (Forschungsmethoden 1990), S. 121-131.

61 So genanntes 'Problem der sozialen Erwünschtheit'; vgl. Mummendey (FragebogenMethode 1995), S. 159-170 sowie Sievers (Forschungsmethoden 1990), S. 324.

62 Vgl. Mummendey (Fragebogen-Methode 1995), S. 78. 
Durch die Rating-Skalen ist die Auswertung der Fragebögen ebenfalls objektiv. Bei der Interpretation der Ergebnisse wird ein Rückbezug auf die zu Grunde liegenden Theorien vorgenommen.

Eine Messung der Retest-Reliabilität ist nahezu unmöglich, da ein zu geringer zeitlicher Abstand zwischen den Erhebungen einen Erinnerungseffekt bringen könnte. Die vorliegenden Daten der sieben Messzeitpunkte wurden zwar einer Korrelationsanalyse unterzogen. Die Ergebnisse können allerdings unter dem Stabilitäts-Aspekt des gemessenen Merkmals interpretiert werden. ${ }^{63}$ Dabei zeigen die Fragen zu eher stabilen Persönlichkeitsmerkmalen eine größere Stabilität als Fragen zum Empfinden in der aktuellen Situation. Da aus zeit-ökonomischen Gründen zu jedem Aspekt nur eine Frage vorgegeben wurde und auch keine Störfragen eingebaut waren, ist keine innere Konsistenz messbar.

Dabei stellen die Einschätzungen der Teilnehmenden ihre Subjektiven Theorien über Unterricht dar, d.h. die Validität ihrer Urteile ist sehr unterschiedlich. Die Subjektiven Theorien werden auch durch die Emotionen und Motivationen der Teilnehmenden beeinflusst und sind situationsabhängig, d. h. nicht unbedingt objektiv und eher nicht reliabel. Trotzdem kann es sinnvoll sein, sie zu erheben, um einen Eindruck der subjektiven Einschätzung des Unterrichts zu bekommen und eventuelle Anpassungen vorzunehmen. ${ }^{64}$

Zur Verbesserung der universitären Lehre können folgende Maßnahmen beitragen: Das erlebte Vertrauen zu den Lehrenden begünstigt die Qualität der Lehre, so dass neben der positiven Wahrnehmung der eigenen Ausbildungs-Situation auch die Motivation und die Leistungsbereitschaft gesteigert werden. Neben der fachlichen ist auch eine persönliche Unterstützung für die Studierenden wichtig, wie auch eine empirische Untersuchung zeigt: Dabei spielen der Respekt und die Akzeptanz der Studierenden durch die Lehrenden eine Rolle, d. h. die Einstellung der Lehrenden zur eigenen Professionalität. ${ }^{65}$ Diese Ergebnisse sind sicherlich auf die berufliche Weiterbildung zu übertragen, da die Beruftstätigen im Berufsalltag ihre Kompetenzen erleben und die Rolle als Studierenden ihnen eher fremd ist.

63 Die Ergebnisse werden in Abschnitt 8.4.2 dargestellt, die Korrelations-Koeffizienten befinden sich im Anhang A.26.

64 Vgl. Schweer (Innovation 1996); auch LüdeckE-Plümer (Befindlichkeit 1991).

65 Vgl. SchweEr (Dozentenverhalten 1998). 


\section{Auswertungsmethoden}

\section{$7.1 \quad$ Vorbemerkungen}

In diesem Kapitel sollen die statistischen Methoden überblicksartig vorgestellt werden, die zur Auswertung der Ergebnisse herangezogen wurden. ${ }^{1}$

In Abschnitt 7.2 werden die Charakteristika von statistischen Test beschrieben. Dabei kann grundsätzlich zwischen strukturen-prüfenden und strukturenentdeckenden Verfahren unterschieden werden: ${ }^{2}$ Erstere prüfen Annahmen über die abhängigen und unabhängigen Variablen. Beispiele hierfür sind die Kontingenz-Analysen, zu denen Fisher's exakter Test gehört (Abschnitt 7.3), weiterhin der t-Test (Abschnitt 7.4) und die Varianzanalyse (Abschnitt 7.5). Letztere Verfahren stellen Zusammenhänge zwischen den Variablen fest. Hierzu gehören die Korrelationsanalyse, auf die in Abschnitt 7.6 eingegangen wird, und die Faktorenanalyse, die in Abschnitt 7.7 betrachtet wird.

\section{2 $\quad$ Statistische Tests}

Statistische Tests dienen dazu, empirische Beobachtungen zu analysieren, um sie hinsichtlich verschiedener Merkmale bewerten zu können. ${ }^{3}$ Dabei werden zwei Hy-

\footnotetext{
1 Die einzelnen Methoden werden jedoch nicht umfassend dargestellt. Bei Interesse ist die angegebene Literatur zu studieren.

2 Vgl. Backhaus et al. (Analysemethoden 1996), S. XVIII.

3 Vgl. zu weitergehenden Grundlagen von Messen und Testen STEYER \& Eid (Messen 1993).
} 
pothesen über den Mittelwert $\mu$ aufgestellt, die als Null-und Alternativ-Hypothese bezeichnet werden. Sie lauten allgemein: ${ }^{4}$

Null-Hypothese $H_{0}: \quad \mu=\mu_{0} \quad$ gegen die Alternativ-Hypothese $H_{1}: \quad \mu \neq \mu_{0}$

Diese Tests sind jedoch mit Fehlern behaftet, die nach $\alpha$-Fehler und $\beta$-Fehler unterschieden werden können: ${ }^{5}$

\begin{tabular}{cc|c|c} 
& & \multicolumn{2}{c}{ Es liegt vor } \\
\multirow{2}{*}{$\begin{array}{c}H_{0} \\
\text { für }\end{array}$} & $H_{0}$ & - & $\beta-$ Fehler \\
\cline { 2 - 4 } & $H_{1}$ & $\alpha$-Fehler & -
\end{tabular}

Insbesondere der $\alpha$-Fehler, der die Irrtums-Wahrscheinlichkeit eines Tests angibt, ist bei der Betrachtung der Ergebnisse von statistischen Tests von Bedeutung. Die üblichen Statistik-Verfahren versuchen jedoch, den $\beta$-Fehler bei festem $\alpha$ möglichst klein zu halten. ${ }^{6}$

Die Wahl des Signifikanz-Niveaus $\alpha$ beeinflusst die Ergebnisse der Analysen, auf die wiederum die Stichproben-Größe einen Einfluss hat. ${ }^{7}$ In der vorliegenden Untersuchung wird das Signifikanz-Niveau auf $5 \%$ festgelegt. Über die praktische Bedeutsamkeit muss jedoch eine zusätzliche Entscheidung getroffen werden. Nicht immer bedeutet statistische Signifikanz auch eine praktische Bedeutsamkeit der gefundenen Ergebnisse.

\subsection{Fisher's exakter Test}

Dieser Test ist besonders sinnvoll anzuwenden, wenn die Stichprobe und die Grundgesamtheit nicht sehr groß sind. Der Test erlaubt die Überprüfung des Einflusses eines nur in diskreten Ausprägungen vorliegenden Merkmals auf die abhängige Variable, die ebenfalls in diskreten Ausprägungen vorliegt. Durch die

\footnotetext{
4 Vgl. Hartung, Elpelt \& Kösener (Statistik 1993), S. 133.

5 In Anlehnung an Hartung, Elpelt \& Kösener (Statistik 1993), S. 133.

6 Vgl. Hartung, Elpelt \& Kösener (Statistik 1993), S. 134.

7 Vgl. Hartung, Elpelt \& Kösener (Statistik 1993), S. 137-138.
} 
Art der Berechnung, bei der kein Parameter geschätzt werden muss, wird das Ergebnis nicht verfälscht. Der Test besitzt die besten Güteeigenschaften hinsichtlich $\alpha$ - und $\beta$-Fehler. ${ }^{8}$

Zur Berechnung wird zunächst eine 2x2-Kontingenz-Tafel aufgestellt, die folgende Form hat:

\begin{tabular}{c|c|c|c} 
& $A_{1}$ & $A_{2}$ & \\
\hline 1 & $a$ & $b$ & $n$ \\
\hline 0 & $c$ & $d$ & $N-n$ \\
\hline & $a+c$ & $b+d$ & $N$
\end{tabular}

Kontingenz-Tafel

Der Umfang der Grundgesamtheit wird mit $N$ bezeichnet, $A_{1}$ und $A_{2}$ stellen bestimmte Kategorien dar, nach denen sich die Stichprobe aufteilen lässt. $a+c$ und $b+d$ sind entsprechend die jeweilige Anzahl der Teilnehmenden. $a$ und $b$ sind die Größen der Gruppen, die das betrachtete Merkmal haben (1), $c$ und $d$ die Größen der Gruppen, die es nicht haben (0). Die gesamte Anzahl der Personen, die das Merkmal haben, ist $a+b=n$.

Dem Modell liegt die Annahme zu Grunde, dass das Merkmal innerhalb beider Kategorien zufällig verteilt ist. Dies entspricht einem Auszählen ohne Reihenfolge, also der hypergeometrischen Verteilung. Die Wahrscheinlichkeit, genau $a$ Merkmale in der Kategorie $A_{1}$ und $b=n-k$ Merkmale in der Kategorie $A_{2}$ zu finden, berechnet sich durch

$$
P(a)=\frac{n !(N-n) !(a+c) !(b+d) !}{N ! a ! b ! c ! d !} .
$$

Unter der Annahme, dass das Merkmal innerhalb der Kategorien unabhängig verteilt ist, kann die Hypothese, dass das gefundene Ergebnis zufällig ist, durch diese Berechnung getestet werden. Dazu muss die Wahrscheinlichkeiten für alle $a \leq k$ aufsummiert werden und mit dem gewählten Signifikanz-Niveau $\alpha$ verglichen werden.

8 Vgl. Hays (Statistic 1973), S.737-740; auch Hartung, Elpelt \& Kösener (Statistik 1993), S. 414-416. 


\section{Beispiel:}

Eine denkbare Fragestellung, die mit diesem Test untersucht werden kann, ist in der vorliegenden Untersuchung die Häufigkeit des Auftretens einer bestimmten Proposition im Netzwerk. So haben fünf von 20 Teilnehmenden der modularen Bedingung im Posttest die Proposition “Die Produktionsmenge beeinflusst die Absatzmenge“ gebildet. Insgesamt wurde sie jedoch nur von sechs der insgesamt 45 Teilnehmenden konstruiert. Die Kontingenz-Tafel sieht entsprechend wie folgt aus:

\begin{tabular}{l|c|c|r} 
& modular & herkömmlich & \\
\hline $1=$ Proposition gebildet & $a=5$ & $b=1$ & $n=6$ \\
\hline $0=$ nicht gebildet & $c=15$ & $d=24$ & $N-n=39$ \\
\hline & $a+c=20$ & $b+d=25$ & $N=45$
\end{tabular}

Nachfolgend wird der p-Wert berechnet, indem der p-Werte für den vorliegenden Fall und alle unwahrscheinlicheren Fälle aufsummiert werden:

$$
\begin{aligned}
\mathrm{p} & =\quad P(a=5)+P(a=6) \\
& =\frac{6 ! 39 ! 20 ! 25 !}{45 ! 5 ! 1 ! 15 ! 24 !}+\frac{6 ! 39 ! 20 ! 25 !}{45 ! 6 ! 0 ! 14 ! 25 !}=0.047<\alpha=0.05
\end{aligned}
$$

Da er empirische p-Wert kleiner ist als das gewählte Signifikanz-Niveau, ist das Ergebnis signifikant. Das bedeutet, die Proposition wurde überzufällig häufig von den Teilnehmenden der modulare Bedingung gebildet. Es lässt sich also ein Zusammenhang zwischen der Konstruktion dieser Proposition und der PlanspielBedingung herstellen.

\section{4 t-Test}

Mit Hilfe des t-Tests lassen sich Aussagen über den Mittelwert $\mu$ einer Beobachtungsreihe machen. Als Voraussetzung zur Anwendung dieses Tests müssen die Daten normalverteilt sein. Um zu einer Aussage hinsichtlich der Gültigkeit 
der zu prüfenden Null-Hypothese zu kommen, wird der empirische t-Wert nach folgender Formel berechnet werden: ${ }^{9}$

$$
t=\frac{M-\mu_{0}}{S} \sqrt{n}
$$

Dabei ist $M$ der Mittelwert, $S$ die empirische Standard-Abweichung und $n$ die Größe der Stichprobe.

Sollen zwei Gruppen miteinander verglichen und Hypothesen hinsichtlich beider Mittelwerte gleichzeitig geprüft werden, so betrifft die Null-Hypothese die Differenz der Mittelwerte und lautet: ${ }^{10}$

$$
H_{0}: \mu_{1}-\mu_{2}=\mu_{0} \quad \text { gegen } \quad \mu_{1}-\mu_{2} \neq \mu_{0}
$$

Häufig wird angenommen, dass $\mu_{0}=0$ ist, d.h. kein Unterschied zwischen den Mittelwerten existiert. In diesem Fall gilt $M=M_{1}-M_{2}$, d. h. $M$ ist die Differenz zwischen den Mittelwerten der beiden Gruppen, die verglichen werden sollen. Entsprechend berechnet sich bei gleicher Varianz in den beiden Gruppen der empirische t-Wert durch

$$
t=\frac{M_{1}-M_{2}}{S} \sqrt{n}
$$

Der ermittelte empirische t-Wert wird mit dem zugehörigen theoretischen Wert der t-Verteilung verglichen. Er ist abhängig von der Stichproben-Größe $n$ und dem Signifikanz-Niveau $\alpha .{ }^{11}$ Die Null-Hypothese wird verworfen, wenn gilt:

$$
|t|>t_{n-1 ; \alpha}
$$

Das bedeutet, dass zum Signifikanz-Niveau die Unterschiede zwischen den Mittelwerten der beiden zu vergleichenden Gruppen statistisch bedeutend sind.

\footnotetext{
9 Vgl. Backhaus et al. (Analysemethoden 1996), S. 28.

10 Vgl. Hartung, Elpelt \& Kösener (Statistik 1993), S. 179.

11 Die Werte sind in den gängigen Lehrbüchern zur Statistik tabelliert.
} 


\subsection{Varianzanalyse}

\section{Modell der Varianzanalyse}

Die Varianzanalyse von den gleichen Voraussetzungen wie der t-Test aus, hier können jedoch mehrere Gruppen und Faktoren gleichzeitig betrachtet werden. ${ }^{12}$ Das allgemeine Modell der Varianzanalyse lautet ${ }^{13}$

$$
y_{i j}=\underbrace{\mu+\tau_{i}}_{\mu_{i}}+\epsilon_{i j} .
$$

Dabei gibt $\mu_{i}$ den Mittelwert der Gruppe $i$ mit $i=1, \ldots, k, \tau_{i}$ den Effekt der Behandlung $i, \epsilon_{i j}$ den zufälligen Versuchsfehler und $j=1, \ldots, n_{i}$ die Beobachtungen in der i-ten Gruppe an.

Die zu prüfende Null-Hypothese besagt, dass es keinen Unterschied zwischen den Gruppenmittelwerten gibt, d.h. es gilt ${ }^{14}$

$$
\mu_{1}=\mu_{2}=\ldots=\mu_{k}
$$

Die empirische Varianz berechnet sich als $S S G=\sum_{i, j}\left(\overline{y_{i}}-y_{i j}\right)^{2}$ mit dem empirischen Mittelwert $\overline{y_{i}}$ der Variable $Y_{i}$. Die Varianzanalyse spaltet diese empirische Varianz auf in die Varianz zwischen den Gruppen und die Fehlervarianz:

$$
S S G=S S T+S S E \text { mit }
$$

$S S G=$ Grand Sum of Sqares / Gesamt-Quadratsumme

$S S T=$ Sum of Squares for Treatments / Quadratsumme der Behandlungen

$S S E=$ Sum of Squares for Errors / Quadratsumme der Fehler

Nachfolgend wird der empirische F-Wert ermittelt:

$$
\begin{gathered}
F=\frac{M S T}{M S E} \\
\text { mit } M S T=\frac{S S T}{k-1} \text { und } M S E=\frac{S S E}{N-k}, \quad \text { wobei }
\end{gathered}
$$

12 Zur Auswertung wurde das Programm $2 V$ aus dem Paket BMDP herangezogen; vgl. Dixon (Software 1985), S. 359-356.

13 Vgl. Backhaus et al. (Analysemethoden 1996), S. 56.

14 Vgl. im Folgenden Hartung, Elpelt \& Kösener (Statistik 1993), S. 610-613 sowie BACKHAus et al. (Analysemethoden 1996), S. 58-66. Ausführlich zur Varianzanalyse LindMAN (Analysis 1974). 
$M S T=$ Mean Sum of Squares for Treatments / Mittlere Quadratsumme der Behandlungen

$M S E=$ Mean Sum of Squares for Errors / Mittlere Quadratsumme der Fehler

Dieser empirische F-Wert wird mit dem zugehörigen theoretischen F-Wert verglichen, der sich aus der Anzahl der Freiheitsgrade $k-1$ für die Treatments und $N-k$ für die Fehler sowie aus dem gewählten Signifikanz-Niveau $\alpha$ ergibt. ${ }^{15}$ Ist der empirische F-Wert größer als der theoretische, gilt also

$$
F>F_{k-1 ; N-k ; \alpha},
$$

so wird die Null-Hypothese zum Niveau $\alpha$ verworfen. Ist er jedoch kleiner, wird die Hypothese nicht verworfen. Der F-Test schließt demnach auf einen signifikanten Unterschied zwischen den Mittelwerten der Messreihen, wenn die Varianz zwischen den Messreihen wesentlich größer ist als die Varianz innerhalb der Reihen.

\section{Mehrfaktorielle Varianzanalyse und Messwiederholungen}

Soll der Einfluss mehrerer verschiedener Faktoren auf eine abhängige Variable betrachtet werden, kann die mehrfaktorielle Varianzanalyse zur Bewertung der Effekte herangezogen werden. ${ }^{16}$

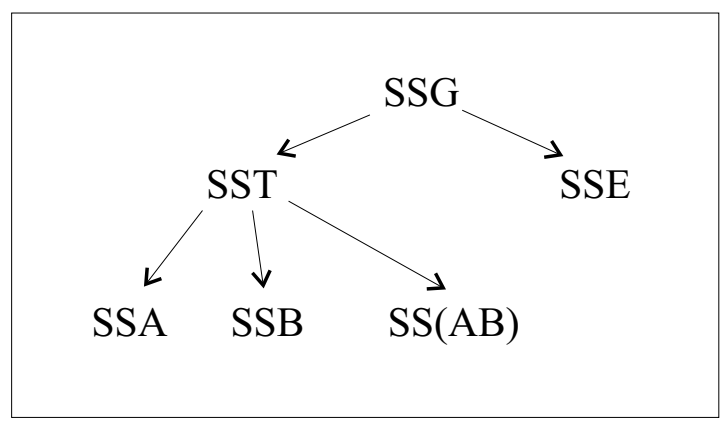

Abbildung 7.1: Mehrfaktorielle Varianzanalyse: Aufspaltung der Varianz bei zwei Faktoren $^{17}$

15 Die theoretischen F-Werte sind ebenfalls in vielen Lehrbüchern zur Statistik tabelliert.

16 Vgl. Backhaus et al. (Analysemethoden 1996), S. 66-75.

17 In Anlehnung an: BackHaus et al. (Analysemethoden 1996), S. 69. 
Abbildung 7.1 zeigt die Aufspaltung der Quadratsumme der Behandlungen (SST) nach dem Einfluss der Faktoren A und B (SSA und SSB) sowie der Wechselwirkung zwischen $\mathrm{A}$ und $\mathrm{B}(\mathrm{SS}(\mathrm{AB})){ }^{18}$

Werden die Beobachtungen unter den gleichen Versuchsbedingungen zu zwei oder mehreren Messzeitpunkten wiederholt, wird von 'Repeated Measures' oder 'Replication' gesprochen. ${ }^{19}$ In diesem Fall könnte zwar die Messwiederholung als ein Faktor angesehen und eine einfaktorielle Varianzanalyse gerechnet werden. Für den Fall aber, dass der Einfluss zusätzlicher Faktoren auf das Ergebnis der Messwiederholung getestet werden soll - wie in der vorliegenden Untersuchung -, bietet es sich an, ein spezielles Modell aufzustellen, dass die Messwiederholungen berücksichtigt und den Vorteil der geringeren Varianz auf Grund der Messwiederholung nutzt. ${ }^{20}$

\subsection{Korrelationsanalyse}

Der Korrelations-Koeffizient $r_{x y}$ zwischen zwei Variablen $X$ und $Y$ gibt den Grad des linearen Zusammenhangs an ${ }^{21} \mathrm{~d}$.h. es ist eine Aussage über die relative Ausprägung einer zweiten Variablen möglich, wenn die erste bekannt ist. Der Koeffizient berechnet sich nach der Formel ${ }^{22}$

$$
r_{x y}=\frac{\frac{1}{N}\left(\sum_{i} x_{i} y_{i}\right)-M_{x} M_{y}}{S_{x} S_{y}} .
$$

Dabei sind $x_{i}$ und $y_{i}$ die i-te Ausprägungen der Variablen $X$ und $Y, M_{x}$ und $M_{y}$ die Mittelwerte und $S_{x}$ und $S_{y}$ die Standard-Abweichungen der beiden Variablen. Der Korrelations-Koeffizient zweier Variablen kann als Cosinus des Winkels zwischen den beiden Vektoren $X=\left(x_{i}\right)_{i=1, \ldots, n}$ und $Y=\left(y_{i}\right)_{i=1, \ldots, n}$ interpretiert werden (Abbildung 7.2).

\footnotetext{
18 Vgl. Hartung, Elpelt \& Kösener (Statistik 1993), S. 624-627.

19 Vgl. Hays (Statistic 1973), S. 499.

20 Vgl. Lindman (Analysis 1974), S. 133. Zur Auswertung wurde das Programm $4 \mathrm{~V}$ aus dem Paket BMDP herangezogen; vgl. Dixon (Software 1985), S. 388-394.

21 Vgl. Backhaus et al. (Analysemethoden 1996), S. 199-203; auch Hartung \& Elpelt (Statistik 1984), S. 143.

22 Vgl. HaYs (Statistic 1973), S.631.
} 


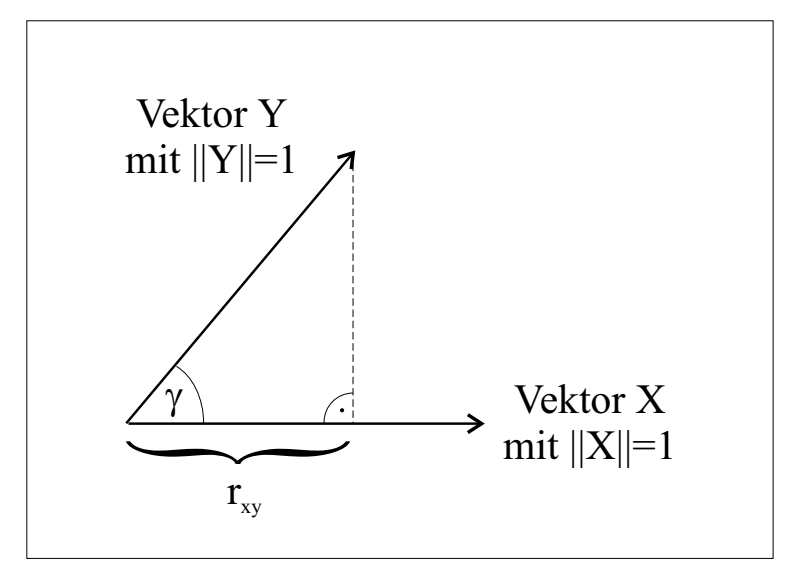

Abbildung 7.2: Interpretation des Korrelations-Koeffizienten

Wird das Lot von Vektor $Y$ auf Vektor $X$ gefällt, so gibt die Länge der Projektion gerade den Korrelations-Koeffizienten $r_{x y}$ an. Er kann auch als $\cos (X, Y)$ interpretiert werden, wenn beide Vektoren auf die Länge 1 normiert sind. Folgende Aussagen sind über den Koeffizienten im Allgemeinen möglich:

- Ist der Koeffizient +1, so haben die Vektoren die gleichen Ausprägungen und zeigen in die gleiche Richtung. Der eine Vektor ist also ein positives Vielfaches des anderen, verschoben um eine Konstante. Der Winkel zwischen ihnen ist $0^{\circ}$. Entsprechend ist auch $r_{x x}=1$.

- Ist der Koeffizient-1, so zeigen die Vektoren genau in entgegengesetzte Richtungen und sind ein negatives Vielfaches des anderen. Der Winkel zwischen ihnen beträgt $180^{\circ}$. Entsprechend ist $r_{x,-x}=-1$.

- Ist $r_{x y}=0$, so sind die beiden Variablen voneinander unabhängig und stehen orthogonal aufeinander. Der Winkel zwischen ihnen beträgt in diesem Fall $90^{\circ}$.

- Für Koeffizienten mit $r_{x y} \in$ ]0; 1[ kann auf einen positiven Zusammenhang zwischen ihnen geschlossen werden: Wird der Wert der Variable $x$ tendenziell größer, so wird auch der Wert der Variablen $y$ tendenziell größer und umgekehrt. 
- Ist $\left.r_{x y} \in\right]-1 ; 0[$, so sind die Variablen negativ korreliert, d. h. die Ausprägungen der Variablen laufen entgegengesetzt: Wird der Wert der Variable $x$ größer, so wird der Wert von $y$ tendenziell kleiner und umgekehrt.

Zur besseren Beurteilung eines Korrelations-Koeffizienten kann das Bestimmtheitsmaß betrachtet werden, der sich als Quadrat des Koeffizienten berechnet. Es gibt an, welcher Anteil der Varianz der Variable $y$ durch die Variable $x$ erklärt wird. ${ }^{23}$ Eine Korrelation von $r_{x y}=0.6$ erklärt also gerade einen Varianz-Anteil von $36 \%$. Erst ab Korrelations-Koeffizienten von $r_{x y}>0.7$ wird mehr als die Hälfte der Varianz durch die Variable erklärt.

Korrelations-Koeffizienten haben jedoch nur eine Aussagekraft bei einem linearen Zusammenhang zwischen zwei Variablen. Ist der Zusammenhang höherer Ordnung, so hat der Wert keine Aussagekraft. Es sollte also neben den rechnerischen Ergebnissen immer auch die zugehörige Punktwolke im Korrelations-Diagramm betrachtet werden. ${ }^{24}$ Dabei werden an den Achsen die Ausprägungen der Variablen gegeneinander aufgetragen. Nachfolgend werden die paarweisen Beobachtungen der beiden Variablen in das Diagramm eingetragen. Lässt sich durch die Punktwolke eine Gerade legen, ist also ein linearer Verlauf erkennbar, so kann die Korrelation als gesichert angesehen werden. Andernfalls sind andere Auswertungsmethoden heranzuziehen.

Eine weitere Möglichkeit der Beurteilung von Korrelations-Koeffizienten ist ein zweiseitiger t-Test auf Unabhängigkeit der betrachteten zwei Variablen. Hierbei wird die ermittelte Korrelation mit einem kritischen Wert verglichen, der sich nach folgender Formel berechnet: ${ }^{25}$

$$
Q:=\frac{|t|}{\sqrt{\left(N-2-t^{2}\right)}} .
$$

Dabei ist $t$ der Wert der zweiseitigen t-Verteilung zum Signifikanz-Niveau $\alpha$ bei einem Stichproben-Umfang $N$. Der ermittelte Korrelations-Koeffizient muss größer als das entsprechende $Q$, um als signifikant zu gelten. Für ein $N$ von 32, wie es beispielsweise bei der Korrelationsanalyse des Fragebogens zum situativen Erleben vorliegt, und einem $\alpha$ von $5 \%$ ergibt sich ein $Q$-Wert von 0.2959 , d.h.

\footnotetext{
23 Vgl. Hartung \& Elpelt (Statistik 1984), S. 145.

24 Vgl. Lienert \& Eye (Statistik 1994), S. 95-98.

25 Vgl. Hays (Statistic 1973), S.661; auch Hartung, Elpelt \& Kösener (Statistik 1993), S. 153 .
} 
Korrelations-Koeffizienten von 0.30 und größer sind zu dem Niveau bereits signifikant. Ihr Aussagewert ist jedoch nicht sehr hoch.

\subsection{Faktorenanalyse}

Die Faktorenanalyse ist eine multivariate Analysemethode, die besonders sinnvoll ist, um eine Vielzahl von Variablen zu einer bestimmten Fragestellung auf einige zentrale Faktoren zu verdichten. Das heißt, die Anzahl der Faktoren $i$ kleiner als die Anzahl der Variablen $k$. Die Faktorenanalyse geht davon aus, dass eine Beschreibung der beoachteten Variablen durch nicht-beobachtbare Faktoren möglich ist. ${ }^{26}$ An diese Faktoren wird die Forderung der Orthogonalität gestellt, d. h. sie sind stochastisch voneinander unabhängig und die Faktoren $Y_{i}$ lassen sich als Linearkombinationen verschiedener Variablen $X_{j}$ zusammenfassen. ${ }^{27}$

$$
\text { Linearkombination: } \quad Y_{i}=c_{1} X_{1}+c_{2} X_{2}+\cdots+c_{k} X_{k}
$$

Ausgangspunkt dieser Methode ist die Korrelationsmatrix der Beobachtungswerte. ${ }^{28}$ Aufgrund der Korrelationen werden die Faktoren extrahiert: Hierbei wird versucht, ein neues Koordinaten-System für die Beobachtungswerte zu finden, wobei mehrere Variablen zu einem Faktor zusammengefasst werden. Die Varianz des ersten Faktors wird dabei maximiert. Nachfolgend wird ein zweite Faktor orthogonal zum ersten gesucht, so dass auch dieser maximale Varianz hat. Die Anzahl der Faktoren wird mehr oder weniger willkürlich durch die oder den Analysierende/n festgelegt, wobei sich im Allgemeinen an den Eigenwerten der Faktoren orientiert wird. Als Ausschluss-Kriterium können die Eigenwerte der Faktoren herangezogen werden. Nach dem häufig verwendeten 'Kaiser-Kriterium' sollen die Faktoren einen Eigenwert größer gleich 1 haben. ${ }^{29}$ Anhand der Kommunalitäten der Faktoren, die ebenfalls berechnet werden, lässt sich die erklärte Varianz ablesen. ${ }^{30}$ Die

26 Zur Auswertung wurde das Programm $4 M$ aus dem Paket BMDP herangezogen; vgl. DiXON (Software 1985), S. 479-499.

27 Vgl. im Folgenden Hartung \& Elpelt (Statistik 1984), S.505-591; Backhaus et al. (Analysemethoden 1996), S. 208-249.

28 Vgl. Backhaus et al. (Analysemethoden 1996), S. 199.

29 Vgl. Backhaus et al. (Analysemethoden 1996), S. 226-227.

30 Vgl. Backhaus et al. (Analysemethoden 1996), S. 210-211. 
Faktorladungen, die die Koeffizienten $c_{1}, \ldots, c_{n}$ der Linearkombination $Y$ darstellen, werden in einer Matrix aufgetragen und müssen nachfolgend durch die oder den Forschenden inhaltlich interpretiert werden. 


\section{Ergebnisse der Planspiel-Phase}

\subsection{Vorbemerkungen}

Inhalt dieses Kapitels ist die Darstellung der Ergebnisse der Planspiel-Phase. Zunächst wird in Abschnitt 8.2 auf der Basis der Ausgangslage der Testphase eine optimale Entscheidung entwickelt. Sie dient zur nachfolgenden Beurteilung der Leistungen. In der Testphase wurde das Unternehmensplanspiel als Erhebungsinstrument eingesetzt, um die Fähigkeit der Teilnehmenden zu erheben, in einer komplexen Anforderung Entscheidungen treffen zu können. ${ }^{1}$ Weiterhin werden in Abschnitt 8.3 die Ergebnisse getrennt nach den Bedingungen in der experimentellen Phase und hinsichtlich eines Einflusses der Komplexität der Anforderungssituation auf den Lernerfolg betrachtet. ${ }^{2}$

In der gesamten Planspiel-Phase wurden zwei verschiedene Fragebögen zur Erhöhung der Reflexivität gekreuzt eingesetzt, ${ }^{3} \mathrm{~d}$. h. es gab Gruppen beider experimenteller Bedingungen, in denen kein Fragebogen oder nur einer der Fragebögen eingesetzt wurde, sowie Gruppen, die beide Fragebögen zur Bearbeitung vorgelegt bekamen. Ihr Einfluss auf den Lernerfolg wird in Abschnitt 8.4 näher betrachtet.

\footnotetext{
1 Zu den Lernzielen der Testphase vgl. Abschnitt 6.4.3.

2 Zu den experimentellen Bedingungen vgl. Abschnitt 6.3.

3 Zum Versuchsplan vgl. Abschnitt 4.9.
} 


\subsection{Entwicklung einer optimalen Entscheidung in der Testphase}

\subsubsection{Unternehmensplanung}

In einem Unternehmensplanspiel ist es kaum möglich, die optimale Entscheidung schlechthin zu ermitteln, da die Teilziele zum Teil konträr laufen und isoliert betrachtet durchaus zu unterschiedlichen Entscheidungen führen können. ${ }^{4} \mathrm{Im}$ Folgenden soll auf Grund der Ausgangslage der Testphase ${ }^{5}$ für das von den Teilnehmenden zu führende Unternehmen ${ }^{6}$ eine Unternehmensplanung durchgeführt werden $^{7}$ und eine zieladäquate Entscheidung getroffen werden.

Als Unternehmensziel wurde den Teilnehmenden die Maximierung des kumulierten Gewinns zum Ende der Testphase vorgegeben. Diese Kennzahl wird über die Unternehmens-Ergebnisse in den Planspiel-Unterlagen zurückgemeldet. ${ }^{8}$

Grundlage der folgenden Überlegungen ist die konjunkturelle Lage: Die 8. Periode, in der sich das Modellunternehmen zu Beginn der Testphase befindet, ist durch einen Rückgang der Nachfrage für beide Produkte auf beiden Märkten gekennzeichnet. Oberstes Ziel ist die Gewinn-Maximierung, das den Teilnehmenden im Planspiel vorgegeben wurde. Um dieses Ziel zu erreichen, müssen in den Funktionsbereichen einer Unternehmung jeweils verschiedene Ziele verfolgt werden, die in einem Ziel-System dargestellt werden können. Innerhalb der Funktionsbereiche werden die Ziele weiter aufgeschlüsselt, bis aus ihnen konkrete Handlungen abgeleitet werden können. Dabei treten u. U. Zielkonflikte zwischen verschiedenen Teilzielen auf. ${ }^{9}$

4 Zur Ermittlung einer optimalen Lösung in einem Unternehmensplanspiel mit Hilfe verschiedener Verfahren des Operations Research vgl. Petzing (Untersuchung 1993), S.87-96.

5 Vgl. Abschnitt 6.4.2.

6 Dabei sind die fünf Konkurrenz-Unternehmen durchnummeriert. Das von den Teilnehmenden geführte Unternehmen U1 wird im Folgenden als 'Modellunternehmen' bezeichnet.

7 Dabei wird sich an den theoretischen Überlegungen aus Abschnitt 3.3 orientiert. Vgl. auch Just (Beurteilung 1998), S. 54-71.

8 Die Planspiel-Unterlagen befinden sich im Anhang A.20.

9 Vgl. CarL \& Kiesel (Unternehmensführung 1996), S. 28-30. 
Grundsätzlich sind im Planspiel EpUS folgende Teilziele zur Maximierung des Gewinns zu verfolgen: ${ }^{10} \mathrm{Im}$ Bereich der Produktion sollte die vorhandene MaschinenKapazität voll ausgelastet werden, im Bereich der Beschaffung sind die Rohstoffmengen so zu planen, dass der Lagerbestand der 9. Periode genau zur Produktion ausreicht. Die Marketing-Instrumente sind so zu setzen, dass bei Produkt 1 die Lagerbestände auf Grund der rückläufigen Nachfrage abgebaut werden, bei Produkt 2 keine weiteren Lagerbestände aufgebaut werden. Schließlich ist im Bereich der Finanzierung ein positiver Kassenbestand zu planen. Tabelle 8.1 stellt die Subziele dar und geht auf die Mittel zu ihrer Erreichung ein.

\begin{tabular}{|c|c|c|c|}
\hline \multicolumn{4}{|c|}{ Gewinn-Maximierung } \\
\hline $\begin{array}{c}\text { Produktion } \\
\text { Kapazitätsauslastung } \\
=100 \%\end{array}$ & $\begin{array}{c}\text { Rohstoff-Lager: } \\
\text { Diff. }^{11}=0\end{array}$ & $\begin{array}{c}\text { Produkt-Lager: } \\
\text { Bestände abbauen }\end{array}$ & Kasse $\geq 0$ \\
\hline $\begin{array}{c}\rightarrow \text { Produktions- } \\
\text { mengen-Planung }\end{array}$ & $\begin{array}{c}\text { Rohstoff- } \\
\text { einkauf }\end{array}$ & $\begin{array}{c}\rightarrow \text { Preise, Werbung, } \\
\text { Kundendienst }\end{array}$ & $\begin{array}{c}\rightarrow \text { Kredit- } \\
\text { aufnahme }\end{array}$ \\
\hline
\end{tabular}

Tabelle 8.1: Subziele und Mittel zur Gewinn-Maximierung im Planspiel EpUS

\subsubsection{Absatzplanung}

Für die Absatzplanung in der Ausgangslage der Testphase bietet es sich an, zunächst die Daten des Konkurrenz-Unternehmens U3 zum Vergleich heranzuziehen. ${ }^{12}$ Die Absatzmengen liegen hier etwa in gleicher Höhe wie beim Modellunternehmen. U3 hat bei etwas höheren Marktpreisen und niedrigeren Aufwendungen für Werbung und Kundendienst einen leicht niedrigeren Umsatz als das Modellunternehmen. Demgegenüber steht ein höherer Jahresüberschuss. Ein Vergleich der gesamten Ausgaben für Werbung und Kundendienst in Relation zum Umsatz zeigt, dass offensichtlich die höheren Kundendienst-Aufwendungen einen stärkeren Einfluss auf die Absatzmengen haben und das Unternehmen damit bei leicht höheren Produktpreisen einen höheren Umsatz erzielt. So kann es für das

\footnotetext{
10 Vgl. Abschnitt 6.2.

11 Diff. = Lagerbestand $(\mathrm{t}=8)+$ Lagerzugang $(\mathrm{t}=8)-\operatorname{Lagerabgang}(\mathrm{t}=9)$.

12 Die Verkaufspreise, die gesamten Aufwendungen für Werbung und Kundendienst, die Absatzmengen sowie der Jahresüberschuss der fünf Unternehmen liegt den Teilnehmenden in Form einer Marktforschung vor; vgl. Anhang A.20.
} 
Modellunternehmen sinnvoll sein, einen Teil der Ausgaben für Werbung in den Kundendienst zu investieren. Differenziert nach beiden Produkten ergibt sich folgende Strategie:

Produkt 1: Da sich dieses Produkt bereits in der Degenerationsphase befindet, sollten die vorhandenen Lagerbestände abgebaut werden. Die Preise sind nahezu stabil zu halten, um noch einen Umsatz mit dem Verkauf zu erzielen. Es sollte jedoch wesentlich weniger in Werbung und Kundendienst investiert werden. Die Produktionsmengen sind entsprechend zu reduzieren. Die frei werdenden Geldmittel können in die Marketing-Instrumente für Produkt 2 investiert werden.

Produkt 2: In der letzten Periode trat bei diesem Produkt ein Nachfrage-Überhang auf, d.h. die Nachfrage konnte durch den vorhandenen Lagerbestand nicht gedeckt werden. Für die Folgeperiode wird daher vorgeschlagen, die Preise leicht zu erhöhen, um den Umsatz zu erhöhen. Allerdings sollten trotzdem die Aufwendungen für Werbung leicht und für Kundendienst stärker erhöht werden, um den Absatz zu fördern.

Die entsprechenden Zahlen für die Gestaltung der Marketing-Instrumente für die 9. Periode können nach diesen Überlegungen wie folgt lauten:

\begin{tabular}{|l|c|c|c|c|}
\hline Produkt Markt & P1 M1 & P1 M2 & P2 M1 & P2 M2 \\
\hline Verkaufspreis & $520,-$ & $510,-$ & $700,-$ & $700,-$ \\
\hline Werbung & 10.000 & 10.000 & 100.000 & 100.000 \\
\hline Kundendienst & 10.000 & 10.000 & 120.000 & 120.000 \\
\hline
\end{tabular}

Tabelle 8.2: Optimale Entscheidung: Marketing-Instrumente

Für die folgenden Perioden werden aufgrund der gesetzten Marketing-Instrumente die Absatzmengen prognostiziert. In Abbildung 8.1 sind die Bereiche der Prognosen dargestellt. Die obere Linie zeigt eine eher optimistische Erwartung, die untere Linie eine eher pessimistische Erwartung bezüglich der Entwicklung der Nachfrage. Auf dieser Basis wird im Folgenden die Planung der Produktions- und Rohstoffmengen vorgenommen. 

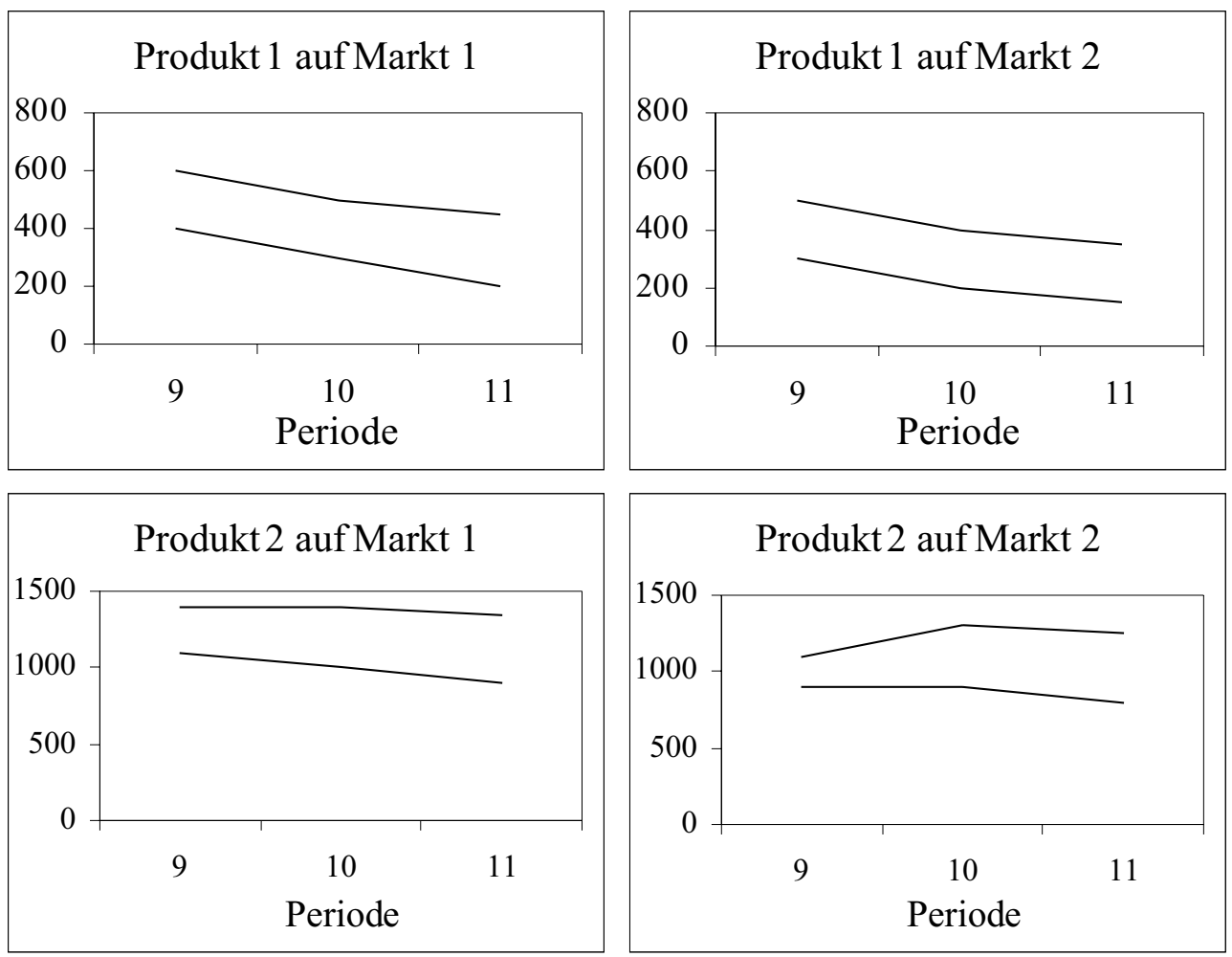

Abbildung 8.1: Optimale Entscheidung: Absatzprognosen

obere Linie = optimistische Erwartung

untere Linie = pessimistische Erwartung

\subsubsection{Produktions- und Beschaffungsplanung}

Im Modellunternehmen stehen für die 9. Periode 32 Maschinen und entsprechend 64 Arbeitnehmer in der Produktion zur Verfügung. In der letzten Periode wurden die Maschinen voll ausgelastet und 2125 Stück von Produkt 1 sowie 2140 Stück von Produkt 2 hergestellt. Die Absatzmenge bei Produkt 1 lag nur bei 1727, d. h. es wurde hier mehr produziert als verkauft, während bei Produkt 2 alle Produkte abgesetzt werden konnten. Im Lager sind nun 2498 Stück von Produkt 1 und 2140 Stück von Produkt2, die verkauft werden können.

Aufgrund einer im Planspiel-Modell abgebildeten 'Rationalisierungs-Maßnahme' zum Ende der 8. Periode sinken die zur Produktion notwendigen Leistungseinheiten je Stück bei beiden Produkten. Dadurch kann in der 9. Periode bei gleichem Maschinenbestand eine größere Menge produziert werden. Diesem erhöhten An- 
gebot auf dem Markt würde jedoch keine ausreichende Nachfrage gegenüberstehen. Mittelfristig sollte die Kapazität entsprechend verringert werden. Nach den Absatzprognosen werden Absatzmengen bei Produkt 1 zwischen 700 und 1.100 Stück erwartet, bei Produkt 2 wird der Absatz bei einer Menge zwischen 2.000 und 2.500 Stück stagnieren. ${ }^{13}$ Bei einer eher optimistischen Erwartung wird eine Kapazität von 22 Maschinen benötigt, so dass 10 Maschinen verkauft und entsprechendes Personal entlassen werden sollte. Der Absatz wird durch die Gestaltung der Marketing-Instrumente gesichert werden.

Für die aktuelle Periode, in der die 32 Maschinen und das entsprechende Personal noch zur Verfügung stehen, ist in der Produktionsplanung eine Entscheidung zwischen einer Unterauslastung der Maschinen mit einer Produktionsmenge, die sich an der prognostizierten Absatzmenge orientiert, und voller Auslastung und damit einer Produktion über die Nachfrage hinaus zu treffen. Zur Verdeutlichung der Auswirkung dieser Entscheidung auf das Unternehmensergebnis werden im Folgenden zwei Fälle beispielhaft dargestellt:

Unterauslastung: Die Produktionsmengen werden im ersten Fall mit 1.000 Stück bei Produkt 1 und 2.500 Stück bei Produkt 2 festgesetzt. Es ergäbe sich eine Maschinenauslastung von rund $70 \%$.

Volle Auslastung: Im zweiten Fall werden Produktionsmengen von 1.000 bzw. 3.925 Stück geplant, wodurch die Maschinen zu 100\% ausgelastet würden.

Dazu werden die variablen Aufwendungen der Gewinn- und Verlustrechnung des Modellunternehmens, also die von der Produktionsmenge abhängigen Aufwendungen, näher betrachtet (Abbildung 8.3). Sie ist im Planspiel EpUS im Gesamtkostenverfahren dargestellt. ${ }^{14}$

Der Verbrauch an Rohstoffen orientiert sich an der Stückliste, allerdings ist in der 9. Periode bei voller Auslastung der Maschinen eine Notbeschaffung auf Grund falscher Disposition der Vorperiode erforderlich. Der Verbrauch an Hilfsstoffen ist abhängig von den zur Produktion benötigten Leistungseinheiten der Maschinen.

\footnotetext{
13 Vgl. Abbildung 8.1.

14 Zur Kritik am traditionellen Rechnungswesen und zu einem wirtschaftsinstrumentellen Ansatz mit entsprechenden Auswirkungen auf die Darstellung der Gewinn- und Verlustrechnung vgl. Preiss (Didaktik 1999).
} 


\begin{tabular}{|l|r|r|}
\hline Aufwendungen & Unterauslastung & volle Auslastung \\
\hline Rohstoffe & 504.680 & 332.000 \\
Hilfsstoffe & 96.000 & 67.500 \\
Instandhaltung & 217.600 & 200.500 \\
Lageraufwand & 92.853 & 85.611 \\
Bestandsveränderungen & -479.580 & -64.203 \\
\hline Summe & 431.553 & 621.408 \\
\hline
\end{tabular}

Tabelle 8.3: Auswirkungen unterschiedlicher Kapazitätsauslastungen im Planspiel EpUS

Die Aufwendungen für die Instandhaltung der Maschinen sind überwiegend fix, da sie proportional zum Maschinenbestand anfallen. Ein Teil des Aufwands hängt jedoch auch von den verbrauchten Leistungseinheiten ab.

Die Berechnung des Lageraufwands orientiert sich an den durchschnittlichen Lagerbeständen der Produkte und Rohstoffe. Er ist durch eine Minimierung der Bestände zu reduzieren, aber auf Grund der Zeitverzögerung bei Beschaffung, Produktion und Absatz nicht zu vermeiden. Da die geplanten Produktionsmengen frühstens in der nächsten Periode verkauft werden können, beeinflusst ihre Planung den Lageraufwand. Ebenso gehen die eingekauften Rohstoffe zunächst auf Lager, bevor sie in der Produktion eingesetzt werden. Entsprechend unterscheiden sich die Lageraufwendungen in beiden Fällen. Sie sind für beide Produkte und Rohstoffe zusammen angegeben.

Die Bestandsveränderungen der Produkte zeigen in beiden Fällen mit einem positiven Wert eine größere Produktions- als Absatzmenge an. Der Unterschied in den Produktionsmengen von 1425 Stück von Produkt 2 zeigt sich in entsprechend höheren Bestandsveränderungen im Fall einer vollen Kapazitätsauslastung.

\section{Fazit der Überlegungen}

Durch die volle Auslastung der Kapazität sind zwar die Aufwendungen für Rohund Hilfsstoffe sowie für Instandhaltung und Lagerhaltung höher als bei einer geringeren Auslastung. Ihnen stehen jedoch erhöhte Bestandsveränderungen durch die Produktion auf Lager gegenüber. Auf Grund dieser Überlegungen sollten die Maschinen in der 9. Periode voll ausgelastet werden. Entsprechend sind die Pro- 
duktionsmengen zu planen: Bei Produkt 1 sollte die Menge bei 1.000 Stück liegen, damit hier die Bestandsveränderungen negativ werden, d.h. weniger produziert als abgesetzt wird. Bei voller Kapazitätsauslastung ergibt sich für Produkt 2 eine Produktionsmenge von 3.925 Stück. In der folgenden Periode sollten mit der reduzierten Kapazität von 22 Maschinen entsprechend der Absatzprognosen ${ }^{15}$ bei Produkt 1 noch 800 Stück und bei Produkt 2 entsprechend 2.600 Stück zur Auslastung der Maschinen hergestellt werden. Die zu beschaffenden Rohstoffmengen ergeben sich entsprechend auf der Basis der Stückliste sowie der Lagerbestände.

\subsubsection{Kostenrechnung}

Zur Unterstützung der Planung im Beschaffungs-, Produktions- und Absatzbereich kann eine Vorkalkulation der Selbstkosten für die nächste Periode durchgeführt werden. ${ }^{16}$ Sie orientiert sich an den Kostenarten, die zunächst identifiziert werden müssen. In den Planspiel-Unterlagen befindet sich bereits die Darstellung der Nachkalkulation der Herstellungskosten, mit denen die Produkt-Bestände bewertet werden. Die Materialkosten berechnen sich aus dem Verbrauch an Rohstoffen, die Fertigungskosten beinhalten die Instandhaltungskosten der Maschinen, die Löhne sowie den Verbrauch an Hilfsstoffen. Als Gemeinkosten kommen die Lagerkosten der Produkte hinzu. ${ }^{17}$

Die Summe aus Fertigungs-, Material- und Gemeinkosten ergibt die Herstellkosten der Produkte. Zur Ermittlung der Herstellungskosten wird ein Teil der Verwaltungs-Gemeinkosten - bestehend aus eventuell angefallenen Einstellungsbzw. Entlassungsaufwendungen, den Abschlusskosten der Bilanz sowie den fixen und variablen Verwaltungskosten - proportional auf der Basis der Herstellkosten verrechnet. $^{18}$

\footnotetext{
$15 \quad$ Vgl. Abbildung 8.1.

16 Vgl. Bloech (Kostenmanagement 1997); zur Zuschlagskalkulation vgl. Freidank (Kostenrechnung 1994), S. 160.

17 In die Material-Gemeinkosten müssten eigentlich auch die Lagerkosten der Rohstoffe eingerechnet werden (vgl. Freidank (Kostenrechnung 1994), S. 96). Da dies im Planspiel-Modell jedoch nicht vorgenommen wird, soll auch an dieser Stelle so vorgegangen werden, um die Vergleichbarkeit der Berechnungen mit den Simulations-Ergebnissen zu gewährleisten.

18 Vgl. Freidank (Kostenrechnung 1994), S.169-172; auch Wöhe (Einführung 1996), S. 1077-1078.
} 
Die so ermittelten Herstellungskosten sind die der Erzeugung, beziehen sich also auf die Produktionsmengen. Da bei Produkt 2 jedoch wesentlich mehr produziert wurde, als voraussichtlich abgesetzt wird, muss hier eine Korrektur vorgenommen werden. Die Differenz zwischen der Produktion von 3.925 Stück und dem Absatz von ca. 2.300 Stück stellt die Bestandsveränderungen dar, die mit den ermittelten Herstellungskosten bewertet und von den Herstellungskosten der Erzeugung abgezogen werden. Man erhält die Herstellungskosten des Umsatzes. ${ }^{19}$

Zur Berechnung der Selbstkosten müssen die übrigen Gemeinkosten anteilig verteilt werden. Dazu gehören die Kosten der Lagerhaltung der Rohstoffe, die Aufwendungen für Werbung und Kundendienst sowie die Informationsbeschaffung. Hier müssen auch die anfallenden Zinsen für die laufenden Kredite eingerechnet werden. Weiterhin sollten die kalkulatorische Zinsen auf das betriebsnotwendige Kapital einbezogen. Es besteht im Allgemeinen aus dem betriebsnotwendigen Anlage- und Umlaufvermögen, das um das 'Abzugskapital' vermindert wird. ${ }^{20}$

Im Planspiel EpUS besteht das betriebsnotwendige Kapital aus dem Anlagevermögen in Form der zur Fertigung benötigten Maschinen. Sie können mit den halben Anschaffungskosten bewertet werden. ${ }^{21}$ Zum betriebsnotwendigen Umlaufvermögen zählen im Planspiel die durchschnittlichen Bestände an Rohstoffen sowie an Produkten. Letztere können maximal zu Herstellungskosten bewertet werden. Anlage- und Umlaufvermögen zusammen ergeben das betriebsnotwendige Vermögen.

Das Abzugskapital besteht im vorliegenden Fall aus den Verbindlichkeiten aus Lieferung und Leistung, also den Lieferanten-Krediten, die dem Unternehmen zinslos zur Verfügung gestellt werden. Das so ermittelte betriebsnotwendige Kapital wird mit $8 \%$ verzinst. Der Zinssatz entspricht den Fremdkapital-Zinsen der aktuellen Periode.

Im Planspiel-Modell sind Abschreibungen nicht direkt abgebildet. Die explizite Berücksichtigung von kalkulatorischen Abschreibungen ist hier nicht notwendig, da die fixen Instandhaltungskosten je Maschine sowie die Wartungskosten je an-

19 Vgl. Schmolke \& Deitermann (Rechnungswesen 1999), S. 351.

20 Vgl. WöHE (Einführung 1996), S. 1264-1267 sowie Freidank (Kostenrechnung 1994), S. 125-129.

21 Dies entspricht einer linearen Amortisation, die der Einfachheit halber gewählt wird, aber nicht der Realität entspricht; vgl. PERridon \& STEIner (Finanzwirtschaft 1995), S. 38-39. 
gefallener Leistungseinheit der Werterhaltung der Maschinen dienen und in den Fertigungskosten enthalten sind. ${ }^{22}$

Den so ermittelten Selbstkosten je Produktart stehen die Verkaufspreise von 520,(Markt 1) und 510,- (Markt 2) bei Produkt 1 bzw. 700,- bei Produkt 2 (beide Märkte) gegenüber. Die Stück-Deckungsbeiträge je Produktart ergeben sich aus der Differenz zwischen den Preisen und den Herstellungskosten und belaufen sich auf 25,65 bzw. 15,65 bei Produkt 1 und auf 179,32 bei Produkt 2, sind also insgesamt in dieser Situation bei Produkt 1 nicht sehr hoch. Insgesamt ergibt sich bei Produkt 1 ein geringfügig positives Betriebsergebnis, bei Produkt 2 liegt es wesentlich höher. Tabelle 8.4 zeigt die Berechnung für beide Produkte gesamt und pro Stück.

\begin{tabular}{|c|c|c|c|c|c|}
\hline \multirow{2}{*}{\multicolumn{2}{|c|}{ Kostenart }} & \multicolumn{2}{|c|}{ Produkt 1} & \multicolumn{2}{|c|}{ Produkt 2} \\
\hline & & gesamt & je St. & gesamt & je St. \\
\hline & Materialkosten & $107.906,-$ & 107,91 & $396.773,-$ & 101,09 \\
\hline+ & Fertigungskosten & $102.083,-$ & 102,08 & $457.917,-$ & 116,67 \\
\hline+ & Gemeinkosten & $6.000,-$ & 6,00 & $23.550,-$ & 6,00 \\
\hline$=$ & Herstellkosten & 215.989,-- & 215,99 & $878.240,-$ & 223,76 \\
\hline+ & Verwaltungs-Gemeinkosten & 41.691,- & 41,69 & $187.012,-$ & 47,65 \\
\hline$=$ & Herstellungskosten d. E. & $257.680,-$ & 257,68 & 1.065.252,- & 271,41 \\
\hline - & Bestandsveränderungen & $0,-$ & & $-441.028,-$ & 271,41 \\
\hline$=$ & Herstellungskosten d. U. & $257.680,-$ & 257,68 & $624.224,-$ & 271,41 \\
\hline+ & übrige Gemeinkosten & $255.166,-$ & 255,17 & $618.136,-$ & 268,75 \\
\hline$=$ & Selbstkosten d. U. & $512.847,-$ & 512,85 & 1.242.360,- & 540,16 \\
\hline & Umsatzerlöse Markt 1 & $286.000,-$ & 520,00 & $910.000,-$ & 700,00 \\
\hline & Umsatzerlöse Markt 2 & $229.500,-$ & 510,00 & $700.000,-$ & 700,00 \\
\hline & Betriebsergebnis & 2.6 & & $36 \%$ & \\
\hline
\end{tabular}

Tabelle 8.4: Optimale Entscheidung: Vorkalkulation der Selbstkosten und Berechnung des Betriebsergebnisses

d. E. = der Erzeugung, d. U. = des Umsatzes

22 Vgl. Bloech \& Rüscher (Modellbeschreibung 1995), S. 78. 


\subsubsection{Finanzplanung}

Durch die Finanzplanung sind Zinsaufwendungen für einen kurzfristigen Kredit zu vermeiden und etwaige Einzahlungs-Überschüsse zur Tilgung langfristiger Kredite zu nutzen. Für die 9. Periode wird eine Liquiditätsplanung durchgeführt: ${ }^{23}$ Dabei werden die Ein- und Auszahlungen der 9. Periode gegenübergestellt. Mit dem Anfangsbestand an Zahlungsmitteln, der im Planspiel dem Kassenbestand entspricht, wird der Endbestand an Zahlungsmitteln bzw. bei rechnerisch negativem Endbestand der Bedarf zusätzlicher Zahlungsmittel berechnet. Tabelle 8.5 zeigt die Zahlen für die 9. Periode im Überblick. ${ }^{24}$

Dabei sind nicht alle Daten sicher zu ermitteln. So hängen die Umsatzerlöse und der Lageraufwand für Produkte von den tatsächlichen Absatzmengen ab. Die Auszahlungen für Steuern richten sich nach dem erzielten Gewinn in der Periode. Hier können nur geschätzte Zahlen zur Finanzplanung herangezogen werden, die in der Tabelle durch ein Sternchen $(*)$ markiert sind.

Der Gewinn wird mit Hilfe einer Vorkalkulation geschätzt, wobei die kalkulatorischen Kosten herausgerechnet werden. Grundsätzlich unsicher sind auch alle Parameter wie die Einkaufspreise der Rohstoffe oder die Löhne. Ihre Entwicklung wird den Teilnehmenden zwar in den Newslettern in jeder Periode mitgeteilt, die tatsächliche Höhe steht aber erst nach Abschluss der Periode fest.

Für die 9. Periode sind keine Steigerungen der Preise oder Kosten zu erwarten, so dass mit den Zahlen der 8. Periode gerechnet wird. Es ergibt sich ein Kapitalbedarf, der durch die Aufnahme eines langfristigen Kredits in Höhe von 200.000 ausgeglichen werden soll.

Die in diesem Abschnitt entwickelte Entscheidung wird im Folgenden zur Bewertung der Leistungen der Teilnehmenden herangezogen.

23 Vgl. Perridon \& Steiner (Finanzwirtschaft 1995), S.631-636; auch Olfert (Finanzierung 1994), S. 102-126.

24 Die Erläuterung zur Berechnung der einzelnen Posten befindet sich im Anhang A.21. 


\section{Auszahlungen}

\begin{tabular}{|l|l|r|}
\hline Auszahlungsart & Berechnungs-Grundlage & Betrag \\
\hline \multirow{2}{*}{ Rohstoffe } & Beschaffung & $413.630,00$ \\
& Notbeschaffung & $91.050,00$ \\
\hline Hilfsstoffe & $2,50 \times 26.400$ Leistungseinheiten (LE) & $66.000,00$ \\
\hline Löhne & $2.750,-\times 64$ Angestellte & $176.000,00$ \\
\hline Sozialaufwand & $40 \%$ der Löhne & $70.400,00$ \\
\hline \multirow{2}{*}{ Zinsaufwand } & $8 \%$ vom Kredit & $148.569,20$ \\
& $12 \%$ vom Kontokorrentkredit & $5.577,29$ \\
\hline \multirow{2}{*}{ Instandhaltung } & $5.000,-\times 32$ Maschinen & $160.000,00$ \\
& $11,50 \times 38.400$ LE & $57.600,00$ \\
\hline \multirow{2}{*}{ Verwaltung } & fixe Kosten & $100.000,00$ \\
& $175,-\times 64$ Angestellte & $11.200,00$ \\
& $0,5 \%$ der Bilanzsumme in t=8 & $21.001,82$ \\
\hline \multirow{2}{*}{ Lageraufwand } & Lixe Kosten & $25.000,00$ \\
& Lager Produkte* & $34.492,50$ \\
& $50 \%$ vom Gewinn* & $33.420,00$ \\
\hline Steuern & & $80.000,00$ \\
\hline Werbung & für Marktforschung & $220.000,00$ \\
\hline Kundendienst & Bilanzgewinn in t=8 & $260.000,00$ \\
\hline Information & Rückzahlung des Kredits & $10.000,00$ \\
\hline Ausschüttung & $60.361,85$ \\
\hline Kontokorrent & $46.477,40$ \\
\hline
\end{tabular}

Einzahlungen

\begin{tabular}{|l|l|r|}
\hline Einzahlungsart & Berechnungs-Grundlage & Betrag \\
\hline Umsatzerlöse & $60 \%$ des Umsatzes* & $1.056 .600,00$ \\
\hline Forderungen & aus $\mathrm{t}=8$ & $842.420,00$ \\
\hline
\end{tabular}

\section{Ermittlung des Kassenbestands}

\begin{tabular}{|l|r|}
\hline \multicolumn{1}{|c|}{ Summe Einzahlungen } & $1.899 .020,00$ \\
\hline- Summe Auszahlungen & $-2.090 .780,06$ \\
\hline \hline$=$ Kapitalbedarf & $-191.760,06$ \\
\hline$\rightarrow$ Kreditaufnahme & $200.000,00$ \\
\hline
\end{tabular}

Tabelle 8.5: Optimale Entscheidung: Liquiditätsplanung 


\subsection{Vergleich der experimentellen Bedingungen}

\subsubsection{Produktions- und Beschaffungsplanung}

In diesem Abschnitt werden die Leistungen der Teilnehmenden in der Testphase betrachtet, wobei hinsichtlich der experimentellen Bedingungen differenziert wird. Es wurden die Ergebnisse von 10 Gruppen der herkömmlichen und 9 Gruppen der modularen Bedingung zur varianzanalytischen Auswertung herangezogen. ${ }^{25}$ Bei der Betrachtung der Ergebnisse muss beachtet werden, dass die Teilnehmenden ihre zweite Entscheidung in der Testphase für zwei Perioden in Folge trafen, also nicht auf Grund der rückgemeldeten Simulations-Ergebnisse, sondern auf Grund der erwarteten Ergebnisse.

Die Komplexität der Entscheidung wurde in der modularen Bedingung stufenweise erhöht, wobei sich die Erhöhung an den individuellen Leistungen der Gruppen orientierte. ${ }^{26}$ Vier Gruppen erreichten bereits in der 4. Entscheidung die höchste Komplexitäts-Stufe, während vier Gruppen die letzte Entscheidung nur auf der zweithöchsten Stufe trafen. ${ }^{27}$ Dies wird bei der Betrachtung der Ergebnisse ebenfalls zur Erklärung von Unterschieden herangezogen.

In der Ausgangslage werden die vorhandenen Maschinen voll ausgelastet. Um die Maschinen in der 9. Periode ebenfalls voll auszulasten, ist eine Notbeschaffung an Rohstoffen zu höheren Einkaufspreisen erforderlich, da der Lager-Anfangsbestand zur Produktion an der Kapazitätsgrenze nicht ausreicht. ${ }^{28}$ Eine hohe Kapazitätsauslastung ist jedoch anzustreben, um die Wartungskosten der Maschinen und die Löhne der Arbeitnehmenden durch den Aufbau eines Lagerbestands an verkaufsfähigen Produkten zu kompensieren. ${ }^{29}$ Die Abbildungen 8.2 und 8.3 zeigen die Ergebnisse bei der Differenz zwischen dem Lager-Anfangsbestand und dem Lager-Abgang in die Produktion für beide Rohstoffe sowie bezüglich des Auslastungsgrads der Maschinen. ${ }^{30}$

\footnotetext{
25 Die Ergebnisse der Varianzanalyse befinden sich in Anhang A.25.

26 Vgl. Abschnitt 6.3.2.

27 Vgl. Anhang A.4.

28 Vgl. Abschnitt 6.4.1.

29 Vgl. Abschnitt 8.2.

30 Jedes Zeichen markiert ein Ergebnis einer Gruppe, die Linien sollen die Entwicklungen verdeutlichen. Die Zeichen sind in den folgenden Abbildungen für die einzelnen Gruppen gleich, so dass ein direkter Vergleich möglich ist.
} 


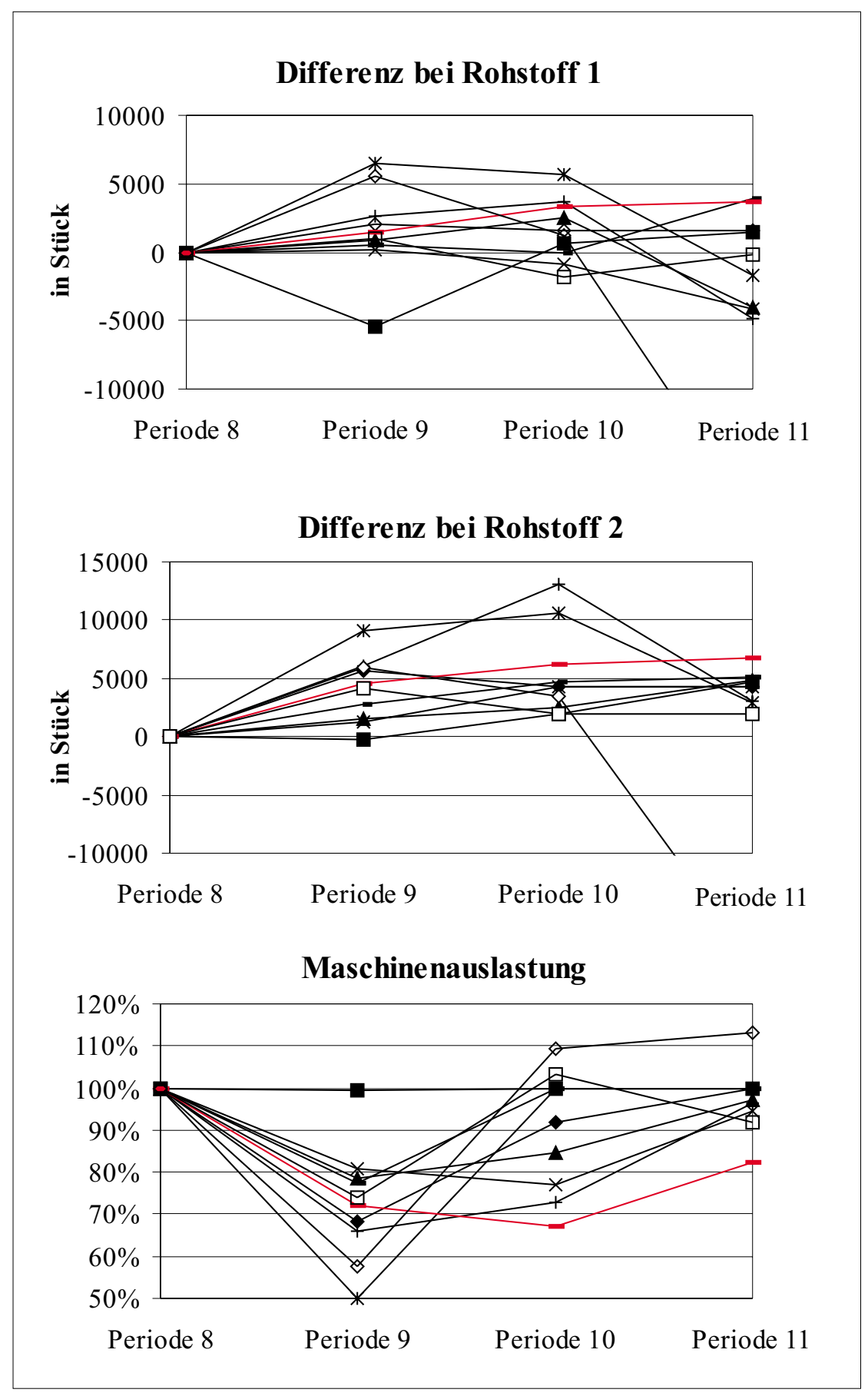

Abbildung 8.2: Testphase: Beschaffungs- und Produktionsplanung, herkömmliche Bedingung 


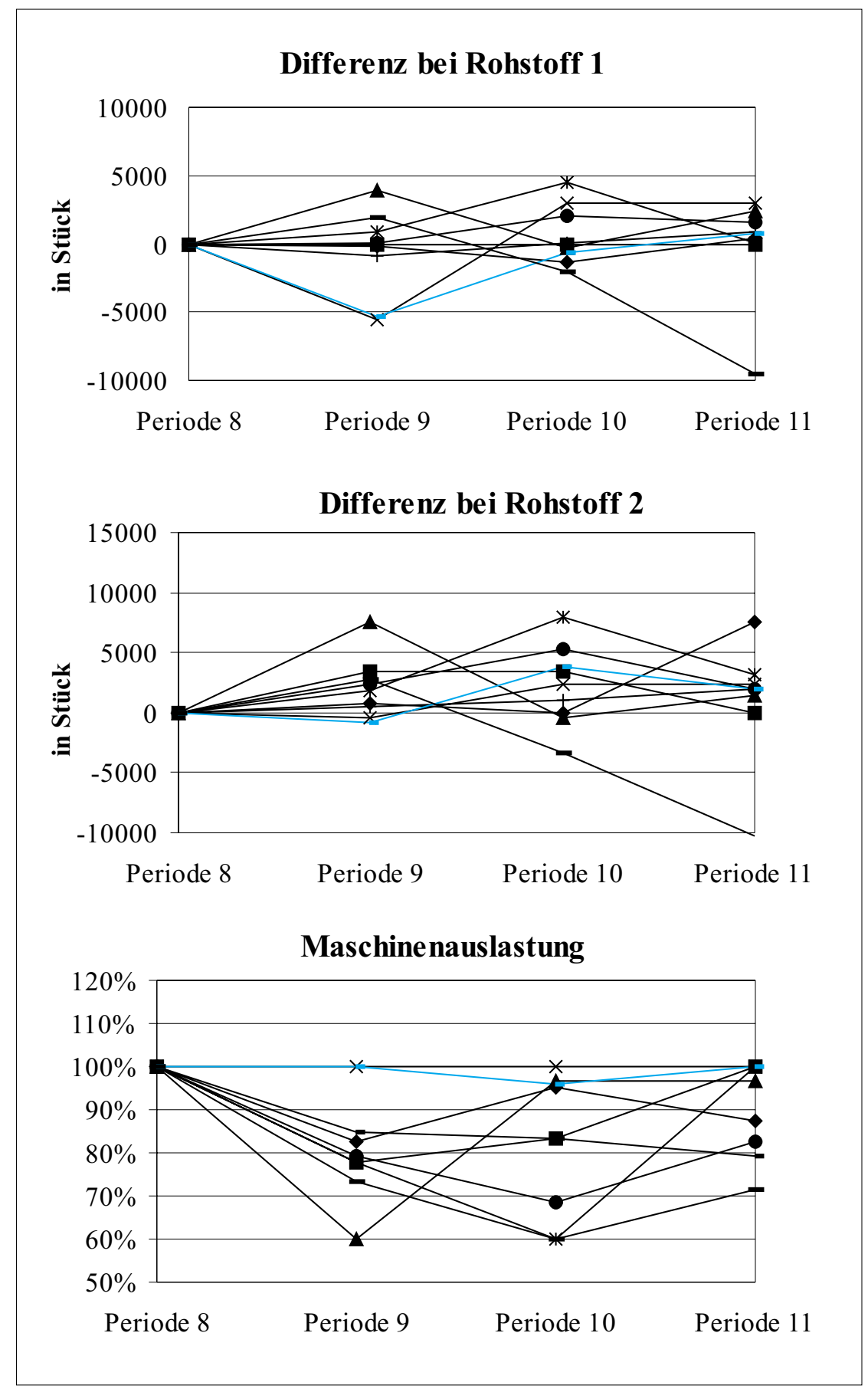

Abbildung 8.3: Testphase: Beschaffungs- und Produktionsplanung, modulare Bedingung 
In der Ausgangslage (Periode 8) war die Differenz bei beiden Rohstoffen gleich Null und die Maschinenauslastung $100 \%$, d.h. hier wurde optimal geplant. Zwei Gruppen der modularen und eine Gruppe der herkömmlichen Bedingung lasten ihre Maschinen voll aus und nehmen die entsprechend hohe Notbeschaffung in Kauf, die sich in einer negativen Differenz insbesondere bei Rohstoff 1 niederschlägt. Diese drei Gruppen liegen mit ihrem Jahresüberschuss entsprechend deutlich über dem Durchschnitt.

Für die Planung der Rohstoffmengen ist in EpUS grundsätzlich kein Sicherheitsbestand zu berücksichtigen. Auf Grund der Unsicherheit der Entscheidung bzw. möglicher Änderungen der Strategien sind Lagerbestände bis 1.000 Stück noch akzeptabel. In diesem Bereich zwischen 0 und 1.000 Stück je Rohstoffart liegt in der 9. Periode jede dritte der modularen Gruppen, in der 11. Periode sogar mehr als die Hälfte der Gruppen. Bei den Gruppen der herkömmmlichen Bedingung erreichen zwar in der 9. Periode auch ein Drittel diese Werte, in den nachfolgenden Perioden bleibt nur eine einzige Gruppe in diesem Bereich.

Wie in den Abbildungen 8.2 und 8.3 zu sehen ist, können die Teilnehmenden der modularen Bedingung die Planung hinsichtlich der Vermeidung einer Notbeschaffung, die sich in negativen Differenzen äußert, besser durchführen. Der Bereich der Beschaffung war der erste, über den die Teilnehmenden dieser Bedingung in der experimentellen Phase zu entscheiden hatten. Insbesondere aus der Planung der letzten Periode resultierte lediglich bei einer Gruppe eine negative Differenz. Bei den Gruppen der herkömmlichen Bedingung ist dies immerhin in der letzten Periode bei Rohstoff 1 bei mehr als der Hälfte der Gruppen der Fall. Dies ist besonders negativ zu bewerten, da die Entscheidungen für die letzten beiden Perioden zusammen getroffen wurden. Die Teilnehmenden planten also die Rohstoffbeschaffung gemeinsam mit den Produktionsmengen für die 11. Periode. Ein Einfluss der Leistung der modularen Gruppen in der experimentellen Phase ist nicht festzustellen.

Bei der Maschinenauslastung fällt auf, dass die Gruppen der herkömmlichen Bedingung ihre Maschinen in der 9. Periode im Durchschnitt geringer auslasten als die Gruppen der modularen Bedingung. Die Streuung ist bei den Ergebnissen der herkömmlichen Bedingung größer. In der 10. und 11. Periode planen zwei bzw. 
eine Gruppe die Produktionsmengen sogar zu hoch, so dass ein automatischer Einkauf von Produkten induziert wird. ${ }^{31}$

Die Teilnehmenden der herkömmlichen Bedingung entscheiden sich aber auch häufiger und in größerem Ausmaß dafür, in der 9. Periode Maschinen zu verkaufen (Abbildung 8.4 und 8.5): Fast alle Gruppen verkaufen einen Teil ihrer Maschinen in der 9. Periode, mehr als die Hälfte in der 10. Periode. Die modularen Gruppen sind eher vorsichtiger: In der 9. Periode verkaufen fünf Gruppen einige Maschinen, in der 10. Periode entscheidet sich ebenfalls die Hälfte der Gruppen für einen Verkauf. Vier dieser Gruppen gehören zu den Erfolgreichen in der experimentellen Phase, wurden also nach der ersten Entscheidung auf die dritte Stufe erhöht und erreichten bereits zur vierten Entscheidung die höchste Stufe.

In der letzten Periode verkauften noch zwei Gruppen der modularen Bedingung und drei der herkömmlichen Bedingung einen Teil ihrer Maschinen, obwohl die Auswirkung dieser Entscheidung auf die Kapazität nicht mehr für sie wirksam wurde. Auf Grund der konjunkturellen Lage wäre es jedoch sinnvoller, bereits in der 9. Periode einen Teil der Maschinen zu verkaufen und die entsprechende Anzahl Personal zu entlassen. Das Verhältnis zwischen den vorhandenen Maschinen und dem Personal ist in allen Gruppen zu jedem Zeitpunkt optimal.

Die Planung der Beschaffung und der Produktion hat einen direkten Einfluss auf die Höhe der Herstellungskosten der Produkte. Da durch die in der 9. Periode wirksam gewordene Rationalisierungsmaßnahme weniger Leistungseinheiten der Maschinen zur Produktion eines Stücks benötigt werden, sinken die Fertigungskosten zunächst in der 9. gegenüber der 8. Periode. Die Maßnahme greift bei Produkt 2 stärker als bei Produkt1, da hier die Zahl der benötigten Leistungseinheiten proportional stärker gesenkt wurde.

Die Gruppen der modularen Bedingung sind eher erfolgreicher und erzielen geringere Herstellungskosten bei beiden Produkten (Abbildung 8.4 und 8.5). Dies spiegelt die geringere Notbeschaffung der Rohstoffe, aber auch die geringere Desinvestition und entsprechende Entlassung von Personal wider. Durch Entlassung entstehen Verwaltungskosten, die anteilig zu den Herstellungskosten gerechnet werden. Entsprechend ist der teilweise starke Anstieg der Herstellungskosten

$\overline{31}$ In den Abbildungen 8.2 und 8.3 durch Auslastungsgrade über $100 \%$ ausgedrückt. 


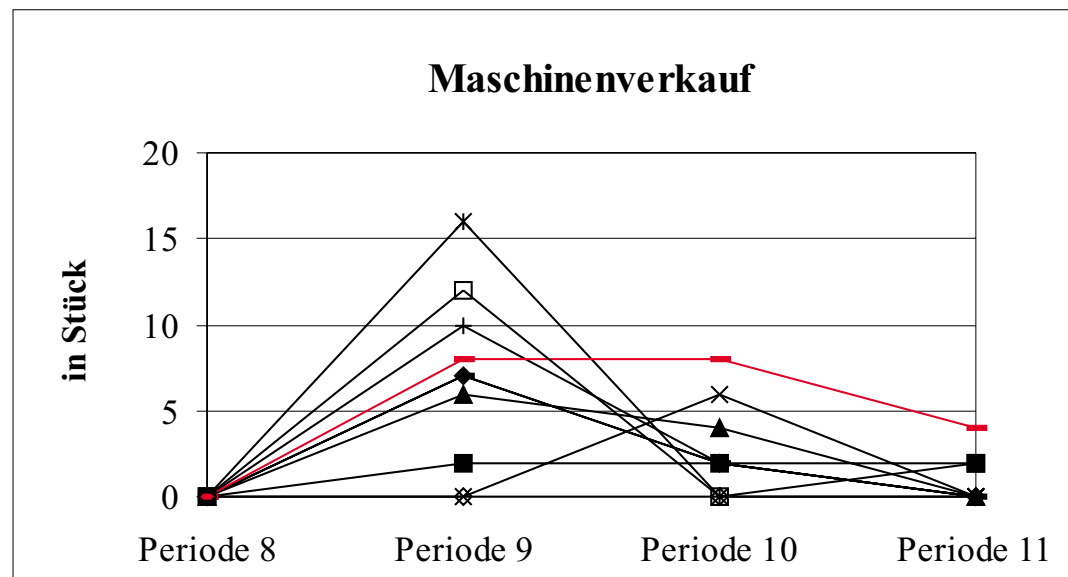

\section{Herstellungskosten Produkt 1}

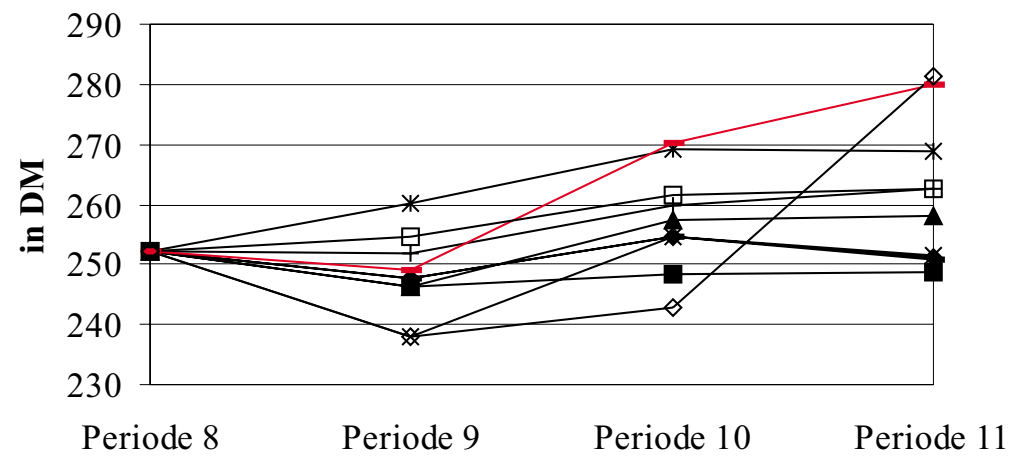

\section{Herstellungskosten Produkt 2}

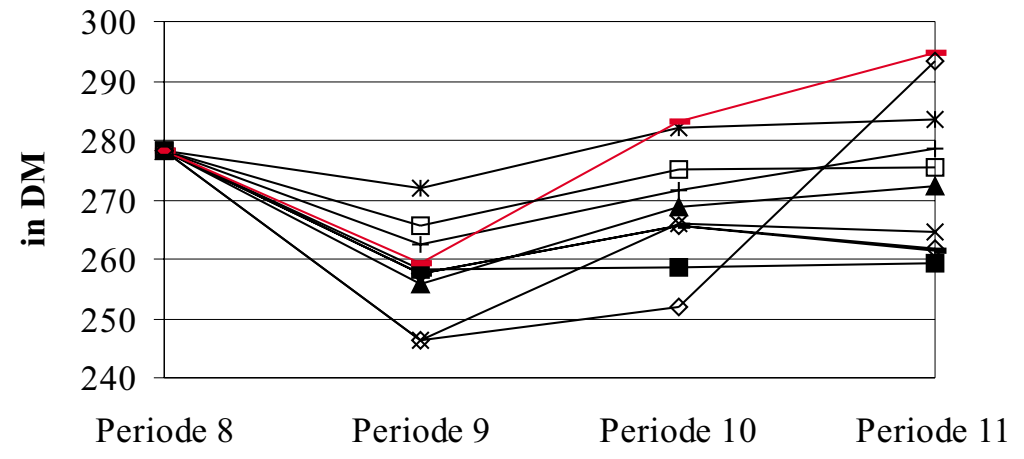

Abbildung 8.4: Testphase: Investitionsplanung und Herstellungskosten, herkömmliche Bedingung 


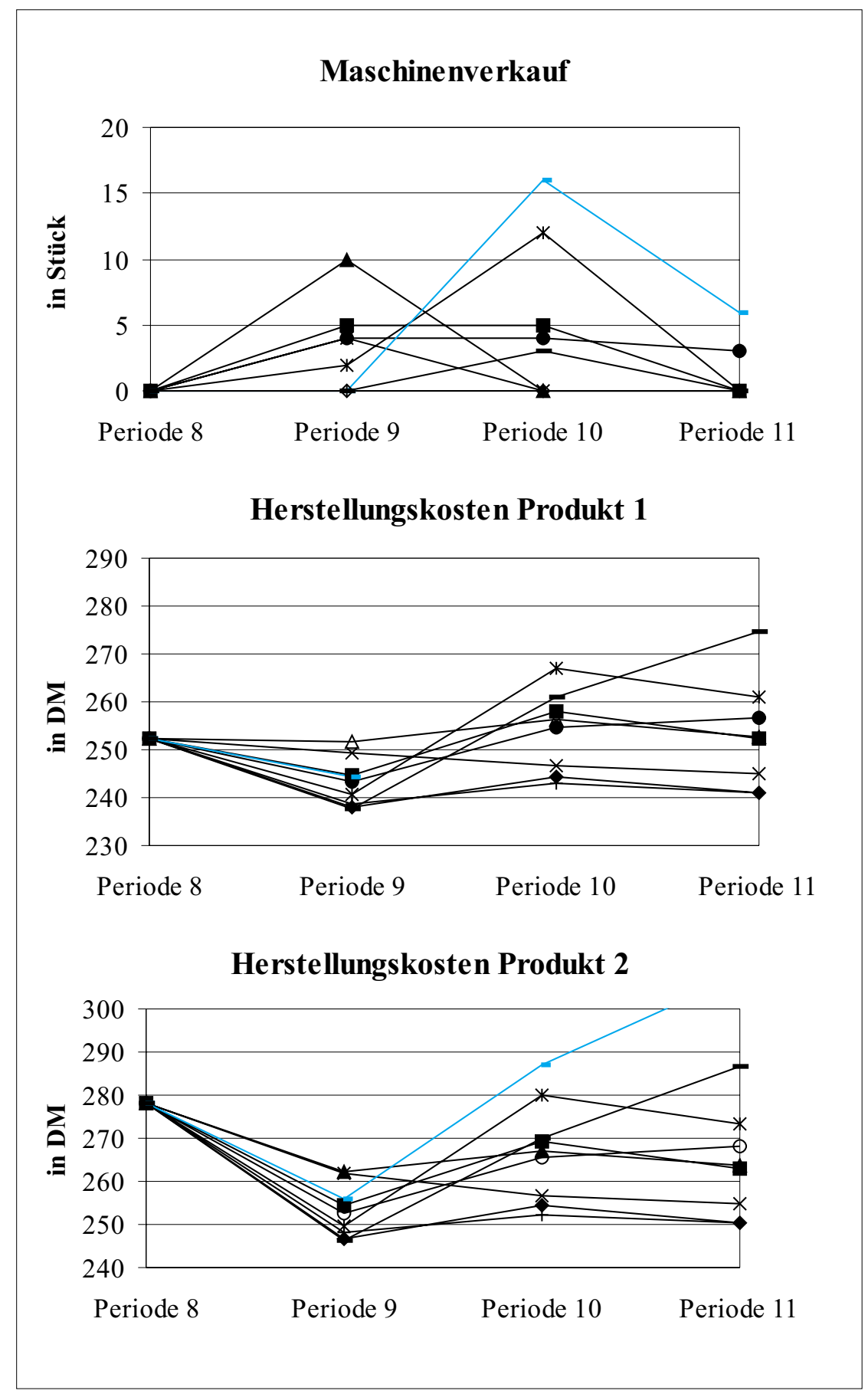

Abbildung 8.5: Testphase: Investitionsplanung und Herstellungskosten, modulare Bedingung 
zwischen der 9. und 10. Periode zu interpretieren. Gleichzeitig erhöhen sich zur 10. Periode hin die Löhne, die in die Fertigungskosten einfließen.

Bei den Herstellungskosten ist der Effekt der Hochstufung bei der modularen Bedingung in der experimentellen Phase umgekehrt: Die Gruppen, die nicht zur letzten Entscheidung die höchste Stufe erreichten, erzielen geringere Herstellungskosten in der Testphase. Jeweils drei Gruppen in beiden Bedingungen gelang es, die Maschinen zu rund $80 \%$ auszulasten und gleichzeitig auf eine Notbeschaffung bei den Rohstoffen zu verzichten. Ihre Herstellungskosten liegen entsprechend unter dem Durchschnitt. Die Auswirkung auf den Jahresüberschuss ist jedoch nicht eindeutig: Jeweils zwei Gruppen liegen über dem durchschnittlichen Gewinn, jeweils eine Gruppe deutlich darunter. Hier müssen die Entscheidungen bei den Marketing-Instrumenten näher betrachtet werden.

\subsubsection{Absatzplanung}

Als Kriterium zur Beurteilung der Entscheidungen, die einen Einfluss auf den Absatzbereich des Modellunternehmens haben, wird der Lagerbestand der Produkte herangezogen. Er ist für beide Produkte in der Abbildung 8.6 (S.232) für die Gruppen der herkömmlichen Bedingungen und in der Abbildung 8.7 (S. 233) für die Gruppen der modularen Bedingung dargestellt. Die Unterschiede zwischen den experimentellen Bedingungen sind nur geringfügig. Der Abbau der Lagerbestände gelingt den meisten Gruppen tendenziell bei Produkt1, nicht aber bei Produkt 2.

Es fällt auf, dass drei Gruppen der herkömmlichen Bedingung bei beiden Produkten Lagerbestände aufbauen, also immer positive Bestandsveränderungen haben. Bei den Gruppen der modularen Bedingung gibt es nur eine Gruppe, die bei beiden Produkten Lagerbestände aufbaut. Je zwei Gruppen haben nur bei einem Produkt positive Bestandsveränderungen und bauen bei dem anderen Produkt die Lagerbestände durch Verkauf ab. Die Gruppen mit den höchsten Beständen waren in der experimentellen Phase diejenigen, die nicht die höchste Stufe der Komplexität erreichten. 


\subsubsection{Finanzplanung}

Bei der Finanzplanung solten die Teilnehmenden durch Aufnahme bzw. Tilgung eines langfristigen Kredits sowie der Anlage von Einzahlungs-Überschüssen in einer kurzfristigen Terminanlage eine möglichst geringe Zinsbelastung erzielen. ${ }^{32}$ Zur Beurteilung wird der Kassenbestand herangezogen (Abbildung 8.8, S. 234).

Bei den Gruppen der modularen Bedingung fällt eine Gruppe auf, die in allen drei Perioden einen hohen positiven Kassenbestand erreicht. Diese Teilnehmenden entschieden sich, für die 9. Periode einen Kredit in Höhe von 1,8 Mio. aufzunehmen, und zahlten erst in der 11. Periode 0,5 Mio. davon zurück. Die Zinsbelastung war entsprechend hoch, was sich auf die Herstellungskosten der Produkte und auf den Jahresüberschuss auswirkt. ${ }^{33}$ Bei den Gruppen der herkömmlichen Bedingung fällt eine Gruppe mit einem hohen Kontokorrentkredit auf, der insbesondere durch eine Fehlplanung der Rohstoffe in der 10 . Periode verursacht wird. ${ }^{34}$

Die Entscheidungen der modularen Gruppen führten nur bei einer Gruppe in der 9. Periode zu einem Kontokorrentkredit. Alle anderen Gruppen erreichten positive Werte, wobei der Durchschnitt bei 56.379 liegt. Im Gegensatz dazu liegt der Durchschnitt bei den Gruppen der herkömmlichen Bedingung bei -12.705. In den beiden darauf folgenden Perioden liegen die Mittelwerte in der modularen Bedingung bei knapp 120.000 und gut 200.000, während sie in der herkömmlichen Bedingung mit knapp 400.000 und knapp 550.000 wesentlich höher sind.

Die Unterschiede in der Streuung zwischen den beiden experimentellen Bedingungen sind sehr auffällig: Während sie in der herkömmlichen Bedingung in allen drei Perioden gleich bleibt, ist sie in der modularen Bedingung - bis auf den Ausreißer - in der 9. Periode gering und wird zur 11. Periode hin größer. Bei der Entscheidung für die 9. Periode legte nur eine Gruppe einen bestimmten Betrag als Terminanlage an. Erst in der 10. und 11. Periode legen einige Gruppen Gelder als Terminanlage an, nehmen einen Kredit auf oder tilgen ihn. Die Teilnehmenden der herkömmlichen Bedingung entscheiden stärker über den Finanzierungsbereich.

\footnotetext{
32 Vgl. Abschnitt 3.3 sowie 8.2.

$33 \mathrm{Vgl}$. Abbildung 8.5 und 8.9.

$34 \mathrm{Vgl}$. Abbildung 8.2.
} 


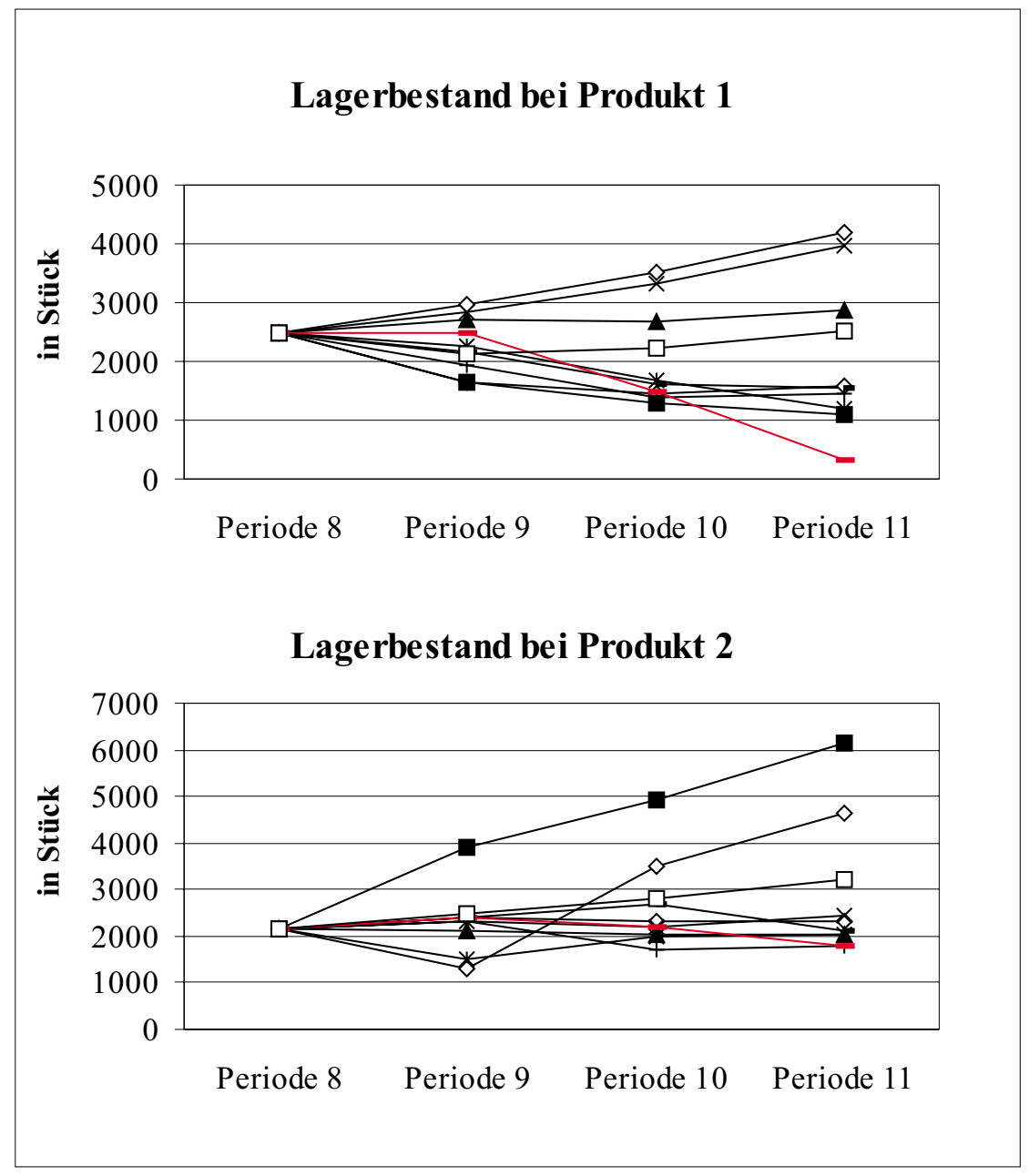

Abbildung 8.6: Testphase: Absatzplanung, herkömmliche Bedingung 


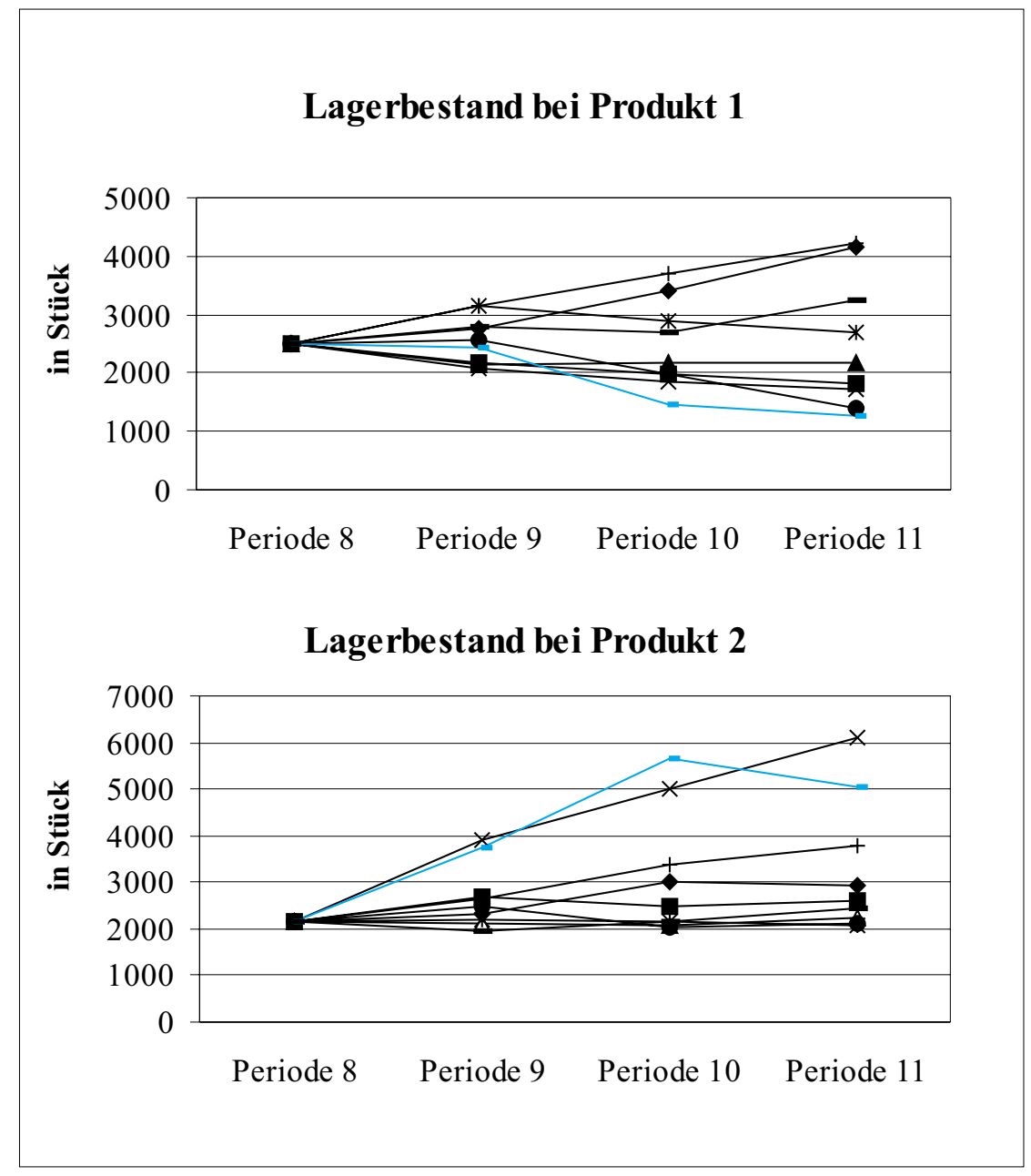

Abbildung 8.7: Testphase: Absatzplanung, modulare Bedingung 


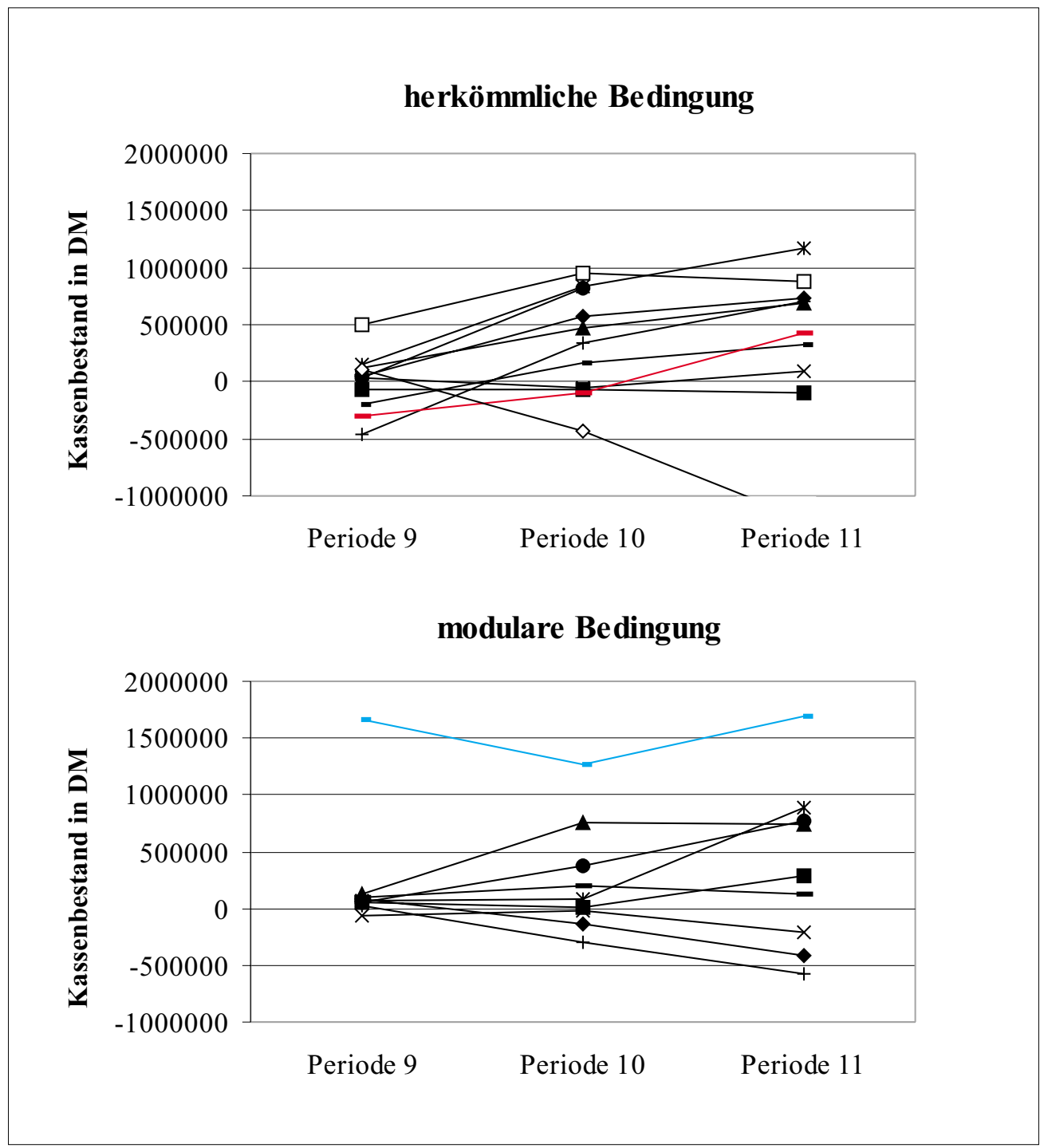

Abbildung 8.8: Testphase: Kassenbestand 
Wird zur Erklärung die Hochstufung in der experimentellen Phase herangezogen, so haben die drei Gruppen, die in der 11. Periode einen Kontokorrentkredit erreichen, in der experimentellen Phase nicht die fünfte Stufe erreicht und damit in der ersten Phase des Planspiels noch nicht über den Finanzierungsbereich entschieden. Auch die Gruppe, die in der 9. Periode einen sehr hohen Kredit aufnimmt, wurde nur bis zur vierten Stufe hochgesetzt. ${ }^{35}$ Hier zeigt sich also deutlich ein Effekt bei der Entscheidung über zwei Perioden. Die Teilnehmenden, die bereits in der experimentellen Phase über den Finanzierungsbereich entschieden haben, können die finanziellen Auswirkungen ihrer Entscheidungen besser abschätzen und entsprechend reagieren.

\subsubsection{Unternehmenserfolg}

Schließlich erfolgt eine Betrachtung der Jahresüberschüsse bzw. -fehlbeträge, die die Gruppen in der Testphase erzielten (Abbildung 8.9, S. 236): Hier wird deutlich, dass es den modularen im Vergleich zu den herkömmlichen Gruppen wesentlich besser gelingt, ein positives Periodenergebnis zu erzielen. Weiterhin ist die Streuung der Werte zwischen den modularen Gruppen nicht so grof wie bei den herkömmlichen. In der 9. Periode erzielen die Teilnehmenden der modularen Bedingung im Durchschnitt einen höheren Gewinn. ${ }^{36}$ In den darauf folgenden beiden Perioden sind die Unterschiede in den Mittelwerte nicht mehr so groß.

Diese Entwicklung wird noch deutlicher bei der Betrachtung der kumulierten Werte, die sich durch Aufsummierung der Jahresüberschüsse ergeben (Abbildung 8.10, S. 237). Die Streuung der Werte ist bei den Gruppen der herkömmlichen Bedingung wesentlich größer als bei denen der modularen Bedingung. Offensichtlich scheint die modulare Bedingung ein gleichartigeres Entscheidungsverhalten zu induzieren. ${ }^{37}$ Überraschenderweise erzielten die Teilnehmenden, die in der experimentellen Phase erfolgreich waren und entsprechend früh über alle Variablen zu entscheiden hatten, einen geringeren kumulierten Gewinn als diejenigen, die zum Ende der Phase noch auf der vierten Stufe waren. ${ }^{38}$

$35 \quad$ Fisher's Test (vgl. Abschnitt 7.3) ergibt eine Wahrscheinlichkeit von $\mathrm{p}=0.0793$.

36 Mittelwerte: herkömmliche Bedingung 2.618, modulare Bedingung 104.928, $\mathrm{p}=0.01347$.

37 Dies zeigte sich bereits in den Erhebungen des DFG-Projekts; vgl. BLoEch et al. (Einsatz 1998), S. 26-29.

38 Das Ergebnis ist allerdings nicht signifikant. 


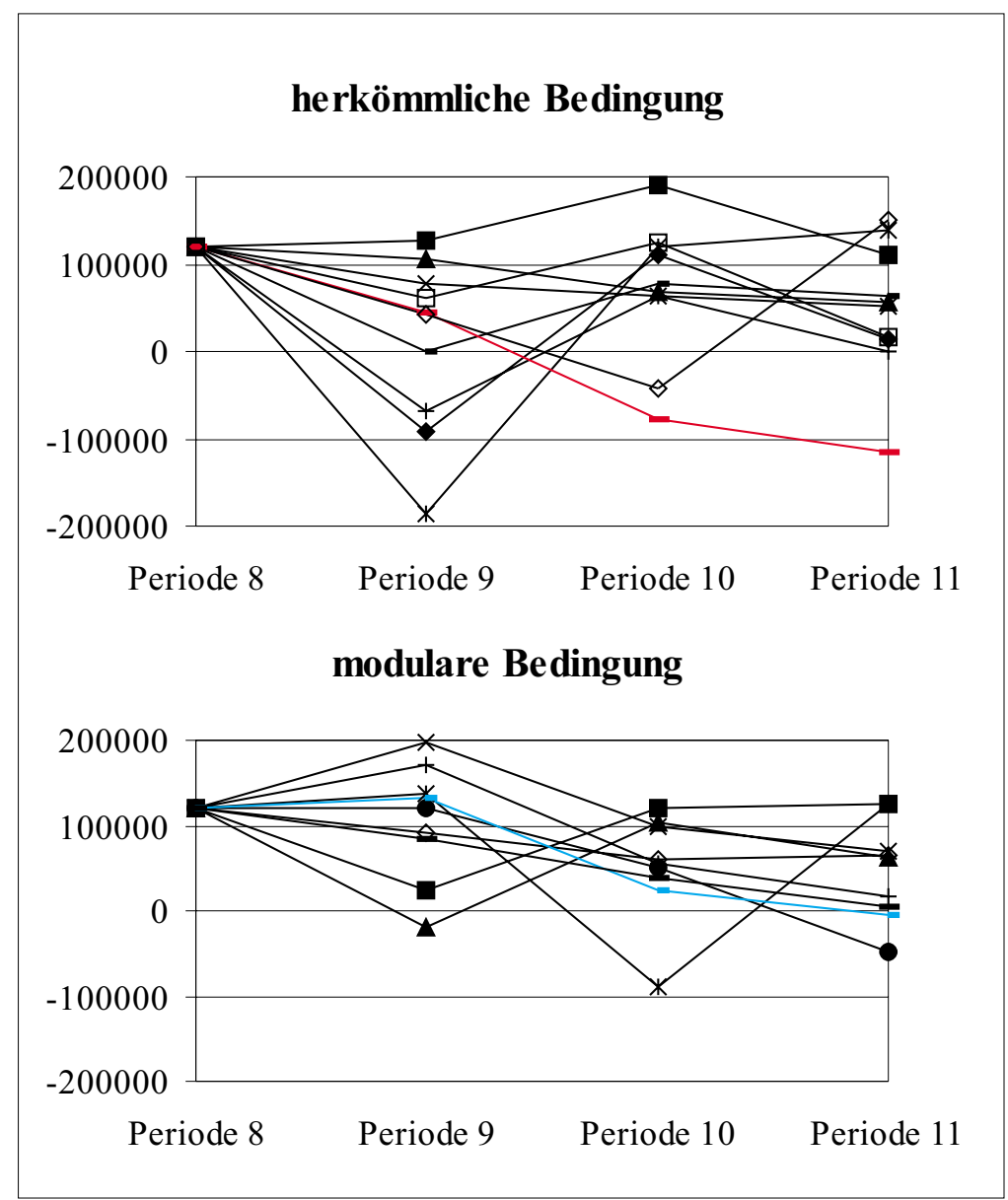

Abbildung 8.9: Testphase: Erzielte Gewinne 


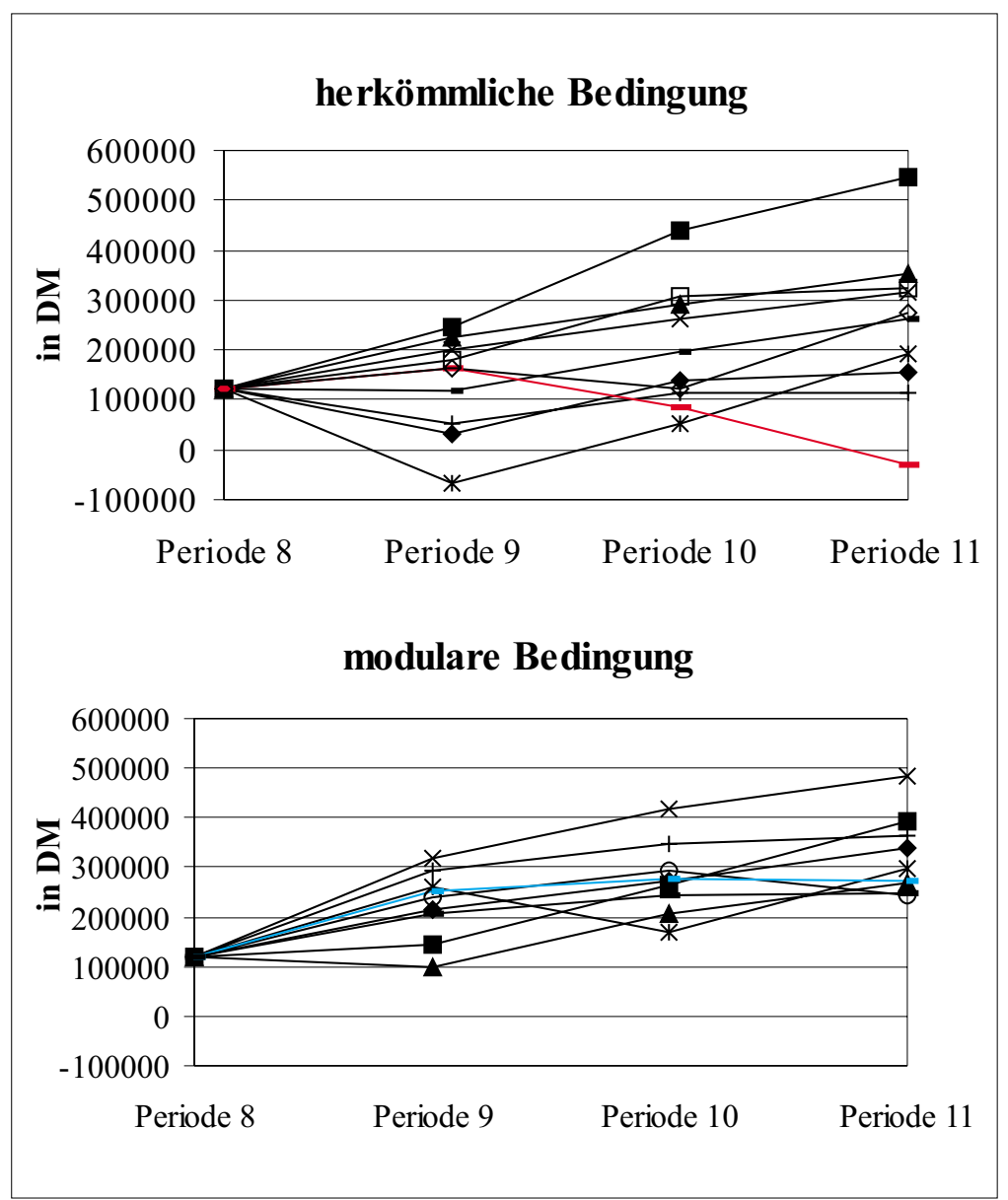

Abbildung 8.10: Testphase: Kumulierte Gewinne 


\subsubsection{Fazit}

Zusammenfassend lässt sich feststellen, dass die modularen Gruppen im Vergleich bessere Ergebnisse erzielen. Die sukzessive Steigerung der Komplexität der Entscheidungssituation scheint entsprechend der aufgestellten Hypothese in Abschnitt 4.8.4 zu fundierteren Entscheidungen zu führen, die sich in besseren Ergebnissen niederschlagen. Allerdings sind die modularen Gruppen insgesamt weniger risikofreudig und treffen insbesondere in der neuen Situation der Testphase eher vorsichtigere Entscheidungen. Die modulare Bedingung scheint weiterhin ein homogeneres Entscheidungsverhalten zu induzieren. Weiterhin wird ein Einfluss der Leistungen der Gruppen in der experimentellen Phase, also hinsichtlich der Höherstufung in der modularen Bedingung zum Teil sichtbar.

\subsection{Vollständiges Design}

\subsubsection{Einsatz des Fragebogens zur Entscheidung}

\section{Deskriptiver Vergleich zweier Gruppen}

Zunächst ist festzustellen, dass nicht alle Gruppen, die den Fragebogen zur Entscheidung bekamen, ihn auch in jeder Planspiel-Sitzung ausgefüllt haben. Jeweils eine Gruppe der herkömmlichen und der modularen Bedingung schrieben während der gesamten Planspiel-Phase ihre Ziele und Maßnahmen auf. ${ }^{39}$ Dabei waren beide Gruppen von Anfang an in der Lage, ihre Ziele und Maßnahmen zu formulieren und durch entsprechende Entscheidungen auch umzusetzen. In der Testphase verfolgen sie unterschiedliche Ziele: Während die Teilnehmenden der Gruppe 18 sich für eine volle Auslastung der Kapazitäten entscheiden sowie erst in der 10. Periode die Hälfte der Maschinen verkaufen und entsprechendes Personal entlassen, reduziert die Gruppe 16 ihre Produktionsmengen bereits in der 9. Periode und verkauft dreimal hintereinander einen Teil ihrer Maschinen. Allerdings sind beide Gruppen sehr gut in der Lage, den Lagerbestand bei Produkt 1 zu reduzieren. Gruppe 16 gelingt dies auch bei Produkt2, hier ist Gruppe 18 nicht

39 Gruppe 16 der herkömmlichen Bedingung (Symbol-), Gruppe 18 der modularen Bedingung (Symbol -). In den Abbildungen durch graue Linien gekennzeichnet. 
so erfolgreich. Der Grund hierfür ist sicherlich in der unterschiedlichen Auslastung der Maschinen zu suchen, da Gruppe 18 die Herstellung von Produkt 1 reduziert und die freien Kapazitäten für Produkt 2 verwendet. Der Kassenbestand ist bei Gruppe 18 sehr hoch, weil sie in der 9. Periode einen Kredit in Höhe von 1,8 Mio. aufnahmen, der jedoch fast nicht notwendig war. Eine Rückzahlung in Höhe von 0,5 Mio. erfolgte jedoch erst in der 11. Periode. Gruppe 16 zahlt über die drei Perioden der Testphase hinweg insgesamt 0,8 Mio. des langfristigen Kredits zurück und erreicht mit der letzten Entscheidung sogar einen Kassenbestand von über 400.000. Ein vergleichbares Ergebnis hätte Gruppe 18 ebenfalls erreichen können.

Die erzielten Jahresüberschüsse der beiden Unternehmen sind sehr niedrig. Allerdings ist der Gewinn bei Gruppe 18 in der 9. Periode noch relativ hoch, weil sie ihre Maschinen voll auslastet. Erst das Ergebnis in der 11. Periode ist knapp negativ, während die Gruppe 16 in der 10. und 11. Periode einen Jahresfehlbetrag erzielt. Beim kumulierten Gewinn schneidet die Gruppe 16 im Vergleich zu den anderen herkömmlichen Gruppen sehr schlecht ab. Gruppe 18 nimmt im Gegensatz dazu den mittleren Platz der modularen Gruppen ein.

\section{Statistische Analysen}

Um die Auswirkungen des Einsatzes des Fragebogens zur Entscheidung auf die Art der Entscheidungsfindung in der Testphase analysieren zu können, wurden zweifaktorielle Varianzanalysen mit Messwiederholungen gerechnet. ${ }^{40}$ Die Faktoren sind der Einsatz des Fragebogens zur Entscheidung sowie als zweiter Faktor die Planspiel-Durchführung in der experimentellen Phase. Als abhängige Variablen wurden die verschiedenen Kennzahlen herangezogen, die bisher betrachtet wurden.

Ein signifikantes Ergebnis hinsichtlich des Einsatzes des Fragebogens ergab sich bei der Planung der Rohstoffbeschaffung, ${ }^{41}$ der sich als Differenz aus dem LagerAnfangsbestand und dem -Abgang in die Produktion berechnet. Es ist auffällig, dass bei beiden Rohstoffen die gleiche Entwicklung zu beobachten ist. Während die Differenz bei den Gruppen, in denen der Fragebogen zur Entscheidung ein-

40 Vgl. Abschnitt 7.5.

41 Mittelwert-Differenz bei Rohstoff 1: $\mathrm{F}=5.02, \mathrm{p}=0.0160$, $\mathrm{df}=26$, bei Rohstoff 2: $\mathrm{F}=3.46$, $\mathrm{p}=0.0493, \mathrm{df}=26$. 
gesetzt wurde, zum Ende der Testphase hin zunimmt, wird die Differenz bei den Gruppen ohne Fragebogen im Durchschnitt negativ, führt also zu einer Notbeschaffung. Dabei ist eine einzelne Gruppe als Ausreißer zu bezeichnen, weil sie für die 11. Periode keine Rohstoffe mehr einkaufte, aber produzierte. Wird diese Gruppe aus der Mittelwert-Berechnung herausgenommen, ergibt sich jedoch fast das gleiche Bild (gestrichelte Linie in Abbildung 8.11).

Die Rohstoffbeschaffung hängt mit der Planung der Produktionsmengen zusammen, die wiederum die Auslastung der vorhandenen Kapazität beeinflusst. Bei der Kennzahl der Maschinenauslastung ergab sich zwar kein signifikanter Unterschied hinsichtlich des Einsatzes des Fragebogens, die Mittelwert-Unterschiede können jedoch zur Erklärung herangezogen werden. Die negative Differenz bei den Rohstoffen in der 9. Periode spiegelt sich in der höheren Maschinenauslastung wider. Die Gruppen ohne Fragebogen entscheiden sich, ihre Maschinen durchschnittlich mit $73 \%$ auszulasten, während die Gruppen, bei denen der Fragebogen eingesetzt wurde, ihre Maschinen zu $82 \%$ auslasten, was eine entsprechende Notbeschaffung an Rohstoffen erfordert.

Als Zwischenfazit lässt sich sagen, dass der Einsatz des Fragebogens zur Entscheidung offensichtlich einen Einfluss auf das methodische Arbeiten hat.

Weiterhin ergab sich ein signifikantes Ergebnis bezüglich der Wechselwirkung zwischen der experimentellen Bedingung und dem Einsatz des Fragebogens bei den Bestandsveränderungen von Produkt1 (Abbildung 8.12). Die Bestandsveränderungen berechnen sich aus dem Lager-Abgang durch Verkauf und -Zugang durch Produktion. Die Nachfrage nach diesem Produkt ging in der Testphase erheblich zurück, worauf die Teilnehmenden entsprechend reagieren mussten. Auf Grund der konjunkturellen Lage ist es sinnvoll, negative Bestandsveränderungen zu erzielen, also die Lagerbestände zu reduzieren.

Am erfolgreichsten zeigen sich hier die Gruppen der herkömmlichen Bedingung, bei denen der Fragebogen eingesetzt wurde. Sie erzielten mit ihren Entscheidungen in allen drei Perioden negative Bestandsveränderungen und bauten den Lagerbestand ab. Auch die Gruppen der modularen Bedingung, in denen der Fragebogen nicht eingesetzt wurde, erreichten negative Bestandsveränderungen und einen Abbau des Lagerbestands. Die Entwicklung beim Lagerbestand in der 9. und 10. Periode verläuft im Durchschnitt ähnlich wie bei den Gruppen der herkömmlichen 


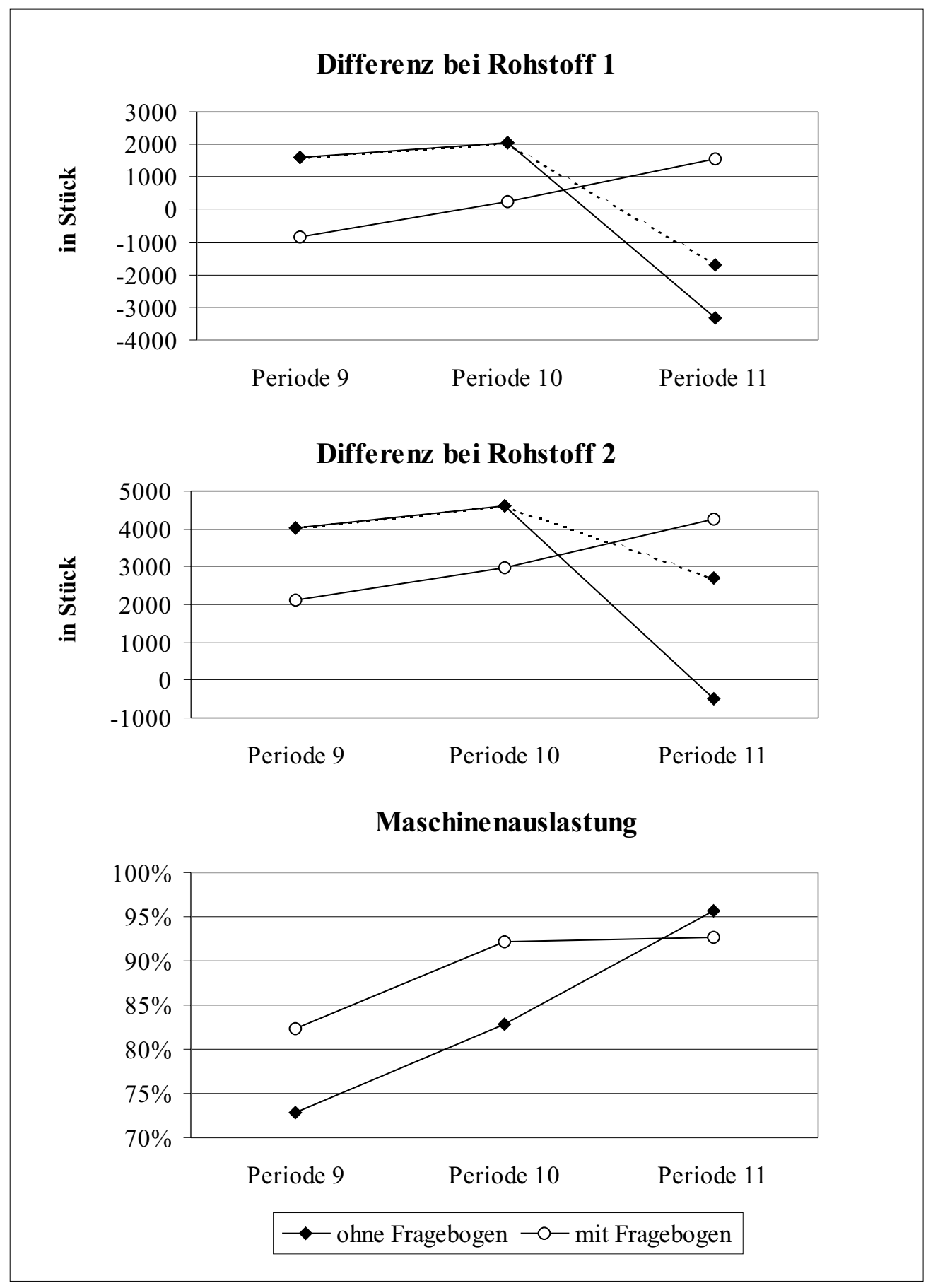

Abbildung 8.11: Testphase mit vollständigem Design: Rohstoffbeschaffung, Einsatz des Fragebogens zur Entscheidung 


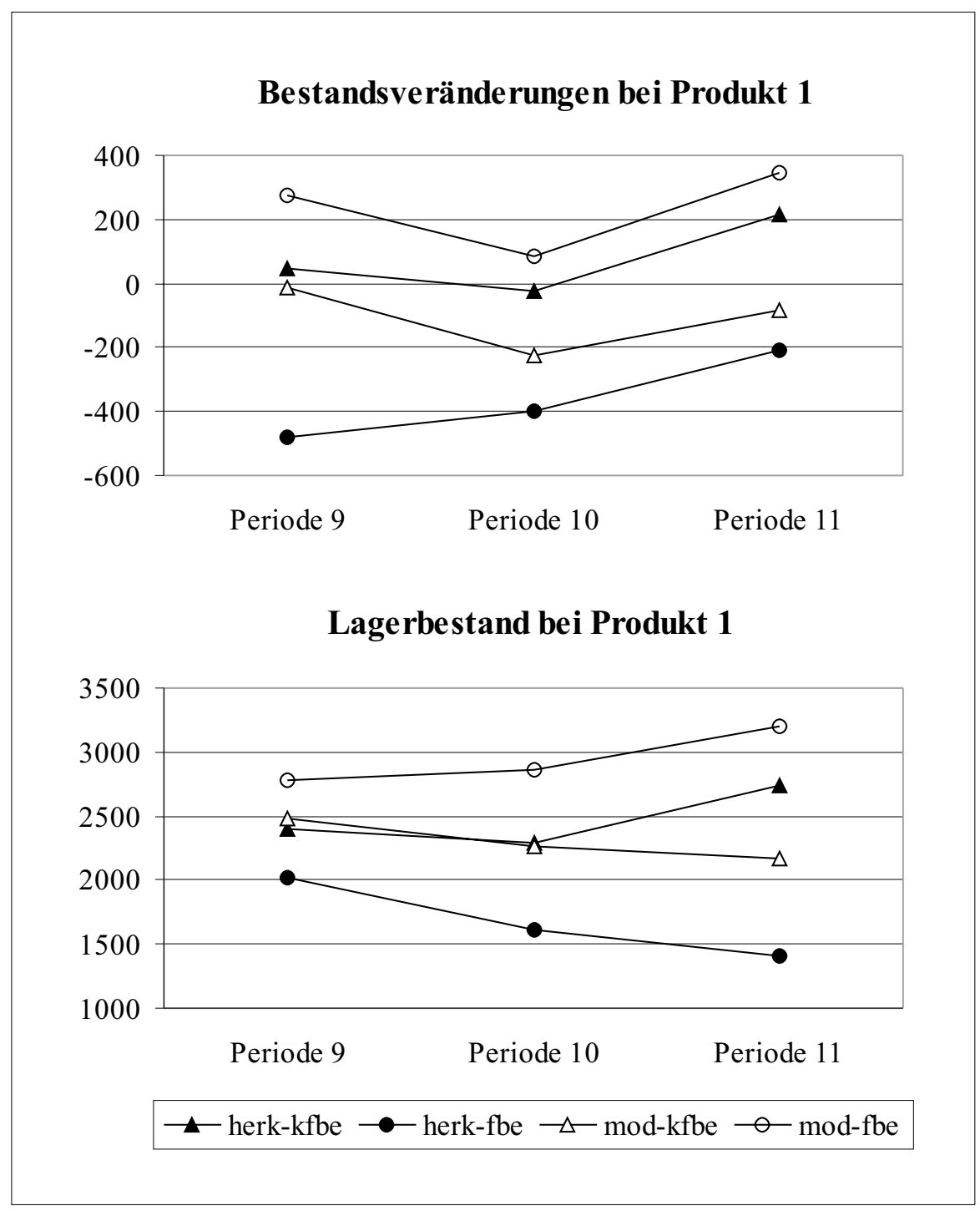

Abbildung 8.12: Testphase mit vollständigem Design: Bestandsveränderungen und Lagerbestand

herk = herkömmliche Bedingung

$\bmod =$ modulare Bedingung

kfbe $=$ kein Einsatz des Fragebogens zur Entscheidung

$\mathrm{fbe}=$ Einsatz des Fragebogens zur Entscheidung 
Bedingung ohne Fragebogen. Entsprechend gering sind die Bestandsveränderungen. Ihre Entscheidungen über die Marketing-Instrumente in Kombination mit den Produktionsmengen für die 11. Periode war jedoch nicht mehr erfolgreich und erhöhte den Lagerbestand. Am wenigsten erfolgreich scheint im Hinblick auf diese Planung dieser Kennzahl die Kombination des Fragebogens zur Entscheidung mit der modularen Bedingung. Bei ihnen sind die Bestandsveränderungen in allen drei Perioden positiv und führen zu einem hohen Lagerbestand am Ende der Testphase.

\subsubsection{Fragebogen zum situativen Erleben}

\section{Gesamte Stichprobe}

Der Fragebogen zum situativen Erleben wurde während der gesamten PlanspielPhase bei der Hälfte der Teilnehmenden eingesetzt, ${ }^{42}$ wodurch sich sieben Messwiederholungen ergeben. Zur Auswertung wurden die Ergebnisse derjenigen herangezogen, von denen mindestens fünf ausgefüllte Fragebögen vorlagen. Die fehlenden Antworten wurden durch das BMDP-Programm am ersetzt. ${ }^{43}$

In diesem Abschnitt werden die Auswertungs-Ergebnisse hinsichtlich der Einschätzungen sowie ihrer Stabilität dargestellt. Die ersten fünf Messzeitpunkte fanden in der experimentellen Phase statt, die letzten beiden in der Testphase. Die Aussagen sollten von den Teilnehmenden auf einer Skala von 1 bis 5 bewertet werden. Ein durchschnittlicher Wert von 3 bezeichnet also die Mitte der Skala. Zur Bewertung der Stabilität werden die Korrelations-Koeffizienten zwischen aufeinander folgenden Messzeitpunkten betrachtet. ${ }^{44}$ In den Abbildungen 8.13 bis 8.14 sind die Mittelwerte der ersten sechs Items für die gesamte Stichprobe im Zeitverlauf dargestellt.

42 Vgl. Anhang A.18.

43 Vgl. Dixon (Software 1985), S. 217-234. Das Programm berechnet zunächst die Korrelations-Matrix aufgrund der vorhandenen Items und führt eine Faktorenanalyse durch. Die Ersetzungen für die fehlenden Items werden in mehreren Iterationsschritten derart berechnet, dass der Faktorraum erhalten bleibt, also auch die Mittelwerte und Varianzen der Items nur unwesentlich verändert werden. Mit der durch die Ersetzungen ausgefüllten Matrix mit Item-Werten wurden nachfolgend die Auswertungen durchgeführt.

44 Die Ergebnisse befinden sich im Anhang A.26. 
Heute Abend fühle ich mich...

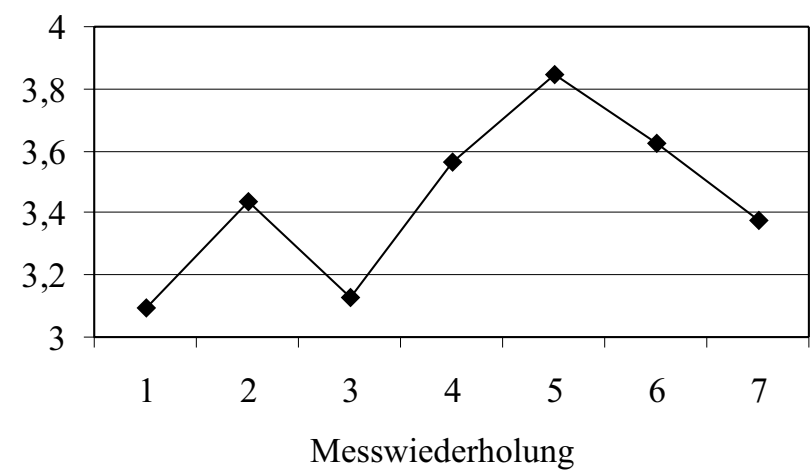

Den Unterricht finde ich...

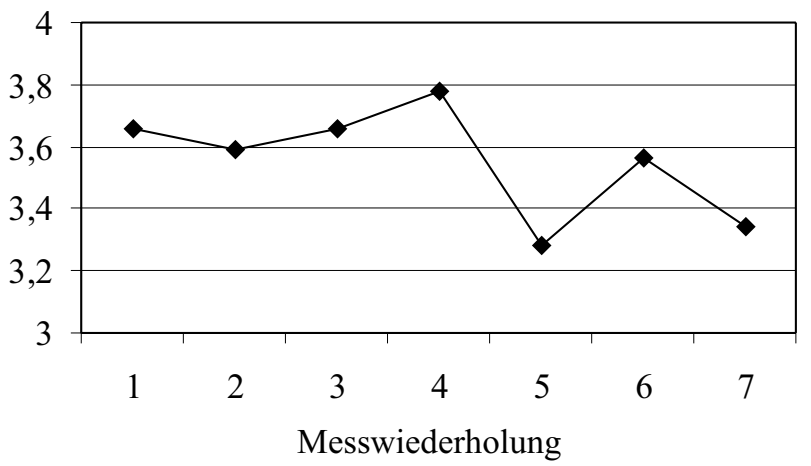

Ich bin im Planspiel heute...

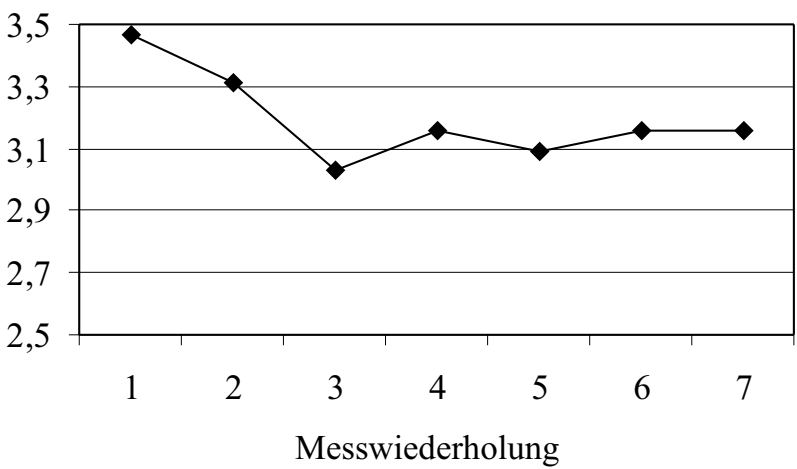

Abbildung 8.13: Ergebnisse des Fragebogens zum situativen Erleben (Item 1-3) 


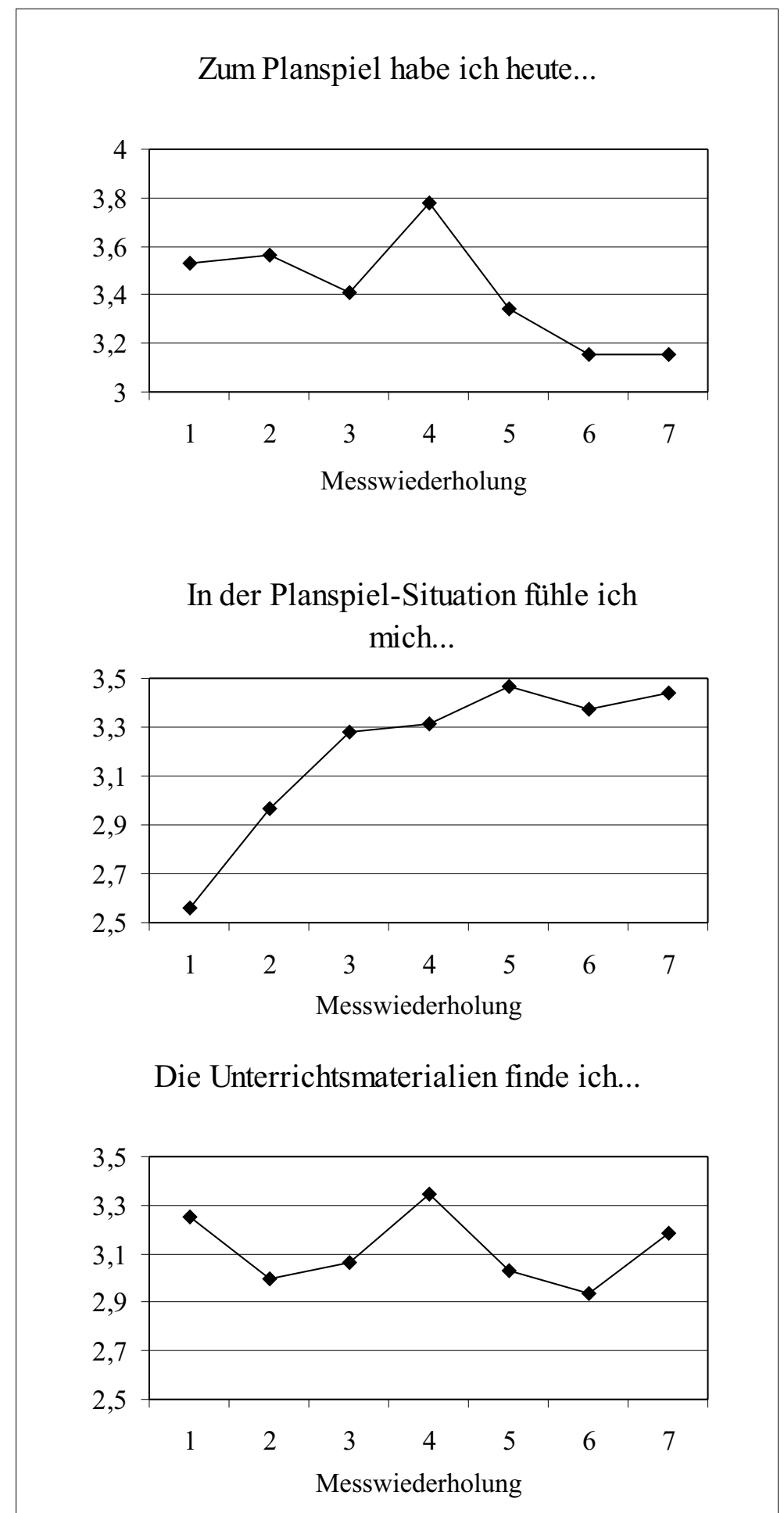

Abbildung 8.14: Ergebnisse des Fragebogens zum situativen Erleben (Item 4-6) 
Bei der ersten Frage nach dem Wohlbefinden an dem entsprechenden Abend waren die Ergebnisse erwartungsgemäf relativ unterschiedlich. Diese Frage diente mehr dazu, die Teilnehmenden zur Reflexion anzuregen, als dass ein Rückschluss auf Merkmale der aktuellen Unterrichtssituation vorgenommen werden sollte. Die Korrelationen liegen entsprechend bei Werten um Null.

Die zweite Frage betraf die Lust oder Unlust, die die Teilnehmenden zum Planspiel an dem aktuellen Abend hatten. Die Mittelwerte liegen über dem Durchschnitt von 3, nehmen allerdings zum Ende hin ab. Ein Grund könnte hier der längere Zeitraum von vier Wochen zwischen der 4. und 5. Entscheidung sowie Weihnachten und der Jahreswechsel zwischen der experimentellen Phase und der Testphase sein. Die Korrelations-Koeffizienten erreichen nur zwischen der 2. und 3. sowie der 3. und 4. Entscheidung Werte bei 0.30, sonst liegen sie niedriger.

Einen ähnlichen Einbruch hat auch das Interesse am Unterricht zu verzeichnen, das mit der dritten Frage erhoben wurde. Das Interesse an der Testphase ist zwar wiederum höher als das Interesse an der fünften Entscheidung der experimentellen Phase. Es werden aber nicht mehr die Werte wie zu Beginn des Unterrichts erreicht. Die Korrelations-Koeffizienten liegt zwischen den letzten drei Messzeitpunkten bei 0.30 bzw. 0.37 , davor bei Werten um Null.

Die vierte Frage nach dem Gefühl der Sicherheit in der Planspiel-Situation bringt zunehmend stabile Ergebnisse, die in der Testphase wieder abnehmen. Die Werte in der Testphase liegen jedoch fast so hoch wie die der experimentellen Phase.

Mit der Über- oder Unterforderung im Planspiel beschäftigte sich die fünfte Frage. Erwartungsgemäß nimmt das Gefühl der Überforderung im Laufe des Planspiels ab. Im Schnitt fühlen sich die Teilnehmenden durch den Unterricht weder über- noch unterfordert. Die individuellen Einschätzungen sind jedoch nicht stabil, schwanken also zwischen den einzelnen Abenden.

Die Bewertung der Unterrichtsmaterialien durch die sechste Frage fällt zu Beginn des Unterrichts eher positiv aus, der Korrelations-Koeffizient zwischen der zweiten und dritten Entscheidung liegt bei 0.61. Insgesamt sind die Einschätzungen bei diesem Item jedoch nicht sehr stabil.

Die folgenden beiden Abbildungen 8.15 bis 8.16 zeigen die Mittelwerte der nächsten sechs Items für die gesamte Stichprobe im Zeitverlauf. 


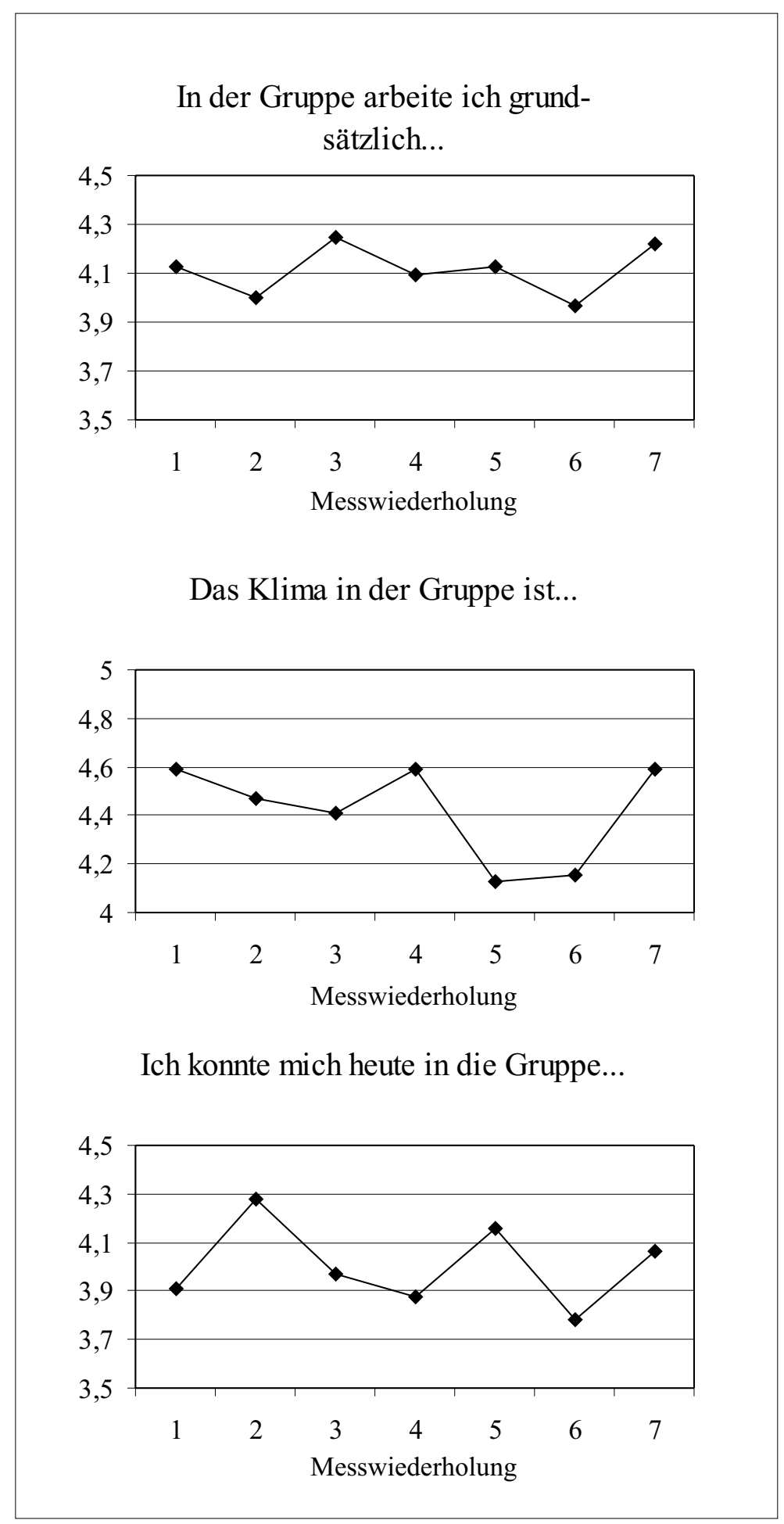

Abbildung 8.15: Ergebnisse des Fragebogens zum situativen Erleben (Item 7-9) 


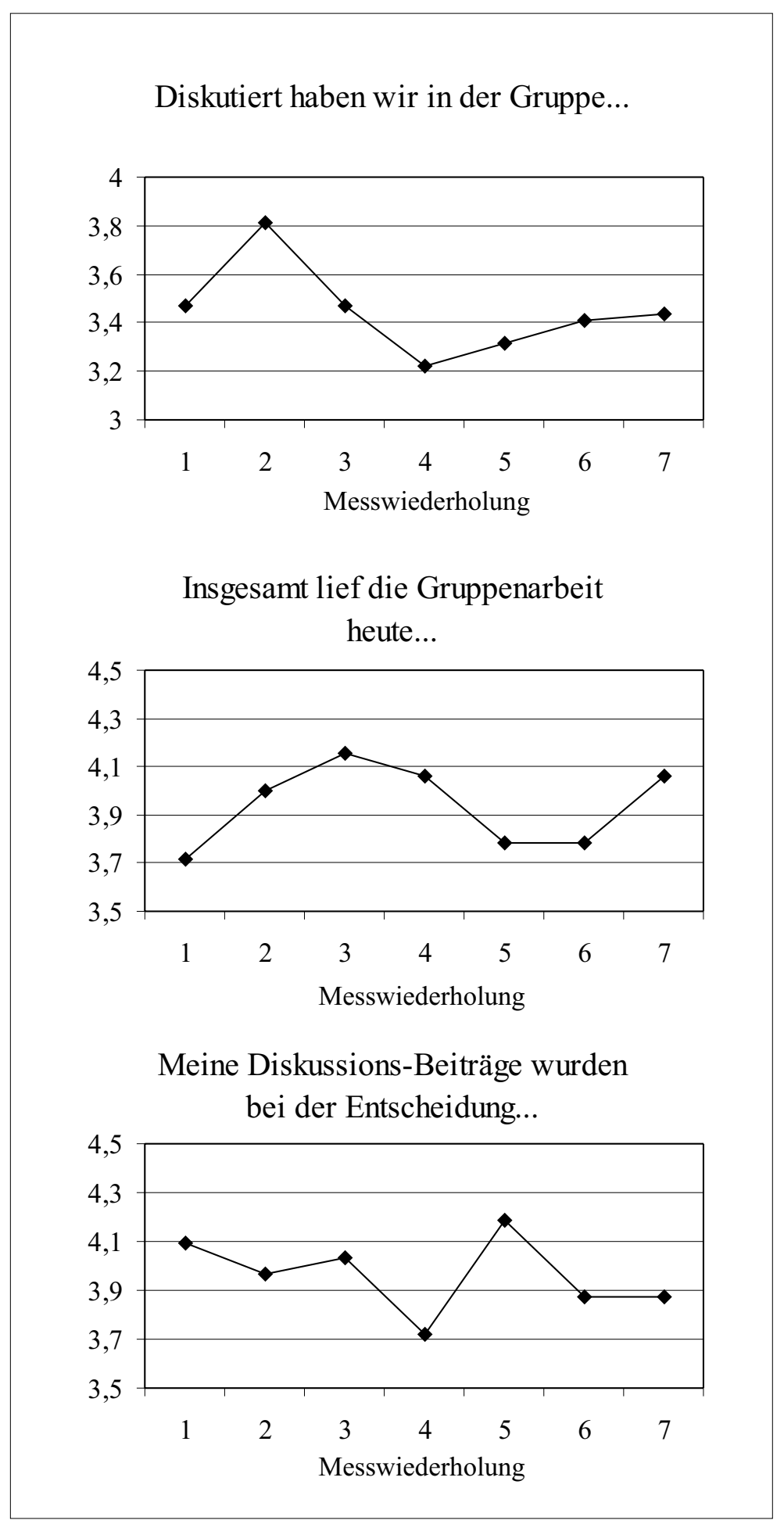

Abbildung 8.16: Ergebnisse des Fragebogens zum situativen Erleben (Item 10-12) 
Die siebte Frage nach der grundsätzlichen Präferenz von Gruppenarbeit bringt stabile Ergebnisse. Die Korrelations-Koeffizienten liegen zwischen 0.31 und 0.60. Die Mittelwerte liegen um den Wert 4 herum, zeigen also durchweg eine eher positive Einstellung gegenüber der Arbeit in Gruppen. Es ist allerdings nicht auszuschließen, dass die Teilnehmenden mit einer eher negativen Einstellung gegenüber Gruppenarbeit nicht oder seltener zum Unterricht erschienen sind.

Die Einschätzung der Intensität der Gruppen-Diskussion in der achten Frage ist sehr instabil, die Korrelations-Koeffizienten liegen unter 0.30. Den Ergebnissen zufolge wurde eher zu viel als zu wenig diskutiert.

Die neunte und zehnte Frage bezogen sich ebenfalls auf die Gruppenarbeit und erreichten vergleichbare Ergebnisse hinsichtlich der Stabilität. Bei der neunten Frage ist die Einschätzung während der gesamten experimentellen Phase stabil, die Koeffizienten liegen zwischen 0.40 und 0.61. Das Klima in der Gruppe änderte sich jedoch nach der vierwöchigen Pause am Ende der experimentellen Phase und zu Beginn der Testphase. Auch hier scheint sich die längere Zeitspanne eher negativ auszuwirken. Insgesamt ist die Einschätzung des Klimas in den Gruppen jedoch positiv. Entsprechend fällt die Bewertung der Gruppenarbeit in der zehnten Frage aus: Zwischen den ersten drei Einschätzungen sind noch Zusammenhänge festzustellen, die Korrelations-Koeffizienten liegen bei 0.59 bzw. 0.44. Danach ist bis auf die beiden Abende in der Testphase kein Zusammenhang zwischen den Messzeitpunkten festzustellen.

Bei der Arbeit in Gruppen ist auch entscheidend, inwieweit sich die/der Einzelne in die Gruppe einbringen kann bzw. inwieweit die Diskussions-Beiträge in der Entscheidung auch berücksichtigt werden. Bei beiden Fragen ist keine Tendenz zu erkennen. Die Werte liegen im Schnitt jedoch eher im positiven Bereich. Die Einschätzungen zwischen der ersten und zweiten Entscheidung liegen mit Korrelations-Koeffizienten von 0.60 bzw. 0.35 im signifikanten Bereich. Bei der zwölften Frage wurde auch zwischen der vierten und fünften Entscheidung ein Koeffizient von 0.46 erreicht. Insgesamt sind die Einschätzungen jedoch eher instabil und von der aktuellen Situation abhängig, was auch zu vermuten war.

Die abschließende Frage 13 bezog sich auf die Sicherheit der Entscheidung. Die Teilnehmenden waren sich über die erste Entscheidung durchschnittlich eher unsicher, was durchaus auch zu erwarten ist. Im Laufe des Planspiels wurden sie sich 
jedoch über die Entscheidungen sicherer. Aber auch hier fällt der fünfte Abend durch einen eher negativen Wert heraus. Entsprechend liegt der KorrelationsKoeffizient zwischen der letzten Entscheidung der experimentellen Phase und der ersten Entscheidung der Testphase bei einem Wert um Null. Die anderen Koeffizienten liegen zwischen 0.43 und 0.57 .

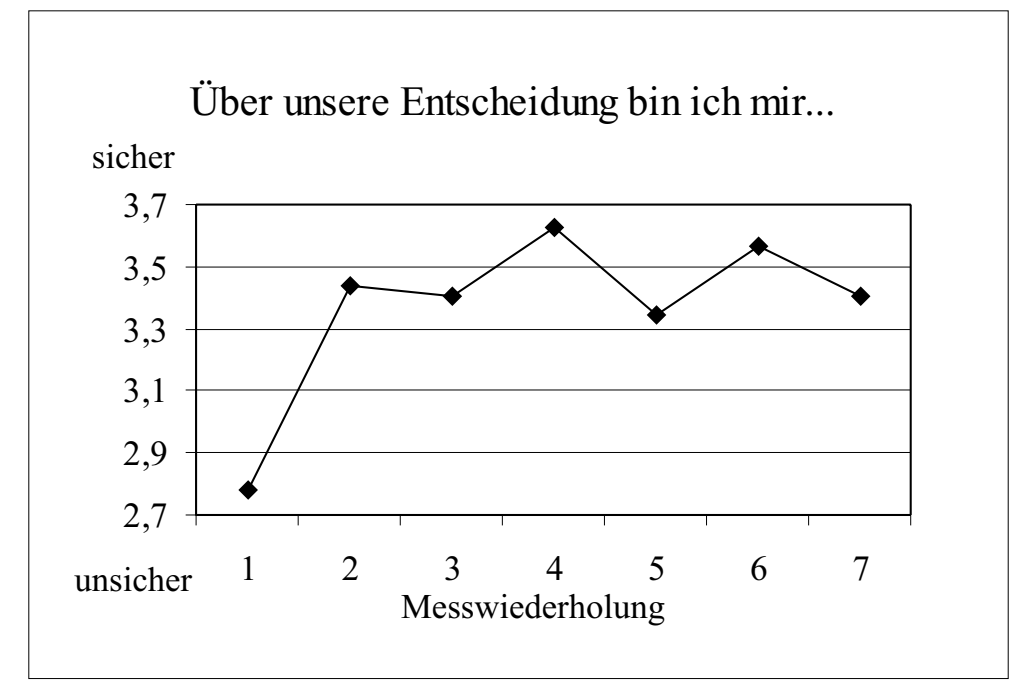

Abbildung 8.17: Ergebnisse des Fragebogens zum situativen Erleben (Item 13)

Im folgenden Abschnitt wird untersucht, ob ein Vergleich der experimentellen Bedingungen eine zusätzliche Erklärung der Schwankungen in den Einschätzungen der Teilnehmenden bringt.

\section{Vergleich der experimentellen Bedingungen}

Bei vier Fragen ergaben sich hinsichtlich der Beantwortung signifikante Unterschiede zwischen den experimentellen Bedingungen, die im Folgenden näher betrachtet werden (Abbildung 8.18).

Die Teilnehmenden der modularen Bedingung fühlen sich in der EntscheidungsSituation am ersten Abend deutlich sicherer als die der herkömmlichen Bedingung. Bei den folgenden Entscheidungen liegen jedoch die Einschätzungen der Teilnehmenden der herkömmlichen Bedingung teilweise über denen der modula- 


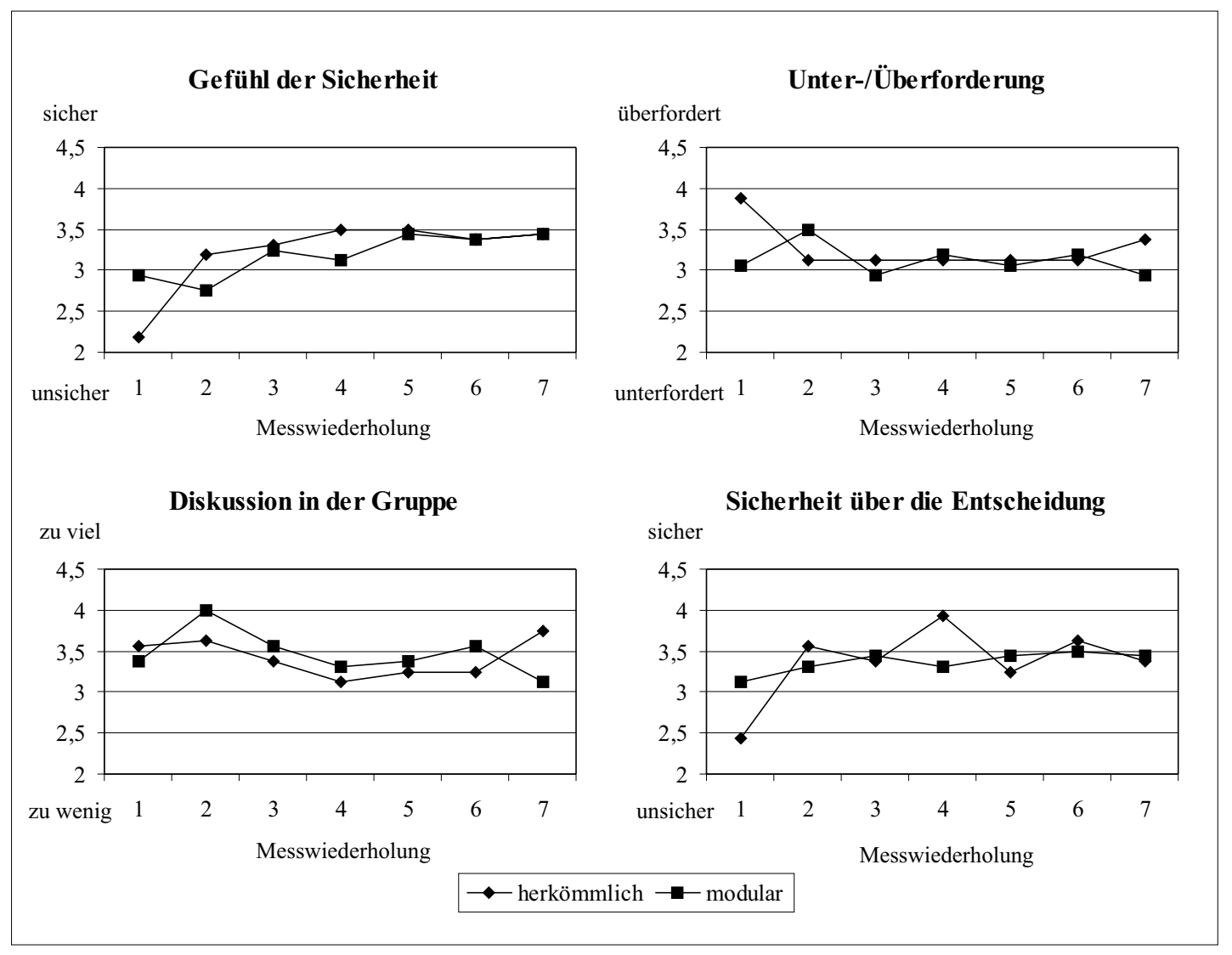

Abbildung 8.18: Vergleich der experimentellen Bedingungen: Einsatz des Fragebogens zum situativen Erleben

ren Bedingung. In der Testphase erreichen sie sogar gleiche Werte. Insgesamt sind die Werte jedoch nicht sehr hoch. ${ }^{45}$

Hinsichtlich der Über- bzw. Unterforderung lassen sich ähnliche Aussagen treffen: ${ }^{46}$ Während sich die Teilnehmenden der modularen Bedingung in der ersten Entscheidung weder über- noch unterfordert fühlten, waren die Teilnehmenden der herkömmlichen Bedingung doch eher überfordert. Auch hier gleichen sich die Mittelwerte im Verlauf des Planspiels an. Nur in der letzten Entscheidung der Testphase fühlen sich die Teilnehmenden der herkömmlichen Bedingung mit

$\begin{array}{ll}45 & \mathrm{~F}=2.27, \mathrm{p}=0.0385, \mathrm{df}=180 \\ 46 & \mathrm{~F}=3.43, \mathrm{p}=0.0031, \mathrm{df}=180\end{array}$ 
der Entscheidung für zwei Perioden ${ }^{47}$ eher überfordert als die Teilnehmenden der modularen Bedingung.

Bei der Bewertung der Gruppen-Diskussion scheinen die Teilnehmenden der modularen Bedingung nach eigener Einschätzung eher zu viel zu diskutieren. Die Mittelwerte liegen bei der herkömmlichen Bedingung bis auf die erste und letzte Entscheidung unter den Werten der modularen Bedingung. Dieses Verhältnis kehrt sich in der letzten Entscheidung jedoch um. Hier scheint in den Gruppen der herkömmlichen Bedingung der Diskussionsbedarf zu steigen. ${ }^{48}$

Die Ergebnisse hinsichtlich des Gefühls der Sicherheit in der aktuellen PlanspielSituation spiegeln sich in denen zur Sicherheit über die getroffene Entscheidung wider. ${ }^{49}$ Der Verlauf der Einschätzungen ist bei den Teilnehmenden der modularen Bedingung nicht so sprunghaft wie bei denen der herkömmmlichen Bedingung. In der Testphase gleichen sich die Werte an.

Weiterhin wurden die Auswirkungen des Einsatzes des Fragebogens auf die Ergebnisse in der Testphase mit Hilfe einer mehrfaktoriellen Varianzanalyse mit Messwiederholungen untersucht. Es ergaben sich keine signifikanten Ergebnisse. Der Einsatz des Fragebogens hat jedoch teilweise einen Einfluss auf die Prä-PostErgebnisse, die im nächsten Kapitel dargestellt werden.

\footnotetext{
$47 \quad$ Vgl. Abschnitt 4.9.2.

$48 \mathrm{~F}=2.03, \mathrm{p}=0.0642, \mathrm{df}=180$.

$49 \mathrm{~F}=2.36, \mathrm{p}=0.0322, \mathrm{df}=180$.
} 


\section{Ergebnisse des Prä- und Post- tests}

\subsection{Vorbemerkungen}

In diesem Kapitel erfolgt eine Darstellung der Ergebnisse, die mit den Instrumenten zum betriebswirtschaftlichen Wissen im Prä- und Posttest erhoben wurden. Dabei werden zunächst in Abschnitt 9.2 die Ergebnisse der gesamten Stichprobe betrachtet. Dabei kamen ein Multiple-Choice-Fragebogen zum Faktenwissen, eine Netzwerk-Erhebung zum Zusammenhangswissen sowie eine Fallstudie zur Transferleistung zum Einsatz. ${ }^{1}$

Nachfolgend wird nach den experimentellen Bedingungen der Planspiel-Phase differenziert (Abschnitt 9.3) und der Einfluss des Einsatzes der zusätzlichen Fragebögen sowie die Wechselwirkung der Planspiel-Durchführungen mit den beiden Fragebögen untersucht (Abschnitt 9.4). Zum Teil werden die Ergebnisse auf Grund der Datenlage und der auszuwertenden Fragestellung deskriptiv dargestellt, zum Teil wurden mittels Varianzanalysen mit Messwiederholung ausgewertet. $^{2}$

1 Zur Darstellung der Erhebungsinstrumente vgl. Kapitel 5.

2 Vgl. Abschnitt 7.5. 


\subsection{Betrachtung der gesamten Stichprobe}

\subsubsection{Multiple-Choice-Fragebogen}

Der eingesetzte Multiple-Choice-Fragebogen diente zur Erhebung des betriebswirtschaftlichen Faktenwissens. ${ }^{3}$ Zur Bewertung des Lernerfolgs wird die Anzahl der richtigen Antworten im Prä- und Posttest ausgezählt und unter individueller Bezugsnorm paarweise verglichen. ${ }^{4}$ In Abbildung 9.1 ist die Anzahl des Prätests gegen die des Posttests abgetragen $(n=45)$. Zur Verdeutlichung wurde die $45^{\circ}-$ Linie eingezeichnet:

- Punkte oberhalb dieser Linie markieren Teilnehmende, die im Posttest weniger richtige Antworten gaben als im Prätest. Dies war bei $27 \%$ der Teilnehmenden der Fall.

- Punkte, die auf der Linie liegen, zeigen an, dass die Anzahl der richtigen Anworten zu beiden Testzeitpunkten gleich groß war (20\%).

- Bei mehr als der Hälfte der Teilnehmenden war die Anzahl der richtigen Antworten jedoch im Posttest größer als im Prätest (53\%).

Die Stärke der Punkte zeigt die Anzahl der Teilnehmenden an, die diese Kombination der richtigen Antworten hatten. Das Ergebnis spiegelt insgesamt zum Teil auch die eher geringe Reliabilität des Instruments wider. ${ }^{5}$

Eine einfaktorielle Varianzanalyse brachte zwar ein signifikantes Ergebnis: ${ }^{6}$ Die Teilnehmenden machten im Posttest weniger Fehler im Fragebogen als im Prätest. Die Differenz beträgt jedoch nur 5,8 Prozentpunkte, so dass der Unterschied wenig praktische Bedeutsamkeit hat. Dies stützt die aufgestellte Vermutung, nach der von einem eher geringen Wissenszuwachs augegangen wurde. ${ }^{7}$

\footnotetext{
3 Vgl. Abschnitt 5.2.

4 Vgl. Abschnitt 4.6.3.

5 Vgl. Abschnitt 5.2.3

6 Mittelwerte: Prätest 37,2\%, Posttest 31,4\% ( $\mathrm{F}=6.09, \mathrm{p}=0.0158, \mathrm{df}=80)$.

7 Vgl. Abschnitt 4.8.4.
} 


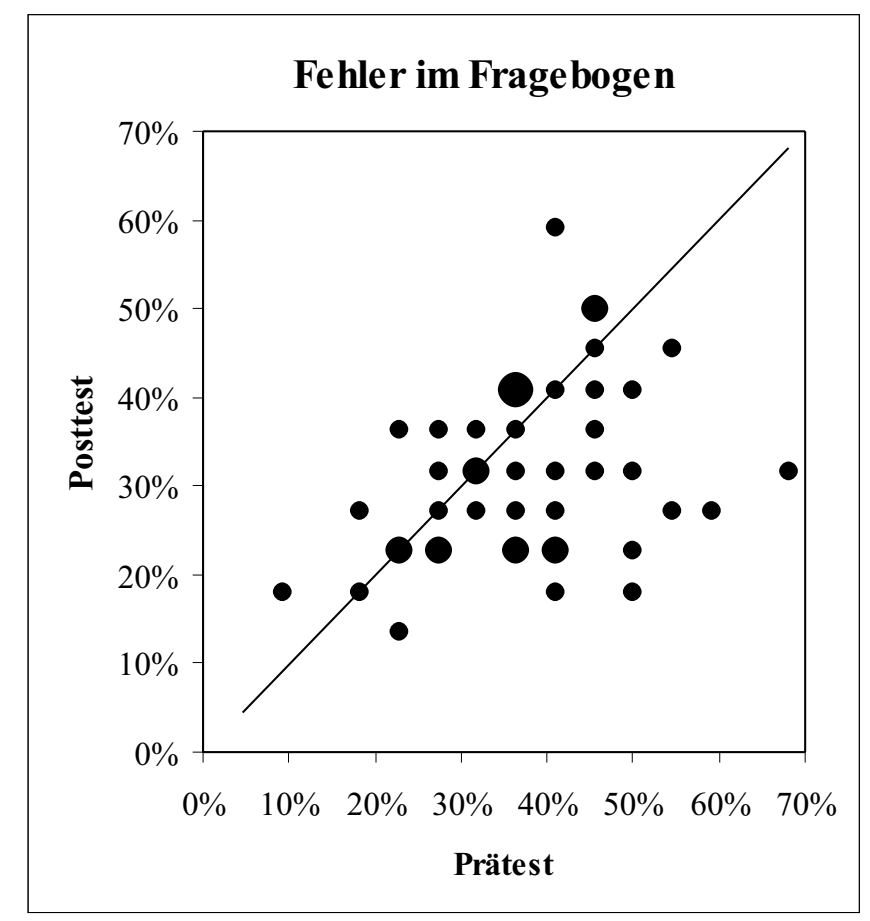

Abbildung 9.1: Prä-Post-Vergleich: Anzahl richtiger Antworten im Fragebogen

\subsubsection{Netzwerk-Erhebung}

\section{Erste Auswertungen}

Die Konstruktion der Netzwerke wurde zur Erhebung des Zusammenhangswissens der Teilnehmenden eingesetzt. Zur Beurteilung kann zunächst die Größe der Netzwerke, die sich in der Anzahl der Propositionen zeigt, herangezogen werden. Der Lernerfolg unter individueller Bezugsnorm kann auch hier durch paarweisen Vergleich der Ergebnisse aus dem Prä- und Posttest bestimmt werden. ${ }^{8}$ Wie Abbildung 9.2 zeigt, bilden die meisten Teilnehmenden eine höhere Anzahl Propositionen im Posttest als im Prätest (links von der $45^{\circ}$-Linie). Nur neun Teilnehmende konstruieren im Posttest weniger Propositionen als im Prätest. ${ }^{9}$ Der

\footnotetext{
8 Vgl. Abschnitt 4.6.3.

9 Dabei kann jedoch auch die Tatsache einen Einfluss haben, dass insbesondere im Prätest nicht alle gebildeten Propositionen aufgrund mangelnder Beschriftung oder fehlender Pfeile erfasst werden konnten.
} 
Zuwachs ist statistisch signifikant und beträgt mit durchschnittlich 6,13 Propositionen fast ein Drittel. ${ }^{10}$

Dies zeigt sich auch in der Ordnung der Netzwerke: ${ }^{11}$ Im Prätest wurde durchschnittlich weniger als die Hälfte der vorgegebenen Konzepte benutzt, im Posttest immerhin knapp zwei Drittel. Dabei muss jedoch der Effekt der Messwiederholung beachtet werden. ${ }^{12}$

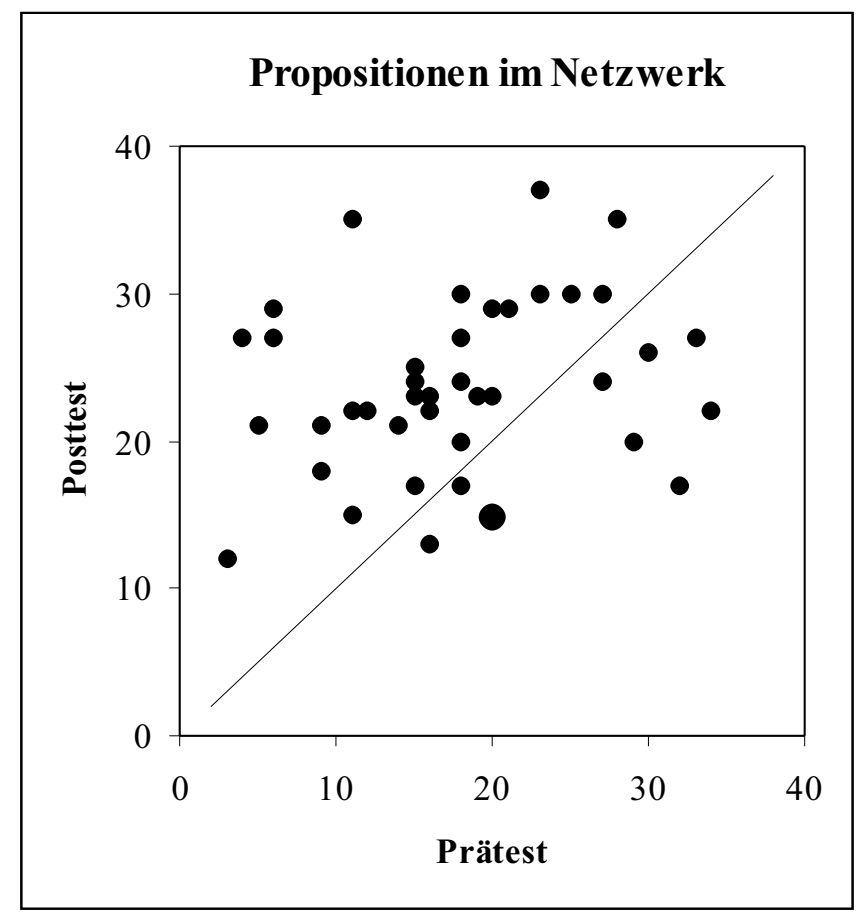

Abbildung 9.2: Prä-Post-Vergleich: Größe der Netzwerke

Neben der isolierten Betrachtung der Größe und der Ordnung der Netzwerke kann auch der Zusammenhange zwischen beiden Kennzahlen betrachtet werden. ${ }^{13}$ Die folgende Abbildung 9.3 zeigt sehr anschaulich, wie sich sowohl die Anzahl der benutzten Konzepte als auch die Anzahl der gebildeten Propositionen im Posttest im Vergleich zum Prätest erhöht hat.

10 Mittelwerte: Prätest 17,82, Posttest 23,95 ( $\mathrm{F}=14.39, \mathrm{p}=0.0003, \mathrm{df}=80)$.

11 Mittelwerte: Prätest 15,63, Posttest 21,87 ( $\mathrm{F}=20.50, \mathrm{p}=0.0000, \mathrm{df}=80)$.

12 Vgl. Abschnitt 5.3.3.

13 Vgl. Wilbers (Netzwerke 1997), S. 68. 


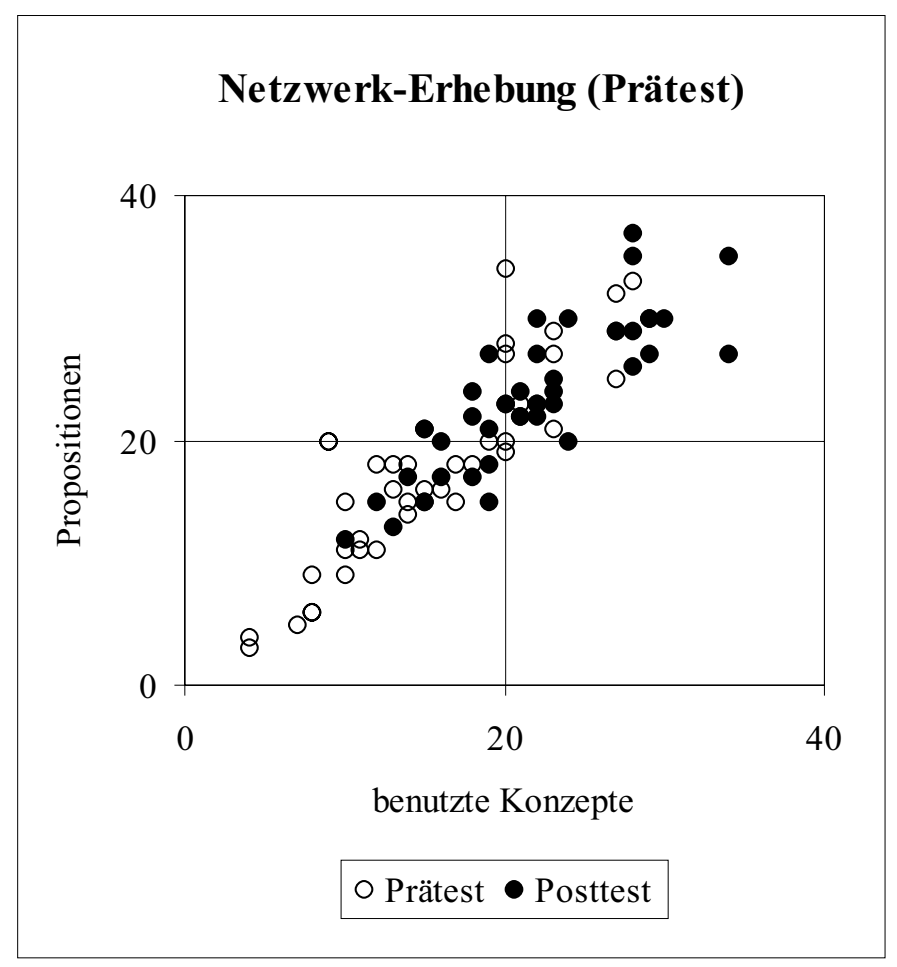

Abbildung 9.3: Prä-Post-Vergleich: Größe und Ordnung der Netzwerke

Weiterhin kann die Dichte der Netzwerke bestimmt werden: ${ }^{14}$ Sie berechnet sich aus dem Quotienten zwischen der Anzahl der Kanten und der Anzahl der möglichen Kanten, wobei sich auf ein Referenznetz als Leistungskriterium bezogen wird. Hier wird ein 'Expertennetz' herangezogen, das als Modales Netzwerk durch Aggregation von Netzwerken entsteht, die mehrere Diplom-Kaufleute konstruierten. ${ }^{15}$ Sie bildeten zusammen 307 Propositionen, davon 219 verschiedene. Die Hälfte von ihnen benutzte alle 35 Konzepte, zwei ließen eins aus, eine/r benutzte nur 31 Konzepte. Dabei wurde das Konzept 'Forderungen/Verbindlichkeiten' zweimal nicht benutzt, die Konzepte 'Lageraufwand für Produkte', 'Rücklagen', 'Terminanlage' und 'Zinsaufwand/-ertrag' jeweils einmal nicht. Das Referenznetz enthält 52 Propositionen, also alle Propositionen, die von mindestens zwei Personen gebildet wurden. Diese Größe erreichten die Teilnehmenden der Untersuchung

14 Vgl. Wilbers (Netzwerke 1997), S. 63-65.

15 Hierbei ist den sechs Mitarbeiterinnen und Mitarbeitern des Instituts für betriebswirtschaftliche Produktions- und Investitionsforschung, Abteilung für Unternehmensplanung, der Universität Göttingen, für ihre Hilfe im Sommer 1998 zu danken. 
mit ihren Netzwerken nicht. Das größte Netzwerk des Posttests bestand aus 40 Propositionen.

Da die Dichte aus der Größe der Netzwerke durch einfache Transformation mittels Division hervorgeht, ist der Effekt vergleichbar: Während im Prätest die Dichte durchschnittlich nur bei $34,3 \%$ liegt, erreicht sie im Posttest 46,0\%. Es kann sicherlich nicht davon ausgegangen werden, dass die Teilnehmenden einer nebenberuflichen Fortbildung durch den einsemestrigen Einsatz eines Unternehmensplanspiels den gleichen Grad der Vernetztheit des betriebswirtschaftlichen Wissens erreichen wie Diplom-Kaufleute nach mehreren Jahren Vollzeit-Studium. Die Steigerung von einem Drittel auf die Hälfte ist jedoch bedeutsam. Insgesamt kann die Hypothese aus Abschnitt 4.8.4 durch die Ergebnisse als bestätigt angesehen werden.

\section{Modale Netzwerke}

Zur weiteren Beurteilung werden die Modalen Netzwerke des Prä- und Posttests miteinander verglichen. Ein Modales Netzwerk entsteht durch Aggregation mehrerer Netzwerke einer Gruppe, in diesem Fall aller konstruierter Netzwerke des Prätests sowie des Posttests. Die Anzahl der Propositionen, aus denen ein Modales Netzwerk besteht, orientiert sich an der durchschnittlichen Anzahl gebildeter Propositionen in der Gruppe. ${ }^{16}$ Dies entspricht einer sozialen Bezugsnorm, da ausschließlich auf die Leistungen der Gruppe Bezug genommen wird. ${ }^{17}$ Abbildung 9.4 (S. 260) zeigt das Modale Netzwerk des Prätests, Abbildung 9.5 (S. 261) das des Posttests.

Die Konzepte, die in den beiden Abbildungen in den Kästchen stehen, sowie die Relationen, die durch Pfeile dargestellt werden, wurden in dem Instrument vorgegeben. ${ }^{18}$ Die Stärke der Pfeile spiegelt die Häufigkeit der Nennung dieser Proposition wider. ${ }^{19}$

16 Vgl. Becker, Oldenbürger \& Piehl (Motivation 1987), S.464; auch Oldenbürger (Datenanalyse 1996). Zur Auswertung wurde das Programm nwz1 herangezogen; vgl. OLDENBÜRgER (Netz-Werk-Zeug 1992).

17 Vgl. Abschnitt 4.6.3.

18 Vgl. Anhang A.12.

19 Die Legende zu den Modalen Netzwerken befindet sich im Anhang A.13. 
Auf den ersten Blick erscheint das Modale Netzwerk des Posttests aufgrund der größeren Anzahl Propositionen gegenüber dem des Prätests unübersichtlicher, ${ }^{20}$ denn auch die Anzahl der benutzten Konzepte hat sich erhöht. ${ }^{21}$ Bis auf die 'Maschinenauslastung' kommen alle Konzepte des Modalen Netzwerks des Prätests auch im Modalen Netzwerk des Posttests vor. Acht Propositionen des Prätests finden sich im Netzwerk des Posttests wieder, drei davon mit dem Konzept 'Absatz'.

Während im Modalen Netzwerk des Prätests die Konzepte bis auf eine isolierte Proposition zusammenhängen, sind im Netzwerk des Posttests drei Bereiche identifizierbar: der Personalbereich, der Absatz- und Beschaffungsbereich sowie der Produktionsbereich. Sie werden erst durch Propositionen verbunden, die nicht mehr in dem Modalen Netzwerk auftauchen und eine Häufigkeit von weniger als $15 \%$ haben.

Die häufigste Proposition im Prätest ist "Der Umsatz beeinflusst den Gewinn“. Sie wurde von $22 \%$ der Teilnehmenden konstruiert. Im Posttest konstruierten nur noch $18 \%$ diese Proposition. Der 'Umsatz' bleibt jedoch im Zentrum des Interesses der Teilnehmenden: Sechsmal ist er mit anderen Konzepten verbunden und ist damit der Schwerpunkt des Netzwerks. Im Prätest hingegen liegt das Gewicht auf der 'Absatzmenge' und dem 'Gewinn'.

Insgesamt ist festzustellen, dass die Zusammenhänge im Posttest gegenüber dem Prätest differenzierter dargestellt werden. Die Proposition 'Der Verkaufspreis der Produkte beeinflusst den Gewinn', die im Prätest von $15 \%$ der Teilnehmenden genannt wurde, wird im Posttest durch 'Der Verkaufspreis beeinflusst den Umsatz' und 'Der Umsatz beeinflusst den Gewinn' präzisiert. Die isolierte Proposition 'Der Einkaufspreis der Rohstoffe beeinflusst die Herstellungskosten' wird im Posttest durch 'Die Herstellungskosten beeinflussen den Verkaufspreis der Produkte' mit dem Absatzbereich des Netzwerks verbunden. Der Beschaffungsbereich wird durch die Ursachen-Kette von 'Rohstoffbeschaffung', 'Rohstoffbestand' und 'Lageraufwand für Rohstoffe' stärker betont. Aber auch im Absatzbereich sind die Konzepte insgesamt wesentlich stärker vernetzt als noch im Modalen Netzwerk des Prätests.

\footnotetext{
20 Propositionen in den Modalen Netzwerken: Prätest $17(n=41)$, Posttest $26(n=45)$.

21 Anzahl der Konzepte in den Modalen Netzwerken: Prätest 16, Posttest 24.
} 


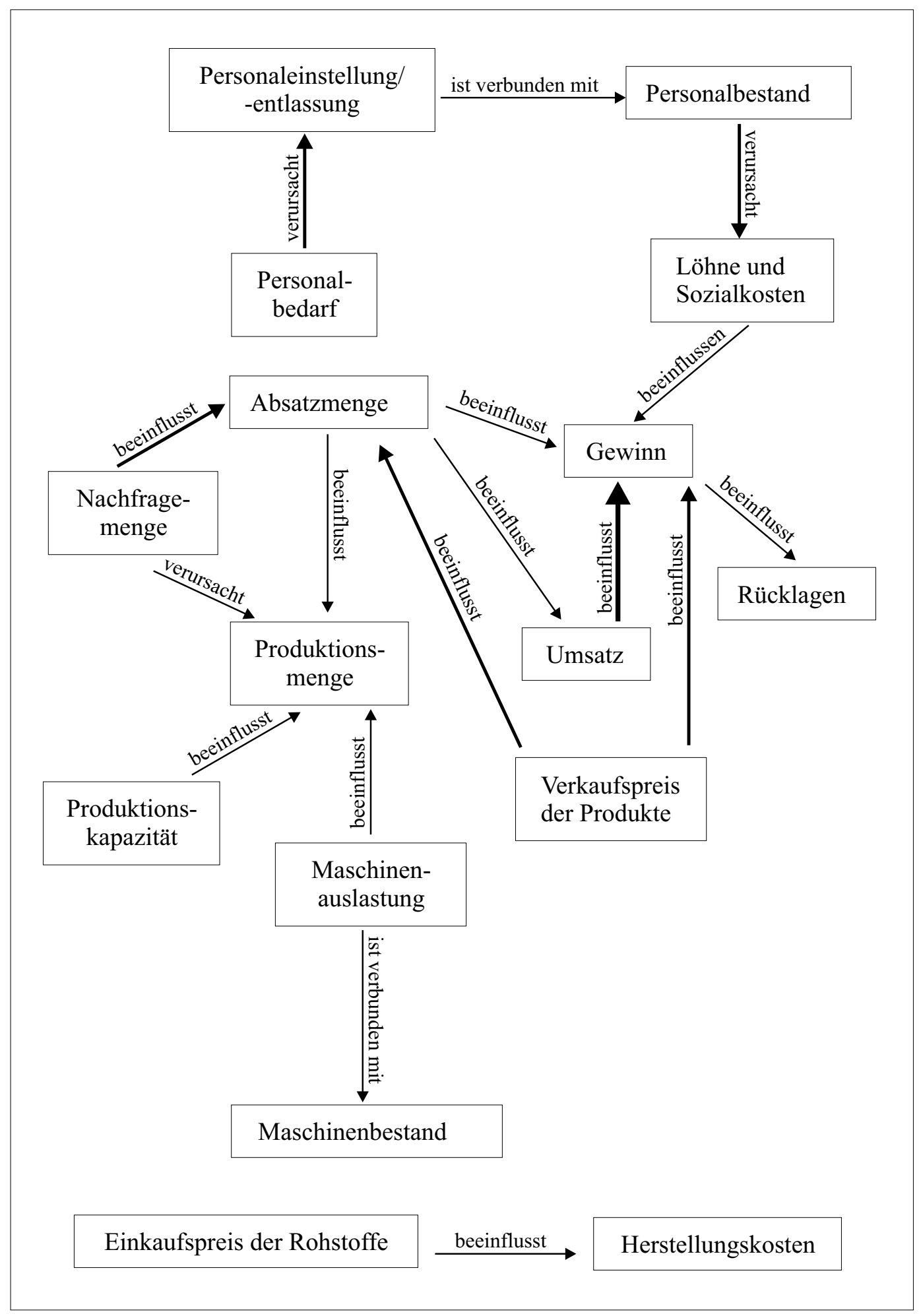

Abbildung 9.4: Prä-Post-Vergleich: Modales Netzwerk des Prätests 


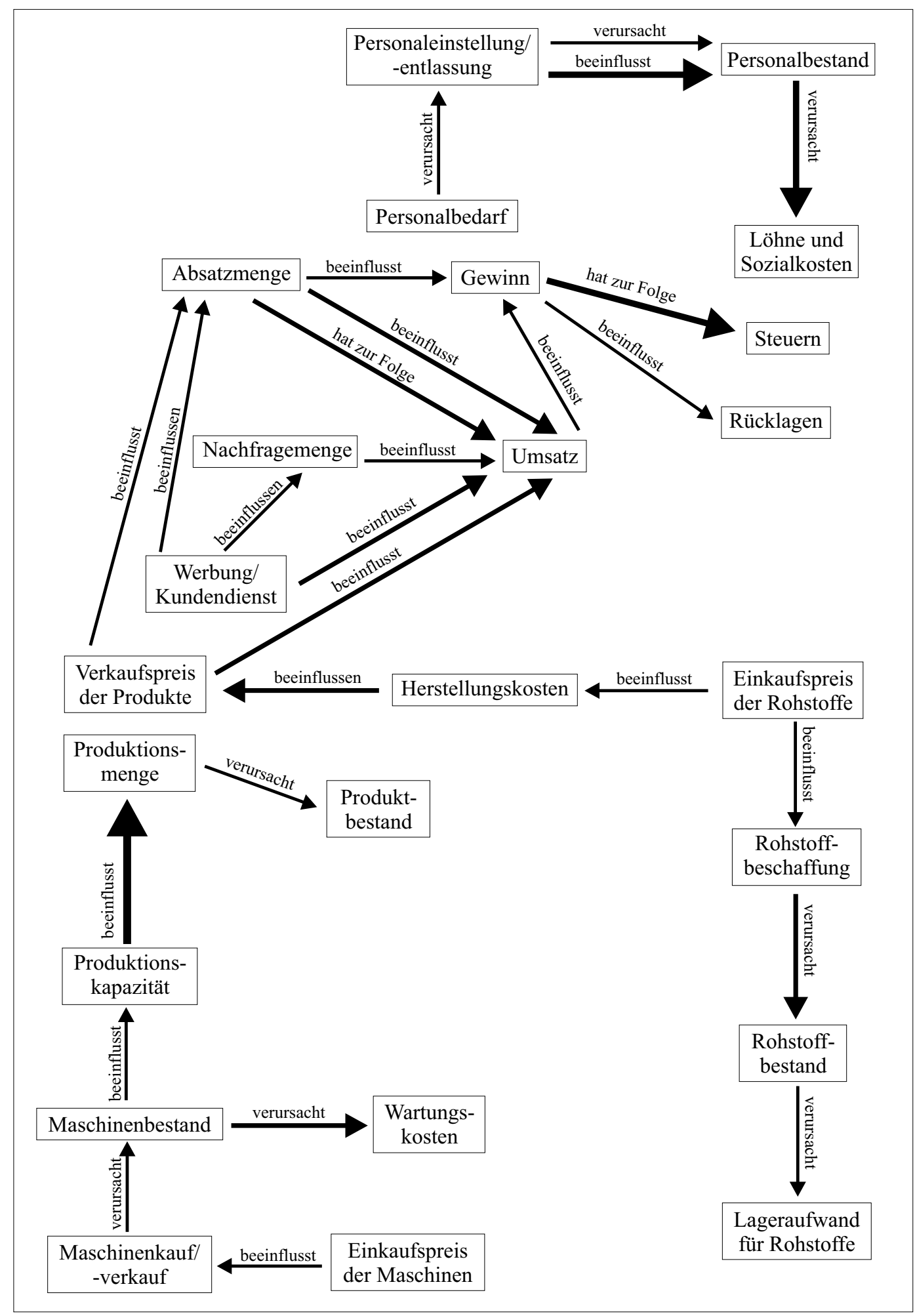

Abbildung 9.5: Prä-Post-Vergleich: Modales Netzwerk des Posttests 


\section{Vergleich mit Referenznetz}

Neben der eher grafischen Analyse der Netzwerke kann auch eine inhaltliche Bewertung durchgeführt werden. Dazu wird die Abbildungsleistung der Teilnehmenden beurteilt, indem die Anzahl der Propositionen des Referenznetzes gezählt werden, die sich in den individuellen Netzwerken wiederfinden. Dies entspricht der sachlichen Bezugsnorm, da eine Orientierung am Referenznetz als Kriterium erfolgt. Das Lernziel wäre entsprechend eine Annäherung an das Referenznetz. ${ }^{22}$ Die sechs Diplom-Kaufleute, aus deren Netzwerken das Referenznetz erstellt wurde, erreichen eine Abbildungsleistung von $40 \%$ bis $52 \%$. Das bedeutet, dass aus jedem ihrer Netzwerke etwa die Hälfte der Propositionen in das Referenznetz übernommen wurde. Abbildung 9.6 zeigt die 22 Propositionen mit einer Häufigkeit von drei und mehr. ${ }^{23}$

Im Prätest haben acht Teilnehmende keine der Proposition aus dem Referenznetz in ihrem Netzwerk. Im Posttest kommt dies nur noch bei einem Netzwerk vor. Die Abbildungsleistung liegt im Prätest bei durchschnittlich 8,62\%, im Posttest bei $11,02 \% .^{24}$

\footnotetext{
22 Vgl. Abschnitt 4.6.3.

23 Die Darstellung des gesamten Referenznetzes mit 52 Propositionen wäre zu unübersichtlich. $24 \mathrm{~F}=16.96, \mathrm{p}=0.0002, \mathrm{df}=40$.
} 


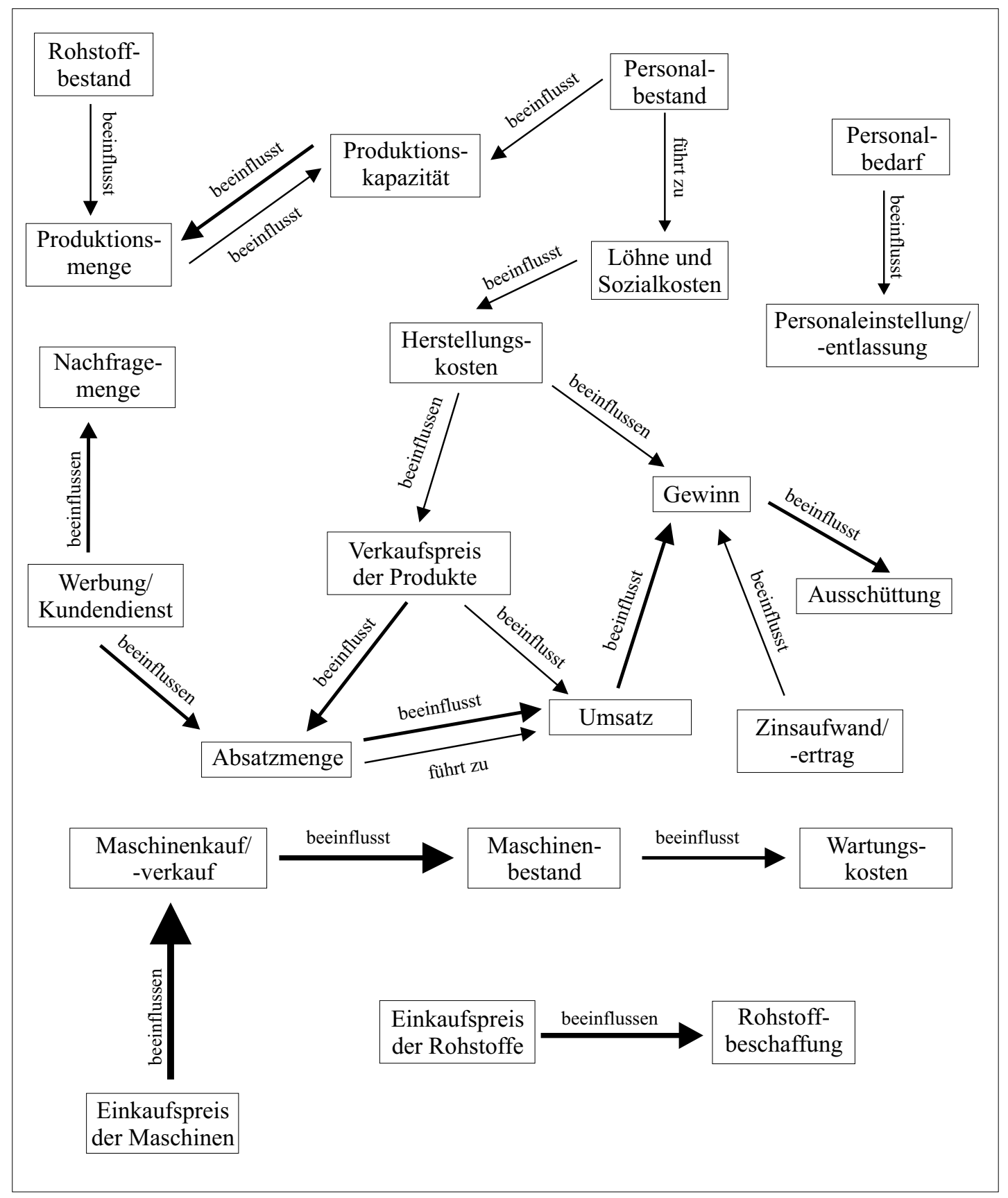

Abbildung 9.6: Referenznetz 


\subsubsection{Fallstudie}

Die Fallstudie wurde zur Beurteilung des methodischen Arbeitens sowie der Transferleistung eingesetzt. Bei der Auswertung wird zunächst die Zielgröße Gewinn herangezogen $(n=41)$. In Abbildung 9.7 sind die erzielten Gewinne im Prätest denen des Posttests bei gleicher Skalierung gegenüber gestellt. Teilnehmende, die im Posttest durch ihre Entscheidungen einen höheren Gewinn erzielten als im Prätest, erscheinen als Punkt links von der $45^{\circ}$-Linie $(73 \%)$. Einen geringeren Gewinn im Posttest erreichten elf Teilnehmende. Bei vier Teilnehmenden bleibt der Gewinn in etwa gleich. Der Unterschied ist statistisch signifikant ${ }^{25}$ und in diesem Fall auch praktisch bedeutsam, da sich der Gewinn im Durchschnitt verdoppelt.

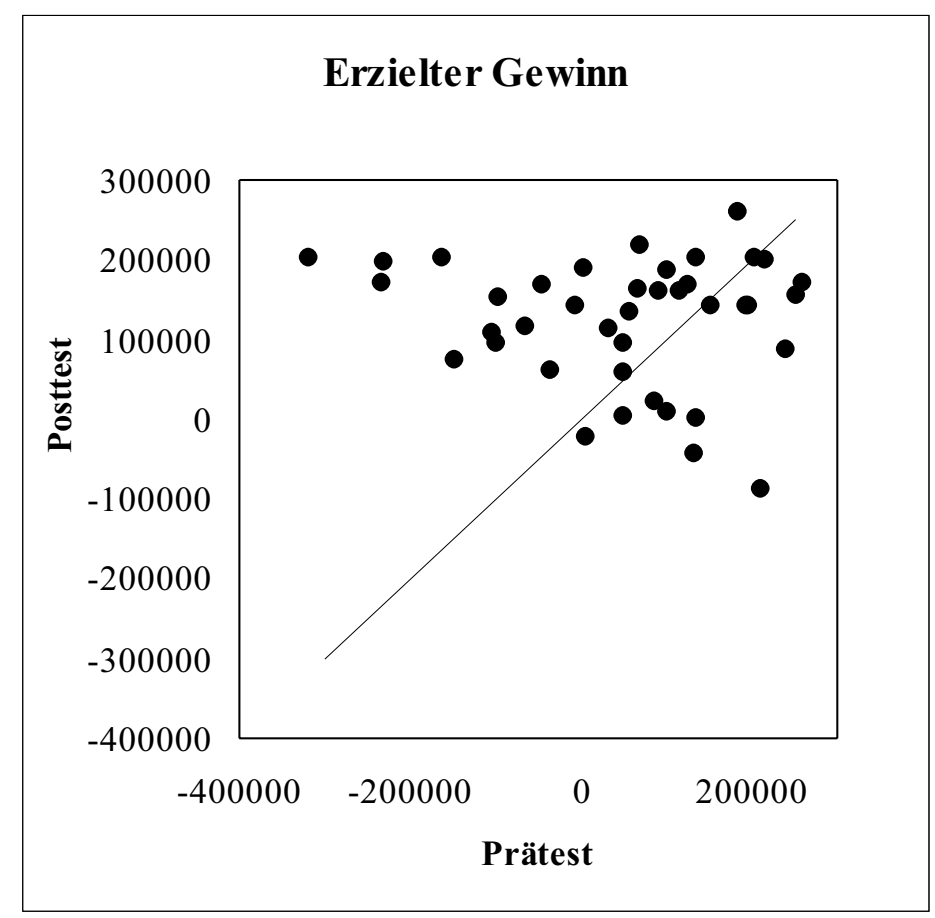

\section{Abbildung 9.7: Prä-Post-Vergleich: Gewinne in der Fallstudie}

Zur genaueren Betrachtung der Leistungen in der Fallstudie kann der Auslastungsgrad der Maschinen herangezogen werden. Die Teilnehmenden sind bereits im Prätest relativ gut in der Lage, die Auslastung zu planen. Der Unterschied

25 Mittelwerte: Prätest 60.866, Posttest 124.300 (F=7.92, p=0.0061, df=80.) 
der Mittelwerte zwischen Prä- und Posttest ist dennoch signifikant. ${ }^{26}$ Im Posttest lasten nur noch knapp ein Fünftel der Teilnehmenden die Maschinen nicht voll aus. Insgesamt 15 Teilnehmende planen sowohl im Prä- als auch im Posttest einen Auslastungsgrad von $100 \%$. Wie im Planspiel EpUS ${ }^{27}$ ist es auch in der Fallstudie sinnvoll, die Kapazität der vorhandenen Maschinen voll auszulasten. In der Fallstudie wird zwar ein Rückgang der Nachfrage prognostiziert, die bei voller Kapazitäts-Auslastung produzierten Mengen können jedoch durch entsprechende Gestaltung der Verkaufspreise und der Werbung am Markt abgesetzt werden.

Ein signifikanter Unterschied ergibt sich auch hinsichtlich der Absatzmengen, die in Abbildung 9.8 dargestellt sind: Zwei Dritteln der Teilnehmenden gelang es im Posttest besser, den Absatz durch entsprechende Entscheidungen im MarketingBereich zu fördern. Der Mittelwert-Unterschied ist allerdings nicht sehr groß. ${ }^{28}$

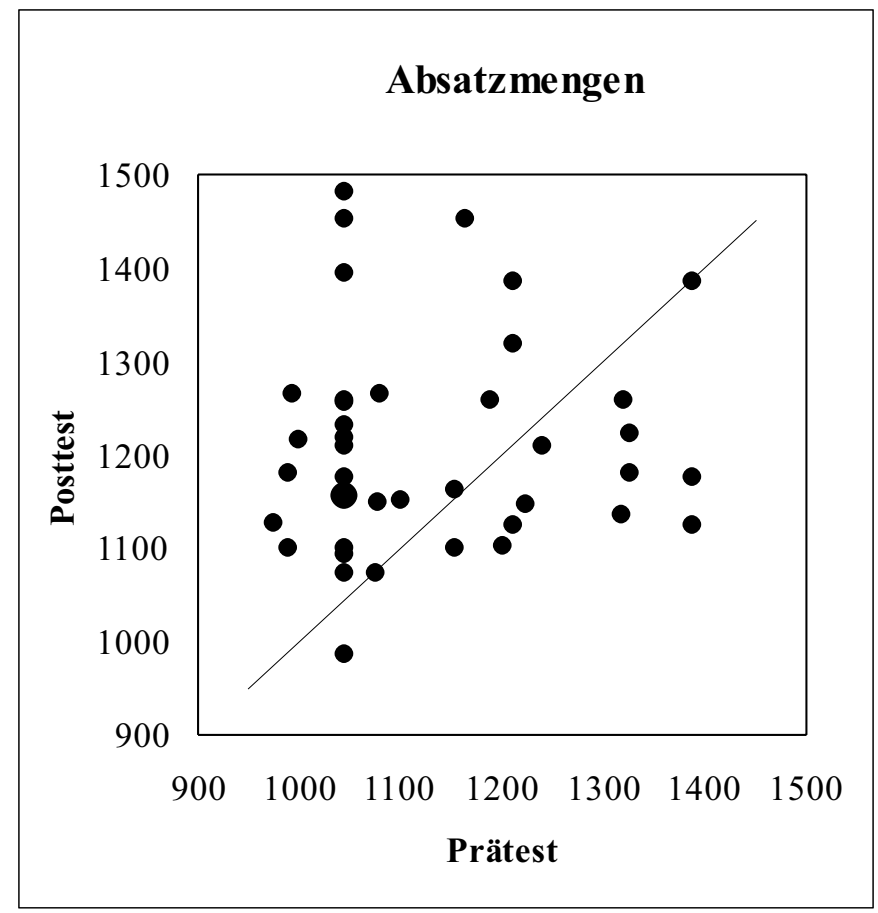

Abbildung 9.8: Prä-Post-Vergleich: Absatzmengen in der Fallstudie

\footnotetext{
26 Mittelwerte: Prätest 88,87\%, Posttest 96,16\% $(\mathrm{F}=8.43, \mathrm{p}=0.0048, \mathrm{df}=80)$.

27 Vgl. Abschnitt 6.2.

28 Mittelwerte: Prätest 1.130, Posttest $1.210(\mathrm{~F}=8.90, \mathrm{p}=0.0038, \mathrm{df}=80)$.
} 
Insgesamt lässt sich feststellen, dass die Teilnehmenden nach dem Einsatz des Unternehmensplanspiels besser in der Lage sind, durch methodisches Arbeiten bessere Leistungen in der Fallstudie zu erbringen. Dies bestätigt die in Abschnitt 4.8.4 aufgestellte Hypothese.

\section{Investitionsrechnung in der Fallstudie}

In der Fallstudie kann der Bestand von zehn Maschinen des Typs S 500 durch Investitionen oder Desinvestitionen der geplanten Produktionsmenge angepasst werden. Für Investitionen stehen zwei verschiedene Maschinentypen zur Auswahl, die durch folgende Zahlen gekennzeichnet sind: ${ }^{29}$

\begin{tabular}{l|r|r} 
& Typ S 500 & \multicolumn{1}{|c}{ Typ S 510 } \\
\hline Anschaffungspreis: & $100.000 \mathrm{DM}$ & $150.000 \mathrm{DM}$ \\
Liquidationserlös: & $20.000 \mathrm{DM}$ & $30.000 \mathrm{DM}$ \\
Kapazität: & $2.500 \mathrm{Std} / \mathrm{Jahr}$ & $2.500 \mathrm{Std} / \mathrm{Jahr}$ \\
Instandhaltungskosten: & $30.000 \mathrm{DM}$ p. a. & $25.000 \mathrm{DM}$ p. a. \\
Nutzungsdauer: & 5 Jahre & 6 Jahre
\end{tabular}

Bei der Erreichung des Unternehmensziels der Gewinn-Maximierung stellt die Minimierung der Kosten ein Unterziel dar. Zur Beurteilung der beiden Alternativen wird daher eine Kostenvergleichsrechnung vorgenommen, in der die jährlich anfallenden Kosten beider Alternativen gegenübergestellt werden. Da beide Maschinen die gleiche Kapazität haben, ist eine Betrachtung der gesamten Kosten ausreichend. Die kostengünstigere Alternative ist entsprechend die relativ vorteilhaftere. ${ }^{30}$

Zunächst werden die in der Aufgabenstellung vorgegebenen Kosten der Instandhaltung der Maschinentypen herangezogen. Weiterhin sind die jährlichen $A b$ schreibungen nach dem linearen Abschreibungsverfahren, das ebenfalls vorgegeben ist, wie folgt $\mathrm{zu}$ berechnen: ${ }^{31}$

29 Zur Investitionsplanung für simulierte Unternehmen vgl. auch BLoEcH (Investitionsplanung 1992).

30 Vgl. Götze \& Blozch (Investitionsrechnung 1995), S. 53-54.

31 Vgl. Wöнe (Einführung 1996), S. 1088-1090. 


$$
\begin{aligned}
\text { Abschreibung } & =\frac{\text { Anschaffungskosten }- \text { Liquidationslös }}{\text { Nutzungsdauer }} \\
\text { Typ S } 500 & =\frac{100.000-20.000}{5}=16.000 \mathrm{DM} / \mathrm{Jahr} \\
\text { Typ S 510 } & =\frac{150.000-30.000}{6}=20.000 \mathrm{DM} / \mathrm{Jahr}
\end{aligned}
$$

Somit ergäbe sich eine relative Vorteilhaftigkeit des alternativen Maschinentyps. Berechnungen dieser Art nehmen auch die Teilnehmenden vor, soweit das aus den Erläuterungen zu ihren Entscheidungen hervorgeht. Neun Teilnehmende nehmen im Prätest, acht im Posttest eine Ersatzinvestition durch Verkauf vorhandener Maschinen und Einkauf des neuen Typs vor. Im Durchschnitt wird sowohl im Prä- als auch im Posttest knapp eine Maschine verkauft. Nur zwei Teilnehmende vergrößern den Bestand an Maschinen im Prätest, im Posttest ist es nur noch eine/r.

Zur Beurteilung der Vorteilhaftigkeit sollte jedoch auch die Zinsbelastung durch das durchschnittlich gebundene Kapitals herangezogen werden. Es berechnet sich unter der Annahme einer kontinuierlichen Amortisation nach folgender Formel: ${ }^{32}$

$$
\begin{aligned}
\text { gebundenes Kapital } & =\frac{\text { Anschaffungskosten }+ \text { Liquidationslös }}{2} \\
\text { Typ S } 500 & =\frac{100.000+20.000}{2}=60.000 \mathrm{DM} / \mathrm{Jahr} \\
\text { Typ S } 510 & =\frac{150.000+30.000}{2}=90.000 \mathrm{DM} / \mathrm{Jahr}
\end{aligned}
$$

Die jährlichen Zinsen werden durch Verzinsung des durchschnittlich gebundenen Kapital berechnet. Es ergeben sich folgende Zahlen:

32 Dabei könnte auch von einer nicht-linearen Amortisation ausgegangen werden, die eher der Realität entspricht; vgl. PERridon \& STEInER (Finanzwirtschaft 1995), S. 38-39. 


\begin{tabular}{l|r|r} 
& Typ S500 & \multicolumn{1}{|c}{ Typ S510 } \\
\hline Instandhaltung & $30.000 \mathrm{DM}$ & $25.000 \mathrm{DM}$ \\
Abschreibungen & $16.000 \mathrm{DM}$ & $20.000 \mathrm{DM}$ \\
\hline Summe & $46.000 \mathrm{DM}$ & $45.000 \mathrm{DM}$ \\
Zinsen & $i \%$ von $60.000 \mathrm{DM}$ & $i \%$ von $90.000 \mathrm{DM}$
\end{tabular}

In der Fallstudie ist kein Zinssatz vorgegeben, da der Finanzierungsbereich zur Reduzierung der Komplexität und damit der Bearbeitungszeit ausgeblendet wurde. Zur Ermittlung des kritischen Zinssatzes $i_{k r i t}$, ab dem eine Alternative vorteilhafter gegenüber der anderen ist, wird folgende Gleichung aufgestellt und gelöst:

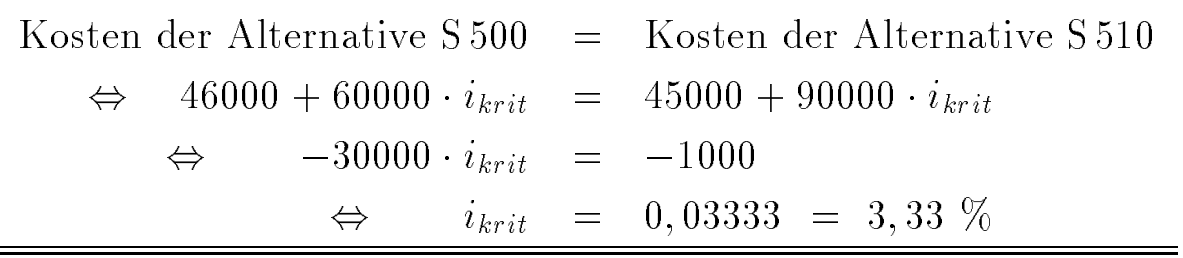

Ein Zinssatz von 3,33\% ist jedoch für eine Fremdfinanzierung unrealistisch. Insofern ist bei einem höheren Zinssatz der vorhandene Maschinentyp in der sich ergebenden Summe der Kosten günstiger. Bei einem Zinssatz von beispielsweise $10 \%$ ergeben sich Kosten von 52.000 DM für den Typ S 500 und 54.000 DM für den Typ S 510 und damit eine relative Vorteilhaftigkeit des vorhandenen Maschinentyps.

\subsection{Vergleich der experimentellen Bedingungen}

\subsubsection{Netzwerk-Erhebung}

Zur Beurteilung der Unterschiede zwischen den beiden experimentellen Bedingungen wurden einfaktorielle Varianzanalysen mit dem Faktor 'PlanspielDurchführung' und den Ergebnissen der Instrumente des Prä- und Posttests als abhängige Variablen gerechnet.

Die Anzahl der Fehler im Multiple-Choice-Fragebogen brachte keine signifikanten Unterschiede zwischen den experimentellen Bedingungen. Beim betriebswirtschaftlichen Zusammenhangswissen zeigte sich in der Netzwerk-Erhebung ein 
größerer Zuwachs bei der herkömmlichen Bedingung, während sich die Teilnehmenden der modularen Bedingung in der Fallstudie als erfolgreicher erwiesen.

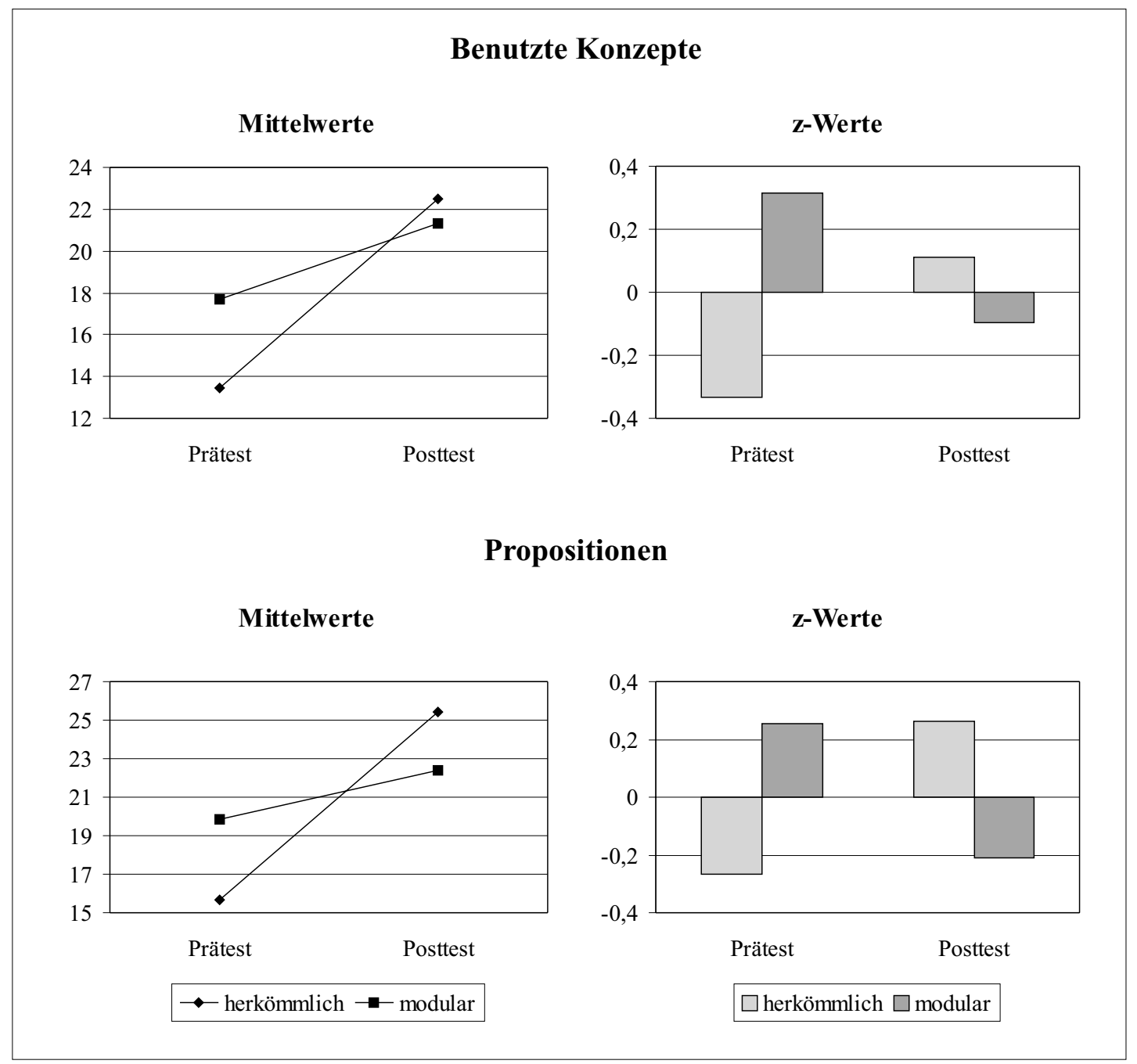

Abbildung 9.9: Prä-Post-Vergleich mit Planspiel-Durchführung: Netzwerk

In der Netzwerk-Erhebung ergab sich bei der Anzahl der benutzten Konzepte ein signifikanter Unterschied zwischen den beiden Planspiel-Durchführungen: ${ }^{33}$ Von den 35 vorgegebenen Konzepten benutzten im Prätest die Teilnehmenden der modulare Bedingung ungefähr die Hälfte, während die Teilnehmenden der herkömmlichen Bedingung nur knapp $40 \%$ benutzen. Dieser Unterschied gleicht $33 \mathrm{~F}=9.24, \mathrm{p}=0.0046, \mathrm{df}=70$. 
sich im Posttest an, wobei die Anzahl in der modularen Bedingung knapp unter der der herkömmlichen liegt (64\% gegenüber $61 \%$ ).

Ein ähnliches Bild ergibt sich bei der durchschnittlichen Anzahl Propositionen in den Netzwerken: Hier lag der Wert der Teilnehmenden der herkömmlichen Bedingung im Prätest ebenfalls unter dem der modularen Bedingung (15,65 gegenüber 19,85) und im Posttest um drei Propositionen über dem der modularen Bedingung (25,4 bzw. 22,4). ${ }^{34}$

Abbildung 2.9 zeigt die Mittelwerte und z-Werte für beide Maßzahlen. Dabei ist die Varianz insbesondere bei der Anzahl der Propositionen im Posttest geringer, so dass die Unterschiede zwischen den Mittelwerten bei den standardisierten zWerten stärker werden.

Das Ergebnis deutet daraufhin, dass die herkömmliche Bedingung in der vorliegenden Untersuchung den Erwerb von Zusammenhangswissen stärker fördert als die modulare Bedingung. Dies wiederspricht der in Abschnitt 4.8.4 aufgestellten Hypothese, nach der die modulare Durchführung des Planspiels einen größeren Lernerfolg erbringen soll.

\subsubsection{Fallstudie}

In der Fallstudie ergeben sich ebenfalls signifikante Unterschiede zwischen den beiden experimentellen Gruppen (Abbildung 9.10, S.272): Der MittelwertsUnterschied bei den Bestandsveränderungen ist im Prätest groß, gleicht sich im Posttest jedoch an. Dabei sind die Teilnehmenden der modularen Bedingung im Prätest besser als im Posttest, sind also nach dem Einsatz des Planspiels weniger erfolgreich als vorher. Die Teilnehmenden der herkömmlichen Bedingung dagegen planen im Posttest besser und haben einen geringen Lernerfolg zu verzeichnen. ${ }^{35}$

Zur Erklärung der Unterschiede können die geplanten und tatsächlichen Produktionsmengen herangezogen werden: ${ }^{36}$ In der Fallstudie war - im Unterschied zum eingesetzten Unternehmensplanspiel EpUS - eine sofortige Anpassung der

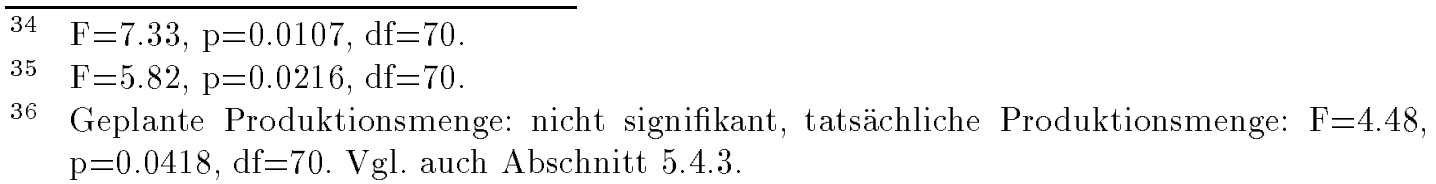


Produktions-Kapazität an die Nachfrage durch Desinvestition bzw. Investition möglich. Im Prätest planen fünf Teilnehmende der herkömmlichen Bedingung und sechs der modularen Bedingung die Produktionsmengen falsch und erreichen trotz teilweisem Kauf zusätzlicher Maschinen nicht die geplanten Mengen. Die Fehlerquelle in der Berechnung liegt zum einen in der Angabe des gesamten Marktvolumens und des eigenen prozentualen Anteils, der erst in eine Menge in Stück umgerechnet werden muss, zum anderen in der nicht oder falsch durchgeführten Berechnung der Maschinenauslastung. Im Posttest hingegen sind es vier andere Teilnehmende der herkömmlichen und ein/e andere/r Teilnehmende/r der modularen Bedingung, die eine zu hohe Produktionsmenge berechnen. Hinsichtlich der Planung der Produktionsmenge kann den Teilnehmenden der modularen Bedingung ein größerer Lernerfolg zugeschrieben werden, der sich in der Maßzahl der Bestandsveränderungen widerspiegelt. Ein entsprechendes Ergebnis zeigt sich bei dem Auslastungsgrad der Maschinen (Abbildung 9.11, S. 273). ${ }^{37}$

Im Prätest lasten die Teilnehmenden der modularen Bedingung ihre Maschinen wesentlich geringer aus als die der herkömmlichen Bedingung. Dieses Verhältnis kehrt sich im Posttest um, die Teilnehmenden der modularen Bedingung erreichen jedoch nur einen geringfügig höheren Auslastungsgrad. Gleichzeitig wird hier die Varianz sehr klein, weil sehr viele Teilnehmende ihre Maschinen voll auslasten. Entsprechend groß sind die z-Werte.

Die bisherigen Unterschiede haben auch eine Auswirkung auf die Höhe des erzielten Gewinns: ${ }^{38}$ Der Gewinn liegt im Prätest bei den Teilnehmenden der modularen Bedingung wesentlich niedriger als bei denen der herkömmlichen Bedingung und auch hier kehrt sich das Verhältnis im Posttest um, wobei die Varianz geringer wird. Insgesamt kann von einem größeren Lernerfolg bei den Teilnehmenden der modularen Bedingung gesprochen werden.

\footnotetext{
$37 \quad \mathrm{~F}=9.87, \mathrm{p}=0.0035, \mathrm{df}=70$.

$38 \mathrm{~F}=4.36, \mathrm{p}=0.0446, \mathrm{df}=70$.
} 


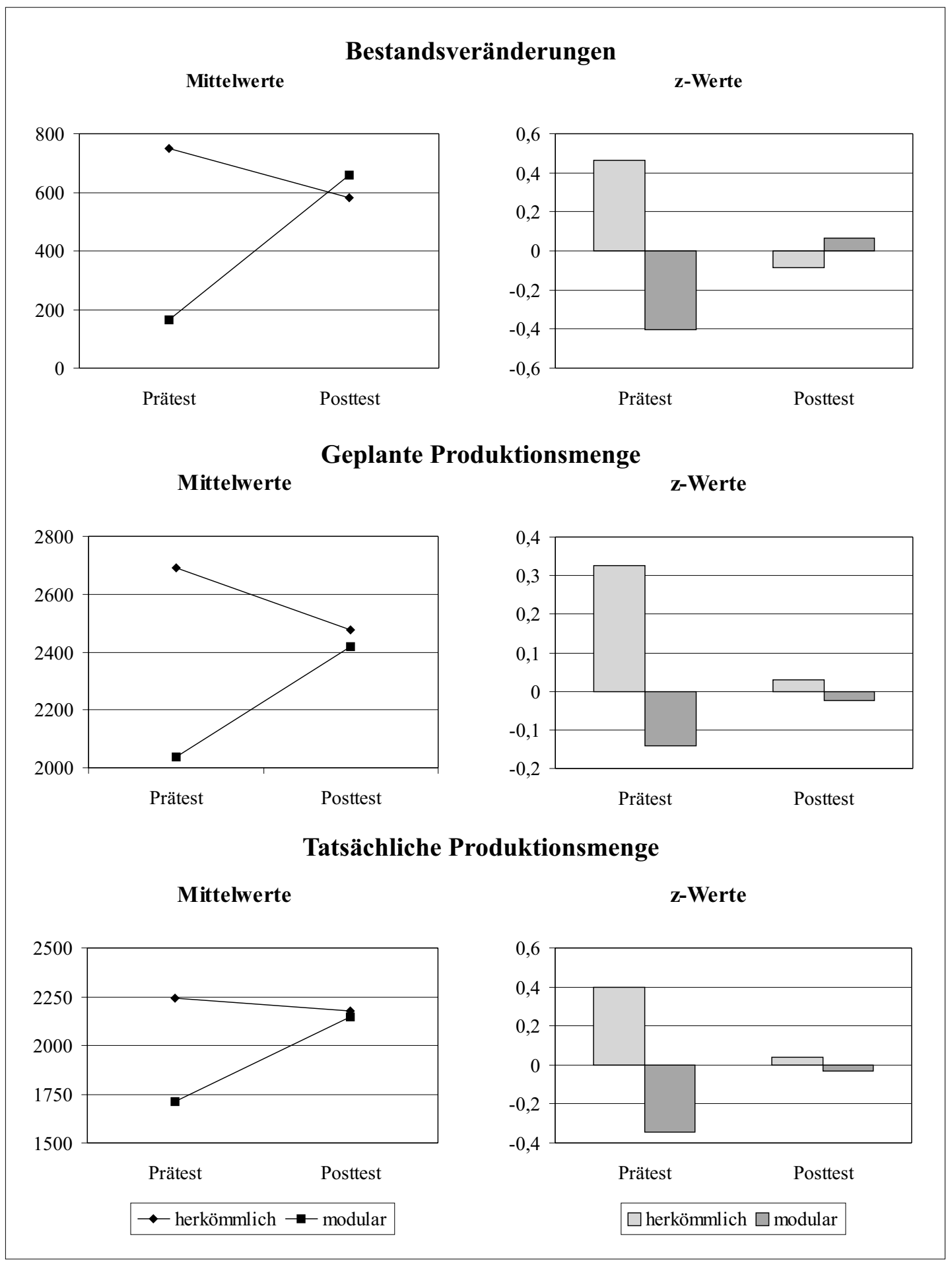

Abbildung 9.10: Prä-Post-Vergleich mit Planspiel-Durchführung: Fallstudie (1) 


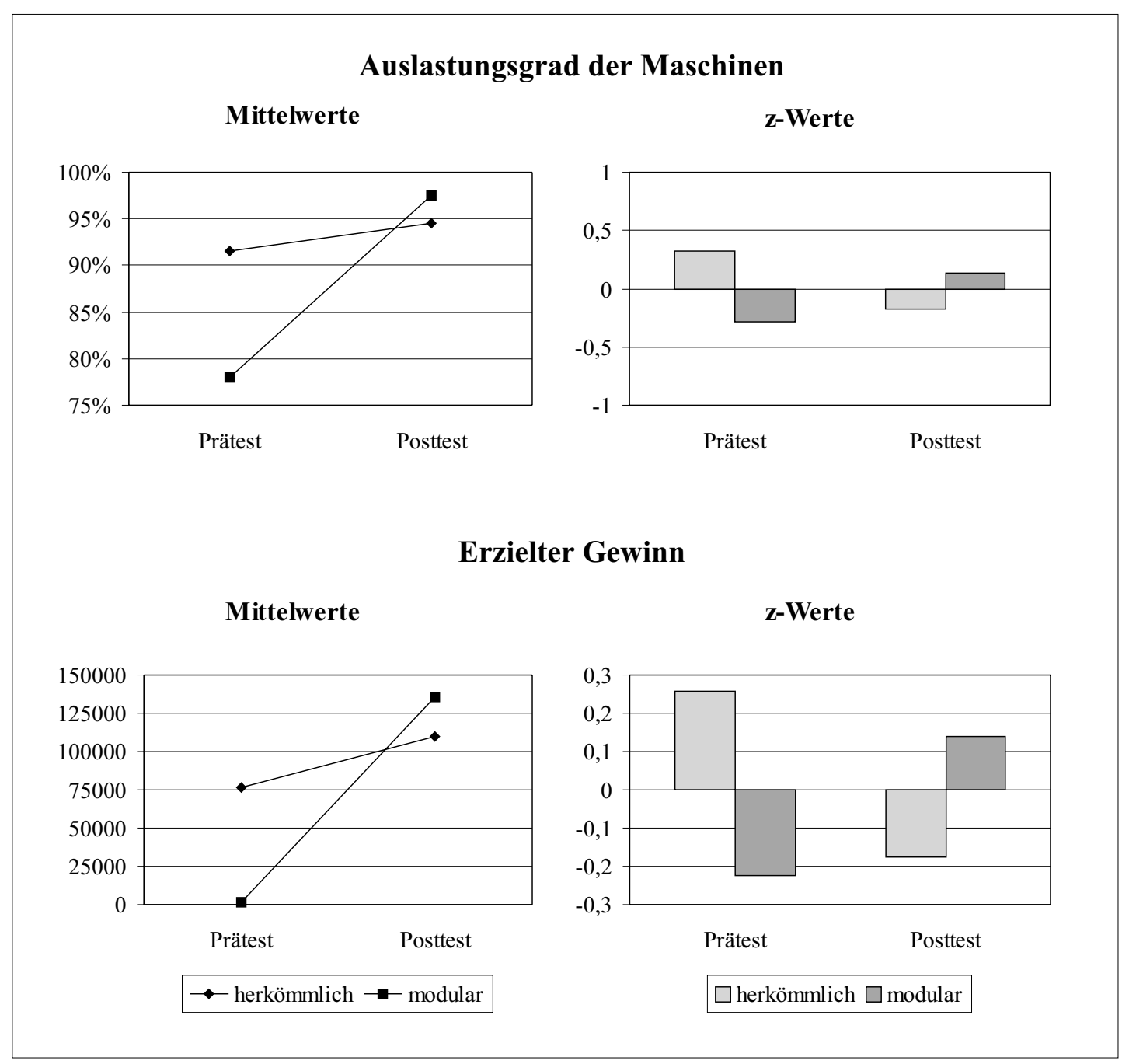

Abbildung 9.11: Prä-Post-Vergleich mit Planspiel-Durchführung: Fallstudie (D) 


\subsection{Vollständiges Untersuchungsdesign}

\subsubsection{Multiple-Choice-Fragebogen}

Dieser Abschnitt zeigt die Ergebnisse der Prä-Post-Erhebung hinsichtlich des vollständigen Untersuchungsdesigns. Dabei wurden dreifaktorielle Varianzanalysen mit Messwiederholung und allen drei Faktoren - 'experimentelle Bedingung' sowie beide Fragebögen der Planspiel-Phase - gerechnet. Die Faktoren haben jeweils dichotome Ausprägung, ${ }^{39}$ so dass sich ein $2 \times 2 \times 2$-Design ergab. Dabei werden die isolierten Einflüsse der Faktoren auf die abhängige Variable sowie die Wechselwirkungen zwischen jeweils zwei Faktoren und allen drei Faktoren berechnet.

Beim Multiple-Choice-Fragebogen waren auch hier keine Effekte feststellbar.

\subsubsection{Netzwerk-Erhebung}

\section{Fragebogen zur Entscheidung}

Bei der Netzwerk-Erhebung ergab sich hinsichtlich der Größe und der Ordnung kein Effekt des Einsatzes der zusätzlichen Fragebögen. Interessanterweise hatte jedoch der Einsatz des Fragebogens zur Entscheidung ${ }^{40}$ einen Einfluss auf die Anzahl der Propositionen, die von den Teilnehmenden mit dem Konzept 'Gewinn' gebildet wurden (Abbildung 9.12): ${ }^{41}$ Im Prätest betrug der Unterschied knapp eine Proposition, wobei der Mittelwert derjenigen, in deren Planspiel-Gruppe der Fragebogen eingesetzt wurde, größer war als der der Gruppen ohne Fragebogen. Im Posttest bilden diejenigen ohne Einsatz des Fragebogens jedoch im Durchschnitt 1,3 Propositionen mehr mit dem Konzept 'Gewinn' als diejenigen mit Fragebogen. Bei ihnen rückt der Gewinn stärker aus dem Mittelpunkt der Betrachtungen, während der Fragebogen offensichtlich einen weiteren Blick für die Zusammenhänge zwischen den betriebswirtschaftlichen Funktionsbereichen fördert.

\footnotetext{
39 Vgl. Abschnitt 4.9.

40 Vgl. Abschnitt 6.5.

$41 \quad \mathrm{~F}=6.09, \mathrm{p}=0.0185, \mathrm{df}=74$.
} 


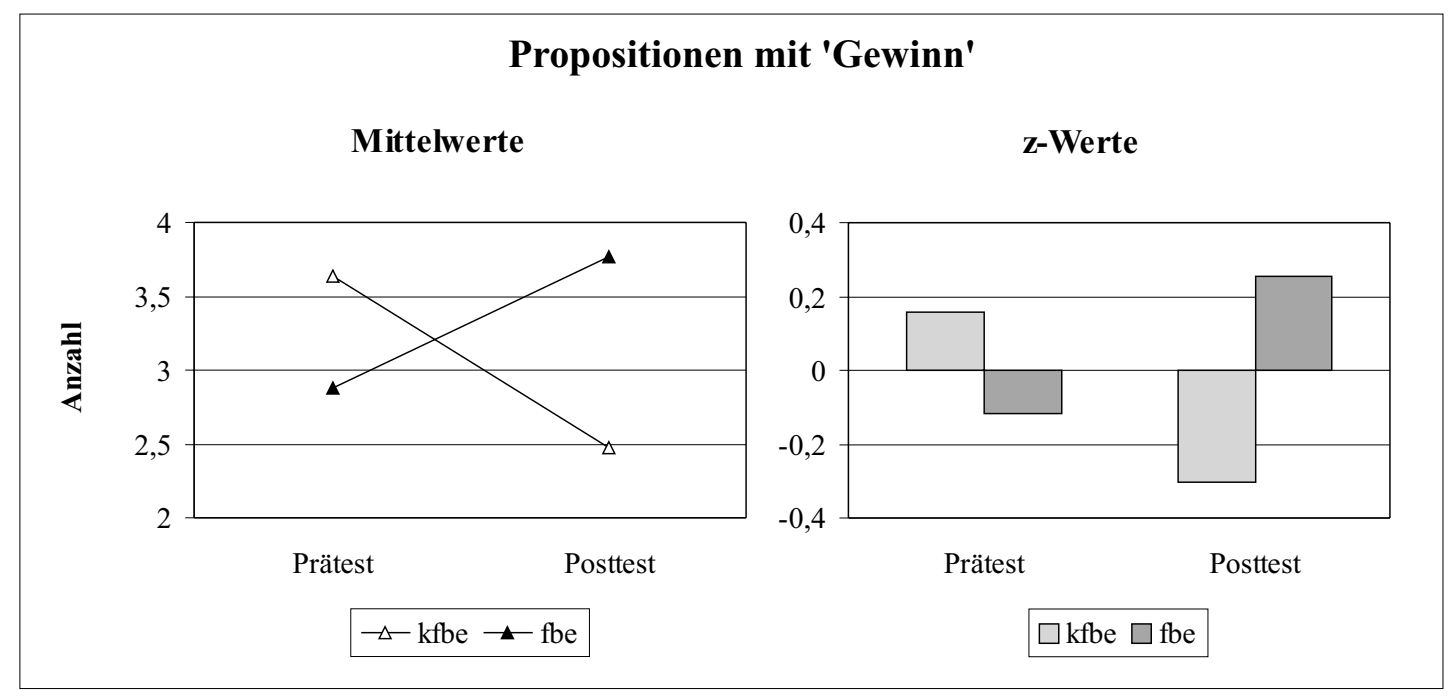

Abbildung 9.12: Vollständiges Design: Netzwerk, Propositionen mit 'Gewinn' kfbe $=$ kein Einsatz des Fragebogens zur Entscheidung fbe $=$ Einsatz des Fragebogens zur Entscheidung

Auf Grund dieses Ergebnisses scheint es interessant, die Modalen Netzwerke des Posttests der Gruppen mit bzw. ohne Fragebogen zur Entscheidung (FBE) näher zu betrachten (Abbildung 9.13, S.276 und 9.14, S.277). Die Anzahl der dargestellten Propositionen in beiden Netzwerken ist vergleichbar und liegt bei der Gruppe ohne Fragebogen bei $19(n=25)$ und bei der Grupppe mit Fragebogen bei $21(n=16)$. Die Anzahl der Konzepte liegt bei der ersten Gruppe bei 16, bei der anderen Gruppe jedoch bei 19. Beide Netzwerke bestehen aus nur einem Teilnetz, wobei in dem Netzwerk der Gruppe mit Fragebogen die Funktionsbereiche wesentlich stärker miteinander verbunden sind. Insbesondere fällt die Verknüpfung des Konzepts 'Herstellungskosten' mit dem Personalbereich, der Rohstoff-Beschaffung und dem Absatzbereich auf. Im Vergleich zum Netzwerk der Gruppe ohne Fragebogen steht hier der Umsatz stärker im Mittelpunkt als der Gewinn. Dies spiegelt auch das Ergebnis der vorherigen Auswertung (Abbildung 9.12 ) wider. 


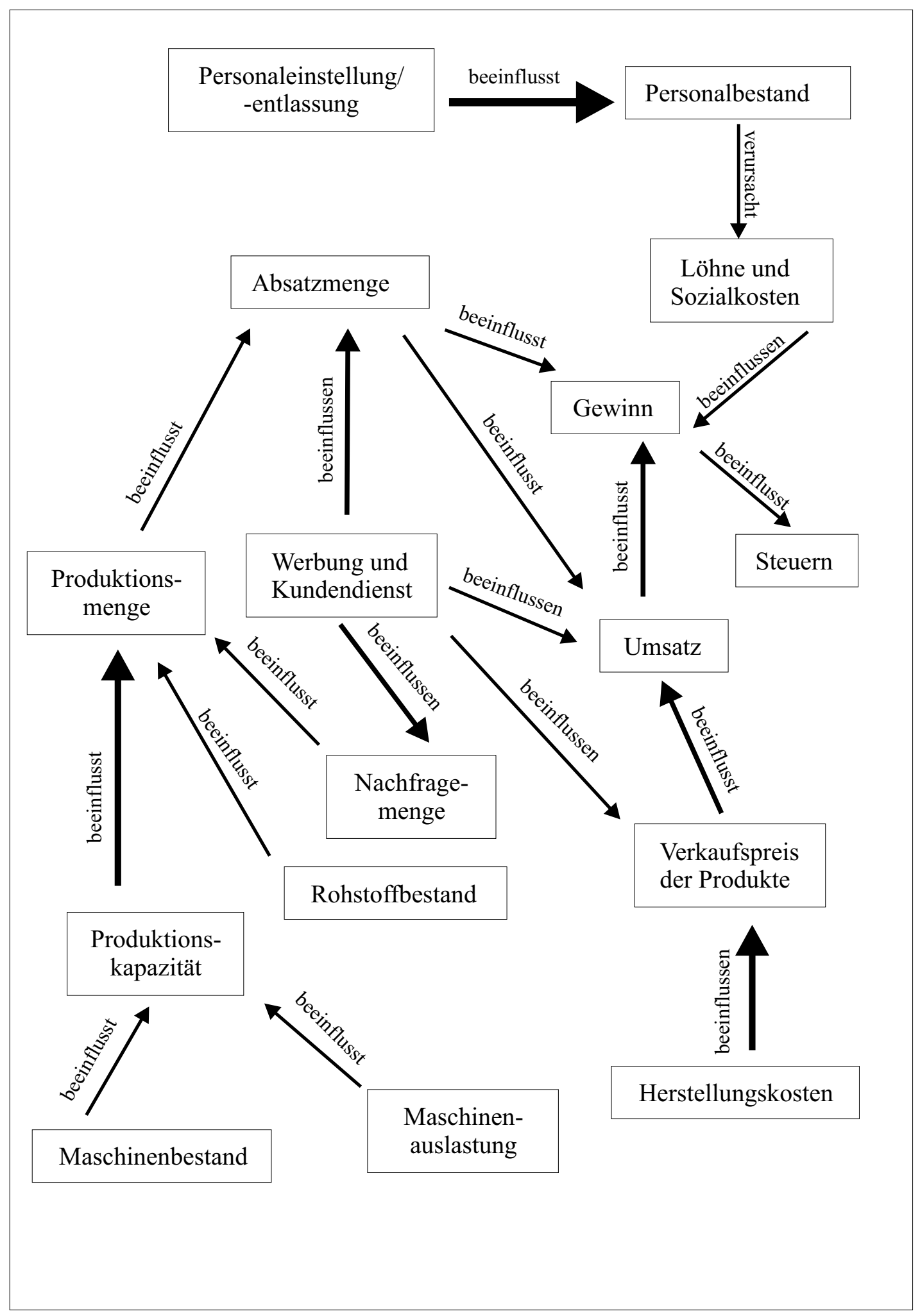

Abbildung 9.13: Vollständiges Design: Modales Netzwerk der Gruppe ohne FBE 


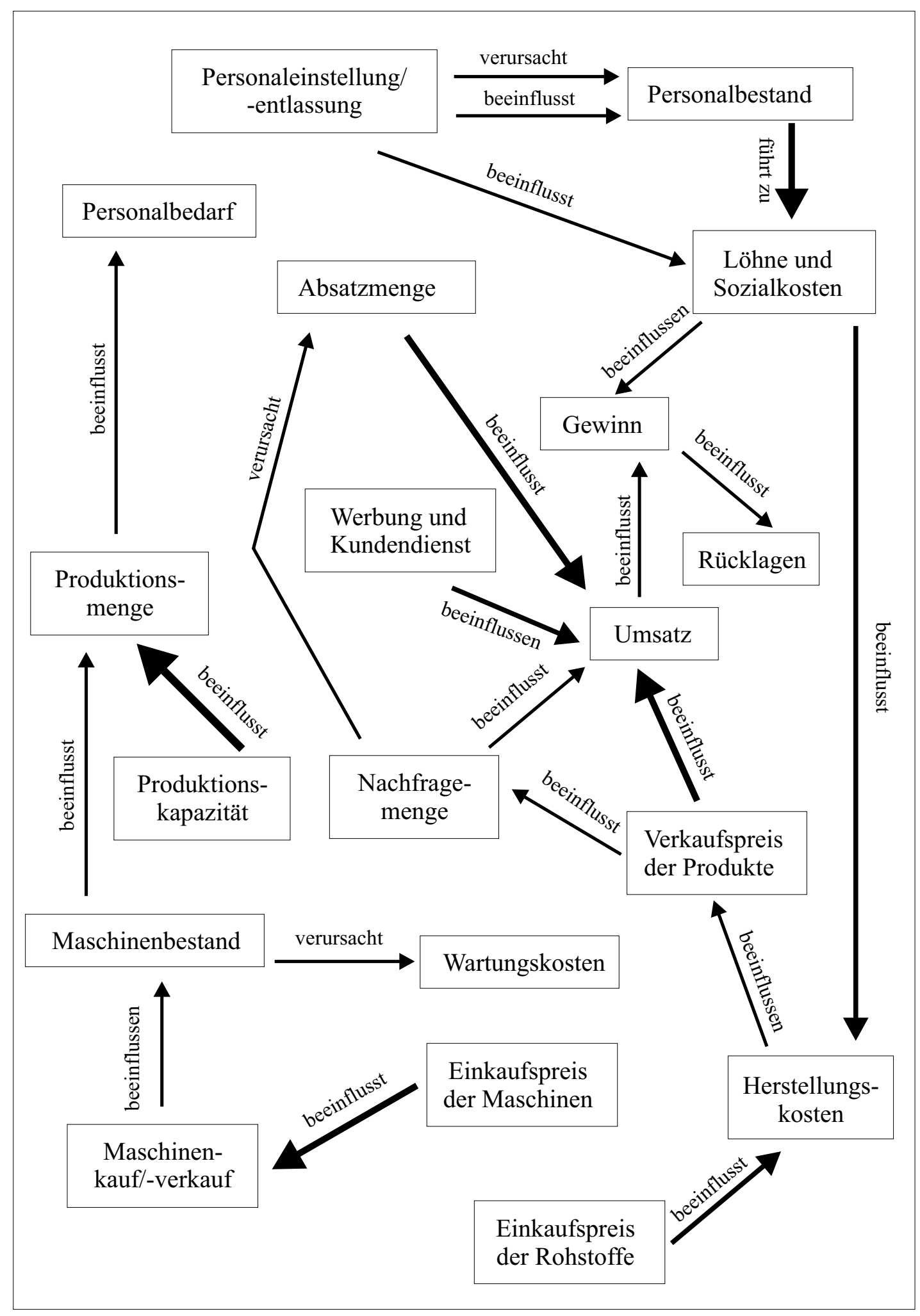

Abbildung 9.14: Vollständiges Design: Modales Netzwerk der Gruppe mit FBE 


\subsubsection{Fallstudie}

Bei den Ergebnissen der Fallstudie ergaben sich verschiedene Effekte, die zum Teil auf große Unterschiede der Werte im Prätest zurückzuführen sind. Dabei sind die Wirkungsketten zwischen den verschiedenen Größen vergleichbar mit der im Abschnitt 9.3.2 dargestellten. In diesem Abschnitt werden daher nur die zentralen Größen 'Erzielte Gewinne' und 'Auslastungsgrad der Maschinen' betrachtet. ${ }^{42}$

Fragebogen zur Entscheidung

Zunächst soll die signifikante Wechselwirkung zwischen der Art der PlanspielDurchführung und dem Einsatz des Fragebogens zur Entscheidung auf den Gewinn als Zielgröße betrachtet werden. ${ }^{43}$ In Abbildung 9.15 sind in der oberen Abbildung die Werte des Prä- und Posttests gegeneinander abgetragen. Zur besseren Übersicht ist die $45^{\circ}$-Linie eingezeichnet.

Hier fällt besonders die Gruppe der Teilnehmenden auf, die in der PlanspielPhase unter herkömmlicher Bedingung spielten und bei denen der Fragebogen zur Entscheidung eingesetzt wurde. Die Streuung der Werte ist im Prätest besonders groß, während die Werte im Posttest relativ eng zusammen liegen. Die Unterschiede zwischen den Mittelwerten sind jedoch eher gering. Einen ähnlichen Verlauf, wenn auch mit niedrigeren Werten, haben die Gruppen der modularen Bedingung mit Fragebogen und die der herkömmlichen Bedingung ohne Fragebogen. Den größten Zuwachs haben die Teilnehmenden der modularen Bedingung, bei denen kein Fragebogen eingesetzt wurde, zu verzeichnen. Sie sind auch für die großen Unterschiede zwischen den experimentellen Bedingungen verantwortlich. ${ }^{44}$

Bezüglich der z-Werte ergibt sich ein differenzierteres Bild: Hier schneidet die Gruppe der modularen Bedingung ohne Fragebogen zwar auch am besten ab, aber die Mittelwerte der Gruppe der herkömmlichen Bedingung ohne Fragebogen weicht zu beiden Testzeitpunkten nach unten vom Durchschnitt ab. Die anderen beiden Gruppen erreichen im Prätest positive z-Werte, jedoch im Posttest negative z-Werte. Ein vergleichbares Bild ergibt sich beim Auslastungsgrad der Maschinen. ${ }^{45}$

\footnotetext{
42 Die gesamten Ergebnisse befinden sich im Anhang A.23.

$43 \mathrm{~F}=5.62, \mathrm{p}=0.0237, \mathrm{df}=70$.

44 Vgl. Abschnitt 9.3.

$45 \mathrm{~F}=4.83, \mathrm{p}=0.0351, \mathrm{df}=70$. Auf eine grafische Darstellung wird verzichtet.
} 


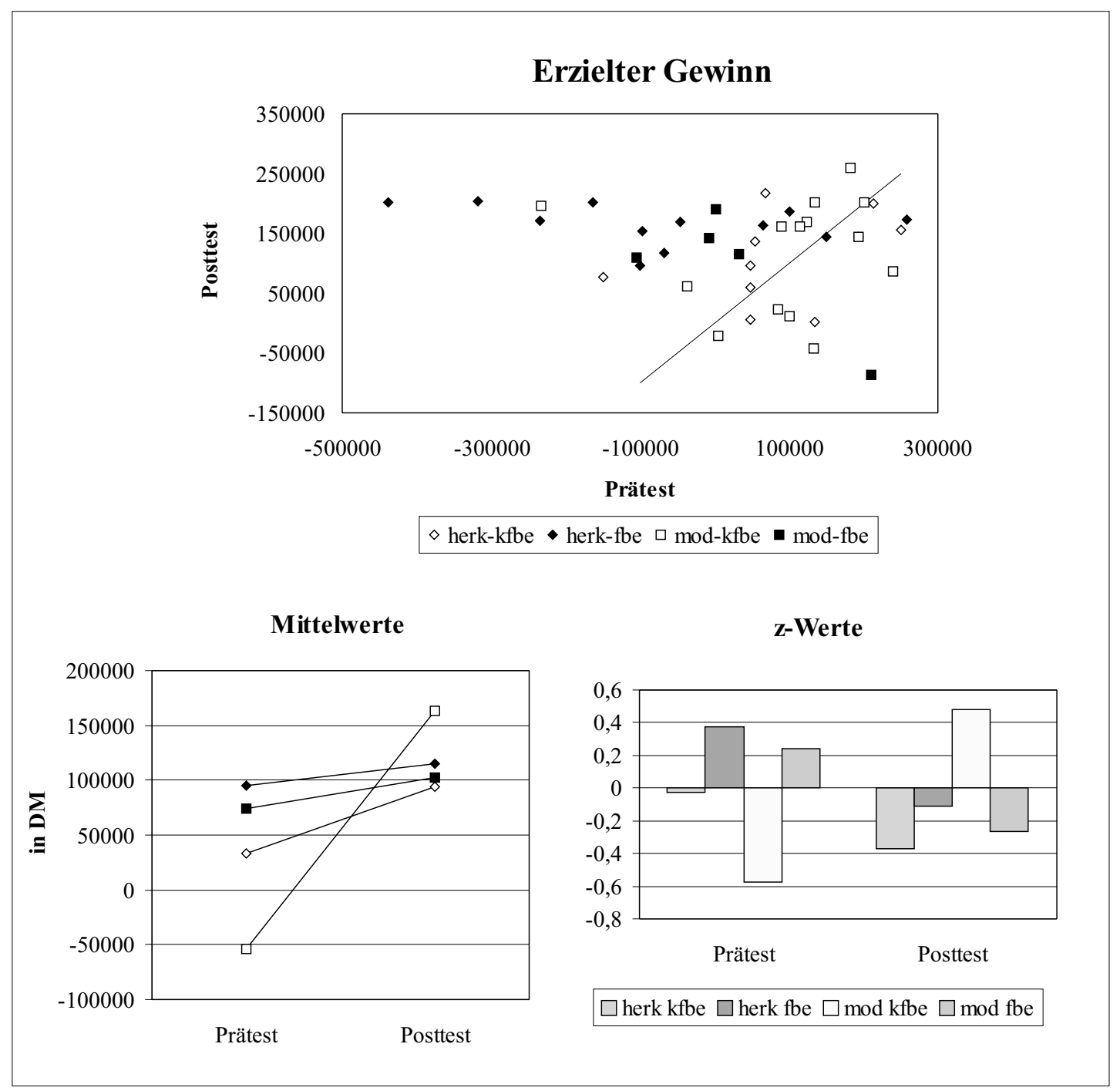

Abbildung 9.15: Vollständiges Design: Fallstudie, Planspiel-Durchführung und

Einsatz des Fragebogens zur Entscheidung

Fragebogen zum situativen Erleben

Aber auch der Fragebogen zum situativen Erleben hat einen Einfluss auf den Lernerfolg in der Fallstudie (Abbildung 9.16): ${ }^{46}$ Die Teilnehmenden, bei denen in der Planspiel-Phase der Fragebogen eingesetzt wird, erzielen im Prätest durchschnittlich geringere, sogar negative Gewinne, während die Teilnehmenden mit Fragebogen bereits im Prätest relativ hohe Gewinne und nur eine geringe Steigerung zum Posttest hin erzielen. Die Varianz ist im Prätest fast doppelt so

$46 \quad \mathrm{~F}=6.84, \mathrm{p}=0.0134, \mathrm{df}=70$. 
groß wie im Posttest, entsprechend werden die Abweichungen vom Mittelwert in den z-Werten vergrößert. Es zeigt sich jedoch auch, dass die Teilnehmenden ohne Fragebogen im Prätest vom Mittelwert der gesamten Stichprobe positiv und im Posttest negativ abweichen. Hinsichtlich des Auslastungsgrads der Maschinen ergibt sich ein vergleichbares Bild. ${ }^{47}$ Dabei muss jedoch berücksichtigt werden, dass Teilnehmende mit einem bereits im Prätest guten Ergebnis einen geringeren Lernerfolg erzielen können als Teilnehmende, die im Prätest noch relativ schlecht sind. ${ }^{48}$

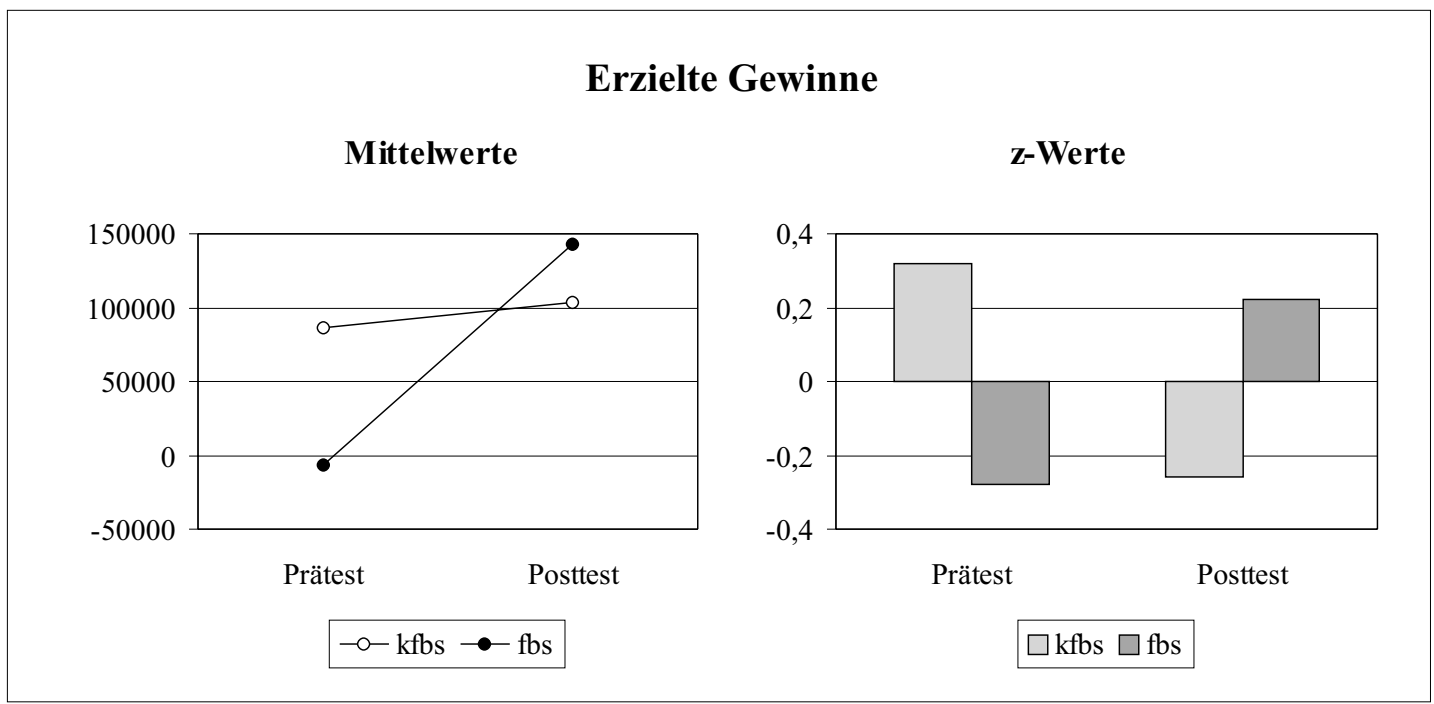

Abbildung 9.16: Vollständiges Design: Fallstudie, Einfluss des Fragebogens zum situativen Erleben

Entsprechend der bisher dargestellten Ergebnisse ergibt sich auch ein Effekt zwischen den beiden eingesetzten Fragebögen hinsichtlich der erzielten Gewinne sowie des Auslastungsgrads der Maschinen: ${ }^{49}$ Die Teilnehmenden, bei denen kein Fragebogen zur Entscheidung, aber der Fragebogen zum situativen Erleben eingesetzt wurde, haben als einzige einen stark positiven Zuwachs hinsichtlich des Mittelwerts der gesamten Stichprobe zu verzeichnen.

$\overline{47} \mathrm{~F}=11.09, \mathrm{p}=0.0021, \mathrm{df}=70$. Auf eine grafische Darstellung wird verzichtet.

48 So genannter 'Decken-Effekt'; vgl. KLeBer (Diagnostik 1992), S. 193.

49 Gewinn: $\mathrm{F}=9.49, \mathrm{p}=0.0041, \mathrm{df}=70$. Auslastungsgrad: $\mathrm{F}=18.39, \mathrm{p}=0.0001, \mathrm{df}=70$. Auf eine grafische Darstellung wird verzichtet. 
An dieser Stelle sollen die Bestandsveränderungen näher betrachtet werden. Es ergab sich ein signifikanter Einfluss der Wechselwirkung der beiden eingesetzten Fragebögen, ${ }^{50}$ wobei der Einsatz des Fragebogens zum situativen Erleben wie bei den erzielten Gewinnen einen stärkeren Effekt als der Fragebogen zur Entscheidung hatte (Abbildung 9.17). Positive Bestandsveränderungen wirken sich als Lagerleistung positiv auf den Gewinn aus. ${ }^{51}$ Ein Lernerfolg zeigt sich bei Bestandsveränderungen jedoch, wenn die Zahlen geringer werden, also die Produktionsmenge in etwa der Absatzmenge entspricht. Von den vier Gruppen erreichen dies am stärksten die Gruppen, bei denen beide Fragebögen eingesetzt wurden. Hinsichtlich der Abweichung der Mittelwerte der Gruppen vom gesamten Mittelwert (z-Werte) erweisen sich die beiden Gruppen, in denen der Fragebogen zur Entscheidung eingesetzt wurde, als erfolgreich. Die Gruppe, bei der nur der Fragebogen zum situativen Erleben eingesetzt wurde, hat einen großen Zuwachs bei den Bestandsveränderungen zu verzeichnen, schneidet also entsprechend schlecht ab. Dies spiegelt das Ergebnis hinsichtlich des Einflusses des Fragebogens auf die erzielten Gewinne wider.

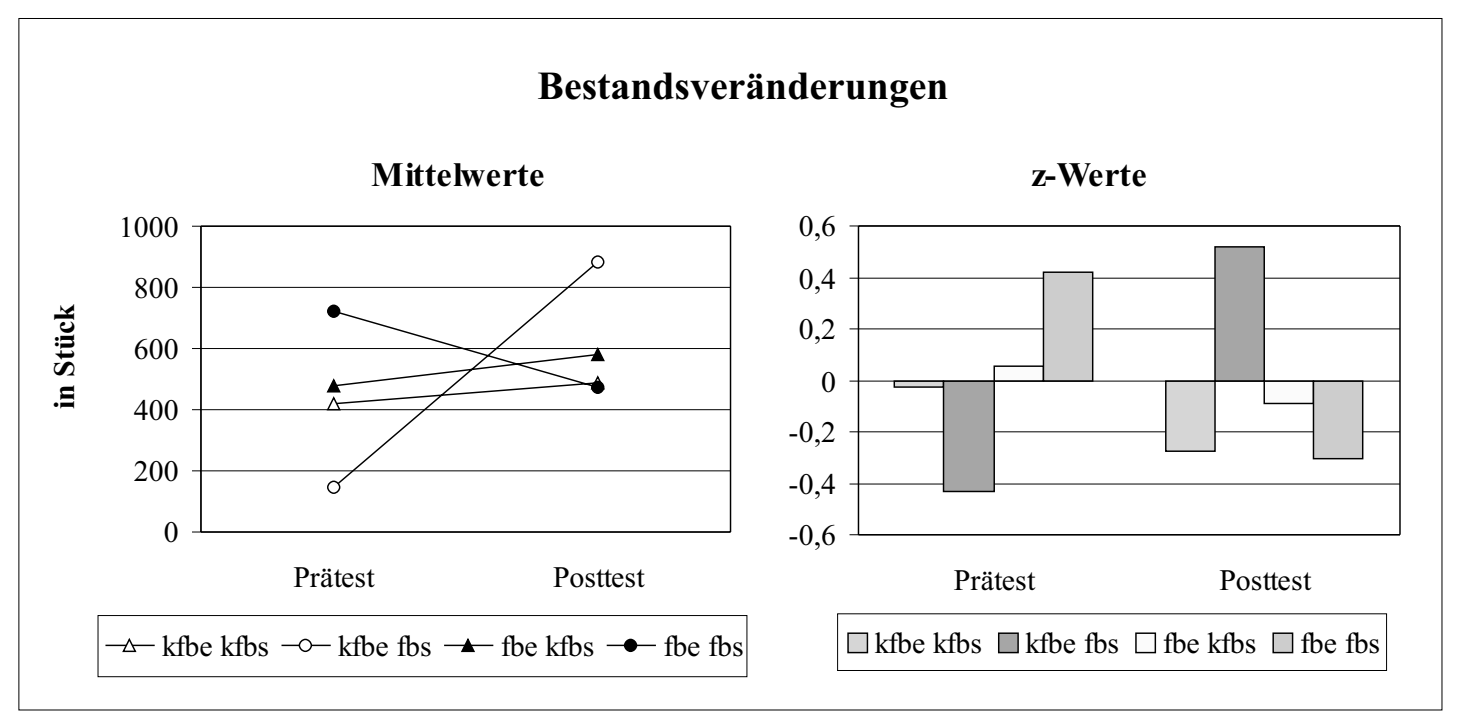

Abbildung 9.17: Vollständiges Design: Fallstudie, Einfluss der zusätzlichen Fragebögen

\footnotetext{
$50 \quad \mathrm{~F}=6.56, \mathrm{p}=0.0152, \mathrm{df}=70$.

51 Vgl. Abschnitt 8.2.3.
} 
$9 \quad$ Ergebnisse des Prä- und Posttests 


\section{Zusammenfassung}

In der vorliegenden Arbeit wurde aus einem Forschungsprojekt heraus, das im Rahmen des Schwerpunktprogramms 'Lehr-Lern-Prozesse in der kaufmännischen Erstausbildung"1 von der Deutschen Forschungsgemeinschaft finanziert wurde, ein weitergehendes Konzept zur Förderung der Lerneffizienz beim Einsatz eines Unternehmensplanspiels entwickelt. ${ }^{2}$ Der interdisziplinäre Ansatz der Konzeption führt die System- und Entscheidungsorientierung der Betriebswirtschaftslehre, die wirtschaftspädagogische Theorie der Handlungsorientierung und die Konzeption komplexer Lehr-Lern-Arrangements sowie ein psychologisches Modell des Zusammenhangs zwischen Kognition, Emotion und Motivation zusammen.

Ziel der Arbeit war die Entwicklung des Einsatzes für die kaufmännische Fortbildung berufstätiger Erwachsener. Zielgruppe waren die Teilnehmenden der Verwaltungs- und Wirtschaftsakademie Göttingen, deren Ausbildung als Aufstiegsfortbildung im Sinne einer Qualifikations-Erweiterung einzuordnen ist. Ein Schwerpunkt der Arbeit lag auf der Untersuchung des Einflusses der Entscheidungs-Komplexität auf den Lernerfolg. Zu diesem Zweck wurde in einer experimentellen Phase die Komplexität der Anforderungssituation variiert und das Planspiel in zwei Durchführungsformen eingesetzt: In der modularen Form wurde ausgehend von einer geringen Komplexität die Anzahl der Entscheidungsvariablen stufenweise erhöht, wobei jeweils ein neuer Funktionsbereich des zu führenden Unternehmens hinzu kam. Gemäß der dargestellten Theorie sollte diese Durchführungsform im Gegensatz zur herkömmlichen Form mit konstanter Komplexität einen stärkeren Einfluss auf den Lernerfolg haben, da die Teilneh-

\footnotetext{
1 Vgl. Deutsche Forschungsgemeinschaft (Berufsbildungsforschung 1990).
}

2 Vgl. Kapitel 1. 
menden die Struktur der zu Grunde liegenden Entscheidungssituation durch die zunächst geringere Anzahl von Variablen besser erkennen können. Der PlanspielEinsatz wurde grundsätzlich wie in den bisherigen Untersuchungen des DFGForschungsprojekts durchgeführt. Auf diese Weise konnte die Konstanz der Forschung gesichert und ein quasi-experimenteller Vergleich der verschiedenen Erhebungen durchgeführt werden.

Ein weiterer Aspekt der Untersuchung war der Einsatz zusätzlicher Maßnahmen in der Planspiel-Phase, die die Reflexivität der Teilnehmenden anregen und somit die Lerneffizienz erhöhen sollten. Zum einen wurde sich auf die zu treffenden Entscheidungen bezogen, wobei ihnen ein sechstufiges Modell des Planungsprozesses vorgegeben wird. Die Teilnehmenden sollten in den Planspiel-Gruppen ihren Entscheidungsprozess kontrollieren und sich zunehmend an Strategien der Unternehmensplanung orientieren. Zum anderen wurden die Teilnehmenden aufgefordert, in der aktuellen Anforderungssituation ihre Emotionen und Motivationen zu reflektieren. Diese Maßnahme lässt Rückschlüsse auf die Wahrnehmung des Planspiel-Einsatzes zu, es wurde jedoch auch von einem Lernerfolg in den Erhebungsinstrumenten des Prä- und Posttests sowie in der Testphase durch die Förderung der Reflexivität ausgegangen.

Zur Überprüfung der Effekte der Variation der Komplexität sowie der zusätzlichen Maßnahmen wurde der Versuchsplan dreifaktoriell konzipiert, wobei alle drei Faktoren vollständig gekreuzt wurden. Der Lernerfolg wurde mit verschiedenen Instrumenten im Prä-Post-Design erhoben: Zur Erhebung des Faktenwissens wurde ein Multiple-Choice-Fragebogen eingesetzt. Das Zusammenhangswissen wurde mit Hilfe einer Netzwerk-Erhebung erfasst, die aus vorgegebenen Begriffen und Relationen aus der allgemeinen Betriebswirtschaftslehre bestand, die die Teilnehmenden in einen Zusammenhang bringen sollten. Dabei wurden bezüglich der Struktur keine Vorgaben gemacht, um eine Re-Aktivität auf das Instrument zu minimieren. Die Transferleistung soll sich in einer Fallstudie zeigen, die eine komplexe betriebliche Situation präsentiert, in der die Teilnehmenden über verschiedene Größen zu entscheiden haben. Die Fallstudie basiert auf einem Unternehmensplanspiel, so dass die Entscheidungen simuliert werden können, was die Auswertung der Ergebnisse erleichtert und die Vergleichbarkeit erhöht. 
Weiterhin wurde aber auch das Unternehmensplanspiel selbst in einer Testphase als Erhebungsinstrument eingesetzt, um die Fähigkeit zu erfassen, in einer komplexen betrieblichen Situation eine Entscheidung in einer Gruppe treffen zu können. Allen Gruppen wurde das Planspiel in voller Entscheidungs-Komplexität eine neue Ausgangslage präsentiert. Insofern unterschied sich die Struktur der Situation nicht von der der experimentellen Phase, wohl aber hinsichtlich der abgebildeten Konjunkturellen Situation sowie der Ausprägungen der Variablen.

Zur Beurteilung der Leistungen der Teilnehmenden in der Testphase des Planspiels wurde auf Grund der theoretischen Überlegungen zur Unternehmensplanung sowie der präsentierten Ausgangslage eine optimale Entscheidung, die zieladäquat alle Zielgrößen gleichzeitig zu berücksichtigen versucht, entwickelt. Sie bildete die Grundlage für weitere Analysen. Ein Vergleich der beiden experimentellen Gruppen zeigte, dass die Teilnehmenden der modularen Bedingung durch eine systematischere Analyse der Situation bessere Ergebnisse erzielten und sich stärker einer aus der Ausgangssituation entwickelten Optimal-Lösung annäherten als die Teilnehmenden der herkömmlichen Bedingung.

Anhand der Größe und Ausgestaltung der Netzwerke ließ sich zeigen, dass der Einsatz des Unternehmensplanspiels die Vernetzung des betriebswirtschaftlichen Wissens fördert. Die Annäherung an ein Referenznetz als 'Experten-Urteil' konnte ebenfalls festgestellt werden. In einer Erhebung zum Einfluss eines PlanspielEinsatzes auf Zusammenhangswissen von Schülerinnen und Schülern einer berufsbildenden Schule wurde zwar eine höhere Abbildungsleistung gemessen, hier waren die vorgegebenen Begriffe jedoch planspiel-spezifisch. ${ }^{3}$ Aber auch in der Transferleistung trafen die Teilnehmenden ihre Entscheidungen nach dem PlanspielEinsatz wesentlich fundierter und zieladäquater als vorher. Da der Zeitraum zwischen Prä- und Posttest vier Monate umfasste, kann von mehr als nur einem kurzfristigen Erfolg oder einem bloßen Erinnerungseffekt an die Art der Testbearbeitung gesprochen werden. Zu vergleichbaren Ergebnissen kamen auch die im Rahmen des DFG-Projekts durchgeführten Erhebungen. ${ }^{4}$

Ein Vergleich der experimentellen Bedingungen gestattete ein differenzierteres Bild hinsichtlich der Vorgehensweise bei der Entscheidungs-Vorbereitung sowohl

\footnotetext{
3 Vgl. Getsch (Möglichkeiten 1990), S. 222-225.

4 Vgl. Bloech, Hartung \& Orth (Unternehmensplanspiele 1998).
} 
in der Testphase des Planspiels als Gruppen-Entscheidung sowie in der Fallstudie als Einzel-Entscheidung: Die Teilnehmenden der modularen Bedingung zeigten ein analytischeres Vorgehen und methodisches Arbeiten. Allerdings hatten sie eher Schwierigkeiten, den Gesamtzusammenhang zu erkennen. Dies spiegelte sich auch in den Ergebnissen der Netzwerk-Erhebung wider: Die Netzwerke der Teilnehmenden der herkömmlichen Bedingung waren im Posttest wesentlich größer, enthalten also mehr Propositionen als die Netzwerke der Teilnehmenden der modularen Bedingung. Es ergaben sich zwar keine inhaltlichen Unterschiede zwischen den modalen Netzwerken beider experimenteller Bedingungen, doch scheint die herkömmliche Durchführung des Planspiels die Teilnehmenden eher zu befähigen, den Überblick über das System 'Unternehmen' als Ganzes zu behalten als die Entscheidungen besonders methodisch zu treffen. Dies zeigt sich insbesondere in der Testphase des Planspiels. Aber auch in der Fallstudie waren die Unterschiede in der Ausgestaltung der Entscheidungen deutlich. Dabei konnten die Teilnehmenden der modularen Bedingung einen größeren Lernerfolg verzeichnen.

Der Einsatz des zusätzlichen Fragebogens zur Entscheidung ergab ebenfalls verschiedene Effekte. Zu vergleichbaren Ergebnissen kommt eine Untersuchung zum Planspiel-Einsatz, bei dem ein Teil der Gruppen zusätzliche Informationen zur Struktur des Planspiel-Modells bekamen. ${ }^{5}$ Zunächst hatte der Einsatz des Fragebogens einen Einfluss auf das Zusammenhangswissen der Teilnehmenden: Die Anzahl der Propositionen mit dem Konzept 'Gewinn' in der Netzwerk-Erhebung unterschied sich im Posttest. Für die Teilnehmenden, in deren Planspiel-Gruppe der Fragebogen eingesetzt wurde, scheint durch die Fokussierung auf die Planung der Entscheidung diese Zielgröße unternehmerischer Tätigkeiten aus dem Mittelpunkt der Betrachtung zu rücken. Weiterhin zeigte die Wechselwirkung zwischen diesem Fragebogen und der Planspiel-Durchführung einen Einfluss auf die Planung in der Fallstudie: Die Gruppe der Teilnehmenden der modularen Bedingung, bei denen der Fragebogen eingesetzt wurde, erzielten im Prätest im Vergleich zu den anderen drei experimentellen Gruppen wesentlich schlechtere Ergebnisse. Im Posttest erzielten sie jedoch überdurchschnittlich gute Leistungen, haben also einen besonders großen Lernerfolg zu verzeichnen. Bezüglich des Mittelwerts der gesamten Stichprobe kann sogar nur bei dieser Gruppe von einer Verbesserung

$5 \quad$ Vgl. Petzing (Untersuchung 1993), S. 113-128. 
der Leistung gesprochen werden. Eine Untersuchung zum Planspiel-Einsatz bei Schülerinnen und Schülern berufsbildender Schulen kam zu vergleichbaren Ergebnissen. ${ }^{6}$

Aber auch der Einsatz des Fragebogens zum situativen Erleben wirkte sich auf die Ergebnisse aus: Die Teilnehmenden, in deren Gruppe der Fragebogen eingesetzt wurde, die also die Wahrnehmung der Anforderungssituation reflektieren sollten, sind nach dem Planspiel-Einsatz wesentlich erfolgreicher als vorher. Die Teilnehmenden der Gruppen, die diesen Fragebogen nicht bekamen, waren zwar im Posttest vergleichsweise besser, konnten ihre Leistungen aber kaum steigern. Im Vergleich zum Mittelwert der gesamten Stichprobe verschlechterten sie sich sogar. Bezüglich der Wechselwirkung zwischen dem Einsatz beider Fragebögen kann festgehalten werden, dass die Kombination beider Maßnahmen zum höchsten Lernerfolg in der Fallstudie führte.

Bezüglich der eingesetzten Erhebungsinstrumente kann gesagt werden, dass sich die Netzwerk-Erhebung zur Rekonstruktion der kognitiven Strukturen bewährt hat. Der Zeitaufwand zur Konstruktion des Instruments sowie bei der Auswertung ist zwar wesentlich höher als bei einem geschlossenen Fragebogen. Der Einsatz kann jedoch auch bei einer großen Stichprobe vorgenommen werden. Durch diese Art der Erhebung können aufschlussreiche Ergebnisse zum Zusammenhangswissen struktureller und inhaltlicher Art gewonnen werden.

Die eingesetzte betriebswirtschaftliche Fallstudie ist geeignet, um Anwendung von Methoden zu erheben, aber auch um eine Transferleistung zu erfassen. Da sie auf einem Unternehmensplanspiel basiert, kann die Auswertung der Ergebnisse mit dem Simulationsmodell vorgenommen werden. Die Interpretation der Ergebnisse orientiert sich an gegebenen Kennzahlen, wobei ein Rückgriff auf die zu Grunde liegende Theorie der Betriebswirtschaftslehre gezogen wird. Die Beurteilung der Leistung könnte sich auch an den von den Teilnehmenden formulierten Zielen orientieren und über eine Rückmeldung von den Teilnehmenden selbst durchgeführt werden.

Das eingesetzte Unternehmensplanspiel EpUS bildet die grundlegenden betrieblichen Zusammenhänge im Modell ab. ${ }^{7}$ Eine Reduzierung auf die Maximierung

\footnotetext{
6 Vgl. STark et al. (Förderung 1995).

7 Vgl. Bloech \& Rüscher (Unternehmensplanspiel 1992).
} 
des Gewinns als Zielgröße vernachlässigt jedoch Aspekte wie der MitarbeiterMotivation oder des Umweltschutzes. Darüber hinaus werden sozial- und arbeitsrechtliche Aspekte der Beschäftigungspolitik in diesem Planspiel nicht berücksichtigt. Insbesondere im Hinblick auf die aktuelle Situation auf dem Arbeitsmarkt sollten diese Aspekte zumindest in der begleitenden Lehre problematisiert werden. ${ }^{8}$

Abschließend lässt sich sagen, dass beide Planspiel-Durchführungen in der Weiterbildung ihre Berechtigung haben: Während die modulare Durchführung eher das Analysieren der Entscheidungssituation und ein methodisches Arbeiten fördert, scheint die herkömmliche Durchführung die Berücksichtigung des Gesamtzusammenhangs eher in den Mittelpunkt zu stellen. Der Einsatz einer modularen Durchführung erscheint entsprechend geeignet, wenn die Teilnehmenden im Berufsleben Anforderungen zu bewältigen haben, die ein analytisches Vorgehen fordern. Für häufig wechselnde berufliche Anforderungen bringt jedoch die herkömmliche Form des Planspiels bessere Ergebnisse.

Die zusätzlichen Maßnahmen sind ebenfalls in der Lage, die Lerneffizienz des Planspiel-Einsatzes zu fördern: Der Fragebogen zur Entscheidung beeinflusst theorie-konform das systematischere Vorgehen in der Entscheidungssituation. Der Fragebogen zum situativen Erleben gibt den Teilnehmenden die Möglichkeit, über die Wahrnehmung der aktuellen Anforderungssituation zu reflektieren und eventuelle Hemmnisse oder Blockaden - insbesondere hinsichtlich der Gruppenarbeit - zur Sprache zu bringen.

Wird der Einsatz eines Unternehmensplanspiels in der beruflichen Weiterbildung durch zusätzliche Maßnahmen gestützt, eröffnet sich die Möglichkeit, sowohl den Anforderungen im Berufsleben gerecht zu werden als auch die Bedürfnisse der Teilnehmenden zu berücksichtigen. Dabei muss jedoch auf die zeitliche Dauer ein Augenmerk gelegt werden. Ein Problem in der vorliegenden Untersuchung bestand in den zum Teil großen Abständen zwischen zwei Unterrichts-Abenden, die teilweise einen negativen Einfluss auf die Lernmotivation und das Interesse an der Fortbildungs-Maßnahme hatten. Grundsätzlich sollte der Einsatz jedoch über ein gesamtes Halbjahr oder Semester hinweg durchgeführt werden, um auch längerfristige Effekte zu haben.

8 Vgl. Kaiser \& Kaminski (Methodik 1994), S. 180. 


\section{Literaturverzeichnis}

ABEle, A.: Stimmung und Leistung. Allgemein- und sozialpsychologische Perspektive. Göttingen u. a. 1995.

ABELE, A.: Zum Einfluß positiver und negativer Stimmungen auf die kognitive Leistung. In: Möller, J. \& Köller, O. (Hrsg.): Emotionen, Kognitionen und Schulleistung. Weinheim 1996, S.91-111.

Achtenhagen, F.: Didaktik des Wirtschaftslehreunterrichts. Opladen 1984.

Achtenhagen, F.: Einige Überlegungen zur Bedeutung der Problemlöseforschung für die Didaktik des Wirtschaftslehreunterrichts. In: FEGER, H. (Hrsg.): Wissenschaft und Verantwortung. Festschrift für Karl Josef Klauer zum sechzigsten Geburtstag. Göttingen 1990, S. 109-129.

Achtenhagen, F.: Erträge und Aufgaben der Berufsbildungsforschung. In: BECK, K. \& KELL, A. (Hrsg.): Bilanz der Bildungsforschung. Stand und Zukunftsperspektiven. Weinheim 1991, S. 185-200.

Achtenhagen, F.: Lernen, Denken, Handeln in komplexen ökonomischen Situationen - Sechzehn Aussagen zu Ergebnissen des Göttinger Forschungsprojekts, verbunden mit einem Ausblick für weitere Forschungsarbeit. In: ACHTENHAGEN, F. \& John, E. G. (Hrsg.): Mehrdimensionale Lehr-Lern-Arrangements. Innovationen in der kaufmännischen Aus- und Weiterbildung. Göttingen 1992, S. 39-42.

Achtenhagen, F.: Zum Einsatz von Planspielen im Betriebswirtschaftslehreunterricht. Zeitschrift für Planung 3, 1/1992, S. 3-19.

Achtenhagen, F.: Zur Operationalisierung von Schlüsselqualifikationen. In: Gonon, P. (Hrsg.): Schlüsselqualifikationen kontrovers. Aarau 1996, S. 107-113.

Achtenhagen, F.: Evaluation von Schlüsselqualifikationen. Wirtschaft und Erziehung 50, 1/1998, S. 4-5.

Achtenhagen, F.: Kriterien zur Konstruktion eines 'handlungsorientierten Unterrichts'. Wirtschaft und Erziehung 50, 1/1998, S.3-4.

Achtenhagen, F. \& John, E. G. (Hrsg.): Mehrdimensionale Lehr-Lern-Arrangements. Innovationen in der kaufmännischen Aus- und Weiterbildung. Göttingen 1992. 
Achtenhagen, F.; Nijhof, W. \& Raffe, D.: Feasibility Study: Research Scope for Vocational Education in the Framework of COST Social Science. Berichte aus dem Seminar für Wirtschaftspädagogik der Georg-August-Universität Göttingen, Bd.22, Göttingen 1995.

Achtenhagen, F. \& Preiss, P.: Planspieleinsatz in der betriebswirtschaftlichen Erstausbildung. In: Biethahn, J.; Hummeltenberg, W. \& Schmidt, B. (Hrsg.): Simulation als betriebliche Entscheidungshilfe. Bd. 2, Berlin u. a. 1991, S. 23-34.

Achtenhagen, F.; Tramm, T.; Preiss, P.; Seemann-Weymar, H.; John, E. G. \& Schunck, A.: Lernhandeln in komplexen Situationen. Neue Konzepte der betriebswirtschaftlichen Ausbildung. Wiesbaden 1992.

ADAm, D.: Planung und Entscheidung. Modelle - Ziele - Methoden. 4. Aufl., Wiesbaden 1996.

Aebli, H.: Denken. Das Ordnen des Tuns. Bd. 2: Denkprozesse. Stuttgart 1981.

AEBLI, H.: Grundlagen des Lehrens. Stuttgart 1987.

Albers, J.: Zu Ansätzen, Ergebnissen und Problemen der Evaluation beruflicher Weiterbildung. Hamburg 1987.

AlBert, H.: Traktat über kritische Vernunft. 5. Aufl., Tübingen 1991.

Amann, H. \& Streb, R.: Welches Planspiel soll im betriebswirtschaftlichen Unterricht eingesetzt werden? - Ein Weg aus diesem Dilemma. Wirtschaft und Erziehung 46, 12/1994, S.409-410.

AmT FÜr Berufs- UND Weiterbildung (Hrsg.): Weiterbildung bringt Gewinn! - Wem? Veröffentlichungen zur Weiterbildung, Bd.16, Hamburg 1995.

Anderson, J. R.: The Architecture of Cognition. Cambridge u. a. 1983.

Anderson, J. R.: Kognitive Psychologie. Eine Einführung. Heidelberg 1988.

ARNOLD, R.: Schlüsselqualifikationen - Ziele einer ganzheitlichen Berufsbildung. Kölner Zeitschrift für Wirtschaft und Pädagogik 7, 13/1992, S. 65-88.

Arnold, R.: Weiterbildung und Beruf. In: Tippelt, R. (Hrsg.): Handbuch Erwachsenenbildung/Weiterbildung. Opladen 1994, S. 172-189. 
ArnolD, R.: Qualitative Forschung im Bereich der beruflichen Bildung - eine prospektive Bilanzierung. In: KöNIG, E. \& Zedler, P. (Hrsg.): Bilanz qualitativer Forschung. Bd. I: Grundlagen qualitativer Forschung. Weinheim 1995, S. 169-182.

ArNold, R.: Schlüsselqualifikationen. "Fachwissen in der Krise" oder Erziehung zur Inkompetenz? In: SEyD, W. \& WiTT, R. (Hrsg.): Situation Handlung Persönlichkeit. Kategorien wirtschaftspädagogischen Denkens. Festschrift für Lothar Reetz. Hamburg 1996, S. 57-70.

Arnold, R. \& Krämer-Stürzl, A.: Qualität durch Professionalität. Grundlagen der Weiterbildung 6, 3/1995, S. 134-139.

Arnold, R. \& Müller, H.-J.: Ganzheitliche Berufsbildung. In: Pätzold, G. (Hrsg.): Handlungsorientierung in der beruflichen Bildung. Frankfurt a. M. 1992, S. $97-122$.

BACH, G.: Planspiele im Betriebswirtschaftslehre-Unterricht. Dargestellt am Planspiel 'Disposition'. Erziehungswissenschaft und Beruf 31, 3/1983, S. 257-267.

Bachmann, W.: Theoretische Grundlagen einer handlungstheoretischen Wirtschaftsdidaktik. Bergisch Gladbach 1988.

Backhaus, K.; Erichson, B.; Plinke, W. \& Weiber, R.: Multivariate Analysemethoden. Eine anwendungsorientierte Einführung. 8. Aufl., Berlin u.a. 1996.

Battge, J.: Betriebswirtschaftliche Systemtheorie. Regelungstheoretische Planungs-Überwachungsmodelle für Produktion, Lagerung und Absatz. Opladen 1974.

Bandura, A.: Sozial-kognitive Lerntheorie. Stuttgart 1979.

Barton-Ziemann, M.: Qualitätskriterien für AFG-geförderte berufliche Bildungsmaßnahmen. In: Feuchthofen, J. E. \& Severing, E. (Hrsg.): Qualitätsmanagement und Qualitätssicherung in der Weiterbildung. Neuwied 1995, S. 57-73.

Baumert, J.: Lernstrategien, motivationale Orientierung und Selbstwirksamkeitsüberzeugungen im Kontext schulischen Lernens. Unterrichtswissenschaft 21, 4/1993, S. 327-354. 
BEck, H.: Schlüsselqualifikationen. Bildung im Wandel. 2. Aufl., Darmstadt 1995.

BEck, H.: Handlungsorientierung des Unterrichts. Anspruch und Wirklichkeit im betriebswirtschaftlichen Unterricht. Darmstadt 1996.

BECK, K.: Die empirischen Grundlagen der Unterrichtsforschung. Eine kritische Analyse der deskriptiven Leistungsfähigkeit von Beobachtungsmethoden. Göttingen u. a. 1987.

Becker, D.; Oldenbürger, H.-A. \& Piehl, J.: Motivation und Emotion. In: LüEr, G. (Hrsg.): Allgemeine experimentelle Psychologie. Stuttgart 1987, S. $431-470$.

Beiträge aus der ArbeitsmarkT- und Berufsforschung 195: Diepold, P. (Hrsg.): Berufliche Aus- und Weiterbildung: Konvergenzen - Divergenzen, neue Anforderungen - alte Strukturen. Nürnberg 1996.

Biethahn, J.; Mucksch, H. \& Ruf, W.: Ganzheitliches Informationsmanagement. Bd. I: Grundlage. 4. Aufl., München u. a. 1996.

BirkHan, G.: Die (Un)brauchbarkeit der klassischen Testgütekriterien für Dialog-Konsens-Verfahren. In: ScheELE, B. (Hrsg.): Struktur-Lege-Verfahren als Dialog-Konsens-Methodik. Ein Zwischenfazit zur Forschungsentwicklung bei der rekonstruktiven Erhebung Subjektiver Theorien. Münster 1992, S. 231-293.

BLEICHER, K.: Unternehmungsspiele. Simulationsmodelle für unternehmerische Entscheidungen. Baden-Baden 1962.

BLEICHER, K.: Entscheidungsprozesse an Unternehmungsspielen. Bd. I: Die Darstellung von Unternehmungspolitik und -planung an Idealmodellen. 3. Aufl., Baden-Baden 1974.

Bloech, J.: Modellierung komplexer betrieblicher Situationen und ihre didaktische Vermittlung mit Hilfe neuer Informations- und Kommunikationstechniken. In: Diepold, P. \& Bundesminister Für Bildung und Wissenschaft (Hrsg.): Neue Informationstechniken in kaufmännischen Modellversuchen: Ergebnisse schulischer Modellversuche und Möglichkeiten ihrer Umsetzung in die kaufmännische Ausbildung. Bad Honnef 1989, S. 102-119.

BLoECH, J.: Investitionsplanung für simulierte Unternehmungen in oligopolistischer Konkurrenz. In: Achtenhagen, F. \& John, E. G. (Hrsg.): Mehrdimen- 
sionale Lehr-Lern-Arrangements. Innovationen in der kaufmännischen Aus- und Weiterbildung. Wiesbaden 1992, S. 242-250.

Bloech, J.: Kostenmanagement in Planspielen. In: Freidank, C.-C.; Götze, U.; Huch, B. \& Weber, J. (Hrsg.): Kostenmanagement. Aktuelle Konzepte und Anwendungen, Berlin u. a. 1997, S. 379-399.

Bloech, J.: Lehr-Lern-Effekte bei der Vermittlung des Wissens über die "Produktion" aus der Gutenberg"schen Unternehmenstheorie. In: AlBACH, H.; EYmann, E.; Luhmer, A. \& Steven, M. (Hrsg.): Die Theorie der Unternehmung in Forschung und Praxis. Berlin u. a. 1999, S. 333-352.

Bloech, J.; Bogaschewsky, R.; Götze, U. \& Roland, F.: Einführung in die Produktion. 3. Aufl., Heidelberg 1998.

Bloech, J.; Döhne, S. R.; Hartung, S. \& Orth, C.: Einsatz eines Planspiels in der kaufmännischen Weiterbildung - Verschiedene Arten der Komplexitätssteigerung im Planspiel. Arbeitsbericht des Instituts für betriebswirtschaftliche Produktions- und Investitionsforschung 1/98, Göttingen 1998.

Bloech, J.; Goertzen, H. \& Maurer, U.: Unternehmenssimulation für Produktionssysteme als Ausbildungsinstrument. In: Biethahn, J.; HummeltenBERG, W. \& SchmidT, B. (Hrsg.): Simulation als betriebliche Entscheidungshilfe. Bd.3., Berlin u. a. 1992, S. 283-303.

Bloech, J.; Götze, U.; Huch, B.; Lücke, W. \& Rudolph, F.: Strategische Planung. Instrumente, Vorgehensweisen und Informationssysteme. Heidelberg 1994.

Bloech, J.; Hartung, S.; Kauer, G. \& Orth, C.: Einsatz eines Planspiels in der kaufmännischen Weiterbildung - Untersuchungen zur Förderung unternehmerischer Handlungskompetenz. Arbeitsbericht des Instituts für betriebswirtschaftliche Produktions- und Investitionsforschung 2/96, Göttingen 1996.

Bloech, J.; Hartung, S. \& Orth, C.: Unternehmensplanspiele in der kaufmännischen Ausbildung - Anpassung der Komplexität an den Lernfortschritt. In: BEck, K. \& DuBs, R. (Hrsg.): Kompetenzentwicklung in der Berufserziehung - Kognitive, motivationale und moralische Dimensionen kaufmännischer Qualifizierungsprozesse. Zeitschrift für Berufs- und Wirtschaftspädagogik, Beiheft 14, Stuttgart 1998, S. 38-56. 
Bloech, J.; Hartung, S. \& Orth, C.: Einsatz eines Planspiels in der kaufmännischen Weiterbildung. Wirtschaft und Erziehung 51, 11/1999, S. 389391.

Bloech, J.; Hartung, S. \& Orth, C.: Förderung der unternehmerischen Handlungskompetenz durch Unternehmensplanspiele. In: Schelten, A.; SloANE, P.F.E. \& Straka, G. A. (Hrsg.): Berufs- und Wirtschaftspädagogik im Spiegel der Forschung, Forschungsberichte des DGfE-Kongresses 1998. Opladen 1999, S. 121-132.

Bloech, J. \& Inde, G. B.: Vahlens Großes Logistiklexikon. München 1997.

Bloech, J.; Kauer, G. \& Orth, C.: Unternehmensplanspiele in der kaufmännischen Ausbildung - Untersuchungen zum Wissenserwerb. In: BECK, K. \& HEID, H. (Hrsg.): Lehr-Lern-Prozesse in der kaufmännischen Erstausbildung Wissenserwerb, Motivierungsgeschehen und Handlungskompetenzen. Zeitschrift für Berufs- und Wirtschaftspädagogik, Beiheft 13, Stuttgart 1996, S.37-52.

Bloech, J. \& RÜscher, H.: Unternehmenssimulation SIM-LOG. Computersimulation zur Logistik und Materialwirtschaft mit LOTUS-123. In: BiETHAHN, J.; Hummeltenberg, W. \& Schmidt, B. (Hrsg.): Simulation als betriebliche Entscheidungshilfe, Bd. 2. Berlin u. a.1991, S. 51-80.

Bloech, J. \& Rüscher, H.: Die Unternehmenssimulation PENTA. In: LüCkE, W. \& Schulz, K. (Hrsg.): Umweltschutz und Investition. Wiesbaden 1992, S. 65-93.

Bloech, J. \& Rüscher, H.: PUMA - Produktions- und Marketing-Simulation. Modellbeschreibung zur Unternehmenssimulation. Göttingen 1992.

Bloech, J. \& RÜscher, H.: Unternehmensplanspiel EpUS - eine experimentell ausgerichtete Unternehmenssimulation. In: Biethahn, J.; HummeltenBerg, W. \& SchmidT, B. (Hrsg.): Simulation als betriebliche Entscheidungshilfe. Bd. 3. Berlin u. a. 1992, S. 268-282.

Bloech, J. \& Rüscher, H.: Modellbeschreibung und Bedienungsanweisung zur Unternehmenssimulation EpUS. Ein-Platz-Unternehmens-Simuation, Spielerhandbuch. Göttingen 1995.

Blohm, H.; Beer, T.; Seidenberg, U. \& Silber, H.: Produktionswirtschaft. 3. Aufl., Herne u. a. 1997. 
Bloom, B.S.; Engelhart, M.D.; Furst, E.J.; Hill, W.H. \& KraThwohl, D.R. (Hrsg.): Taxonomie von Lernzielen im kognitiven Bereich. 5. Aufl., Weinheim u. a. 1976.

Böhret, C. \& Wordelmann, P.: Das Planspiel als Methode der Fortbildung. Zur allgemeinen und speziellen Verwendung der Simulationsmethode in der öffentlichen Verwaltung. Bonn 1975.

Bogaschewsky, R. \& Götze, U. (Hrsg.): Unternehmensplanung und Controlling. Festschrift zum 60.Geburtstag von Jürgen Bloech. Heidelberg 1998.

Bojanowski, A.; Brater, M. \& Dedering, H.: Qualifizierung als Persönlichkeitsbildung. Analysen und Ansätze zur Verbindung von Arbeit und Lernen in Schule und Betrieb. Frankfurt a. M. 1991.

Bonato, M.: Wissensstrukturierung mittels Struktur-Lege-Techniken. Eine graphentheoretische Analyse von Wissensnetzen. Europäische Hochschulschriften, Reihe VI, Bd.297, Frankfurt a. M. u. a. 1990.

Boos, M.: Entscheidungsfindung in Gruppen. Eine Prozeßanalyse. Bern u.a. 1996.

Born, A.: Geschichte der Erwachsenenbildungsforschung. Bad Heilbrunn/Obb. 1991.

Bortz, J. \& Döring, N.: Forschungsmethoden und Evaluation. Berlin u.a. 1995.

Bossel, H.: Simulation dynamischer Systeme. Braunschweig u. a. 1989.

Bransford, J.D.; Franks, J. J.; VYe, N. J. \& Sherwood, R. D.: New approaches to instruction. Because wisdom can't be told. In: Vosniadou, S. \& Ortony, A. (Hrsg.): Similarity and analogical reasoning. Cambridge 1989 , S. 470-497.

Bransford, J. D.; Vye, N. J.; Adams, L. T. \& Perfetto, G. A.: Learning skills and the acquisition of knowledge. In: Lesgold, A. M. \& Glaser, R. (Hrsg.): Foundations for a psychology of education. Hillsdale 1989, S. 199-249.

Bransford, J. D.; Sherwood, R. D.; Hasselbring, T.S.; Kinzer, C. K. \& Williams, S. M.: Anchored instruction. Why we need it and how technology 
can help. In: Nix, D. \& Spiro, R. (Hrsg.): Cognition, Education, and Multimedia. Exploration in High Technology. Hilssdale 1990, S. 115-142.

Breyde, C.: Die Schlüsselqualifikationen als endlose Debatte oder der Wunsch nach Klarheit über einen zu Recht vielschichtigen Begriff. In: SEYD, W. \& WITT, R. (Hrsg.): Situation Handlung Persönlichkeit. Kategorien wirtschaftspädagogischen Denkens. Festschrift für Lothar Reetz. Hamburg 1996, S. 237-244.

Bronner, R. \& Kollmannsperger, M.: Planspieleinsatz an deutschen Hochschulen. Zeitschrift für Planung 8, 4/1997, S. 407-419.

Buber, R. \& Enzinger, H.: Das Planspiel beim Führungskräftetraining: Ein Fallbeispiel aus der öffentlichen Verwaltung. München 1992.

Buddensiek, W.: Pädagogische Simulationsspiele im sozio-ökonomischen Unterricht der Sekundarstufe I. Theoretische Grundlegung und Konsequenzen für die unterrichtliche Realisation. Bad Heilbrunn/Obb. 1979.

BudDensiek, W.: Entscheidungstraining im Methodenverbund - Didaktische Begründung für die Verbindung von Fallstudie und Simulationsspiel. In: KaISER, F.-J. (Hrsg.): Die Fallstudie. Theorie und Praxis der Fallstudiendidaktik. Bad Heilbrunn/Obb. 1983, S. 128-147.

BudDEnsiek, W.: Entscheidungstraining im Methodenverbund - Didaktische Begründung für die Verbindung von Fallstudie und Simulationsspiel. In: KEIM, H. (Hrsg.): Planspiel, Rollenspiel, Fallstudie. Köln 1992, S.9-24.

Bundesinstitut Für Berufsbildung (Hrsg.): Laur-Ernst, U., GutSchmidt, F. \& Lietzau, E.: Neue Fabrikstrukturen - Veränderte Qualifikationen. Tagungen und Expertengespräche zur beruflichen Bildung 8, Berlin 1990.

Bundesinstitut für Berufsbildung (Hrsg.): Höpfner, H.-D.: Entwicklung selbständigen Handelns in der beruflichen Aus- und Weiterbildung. Berichte zur beruflichen Bildung 142, Berlin 1991.

Bundesinstitut für Berufsbildung (Hrsg.): Stockmann, R.: Qualitätsaspekte beruflicher Weiterbildung. Berlin 1993.

Bundesinstitut für Berufsbildung (Hrsg.): Alt, C., Sauter, E., TillMANN, H. \& LischKA, I.: Berufliche Weiterbildung in Deutschland. Bielefeld 1994. 
Bundesinstitut Für Berufsbildung (Hrsg.): Lernen heute - Fragen für morgen. Berichte zur beruflichen Bildung 168, Bielefeld 1994.

Bundesverband Deutscher Verwaltungs- und WirtschaftsakadeMIEN (Hrsg.): Rahmen-Studienordnung Rahmen-Prüfungsordnung des Bundesverbandes Deutscher Verwaltungs- und Wirtschaftsakademien für die verwaltungswissenschaftlichen und wirtschaftswissenschaftlichen Fortbildungsstudiengänge an Verwaltungs- und Wirtschaftsakademien. Frankfurt a. M. 1994.

Bunk, G. P.; Kaiser, M. \& ZEDLER, R.: Schlüsselqualifikationen - Intention, Modifikation und Realisation in der beruflichen Aus- und Weiterbildung. Mitteilungen aus der Arbeitsmarkt- und Berufsforschung 2/91, S. 365-374.

Buttler, F.: Tätigkeitslandschaft bis 2010. In: Achtenhagen, F. \& John, E. G. (Hrsg.): Mehrdimensionale Lehr-Lern-Arrangements. Innovationen in der kaufmännischen Aus- und Weiterbildung. Göttingen 1992, S. 163-173.

ButTler, F.: Berufliche Weiterbildung als öffentliche Aufgabe. In: Diepold, P. (Hrsg.): Lernen im Aufbruch, Strukturwandel und Weiterbildung in Europa. Gesellschaft zur Förderung Arbeitsorientierter Forschung und Bildung (Hrsg.): ANSTÖSSE, Materialien für Theorie und Praxis 12, Frankfurt a. M. 1995, S.6191.

Carl, N. \& Kiesel, M.: Unternehmensführung. Moderne Theorien, Methoden und Instrumente. Landsberg/Lech 1996.

CASTI, J.: Connectivity, complexity, and catastrophe in large-scale systems. International Series on Applied Systems Analysis 7, Chichester u. a. 1979.

Christopher, E. M.: Open and closed games. Further observations on their structure and effects. In: Crookall, D.; Klabbers, J.H.G.; Coote, A.; Saunders, D.; Cecchini, A. \& Piane, A. D. (Hrsg.): Simulation-Gaming in Education and Training. Proceedings of the International Simulation and Gaming Association's 18th International Conference. Oxford 1988, S.43-53.

Cohen, K.J. \& Rhenman, E.: Die Rolle von Unternehmungsspielen in Ausbildung und Forschung. In: EisenfüHr, F.; Ordelheide, D. \& Puck, G. (Hrsg.): Unternehmungsspiele in Ausbildung und Forschung. Wiesbaden 1974, S. $13-56$. 
Cook, T. D. \& Campbell, D. T.: Quasi-Experimentation. Design \& Analysis Issues for Field Settings. Chicago 1979.

Collins, A.; Brown, J.S. \& Newman, S. E.: Cognitive apprenticeship. Teaching the crafts of reading, writing, and mathematics. In: RESNICK, L. B. (Hrsg.): Knowing, learning, and instruction. Hillsdale 1989, S. 453-494.

Cognition and Technology Group at Vanderbilt: Anchored Instruction and Situated Cognition Revisited. Educational Technology 33, 3/1993, S. 52-70.

Daniel, A. M.: Das Planspiel als Instrument der Angewandten Gruppendynamik. Zur Bedeutung der Teamkompetenz in Führung und Zusammenarbeit des middle management im Handel. Göttinger handelswissenschaftliche Schriften 42, Göttingen 1996.

DANN, H. D.: Subjektive Theorien. Irrweg oder Forschungsprogramm? Zwischenbilanz eines kognitiven Konstrukts. In: Montada, L.; Reusser, K. \& STEINER, G. (Hrsg.): Kognition und Handeln. Stuttgart 1983, S.77-92.

Deutsche Forschungsgemeinschaft (Hrsg.): Berufsbildungsforschung an den Hochschulen der Bundesrepublik Deutschland. Weinheim u. a. 1990.

Deutscher Bildungsrat (Hrsg.): Empfehlungen der Bildungskommission, Strukturplan für das Bildungswesen. Stuttgart 1970.

Deutscher Bundestag (Hrsg.): Zukünftige Bildungspolitik - Bildung 2000. Schlußbericht der Enquête-Kommission des 11. Deutschen Bundestages und parlamentarische Beratung am 26. Oktober. Bonn 1990.

Deutscher Industrie- Und Handelstag (Hrsg.): Wettbewerb ohne Grenzen. Erfolg durch Weiterbildung. Bonn 1993.

DEwe, B.: Wissensverwendung in der Fort- und Weiterbildung. Zur Transformation wissenschaftlicher Informationen in Praxisdeutungen. Studien zum Umgang mit Wissen 6, Baden-Baden 1988.

Dicke, H., Glismann, H.H. \& Groenn, A.: Der deutsche Markt für berufliche Weiterbildung. Kieler Studien 269, Tübingen 1995.

DiePold, P.: Modellversuch WOKI. Wolfsburger Kooperationsmodell für den Ausbildungsberuf Industriekaufmann/-frau unter besonderer Berücksichtigung neuer Technologien. Göttingen 1991. 
Dient-Becker, A.: Personal-Effectiveness-Trainings für Frauen: Theoretische Fundierung, Durchführung und Evaluierung anhand von Selbstkonzept-Daten. Göttingen 1990.

Dixon, W. J.: BMDP Statistical Software. Berkley 1985.

DörIg, R.: Das Konzept der Schlüsselqualifikationen. Ansätze, Kritik und konstruktivistische Neuorientierung auf der Basis der Erkenntnisse der Wissenspsychologie. Dissertation, St. Gallen 1994.

Dörner, D.: Kognitive Prozesse und die Organisation des Handelns. In: Hacker, W.; Volpert, W. \& Cranach, M. v. (Hrsg.): Kognitive und motivationale Aspekte der Handlung. Bern u. a. 1983, S. 26-37.

Dörner, D.: Problemlösen als Informationsverarbeitung. 3. Aufl., Stuttgart 1987.

Dörner, D.: Die Logik des Mißlingens. Strategisches Denken in komplexen Situationen. Reinbek bei Hamburg 1989.

Dörner, D.; Kreuzig, H.W.; Reither, F. \& Stäudel, F.: Lohhausen. Vom Umgang mit Unbestimmtheit und Komplexität. Bern 1983.

DrukarczyK, J.: Finanzierung. Eine Einführung. 7. Aufl., Stuttgart 1996.

DuBs, R.: Der Anfänger-Unterricht in Betriebswirtschaftslehre. In: AcHTENHAGEN, F. \& John, E. G. (Hrsg.): Mehrdimensionale Lehr-Lern-Arrangements. Innovationen in der kaufmännischen Aus- und Weiterbildung. Göttingen 1992, S. 251-268.

Dubs, R.: Komplexe Lehr-Lern-Arrangements im Wirtschaftsunterricht Grundlagen, Gestaltungsprinzipien und Verwendung im Unterricht. In: BECK, K.; Müller, W.; Deissinger, T. \& Zimmermann, M. (Hrsg.): Berufserziehung im Umbruch. Didaktische Herausforderungen und Ansätze zu ihrer Bewältigung. Weinheim 1996, S. 159-172.

DuBs, R.: Schlüsselqualifikationen - werden wir erneut um eine Illusion ärmer? In: Gonon, P. (Hrsg.): Schlïsselqualifikationen kontrovers. Aarau 1996, S. 49-57.

EBert, G.: Planspiel - Eine aktive und attraktive Lehrmethode. In: KEIM, H. (Hrsg.): Planspiel, Rollenspiel, Fallstudie. Köln 1992, S. 25-42. 
Echterhoff, W.: Lern- und Veränderungsmessung. In: KLAuER, K. J. (Hrsg): Handbuch der Pädagogischen Diagnostik. Bd.1, Düsseldorf 1978, S. 157-176.

Eckert, A.: Kognition und Wissensdiagnose. Die Entwicklung und empirische Überprüfung des computerunterstützten wissensdiagnostischen Instrumentatiums 'Netzwerk Elaborations Technik (NET)'. Dissertation, Mannheim 1997.

EHRHARD, T.: Metakognition im Unterricht: Optimierung des Problemlöseverhaltens durch selbstreflexive Prozesse. Europäische Hochschulschriften, Reihe XI, Bd. 624, Frankfurt a. M. 1995.

EisenfüHr, F.; Ordelheide, D. \& Puck, G. (Hrsg.): Unternehmensplanspiele in Ausbildung und Forschung. Wiesbaden 1974.

Eisenstadt, M. \& Kareev, Y.: Aspekte des menschlichen Problemlösens. Die Benützung innerer Repräsentationen. In: Norman, D. A. \& Rumelhart, D. E.: Strukturen des Wissens. Wege der Kognitionsforschung. Stuttgart 1978, S. 308-344.

Enquête-Kommission ZukÜnftige Bildungspolitik - Bildung 2000 (Hrsg.): Block, R.: Bildungsbeteiligung in der beruflichen Weiterbildung. Bonn 1991.

ERPENBEcK, J.: Kompetenz und kein Ende? Bulletin, Qualifikations-Entwicklungs-Management 1/1996, S.9-13.

FABEL, M.: Fortentwicklungsperspektiven für Unternehmungsplanspiele. Zeitschrift für Planung 4, 1/1993, S. 49-64.

Fassheber, P.: Planspiele. In: Sarges, W. (Hrsg.): Management-Diagnostik. 2. Aufl., Göttingen 1995, S. 608-617.

Faulstich, P.: Lernkultur 2006: Erwachsenenbildung und Weiterbildung. München 1990.

Faulstich, P.: Qualitätskriterien für die Erwachsenenbildung als ein Fokus der Berufsbildungsforschung. Zeitschrift für Berufs- und Wirtschaftspädagogik 87, 7/1991, S. $572-581$.

Faulstich, P., Bayer, M. \& Krohn, M. (Hrsg.): Zukunftskonzepte der Weiterbildung. Projekte und Innovationen. Weinheim u. a. 1998. 
Feger, H. \& Bredenk amp, J. (Hrsg.): Datenerhebung. Enzyklopädie der Psychologie, Themenbereich B: Methodologie und Methoden, Serie I: Forschungsmethoden der Psychologie, Bd. 2, Göttingen u. a. 1983.

Feuchthofen, J. E. \& Severing, E. (Hrsg.): Qualitätsmanagement und Qualitätssicherung in der Weiterbildung. Neuwied 1995.

Fisch, R. \& Boos, M. (Hrsg.): Vom Umgang mit Komplexität in Organisationen. Konzepte, Fallbeispiele, Strategien. Konstanz 1990.

Flothow, K.: Förderung von Handlungskompetenz in der beruflichen Erstausbildung durch Lern- und Arbeitstechniken. Wirtschafts- und Berufspädagogische Schriften 10, Bergisch Gladbach 1992.

Foster, H.: Politische Bildung zwischen Tradition und Aktualität. Berufsbildung in Wissenschaft und Praxis 19, 2/1990, S.11-16.

Freidank, C.-C.: Kostenrechnung. Einführung in die begrifflichen, theoretischen, verrechnungstechnischen sowie planungs- und kontrollorientierten Grundlagen des innerbetrieblichen Rechnungswesens. 4. Aufl., München u. a. 1994.

Friedrich, H. F.: Analyse und Förderung kognitiver Lernstrategien. Empirische Pädagogik 9, 2/1995, S. 115-153.

Friedrich, H. F. \& Mandl, H. (Hrsg.): Lern- und Denkstrategien. Analyse und Intervention. Göttingen u. a. 1992.

Friedrichs, J.: Methoden empirischer Sozialforschung. 14. Aufl., Opladen 1990.

Funrer, U.: Mehrfachhandeln in dynamischen Umfeldern. Göttingen 1985.

Funke, J.: Komplexes Problemlösen. Bestandsaufnahme und Perspektiven. Lehr- und Forschungstexte Psychologie 18, Berlin 1986.

Funke, J.: Probleme komplexer Problemlöseforschung. In: Fisch, R. \& Boos, M. (Hrsg.): Vom Umgang mit Komplexität in Organisationen. Konzepte, Fallbeispiele, Strategien. Konstanz 1990, S. 95-105.

Fürstenau, B.: Komplexes Problemlösen im betriebswirtschaftlichen Unterricht. Wiesbaden 1994.

FüsER, K.: Modernes Management. Lean Management, Business Reengineering, Benchmarking und viele andere Methoden. 2. Aufl., München u. a. 1999. 
Gardner, H.: Dem Denken auf der Spur. Der Weg der Kognitionswissenschaft. Stuttgart 1989.

GeilhardT, T. \& MÜhlbradt, T.: Konzepte und Trends im Überblick. In: Geilhardt, T. \& Mühlbradt, T. (Hrsg.): Planspiele im Personal- und Organisationsmanagement. Göttingen 1995, S.9-18.

Georg, W.: Berufs- und wirtschaftspädagogische Perspektiven. Bildung und Erziehung 51, 1/1998, S. 59-65.

Gerjets, P. \& Westermann, R.: Theorien der kognitiven Psychologie und das Problem der Intentionalität. In: KLuwE, R. H. (Hrsg.): Strukturen und Prozesse intelligenter Systeme. Wiesbaden 1997, S. 255-279.

Gerlach, H.H.: Betriebliche Weiterbildung in Europa durch modulare Planspiele. Die Berufsbildende Schule 48, 12/1996, S. 368-374.

Gerstenmaier, J. \& MandL, H.: Wissenserwerb unter konstruktivistischer Perspektive. Zeitschrift für Pädagogik 41, 6/1995, S. 867-887.

Getsch, U.: Möglichkeiten einer Förderung von betriebswirtschaftlichem Zusammenhangswissen - eine empirische Untersuchung mit Hilfe eines Unternehmensplanspiels bei angehenden Industriekaufleuten. Berichte aus dem Seminar für Wirtschaftspädagogik der Georg-August-Universität Göttingen, Bd.13, Göttingen 1990.

Geuting, M.: Planspiel und soziale Simulation im Bildungsbereich, Frankfurt a. M. 1992.

Gigerenzer, G.: Messung und Modellbildung in der Psychologie. München/Basel 1981.

Götze, U. \& Blozch, J.: Investitionsrechnung. Modelle und Analysen zur Beurteilung von Investitionsvorhaben. 2. Aufl., Berlin u. a. 1995.

Götze, U. \& RudolPh, F.: Instrumente der strategischen Planung. In: Bloech, J.; Götze, U.; Huch, B.; Lücke, W. \& Rudolph, F. (Hrsg.): Strategische Planung. Instrumente, Vorgehensweisen und Informationssysteme. Heidelberg 1994, S.156.

Goetze, W.: Von SQ (Schlüsselqualifikationen) zu QS (Qualitätssicherung): Sollten wir mehr über Qualität von Bildungsangeboten reden - und weniger über 
Schlüsselqualifikationen? In: GonON, P. (Hrsg.): Schlüsselqualifikationen kontrovers. Aarau 1996, S. 58-66.

Gonon, P. (Hrsg.): Schlüsselqualifikationen kontrovers. Aarau 1996.

Groeben, N.: Die Handlungsperspektive als Theorierahmen für Forschung im pädagogischen Feld. In: HofER, M. (Hrsg.): Informationsverarbeitung und Entscheidungsverhalten von Lehrern. München 1981.

Groeben, N.: Handeln, Tun, Verhalten als Einheiten einer verstehend-erklärenden Psychologie. Wissenschaftstheoretischer Überblick und Programmentwurf zur Integration von Hermeneutik und Empirismus. Tübingen 1986.

Groeben, N.: Zur Kritik einer unnötigen, widersinnigen und destruktiven Radikalität. In: Fischer, H. R. (Hrsg.): Die Wirklichkeit des Konstruktivismus. Heidelberg 1995, S. 149-159.

Groeben, N. \& Scheele, B.: Argumente für eine Psychologie des reflexiven Subjekts. Darmstadt 1977.

Groeben, N.; Wahl, D.; Schlee, J. \& Scheele, B.: Das Forschungsprogramm Subjektive Theorien. Eine Einführung in die Psychologie des reflexiven Subjekts. Tübingen 1988.

GrünER, H.: Evaluation betrieblicher Weiterbildung. Formatives oder summatives Vorgehen? Grundlagen der Weiterbildung 4, 6/1993, S. 348-353.

Gutenberg, E.: Grundlagen der Betriebswirtschaftslehre. Bd. 1: Die Produktion. 24. Aufl., Berlin u. a. 1983.

Hager, W.: Grundlagen einer Versuchsplanung zur Prüfung empirischer Hypothesen in der Psychologie. In: LüER, G. (Hrsg.): Allgemeine experimentelle Psychologie. Stuttgart 1987, S. 43-264.

HAHNE, H.: Schlüsselqualifikationen über PC-Planspiele-dargestellt am Beispiel von Unternehmensplanspielen zunehmender Komplexität aus Modellversuchen in Baden-Württemberg. Wirtschaft und Erziehung 41, 12/1989, S. 391-397.

HALFPAP, K.: Ganzheitliches Lernen im Unterricht kaufmännischer beruflicher Schulen. Erziehungswissenschaft und Beruf, 3/1991, S. 235-251. 
Hamel, W.: CISMET. Computerunterstütztes Intensiv-Seminar für Management-Entscheidungs-Training. Düsseldorfer Schriften zur Unternehmensführung 6/1995, 7. Aufl., Düsseldorf 1996.

Hamm, H.-J.: Praxisbezug im Betriebswirtschaftslehrestudium und betriebliches Einstellungsverhalten. Europäische Hochschulschriften, Reihe V, Bd. 1355, Frankfurt a. M. 1993.

Hammer, R. M.: Unternehmensplanung. Lehrbuch der Planung und strategischen Unternehmensführung. 7. Aufl., München u.i998.

Hartung, J. \& Elpelt, B.: Multivariate Statistik. Lehr- und Handbuch der angewandten Statistik, München 1984.

Hartung, J.; Elpelt, B. \& Klösener, K.-H.: Statistik. Lehr- und Handbuch der angewandten Statistik, 9. Aufl. München 1993.

Harney, K.; JÜtTing, D. \& Koring, B.: Professionalisierung in der Erwachsenenbildung. Studien zur Erwachsenenbildung, Bd.1, Frankfurt a. M. 1987.

Hasselmann, D.: Computersimulierte komplexe Problemstellungen in der Management-Diagnostik. Die Theorie zum Konzept Herausforderung Komplexität. Hamburg 1993.

Hauschildt, J.: Komplexität, Zielbildung und Effizienz von Entscheidungen in Organisationen. In: Fisch, R. \& Boos, M. (Hrsg.): Vom Umgang mit Komplexität in Organisationen: Konzepte, Fallbeispiele, Strategien. Konstanz 1990, S. 131-147.

HaYs, W. L.: Statistic for the social sciences. 2. Aufl., London 1973.

Heckhausen, H.: Motivation und Handeln. 2. Aufl., Berlin 1989.

Heckhausen, H., Gollwitzer, P. M. \& Weinert, F. E. (Hrsg.): Jenseits des Rubikon. Der Wille in den Humanwissenschaften, Heidelberg 1987.

Heckhausen, H. \& Rheinberg, F.: Lernmotivation im Unterricht erneut betrachtet. Unterrichtswissenschaft 8, 1/1980, S. 7-47.

HEID, H.: Schlüsselqualifikationen - ideologiekritische Anmerkungen zu einer berufspädagogischen Konzeption. In: Metzger, C. \& Seitz, H. (Hrsg.): Wirtschaftliche Bildung. Träger, Inhalte, Prozesse, Zürich 1995, S. 49-65. 
HEID, H.: Erfordernis und Problematik einer Unterscheidung zwischen Verhalten und Verhaltensdisposition. - Beitrag zur Zielbestimmung erziehungspraktischen Handelns. In: Beck, K.; Müller, W.; Deissinger, T. \& Zimmermann, M. (Hrsg.): Berufserziehung im Umbruch. Didaktische Herausforderungen und Ansätze zu ihrer Bewältigung. Weinheim 1996, S. 79-85.

HeID, H.: Berufliche Bildung im Spannungsfeld. In: BEITRÄGE AUS DER Arbeitsmarkt- und Berufsforschung 214: Euler, D. (Hrsg.): Berufliches Lernen im Wandel - Konsequenzen für die Lernorte? Nürnberg 1998, S. 34-54.

HeIDACK, C.: Lerninstrument an Hochschulen und in der Wirtschaft. In: Graf, J. (Hrsg.): Planspiele - simulierte Realitäten für den Chef von morgen. Speyer 1992, S. 45-58.

Heimann, P.; Otto, G. \& Schulz, W. (Hrsg.): Unterricht - Analyse und Planung. Hannover 1979.

Heinecke, A. \& Oelsnitz, D. v. D.: Computergestützte Planspiele. Integration in die betriebliche Aus- und Weiterbildung. Personal 46, 4/1994, S. 168-173.

Hempel, C. G. \& Oppenheim, P.: Studies in the Logic of Explanation. Philosophy of Science 15, 1948, S. 135-175.

Henz, H.: Lehrbuch der systematischen Pädagogik. Freiburg u. a. 1964.

Hesse, F.-W.: Review: John R. Anderson (1983), The Architecture of Cognition. Sprache und Kognition 4, 1985, S. 231-237.

Hesse, F.-W.; SpIEs, K. \& LüER, G.: Einfluß motivationaler Faktoren auf das Problemlöseverhalten im Umgang mit komplexen Problemen. Zeitschrift für experimentelle und angewandte Psychologie XXX, 3/1983, S.400-424.

Högsdal, B.: Planspiele. Einsatz von Planspielen in der Aus- und Weiterbildung. Praxiserfahrungen und bewährte Methoden. Bonn 1996.

Hösch, G.: Evaluation eines computergestützten Planspiels. Dissertation, Frankfurt a. M. 1995.

Hofer, M.; Pekrun, R. \& Zielinski, W.: Die Psychologie des Lerners. In: Weidenmann, B.; Krapp, A.; Hofer, M.; Huber, G. L. \& Mandl, H. (Hrsg.): Pädagogische Psychologie. München u. a., S. 219-276. 
HopfenBEck, W.: Allgemeine Betriebswirtschafts- und Managementlehre. Das Unternehmen im Spannungsfeld zwischen ökonomischen, sozialen und ökologischen Interessen. 7. Aufl., Landsberg/Lech 1992.

HollmanN, H.: Validität in der Eignungsdiagnostik. Göttingen 1991.

Hub, H.: Ganzheitliches Denken im Management. Komplexe Aufgaben PCgestützt lösen. Wiesbaden 1994.

InGENK AMP, K.-H.: Lehrbuch der pädagogischen Diagnostik. 3. Aufl., Weinheim u. a. 1985 .

Jagenlauf, M.; Schulz, M. \& Wolgast, G. (Hrsg.): Weiterbildung als quartärer Bereich. Bestand und Perspektive nach 25 Jahren. Neuwied 1995.

Jank, W. \& Meyer, H.: Didaktische Modelle. 3. Aufl., Frankfurt a. M. 1994.

John, E. G.: Fallstudien und Fallstudienunterricht. In: Achtenhagen, F. \& John, E. G. (Hrsg.): Mehrdimensionale Lehr-Lern-Arrangements. Innovationen in der kaufmännischen Aus- und Weiterbildung. Göttingen 1992, S. 79-91.

Joint Committee on Standards for Educational Evaluation \& SanDERs, J.R. (Hrsg.): Handbuch der Evaluationsstandards. Die Standards des "Joint Committee on Standards for Educational Evaluation". Opladen 1999.

Just, T.: Beurteilung von Lernerfolgen in einem Unternehmensplanspiel. Unveröffentlichte Diplomarbeit am Institut für Betriebswirtschaftliche Produktionsund Investitionsforschung, Göttingen 1998.

KAISER, A.: Schlïsselqualifikationen in der Arbeitnehmerweiterbildung. Landesarbeitsgemeinschaft für katholische Erwachsenenbildung in Nordrhein-Westphalen e. V.. Neuwied 1992.

KaISER, F.-J.: Entscheidungstraining. Die Methoden der Entscheidungsfindung. Fallstudie - Simulation - Planspiel. 2. Aufl., Bad Heilbrunn/Obb. 1976.

Kaiser, F.-J. (Hrsg.): Die Fallstudie. Theorie und Praxis der Fallstudiendidaktik. Bad Heilbrunn/Obb. 1983.

KaISER, F.-J.: Grundlagen der Fallstudiendidaktik - Historische Entwicklung - Theoretische Grundlagen - Unterrichtliche Praxis. In: KaIsER, F.-J. (Hrsg.): Die Fallstudie. Theorie und Praxis der Fallstudiendidaktik. Bad Heilbrunn/Obb. 1983, S. 9-34. 
KAISER, F.-J. (Hrsg.): Handlungsorientiertes Lernen in kaufmännischen Berufsschulen. Didaktische Grundlagen und Realisierungsmöglichkeiten für die Arbeit im Lernbüro. Bad Heilbrunn/Obb. 1987.

KaIser, F.-J. \& Kaminski, H.: Methodik des Ökonomie-Unterrichts: Grundlagen eines handlungsorientierten Lernkonzepts mit Beispielen. Bad Heilbrunn/Obb. 1994.

Kastner, M.: Systemisches Denken. In: Geilhardt, T. \& Mühlbradt, T. (Hrsg.): Planspiele im Personal- und Organisationsmanagement. Göttingen 1995, S. 27-43.

Kelly, G. A.: The Psychology of Personal Constructs. New York 1955.

Keys, B. \& Wolfe, J.: The Role of Management Games and Simulations in Education and Research. Journal of Management 16, 2/1990, S.307-336.

KLAFKI, W.: Zur Unterrichtsplanung im Sinne kritisch-konstruktiver Didaktik. In: ADL-Amini, B. \& KünZli, R. (Hrsg.): Didaktische Modelle und Unterrichtsplanung, Weinheim 1980, S. 11-48.

Klafki, W.: Leistung. In: Lenzen, D. \& Mollenhauer, K. (Hrsg.): Enzyklopädie Erziehungswissenschaft, Bd.1: Theorien und Grundbegriffe der Erziehung und Bildung. 1. Aufl., Stuttgart 1983, S.491-495.

KLAUER, K. J.: Methodik der Lehrzieldefinition und Lehrstoffanalyse. Düsseldorf 1974.

KLAuer, K. J.: Kontentvalidität. In: Klauer, K. J. (Hrsg.): Handbuch der Pädagogische Diagnostik. Bd.1, Düsseldorf 1978, S. 225-255.

Klauer, K.J.: Ein kriteriumsorientiertes Zensierungsmodell. Zeitschrift für Entwicklungspsychologie und Pädagogische Psychologie 14, 1982, S. 65-79.

KLaUer, K. J.: Fördernde Notengebung durch Benotung unter drei Bezugsnormen. In: Olechowski, R. \& Persy, E. (Hrsg.): Fördernde Leistungsbeurteilung. Ein Symposium. Schule - Wissenschaft - Politik 1, Wien u. a. 1987, S. 180-206.

KlaueR, K. J.: Kriteriumsorientierte Tests. Lehrbuch der Theorie und Praxis lehrzielorientierten Messens. Göttingen u. a. 1987. 
KLAUER, K.J.: Teaching for learning-to-learn: a critical appraisal with some proposals. Instructional Science 17, 1988, S. 351-367.

Klauser, F.: Effektive Gestaltung von Lehr- und Lernprozessen in der kaufmännischen Ausbildung - Erfordernisse, neuere Befunde und künftige Forschungsaufgaben. Zeitschrift für Berufs- und Wirtschaftspädagogik 94, 2/1998, S. 248-264.

Klauser, F.: Neuere Befunde zum Lernerfolg beim Einsatz komplexer LehrLern-Arrangements in der kaufmännischen Ausbildung. Wirtschaft und Erziehung 50, 12/1998, S. 406-412.

KLAUSER, F.: Problem-based learning. Zeitschrift für Erziehungswissenschaft 1, 2/1998, S. 273-293.

KLEBER, E. W.: Diagnostik in pädagogischen Handlungsfeldern: Einführung in Bewertung, Beurteilung, Diagnose und Evaluation. Weinheim 1992.

KLIPPERT, H.: Planspiele in Schule und Lehrerfortbildung. In: KEIM, H. (Hrsg.): Planspiel, Rollenspiel, Fallstudie. Köln 1992, S. 219-250.

Knoblich, G. \& Rhenius, D.: Zur Reaktivität Lauten Denkens beim komplexen Problemlösen. Zeitschrift f̈̈ Experimentelle Psychologie 42, 3/1995, S.419454 .

KöHleR, K.: DIN ISO 9000 ff. beim Bildungsträger. München 1995.

KÖNIG, E.: Qualitative Forschung in der Erwachsenenbildung. In: KöNIG, E. \& ZEDLER, P. (Hrsg.): Bilanz qualitativer Forschung. Bd. I: Grundlagen qualitativer Forschung. Weinheim 1995, S. 195-206.

KöNIG, E.: Qualitative Forschung subjektiver Theorien. In: KöNIG, E. \& ZEDLER, P. (Hrsg.): Bilanz qualitativer Forschung. Bd. II: Methoden. Weinheim 1995, S. 11-30.

Konne, T.: Reliabilitätsuntersuchungen zur Netzwerkbildung bei der Erhebung kognitiver Strukturierungen. Unveröffentlichte Diplomarbeit am Seminar für Wirtschaftspädagogik, Göttingen 1992.

Koller, H.: Simulation und Planspieltechnik. Berechnungsexperimente in der Betriebswirtschaft. Wiesbaden 1969. 
KorndörFER, W.: Unternehmensführungslehre. Einführung, Entscheidungslogik, soziale Komponenten. 8. Aufl., Wiesbaden 1995.

Kramer, M.: Probleme der Leistungsbeurteilung im Fach Wirtschaftspraxis der Berufsfachschule - Wirtschaft. Unveröffentlichte Diplomarbeit am Seminar für Wirtschaftspädagogik, Göttingen 1995.

Kruse, C.-H.: Das 'lernende Unternehmen' als strategische Antwort auf den Strukturwandel. In: Diepold, P. (Hrsg.): Lernen im Aufbruch, Strukturwandel und Weiterbildung in Europa. ANSTÖSSE, Materialien für Theorie und Praxis 12, Frankfurt a. M. 1995, S. 15-31.

Kutscha, G.: 'Entberuflichung' und 'Neue Beruflichkeit' - Thesen und Aspekte zur Modernisierung der Berufsbildung und ihrer Theorie. Zeitschrift für Berufsund Wirtschaftspädagogik 88, 7/1992, S. 535-548.

Kuwan, H.; Gnahs, D. \& Seusing, B.: Weiterbildungsstatistik in Deutschland - Ausgangslage und zukünftige Anforderungen. Mitteilungen aus der Arbeitsmarkt- und Berufsforschung 2/91, Nürnberg 1991, S.277-290.

LAUR-ERNST, U.: Schlüsselqualifikationen in Deutschland - ein ambivalentes Konzept zwischen Ungewissheitsbewältigung und Persönlichkeitsbildung. In: GoNON, P. (Hrsg.): Schlüsselqualifikationen kontrovers. Aarau 1996, S. 17-23.

LAVE, J. \& WEngER, E.: Situated Learning. Legitimate peripheral participation. Cambridge 1991.

LEMKE, S.: Transfermanagement. Göttingen 1995.

Leutner, D.: Adaptive Lehrsysteme. Instruktionspsychologische Grundlagen und experimentelle Analysen. Fortschritte der psychologischen Forschung 13, Weinheim 1992.

LienErT, G. A. \& EyE, A. V.: Erziehungswissenschaftliche Statistik. Eine elementare Einführung für pädagogische Berufe. Weinheim 1994.

Lienert, G. A. \& RaAtz, U.: Testaufbau und Testanalyse. 6. Aufl., Weinheim 1998.

Lindman, H. R.: Analysis of Variance in Complex Experimental Designs. San Francisco 1974. 
Lipsmeier, A.: Vom verblassenden Wert des Berufes für das berufliche Lernen. Zeitschrift für Berufs- und Wirtschaftspädagogik 94, 4/1998, S. 481-495.

LüDECKE-PlüMER, S.: Emotionale Befindlichkeit im Kontext von Lernen, Denken, Handeln in komplexen ökonomischen Situationen - Am Beispiel der kaufmännischen Berufsschule. Dissertation, Göttingen 1991.

Malik, F.: Strategie des Managements komplexer Systeme. Ein Beitrag zur Management-Kybernetik evolutionärer Systeme, 3. Aufl., Bern u. a. 1989.

Mandelbrot, B. B.: Die fraktale Geometrie der Natur. Basel u. a. 1987.

Mandl, H.; Gruber, H. \& Renkl, A.: Prozesse der Wissensanwendung beim komplexen Problem-Lösen in einer kooperativen Situation. In: AcHTEnHAgen, F. \& John, E. G. (Hrsg.): Mehrdimensionale Lehr-Lern-Arrangements. Innovationen in der kaufmännischen Aus- und Weiterbildung. Göttingen 1992, S.478490.

Mandl, H.; Gruber, H. \& Renkl, A.: Das träge Wissen. Psychologie heute, 9/1993, S. 64-69.

Mandl, H. \& Reinmann-Rothmeier, G.: Unterrichten und Lernumgebungen gestalten. Forschungsbericht der Ludwigs-Maximilians-Universität 60, München 1995.

Mandl, H. \& Spada, H. (Hrsg.): Wissenspsychologie. München u. a. 1988.

Mayring, P.: Qualitative Inhaltsanalyse. Grundlagen und Techniken. 4. Aufl., Weinheim 1993.

McClelland, J. L.; Rumelhart, D. E. \& PDP Research Group: Parallel distributed processing. Explorations in the Microstructure of Cognition. Bd. 2: Psychological and Biological Models. 4. Aufl., Cambridge 1987.

McClelland, J. L.; Rumelhart, D. E. \& PDP Research Group: Parallel distributed processing. Explorations in the Microstructure of Cognition. Bd. 1: Foundations. 7. Aufl., Cambridge 1988.

MCKenney, J.L.: An evaluation of a business game in an MBA curriculum. Journal of Business 35, 1962, S. 278-286. 
McKinsey Company, Inc. \& Rommel, G.: Einfach überlegen. Das Unternehmenskonzept, das die Schlanken schlank und die Schnellen schnell macht. Stuttgart 1993.

Meer, E.v.D. \& Kolbe, M.: Zur Kodierung von Zeitbezügen in begrifflichen Wissenstrukturen. In: KLuwE, R. H. (Hrsg.): Strukturen und Prozesse intelligenter Systeme. Wiesbaden 1997, S. 107-131.

Meffert, H.: Marketing. Grundlagen der Absatzpolitik. 7. Aufl., Wiesbaden 1991.

Mertens, D.: Schlüsselqualifikationen. Mitteilungen aus der Arbeitsmarkt- und Berufsforschung 7/74, Nürnberg 1974, S. 36-45.

Metzig, W. \& Schuster, M.: Lernen zu lernen. Lernstrategien wirkungsvoll einsetzen. Berlin 1. a. 1993.

Miller, G. A.; Galanter, E. \& Pribram, K.H.: Strategie des Handelns. Stuttgart 1973.

Minsky, M.: Mentopolis. Stuttgart 1990.

MöBus, C.: Zur Modellierung kognitiver Prozesse mit daten- bzw. zielorientierten Regelsystemen. In: MandL, H. \& SPADA, H. (Hrsg.): Wissenspsychologie. München u. a. 1988, S. 423-467.

Mummendey, H. D.: Die Fragebogen-Methode. Grundlagen und Anwendung in Persönlichkeits-, Einstellungs- und Selbstkonzeptforschung. 2. Aufl., Göttingen u. a. 1995.

Newelt, A. \& Simon, H. A.: Human Problem Solving. Englewood Cliffs 1972.

NezEL, I.: Allgemeine Didaktik der Erwachsenenbildung. Bern 1992.

Nieschlag, R.; Dichtl, E. \& Hörschgen, H.: Marketing. 18. Aufl., Berlin 1997.

Norman, D. A. \& Rumelhart, D. E.: Strukturen des Wissens. Wege der Kognitionsforschung. Stuttgart 1978.

Nüse, R., Groeben, N., Freitag, B. \& Schreier, M.: Über Erfindung/en des Radikalen Konstruktivismus. Kritische Gegenargumente aus psychologischer Sicht. Weinheim 1991. 
Oldenbürger, H.-A.: Zur Erhebung und Repräsentation kognitiver Strukturen. Braunschweig 1986.

Oldenbürger, H.-A.: Netz-Werk-Zeug 1. Zählwerk für beliebige Variablenkombinationen. Version $\alpha . z$, Programm und Dokumentation. Göttingen 1992.

OldenBÜRgER, H.-A.: Exploratorische, graphische und robuste Datenanalyse. In: Erdfelder, E.; Mausfeld, R.; Meiser, T. \& Rudinger, G. (Hrsg.): Handbuch Quantitative Methoden. Weinheim 1996, S. 71-86.

Olfert, K.: Finanzierung. 8. Aufl., Ludwigshafen/Rhein 1994.

OpwIS, K. \& LÜER, G.: Modelle der Repräsentation von Wissen. Enzyklopädie der Psychologie, Themenbereich C: Theorie und Forschung, Serie II: Kognition, Bd. 4: Gedächtnis, Göttingen 1994, S.1-81.

OrTH, C.: Entwicklung von Unternehmensplanspielen für die betriebswirtschaftliche Aus- und Weiterbildung. Konzeption eines Planspiels mit variabler Modellkomplexität. In: Becker, F. G. \& Berthel, J. (Hrsg.): Personal-Management 18, Lohmar u. a. 1999.

PäTzold, G. (Hrsg.): Handlungsorientierung in der beruflichen Bildung. Frankfurt a. M. 1992.

PÄTzolD, G.: “Schlüsselqualifikationen"- Didaktisch-methodischer Passepartout für eine moderne Berufsbildung? In: GonON, P. (Hrsg.): Schlüsselqualifikationen kontrovers. Aarau 1996, S. 136-145.

Palm, G.: Modellvorstellungen auf der Basis neuronaler Netzwerke. In: MandL, H. \& Spada, H. (Hrsg.): Wissenspsychologie. München 1988, S. 488-502.

Perridon, L. \& Steiner, M.: Finanzwirtschaft der Unternehmung. 8. Aufl., München 1995.

Peterson, C.; Maier, S. F. \& Seligman, M. E. P.: Learned helplessness. A theory for the age of personal control. Oxford 1993.

Petzing, F.: Untersuchung des Wissenserwerbs bei einem Unternehmensplanspiel mit Verfahren des Operations Research. Schriftenreihe Handeln und Entscheiden in komplexen ökonomischen Situationen, Bd. 8, Heidelberg 1993. 
Pietzsch, K.: Zum Einsatz von Planspielen in Institutionen der kaufmännischen Weiterbildung. Unveröffentlichte Diplomarbeit am Institut für Betriebswirtschaftliche Produktions- und Investitionsforschung, Göttingen 1997.

Popper, K. R.: Logik der Forschung. 9. Aufl., Tübingen 1989.

Posch, P.; Schneider, W. \& Mann, W.: Unterrichtsplanung mit Beispielen für den betriebswirtschaftlichen Unterricht. Wien 1977.

PREHM, H.-J .: Marketing-Unternehmensspiel MARKUS: Modelldarstellung und Instrumente zur Entscheidungsvorbereitung. Wiesbaden u. a. 1995.

Preiss, P.: Komplexität im Betriebswirtschaftslehre-Anfangsunterricht. In: Achtenhagen, F. \& John, E. G. (Hrsg.): Mehrdimensionale Lehr-Lern-Arrangements. Innovationen in der kaufmännischen Aus- und Weiterbildung. Göttingen 1992, S. 58-78.

Preiss, P.: Planspiel Jeansfabrik - Betriebliche Leistungsprozesse. Wiesbaden 1994.

Preiss, P.: Didaktik des wirtschaftsinstrumentellen Rechnungswesens. Lehren und Lernen, München u. a. 1999.

Prenzel, M. \& Schiefele, H.: Konzepte der Veränderung und Erziehung. In: Weidenmann, B.; KrapP, A.; Hofer, M.; Huber, G. L. \& Mandl, H. (Hrsg.): Pädagogische Psychologie. München u. a., S. 105-142.

Probst, G. J. B. \& Gomez, P. (Hrsg.): Vernetztes Denken. Unternehmen ganzheitlich führen. Wiesbaden 1989 .

Putz-Osterloh, W.: Gibt es Experten für komplexe Probleme? Zeitschrift für Psychologie 195, 1/1987, S.63-84.

Putz-Osterloh, W. \& Lüer, G.: Über die Vorhersagbarkeit komplexer Problemlöseleistungen durch Ergebnisse in einem Intelligenztest. Zeitschrift für experimentelle und angewandte Psychologie 28, 1981, S.309-334.

RAIA, A. P.: A study of the educational value of management games. Journal of Business 39, 3/1966, S. 339-352.

REBmann, K.: Komplexität von Lehrbüchern für den Wirtschaftslehreunterricht. Eine empirisch-analytische Studie. Schriftenreihe Handeln und Entscheiden in komplexen ökonomischen Situationen 12, Göttingen 1993. 
Rebmann, K.: Der Planspieleinsatz aus der Sicht von Lehrern und Lehrerinnen sowie Ausbildern und Ausbilderinnen. Zeitschrift für Berufs- und Wirtschaftspädagogik 94, 4/1998, S. 552-565.

REETz, L.: Fälle und Fallstudien im Wirtschaftslehre-Unterricht. Wirtschaft und Erziehung 40, 5/1988, S. 148-156.

REETz, L.: Zum Einsatz didaktischer Fallstudien im Wirtschaftslehreunterricht. Unterrichtswissenschaft 16, 2/1988, S. 38-55.

ReEtz, L.: Zum Konzept der Schlüsselqualifikationen in der Berufsbildung. Berufsbildung in Wissenschaft und Praxis 18, 5-6/1989, S. 3-10 und S.24-30.

ReEtz, L.: Wissen und Handeln. - Zur Bedeutung konstruktivistischer Lernbedingungen in der kaufmännischen Berufsbildung. In: BECK, K.; Müller, W.; Deissinger, T. \& Zimmermann, M. (Hrsg.): Berufserziehung im Umbruch. Didaktische Herausforderungen und Ansätze zu ihrer Bewältigung. Weinheim 1996 , S. 173-188.

Reetz, L. \& Reitmann, T. (Hrsg.): Schlüsselqualifikationen. Dokumentation des Symposiums in Hamburg 'Schlüsselqualifikationen - Fachwissen in der Krise?'. Materialien zur Berufsausbildung 3, Hamburg 1990.

ReEtz, L. \& Sievers, H.-P.: Zur curriculum- und lerntheoretischen Begründung der Fallstudienverwendung im Wirtschaftslehreunterricht der Sekundarstufe II. In: KaISER, F.-J. (Hrsg.): Die Fallstudie. Theorie und Praxis der Fallstudiendidaktik. Bad Heilbrunn/Obb. 1983, S. 75-110.

Reinmann-Rothmeier, G. \& Mandl, H.: Lernen als Erwachsener. Forschungsbericht der Ludwigs-Maximilians-Universität 39, München 1994.

Reisse, W.: Zur Prüfbarkeit von Schlüsselqualifikationen. In: Gonon, P. (Hrsg.): Schlüsselqualifikationen kontrovers. Aarau 1996, S. 114-120.

ReITHER, F.: Schwierigkeiten beim Umgang mit wirtschaftlich-ökologischen Systemen. In: BAlck, H. \& KreiBich, R. (Hrsg.): Evolutionäre Wege in die Zukunft. Wie lassen sich komplexe Systeme managen? Zukunftsstudien 4, Weinheim 1991, S. 128-161.

Reither, F.: Komplexitätsmanagement. Denken und Handeln in komplexen Situationen. München 1997. 
RENkL, A.: Träges Wissen. Die "unerklärliche" Kluft zwischen Wissen und Handeln. Forschungsbericht der Ludwig-Maximilians-Universität, Institut für Pädagogische Psychologie und Empirische Pädagogik 41, München 1994.

Renkl, A.: Vorwissen und Schulleistung. In: Möller, J. \& Köller, O. (Hrsg.): Emotionen, Kognitionen und Schulleistung. Weinheim 1996, S. 175-190.

Rheinberg, F.: Soziale versus individuelle Leistungsvergleiche und ihre motivationalen Folgen in Lehr-Lernsituationen. In: Olechowski, R. \& PERSY, E. (Hrsg.): Fördernde Leistungsbeurteilung. Ein Symposium. Schule - Wissenschaft - Politik 1, Wien u. a. 1987, S. 80-115.

Ripper, J.; ZaChay, A.; Herz, B. \& Merz, W.: Computerstützte Ökonomieplanspiele als Lehr-Lern-Methode: Auswirkungen der Komplexität des Planspiels und des Vorwissens der Benutzer auf den Lernerfolg. In: MöBıUs, C. (Hrsg.): Interne Berichte des Fachbereichs Informatik der Universität Oldenburg. Oldenburg 1993, S. 112-121.

Rohn, W. E.: Führungsentscheidungen im Unternehmensplanspiel. Essen 1964.

Rohn, W.E.: Methodik und Didaktik des Planspiels. In: InstituT DER DEUTSCHEN WirtschafT (Hrsg.): Beiträge zur Gesellschafts- und Bildungspolitik. Köln 1980.

Rohn, W. E.: Management-Lernen durch Planspiele. Eine Bilanz: 30 Jahre Unternehmens-Planspiele in Deutschland. Personalführung 10, 1991, S. 764-773.

Rohn, W. E.: Ursprung und Entwicklung des Planspiels. In: Geilhardt, T. \& Mühlbradt, T. (Hrsg.): Planspiele im Personal- und Organisationsmanagement. Göttingen 1995, S. 57-67.

Rohn, W. E.: Einsatzgebiete und Formen des Planspiels. In: Geilhardt, T. \& Mühlbradt, T. (Hrsg.): Planspiele im Personal- und Organisationsmanagement. Göttingen 1995, S. 69-77.

Rumelhart, D. E. \& Norman, D. A.: Accretion, tuning, and restructuring. Three modes of learning. In: CotTon, J. W. \& Klatzky, R. (Hrsg.): Semantic fractors in cognition. Hillsdale, New York 1978, S. 37-54.

Rumelhart, D. E. \& Norman, D. A.: Das aktive strukturelle Netz. In: Norman, D. A. \& Rumelhart, D. E.: Strukturen des Wissens. Wege der Kognitionsforschung. Stuttgart 1978, S. 51-77. 
Savage, C. M.: Fifth Generation Management. Kreatives Kooperieren durch virtuelles Unternehmertum, dynamische Teambildung und Vernetzung von Wissen. Mensch Technik Organisation 12, Zürich 1997.

SCHEElE, B.: Emotionen als bedürfnisrelevante Bewertungszustände. Grundriß einer epistemologischen Emotionstheorie. Tübingen 1990.

Scheele, B. (Hrsg.): Struktur-Lege-Verfahren als Dialog-Konsens-Methodik. Ein Zwischenfazit zur Forschungsentwicklung bei der rekonstruktiven Erhebung Subjektiver Theorien. Münster 1992.

Scheele, B. \& Groeben, N.: Dialog-Konsens-Methoden zur Rekonstruktion Subjektiver Theorien. Tübingen 1988.

Schlag, B.: Lern- und Leistungsmotivation. Opladen 1995.

Schmager, B. \& Wirth, S.: Planspiel und strategisches Denken - Bedeutung für die betriebliche Weiterbildung. Fortschrittliche Betriebsführung und Industrial Engineering 38, 5/1989, S.278-283.

Schmiel, M.: Die Förderung der Lernmotivation in der beruflichen Weiterbildung. Berlin 1988.

Schmiel, M. \& Sommer, K.-H.: Zur Legitimationsproblematik von Schlüsselqualifikationen. In: GonON, P. (Hrsg.): Schlüsselqualifikationen kontrovers. Aarau 1996, S. 75-80

Schmolke, M. \& Deitermann, S.: Industrielles Rechnungswesen IKR. 27. Aufl., Darmstadt 1999.

SchneIDER, D.: Investition, Finanzierung und Besteuerung. 7. Aufl., Wiesbaden 1992.

Schnell, R.; Hill, P. B. \& Esser, E.: Methoden der empirischen Sozialforschung. 6. Aufl., München 1999.

ScHOEL, K.: Konzeption handelsbetrieblicher Unternehmenssimulationen und Planspiele: Ein Modellentwurf für den mittelständischen Textileinzelhandel. Göttinger handelswissenschaftliche Schriften 57, Göttingen 1999.

ScHunck, A.: Subjektive Theorien von Berufsfachschülern zu einem planspielgestützten Betriebswirtschaftslehre-Unterricht. Berichte aus dem Seminar für 
Wirtschaftspädagogik der Georg-August-Universität Göttingen, Bd.19, Göttingen 1993.

SchweER, M. K. W.: Innovation durch Evaluation? Eine kritische Analyse hochschulpolitischer Maßnahmen zur Verbesserung der Qualität der Lehre. Bildung und Erziehung 49, 2/1996, S.221-231.

SchweER, M.K.W.: Vertrauensförderndes Dozentenverhalten. Ansatzpunkte zur Verbesserung der universitären Lehre. Bildung und Erziehung 51, 2/1998, S. 149-159.

Selg, H.; Klapprott, J. \& Kamenz, R.: Forschungsmethoden der Psychologie. Grundriß der Psychologie 3, Stuttgart 1992.

SEll, R.: Angewandtes Problemlösungsverhalten. Denken und Handeln in komplexen Zusammenhängen. 2. Aufl., Berlin 1989.

SEmBill, D.: Problemlösefähigkeit, Handlungskompetenz und Emotionale Befindlichkeit - Zielgrößen Forschenden Lernens -. Ergebnisse der Pädagogischen Psychologie 10, Göttingen 1992.

SEYFRIED, E.: Evaluation of quality aspects in vocational training programmes. Synthesis report. Luxembourg 1998.

Sievers, W.: Empirische Forschungsmethoden in den Sozialwissenschaften, Buch I: Befragen. Göttingen 1990.

SkINNER, B. K.: Wissenschaft und menschliches Verhalten. München 1973.

Söltenfuss, G. \& Half Pap, K. (Hrsg.): Handlungsorientierte Ausbildung im kaufmännischen Bereich. Ergebnisse der Hochschultage Berufliche Bildung ' 86 in Essen. St. Augustin 1987.

SpIEs, K. \& Hesse, F. W.: Interaktion von Emotion und Kognition. Psychologische Rundschau 37, 1986, S. 75-90.

SPrEmann, K.: Wirtschaft, Investition und Finanzierung. 5. Aufl., München u. a. 1996.

Staudt, E.; Kröll, M. \& Hören, M. v.: Potentialorientierung der strategischen Unternehmensplanung. Unternehmens- und Personalentwicklung als iterativer Prozeß. Die Betriebswirtschaft 53, 1/1993, S. 57-59. 
Stachowiak, H.: Erkenntnisstufen zum Neopragmatismus und zur Modelltheorie. In: Stachowiak, H. (Hrsg.): Modelle und Modelldenken im Unterricht. Bad Heilbrunn/Obb. 1980, S. 87-146.

Stachowiak, H.: Zur Einleitung: Der Weg zum Systematischen Neopragmatismus und das konzept der Allgemeinen Modell-Theorie. In: STACHOWiak, H. (Hrsg.): Modelle und Modelldenken im Unterricht. Bad Heilbrunn/Obb. 1980, S. 9-49.

Stark, R.; Graf, M.; Renkl, A.; Mande, H. \& Gruber, H.: Förderung von Handlungskompetenz durch geleitetes Problemlösen und multiple Lernkontexte. Forschungsbericht der Ludwig-Maximilians-Universität, Institut für Pädagogische Psychologie und Empirische Pädagogik 55, München 1995.

Stark, R.; Gruber, H.; Graf, M.; Renkl, A. \& Mandl, H.: Komplexes Lernen in der kaufmännischen Erstausbildung: Kognitive und motivationale Aspekte. Forschungsbericht der Ludwig-Maximilians-Universität, Institut für Pädagogische Psychologie und Empirische Pädagogik 58, München 1995.

Starker, U. \& Dörner, D.: Kognitive, emotionale und motivationale Determinanten des Handelns. In: KLuwe, R. H. (Hrsg.): Strukturen und Prozesse intelligenter Systeme. Wiesbaden 1997, S. 231-253.

STEnTzEL, M.: Lernschwierigkeiten von Erwachsenen in der beruflichen Weiterbildung. Beiträge zur Arbeits-, Berufs- und Wirtschaftspädagogik 08, Frankfurt a. M. 1986.

Steyer, R. \& Eid, M.: Messen und Testen. Berlin u. a. 1993.

Stiensmeier-Pelster, J. \& Schlangen, B.: Erlernte Hilflosigkeit und Leistung. In: Möller, J. \& Köller, O. (Hrsg.): Emotionen, Kognitionen und Schulleistung. Weinheim 1996, S. 69-90.

STumpF, S.: Diskussionsprozeß und Gruppeneffektivität beim Lösen komplexer ökonomischer Probleme. Schriftenreihe Handeln und Entscheiden in komplexen ökonomischen Situationen, Bd.4, Heidelberg 1992.

Tergan, S.-O.: Modelle der Wissensrepräsentation als Grundlage qualitativer Wissensdiagnostik. Opladen 1986. 
Tergan, S.-O.: Psychologische Grundlagen der Erfassung individueller Wissensrepräsentationen. Teil I: Grundlagen der Wissensmodellierung. Sprache und Kognition 8, 3/1989, S. 152-165.

Tergan, S.-O.: Psychologische Grundlagen der Erfassung individueller Wissensrepräsentationen. Teil II: Methodologische Aspekte. Sprache und Kognition 8, 4/1989, S. 193-202.

Tessaring, M.: Das duale System der Berufsausbildung in Deutschland. Attraktivität und Beschäftigungsperspektiven. Mitteilungen aus der Arbeitsmarktund Berufsforschung 2/93, Nürnberg 1993, S. 131-161.

Tessaring, M.: Qualifikationsentwicklungen bis 2010. Welche Trends bestimmen die langfristige Entwicklung unter besonderer Berücksichtigung von Ausund Weiterbildung? In: BeiträgE Aus Der ArbeitsmarkT- Und BerufsForschung 195: Diepold, P. (Hrsg.): Berufliche Aus- und Weiterbildung: Konvergenzen - Divergenzen, neue Anforderungen - alte Strukturen. Nürnberg 1996 , S. 277-288.

Tietgens, H.: Reflexionen zur Erwachsenendidaktik. Theorie und Praxis der Erwachsenenbildung. Bad Heilbrunn/Obb. 1992.

Tramm, T.: Entwicklungsperspektiven der Übungsfirmen- und Lernbüroarbeit aus der Sicht einer Didaktik handlungsorientierten Lernens. Wirtschaft und Erziehung 43, 7-8/1991, S. 248-258.

Tramm, T.: Konzeption und theoretische Grundlagen einer evaluativ-konstruktiven Curriculumstrategie. Entwurf eines Forschungsprogramms unter der Perspektive des Lernhandelns. Berichte aus dem Seminar für Wirtschaftspädagogik der Georg-August-Universität Göttingen, Bd. 17, Göttingen 1992.

Ulrich, H.: Das Unternehmen als produktives soziales System. 2. Aufl., Bern 1970 .

Ulrich, H. \& Probst, G. J. B.: Anleitung zum ganzheitlichen Denken und Handeln. Ein Brevier für Führungskräfte. Bern 1988.

VerWaltungs- und Wirtschaftsakademie Göttingen: Vorlesungs-Verzeichnis Wintersemester 1997/98. Göttingen 1997.

Wagner, D. \& Krause, A.: Zum Einsatz aktiver Lehr- und Lernmethoden in der Hochschulausbildung. Vermittlung von Schlüsselqualifikationen für die beruf- 
liche Praxis. Beiträge aus dem Fachbereich Pädagogik der Universität der Bundeswehr Hamburg 5, Hamburg 1990.

Walter, J.: Prüfungen und Beurteilungen in der beruflichen Bildung. Kritik der aktuellen Praxis und Entwurf einer Neuorientierung vor dem Hintergrund einer veränderten Qualifikationsentwicklung und neuerer erkenntnistheoretischer und berufspädagogisch-didaktischer Ansätze. Europäische Hochschulschriften, Reihe XI, Bd. 688, Frankfurt a. M. 1. a. 1996.

WEBER, S.: Vorwissen in der betriebswirtschaftlichen Ausbildung. Eine strukturund inhaltsanalytische Studie. Wiesbaden 1994.

Weber, S. \& Schumann, M.: The Concept Mapping Software Tool (COMASOTO) for Teaching and Assessing Structurale Knowledge. Paper presented at the Annual Meeting of the American Educational Research Association, San Diego 1998.

Weidle, R. \& Wagner, A. C.: Die Methode des Lauten Denkens. In: Huber, G. L. \& MANDL, H.: Verbale Daten. Eine Einführung in die Grundlagen und Methoden der Erhebung und Auswertung. Weinheim 1982, S.81-103.

Weinert, F. E.: Lernen lernen und das eigene Lernen verstehen. In: REUSSER, K. \& Reusser-Weyeneth, M. (Hrsg.): Verstehen. Psychologischer Prozeß und didaktische Aufgabe. Bern 1994, S. 183-206.

Wesseler, M.: Evaluation und Evaluationsforschung. In: TiPPELT, R. (Hrsg.): Handbuch Erwachsenenbildung/Weiterbildung. Opladen 1994, S. 671-686.

Weyerich, A.: Aufgabenbezogene Gestaltung von Qualifizierungsprozessen in der beruflichen Weiterbildung. Das Konzept der lerngerechten Entfaltung von Handlungsanforderungen (LEHA). Dissertation, Berlin 1995.

Wiersma, W. \& Jurs, S. G.: Educational measurement and testing. 2. Aufl., Boston 1990.

WiLBERS, K.: Netzwerke in der Wirtschaftspädagogik. Überblick - Graphentheoretische Rekonstruktion - Konzeptionelle Grenzen aus systemtheoretischer Sicht. Dissertation, Köln 1997.

WiLD, K.-P. \& Schief ElE, U.: Induktiv versus deduktiv entwickelte Fragebogenverfahren zur Erfassung von Merkmalen des Lernverhaltens. Unterrichtswissenschaft 21, 4/1993, S. 312-326. 
Wild, K.-P.; Schiefele, U. \& Winteler, A.: LIST. Ein Verfahren zur Erfassung von Lernstrategien im Studium. Arbeiten zur empirischen Pädagogik und Pädagogischen Psychologie 20, Universität der Bundeswehr, Neubiberg 1992.

Will, H.; Winteler, A. \& Krapp, A.: Evaluation in der beruflichen Ausund Weiterbildung. Konzepte und Strategien. Heidelberg 1987.

Wilms, F.E. P.: Entscheidungsverhalten als rekursiver Prozeß. Konzeptuelle Bausteine des systemorientierten Managements. Neue Betriebswirtschaftliche Forschung 148, Wiesbaden 1995.

Windecker, A.-C.: Außerfachliche Kompetenzen und Schlïsselqualifikationen in der beruflichen Bildung. Dissertation, Ludwigsburg 1991.

Winter, A.: Zur Lebens- und Studiensituation der Studierenden. In: Zabeck, J. \& Zimmermann, M. (Hrsg.): Anspruch und Wirklichkeit der Berufsakademie Baden-Württembergs. Eine Evaluationstudie. Weinheim 1995, S. 377-392.

Woene, G.: Einführung in die Allgemeine Betriebswirtschaftslehre. 19. Aufl., München 1996.

Wolfe, J.: The Teaching Effectiveness of Games in Collegiate Business Courses. Simulation \& Games 16, 3/1985, S. 251-288.

Woortmann, G.: Qualität in der Weiterbildung. In: Feuchthofen, J. E. \& Severing, E. (Hrsg.): Qualitätsmanagement und Qualitätssicherung in der Weiterbildung. Neuwied 1995, S. 45-51.

Wottawa, H. \& Thierau, H.: Lehrbuch Evaluation. 2. Aufl., Bern u. a. 1998. Wright, G. H. v.: Erklären und Verstehen. Frankfurt a. M. 1974.

Wunder, D.: Qualitätsmanagement. Das System der DIN ISO 9000 ff. Grundlagen der Weiterbildung 5, 1/1994, S. 33-36.

WuTTKE, E.: Motivation und Lernstrategien in einer selbstorganisationsoffenen Lernumgebung. Eine empirische Untersuchung bei Industriekaufleuten. Europäische Hochschulschriften, Reihe XI, Bd.769, Frankfurt a. M. 1999.

Young, W. H. (Hrsg.): Continuing professional education in transition. Visions for the professions and new strategies for lifelong learning. Malabar/Florida 1998. 


\section{A Anhang}

\section{A.1 Zeitplan der Erhebung}

\begin{tabular}{|c|c|c|c|}
\hline \multicolumn{2}{|l|}{ Datum } & 18:15-19:45 Uhr & $20: 00-21: 30 \mathrm{Uhr}$ \\
\hline 8. Oktober & 1997 & & Vorbesprechung \\
\hline 15. Oktober & 1997 & Prätest & \\
\hline 22. Oktober & 1997 & Einführung EpUS & 1. Entscheidung (EP) \\
\hline 29. Oktober & 1997 & 2. Entscheidung (EP) & begleitende Lehre \\
\hline 5. November & 1997 & 3. Entscheidung (EP) & begleitende Lehre \\
\hline 12. November & 1997 & & \\
\hline 19. November & 1997 & 4. Entscheidung (EP) & \\
\hline 26. November & 1997 & & \\
\hline 3. Dezember & 1997 & & \\
\hline 10. Dezember & 1997 & & \\
\hline 17. Dezember & 1997 & 5. Entscheidung (EP) & \\
\hline 7. Januar & 1998 & & 6. Entscheidung (TP) \\
\hline 14. Januar & 1998 & 7. + 8. Entscheidung (TP) & \\
\hline 21. Januar & 1998 & Posttest & Abschlussbesprechung \\
\hline
\end{tabular}

Erläuterung:

$(\mathrm{EP})=$ experimentelle Phase

$(\mathrm{TP})=$ Testphase 


\section{A.2 Einteilung der Gruppen}

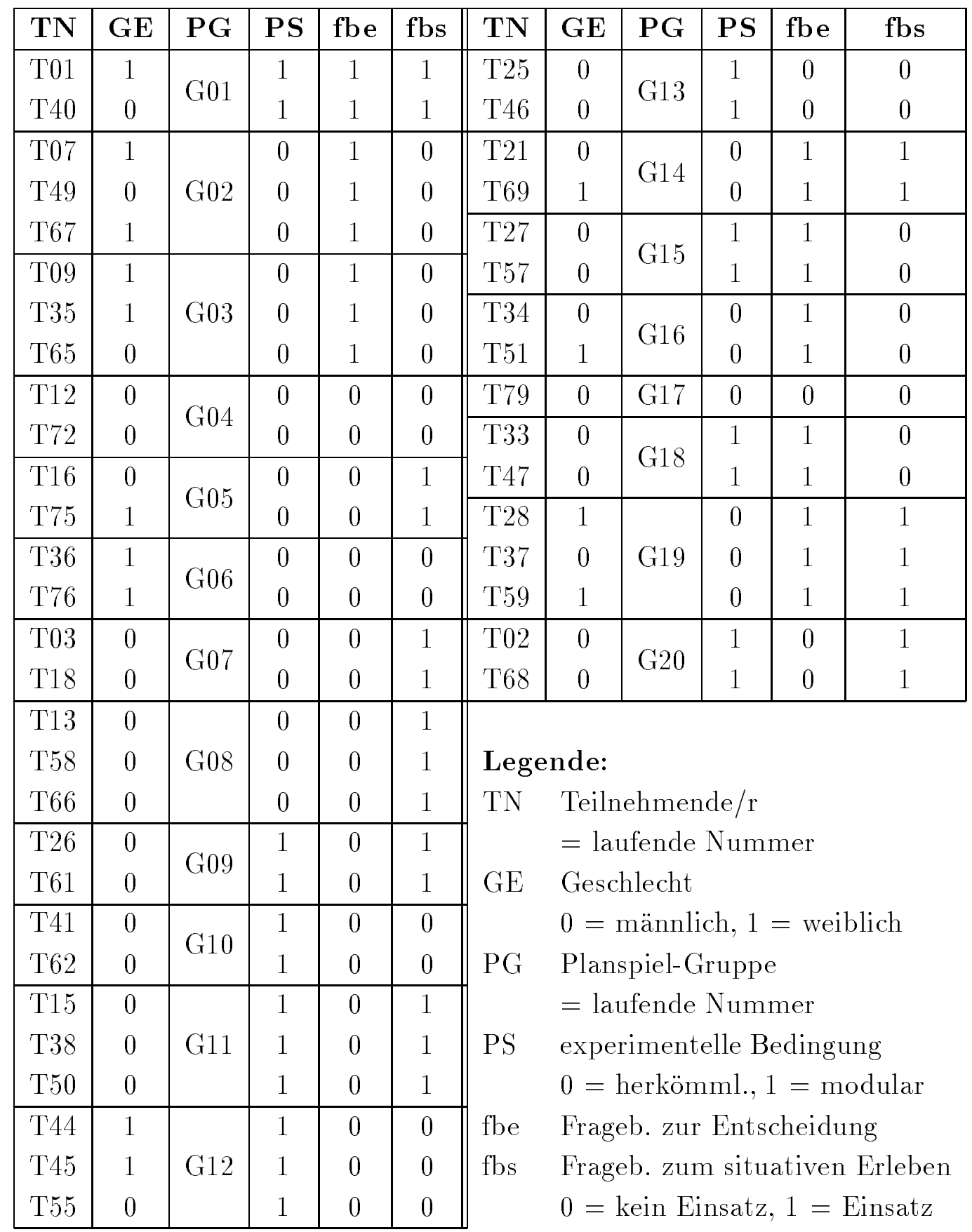




\section{A.3 Reduzierung des Planspiel-Modells bei der Konstruktion der Fallstudie}

Bei der Konstruktion der Fallstudie wurden gegenüber der Vollversion von SIMLOG folgende Reduktionen vorgenommen: ${ }^{1}$

Lieferanten: Zwei der vier Alternativen werden ausgeblendet und je Bestellung und Lieferant gleiche bestellfixe Kosten vorgegeben. Weiterhin gibt es keine Qualitätsunterschiede und keine Kapazitätsbeschränkung.

Rohstoffe: Es ist keine Notbeschaffung sowie keine Lagerstände bei den Rohstoffen vorgesehen, stattdessen wird die Produktionsmenge bei nicht vorhandenen Rohstoffen reduziert.

Transportart: Es werden zwei Modelle (Linie versus Charter) mit unterschiedlichen Kosten zur Auswahl gestellt.

Maschinen: Es ist bereits ein Maschinentyp vorhanden, ein anderer kann alternativ am Markt beschafft werden, wobei eine Kostenvergleichsrechnung erforderlich ist.

Produktion: Es gibt nur ein Hauptprodukt und keine Kuppelproduktion mit Haupt- und Nebenprodukt wie in der Vollversion. Weiterhin werden keine Qualitätsaspekte bei der Produktion berücksichtigt.

Absatz: Hier ist der Inlandspreis festzusetzen sowie eine Entscheidung über einen Export zum Festpreis mit Mengenbeschränkung zu treffen.

Finanzierung: Der Finanzierungsbereich wird ausgeblendet.

1 Zur Modellbeschreibung vgl. BLoEch \& Rüscher (Unternehmenssimulation 1991). 


\section{A.4 Komplexitäts-Steigerung in der experimen- tellen Phase der modularen Durchführung}

Die folgende Abbildung zeigt die Anzahl der Gruppe, die in der experimentellen Phase das Planspiel in der modularen Durchführung spielten, und ihr Übergang auf die nächste Stufe bei den einzelnen Entscheidungen.

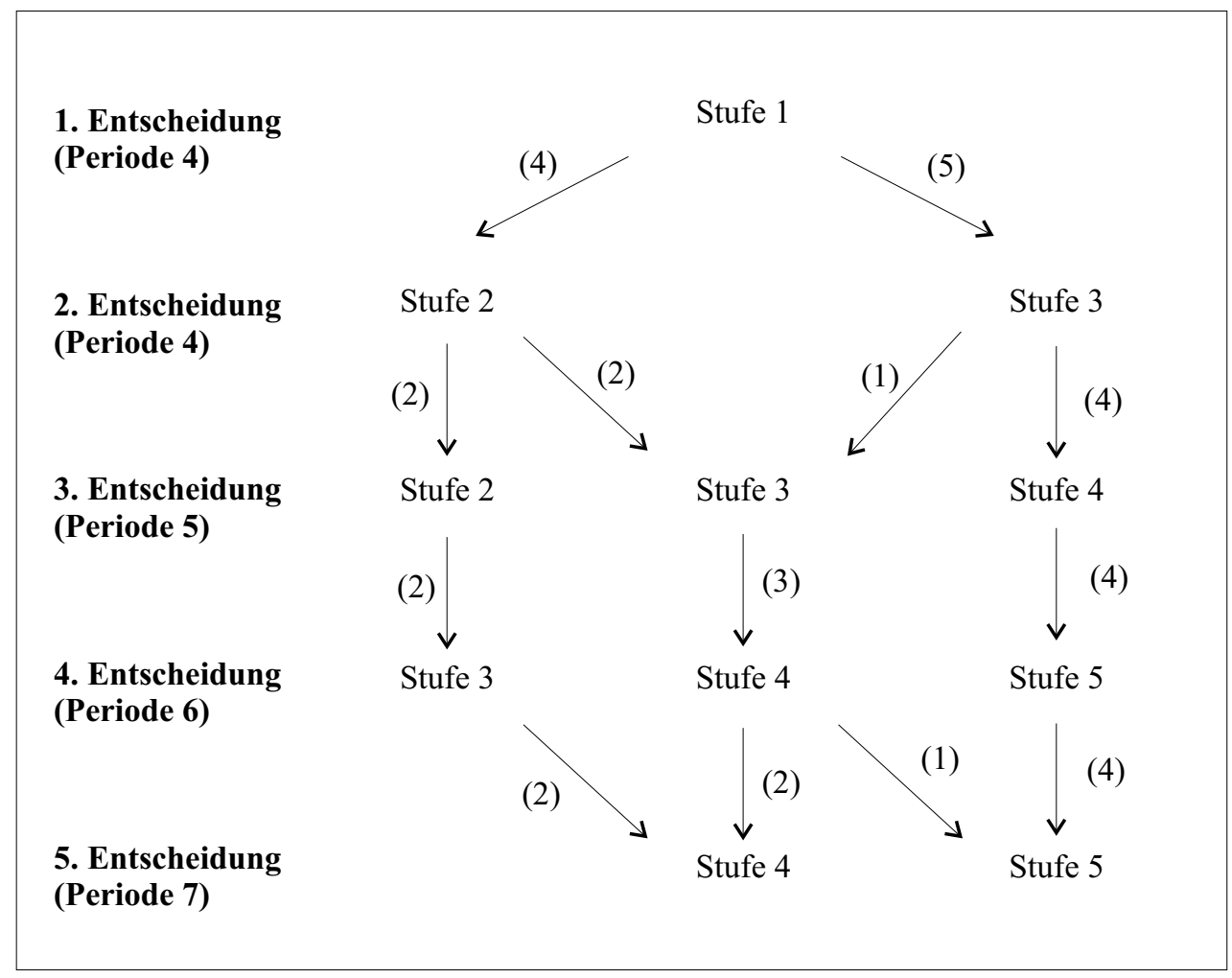

Modulare Bedingung: Anzahl der Gruppen auf den Stufen 


\section{A.5 Instruktion zum Prätest}

\section{Einleitung}

Sie werden in dieser Veranstaltung an einem Unternehmensplanspiel teilnehmen. Dabei führen Sie in Kleingruppen simulierte Unternehmen. In diesem Rahmen haben Sie eine Reihe unternehmerischer Entscheidungen zu treffen.

In einer begleitenden Untersuchung wollen wir den Lernerfolg dieses PlanspielUnterrichts bewerten. Dazu sind wir auf Ihre Mitarbeit angewiesen.

Der heutige Test soll den Stand Ihres betriebswirtschaftlichen Wissens aufgrund Ihrer bisherigen Ausbildung erheben. Zu diesem Zweck wurde eine Fallstudie, ein Fragebogen und eine Netzwerk-Erhebung entwickelt. Über die Bearbeitungszeit für die Teilaufgaben werden Sie informiert.

Vor Ihnen liegt die Mappe mit den Aufgabenstellungen, die in der vorgegebenen Reihenfolge zu bearbeiten sind. Ihre Ergebnisse tragen Sie bitte auf den beiliegenden Lösungsblättern ein. Selbstversändlich bleibt die Anonymität Ihrer Antworten gewahrt. Die Ergebnisse werden von uns gesammelt und nicht an Dritte weitergegeben.

Um einen direkten Vergleich zwischen der heutigen Vorerhebung und der im Anschluss an den Planspiel-Unterricht laufenden Nacherhebung zu gewährleisten, bitten wir Sie, Ihre Lösungsblätter durch ein Kennwort zu markieren. Lassen Sie bitte die Seiten in den Mappen und geben Sie diese am Ende beim Veranstaltungsleiter ab.

Wenn Sie noch Fragen haben, wenden Sie sich bitte direkt an uns.

\section{Vielen Dank für Ihre Mitarbeit und viel Spaß!}

\section{Erläuterungen zur Fallstudie}

Sie übernehmen die Position eines Abteilungsleiters in einem Unternehmen und treffen Entscheidungen, die sich positiv auf den Unternehmenserfolg auswirken sollen. Neben Threr Firma sind noch drei fiktive Konkurrenzunternehmen am Markt. Alle weiteren Informationen können Sie der Fallbeschreibung entnehmen.

Tragen Sie Ihre Entscheidungen auf dem beiliegenden Lösungsblatt ein. Neben den Lösungen in Zahlenform interessiert uns, welche Absichten und Ziele Sie mit Ihren Entscheidungen verfolgen, da diese aus den Zahlen nicht unbedingt ersichtlich sind. Nutzen Sie also bitte den Platz auf dem Entscheidungsblatt zur Fallstudie, um uns Einblicke in Thre Überlegungen zu geben. Faktoren, die nicht in der Fallbeschreibung enthalten sind, sollten Sie auch bei Ihren Entscheidungen unberücksichtigt lassen. 


\section{A.6 Instruktion zum Posttest}

\section{Einleitung}

Sie haben in dieser Veranstaltung an einem Unternehmensplanspiel teilgenommen. In der begleitenden Untersuchung wollen wir den Lernerfolg dieses PlanspielUnterrichts bewerten. Dazu sind wir auf Ihre Mitarbeit angewiesen.

Der heutige Test soll den Stand Ihres betriebswirtschaftlichen Wissens nach diesem Planspiel-Unterricht feststellen. Zu diesem Zweck werden wieder eine Fallstudie, ein Fragebogen und eine Netzwerk-Erhebung eingesetzt. Über die Bearbeitungszeit für die Teilaufgaben werden Sie informiert.

Lassen Sie bitte die Seiten in den Mappen und geben Sie diese am Ende beim Veranstaltungsleiter ab.

Wenn Sie noch Fragen haben, wenden Sie sich bitte direkt an uns.

\section{Vielen Dank für Ihre Mitarbeit und viel Spaß!}

\section{Erläuterungen zur Fallstudie}

Sie übernehmen die Position eines Abteilungsleiters in einem Unternehmen und treffen Entscheidungen, die sich positiv auf den Unternehmenserfolg auswirken sollen. Neben Ihrer Firma sind noch drei fiktive Konkurrenzunternehmen am Markt. Alle weiteren Informationen können Sie der Fallbeschreibung entnehmen. Tragen Sie Ihre Entscheidungen auf dem beiliegenden Lösungsblatt ein. Neben den Lösungen in Zahlenform interessiert uns, welche Absichten und Ziele Sie mit Ihren Entscheidungen verfolgen, da diese aus den Zahlen nicht unbedingt ersichtlich sind. Nutzen Sie also bitte den Platz auf dem Entscheidungsblatt zur Fallstudie, um uns Einblicke in Ihre Überlegungen zu geben. Faktoren, die nicht in der Fallbeschreibung enthalten sind, sollten Sie auch bei Ihren Entscheidungen unberücksichtigt lassen. 


\section{A.7 Fallstudie}

Sie treten zu Beginn des Jahres Ihre Stelle bei der Firma Weiß an. Es handelt sich um ein Büroausstattungsunternehmen, dessen Produktpalette von Büromöbeln bis hin zu Kleinutensilien für den täglichen Bürobedarf reicht. Sie sind in Threr Abteilung für das Produkt 'Schreibtische' zuständig.

Neben Ihrem Unternehmen bieten in Deutschland noch drei weitere Unternehmen ein vergleichbares Schreibtischmodell mit ähnlichen Eigenschaften an. Im letzten Geschäftsjahr bestand für dieses Erzeugnis auf dem Inlandsmarkt eine Gesamtnachfrage von etwa 10.000 Stïck. Aufgrund neuester Marktprognosen ist allerdings mit einer rückläufigen Nachfrage zu rechnen, pessimistische Einschätzungen gehen bereits im laufenden Jahr von einer Halbierung des Absatzpotentials aus.

Mit einem europäischen Handelsunternehmen wurden vertragliche Vereinbarungen getroffen, wodurch sich die Möglichkeit eröffnet, zusätzlich bis zu 500 Schreibtische zu einem Festpreis von 550,- DM/Stïck zu exportieren.

Für die Fertigung der Schreibtische werden vier Bauteile fremdbezogen und maschinell verarbeitet. Die benötigten Komponenten wurden in der Vergangenheit von der Firma Holzmann bezogen. Bei der Herstellung eines Tisches werden folgende Mengen der Bauteile benötigt:

\begin{tabular}{|l|l|l|l|}
\hline Tischplatten & 1 & Tisch beine & 4 \\
\hline Schubläden & 3 & Beschläge & 5 \\
\hline
\end{tabular}

Die Fertigung der Tische erfolgt auf eine Universalmaschine, von der zwei alternative Typen mit unterschiedlichen Leistungsdaten im Handel sind. Die Maschinentypen S 500 und S 510 können sofort und einsatzbereit geliefert werden. Für die Herstellung eines Tisches werden unabhängig von der Maschinenart 10 Maschinenstunden benötigt.

In der Produktion werden zur Zeit 10 Universalmaschinen des Typs S 500 mit einer Restnutzungsdauer von drei Jahren eingesetzt. Der Anschaffungspreis beträgt 100.000 DM, der Liquidationserlös am Ende der Nutzungsdauer beläuft sich auf 20.000 DM. Jede Maschine hat eine Kapazität von 2.500 Stunden pro Jahr. Die Instandhaltungskosten sind unabhängig von der Maschinenauslastung und belaufen sich auf 30.000 DM pro Jahr bei einer Nutzungsdauer von 5 Jahren. Der Verkaufserlös einer Maschine entspricht dem Restbuchungswert. Es wird lineare Abschreibung angesetzt.

Von dem Maschinenlieferanten liegen Leistungsdaten und Preise für einen alternativen Maschinentyp S 510 vor:

$\begin{array}{ll}\text { Anschaffungspreis: } & 150.000 \mathrm{DM} \\ \text { Liquidationserlös: } & 30.000 \mathrm{DM} \\ \text { Kapazität: } & 2.500 \mathrm{Stunden/Jahr} \\ \text { Instandhaltungskosten: } & 25.000 \mathrm{DM} / \mathrm{Jahr} \\ \text { Nutzungsdauer: } & \text { 6 Jahre }\end{array}$


Zur Beurteilung des letzten Geschäftsjahres hat Ihr Assistent einige Grafiken vorbereitet. Die folgende Grafik gibt eine Übersicht über die Marktanteile im letzten Geschäftsjahr. Danach verteilte sich der Gesamtabsatz von ca. 10.000 Stïck wie folgt auf die Unternehmen:

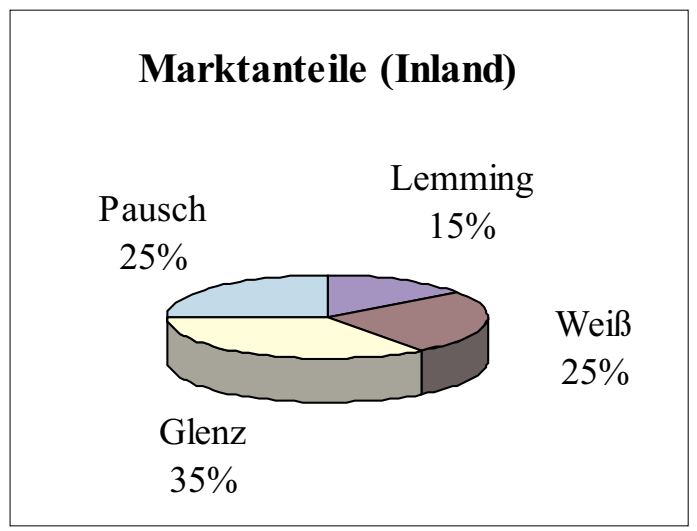

Die benötigten Rohstoffe wurden in der Vergangenheit bei der Firma Holzmann bezogen. Neben deren Preisliste liegt ein zusätzliches Angebot der Grünewald GmbH vor (vgl. folgende Seite). Für eine Bestellung werden von den Lieferanten fixe Verwaltungskosten in Höhe von 1.000 DM in Rechnung gestellt.

\section{Holzmann}

Firma Weiß

Zechenstr. 15

Gartenstr. 10-12

24107 Kiel

25335 Elmshorn

Sehr geehrte Damen und Herren,

bezïglich Ihrer Nachfrage machen wir Ihnen folgendes Angebot:

\section{Tischplatten:}

48,- je Stïck, Rabatt von $9 \%$ ab einer Menge von 2.000 Stïck

Tischbeine:

23,- je Stïck, Rabatt von $9 \%$ ab einer Menge von 8.000 Stïck

schubläden:

18,- je Stïck, Rabatt von $8 \%$ ab einer Menge von 7.000 Stïck

Tischbeine:

14,- je Stück, Rabatt von $6 \%$ ab einer Menge von 10.000 Stïck

Mit freundlichem Gruß

D. $\mathcal{R I E \mathcal { M A N N }}$ 


\section{Grünewald}

Firma Weiß

Gartenstr. 10-12

25335 Elmshorn
Gärtnerstr. 2

23564 Lübeck

Sehr geehrte Damen und Herren, wir danken für Ihr Interesse an unseren Produkten und senden Ihnen hiermit die gew ïnschte Preisliste.

$\begin{array}{lccc} & \text { Einzelpreis } & \text { Mengen rabatt } & \text { ab Bestellmenge } \\ \text { Tischplatten: } & 50,00 & 10 \% & 1.000 \\ \text { Tischbeine: } & 25,00 & 10 \% & 3.000 \\ \text { schubläden: } & 19,00 & 8 \% & 3.000 \\ \text { Tischbeine: } & 15,00 & 8 \% & 5.000 \\ & & \\ \text { Mit freundlichen Grïßen } & & \\ Y . \text { Maier } & & & \end{array}$

Für den Transport der Rohstoffe liegt ein Angebot einer Speditionsfirma vor: 2.000 DM fixe Kosten je Ladung bei einer Ladungsgröße von 1.000 Stück; je Lkw kann nur eine Rohstoffsorte geladen werden. Alternativ können die Rohstoffe auch mit der Bahn transportiert werden: variable Kosten von 2,- DM je Stück. Weitere Aspekte wie z. B. Lagerhaltungskosten oder Kosten des Transports von der Spedition/vom Bahnhof zum Unternehmen sind zu vernachlässigen.

Die unterschiedlichen Werte-Etats der Konkurrenten werden durch folgende Abbildung veranschaulicht: 


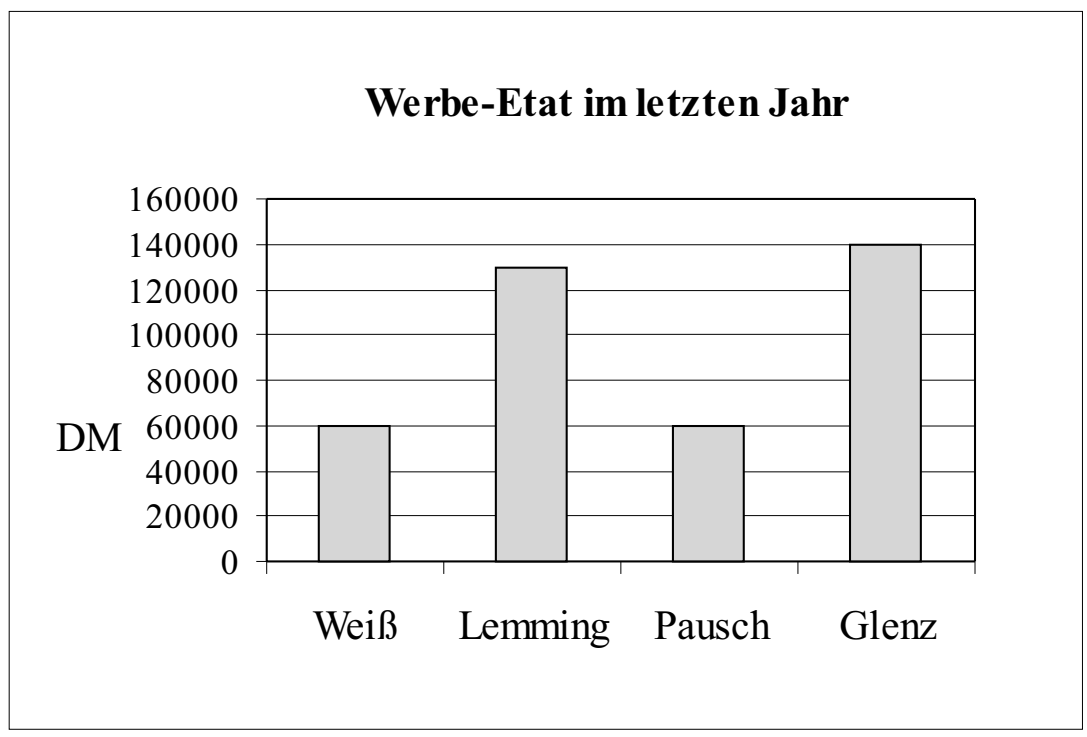

Die nächste Darstellung gibt eine Übersicht über die unterschiedlichen Preispolitiken der Unternehmen. Für die Firma Weiß sind zusätzlich die Herstellungskosten (inkl. anteiligen Verwaltungsgemein kosten) je Tisch eingetragen.

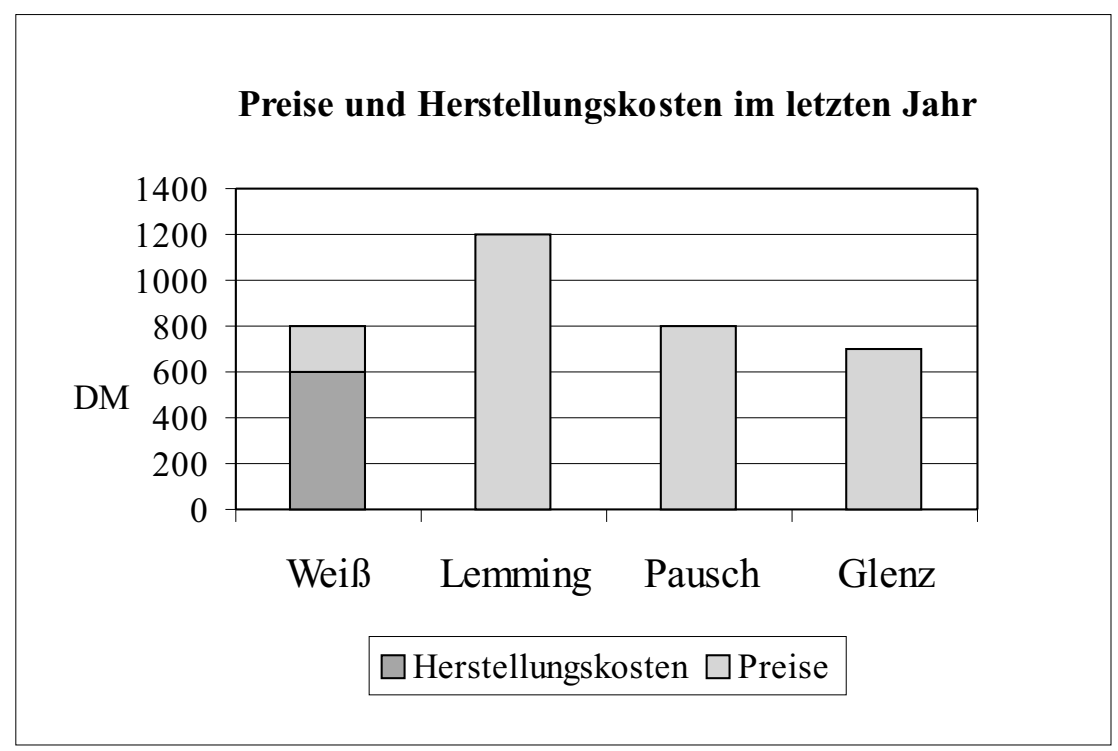

Auf der Basis dieser Daten haben Sie die Aufgabe, Entscheidungen ïber die Beschaffung, die Produktion und die Gestaltung der Marketing-Instrumente für die nächste Periode zu treffen. 


\section{A.8 Lösungsblatt zur Fallstudie}

\begin{tabular}{|l|l|l|l|}
\hline Kennwort: & & & \\
$\begin{array}{l}\text { (= jeweils die ersten zwei Buchstaben } \\
\text { des Vornamens und des Nachnamens) }\end{array}$
\end{tabular}

Bitte tragen Sie in die nachfolgende Tabelle Ihre Entscheidungen zur Fallstudie ein. Erläutern Sie auch die Absichten und Ziele, die Sie mit Ihren Entscheidungen verfolgen.

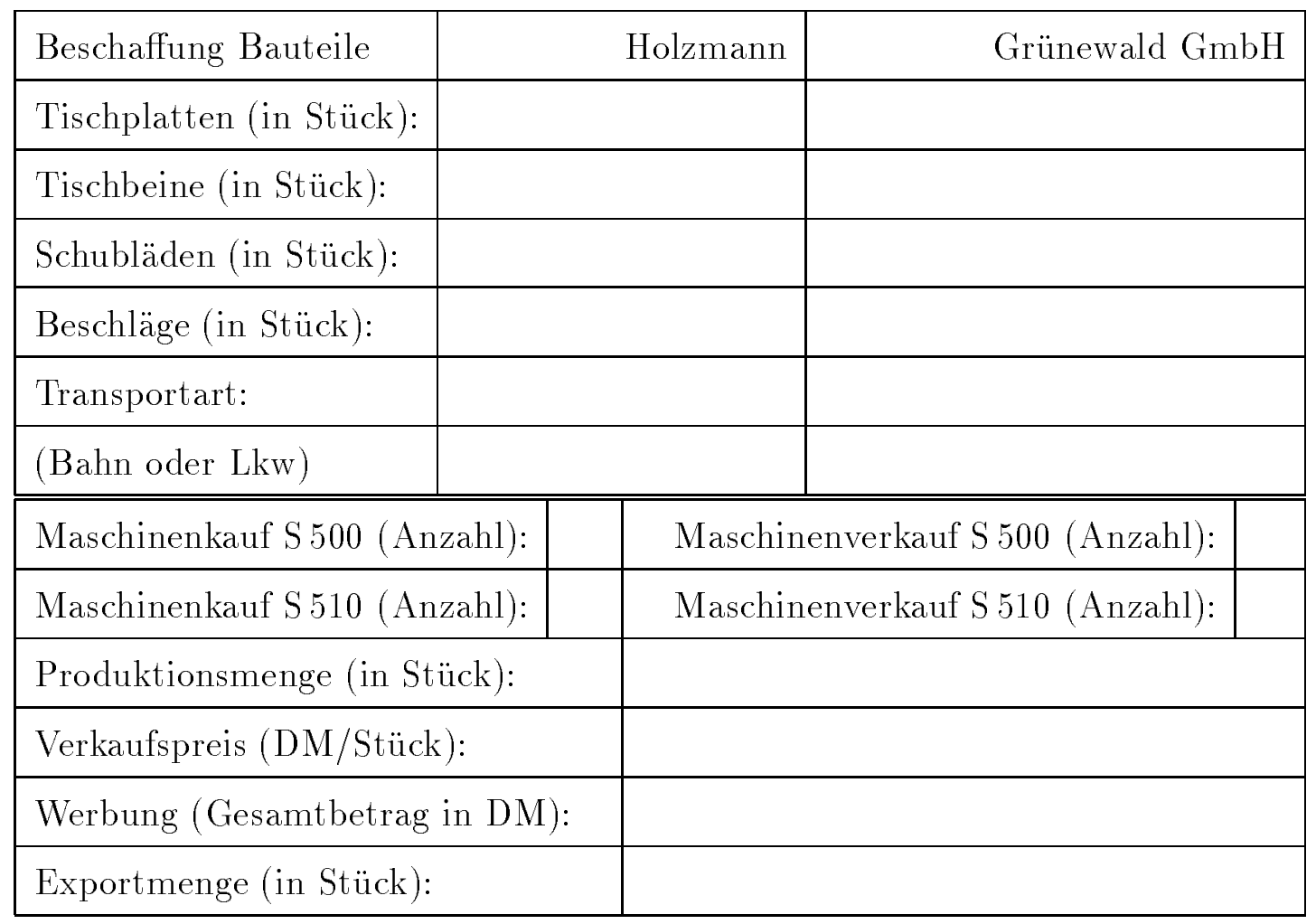




\section{A.9 Multiple-Choice-Fragebogen, Version A}

Die folgenden Fragen beziehen sich auf betriebswirtschaftliche Begriffe und Vorgehensweisen. Es ist jeweils nur eine Antwort richtig, deren Buchstabe ( $a, b$ oder c) Sie bitte auf das Lösungsblatt übertragen.

1) Welche der folgenden Aussage ist richtig?

a) Alle fixen Kosten sind Gemeinkosten.

b) Alle Gemein kosten sind fixe Kosten.

c) Alle variablen Kosten sind Einzelkosten.

2) Komponenten des Anlagevermögens in der Bilanz sind

a) Grundstïcke und Maschinen.

b) Grundstïcke und Vorräte.

c) Grundstücke, Maschinen und Vorräte.

3) Eine Verringerung des Kaufpreises bei einem Produkt wird dessen Absatzmenge

a) eher erhöhen.

b) eher verringern.

c) eher nicht beeinflussen.

4) Zur Außenfinanzierung gehören

a) Selbstfinanzierung und Kreditfinanzierung.

b) Beteiligungsfinanzierung und Selbstfinanzierung.

c) Kreditfinanzierung und Beteiligungsfinanzierung.

5) Bei der Bestimmung der Herstellungskosten eines Produkts

a) werden die Materialkosten und die Fertigungskosten aufsummiert (Herstellkosten) und anschließend die Verwaltungsgemeinkosten auf Basis der Herstellkosten zugerechnet.

b) werden die Materialkosten und die Fertigungskosten aufsummiert (Herstellkosten) und anschließend die Verwaltungs- und Vertriebsgemeinkosten auf Basis der Herstellkosten zugerechnet.

c) werden die Materialkosten, die Fertigungskosten und die Verwaltungskosten aufsummiert (Herstellkosten) und anschließend die Vertriebsgemeinkosten auf Basis der Herstellkosten zugerechnet.

6) Bei der arithmetisch-degressiven Abschreibung von Gegenständen des Anlagevermögens wird der Degressionsbetrag ermittelt, indem man

a) die Differenz aus Restbuchwert und Restwert durch die Jahre der geplanten Nutzung dividiert.

b) die Differenz aus Anschaffungswert und Restwert durch die Jahre der geplanten Nutzung dividiert.

c) den Restbuchwert durch die Jahre der geplanten Nutzung dividiert.

7) Eine Verringerung der Werbeausgaben für ein Produkt wird dessen Absatzmenge

a) eher erhöhen.

b) eher verringern.

c) eher nicht beeinflussen. 
8) Liquidität bedeutet,

a) daß das Vermögen größer ist als das Fremdkapital.

b) daß der Bedarf an Zahlungsmitteln höher als der Zahlungsmittelbestand ist.

c) daß das Unternehmen die fälligen kurzfristigen Verbindlichkeiten durch die flïssigen Mittel decken kann.

9) Ein Verfahren zur Ermittlung der Herstellungskosten eines Produktes ist die

a) Fixkostendeckungsrechnung.

b) Plankosten rechnung.

c) Zuschlagskalkulation.

10) Die Abschreibung hat die Aufgabe,

a) die Anschaffungs- oder Herstellkosten von Wirtschaftsgïtern auf die Zeit der mehrjährigen Nutzung zu verteilen.

b) den Gewinn zu erhöhen.

c) den Buchwert der Anlage zu erhalten.

11) Eine Verringerung der Kundendienstausgaben für ein Produkt wird dessen

Absatzmenge

a) eher erhöhen.

b) eher verringern.

c) eher nicht beeinflussen.

12) Bei der Lagerbestandsbewertung eines Produktes in der Bilanz werden aktiviert: a) die Herstell-, die Verwaltungs- und die Vertriebskosten.

b) die Herstell- und die Vertriebskosten.

c) nur die Herstell- und die Verwaltungskosten.

13) Eine Erhöhung der Investitionen für Maschinen wird die mögliche Produktionsmenge

a) eher erhöhen.

b) eher verringern.

c) eher nicht beeinflussen.

14) Bei der Ermittlung des Jahresüberschusses in der Gewinn- und Verlustrechnung nach dem Gesamtkostenverfahren

a) werden vom Umsatz, erhöht (vermindert) $1 \mathrm{~m}$ die wertmäßige Lagerbestandsänderung, alle angefallenen Aufwendungen abgezogen.

b) werden zum Umsatz die außerordentlichen Erträge addiert und die Aufwendungen abgezogen, die für die verkauften Produkte angefallen sind.

c) werden vom Umsatz der Periode alle angefallenen Auszahlungen abgezogen.

15) Die Bestandsveränderung wird auf dem Gewinn- und Verlustkonto im Soll erfaßt, wenn

a) der Schlußbestand geringer als der Anfangsbestand ist.

b) der Schlußbestand höher als der Anfangsbestand ist.

c) der Schlußbestand und Anfangsbestand ïbereinstimmen. 
16) Das klassische Marketing-Instrumentarium umfaßt

a) Produkt-, Verpackung-, Preis- und Distributionspolitik.

b) Produkt-, Konditionen-, Preis- und Distributionspolitik.

c) Produkt-, Preis-, Distributions- und Kommunikationspolitik.

17) Eine Erhöhung der Investitionen in Maschinen wird die Verwaltungskosten

a) eher erhöhen.

b) eher verringern.

c) eher nicht beeinflussen.

18) Komponenten des Eigenkapitals in der Bilanz sind

a) Gezeichnetes Kapital und Rücklagen .

b) Gezeichnetes Kapital und Rückstellungen.

c) Gezeichnetes Kapital, Rücklagen und Rückstellungen.

19) Wie wirkt eine Preissenkung bei einem Produkt auf das Eigenkapital?

a) höhere Absatzmenge, geringerer Umsatz, geringerer Jahresuiberschuß, geringere Gewinnrücklage $\rightarrow$ geringeres Eigenkapital

b) höhere Absatzmenge, veränderter Umsatz, veränderter Jahresüberschuß, veränderte Gewinnrücklage $\rightarrow$ verändertes Eigenkapital

c) geringere Absatzmenge, geringerer Umsatz, geringerer Jahresïberschuß, geringere Gewinnrücklage $\rightarrow$ geringeres Eigenkapital

20) Bei der Bewertung von Rohstofflagerbeständen nach der LIFO-Methode wird a) die in die Produktion einfließende Rohstoffmenge mit dem Wert der zuletzt eingegangenen Rohstoffe bewertet.

b) die in die Produktion einfließende Rohstoffmenge mit dem Wert der zuerst eingegangenen Rohstoffe bewertet.

c) der Lagerbestand mit dem Wert der zuletzt eingegangenen Rohstoffe bewertet.

21) Erträge aus dem Abgang von Anlagevermögen entstehen, wenn

a) die kalkulatorischen höher als die bilanziellen Abschreibungen sind.

b) der Verkaufspreis über dem bilanziellen Buchwert einer Anlage liegt.

c) der Verkaufspreis ïber dem Tageswert einer Anlage liegt.

22) Die Wertschöpfung einer Aktiengesellschaft

a) muß alle übrigen Aufwendungen des Unternehmens decken.

b) wird als Rïcklage bilanziert.

c) wird an die Aktionäre ausgeschïttet. 


\section{A.10 Multiple-Choice-Fragebogen, Version B}

Die folgenden Fragen beziehen sich auf betriebswirtschaftliche Begriffe und Vorgehensweisen. Es ist jeweils nur eine Antwort richtig, deren Buchstabe ( $a, b$ oder $c)$ Sie bitte auf das Lösungsblatt übertragen.

1) Bei einer Gliederung der Kosten nach ihrer Abhängigkeit vom Beschäftigungsgrad a) unterscheidet man fixe und variable Kosten.

b) unterscheidet man Einzel- und Gemeinkosten.

c) wird zwischen fixen/variablen und Einzel-/Gemeinkosten unterschieden.

2) Komponenten des Umlaufvermögens in der Bilanz sind

a) Vorräte und Maschinen.

b) Vorräte und Wertpapiere.

c) Vorräte, Maschinen und Wertpapiere.

3) Eine Erhöhung des Verkaufspreises bei einem Produkt wird dessen Absatzmenge

a) eher erhöhen.

b) eher verringern.

c) eher nicht beeinflussen.

4) Zur Eigenfinanzierung gehören

a) Selbst- und Kreditfinanzierung.

b) Beteiligungs- und Selbstfinanzierung.

c) Kredit- und Beteiligungsfinanzierung.

5) Bei der Zuschlagskalkulation zur Ermittlung der Selbstkosten für die Preiskalkulation eines Produktes

a) werden die Materialkosten und die Fertigungskosten aufsummiert (Herstellkosten) und anschließend die Verwaltungsgemeinkosten auf Basis der Herstellkosten zugerechnet.

b) werden die Materialkosten, die Fertigungskosten und die Verwaltungskosten aufsummiert (Herstellkosten) und anschließend die Vertriebsgemein kosten auf Basis der Herstellkosten zugerechnet.

c) werden die Materialkosten und die Fertigungskosten aufsummiert (Herstellkosten) und anschließend die Verwaltungs- und Vertriebsgemein kosten auf Basis der Herstellkosten zugerechnet.

6) Bei der Abschreibung von Gegenständen des Anlagevermögens wird im Rahmen der geometrisch-degressiven Abschreibung

a) die Differenz aus Anschaffungswert und Liquidationserlös gleichmäßig auf die Jahre der geplanten Nutzung verteilt.

b) in jeder Periode ein bestimmter Prozentsatz vom Restbuchwert abgeschrieben.

c) die Abschreibungsquote als Quotient aus Restbuchwert und -laufzeit ermittelt.

7) Eine Erhöhung der Werbeausgaben für ein Produkt wird dessen Absatzmenge

a) eher erhöhen.

b) eher verringern.

c) eher nicht beeinflussen. 
8) Illiquidität bedeutet,

a) daß der Zahlungsmittelbestand höher ist als der Bedarf an Zahlungsmitteln.

b) daß das Unternehmen die fälligen kurzfristigen Verbindlichkeiten

durch die flïssigen Mittel decken kann.

c) bei Kapitalgesellschaften ein Konkursgrund.

9) Eine Methode, die Herstellungskosten eines Produktes zu ermitteln, ist die

a) Teilkosten rechnung.

b) Zuschlagskalkulation.

c) Deckungsbeitragsrechnung.

10) Die Berücksichtigung von Abschreibungen als Aufwand in der Gewinn- und Verlustrechnung sorgt dafür,

a) daß die verdienten Gegenwerte als Gewinne erscheinen.

b) daß die verdienten Gegenwerte im Betrieb verbleiben und nicht als Gewinne erscheinen.

c) daß der Gewinn erhöht wird.

11) Eine Erhöhung der Kundendienstausgaben für ein Produkt wird die Absatzmenge

a) eher erhöhen.

b) eher verringern.

c) eher nicht beeinflussen.

12) Für die Preisbildung bei einem Produkt werden folgende Kosten berücksichtigt:

a) Herstellkosten.

b) Herstell- und Vertriebskosten.

c) Herstell-, Verwaltungs- und Vertriebskosten.

13) Eine Reduzierung der Investition in Maschinen wird die mögliche Produktionsmenge

a) eher erhöhen.

b) eher verringern.

c) eher nicht beeinflussen.

14) Bei der Ermittlung des Jahresïberschusses in der Gewinn- und Verlustrechnung nach dem Umsatzkostenverfahren

a) werden von allen in der betrachteten Periode angefallenen Erträgen alle Aufwendungen abgezogen.

b) werden dem Umsatz der periode die Aufwendungen gegenübergestellt, die für die verkauften Produkte angefallen sind.

c) werden vom Umsatz, erhöht bzw. vermindert um die wertmäßige Lagerbestandsänderung, alle angefallenen Auszahlungen abgezogen.

15) Die Bestandsveränderung wird auf dem Gewinn- und Verlustkonto im Haben erfaßt, wenn

a) der Schlußbestand geringer als der Anfangsbestand ist.

b) der Schlußbestand höher als der Anfangsbestand ist.

c) der Schlußbestand und Anfangsbestand ïbereinstimmen. 
16) Eine umfassende Klassifizierung von Marketing-Instrumenten kann nach den unten aufgeführten Politiken erfolgen:

a) Produkt-, Programm-, Kundendienst- und Garantiepolitik.

b) Produkt-, Preis-, Distributions- und Kommunikationspolitik.

c) Preis-, Rabatt-, Konditionen- und Kreditpolitik.

17) Eine Reduzierung der Investitionen in Maschinen wird die Verwaltungskosten

a) eher erhöhen.

b) eher verringern.

c) eher nicht beeinflussen.

18) Komponenten des Fremdkapitals in der Bilanz sind

a) Verbindlichkeiten und Rücklagen.

b) Verbindlichkeiten und Rückstellungen.

c) Verbindlichkeiten, Rücklagen und Rückstellungen.

19) Wie wirkt eine Preiserhöhung bei einem Produkt auf das Eigenkapital?

a) höhere Absatzmenge, geringerer Lagerbestand, geringere Lagerkosten, höherer Jahresïberschuß $\rightarrow$ höheres Eigen kapital

b) geringere Absatzmenge, veränderter Umsatz, veränderter Jahresüberschuß, veränderte Gewinnrüicklage $\rightarrow$ verändertes Eigenkapital

c) geringere Absatzmenge, höherer Umsatz, höherer Jahresüberschuß, geringere Gewinnrücklage $\rightarrow$ geringeres Eigenkapital

20) Bei der FIFO-Methode zur Bewertung von Rohstofflagerbeständen wird

a) der Lagerbestand mit dem Wert der zuletzt eingegangenen Rohstoffe bewertet.

b) die in die Produktion einfließende Rohstoffmenge mit dem Wert der zuletzt eingegangenen Rohstoffe bewertet.

c) die in die Produktion einfließende Rohstoffmenge mit dem Wert der zuerst eingegangenen Rohstoffe bewertet.

21) Aufwendungen aus dem Abgang von Anlagevermögen entstehen, wenn

a) die kalkulatorischen niedriger als die bilanziellen Abschreibungen sind.

b) der Verkaufspreis unter dem Tageswert einer Anlage liegt.

c) der Verkaufspreis unter dem bilanziellen Buchwert einer Anlage liegt.

22) Der Saldo aus Gesamtleistung und eingesetzten Materialien bzw. Handelswaren ist

a) der Rohertrag einer Periode.

b) der Bilanzgewinn einer Periode.

c) das Betriebsergebnis einer Periode. 


\section{A.11 Lösungsblatt zum Fragebogen}

\begin{tabular}{|l|l|l|l|}
\hline Kennwort: & & & $\begin{array}{l}\text { (= jeweils die ersten zwei Buchstaben } \\
\text { des Vornamens und des Nachnamens) }\end{array}$ \\
\hline
\end{tabular}

Bitte tragen Sie den Buchstaben der richtigen Antwort wie folgt ein:

\begin{tabular}{|c|c|}
\hline Frage & 41 \\
\hline Antwort & d \\
\hline
\end{tabular}

\begin{tabular}{|c|l|l|l|l|l|l|l|l|l|l|l|}
\hline Frage & 1 & 2 & 3 & 4 & 5 & 6 & 7 & 8 & 9 & 10 & 11 \\
\hline Antwort & & & & & & & & & & & \\
\hline
\end{tabular}

\begin{tabular}{|c|l|l|l|l|l|l|l|l|l|l|l|}
\hline Frage & 12 & 13 & 14 & 15 & 16 & 17 & 18 & 19 & 20 & 21 & 22 \\
\hline Antwort & & & & & & & & & & & \\
\hline
\end{tabular}




\section{A.12 Netzwerk-Erhebung}

\section{Vorgehensweise}

Der Einsatz eines Unternehmensplanspiels kann u. a. den Erwerb von betriebswirtschaftlichem Zusammenhangswissen fördern. Die heutige Netzwerk-Erhebung soll den Stand Ihres bisher erworbenen Wissens feststellen.

Im folgenden werden Ihnen eine Reihe von Begriffen (sog. 'Konzepte') vorgegeben, die geeignet sind, betriebswirtschaftliche Zusammenhänge darzustellen. Bringen Sie diese Begriffe bitte mit Hilfe der ebenfalls vorgegebenen 'Relationen' in einen Zusammenhang, und stellen Sie sie graphisch dar. Das folgende Beispiel soll Ihnen die Vorgehensweise verdeutlichen.

\section{Beispiel:}

Die Konzepte 'Wald', 'Vögel', 'Rehe' und 'Bäume' können unter Zuhilfenahme verschiedener Relationen wie z. B. 'leben in' in einen Zusammenhang gebracht werden. Ein Beispiel wäre: "Rehe leben in einem Wald". Die Relationen haben eine Richtung, die durch die Pfeilspitze markiert wird. Graphisch kann dies wie folgt dargestellt werden:

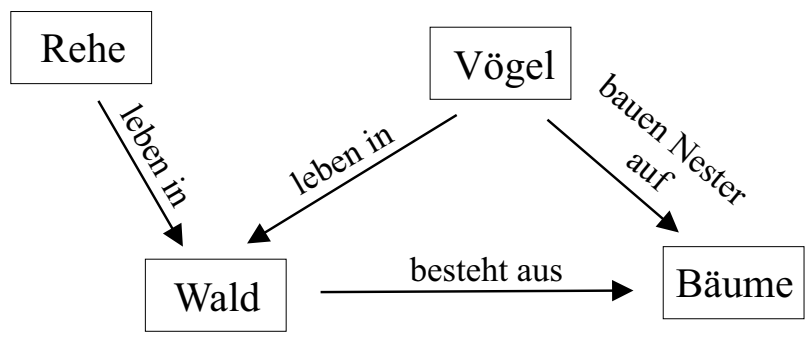

\section{Bezüglich der Struktur gibt es keine Vorgaben. Aus mehreren Bezie- hungen soll sukzessive ein 'Netzwerk' entstehen.}

In unserer Erhebung geht es um die Zusammenhänge in einer Unternehmung. Die folgenden Listen der Konzepte und Relationen dienen dabei als Basis. Sie können von Ihnen nach Bedarf erweitert werden. Benutzen Sie bitte das beiliegende DIN A3-Blatt und sinnvollerweise einen Bleistift für Ihre Konstruktion. 


\section{Liste der Konzepte}

\begin{tabular}{|c|c|c|c|}
\hline K1 & Absatzmenge & $\mathrm{K} 20$ & Personalbestand \\
\hline $\mathrm{K} 2$ & Ausschüttung & $\mathrm{K} 21$ & Personaleinstellung/-entlassung \\
\hline K3 & Einkaufspreis der Maschinen & $\mathrm{K} 22$ & Produktbestand \\
\hline K4 & Einkaufspreis der Rohstoffe & $\mathrm{K} 23$ & Produktionskapazität \\
\hline K5 & Forderungen/Verbindlichkeiten & K24 & Produktionsmenge \\
\hline K6 & Gewinn & K25 & Rohstoffbeschaffung \\
\hline K7 & Herstellungskosten & $\mathrm{K} 26$ & Rohstoffbestand \\
\hline K8 & Kassenbestand & K27 & Rücklagen \\
\hline K9 & Kreditaufnahme/-tilgung & $\mathrm{K} 28$ & Steuern \\
\hline K10 & Lageraufwand für Produkte & K29 & Terminanlage \\
\hline K11 & Lageraufwand für Rohstoffe & K30 & Umsatz \\
\hline K12 & Liquidationserlös der Maschinen & K31 & Verkaufspreis der Produkte \\
\hline K13 & Löhne und Sozialkosten & K 32 & Wartungskosten \\
\hline K14 & Marktforschung & K33 & Werbung/Kundendienst \\
\hline K15 & Maschinenauslastung & K34 & Zinsaufwand/-ertrag \\
\hline K16 & Maschinenbestand & K 35 & Zinssätze \\
\hline K17 & Maschinenkauf/-verkauf & K36 & \\
\hline K18 & Nachfragemenge & K37 & \\
\hline 19 & Personalbedarf & K38 & \\
\hline
\end{tabular}

\section{Liste der Relationen}

R1 A hat B zum Ziel / A hat B zum Zweck

R2 A hat B zur Folge / A führt zu B

R3 A hat Einfluß auf B / A beeinflußt B

R4 A ist Ursache für B / A verursacht B

R5 A ist verbunden mit $\mathrm{B} / \mathrm{A}$ ist verknüpft mit $\mathrm{B}$

R6

R7 


\section{A.13 Legende zur Darstellung der Netzwerke}

\section{Legende der Modalen Netzwerke}

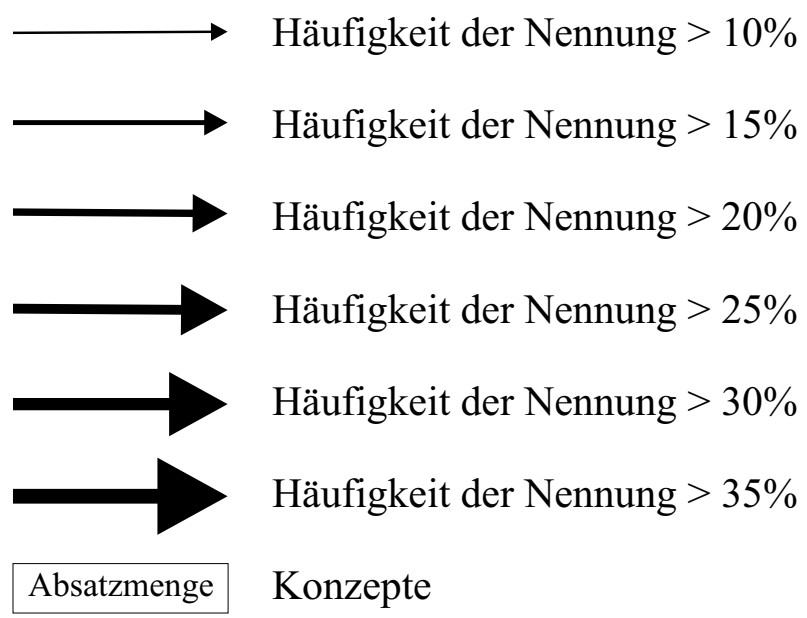

\section{Legende des Referenznetzes}

$\begin{array}{ll}\longrightarrow & \text { Häufigkeit der Nennung }=3 \text { von } 6=50 \% \\ \longrightarrow & \text { Häufigkeit der Nennung }=4 \text { von } 6=67 \% \\ \longrightarrow & \text { Häufigkeit der Nennung }=5 \text { von } 6=83 \% \\ \longrightarrow & \text { Häufigkeit der Nennung }=6 \text { von } 6=100 \% \\ \text { Absatzmenge } & \text { Konzepte }\end{array}$




\section{A.14 Entscheidungsblätter der modularen Be- dingung}

Name Ihrer Unternehmung:

Kennworte der

Gruppenmitglieder

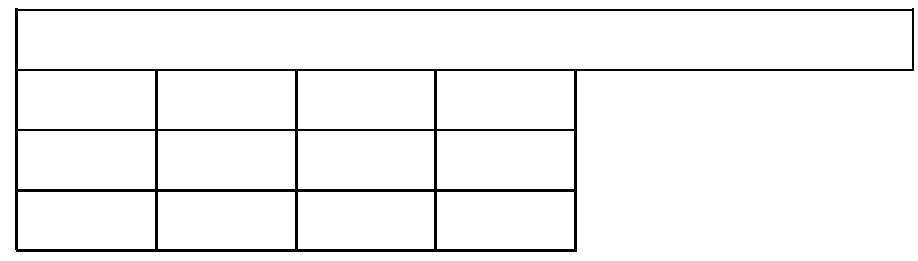

Sie übernehmen die Leitung eines Unternehmens am Ende der Periode 3. Zur Information liegt Ihnen ein Ausdruck der Unternehmensergebnisse vor. Auf der Basis dieser Daten haben Sie Entscheidungen über die Beschaffung von Rohstoffen, Maschinen und Bedienungspersonal zu treffen. Die Produktionsmengen für die Periode 4 sind bereits festgelegt und belaufen sich auf 1.900 Stück von Produkt P1 und 1.840 Stück von Produkt P2.

Es werden Ihnen zwei Szenarien zur Entscheidung vorgelegt.

Viel Spaß und Erfolg bei Ihrer Unternehmensführung!

Sie haben in dem positiven Szenario dafür zu sorgen, daß für die übernächste Periode eine Produktionskapazität von 2.500 Stück bei Produkt P1 und 2.200 Stück bei Produkt P2 geschaffen wird. Bitte tragen Sie Ihre Entscheidungen in die folgende Tabelle ein.

\begin{tabular}{|l|l|r|r|r|l|}
\hline \multicolumn{2}{|c|}{ Rohstoffbeschaffung } & \multicolumn{2}{|c|}{ Investitionen } & \multicolumn{2}{c|}{ Personal } \\
\hline Rohstoff P1: & Maschinenkauf: & & Einstellungen: & \\
\hline Rohstoff P2: & & Maschinenkauf: & & Einstellungen: & \\
\hline
\end{tabular}

Für das pessimistischere Szenario mit einer ungünstigeren Nachfrageentwicklung sollen Sie ebenfalls die Beschaffungsplanung durchführen. Gehen Sie dabei von einer Produktionskapazität von jeweils 2.000 Stück bei Produkt P1 und P2 aus.

\begin{tabular}{|l|l|l|l|l|l|}
\hline \multicolumn{2}{|r|}{ Rohstoffbeschaffung } & \multicolumn{2}{|c|}{ Investitionen } & \multicolumn{2}{c|}{ Personal } \\
\hline Rohstoff P1: & Maschinenkauf: & & Einstellungen: & \\
\hline Rohstoff P2: & Maschinenkauf: & & Einstellungen: & \\
\hline
\end{tabular}




\section{modulare Bedingung: 2. Entscheidung, 2. Stufe}

Name Ihrer Unternehmung:

Kennworte der

Gruppenmitglieder

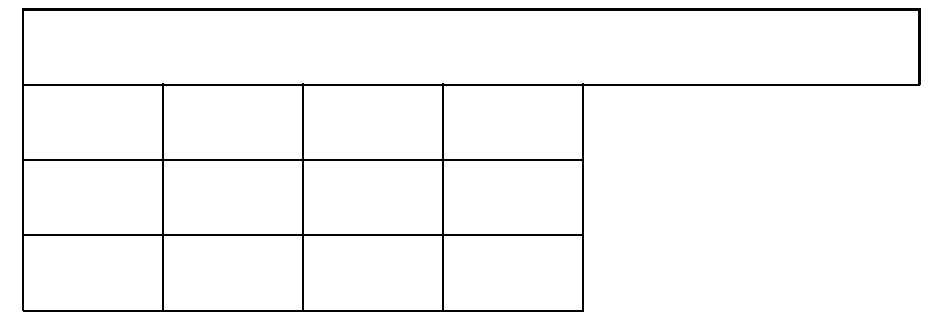

In der letzten Sitzung haben Sie Entscheidungen für die Periode 4 getroffen. Die Auswirkungen dieser Entscheidungen können Sie dem beigefügten Unternehmensbericht entnehmen.

\section{Sie werden nun in die letzte Periode zurückversetzt!}

Auf der Basis des Unternehmensberichts von Periode 3 entscheiden Sie über die Beschaffung der Rohstoffe, der Maschinen sowie des Bedienungspersonals. Zusätzlich treffen Sie nun die Entscheidung über die Produktion der laufenden Periode und die Produktionsmenge der folgenden Periode.

\begin{tabular}{|l|l|}
\hline jetzige Produktionsmenge für Produkt 1: & \\
\hline jetzige Produktionsmenge für Produkt 2: & \\
\hline zukünftige Produktionsmenge für Produkt 1: & \\
\hline zukünftige Produktionsmenge für Produkt 2: & \\
\hline
\end{tabular}

\begin{tabular}{|l|l|l|l|l|l|}
\hline \multicolumn{2}{|c|}{ Rohstoffbeschaffung } & \multicolumn{2}{|c|}{ Investitionen } & \multicolumn{2}{c|}{ Personal } \\
\hline Rohstoff P1: & Maschinenkauf: & & Einstellungen: & \\
\hline Rohstoff P2: & Maschinenkauf: & & Einstellungen: & \\
\hline
\end{tabular}




\section{modulare Bedingung: 2. Entscheidung, 3. Stufe}

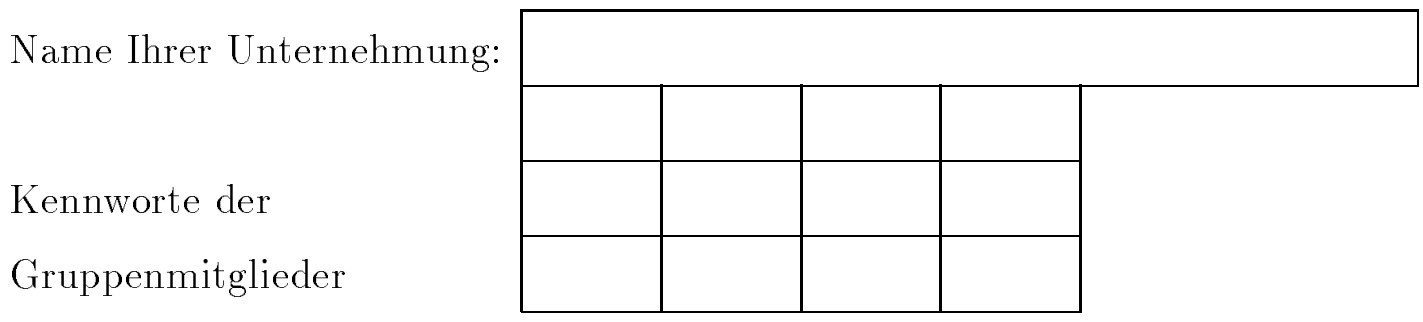

In der letzten Sitzung haben Sie Entscheidungen für die Periode 4 getroffen. Die Auswirkungen dieser Entscheidungen können Sie dem beigefügten Unternehmensbericht entnehmen.

\section{Sie werden nun in die letzte Periode zurückversetzt!}

Auf der Basis des Unternehmensberichts von Periode 3 entscheiden Sie über die Beschaffung der Rohstoffe, der Maschinen sowie des Bedienungspersonals. Zusätzlich treffen Sie nun die Entscheidung über die Produktion der laufenden Periode und die Produktionsmenge der folgenden Periode. Weiterhin sind die Verkaufspreise der Produkte auf beiden Märkten festzulegen.

\begin{tabular}{|l|l|}
\hline jetzige Produktionsmenge für Produkt 1: & \\
\hline jetzige Produktionsmenge für Produkt 2: & \\
\hline zukünftige Produktionsmenge für Produkt 1: & \\
\hline zukünftige Produktionsmenge für Produkt 2: & \\
\hline
\end{tabular}

\begin{tabular}{|l|l|r|r|r|l|}
\hline \multicolumn{2}{|c|}{ Rohstoffbeschaffung } & \multicolumn{2}{|c|}{ Investitionen } & \multicolumn{2}{c|}{ Personal } \\
\hline Rohstoff P1: & Maschinenkauf: & & Einstellungen: & \\
\hline Rohstoff P2: & & Maschinenkauf: & & Einstellungen: & \\
\hline
\end{tabular}

\begin{tabular}{|l|l|l|}
\hline \multicolumn{1}{|c|}{ Verkaufspreise } & Markt 1 & Markt 2 \\
\hline Produkt 1: & & \\
\hline Produkt 2: & & \\
\hline
\end{tabular}




\section{modulare Bedingung: 3. Entscheidung, 2. Stufe}

Name Ihrer Unternehmung:

Kennworte der

Gruppenmitglieder

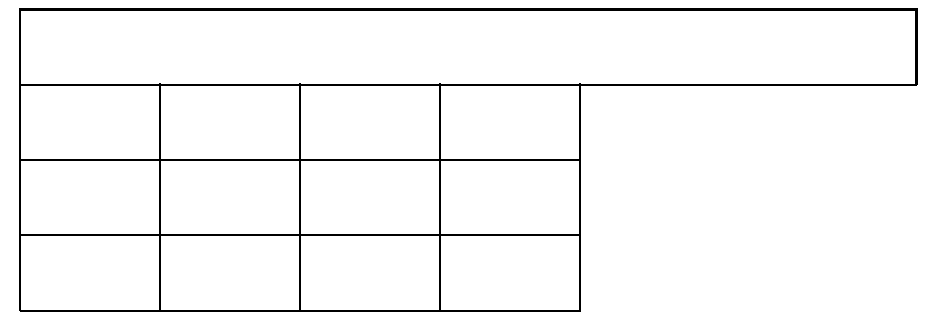

Aufgrund der von Ihnen in der letzten Sitzung getroffenen Entscheidung liegt Ihnen nun der Unternehmensbericht für die Periode 4 vor.

Treffen Sie auf dieser Basis Ihre Entscheidungen für die nächste Periode (Periode 5). Bitte beachten Sie dabei folgende Angaben:

- Die Rohstoffpreise zeigen leicht steigende Tendenz.

- Die Konjunktur zeigt weiterhin erfreuliches Wachstum.

- Das Vorstandsgehalt wurde angehoben.

- Die Lagerhaltungsfixkosten steigen.

- Die Wartungskosten pro LE steigen von 1,25 auf 1,150 DM/LE.

\begin{tabular}{|l|l|}
\hline jetzige Produktionsmenge für Produkt 1: & \\
\hline jetzige Produktionsmenge für Produkt 2: & \\
\hline zukünftige Produktionsmenge für Produkt 1: & \\
\hline zukünftige Produktionsmenge für Produkt 2: & \\
\hline
\end{tabular}

\begin{tabular}{|l|l|r|r|r|}
\hline \multicolumn{2}{|c|}{ Rohstoffbeschaffung } & \multicolumn{2}{|c|}{ Investitionen } & \multicolumn{2}{c|}{ Personal } \\
\hline Rohstoff P1: & Maschinenkauf: & & Einstellungen: & \\
\hline Rohstoff P2: & Maschinenkauf: & & Einstellungen: & \\
\hline
\end{tabular}




\section{modulare Bedingung: 3. Entscheidung, 3. Stufe}

Name Ihrer Unternehmung:

Aufgrund der von Ihnen in der letzten Sitzung getroffenen Entscheidung liegt Ihnen nun der Unternehmensbericht für die Periode 4 vor.

Treffen Sie auf dieser Basis Ihre Entscheidungen für die nächste Periode (Periode 5). Bitte beachten Sie dabei folgende Angaben:

- Die Rohstoffpreise zeigen leicht steigende Tendenz.

- Die Konjunktur zeigt weiterhin erfreuliches Wachstum.

- Das Vorstandsgehalt wurde angehoben.

- Die Lagerhaltungsfixkosten steigen.

- Die Wartungskosten pro LE steigen von 1,25 auf 1,150 DM/LE.

\begin{tabular}{|l|l|l|}
\hline \multicolumn{1}{|c|}{ Produktionsmenge } & für Periode 5 & für Periode 6 \\
\hline Produkt 1: & & \\
\hline Produkt 2: & & \\
\hline
\end{tabular}

\begin{tabular}{|l|l|l|l|l|l|}
\hline \multicolumn{2}{|c|}{ Rohstoffbeschaffung } & \multicolumn{2}{|c|}{ Investitionen } & \multicolumn{2}{c|}{ Personal } \\
\hline Rohstoff P1: & Maschinenkauf: & & Einstellungen: & \\
\hline Rohstoff P2: & & Maschinenkauf: & & Einstellungen: & \\
\hline
\end{tabular}

\begin{tabular}{|l|l|l|}
\hline \multicolumn{1}{|c|}{ Verkaufspreise } & Markt 1 & Markt 2 \\
\hline Produkt 1: & & \\
\hline Produkt 2: & & \\
\hline
\end{tabular}




\section{modulare Bedingung: 3. Entscheidung, 4. Stufe}

Name Ihrer Unternehmung:

Aufgrund der von Ihnen in der letzten Sitzung getroffenen Entscheidung liegt Ihnen nun der Unternehmensbericht für die Periode 4 vor.

Treffen Sie auf dieser Basis Ihre Entscheidungen für die nächste Periode (Periode 5). Bitte beachten Sie dabei folgende Angaben:

- Die Rohstoffpreise zeigen leicht steigende Tendenz.

- Die Konjunktur zeigt weiterhin erfreuliches Wachstum.

- Das Vorstandsgehalt wurde angehoben.

- Die Lagerhaltungsfixkosten steigen.

- Die Wartungskosten pro LE steigen von 1,25 auf 1,150 DM/LE.

\begin{tabular}{|l|l|l|}
\hline \multicolumn{1}{|c|}{ Produktionsmenge } & für Periode 5 & für Periode 6 \\
\hline Produkt $1:$ & & \\
\hline Produkt $2:$ & & \\
\hline
\end{tabular}

\begin{tabular}{|l|l|l|l|l|l|}
\hline \multicolumn{2}{|c|}{ Rohstoffbeschaffung } & \multicolumn{2}{c|}{ Investitionen } & \multicolumn{2}{c|}{ Personal } \\
\hline Rohstoff P1: & Maschinenkauf: & & Einstellungen: & \\
\hline Rohstoff P2: & Maschinenkauf: & & Einstellungen: & \\
\hline
\end{tabular}

\begin{tabular}{|l|l|l|}
\hline \multicolumn{1}{|c|}{ Verkaufspreise } & Markt 1 & Markt 2 \\
\hline Produkt 1: & & \\
\hline Produkt 2: & & \\
\hline
\end{tabular}

Weiterhin entscheiden Sie heute über die Ausgaben für Werbung und Kundendienst.

\begin{tabular}{|l|l|l|}
\hline \multicolumn{1}{|c|}{ Produkt 1 } & Markt 1 & Markt 2 \\
\hline Werbung: & & \\
\hline Kundendienst: & Markt 1 & Markt 2 \\
\hline Produkt 2 & & \\
\hline Werbung: & & \\
\hline Kundendienst: & & \\
\hline
\end{tabular}




\section{modulare Bedingung: 4. Entscheidung, 3. Stufe}

Name Ihrer Unternehmung:

Aufgrund der von Ihnen in der letzten Sitzung getroffenen Entscheidung liegt Ihnen nun der Unternehmensbericht für die Periode 5 vor.

Treffen Sie auf dieser Basis Ihre Entscheidungen für die nächste Periode (Periode 6). Bitte beachten Sie dabei folgende Angaben:

- Die Konjunktur zeigt noch Wachstum, sie nähert sich bei Produkt 1 dem Höhepunkt.

- Das Vorstandsgehalt wurde angehoben.

- Die Lagerhaltungsfixkosten steigen.

- Für die Periode 6 ist mit einer neuen Tarifrunde zu rechnen. Erwartete Lohnerhöhungen um ca. $10 \%$.

- Außerdem tritt in Periode 6 eine kurzfristige Verknappung bei Rohstoff 2 auf, die Preise steigen auf 25,- DM/Stück. Die Verknappung wird nur eine Periode anhalten.

\begin{tabular}{|l|l|l|}
\hline \multicolumn{1}{|c|}{ Produktionsmenge } & für Periode 6 & für Periode 7 \\
\hline Produkt 1: & & \\
\hline Produkt 2: & & \\
\hline
\end{tabular}

\begin{tabular}{|l|l|l|l|l|l|}
\hline \multicolumn{2}{|c|}{ Rohstoffbeschaffung } & \multicolumn{2}{c|}{ Investitionen } & \multicolumn{2}{c|}{ Personal } \\
\hline Rohstoff P1: & & Maschinenkauf: & & Einstellungen: & \\
\hline Rohstoff P2: & Maschinenkauf: & & Einstellungen: & \\
\hline
\end{tabular}

\begin{tabular}{|l|l|l|}
\hline \multicolumn{1}{|c|}{ Verkaufspreise } & Markt 1 & Markt 2 \\
\hline Produkt 1: & & \\
\hline Produkt 2: & & \\
\hline
\end{tabular}




\section{modulare Bedingung: 4. Entscheidung, 4. Stufe}

Name Ihrer Unternehmung:

Aufgrund der von Ihnen in der letzten Sitzung getroffenen Entscheidung liegt Ihnen nun der Unternehmensbericht für die Periode 5 vor. Treffen Sie auf dieser Basis Ihre Entscheidungen für die nächste Periode. Bitte beachten Sie dabei folgende Angaben:

- Die Konjunktur zeigt noch Wachstum, sie nähert sich bei Produkt 1 dem Höhepunkt.

- Das Vorstandsgehalt wurde angehoben.

- Die Lagerhaltungsfixkosten steigen.

- Für die Periode 6 ist mit einer neuen Tarifrunde zu rechnen. Erwartete Lohnerhöhungen um ca. $10 \%$.

- Außerdem tritt in Periode 6 eine kurzfristige Verknappung bei Rohstoff 2 auf, die Preise steigen auf 25,- DM/Stück. Die Verknappung wird nur eine Periode anhalten.

\begin{tabular}{|l|c|c|}
\hline \multicolumn{1}{|c|}{ Produktionsmenge } & für Periode 6 & für Periode 7 \\
\hline Produkt 1: & & \\
\hline Produkt 2: & & \\
\hline
\end{tabular}

\begin{tabular}{|l|l|l|l|l|l|}
\hline \multicolumn{2}{|c|}{ Rohstoffbeschaffung } & \multicolumn{2}{c|}{ Investitionen } & \multicolumn{2}{c|}{ Personal } \\
\hline Rohstoff P1: & Maschinenkauf: & Einstellungen: & \\
\hline Rohstoff P2: & Maschinenkauf: & & Einstellungen: & \\
\hline
\end{tabular}

\begin{tabular}{|l|l|l|}
\hline \multicolumn{1}{|c|}{ Verkaufspreise } & Markt 1 & Markt 2 \\
\hline Produkt 1: & & \\
\hline Produkt 2: & & \\
\hline
\end{tabular}

Weiterhin entscheiden Sie heute über die Ausgaben für Werbung und Kundendienst.

\begin{tabular}{|l|l|l|}
\hline \multicolumn{1}{|c|}{ Produkt 1 } & Markt 1 & Markt 2 \\
\hline Werbung: & & \\
\hline Kundendienst: & Markt 1 & Markt 2 \\
\hline Produkt 2 & & \\
\hline Werbung: & & \\
\hline Kundendienst: & & \\
\hline
\end{tabular}




\section{modulare Bedingung: 4. Entscheidung, 5. Stufe}

Name Ihrer Unternehmung:

Aufgrund der von Thnen in der letzten Sitzung getroffenen Entscheidung liegt Ihnen nun der Unternehmensbericht für die Periode 5 vor. Treffen Sie auf dieser Basis Ihre Entscheidungen für die nächste Periode. Bitte beachten Sie dabei folgende Angaben:

- Die Konjunktur zeigt noch Wachstum, sie nähert sich bei Produkt 1 dem Höhepunkt.

- Das Vorstandsgehalt wurde angehoben.

- Die Lagerhaltungsfixkosten steigen.

- Für die Periode 6 ist mit einer neuen Tarifrunde zu rechnen. Erwartete Lohnerhöhungen um ca. $10 \%$.

- Außerdem tritt in Periode 6 eine kurzfristige Verknappung bei Rohstoff 2 auf, die Preise steigen auf 25,- DM/Stück. Die Verknappung wird nur eine Periode anhalten.

\begin{tabular}{|l|l|l|}
\hline \multicolumn{1}{|c|}{ Produktionsmenge } & für Periode 6 & für Periode 7 \\
\hline Produkt $1:$ & & \\
\hline Produkt $2:$ & & \\
\hline
\end{tabular}

\begin{tabular}{|c|c|c|}
\hline Rohstoffbeschaffung & Investitionen & Personal \\
\hline Rohstoff P1: & Maschinenkauf: & Einstellungen: \\
\hline Rohstoff P2: & Maschinenkauf: & Einstellungen: \\
\hline
\end{tabular}

\begin{tabular}{|l|l|l|}
\hline \multicolumn{1}{|c|}{ Produkt 1 } & Markt 1 & Markt 2 \\
\hline Verkaufspreis: & & \\
\hline Werbung: & & \\
\hline Kundendienst: & Markt 1 & \\
\hline \multicolumn{1}{|c|}{ Produkt 2 } & & Markt 2 \\
\hline Verkaufspreis: & & \\
\hline Werbung: & & \\
\hline Kundendienst: & & \\
\hline
\end{tabular}

\begin{tabular}{|l|l|l|l|}
\hline \multicolumn{3}{|c|}{ Finanzierung } \\
\hline Kreditaufnahme: & & Terminanlage: & \\
\hline Kredittilgung: & & \multicolumn{1}{c}{} \\
\cline { 1 - 3 } & &
\end{tabular}




\section{modulare Bedingung: 5. Entscheidung, 4. Stufe}

Name Ihrer Unternehmung:

Aufgrund der von Thnen in der letzten Sitzung getroffenen Entscheidung liegt Ihnen nun der Unternehmensbericht für die Periode 6 vor. Treffen Sie auf dieser Basis Ihre Entscheidungen für die nächste Periode. Bitte beachten Sie dabei folgende Angaben:

- Beim Produkt 1 scheint die Nachfrage den Höhepunkt erreicht zu haben. Produkt 2 wird noch Nachfragezuwächse bis zur Periode 9 verzeichnen können.

- Das Vorstandsgehalt wurde angehoben.

- Die Lagerhaltungsfixkosten steigen.

- Die Tarifrunde brachte Lohnerhöhungen von $10 \%$.

- Die Rohstoffpreise normalisieren sich auf hohem Niveau. Die Lagerhaltungskosten für Produkte steigen auf 6,- DM/Stück.

\begin{tabular}{|l|l|l|}
\hline \multicolumn{1}{|c|}{ Produktionsmenge } & für Periode 7 & für Periode 8 \\
\hline Produkt 1: & & \\
\hline Produkt 2: & & \\
\hline
\end{tabular}

\begin{tabular}{|c|l|l|l|l|l|}
\hline \multicolumn{2}{|c|}{ Rohstoffbeschaffung } & \multicolumn{2}{c|}{ Investitionen } & \multicolumn{2}{c|}{ Personal } \\
\hline Rohstoff P1: & Maschinenkauf: & & Einstellungen: & \\
\hline Rohstoff P2: & Maschinenkauf: & & Einstellungen: & \\
\hline
\end{tabular}

\begin{tabular}{|l|l|l|}
\hline \multicolumn{1}{|c|}{ Verkaufspreise } & Markt 1 & Markt 2 \\
\hline Produkt 1: & & \\
\hline Produkt 2: & & \\
\hline
\end{tabular}

Weiterhin entscheiden Sie heute über die Ausgaben für Werbung und Kundendienst.

\begin{tabular}{|l|l|l|}
\hline \multicolumn{1}{|c|}{ Produkt 1 } & Markt 1 & Markt 2 \\
\hline Werbung: & & \\
\hline Kundendienst: & Markt 1 & Markt 2 \\
\hline Produkt 2 & & \\
\hline Werbung: & & \\
\hline Kundendienst: & & \\
\hline
\end{tabular}




\section{modulare Bedingung: 5. Entscheidung, 5. Stufe}

Name Ihrer Unternehmung:

Aufgrund der von Ihnen in der letzten Sitzung getroffenen Entscheidung liegt Ihnen nun der Unternehmensbericht für die Periode 6 vor. Treffen Sie auf dieser Basis Ihre Entscheidungen für die nächste Periode. Bitte beachten Sie dabei folgende Angaben:

- Beim Produkt 1 scheint die Nachfrage den Höhepunkt erreicht zu haben. Produkt 2 wird noch Nachfragezuwächse bis zur Periode 9 verzeichnen können.

- Das Vorstandsgehalt wurde angehoben.

- Die Lagerhaltungsfixkosten steigen.

- Die Tarifrunde brachte Lohnerhöhungen von $10 \%$.

- Die Rohstoffpreise normalisieren sich auf hohem Niveau. Die Lagerhaltungskosten für Produkte steigen auf 6,- DM/Stück.

\begin{tabular}{|l|l|l|}
\hline \multicolumn{1}{|c|}{ Produktionsmenge } & für Periode 7 & für Periode 8 \\
\hline Produkt $1:$ & & \\
\hline Produkt $2:$ & & \\
\hline
\end{tabular}

\begin{tabular}{|l|l|l|l|l|l|}
\hline \multicolumn{2}{|c|}{ Rohstoffbeschaffung } & \multicolumn{2}{c|}{ Investitionen } & \multicolumn{2}{c|}{ Personal } \\
\hline Rohstoff P1: & Maschinenkauf: & & Einstellungen: & \\
\hline Rohstoff P2: & Maschinenkauf: & & Einstellungen: & \\
\hline
\end{tabular}

\begin{tabular}{|l|l|l|}
\hline \multicolumn{1}{|c|}{ Produkt 1 } & Markt 1 & Markt 2 \\
\hline Verkaufspreis: & & \\
\hline Werbung: & & \\
\hline Kundendienst: & Markt 1 & Markt 2 \\
\hline \multicolumn{1}{|c|}{ Produkt 2 } & & \\
\hline Verkaufspreis: & & \\
\hline Werbung: & & \\
\hline Kundendienst: & & \\
\hline
\end{tabular}

\begin{tabular}{|l|l|l|l|}
\hline \multicolumn{3}{|c|}{ Finanzierung } \\
\hline Kreditaufnahme: & & Terminanlage: & \\
\hline Kredittilgung: & & \multicolumn{1}{c}{} \\
\cline { 1 - 2 } & &
\end{tabular}




\section{A.15 Entscheidungsblätter der herkömmlichen Bedingung}

Name Ihrer Unternehmung:

Kennworte der

Gruppenmitglieder

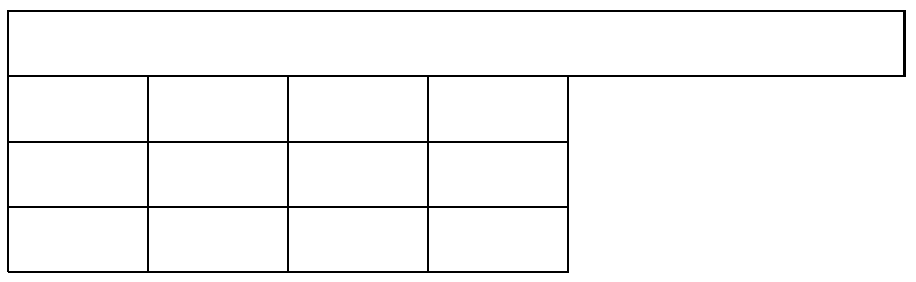

Sie übernehmen die Leitung eines Unternehmens am Ende der Periode 3. Zur Information liegt Ihnen ein Ausdruck der Unternehmensergebnisse vor. Auf der Basis dieser Daten haben Sie Entscheidungen über die Bereiche Beschaffung, Produktions, Marketing und Finanzierung für die nächste Periode.

Diese Entscheidungen treffen Sie zur Probe. In der nächsten Sitzung haben Sie einmalig die Wahl, ob Sie Thre Entscheidungen revidieren möchten oder ob Sie gleich zur nächste Periode übergehen wollen.

Viel Spaß und Erfolg bei Ihrer Unternehmensführung!

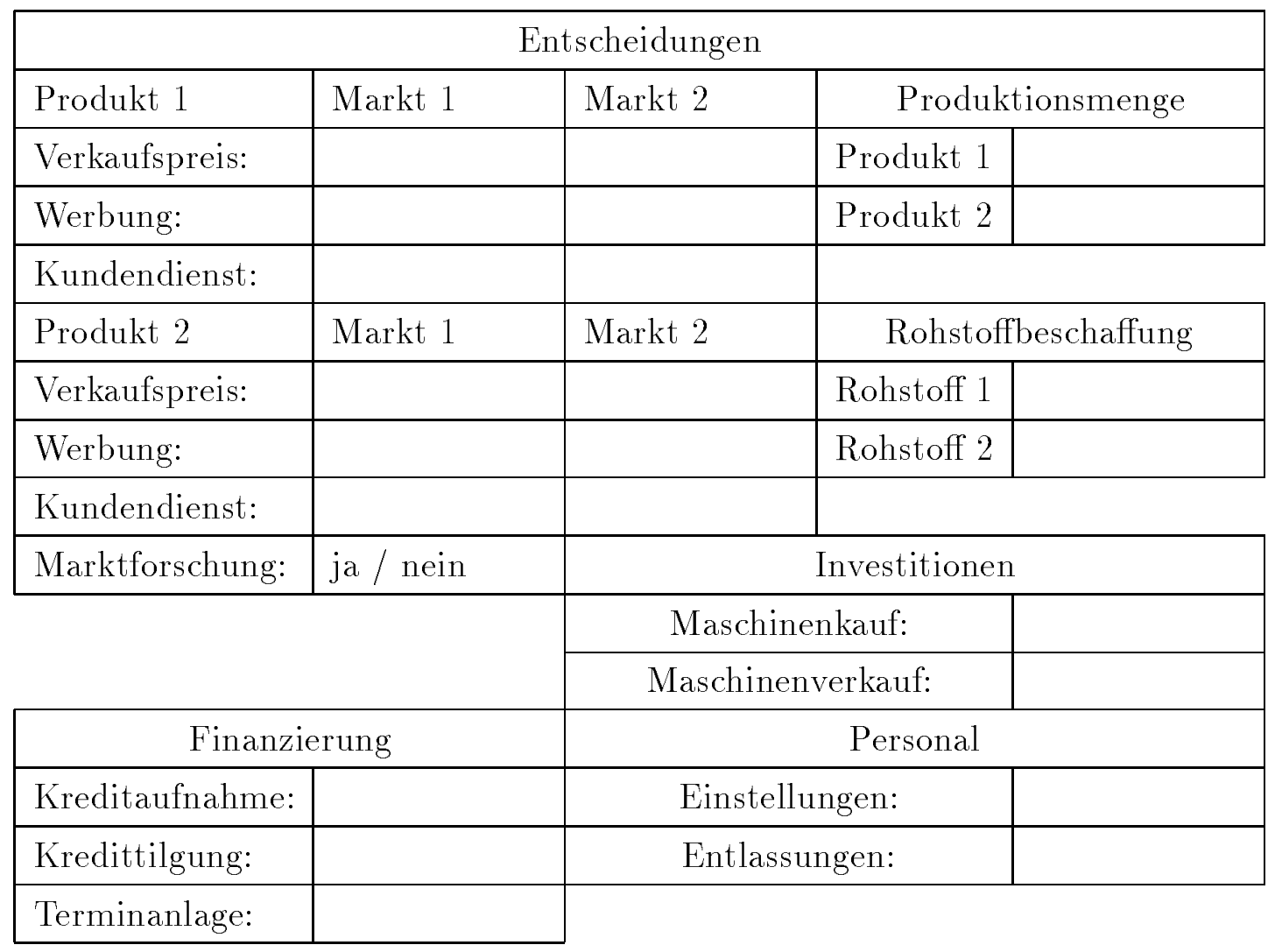




\section{herkömmliche Bedingung: 2. Entscheidung}

Name Ihrer Unternehmung:

In der letzten Sitzung trafen Sie Entscheidungen für die Periode 4. Die Auswirkungen dieser Entscheidungen können Sie dem beigefügten Unternehmensbericht entnehmen.

Sie haben jetzt die Wahl, ob Sie Ihre Entscheidungen revidieren wollen oder gleich Entscheidungen für die Periode 5 treffen wollen. Stützen Sie sich dabei auf den entsprechenden Unternehmensbericht, also bei Wiederholung auf den der Periode 3, bei einer Folgeentscheidung auf den der Periode 4.

Kreuzen Sie bitte an: Wiederholung: $\square \quad$ Folgeentscheidung:

\begin{tabular}{|c|c|c|c|c|}
\hline \multicolumn{5}{|c|}{ Entscheidungen } \\
\hline Produkt 1 & Markt 1 & Markt 2 & \multicolumn{2}{|c|}{ Produktionsmenge } \\
\hline Verkaufspreis: & & & Produkt 1 & \\
\hline Werbung: & & & Produkt 2 & \\
\hline \multicolumn{5}{|l|}{ Kundendienst: } \\
\hline Produkt 2 & Markt 1 & Markt 2 & \multicolumn{2}{|c|}{ Rohstoffbeschaffung } \\
\hline Verkaufspreis: & & & Rohstoff 1 & \\
\hline Werbung: & & & Rohstoff 2 & \\
\hline \multicolumn{5}{|l|}{ Kundendienst: } \\
\hline Marktforschung: & ja / nein & \multicolumn{3}{|c|}{ Investitionen } \\
\hline & & \multicolumn{2}{|c|}{ Maschinenkauf: } & \\
\hline & & \multicolumn{2}{|c|}{ Maschinenverkauf: } & \\
\hline \multicolumn{2}{|c|}{ Finanzierung } & \multicolumn{3}{|c|}{ Personal } \\
\hline Kreditaufnahme: & & \multicolumn{2}{|c|}{ Einstellungen: } & \\
\hline Kredittilgung: & & \multicolumn{2}{|c|}{ Entlassungen: } & \\
\hline Terminanlage: & & & & \\
\hline
\end{tabular}




\section{herkömmliche Bedingung: 3.-5. Entscheidung}

Name Ihrer Unternehmung:

Treffen Sie auf der Basis Ihres aktuellen Unternehmensberichts die Entscheidungen für die nächste Periode.

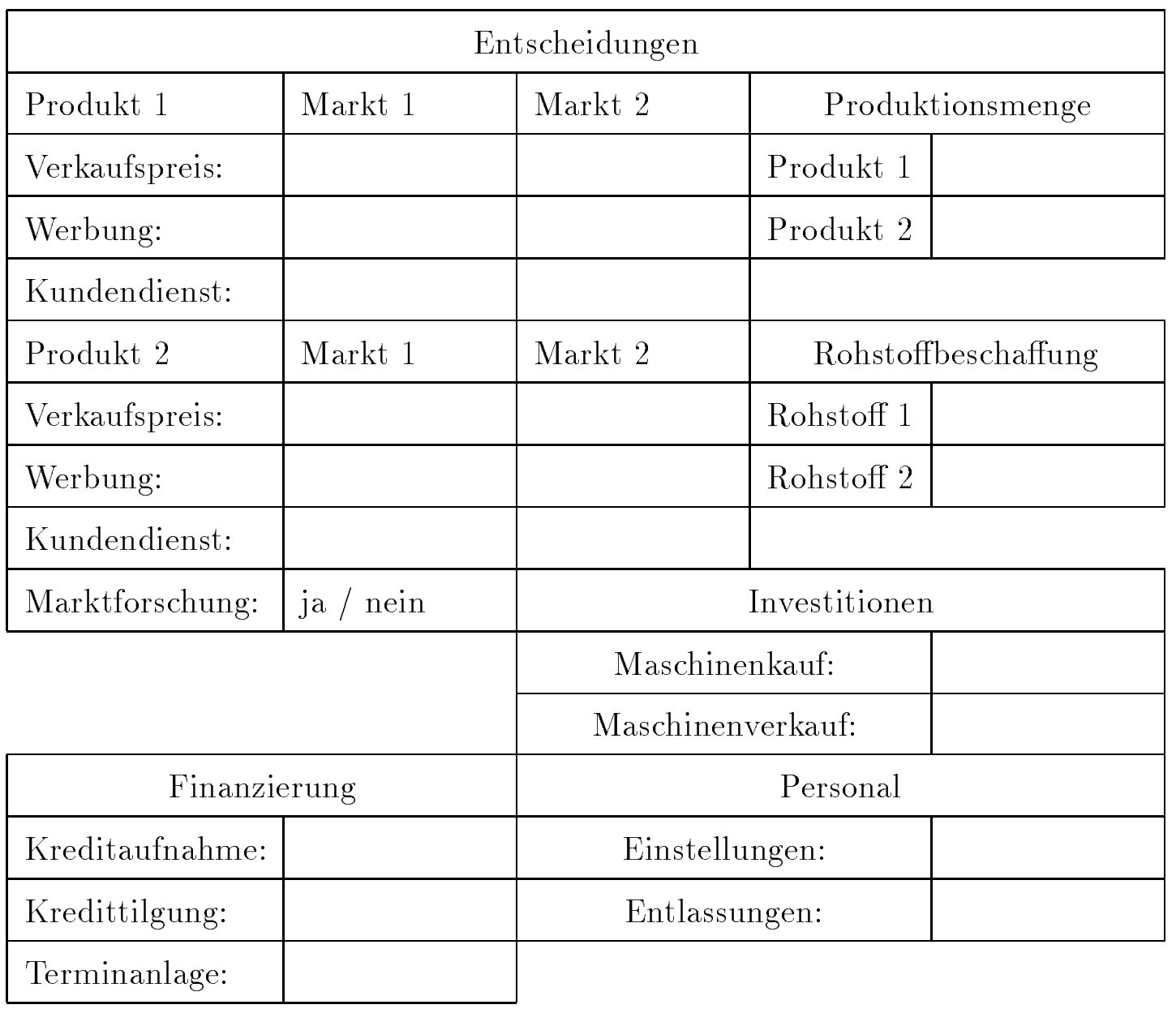




\section{A.16 Entscheidungsblätter in der Testphase}

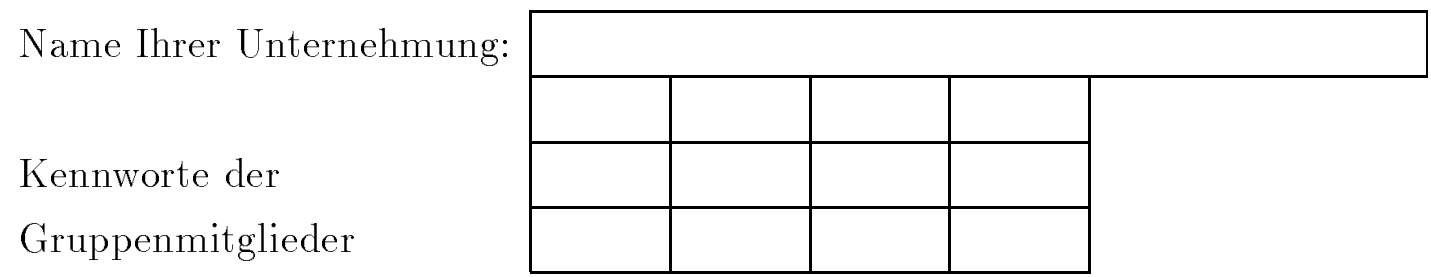

Sie übernehmen die Leitung eines Unternehmens am Ende der Periode 8. Auf der Basis der Ihnen vorliegenden Unternehmensergebnisse treffen Sie Entscheidungen für die nächste Periode.

Zur Erläuterung der neuen Ausgangslage vergleichen Sie bitte die Anmerkungen auf der Rückseite.

Viel Spaß und Erfolg bei Ihrer Unternehmensführung!

\begin{tabular}{|c|c|c|c|c|}
\hline \multicolumn{5}{|c|}{ Entscheidungen } \\
\hline Produkt 1 & Markt 1 & Markt 2 & \multicolumn{2}{|c|}{ Produktionsmenge } \\
\hline Verkaufspreis: & & & Produkt 1 & \\
\hline Werbung: & & & Produkt 2 & \\
\hline \multicolumn{5}{|l|}{ Kundendienst: } \\
\hline Produkt 2 & Markt 1 & Markt 2 & \multicolumn{2}{|c|}{ Rohstoffbeschaffung } \\
\hline Verkaufspreis: & & & Rohstoff 1 & \\
\hline Werbung: & & & Rohstoff 2 & \\
\hline \multicolumn{5}{|l|}{ Kundendienst: } \\
\hline Marktforschung: & ja / nein & \multicolumn{3}{|c|}{ Investitionen } \\
\hline & & \multicolumn{2}{|c|}{ Maschinenkauf: } & \\
\hline & & \multicolumn{2}{|c|}{ Maschinenverkauf: } & \\
\hline \multicolumn{2}{|c|}{ Finanzierung } & \multicolumn{3}{|c|}{ Personal } \\
\hline Kreditaufnahme: & & \multicolumn{2}{|c|}{ Einstellungen: } & \\
\hline Kredittilgung: & & \multicolumn{2}{|c|}{ Entlassungen: } & \\
\hline Terminanlage: & & & & \\
\hline
\end{tabular}




\section{Erläuterungen zur Ausgangslage der Testphase}

Grundlage Ihrer heutigen Entscheidungen ist der beigefügte Unternehmensbericht für die Periode 8. Sie werden darin mit einer neuen Ausgangslage konfrontiert. Bevor Sie Ihre Entscheidungen treffen, analysieren Sie bitte den Unternehmensbericht.

Ergänzend wird auf folgende Punkte hingewiesen:

1. Die Nachfrage nach Produkt 1 ist auf beiden Märkten rückläufig. Bei Produkt 2 ist die Nachfrage auf Markt 1 noch leicht steigend, auf Markt 2 stagnierend. In den kommenden Perioden ist mit einer weiteren Abschwächung zu rechnen.

2. Ihrer Entwicklungsabteilung ist in Zusammenarbeit mit dem Maschinenlieferanten eine entscheidende Rationalisierungsmaßnahme gelungen. Die Bearbeitungszeit pro Produkt ( LE = Leistungseinheiten) konnten gesenkt werden:

$\rightarrow$ Produkt $1=7 \mathrm{LE}$

$\rightarrow$ Produkt $2=8$ Le

Ihre Anlagen wurden in der letzten Periode auf den neuen Stand umgerüstet, so daf bei gleichem Maschinenbestand ab sofort mehr Produkte gefertigt werden können.

Berücksichtigen Sie diese Informationen bei Ihren Entscheidungen für die nächste Periode! 


\section{Testphase: 2. Entscheidung}

\begin{tabular}{l|l|l|l|l|l|}
\hline \multirow{2}{*}{\begin{tabular}{l} 
Name Ihrer Unternehmung: \\
\cline { 2 - 5 } \\
\cline { 2 - 5 } $\begin{array}{l}\text { Kennworte der } \\
\text { Gruppenmitglieder }\end{array}$
\end{tabular}} & & & & \\
\cline { 2 - 5 } & & & & \\
\cline { 2 - 5 }
\end{tabular}

\section{Newsletter für die Perioden 10 und 11:}

Treffen Sie heute auf der Basis Ihres aktuellen Unternehmensberichts die Entscheidungen für die Periode 10. Zusätzlich entscheiden Sie - ohne vorherige Rückmeldung - für die nachfolgende Periode 11.

Berücksichtigen Sie die folgenden Informationen bei Ihren Entscheidungen für die nächsten Perioden:

1. Die Nachfrage hat bei Produkt 2 den Höhepunkt erreicht. In den nächsten Perioden ist mit einem Nachfragerückgang zu rechnen. Produkt 1 zeigt eine weiterhin rückläufige Nachfrage.

2. Die Lohnkosten steigen in der nächsten Periode um ca. $10 \%$, die Einstellungs- und Enlassungskosten steigen auf 5.000,- DM.

3. Die Lagerhaltungskosten für Rohstoffe steigen ebenfalls in der nächsten Periode.

- Die Preise für beide Rohstoffe werden in Periode 11 leicht sinken.

- Die Einkaufspreise für die Produkte (Handelsware) geben weiter nach. 


\begin{tabular}{|c|c|c|c|c|}
\hline \multicolumn{5}{|c|}{ Entscheidungen für Periode 10} \\
\hline Produkt 1 & Markt 1 & Markt 2 & \multicolumn{2}{|c|}{ Produktionsmenge } \\
\hline Verkaufspreis: & & & Produkt 1 & \\
\hline Werbung: & & & Produkt 2 & \\
\hline \multicolumn{5}{|l|}{ Kundendienst: } \\
\hline Produkt 2 & Markt 1 & Markt 2 & \multicolumn{2}{|c|}{ Rohstoffbeschaffung } \\
\hline Verkaufspreis: & & & Rohstoff 1 & \\
\hline Werbung: & & & Rohstoff 2 & \\
\hline \multicolumn{5}{|l|}{ Kundendienst: } \\
\hline Marktforschung: & $\mathrm{ja} /$ nein & \multicolumn{3}{|c|}{ Investitionen } \\
\hline & & \multicolumn{2}{|c|}{ Maschinenkauf: } & \\
\hline & & \multicolumn{2}{|c|}{ Maschinenverkauf: } & \\
\hline \multicolumn{2}{|c|}{ Finanzierung } & \multicolumn{3}{|c|}{ Personal } \\
\hline Kreditaufnahme: & & \multicolumn{2}{|c|}{ Einstellungen: } & \\
\hline Kredittilgung: & & \multicolumn{2}{|c|}{ Entlassungen: } & \\
\hline Terminanlage: & & & & \\
\hline \multicolumn{5}{|c|}{ Entscheidungen für Periode 11} \\
\hline Produkt 1 & Markt 1 & Markt 2 & \multicolumn{2}{|c|}{ Produktionsmenge } \\
\hline Verkaufspreis: & & & Produkt 1 & \\
\hline Werbung: & & & Produkt 2 & \\
\hline \multicolumn{5}{|l|}{ Kundendienst: } \\
\hline Produkt 2 & Markt 1 & Markt 2 & \multicolumn{2}{|c|}{ Rohstoffbeschaffung } \\
\hline Verkaufspreis: & & & Rohstoff 1 & \\
\hline Werbung: & & & Rohstoff 2 & \\
\hline \multicolumn{5}{|l|}{ Kundendienst: } \\
\hline Marktforschung: & ja / nein & \multicolumn{3}{|c|}{ Investitionen } \\
\hline & & \multicolumn{2}{|c|}{ Maschinenkauf: } & \\
\hline & & \multicolumn{2}{|c|}{ Maschinenverkauf: } & \\
\hline \multicolumn{2}{|c|}{ Finanzierung } & \multicolumn{3}{|c|}{ Personal } \\
\hline Kreditaufnahme: & & \multicolumn{2}{|c|}{ Einstellungen: } & \\
\hline Kredittilgung: & & \multicolumn{2}{|c|}{ Entlassungen: } & \\
\hline Terminanlage: & & & & \\
\hline
\end{tabular}




\section{A.17 Fragebogen zur Entscheidung}

\section{Erfassung der Entscheidungsvorgänge im Planspiel}

Dieser Fragebogen soll Sie bei Ihrer Entscheidung im Planspiel unterstützen. Zu diesem Zweck notieren Sie bitte alle Überlegungen, die während der Diskussion in Ihrer Gruppe geäußert werden und die mit der zu treffenden Entscheidung zu tun haben. Benutzen Sie jede Darstellungsform, die Sie für angemessen halten (Skizzen, Tabellen, Stichworte etc.). Die folgenden Fragen dienen dabei Ihrer Orientierung.

Wie schätzen Sie die aktuelle Unternehmenssituation ein? Haben sich Ihre Erwartungen bezüglich der Ergebnisse Ihrer letzten Entscheidungen erfüllt? ${ }^{2}$ Welche Ziele setzen Sie sich für die nächste Periode? Welche Maßnahmen können Sie ergreifen, um diese Ziele zu erreichen? Sehen Sie alternative Möglichkeiten? Versuchen Sie auch, den Zeitablauf Ihrer Vorgehensweise sichtbar zu machen.

2 Dieser Satz wurde im Fragebogen bei der ersten Entscheidung weggelassen. 


\section{A.18 Fragebogen zum situativen Erleben}

Dieser Fragebogen soll uns helfen zu verstehen, wie Sie die Unterrichtssituation während des Planspiel-Einsatzes empfinden, damit wir sie verbessern können. Es ist daher wichtig, daß Sie den Fragebogen in jeder Planspiel-Sitzung ausfüllen. Bitte kreuzen Sie bei jeder Frage das für Sie zutreffende Kästchen an. Weiterführende Kritik oder auch Lob können Sie am Ende des Fragebogens äußern.

Vielen Dank für Ihre Mitarbeit!

1. Heute Abend fühle ich mich ............schlecht

2. Zum Planspiel habe ich heute .........keine Lust

3. Den Unterricht finde ich .......... uninteressant

4. In der Planspiel-Situation fühle ich mich unsicher

$\square \square-\square-\square \quad$ sehr gut.

5. Ich denke, ich bin im Planspiel heute unterfordert

$\square-\square-\square-\square-\square$ große Lust.

6. Die Unterrichtsmaterialien finde ich ..... schlecht

$\square-\square-\square-\square$ interessant.

$\square-\square-\square-\square$ sehr sicher.

7. In Gruppen arbeite ich grundsätzlich ...nicht gern

$\square-\square-\square-\square \quad$ iiberfordert.

$\square \square-\square-\square$ sehr gut.

8. Diskutiert haben wir in der Gruppe .... zu wenig

$\square-\square-\square-\square \quad$ sehr gern.

9. Das Klima in der Gruppe ist .......... schlecht

10. Insgesamt lief die Gruppenarbeit heute .schlecht

11. Ich konnte mich heute in die Gruppe ............. nicht einbringen

12. Meine Beiträge während der Diskussion

wurden bei der Entscheidung nicht berïcksichtigt

13. Über unsere Entscheidung bin ich mir ...unsicher

$\square \square-\square-\square-\square \quad$ zu viel.

$\square \square-\square-\square-\square \quad$ sehr gut.

$\square-\square-\square-\square \quad$ sehr gut.

$\square \square-\square-\square \quad$ einbringen.

berïcksichtigt.

sicher.

\section{Hier ist Platz für weitere Aspekte:}




\section{A.19 Ausgangslage der experimentellen Phase}

Periode 3

BESTANDSRECHNUNGEN

Rohstoff 1

Anfangsbestand

Notbeschaffung

Abgang

Zugang (Ende Periode)

Endbestand

Rohstoff 2

Anfangsbestand

Notbeschaffung

Abgang

Zugang (Ende Periode)

Endbestand
Spielergebnisse der letzten Periode Seite 1/14

Anzahl Wert in DM

$15200 \quad 98700,00$

$0 \quad 0,00$

$14928 \quad 96933,79$

$15000 \quad 105000,00$

$15272 \quad 106766,21$

$\begin{array}{rr}19000 & 171000,00 \\ 0 & 0,00 \\ 18660 & 167940,00 \\ 19000 & 190000,00 \\ 19340 & 193060,00\end{array}$

Spielergebnisse der letzten Periode

Seite $2 / 14$

Anzahl Wert in DM

Produkt 1

Anfangsbestand

Verkauf

Produktion

Automatischer Einkauf

Endbestand

1842419824,44

$1552 \quad 353728,30$

$1866 \quad 395351,91$

$34 \quad 12750,00$

$2190 \quad 474198,05$

Produkt 2

Anfangsbestand

Verkauf

Produktion

Automatischer Einkauf

Endbestand

$1809 \quad 478155,56$

$1450 \quad 383264,54$

$1866 \quad 448224,29$

$34 \quad 16150,00$

$2259 \quad 559265,31$

1033463,36

=二= = 
Periode 3

\section{MASCHINEN}

Anfangsbestand

Zugang

Abgang

Endbestand

Verfïgbare LE des Endbestandes

\section{PERSONAL}

Anfangsbestand

Einstellungen

Entlassungen

Endbestand
Spielergebnisse der letzten Periode Seite 3/14

$\begin{array}{rr}\text { Anzahl } & \text { Wert in DM } \\ 28 & 1400000,00 \\ 0 & 0,00 \\ 0 & 0,00 \\ 28 & 1400000,00\end{array}$

33600

Angestellte

56

0

0

56

0

Periode 3

\section{PRODUKTIONSBERICHT}

Geplante Menge

Verbrauchte LE

Realisierte Menge

Notbeschaffung

Grad der Maschinenauslastung

\section{MATERIALKOSTEN IN DM}

Rohstoff 1

Rohstoff 2

Summe

Gesamtkosten
Produkt 1

1900

14928

1866

34
Produkt 2

1900

18660

1866

34

$99,96 \%$

Produkt 2

Wert

60583,62

67176,00

$11196 \quad 100764,00 \quad 7464$

137114,17

127759,62

264873,79 
Periode 3

\section{FERTIGUNGSKOSTEN IN DM}

Instandhaltung Fix

Wartung

Löhne

Hilfsstoffe

Gesamtkosten

Abgegebene LE

Kosten/LE

VERWALTUNGSKOSTEN IN DM

Personalverwaltung

Abschlußkosten

Allg. Verwaltung

Gesamtkosten
Tatsächliche K.

140000,00

41985,00

196000,00

83970,00

461955,00

$====$

33588

13,75

0,00

14842,50

83400,00

98242,50

$====$

Periode 3

Spielergebnisse der letzten Periode

Seite $6 / 14$

HERSTELLUNGSKOSTEN IN DM

Materialkosten

Materialkosten/Stïck

Produkt 1

Produkt 2

137114,17

73,48

127759,62

68,47

Fertigungskosten

205260,00

256575,00

Fertigungskosten/Stiick

110,00

137,50

Lagerkosten

Lagerkosten/Stück

9330,00

9330,00

5,00

5,00

Ant. Verw. Kosten

43647,74

54559,67

Ant. Verw. Kosten/Stïck

23,39

29,24

Herstellungskosten

Herstellungskosten/Stïck

448224,29

240,21 
Periode 3

Spielergebnisse der letzten Periode
Seite $7 / 14$

\section{ABSATZBERICHT}

Markt 1

Absatzmenge

Verkaufspreis in DM

Umsatz in DM

Marktanteil

Überhangsnachfrage

Kundendienstausgaben/Stïck

Wirksame Werbeausgaben in DM

Gesamtumsatz in DM (Markt 1)

Periode 3
Produkt 1

749

620,00

Produkt 2

657

690,00

464380,00

$20,32 \%$

0

60,61

54000,00

453330,00

$19,33 \%$

0

63,29

47000,00

917710,00

$=====$

Seite $8 / 14$

\section{ABSATZBERICHT}

Markt 2

Absatzmenge

Produkt 1

Produkt 2

Verkaufspreis in DM

803

793

Umsatz in DM

600,00

680,00

Marktanteil

Überhangsnachfrage

481800,00

539240,00

$25,38 \%$

$21,99 \%$

Kundendienstausgaben/Stïck

87,72

Wirksame Werbeausgaben in DM

37000,00

81,82

37000,00

Gesamtumsatz in DM (Markt 1)

1021040,00

$====$ 
Periode 3

Spielergebnisse der letzten Periode

Seite $9 / 14$

AUSZAHLUNGEN

Rohstoffe

Hilfsstoffe

Handelsware

Löhne

Sozialaufwand

Zinsen

Kredittilgung

Instandhaltung

Verwaltung

Periode 3

\section{EINZAHLUNGEN}

Umsatzerlöse

Forderungen aus t-1

Anlagenverkauf

Kreditaufnahme

Zinsen Terminanlage

Rückzahlung Terminanlage

Kapitalerhöhung

Summe

$\begin{aligned} 268500,00 & \text { Lageraufwand } \\ 83970,00 & \text { Steuern } \\ 28900,00 & \text { Werbung } \\ 140000,00 & \text { Kundendienst } \\ 56000,00 & \text { Information } \\ 120000,00 & \text { Anlagenkauf } \\ 181985,00 & \text { Ausschïttung } \\ 181985,00 & \text { Terminanlage } \\ 98242,50 & \end{aligned}$

Summe

Spielergebnisse der letzten Periode

$$
\begin{array}{r}
64486,00 \\
331428,03 \\
190000,00 \\
82500,00 \\
10000,00 \\
200000,00 \\
0,00 \\
0,00
\end{array}
$$

1856011,53

$====$

Seite 10/14

$\begin{array}{rlr}1163250,00 & \text { Kassenanfangsbestand } & 34376,00 \\ 276024,00 & \text { - Summe Auszahlungen } & 1856011,53 \\ 0,00 & + \text { Summe Einzahlungen } & 1689274,00 \\ 250000,00 & \text { Kontokorrentkr. neu } & 132361,53 \\ 0,00 & & - \\ 0,00 & \text { Kassenendbestand } & 0,00 \\ 0,00 & & ====\end{array}$

1689274,00

$====$ 
Periode 3

GEWINN- und VERLUSTRECHNUNG IN DM

Umsätze

1938750,00

Bestandsveränderungen Fertigerzeugnisse

135483,36

Gesamtleistung

2074233,36

Aufw. für Roh-, Hilfs- und Betriebsstoffe

348843,79

Wareneinsatz Fertigprodukte

28900,00

Rohertrag

1696489,57

Zins- und sonstige Erträge

0,00

Erträge a.d. Abgang d. Anlageverm.

0,00

Aufwand a.d. Abgang d. Anlageverm.

0,00

Löhne

140000,00

Sozialaufwand

56000,00

Instandhaltung (Anl.)

181985,00

Zinsaufwendungen

120000,00

Steuern v. Ertrag

331428,03

Periode 3

Spielergebnisse der letzten Periode

Seite $12 / 14$

Verwaltung

98242,50

Lagerhaltung

64486,00

Werbung

190000,00

Kundendienst

82500,00

Informationsbeschaffung

10000,00

Jahresiiberschuß/Fehlbetrag

421848,03

Gewinn-/Verlustvortrag

Ausschïttung

Einstellungen in die Rücklagen

$-90420,00$

0,00

165714,02

Bilanzgewinn/Bilanzverlust

165714,02

Kumulierter Gewinn t-1

$-90420,00$

753276,07

Jahresüberschuß/Fehlbetrag vor Steuern

662856,07

Kumulierter Gewinn 
Periode 3

BILANZ IN DM (AKTIVA)

Maschinen und Anlagen

1400000,00

Rohstoffe 299826,21

Fertige Erzeugnisse

1033463,36

Forderungen aus Lief. u. Leistungen

775500,00

Forderungen aus Anlagenverkauf

0,00

Terminanlage

0,00

Kassenbestand

0,00

Verlustvortrag

0,00

Bilanzverlust der Periode

0,00

Bilanzsumme

3508789,57

二=ニ二=

Periode 3

Seite 14/14

BILANZ IN DM (PASSIVA)

Grundkapital

1000000,00

Rücklagen

165714,02

Verbindlichkeiten aus Lief. und Leist.

295000,00

Verbindlichkeiten aus Anlageneinkauf

0,00

Langfristige Kredite

1750000,00

Kurzfristige Kredite

132361,53

Bilanzgewinn

165714,02

Bilanzsumme

3508789,57

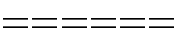




\begin{tabular}{|c|c|c|c|c|c|}
\hline \multirow[t]{2}{*}{ Periode 3} & \multicolumn{5}{|c|}{ Marktforschung } \\
\hline & $\mathrm{U} 1$ & $\mathrm{U} 2$ & $\mathrm{U} 3$ & U 4 & $\mathrm{U} 5$ \\
\hline \multicolumn{6}{|l|}{ Markt 1} \\
\hline Absatz Produkt 1 & 749 & 567 & 670 & 803 & 897 \\
\hline Verkaufspreis & $620,-$ & $650,-$ & $615,-$ & $580,-$ & $550,-$ \\
\hline Absatz Produkt 2 & 657 & 577 & 644 & 709 & 811 \\
\hline Verkaufspreis & $690,-$ & $720,-$ & $690,-$ & $670,-$ & $650,-$ \\
\hline \multicolumn{6}{|l|}{ Markt 2} \\
\hline Absatz Produkt 1 & 803 & 451 & 538 & 656 & 716 \\
\hline Verkaufspreise & $600,-$ & $640,-$ & $610,-$ & $560,-$ & $540,-$ \\
\hline Absatz Produkt 2 & 793 & 489 & 638 & 780 & 906 \\
\hline Verkaufspreise & $680,-$ & $720,-$ & $680,-$ & $650,-$ & $640,-$ \\
\hline Gesamtumsatz & 1938750 & 1424710 & 1618430 & 1815130 & 1986980 \\
\hline Werbeaufwendungen & 190000 & 165000 & 180000 & 195000 & 212500 \\
\hline Kundendienst & 82500 & 60000 & 73000 & 85500 & 101500 \\
\hline J. Übersch./Jf. & 421848,03 & 275339,76 & 328987,43 & 367927,50 & 393341,56 \\
\hline Kumul. Gewinn & 662856,07 & 501179,51 & 518366,86 & 505214,99 & 434949,11 \\
\hline
\end{tabular}


Periode 3

Parameter

Seite $1 / 4$

MÄRKTE

Normalbeschaffungspreis Rohstoff 1 (DM/Stück) 7

Normalbeschaffungspreis Rohstoff 2 (DM/Stuick)

Notbeschaffungspreis Rohstoff 1 (DM/Stuick)

Notbeschaffungspreis Rohstoff 2 (DM/Stuick)

Normalbeschaffungspreis Produkt 1 (DM/Stïck)

375

Normalbeschaffungspreis Produkt 2 (DM/Stuick)

Aufwand für Informationsbeschaffung (DM)

Absatzinfo Absatzmarkt 1

Marktpotential Produkt 1

4200

Marktpotential Produkt 2

Absatzinfo Absatzmarkt 2

Marktpotential Produkt 1

4800

Marktpotential Produkt 2

4300

Periode 3

Parameter

Seite $2 / 4$

\section{SONSTIGE}

Lageraufwand Rohstoffe (DM/Stïck)

Lageraufwand Produkte (DM/Stïck)

Fixe Lagerkosten (DM)

Variable Verwaltungskosten (DM/Angestellter)

Fixe Verwaltungskosten (DM)

75000

Prozentuale Ausschïttung vom Gewinn

$50 \%$

Prozentuale Steuern vom Ertrag

$50 \%$

Aschlußkosten in \% der Bilanzsumme der Vorperiode

$0,5 \%$

Anteil Forderungen von den Umsatzerlösen

Kapitalerhöhung (DM)

$40 \%$

\section{PERSONAL}

Lohn/Angestelltem (DM) 
Periode 3

Parameter

\section{MASCHINEN}

Verfügbare Leistungsein heiten/Aggregat

Personalbedarf/installiertem Aggregat

Instandhaltungskosten/Aggregat in einer Periode (DM)

Wartungskosten für jede angefallene LE (DM)

Kosten für Hilfsstoffe/angefallener LE (DM)

Einkaufspreis für ein Aggregat (DM)

Verkaufspreis für ein Aggregat (DM)

\section{FINANZEN}

Zinssatz Normalkredit

Habenzinssatz Anlage

Zinssatz Kontokorrentkredit

Kredithöchstbetrag (DM)

Periode 3

Parameter

STÜCKLISTE

Produkt 1

Menge Rohstoff 1/Stuick 3

Menge Rohstoff 2/Stïck

Notwendige Leistungseinheiten (LE)/Stück

\section{Produkt 2}

Menge Rohstoff $1 /$ Stïck

Menge Rohstoff 2/Stïck

4

Notwendige Leistungseinheiten (LE)/Stück
Seite $3 / 4$

1200

2

5000

1,25

2,50

50000

40000

\section{$8 \%$ \\ $5 \%$ \\ $12 \%$}

4000000

Seite $4 / 4$ 


\section{A.20 Ausgangslage der Testphase}

Periode 8

BESTANDSRECHNUNGEN

Rohstoff 1

Anfangsbestand

Notbeschaffung

Abgang

Zugang (Ende Periode)

Endbestand

Rohstoff 2

Anfangsbestand

Notbeschaffung

Abgang

Zugang (Ende Periode)

Endbestand
Spielergebnisse der letzten Periode Seite 1/14

Anzahl Wert in DM

$17075 \quad 136600,00$

$0 \quad 0,00$

$17075 \quad 136600,00$

$17075 \quad 136600,00$

$17075 \quad 136600,00$

$\begin{array}{rr}21310 & 277030,00 \\ 0 & 0,00 \\ 21310 & 277030,00 \\ 21310 & 277030,00 \\ 21310 & 277030,00\end{array}$

Spielergebnisse der letzten Periode Seite 2/14

Anzahl Wert in DM

Produkt 1

Anfangsbestand

Verkauf

Produktion

Automatischer Einkauf

Endbestand

$2100 \quad 681266,86$

$1727 \quad 560260,89$

$2125 \quad 536036,99$

$0 \quad 0,00$

$2498 \quad 657042,96$

Produkt 2

Anfangsbestand

Verkauf

Produktion

Automatischer Einkauf

Endbestand

$2160 \quad 705274,53$

$2160 \quad 705274,53$

$2140 \quad 595595,97$

$0 \quad 0,00$

$2140 \quad 595595,97$

Gesamtwert des Endbestands
1252638,93

二=ニ二= 
Periode 8

Spielergebnisse der letzten Periode

\section{MASCHINEN}

Anfangsbestand

Zugang

Abgang

Endbestand

Verfügbare LE des Endbestandes

\section{PERSONAL}

Anfangsbestand

Einstellungen

Entlassungen

Endbestand

Seite $3 / 14$

$\begin{array}{rr}\text { Anzahl } & \text { Wert in DM } \\ 32 & 1600000,00 \\ 0 & 0,00 \\ 0 & 0,00 \\ 32 & 1600000,00\end{array}$

38400

Angestellte

64

0

0

64

Aushilfskräfte in der letzten Periode 0
Seite 4/14

Produkt 2

2140

21400

2140

17000

2125

0

Grad der Maschinenauslastung

MATERIALKOSTEN IN DM

Rohstoff 1

Rohstoff 2

Produkt 1

Anzahl Wert Anzahl

10700

8560

$12750 \quad 165750,00$

216750,00

Summe

Gesamtkosten
Produkt 2

Wert

85600,00

111280,00

196880,00

413630,00 
Periode 8

\section{FERTIGUNGSKOSTEN IN DM}

Instandhaltung Fix

Wartung

Löhne

Hilfsstoffe

Gesamtkosten

Abgegebene LE

Kosten/LE

VERWALTUNGSKOSTEN IN DM

Personalverwaltung

Abschlußkosten

Allg. Verwaltung

Gesamtkosten

Periode 8

Spielergebnisse der letzten Periode

\section{HERSTELLUNGSKOSTEN IN DM}

Materialkosten

Materialkosten/Stïck

Fertigungskosten

Fertigungskosten/Stiick

Lagerkosten

Lagerkosten/Stück

Ant. Verw. Kosten

Ant. Verw. Kosten/Stück

Herstellungskosten

Herstellungskosten/Stïck

\begin{tabular}{rr} 
Tatsächliche K. & Anteilige K. \\
160000,00 & 160000,00 \\
57600,00 & 57600,00 \\
246400,00 & 246400,00 \\
96000,00 & 96000,00 \\
\hline 560000,00 & 560000,00 \\
$=====$ & $=====$ \\
38400 & 38400 \\
14,58 & 14,58
\end{tabular}

0,00

21212,96

111200,00

132412,96

$====$

Seite 6/14

Produkt 1

Produkt 2

216750,00

196880,00

$102,00 \quad 92,00$

247916,67

116,67

312083,33

145,83

12750,00

12840,00

6,00

6,00

58620,32

73792,64

27,59

34,48

536036,99

252,25

595595,97

278,32

=三二= 
Periode 8

Spielergebnisse der letzten Periode
Seite $7 / 14$

\section{ABSATZBERICHT}

Markt 1

Absatzmenge

Produkt 1

Produkt 2

Verkaufspreis in DM

1032

550,00

1166

Umsatz in DM

567600,00

$20,48 \%$

Marktanteil

Überhangsnachfrage

0

Kundendienstausgaben/Stïck

42,62

Wirksame Werbeausgaben in DM

60000,00

640,00

746240,00

$19,08 \%$

0

28,17

80000,00

Gesamtumsatz in DM (Markt 1)

1313840,00

$=====$

Periode 8

Spielergebnisse der letzten Periode

Seite $8 / 14$

\section{ABSATZBERICHT}

Markt 2

Absatzmenge

Produkt 1

Produkt 2

Verkaufspreis in DM

695

994

Umsatz in DM

540,00

650,00

Marktanteil

375300,00

$20,08 \%$

Überhangsnachfrage

0

Kundendienstausgaben/Stück

45,92

Wirksame Werbeausgaben in DM

50000,00

646100,00

$18,89 \%$

0

30,40

70000,00

Gesamtumsatz in DM (Markt 1)

1021400,00 
Periode 8

Spielergebnisse der letzten Periode

Seite $9 / 14$

AUSZAHLUNGEN

Rohstoffe

Hilfsstoffe

Handelsware

Löhne

Sozialaufwand

Zinsen

Kredittilgung

Instandhaltung

Verwaltung

Periode 8

\section{EINZAHLUNGEN}

Umsatzerlöse

Forderungen aus t-1

Anlagenverkauf

Kreditaufnahme

Zinsen Terminanlage

Rückzahlung Terminanlage

Kapitalerhöhung

Summe

$\begin{aligned} 413630,00 & \text { Lageraufwand } \\ 96000,00 & \text { Steuern } \\ 0,00 & \text { Werbung } \\ 176000,00 & \text { Kundendienst } \\ 70400,00 & \text { Information } \\ 163768,17 & \text { Anlagenkauf } \\ 126658,07 & \text { Ausschüttung } \\ 217600,00 & \text { Terminanlage } \\ 132412,96 & \end{aligned}$

Summe

Spielergebnisse der letzten Periode

$$
\begin{array}{r}
90079,00 \\
120723,70 \\
260000,00 \\
33000,00 \\
10000,00 \\
0,00 \\
82769,50 \\
0,00
\end{array}
$$

2290041,40

$====$

Seite 10/14

$\begin{array}{rlr}1401144,00 & \text { Kassenanfangsbestand } & 0,00 \\ 842420,00 & \text { - Summe Auszahlungen } & 2290041,40 \\ 0,00 & \text { + Summe Einzahlungen } & 2243564,00 \\ 0,00 & \text { Kontokorrentkr. neu } & 46477,40 \\ 0,00 & & - \\ 0,00 & \text { Kassenendbestand } & 0,00 \\ 0,00 & & ====\end{array}$

2243564,00

$====$ 
Periode 8

GEWINN- und VERLUSTRECHNUNG IN DM

Umsätze

2335240,00

Bestandsveränderungen Fertigerzeugnisse

$-133902,47$

Gesamtleistung

Aufw. für Roh-, Hilfs- und Betriebsstoffe

2201337,53

Wareneinsatz Fertigprodukte

509630,00

0,00

Rohertrag

1691707,53

Zins- und sonstige Erträge

Erträge a.d. Abgang d. Anlageverm.

0,00

0,00

Aufwand a.d. Abgang d. Anlageverm.

0,00

Löhne

176000,00

Sozialaufwand

70400,00

Instandhaltung (Anl.)

217600,00

Zinsaufwendungen

163768,17

Steuern v. Ertrag

120723,70

Periode 8

Spielergebnisse der letzten Periode

Seite $12 / 14$

Verwaltung

132412,96

Lagerhaltung

90079,00

Werbung

260000,00

Kundendienst

33000,00

Informationsbeschaffung

10000,00

Jahresiiberschuß/Fehlbetrag

120723,70

$====$

Gewinn-/Verlustvortrag

82769,50

Ausschïttung

82769,50

Einstellungen in die Rücklagen

60361,85

Bilanzgewinn/Bilanzverlust

60361,85

Kumulierter Gewinn t-1

3049675,30

Jahresüberschuß/Fehlbetrag vor Steuern

241447,41

Kumulierter Gewinn

3291122,71 
Periode 8

BILANZ IN DM (AKTIVA)

Maschinen und Anlagen

1600000,00

Rohstoffe

413630,01

Fertige Erzeugnisse

1252638,93

Forderungen aus Lief. u. Leistungen

934096,00

Forderungen aus Anlagenverkauf

0,00

Terminanlage

0,00

Kassenbestand

0,00

Verlustvortrag

0,00

Bilanzverlust der Periode

0,00

Bilanzsumme

4200364,93

$====$

Periode 8

Seite 14/14

BILANZ IN DM (PASSIVA)

Grundkapital

1000000,00

Ruicklagen

822780,68

Verbindlichkeiten aus Lief. und Leist.

413630,00

Verbindlichkeiten aus Anlageneinkauf

0,00

Langfristige Kredite

1857115,00

Kurzfristige Kredite

46477,40

Bilanzgewinn

60361,85

Bilanzsumme

4200364,93

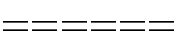




\begin{tabular}{|c|c|c|c|c|c|}
\hline \multirow[t]{2}{*}{ Periode 8} & \multicolumn{5}{|c|}{ Marktforschung } \\
\hline & $\mathrm{U} 1$ & $\mathrm{U} 2$ & U 3 & U 4 & $\mathrm{U} 5$ \\
\hline \multicolumn{6}{|l|}{ Markt 1} \\
\hline Absatz Produkt 1 & 1032 & 687 & 897 & 1110 & 1313 \\
\hline Verkaufspreis & $550,-$ & $610,-$ & $560,-$ & $530,-$ & $510,-$ \\
\hline Absatz Produkt 2 & 1166 & 866 & 1101 & 1362 & 1617 \\
\hline Verkaufspreis & $640,-$ & $690,-$ & $660,-$ & $630,-$ & $610,-$ \\
\hline \multicolumn{6}{|l|}{ Markt 2} \\
\hline Absatz Produkt 1 & 695 & 423 & 600 & 783 & 960 \\
\hline Verkaufspreise & $540,-$ & $610,-$ & $560,-$ & $530,-$ & $510,-$ \\
\hline Absatz Produkt 2 & 994 & 618 & 957 & 1173 & 1519 \\
\hline Verkaufspreise & $650,-$ & $700,-$ & $660,-$ & $640,-$ & $610,-$ \\
\hline Gesamtumsatz & 2335240 & 1707240 & 2196600 & 2612070 & 3072190 \\
\hline Werbeaufwendungen & 260000 & 195000 & 240000 & 280000 & 315000 \\
\hline Kundendienst & 330000 & 215000 & 320000 & 440000 & 565000 \\
\hline J. Übersch./Jf. & 120723,70 & 177629,16 & 178112,56 & 146987,16 & 123521,31 \\
\hline Kumul. Gewinn & 3291123 & 3003394 & 3258051 & 3287717 & 3287013 \\
\hline
\end{tabular}


Periode 8

Parameter

Seite $1 / 4$

MÄRKTE

Normalbeschaffungspreis Rohstoff 1 (DM/Stück) 8

Normalbeschaffungspreis Rohstoff 2 (DM/Stuick)

Notbeschaffungspreis Rohstoff 1 (DM/Stück)

Notbeschaffungspreis Rohstoff 2 (DM/Stuick)

Normalbeschaffungspreis Produkt 1 (DM/Stïck)

350

Normalbeschaffungspreis Produkt 2 (DM/Stuick)

Aufwand für Informationsbeschaffung (DM)

Absatzinfo Absatzmarkt 1

Marktpotential Produkt 1

4500

Marktpotential Produkt 2

Absatzinfo Absatzmarkt 2

Marktpotential Produkt 1

4300

Marktpotential Produkt 2

6500

Periode 8

Parameter

Seite $2 / 4$

\section{SONSTIGE}

Lageraufwand Rohstoffe (DM/Stück)

Lageraufwand Produkte (DM/Stuick)

Fixe Lagerkosten (DM)

Variable Verwaltungskosten (DM/Angestellter)

25000

Fixe Verwaltungskosten (DM)

Prozentuale Ausschïttung vom Gewinn

100000

Prozentuale Steuern vom Ertrag

$50 \%$

$50 \%$

Aschlußkosten in \% der Bilanzsumme der Vorperiode

$0,5 \%$

Anteil Forderungen von den Umsatzerlösen

Kapitalerhöhung (DM)

\section{PERSONAL}

Lohn/Angestelltem (DM)

2750

Sozialkostensatz

$40 \%$

Lohn/Aushilfskraft (DM)

5500

Einstellungs- und Entlassungsaufwand/Angestelltem (DM)

4000 
Periode 8

Parameter

Seite $3 / 4$

\section{MASCHINEN}

Verfügbare Leistungsein heiten/Aggregat 1200

Personalbedarf/installiertem Aggregat

Instandhaltungskosten/Aggregat in einer Periode (DM)

Wartungskosten für jede angefallene LE (DM)

Kosten für Hilfsstoffe/angefallener LE (DM)

Einkaufspreis für ein Aggregat (DM)

Verkaufspreis für ein Aggregat (DM)

\section{FINANZEN}

Zinssatz Normalkredit

Habenzinssatz Anlage

Zinssatz Kontokorrentkredit

Kredithöchstbetrag (DM)

Periode 8

Parameter

STÜCKLISTE

\section{Produkt 1}

Menge Rohstoff 1/Stïck 3

Menge Rohstoff 2/Stïck

Notwendige Leistungseinheiten (LE)/Stück

\section{Produkt 2}

Menge Rohstoff 1/Stück

Menge Rohstoff 2/Stück

4

Notwendige Leistungseinheiten (LE)/Stück 


\section{A.21 Finanzplanung in EpUS}

\section{Berechnung der Auszahlungen der Periode $\mathbf{k}$}

\begin{tabular}{|c|c|}
\hline Rohstoffe & $\begin{array}{l}=\text { Verbindlichkeiten aus Lief. und Leist. (k-1) } \\
+ \text { Notbeschaffung }(\mathrm{k})\end{array}$ \\
\hline Hilfsstoffe & $=$ Kosten je angefallener LE $(\mathrm{k}) \times$ angefallene LE $(\mathrm{k})$ \\
\hline Handelsware & $=$ automatischer Einkauf $(\mathrm{k})$ \\
\hline Löhne & $\begin{array}{l}=\text { Lohn/Angestellte }(\mathrm{k}) \times(\text { Personal }(\mathrm{k}-1)+\text { Zugang }) \\
+ \text { Lohn/Aushilfskraft }(\mathrm{k})\end{array}$ \\
\hline Sozialaufwand & $=$ Löhne $(\mathrm{k}) \times$ Sozialkostensatz $(\mathrm{k})$ \\
\hline Zinsen & $\begin{array}{l}=\text { langfristiger Kredit }(\mathrm{k}-1) \times \operatorname{Zinssatz}(\mathrm{k}) \\
+ \text { Kontokorrent }(\mathrm{k}-1) \times \operatorname{Zinssatz}(\mathrm{k})\end{array}$ \\
\hline Kredittilgung & $=$ Kreditrückzahlung $(\mathrm{k})+$ Kontokorrent $(\mathrm{k}-1)$ \\
\hline Instandhaltung & $\begin{array}{l}=\text { Endbestand Maschinen }(\mathrm{k}) \times \text { Instandhaltung } / \text { Masch. } \\
+ \text { Wartungskosten/angefallene LE }(\mathrm{k}) \times \text { angef. LE }(\mathrm{k})\end{array}$ \\
\hline Verwaltung & $\begin{array}{l}=\text { fixe Verwaltungskosten }(\mathrm{k}) \\
+ \text { Verw.kosten/Angestellte }(\mathrm{k}) \times \text { Angestellte }(\mathrm{k}) \\
+ \text { Abschlusskosten }(\mathrm{k}) \times \text { Bilanzsumme }(\mathrm{k}-1)\end{array}$ \\
\hline Lageraufwand & $\begin{array}{l}=\text { Fixe Lagerkosten }(\mathrm{k}) \\
+ \text { Lageraufwand } R_{1}(\mathrm{k}) \times \text { durchschn. Bestand } R_{1}(\mathrm{k}) \\
+ \text { Lageraufwand } R_{2}(\mathrm{k}) \times \text { durchschn. Bestand } R_{2}(\mathrm{k}) \\
+ \text { Lageraufwand } P_{1}(\mathrm{k}) \times \text { durchschn. Bestand } P_{1}(\mathrm{k}) \\
+ \text { Lageraufwand } P_{2}(\mathrm{k}) \times \text { durchschn. Bestand } P_{1}(\mathrm{k}) \\
\text { mit durchschn. Bestand }=(\text { Anfangs- }+ \text { Endbestand }) / 2\end{array}$ \\
\hline Steuern & $=$ Steuersatz $\times$ Jahresüberschuss $(\mathrm{k})$ \\
\hline Werbung & $=$ Ausgaben für Werbung $(\mathrm{k})$ \\
\hline Kundendienst & $=$ Ausgaben für Kundendienst $(\mathrm{k})$ \\
\hline Information & $=10.000$ (bei Entscheidung für Marktforschung) \\
\hline Anlagenkauf & $=$ Verbindlichkeiten aus Anlageneinkauf (k-1) \\
\hline Ausschüttungen & $=$ Bilanzgewinn $(\mathrm{k}-1)$ \\
\hline Terminanlage & $=$ Rückzahlung der Terminanlage $(\mathrm{k})$ \\
\hline Summe & $=$ Summe der Auszahlungen \\
\hline
\end{tabular}




\section{Finanzplanung in EpUS}

\section{Berechnung der Einzahlungen der Periode $\mathbf{k}$}

\begin{tabular}{|c|l|}
\hline \multirow{2}{*}{ Umsatzerlöse } & $\begin{array}{l}=(100-\text { Anteil Forderungen }) \times \text { Umsatz }(\mathrm{k}) \\
\text { Umsatz }=\sum_{i, j}\left(a_{i j} \cdot p_{i j}\right) \text { mit } a_{i j}=\text { Absatzmenge } P_{i} M_{j} \\
\end{array}$ \\
\hline Forderungen aus t-1 & $=$ Forderungen aus Lief. und Leistg. $(\mathrm{k}-1)$ \\
\hline Anlagenverkauf & $=$ Forderungen aus Anlagenverkauf $(\mathrm{k}-1)$ \\
\hline Kreditaufnahme & $=$ Kreditaufnahme $(\mathrm{k})$ \\
\hline Zinsen Terminanlage & $=$ Habenzinssatz Anlage $(\mathrm{k}) \times$ Terminanlage $(\mathrm{k}-1)$ \\
\hline Rückz. Terminanlage & $=$ Einzahlung Terminanlage $(\mathrm{k}-1)$ \\
\hline Kapitalerhöhung & (entfällt $)$ \\
\hline \hline Summe & $=$ Summe der Einzahlungen $(\mathrm{k})$ \\
\hline
\end{tabular}

\section{Berechnung des Kassenbestands der Periode $\mathbf{k}$}

\begin{tabular}{|l|l|}
\hline Kassenanfangsbestand & $=$ Kassenendbestand (k-1) \\
\hline - Summe der Auszahlungen & $=$ Summe der Auszahlungen (k) \\
\hline+ Summe der Einzahlungen & $=$ Summe der Einzahlungen (k) \\
\hline \hline = Kassenendbestand & $=$ Kassenendbestand (k) \\
\hline
\end{tabular}




\section{A.22 Korrelations-Koeffizienten der Fallstudie}

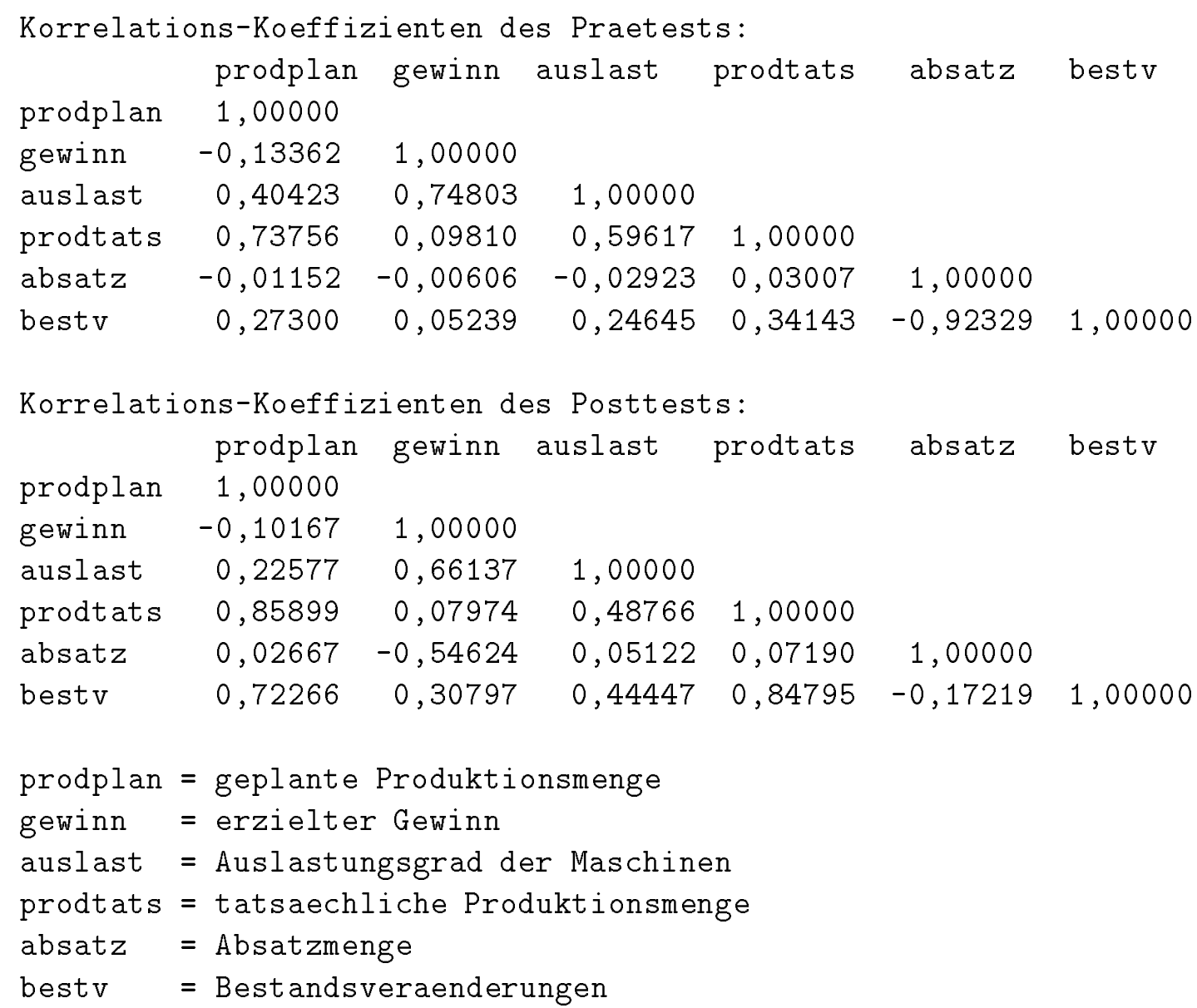




\section{A.23 Auswertungsergebnisse des Prä-Post-Ver- gleichs}

\section{Einfluss der Planspiel-Durchführung}

\begin{tabular}{l|r|r|r|} 
& \multicolumn{2}{|c|}{$\begin{array}{l}\text { Mittelwerte der Bedingung } \\
\text { herkömmlich }\end{array}$} & $\begin{array}{r}\text { Varianz } \\
\text { modular }\end{array}$ \\
gesamt \\
\hline Prätest & 15,7 & 19,9 & 8,08 \\
\hline Posttest & 25,4 & 22,4 & 6,31 \\
\hline
\end{tabular}

Netzwerk-Erhebung: Propositionen

\begin{tabular}{l|r|r|r|} 
& \multicolumn{2}{|c|}{ Mittelwerte der Bedingung } & Varianz \\
& herkömmlich & modular & gesamt \\
\hline Prätest & 13,5 & 17,7 & 6,58 \\
\hline Posttest & 22,5 & 21,3 & 5,76 \\
\hline
\end{tabular}

Netzwerk-Erhebung: Konzepte

\begin{tabular}{l|r|r|r|} 
& \multicolumn{2}{|c|}{ Mittelwerte der Bedingung } & Varianz \\
& herkömmlich & modular & gesamt \\
\hline Prätest & 750 & 166 & 674,4 \\
\hline Posttest & 585 & 658 & 495,5 \\
\hline
\end{tabular}

Fallstudie: Bestandsveränderungen

\begin{tabular}{l|r|r|r|} 
& \multicolumn{2}{|c|}{ Mittelwerte der Bedingung } & Varianz \\
& herkömmlich & modular & gesamt \\
\hline Prätest & 2241 & 1710 & 717,4 \\
\hline Posttest & 2176 & 2148 & 414,0 \\
\hline
\end{tabular}

Fallstudie: Tatsächliche Produktionsmenge

\begin{tabular}{l|r|r|r|} 
& \multicolumn{2}{|c|}{ Mittelwerte der Bedingung } & Varianz \\
& herkömmlich & modular & gesamt \\
\hline Prätest & 91,5 & 78,0 & 21,9 \\
\hline Posttest & 94,5 & 97,5 & 9,4 \\
\hline
\end{tabular}

Fallstudie: Auslastungsgrad der Maschinen 


\begin{tabular}{l|r|r|r|} 
& \multicolumn{2}{|c|}{ Mittelwerte der Bedingung } & Varianz \\
& herkömmlich & modular & gesamt \\
\hline Prätest & 76.650 & 1.864 & 156.110 \\
\hline Posttest & 109.840 & 135.740 & 81.770 \\
\hline
\end{tabular}

Fallstudie: Erzielter Gewinn

\section{Einfluss der zusätzlichen Fragebögen}

\begin{tabular}{|c|c|c|c|}
\hline & $\begin{array}{l}\text { Mittelw } \\
\text { kein Fragebogen } \\
\text { zur Entsch }\end{array}$ & ogen & $\begin{array}{l}\text { Varianz } \\
\text { gesamt }\end{array}$ \\
\hline Prätest & 3,63 & 2,88 & 2,72 \\
\hline Posttest & 2,47 & 3,77 & 2,32 \\
\hline
\end{tabular}

Netzwerk-Erhebung: Propositionen mit 'Gewinn'

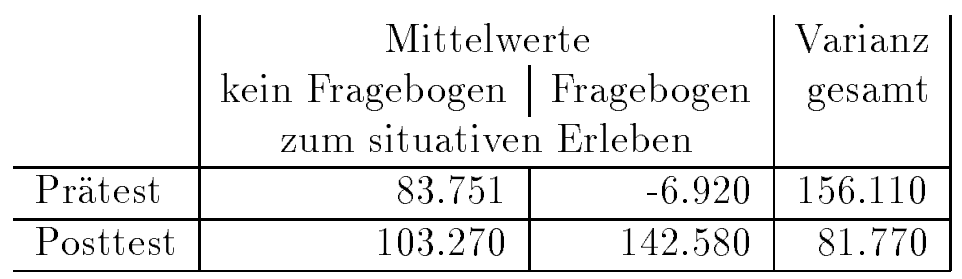

Fallstudie: Erzielte Gewinne

\begin{tabular}{|c|c|c|c|}
\hline & \multicolumn{2}{|c|}{ Mittelwerte } & \multirow{2}{*}{$\begin{array}{l}\text { Varianz } \\
\text { gesamt }\end{array}$} \\
\hline & $\begin{array}{l}\text { kein Fragebogen } \\
\text { zum situative }\end{array}$ & $\begin{array}{l}\text { agebogen } \\
\text { rleben }\end{array}$ & \\
\hline Prätest & $89,59 \%$ & $79,39 \%$ & \\
\hline Posttest & $93,36 \%$ & $98,61 \%$ & \\
\hline
\end{tabular}

Fallstudie: Auslastungsgrad der Maschinen

\begin{tabular}{cl|r|r|} 
& & \multicolumn{2}{|c|}{\begin{tabular}{c} 
Mittelwerte \\
kein Fragebogen \\
zum situativen Eragebogen \\
\multirow{2}{*}{ Prätest }
\end{tabular}} \\
\cline { 2 - 4 } & kein Fragebogen zur Ensch. & 88.377 & -93.181 \\
\cline { 2 - 4 } & Fragebogen zur Entscheidung & 85.875 & 87.183 \\
\hline \multirow{2}{*}{ Posttest } & kein Fragebogen zur Entsch. & 114.530 & 159.180 \\
\cline { 2 - 4 } & Fragebogen zur Entscheidung & 98.450 & 124.480 \\
\hline
\end{tabular}

Fallstudie: Erzielte Gewinne 


\begin{tabular}{|c|c|c|c|}
\hline & \multicolumn{2}{|c|}{ Mittelwerte } \\
\hline & & $\begin{array}{l}\text { kein Fragebogen } \\
\text { zum situativer }\end{array}$ & $\begin{array}{l}\text { Fragebogen } \\
\text { n Erleben }\end{array}$ \\
\hline \multirow{2}{*}{ Prätest } & kein Fragebogen zur Ensch. & $95,6 \%$ & $63,2 \%$ \\
\hline & Fragebogen zur Entscheidung & $86,9 \%$ & $97,1 \%$ \\
\hline \multirow{2}{*}{ Posttest } & kein Fragebogen zur Entsch. & $92,2 \%$ & $99,1 \%$ \\
\hline & Fragebogen zur Entscheidung & $93,9 \%$ & $98,1 \%$ \\
\hline
\end{tabular}

Fallstudie: Auslastungsgrad der Maschinen

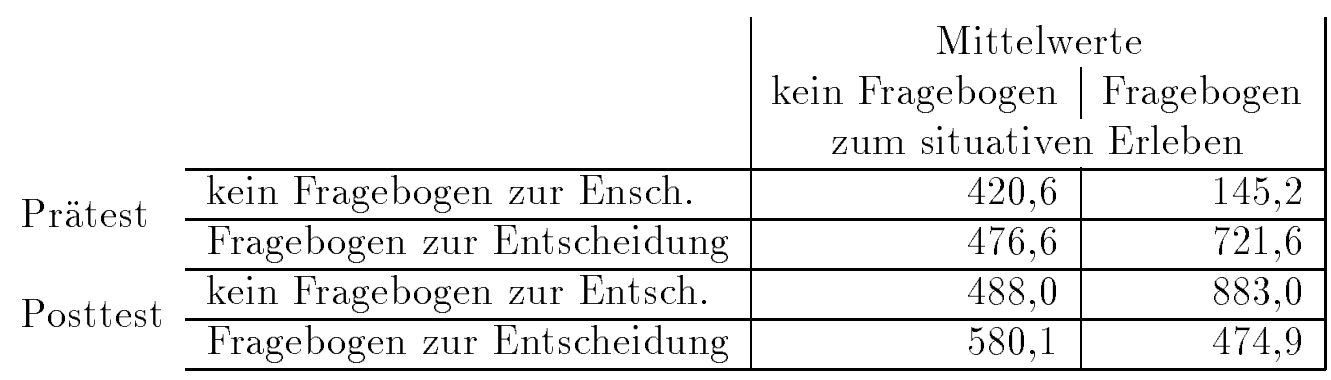

Fallstudie: Bestandsveränderungen

\begin{tabular}{cl|r|r|} 
& & \multicolumn{2}{|c|}{$\begin{array}{c}\text { Mittelwerte } \\
\text { kein Fragebogen } \\
\text { zum situativen Erleben }\end{array}$} \\
\cline { 2 - 4 } Prätest & kein Fragebogen zur Ensch. & 1892,9 & 1715,0 \\
\cline { 2 - 4 } & Fragebogen zur Entscheidung & 1913,4 & 2313,6 \\
\hline \multirow{2}{*}{ Posttest } & kein Fragebogen zur Entsch. & 1883,3 & 2291,7 \\
\cline { 2 - 4 } & Fragebogen zur Entscheidung & 2200,0 & 2118,2 \\
\hline
\end{tabular}

Fallstudie: tatsächliche Produktionsmenge $(F=5.06, p=0.0312, d f=70)$

\section{Wechselwirkung zwischen Planspiel-Durchführung und zusätzlichen Fragebögen}

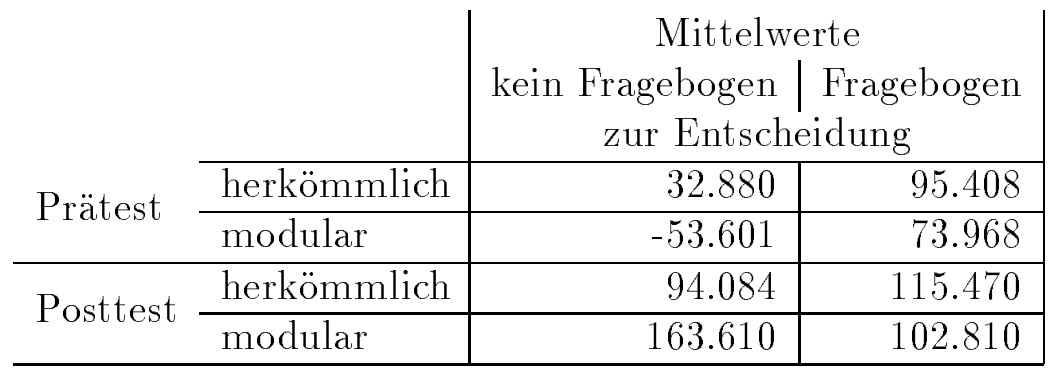

Fallstudie: Erzielte Gewinne 


\begin{tabular}{cl|r|r|} 
& & \multicolumn{2}{|c|}{$\begin{array}{c}\text { Mittelwerte } \\
\text { kein Fragebogen }\end{array}$} \\
& & \multicolumn{1}{|c|}{$\begin{array}{c}\text { Fragebogen } \\
\text { zur Entscheidung }\end{array}$} \\
\cline { 2 - 4 } Prätest & herkömmlich & $85,3 \%$ & $94,1 \%$ \\
\cline { 2 - 4 } & modular & $70,4 \%$ & $88,0 \%$ \\
\hline \multirow{2}{*}{ Posttest } & herkömmlich & $90,6 \%$ & $95,9 \%$ \\
\cline { 2 - 4 } & modular & $99,1 \%$ & $95,5 \%$ \\
\hline
\end{tabular}

Fallstudie: Auslastungsgrad der Maschinen

\begin{tabular}{cl|r|r|} 
& & \multicolumn{2}{|c|}{$\begin{array}{c}\text { Mittelwerte } \\
\text { kein Fragebogen } \\
\text { zum situativen Erleben }\end{array}$} \\
\cline { 2 - 4 } Prätest & Fragebogen \\
\cline { 2 - 4 } & herkömmlich & 2430,6 & 2904,5 \\
\hline \multirow{2}{*}{ Posttest } & hodular & 2363,6 & 1761,5 \\
\cline { 2 - 4 } & modümmlich & 2727,8 & 2272,7 \\
\hline
\end{tabular}

Fallstudie: geplante Produktionsmenge $(F=4.31, p=0.0451, d f=70)$ 


\section{A.24 Faktorenanalyse der Ergebnisse der Test- phase}

\begin{tabular}{|c|c|c|c|c|c|}
\hline - Fakt & & 2. Fakt & tor & 3. Fakt & tor \\
\hline dst 22 & 0.929 & absp1m1 & 10.882 & $\operatorname{prp} 1 \mathrm{~m} 1$ & 0.913 \\
\hline st12 & 0.847 & $\operatorname{map} 1 \mathrm{~m} 1$ & 0.823 & $\operatorname{prp} 2 \mathrm{~m} 2$ & 0.883 \\
\hline st21 & 0.840 & bsp1m2 & 20.818 & prp1m2 & 0.780 \\
\hline dst 11 & 0.764 & $\operatorname{map} 1 \mathrm{~m} 2$ & 0.783 & pdiff 1 & 0.498 \\
\hline $\mathrm{p} 2 \mathrm{~m} 2$ & 0.702 & $\operatorname{diff} 2$ & -0.657 & maus 1 & -0.438 \\
\hline osp $2 \mathrm{~m} 2$ & 0.566 & umsm2 & 0.472 & $\operatorname{map} 2 \mathrm{~m} 2$ & -0.387 \\
\hline $1 \mathrm{sm} 2$ & 0.556 & umsm 1 & 0.418 & prodm 1 & 0.339 \\
\hline $\operatorname{ap} 1 \mathrm{~m} 2$ & 0.375 & dst 11 & 0.362 & kasse & -0.299 \\
\hline $\operatorname{ap} 2 \mathrm{~m} 1$ & 0.338 & termin & 0.322 & $f f 2$ & -0.286 \\
\hline ef 1 & -0.272 & pdiff 1 & -0.312 & $\operatorname{absp} 2 \mathrm{~m} 2$ & 273 \\
\hline & & kdst 12 & 0.281 & & \\
\hline & & hk2 & -0.259 & & \\
\hline & & pbv2 & -0.254 & & \\
\hline VP & 4.792 & VP & 4.333 & VP & 3.60 \\
\hline Fakt & & 5. Fakt & & 6. Fakt & tor \\
\hline $\mathrm{m} 1$ & 0.906 & rohst 1 & 0.781 & \pm 1 & 0.912 \\
\hline $\mathrm{p} 2 \mathrm{~m} 1$ & 0.799 & rohst 2 & 0.693 & rdiff 2 & 0.870 \\
\hline & 0.750 & $h k 2$ & -0.682 & is 1 & -0.433 \\
\hline osp $2 \mathrm{~m} 2$ & 0.545 & kasse & -0.660 & prodm 2 & -0.395 \\
\hline $\operatorname{ap} 2 \mathrm{~m} 2$ & 0.464 & prodm 1 & 0.624 & gewinn & -0.374 \\
\hline ohst 1 & 0.387 & $\mathrm{pbv1}$ & 0.624 & hk2 & -0.354 \\
\hline $\mathrm{msm}$ & 0.361 & prodm 2 & 0.295 & rohst 1 & -0.277 \\
\hline ewinn & 0.310 & $\mathrm{absp} 1 \mathrm{~m} 1$ & 10.280 & & \\
\hline $\mathrm{v} 1$ & -0.281 & & & & \\
\hline ov2 & -0.276 & & & & \\
\hline 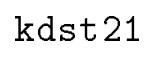 & 0.26 & & & & \\
\hline & .50 & VP & 3.475 & VP & .626 \\
\hline
\end{tabular}




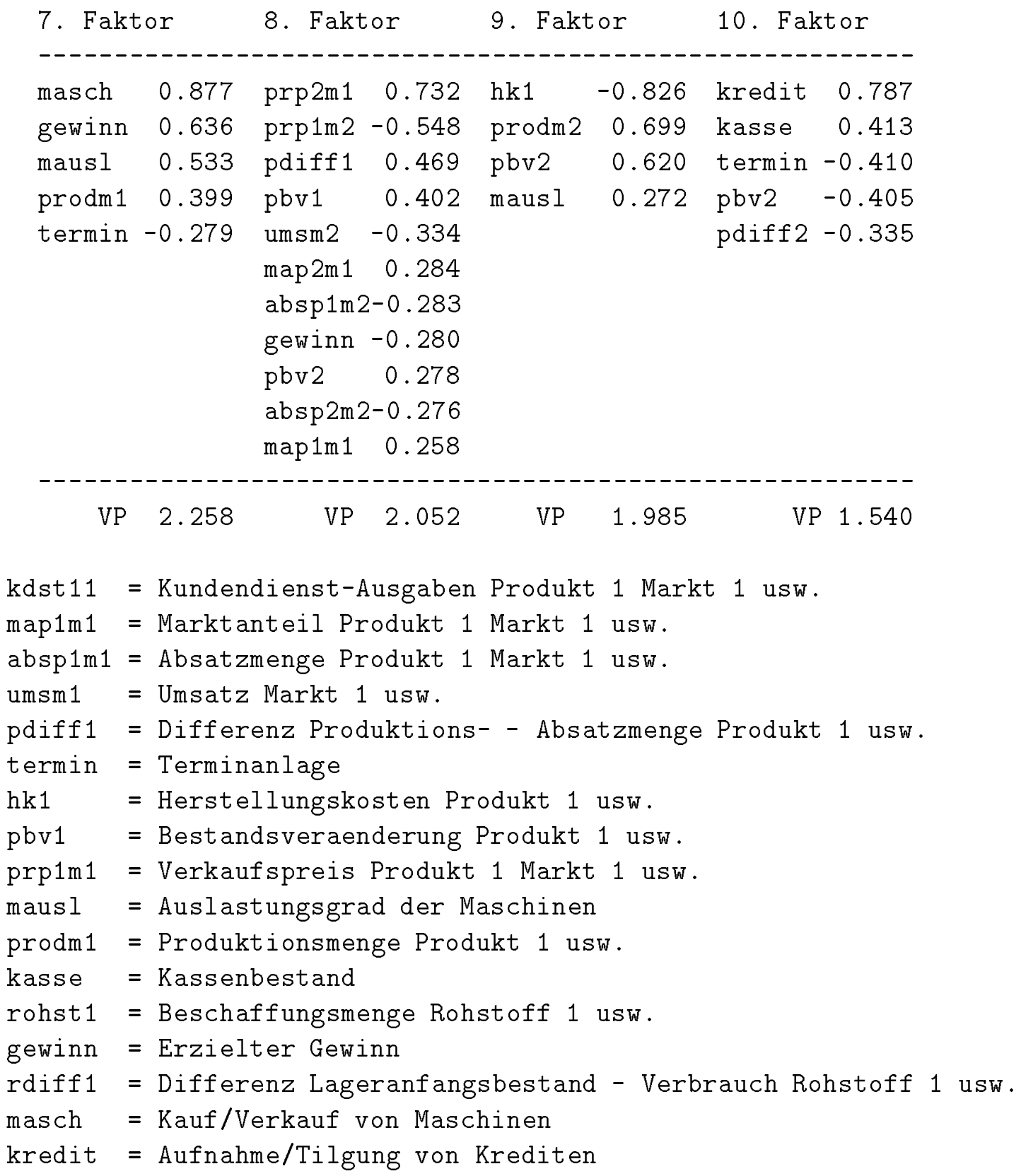




\section{A.25 Auswertungsergebnisse Testphase}

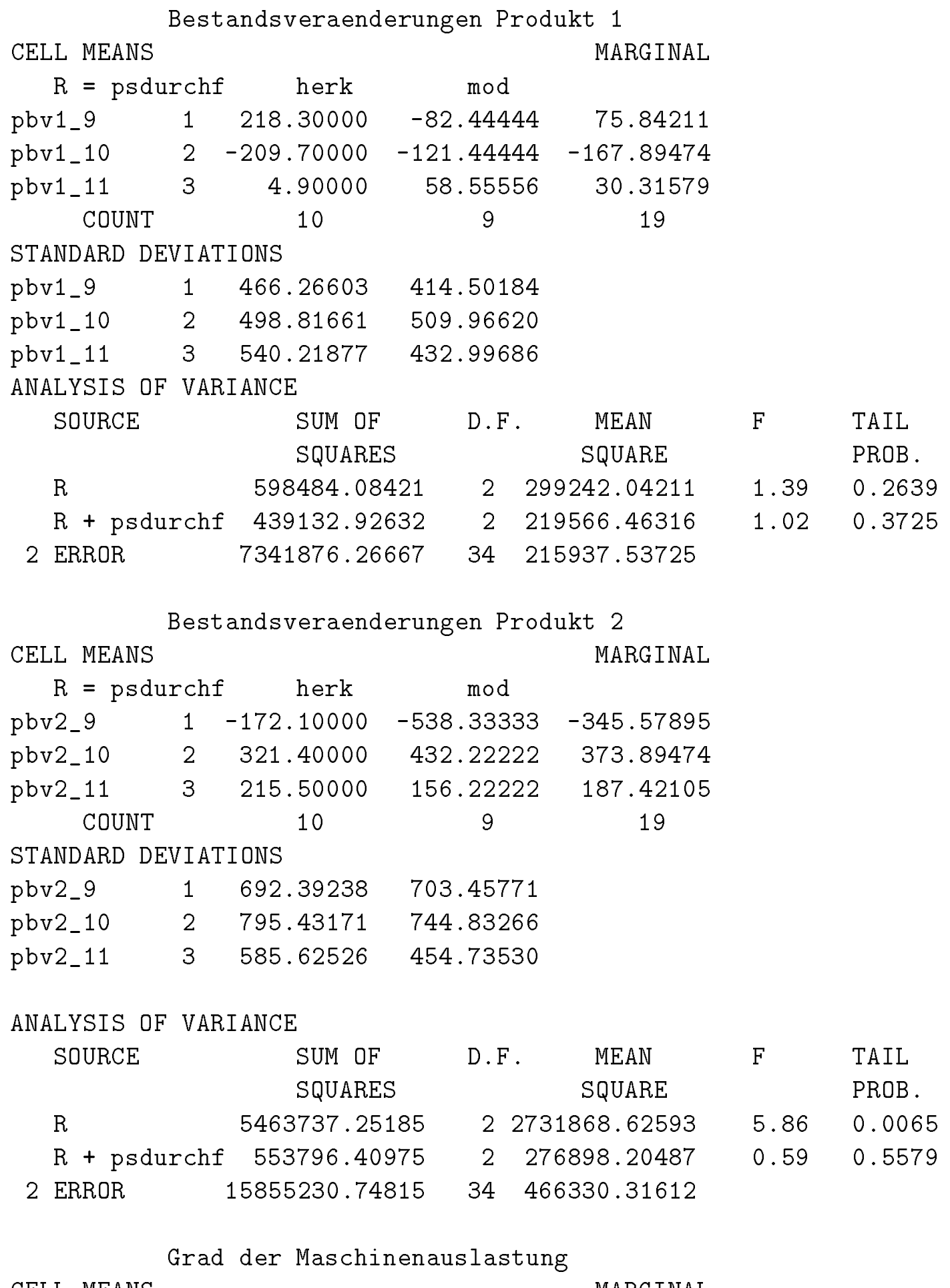




\begin{tabular}{|c|c|c|c|c|}
\hline \multicolumn{2}{|c|}{$R=\operatorname{psdurchf}$} & \multicolumn{2}{|r|}{$\bmod$} & \multirow[b]{2}{*}{76.85005} \\
\hline maus 109 & 1 & 72.38672 & 81.80932 & \\
\hline maus 110 & 2 & 90.57771 & 82.52791 & 86.76465 \\
\hline maus 111 & 3 & 97.59176 & 90.82415 & 94.38605 \\
\hline \multicolumn{2}{|c|}{ COUNT } & 10 & 9 & 19 \\
\hline \multicolumn{5}{|c|}{ STANDARD DEVIATIONS } \\
\hline maus 109 & 1 & 13.49699 & 12.48475 & \\
\hline maus 110 & 2 & 14.41553 & 16.05715 & \\
\hline maus 111 & 3 & 7.78110 & 10.90094 & \\
\hline
\end{tabular}

\begin{tabular}{lcrrrl} 
ANALYSIS OF VARIANCE & \multicolumn{1}{c}{ MEAN } & \multicolumn{1}{l}{ F } & \multicolumn{1}{l}{ TAIL } \\
SOURCE & SUM OF & D.F. & \multicolumn{1}{c}{ MEANORE } \\
& SQUARES & & SQUARE & & PROB \\
R & 2783.64774 & 2 & 1391.82387 & 10.89 & 0.0002 \\
R + psdurchf & 898.50237 & 2 & 449.25118 & 3.52 & 0.0409 \\
2 ERROR & 4343.91908 & 34 & 127.76233 & &
\end{tabular}

Herstellungskosten Produkt 1

CELL MEANS

$$
\mathrm{R}=\operatorname{psdurchf} \text { herk } \bmod
$$

\begin{tabular}{lcccc} 
hk1_9 & 1 & 247.87300 & 243.22111 & 245.66947 \\
hk1_10 & 2 & 257.33200 & 225.68111 & 242.33947 \\
hk1_11 & 3 & 261.56000 & 257.31111 & 259.54737 \\
\multicolumn{1}{c}{ COUNT } & 10 & 9 & 19
\end{tabular}

STANDARD DEVIATIONS

$\begin{array}{llrr}h k 1 \_9 & 1 & 6.82281 & 4.96351 \\ h k 1 \_10 & 2 & 8.44157 & 85.00591 \\ h k 1 \_11 & 3 & 11.95194 & 16.63100\end{array}$

ANALYSIS OF VARIANCE

\begin{tabular}{lcrcrl} 
SOURCE & \multicolumn{1}{c}{ SUM OF } & D.F. & MEAN & $F$ & TAIL \\
& SQUARES & & SQUARE & & PROB. \\
$R$ & 3351.56905 & 2 & 1675.78452 & 1.22 & 0.3091 \\
$R+$ psdurchf & 2336.80736 & 2 & 1168.40368 & 0.85 & 0.4374 \\
2 ERROR & 46878.07383 & 34 & 1378.76688 & &
\end{tabular}

Herstellungskosten Produkt 2

CELL MEANS

MARG INAL

$$
R=\operatorname{psdurchf} \text { herk } \bmod
$$

$\begin{array}{lllll}\text { hk2_9 } & 1 & 258.09600 & 253.06222 & 255.71158 \\ h k 2 \_10 & 2 & 268.87000 & 266.93333 & 267.95263 \\ h k 2 \_11 & 3 & 274.48800 & 268.66667 & 271.73053\end{array}$


$\begin{array}{llll}\text { COUNT } & 10 & 9 & 19\end{array}$

STANDARD DEVIATIONS

$\begin{array}{lrrr}\text { hk2_9 } & 1 & 7.77134 & 6.07862 \\ \text { hk2_10 } & 2 & 9.72634 & 11.56871 \\ \text { hk2_11 } & 3 & 13.00563 & 18.71160\end{array}$

ANALYSIS OF VARIANCE

\begin{tabular}{|c|c|c|c|c|c|}
\hline SOURCE & $\begin{array}{l}\text { SUM OF } \\
\text { SQUARES }\end{array}$ & D.F. & $\begin{array}{c}\text { MEAN } \\
\text { SQUARE }\end{array}$ & F & $\begin{array}{l}\text { TAIL } \\
\text { PROB. }\end{array}$ \\
\hline$R$ & 2660.83556 & 2 & 1330.41778 & 14.95 & 0.0000 \\
\hline$R+$ psdurchf & 39.95216 & 2 & 19.97608 & 0.22 & 0.8001 \\
\hline ERROR & 3026.07877 & 34 & 89.00232 & & \\
\hline
\end{tabular}

Kassenbestand

CELL MEANS

MARGINAL

$$
\mathrm{R}=\text { psdurchf herk } \bmod
$$

$\begin{array}{lllll}\text { kasse9 } & 1 & -5007.45273 & 234397.98177 & 108395.12150\end{array}$

$\begin{array}{llllll}\text { kasse10 } & 2 & 271230.49727 & 246315.07742 & 259428.45629\end{array}$

kasse11 3371136.17500369059 .29774370152 .39104

$\begin{array}{llll}\text { COUNT } & 10 & 9 & 19\end{array}$

STANDARD DEVIATIONS

\begin{tabular}{|c|c|c|c|c|c|}
\hline kasse9 & \multicolumn{5}{|c|}{1266969.94535536447 .20962} \\
\hline kasse10 & \multicolumn{5}{|c|}{2443591.80583489926 .52875} \\
\hline kasse11 & \multicolumn{5}{|c|}{$3668917.28796 \quad 729168.28063$} \\
\hline \multicolumn{6}{|c|}{ ANALYSIS OF VARIANCE } \\
\hline SOURCE & $\begin{array}{l}\text { SUM OF } \\
\text { SQUARES }\end{array}$ & D.F. & $\begin{array}{c}\text { MEAN } \\
\text { SQUARE }\end{array}$ & $\mathrm{F}$ & $\begin{array}{l}\text { TAIL } \\
\text { PROB. }\end{array}$ \\
\hline$R$ & $* * * * * * * * * * * * * * *$ & $2 *$ & $* * * * * * * * * * * * *$ & 2.85 & 0.0716 \\
\hline$R G$ & $* * * * * * * * * * * * * * *$ & $2 *$ & $* * * * * * * * * * * * *$ & 0.93 & 0.4032 \\
\hline 2 ERROR & $* * * * * * * * * * * * * * *$ & $34 *$ & $* * * * * * * * * * * * *$ & & \\
\hline
\end{tabular}

CELL MEANS

Gewinn

$$
R=\text { psdurchf herk mod }
$$

gewinn9 $\quad 111026.04894104928 .2102955506 .02010$

gewinn10 $270149.60312 \quad 51547.8125061338 .22862$

gewinn11 $348620.89296 \quad 46398.53106 \quad 47568.19522$

$\begin{array}{llll}\text { COUNT } & 10 & 9 & 19\end{array}$

STANDARD DEVIATIONS

gewinn9 $\quad 198426.8189268813 .35252$ 


\begin{tabular}{|c|c|c|c|c|c|}
\hline gewinn10 & 278872.4792161 & .073 & 311 & & \\
\hline gewinn 11 & 377563.8930359 & 2.856 & 619 & & \\
\hline \multicolumn{6}{|c|}{ ANALYSIS OF VARIANCE } \\
\hline SOURCE & $\begin{array}{l}\text { SUM OF } \\
\text { SQUARES }\end{array}$ & D.F & $\begin{array}{c}\text { MEAN } \\
\text { SQUARE }\end{array}$ & F & $\begin{array}{l}\text { TAIL } \\
\text { PROB. }\end{array}$ \\
\hline$R$ & 1867841694.60452 & $2 *$ & $<* * * * * * * * * * * * *$ & 0.16 & 0.8565 \\
\hline$R G$ & $* * * * * * * * * * * * * * * *$ & $2 *$ & $* * * * * * * * * * * * *$ & 2.92 & 0.0678 \\
\hline 2 ERROR & $* * * * * * * * * * * * * * * *$ & $34 *$ & $<* * * * * * * * * * * * *$ & & \\
\hline
\end{tabular}




\section{A.26 Auswertungsergebnisse Fragebogen zum situativen Erleben}

CELL MEANS

\begin{tabular}{lcccc} 
& messwdh & herk & mod & MARGINAL \\
sit1_4 & 1 & 2.18750 & 2.93750 & 2.56250 \\
sit2_4 & 2 & 3.18750 & 2.75000 & 2.96875 \\
sit3_4 & 3 & 3.31250 & 3.25000 & 3.28125 \\
sit4_4 & 4 & 3.50000 & 3.12500 & 3.31250 \\
sit5_4 & 5 & 3.50000 & 3.43750 & 3.46875 \\
sit6_4 & 6 & 3.37500 & 3.37500 & 3.37500 \\
sit7_4 & 7 & 3.43750 & 3.43750 & 3.43750 \\
\multicolumn{2}{r}{ MARGINAL } & 3.21429 & 3.18750 & 3.20089 \\
\multicolumn{2}{c}{ COUNT } & 16 & 16 & 32
\end{tabular}

\begin{tabular}{lccc} 
STANDARD & \multicolumn{3}{l}{ DEVIATIONS } \\
& messwdh & herk & mod \\
sit1_4 & 1 & 0.83417 & 1.18145 \\
sit2_4 & 2 & 0.83417 & 1.18322 \\
sit3_4 & 3 & 0.60208 & 0.77460 \\
sit4_4 & 4 & 1.21106 & 0.88506 \\
sit5_4 & 5 & 0.73030 & 0.72744 \\
sit6_4 & 6 & 0.50000 & 0.95743 \\
sit7_4 & 7 & 0.89209 & 0.72744
\end{tabular}

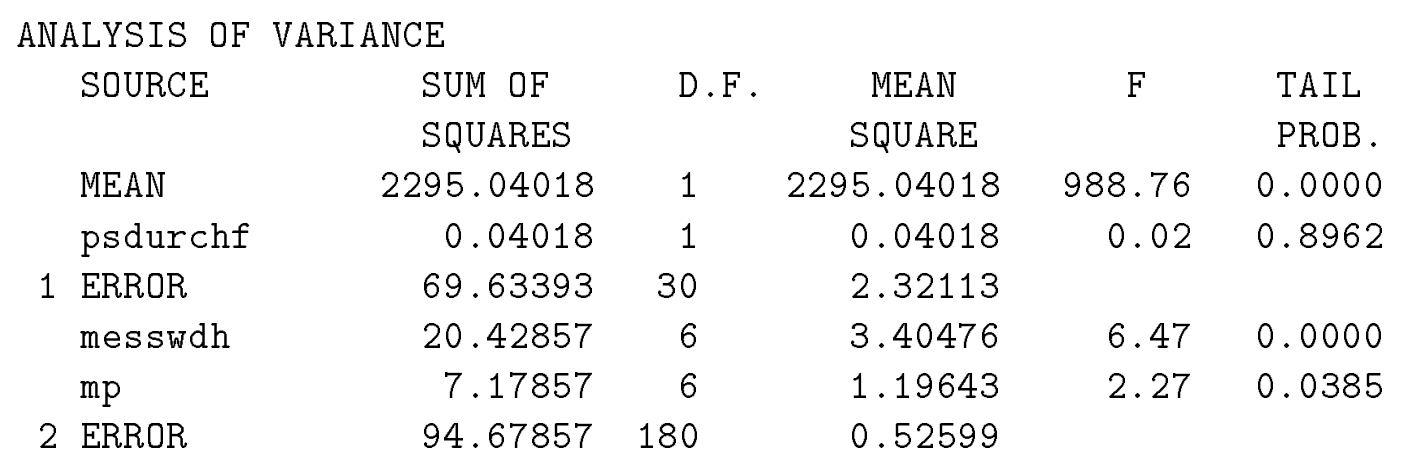


CELL MEANS

\begin{tabular}{|c|c|c|c|c|}
\hline & messwdh & herk & $\bmod$ & MARGINAI \\
\hline sit1_5 & 1 & 3.87500 & 3.06250 & 3.46875 \\
\hline sit2_5 & 2 & 3.12500 & 3.50000 & 3.31250 \\
\hline sit3_5 & 3 & 3.12500 & 2.93750 & 3.03125 \\
\hline sit4_5 & 4 & 3.12500 & 3.18750 & 3.15625 \\
\hline sit5_5 & 5 & 3.12500 & 3.06250 & 3.09375 \\
\hline sit6_5 & 6 & 3.12500 & 3.18750 & 3.15625 \\
\hline sit7_5 & 7 & 3.37500 & 2.93750 & 3.15625 \\
\hline \multicolumn{2}{|c|}{ MARGINAL } & 3.26786 & 3.12500 & 3.19643 \\
\hline \multicolumn{2}{|c|}{ COUNT } & 16 & 16 & 32 \\
\hline
\end{tabular}

STANDARD DEVIATIONS

$\begin{array}{lccc} & \text { messwdh } & \text { herk } & \text { mod } \\ \text { sit1_5 } & 1 & 0.80623 & 0.77190 \\ \text { sit2_5 } & 2 & 0.34157 & 0.89443 \\ \text { sit3_5 } & 3 & 0.34157 & 0.77190 \\ \text { sit4_5 } & 4 & 0.34157 & 0.54391 \\ \text { sit5_5 } & 5 & 0.71880 & 0.44253 \\ \text { sit6_5 } & 6 & 0.61914 & 0.75000 \\ \text { sit7_5 } & 7 & 0.50000 & 0.25000\end{array}$

ANALYSIS OF VARIANCE

\begin{tabular}{lrrrrr} 
SOURCE & \multicolumn{1}{c}{ SUM OF } & D.F. & \multicolumn{1}{c}{ MEAN } & F & \multicolumn{1}{c}{ TAIL } \\
& SQUARES & & SQUARE & & PROB. \\
MEAN & 2288.64286 & 1 & 2288.64286 & 4234.49 & 0.0000 \\
psdurchf & 1.14286 & 1 & 1.14286 & 2.11 & 0.1563 \\
1 ERROR & 16.21429 & 30 & 0.54048 & & \\
messwdh & 4.16964 & 6 & 0.69494 & 2.00 & 0.0684 \\
mp & 7.16964 & 6 & 1.19494 & 3.43 & 0.0031 \\
2 ERROR & 62.66071 & 180 & 0.34812 & &
\end{tabular}


CELL MEANS

\begin{tabular}{|c|c|c|c|c|}
\hline & messwdh & herk & $\bmod$ & MARGINAL \\
\hline sit1_8 & 1 & 3.56250 & 3.37500 & 3.46875 \\
\hline sit2_8 & 2 & 3.62500 & 4.00000 & 3.81250 \\
\hline sit3_8 & 3 & 3.37500 & 3.56250 & 3.46875 \\
\hline sit4_8 & 4 & 3.12500 & 3.31250 & 3.21875 \\
\hline sit5_8 & 5 & 3.25000 & 3.37500 & 3.31250 \\
\hline sit6_8 & 6 & 3.25000 & 3.56250 & 3.40625 \\
\hline sit7_8 & 7 & 3.75000 & 3.12500 & 3.43750 \\
\hline \multicolumn{2}{|c|}{ MARGINAL } & 3.41964 & 3.47321 & 3.44643 \\
\hline \multicolumn{2}{|c|}{ COUNT } & 16 & 16 & 32 \\
\hline
\end{tabular}

\begin{tabular}{lccc}
\multicolumn{4}{l}{ STANDARD } \\
& DEVIATIONS & \\
messwdh & herk & mod \\
sit1_8 & 1 & 0.81394 & 0.88506 \\
sit2_8 & 2 & 0.50000 & 0.73030 \\
sit3_8 & 3 & 0.61914 & 0.62915 \\
sit4_8 & 4 & 0.95743 & 0.47871 \\
sit5_8 & 5 & 0.93095 & 0.61914 \\
sit6_8 & 6 & 0.68313 & 0.51235 \\
sit7_8 & 7 & 0.77460 & 0.61914
\end{tabular}

ANALYSIS OF VARIANCE

\begin{tabular}{lrrrrl} 
SOURCE & \multicolumn{1}{c}{ SUM OF } & D.F. & \multicolumn{1}{c}{ MEAN } & \multicolumn{1}{c}{ F } & \multicolumn{1}{l}{ TAIL } \\
SQUARES & & SQUARE & & PROB. \\
MEAN & 2660.64286 & 1 & 2660.64286 & 3924.39 & 0.0000 \\
psdurchf & 0.16071 & 1 & 0.16071 & 0.24 & 0.6299 \\
1 ERROR & 20.33929 & 30 & 0.67798 & & \\
mesSwdh & 6.60714 & 6 & 1.10119 & 2.29 & 0.0370 \\
mp & 5.83929 & 6 & 0.97321 & 2.03 & 0.0642 \\
2 ERROR & 86.41071 & 180 & 0.48006 & &
\end{tabular}


CELL MEANS

\begin{tabular}{lcccc} 
& messwdh & herk & mod & MARGINAL \\
sit1_13 & 1 & 2.43750 & 3.12500 & 2.78125 \\
sit2_13 & 2 & 3.56250 & 3.31250 & 3.43750 \\
sit3_13 & 3 & 3.37500 & 3.43750 & 3.40625 \\
sit4_13 & 4 & 3.93750 & 3.31250 & 3.62500 \\
sit5_13 & 5 & 3.25000 & 3.43750 & 3.34375 \\
sit6_13 & 6 & 3.62500 & 3.50000 & 3.56250 \\
sit7_13 & 7 & 3.37500 & 3.43750 & 3.40625 \\
\multicolumn{2}{r}{ MARGINAL } & 3.36607 & 3.36607 & 3.36607 \\
\multicolumn{2}{r}{ COUNT } & 16 & 16 & 32
\end{tabular}

STANDARD DEVIATIONS

$\begin{array}{lccc} & \text { messwdh } & \text { herk } & \text { mod } \\ \text { sit1_13 } & 1 & .89209 & 1.25831 \\ \text { sit2_13 } & 2 & 1.03078 & 1.35247 \\ \text { sit3_13 } & 3 & 1.14746 & 0.89209 \\ \text { sit4_13 } & 4 & 0.68007 & 1.07819 \\ \text { sit5_13 } & 5 & 1.12546 & 0.81394 \\ \text { sit6_13 } & 6 & 0.95743 & 0.81650 \\ \text { sit7_13 } & 7 & 0.88506 & 0.89209\end{array}$

ANALYSIS OF VARIANCE

\begin{tabular}{lrrrrl} 
SOURCE & \multicolumn{1}{c}{ SUM OF } & D.F. & \multicolumn{1}{c}{ MEAN } & F & \multicolumn{1}{l}{ TAIL } \\
& \multicolumn{1}{c}{ SQUARES } & & SQUARE & & \multicolumn{1}{l}{ PROB } \\
MEAN & 2538.01786 & 1 & 2538.01786 & 683.42 & 0.0000 \\
psdurchf & 0.00000 & 1 & 0.00000 & 0.00 & 1.0000 \\
1 ERROR & 111.41071 & 30 & 3.71369 & & \\
messwdh & 14.60714 & 6 & 2.43452 & 4.38 & 0.0004 \\
mp & 7.87500 & 6 & 1.31250 & 2.36 & 0.0322 \\
2 ERROR & 100.08929 & 180 & 0.55605 & &
\end{tabular}




\section{A.27 Korrelationsanalyse der Items des Frage- bogens zum situativen Erleben}

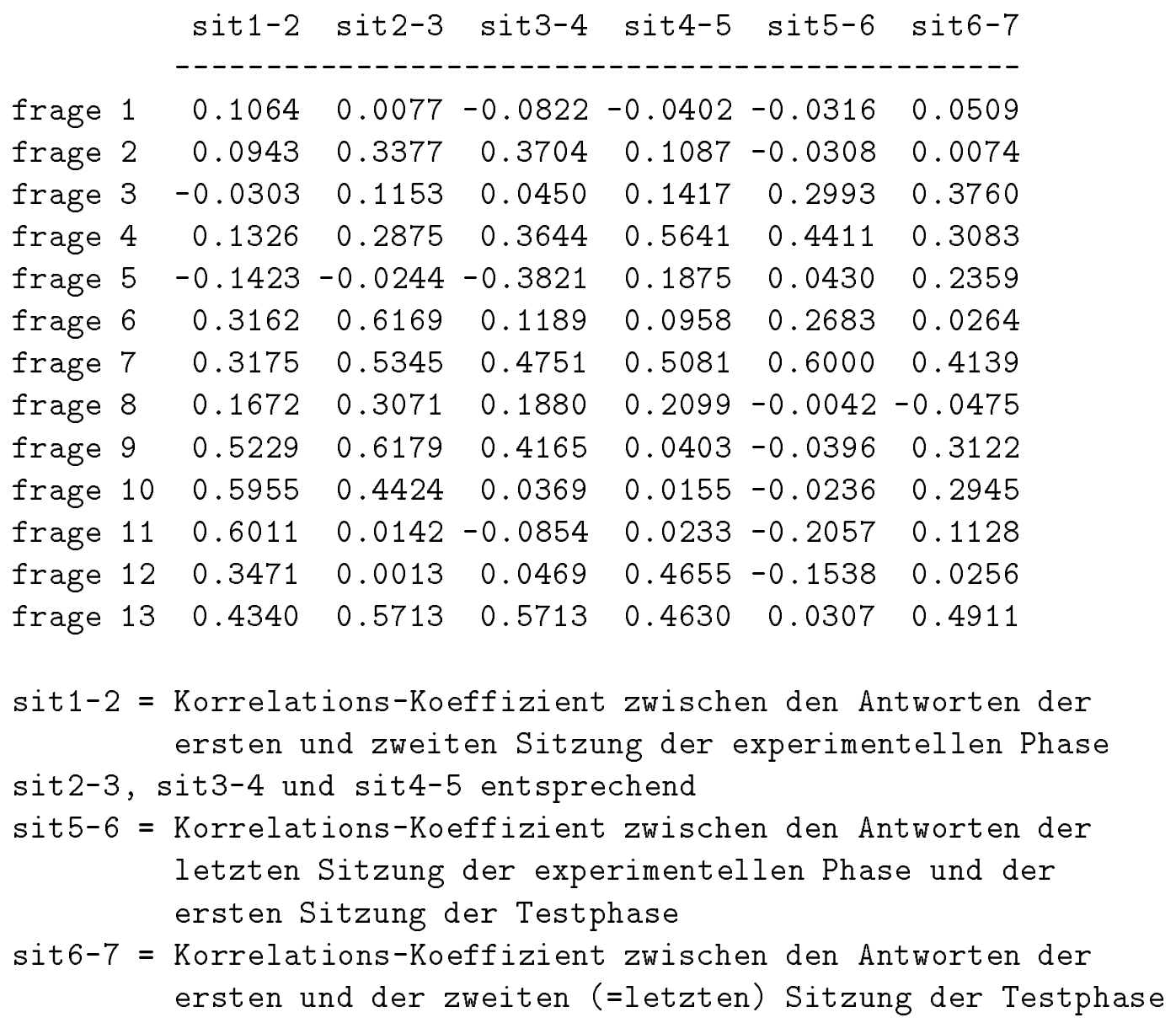




\section{Literaturverzeichnis}

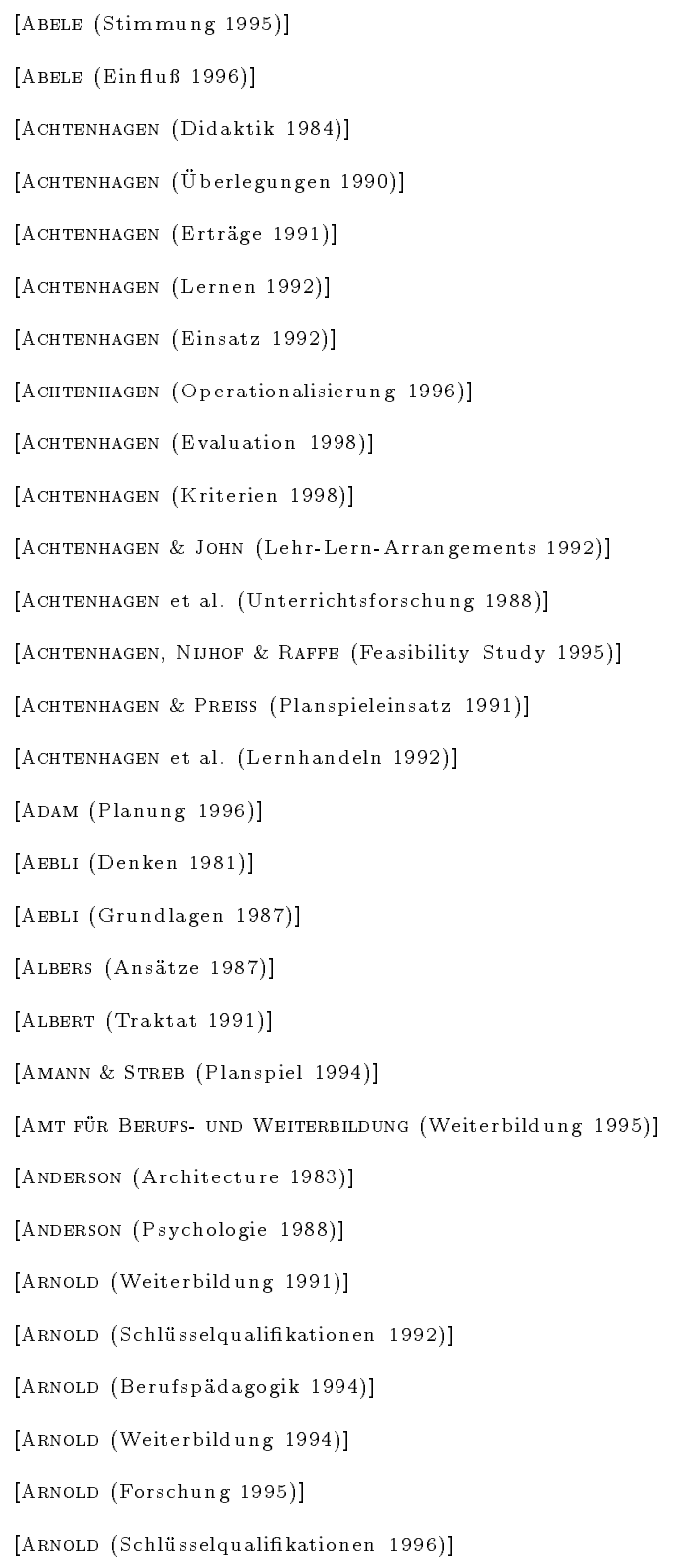


[ARNOLD \& KRÄMER-STÜRZL (Erfolgョkontrolle 1993)]

[ARNOLD \& KRÄMER-STÜRZL (Qualität 1995)]

[ARNOLD \& MÜLLER (Berufßbildung 1992)]

[BACH (Flanspiele 1983)]

[BACHMANN (Grundlagen 1988)]

[BACKHAUS et al. (Analyæemethoden 1996)]

[BAETGE (Systemtheorie 1974)]

[BAETHGE \& OBERBECK (Zukunft 1986)]

[BanduRa (Lerntheorie 1979)]

[BARTON-ZIEMANN (Qualitätıkriterien 1995)]

[BAUMERT (Lernatrategien 1993)]

[BECK (Schlüョselqualifikationen 1995)]

[BECK (Handlungsorientierung 1996)]

[BECK (Grundlagen 1987)]

[BECK et al. (Berufßerziehung 1996)]

[BECKER (Qualität 1991)]

[Becker, Oldenbürger \& Piehl (Motivation 1987)]

[BeitrAB 170 (Bestand 1993)]

[BEITRAB 177 (Einheitlichkeit 1993)]

[BeItRAB 192 (Weiterbildung 1995)]

[BEITRAB 195 (Weiterbildung 1996)]

[BEITRAB 214 (Lernen 1998)]

[BIETHAhN, MuCKsch \& RuF (Informationæmanagement 1996)]

[BIRKHAN ((Un) brauchbarkeit 1992)]

[BleICHER (Unternehmungøapiele 1962)]

[Bleicher (Entacheidungaproze日se 1974)]

[BLOECH (Modellierung 1989)]

[BLOECH (Investitionøplanung 1992)]

[BLOECH (Kostenmanagement 1997)]

[BLOECH (Lehr-Lern-Effekte 1999)]

[BLOECH et al. (Einführung 1998)]

[BLOECH et al. (Einsatz 1998)]

[BLOECH, GOERTZEN \& MAURER (Unternehmensimulation 1992)]

[BLOECH et al. (Planung 1994)]

[BLOECH et al. (Einaatz 1996)]

[BloECh, HaRTUNG \& ORTh (Unternehmenəplanıpiele 1998)]

[Bloech, Hartung \& Orth (Einsatz 1999)]

[Bloech, HaRTung \& ORTh (Förderung 1999)]

[BlOECH \& IHDE (Logistiklexikon 1997)]

[BLOECH, K AUER \& ORTH (Unternehmenæplanıpiele 1996)] 
[BLOECH \& RÜSCHER (Unternehmenasimulation 1991)]

[BLOECH \& RÜSCHER (Unternehmensaimulation 1992)]

[BLOECH \& RÜSCHER (Marketing-Simulation 1992)]

[BLOECH \& RÜSCHER (Unternehmenミplanョpiel 1992)]

[BLOECH \& RÜSCHER (Modellbeachreibung 1995)]

[Blohm et al. (Produktionswirtachaft 1997)]

[BLoom et al. (Taxonomie 1976)]

[BÖHREt \& Wordelmann (Flanıpiel 1975)]

[BODENHOEFER (Ansätze 1994)]

[BOGASCHEWSKY \& GöTZE (Unternehmenəplanung 1998)]

[BojanowsKi, Brater \& DEDERING (Qualifizierung 1991)]

[BONATO (WiBsensstrukturierung 1990)]

[Boos (Enticheidungafindung 1996)]

[Born (Geachichte 1991)]

[BoRTz \& DöRING (Forachungamethoden 1995)]

[Bossel (Simulation 1989)]

[BRANSFORD et al. (Approachea 1989)]

[BRANSFORD et al. (Learning akillı 1989)]

[BRANSFORD et al. (Anchored instruction 1990)]

[BREYDE (Schlüsselqualifikationen 1996)]

[BRonNer \& KOllmanNSPERger (Planəpieleinョatz 1997)]

[Buber \& Enzinger (Flanıpiel 1992)]

[BUDDENSIEK (Simulationsıpiele 1979)]

[BUDDENSIEK (Entscheidungstraining 1983)]

[BUDDENSIEK (Entacheidungatraining 1992)]

[BUNDESANSTALT FÜR ARBEIT (Weiterbildung 1993)]

[BUNDESANSTALT FÜR ARBEIT (Weiterbildung 1994)]

[BUNDESINSTITUT FÜR BERUFSBILDUNG (Fortbildungæmaterialien 1989)]

[BUNDESINSTITUT FÜR BERUFSBILDUNG (Fabrikstrukturen 1990)]

[BUNDESINSTITUT FÜR BERUFSBILDUNG (Entwicklung 1991)]

[BUNDESINSTITUT FÜR BeRUfSBILdUNG (Qualitätıaspekte 1993)]

[BUNDESINSTITUT FÜR BeRUFSBILDUNG (Weiterbildung 1994)]

[BUNDESINSTITUT FÜR BERUFSBILdUNG (Lernen 1994)]

[Bundesverband Deutscher VWA (Rahmen-Studienordnung 1994)]

[BuNK (Kompetenzvermittlung 1994)]

[BUNK, KAISER \& ZEDLER (Schlüョaelqualifikationen 1991)]

[BUtTLER (Tätigkeitılandıchaft 1992)]

[BUtTleR (Weiterbildung 1995)]

[CARL \& KIESEL (Unternehmensführung 1996)]

[CASTI (Connectivity 1979)] 
Nicht ausdrucken!!

[CAVALli et al. (Strukturwandel 1995)]

[CHRISTOPHeR (Gamea 1988)]

[COHEN \& RHENMAN (Rolle 1974)]

[CoOK \& CAMPBell (QuaBi-Experimentation 1979)]

[Collins, Brown \& Newman (Cognitive apprenticeship 1989)]

[COGNITION AND TEChNOLOGY GROUP at VANDERBILT (Ingtruction 1993)]

[CZAYKa (Grundzüge 1972)]

[DANIEL (Planıpiel 1996)]

[DanN (Subjektive Theorien 1983)]

[DANSEREAU (Learning atrategy 1985)]

[DANSEREAU (Learning Strategies 1988)]

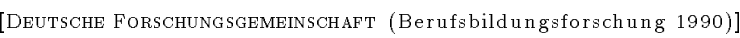

[DEUTSCher BiLdUNGSRAT (Empfehlungen 1970)]

[Deutscher Bundestag (Bildungepolitik 1990)]

[DeUtscher Industrie- und Handelstag (Wettbewerb 1993)]

[DEWE (Wissensverwendung 1988)]

[Dicke, Glismann \& Groenn (Markt 1995)]

[DIEPolD (Modellverauch 1991)]

[DIEPOLD (Lernen 1995)]

[DIEHL-BECKER (Fersonal-Effectivenes:-Traininga 1990)]

[DIXON (Software 1985)]

[DöRIG (Konzept 1994)]

[DöRner (Frozęвe 1983)]

[DöRNER (Problemlösen 1987)]

[DöRNER (Logik 1989)]

[DöRNER et al. (Lohhauıen 1983)]

[DRUKARCZYK (Finanzierung 1996)]

[DuBs (Denken 1989)]

[DuBs (Stellenwert 1989)]

[DuBs (Anfänger-Unterricht 1992)]

[DuBs (Lehr-Lern-Arrangement: 1996)]

[DuBS (Schlüвselqualifikationen 1996)]

[Dulisch (Lernen 1986)]

[Ebert (Planspiel 1992)]

[ECHTERHOFF (Veränderungsmeasung 1978)]

[ECKERT (Kognition 1997)]

[EHRHARD (Metakognition 1995)]

[EISENFÜHR, ORDELHEIDE \& PUCK (Unternehmenミplanıpiele 1974)]

[EISENSTADT \& KareEv (Aspekte 1978)]

[ENDL (Aspekte 1992)] 
[ENQUÊTE-KOMMISSION (Bildungøpolitik 1991)]

[ERB (Gegenstandakonstituierung 1997)]

[ERPENBECK (Kompetenz 1996)]

[FABEL (Fortentwicklungøperæpektiven 1993)]

[FAssheBer (Flanıpiele 1995)]

[FAULSTiCh (Lernkultur 1990)]

[FALLSTICH (Qualitätıkriterien 1991)]

[FAULSTich, Bayer \& Krohn (Zukunftakonzepte 1998)]

[FEgER \& BREDENKAMP (Datenerhebung 1983)]

[FEUCHTHOFEN \& SEVERING (Qualitätımanagement 1995)]

[FISCH \& Boos (Umgang 1990)]

[FLothow (Förderung 1992)]

[FOERSTER (Sicht 1985)]

[Foerster (Wiagen 1993)]

[Foster (Bildung 1990)]

[FREIDANK (Kostenrechnung 1994)]

[FRIEDE (Sozialkompetenz 1994)]

[FRIEDRICH (Analyse 1995)]

[FRIEDRICH \& MANDL (Denkatrategien 1992)]

[FrIEDRICHS (Methoden 1990)]

[FuHRER (Mehrfachhandeln 1985)]

[FUNKE (Froblemlöョen 1986)]

[Funke (Probleme 1990)]

[FüRstenau (Froblemlößen 1994)]

[FÜSER (Management 1999)]

[GARDNER (Denken 1989)]

[Gemlardt \& MÜhlbRadt (Planıpiele 1995)]

[GemhaRdT \& MÜHLbRadt (Konzepte 1995)]

[GEISSLER \& ORTHEY (Schlüвョelqualifikationen 1993a)]

[GEISSLER \& ORTHEY (Schlüвselqualifikationen 1993b)]

[GEISSLER \& W WTTWER (Entwicklung 1989)]

[Georg (Peræpektiven 1998)]

[GERDS (Selbatregulation 1995)]

[GER.Jets \& WestermanN (Theorien 1997)]

[GERLACH (Weiterbildung 1996)]

[Gerstenmaier \& MandL (Wiagenserwerb 1995)]

[GETSCH (Möglich keiten 1990)]

[GeUt Ing (Flanıpiel 1992)]

[Gigerenzer (Messung 1981)]

[GNAHS (Qualitätıaicherung 1995)] 
Nicht ausdrucken!!

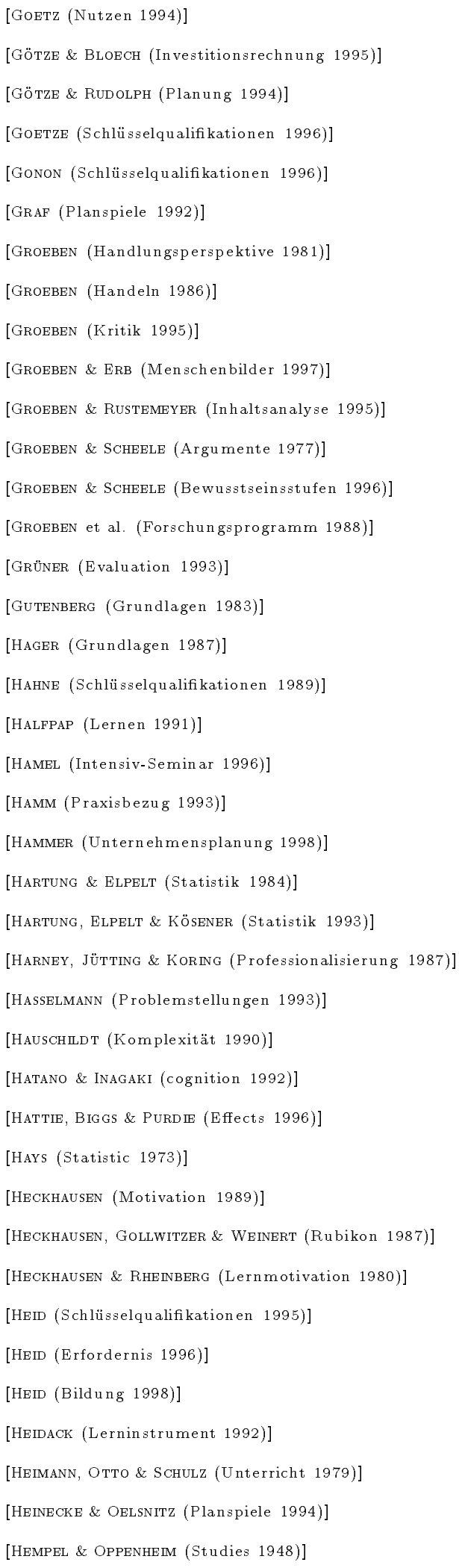


[HENZ (Lehrbuch 1964)]

[Hermann, HöWelmann \& Ullal (Unternehmenəplanョpiele 1987)]

[HERZER, DYBOWSKI \& BAUER (Rationalisierungs-Kuratorium 1990)]

[Hesse (Review 1985)]

[Hesse, SpIEs \& LÜER (Einfluf 1983)]

[HIRSHLETfER \& Riley (analy tica 1992)]

[HöGSDAL (Planミpiele 1996)]

[Hösch (Evaluation 1995)]

[HoEts (Förderung 1993)]

[Hötте (Lernen 1983)]

[Hofer, Pekrun \& Zielinski (Paychologie 1986)]

[HOFMANN \& REgNET (Weiterbildungョkonzepte 1994)]

[HOPFENBECK (Managementlehre 1992)]

[HollmanN (Validität 1991)]

[Hub (Denken 1994)]

[Hussy (Intelligenz 1989)]

[INGENKAMP (Wert 1981)]

[INGENKAMP (Diagnostik 1989)]

[INGENKAMP (Fragwürdigkeit 1995)]

[INGENKAMP (Lehrbuch 1995)]

[INGENKAMP et al. (Pädagogik 1992)]

[JAGENLAUF, SCHUlZ \& Wolgast (Weiterbildung 1995)]

[JANK \& MeYER (Modelle 1994)]

[JoHN (Fallatudien 1992)]

[JoINT CoMmitTeE \& SANDERS (Handbuch 1999)]

[Just (Beurteilung 1998)]

[KAHLE (Anョätze 1998)]

[Kahle \& AChtenhagen (Evaluation 1979)]

[KAISER (Schlü̈вelqualifikationen 1992)]

[KAISER (Entscheidungatraining 1976)]

[KaISER (Fallatudie 1983)]

[KAISER (Grundlagen 1983)]

[KAISER (Lernen 1987)]

[KAISER \& KAMINSKI (Methodik 1994)]

[KASTNER (Denken 1995)]

[KeIM (Planıpiel 1992)]

[Kelly (Faychology 1955)]

[KEYS \& WOLFE (Role 1990)]

[KLAFKI (Unterrichtsplanung 1980)]

[KLAFKI (Leistung 1983)] 
Nicht ausdrucken!!

[KLAUER (Methodik 1974)]

[KLAUER (Kontentvalidität 1978)]

[KLAUER (Zenaierungamodell 1982)]

[KLAUER (Notengebung 1987)]

[KLAUER (Testa 1987)]

[KLAUER (Teaching 1988)]

[KLAUSER (Gestaltung 1998)]

[KlaUSER (Befunde 1998)]

[KLAUSER (Froblem-based learning 1998)]

[KLEBER (Diagnostik 1992)]

[KliPPERT (Planıpiele 1992)]

[KNOBLICH \& RHENIUS (Reaktivität 1992)]

[K ÖHLER (DIN ISO $9000 \mathrm{ff} .1995$ )]

[KöNIG (Erwachsenenbildung 1995)]

[KöNIG (Forachung 1995)]

[KOHNE (Reliabilitätıunterauchungen 1992)]

[KOLLER (Simulation 1969)]

[KORNDÖRFER (Unternehmenョführungalehre 1995)]

[Kramer (Probleme 1995)]

[KRUSE (Unternehmen 1995)]

[KuWan, GNahs \& SEUSING (Weiterbildungastatiıtik 1991)]

[LAIB (Managementdiagnostik 1993)]

[LANDSBERG \& WeISS (Bildungscontrolling 1992)]

[LAUR-ERNST (Schlü:selqualifikationen 1996)]

[LAVE \& Wenger (Situated Learning 1991)]

[Lehmann \& Moog (Rechnungswesen 1996)]

[LeMKE (Tranョfermanagement 1995)]

[LEUTNER (Lehrayateme 1992)]

[LIENERT \& EYe (Statistik 1994)]

[LiENERT \& RAATZ (Testaufbau 1998)]

[Lindman (Analyiis 1974)]

[LIPSMEIER (Wert 1998)]

[LÜDECKE-PLüMER (Befindlichkeit 1991)]

[MaLiK (Strategie 1989)]

[MANDElBRot (Geometrie 1987)]

[MandL, Gruber \& Renkl (Prozesıe 1992)]

[MandL, Gruber \& RenkL (Wigsen 1993)]

[MANDL \& ReINMANN-RothmeIER (Unterrichten 1995)]

[MANDL \& SPADA (Wiäenæpвychologie 1988)]

[Manturana, Varela \& Uribe (Autopoiëse 1982)] 
[MAYRING (Inhaltianalyıe 1993)]

[MoClelland, Rumelhart \& PDP Research Group (Explorations 1987)]

[MCClelland, Rumelhart \& PDP Research Group (Explorationa 1988)]

[MCKENNEY (Evaluation 1962)]

[MCK INSEY COMPANY INC. \& RoMmel. (Unternehmenøkonzept 1993)]

[MeER \& Kolbe (Kodierung 1997)]

[MefFerT (Marketing 1991)]

[Merten (Inhaltaanalyse 1983)]

[MERTENS (Schlü̈aelqualifikationen 1974)]

[Metzig \& SChuster (Lernen 1993)]

[MIDDENDORF (Anmerkungen 1991)]

[Miller, Galanter \& Pribram (Strategie 1973)]

[MINSKY (Mentopolia 1990)]

[MöBus (Modellierung 1988)]

[Mummendey (Fragebogen-Methode 1995)]

[NAGEL (Weiterbildung 1992)]

[NEWELL \& SiMON (Problem Solving 1972)]

[NEzEL (Didaktik 1992)]

[Nieschlag, Dichtl \& Hörschgen (Marketing 1997)]

[NoRMan \& RumelhaRT (Strukturen 1978)]

[NÜSE et al. (Erfindung/en 1991)]

[NuissL (Lernökologie 1992)]

[OLDENBÜRger (Erhebung 1986)]

[OLdenbüRger (Netz-Werk-Zeug 1992)]

[OLDENBürger (Datenanalyıe 1996)]

[OLFERT (Finanzierung 1994)]

[OPwIS \& LÜER (Modelle 1994)]

[ORTH (Entwicklung 1998)]

[PätzolD (Handlungøorientierung 1992)]

[P ̈̈̈тоLD (Schlüвselqualifikationen 1996)]

[PALM (Modellvoratellungen 1988)]

[PAULSEN (Erfahrungalernen 1993)]

[PERRIDON \& Steiner (Finanzwirtachaft 1995)]

[Peterson, Maier \& Seligman (Helpleasneas 1993)]

[Petzing (Untersuchung 1993)]

[PIETzSch (Einョatz 1997)]

[POPPER (Logik 1989)]

[POSCH, SCHNEIDER \& MANN (Unterrichtøplanung 1977)]

[PREHM (Marketing-Unterneh mens:piel 1995)]

[Preiss (Komplexität 1992)] 
Nicht ausdrucken!!

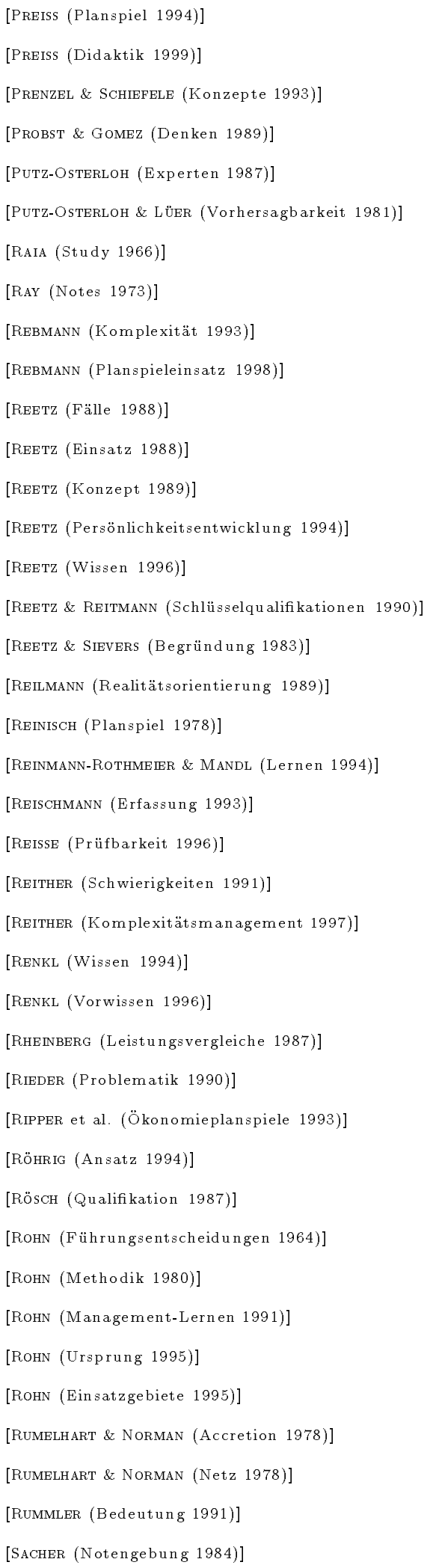


[SAUTER (Weiterbildungsforschung 1991)]

[SAVAGE (Management 1997)]

[Schaumann (Feripektiven 1993)]

[SCHEELE (Emotionen 1990)]

[SCHEELE (Struktur-Lege-Verfahren 1992)]

[SCheEle \& Groeben (Dialog-Konseni-Methoden 1988)]

[SCHLAG (LeiBtungsmotivation 1995)]

[SChMAGer \& WIRTH (Flanıpiel 1990)]

[SCHMIEL (Förderung 1988)]

[SCHMIEL \& SOMMER (Legitimationəproblematik 1996)]

[Schmolke \& DeitermanN (Rechnungıwesen 1999)]

[SCHNEIDER (Investition 1992)]

[SCHNELl, HILL \& ESSER (Methoden 1999)]

[SCHOEL (Konzeption 1998)]

[SCHULER (Funktionakreis 1991)]

[SCHUNCK (Subjektive Theorien 1993)]

[SCHWARze (Netzplantechnik 1994)]

[SCHWEER (Innovation 1996)]

[SCHWEER (Dozentenverhalten 1998)]

[SELG, Klapprott \& Kamenz (Forachungamethoden 1992)]

[SELL (Froblemlöョungəverhalten 1989)]

[SEMBILl (Froblemlöョefähigkeit 1992)]

[SEYFRIED (Evaluation 1998)]

[SIEvers (Forachungæmethoden 1990)]

[SKINNER (Wiäenachaft 1973)]

[SÖLTENFUSS \& HALFPAP (Außbildung 1987)]

[SPIES \& HESSE (Interaktion 1986)]

[SPREMANN (Investition 1996)]

[STAUDT, KRÖLL \& HÖREN (Fotentialorientierung 1993)]

[STÄUdel (Froblemlößen 1987)]

[STACHOWIAK (Erkenntniøatufen 1980)]

[STACHOWIAK (Einleitung 1980)]

[STARK et al. (Förderung 1995)]

[STARK et al. (Lernen 1995)]

[STARKER \& DöRNER (Determinanten 1997)]

[SteinmanN \& Weber (Methoden 1995)]

[STENDER (Weiterbildungøbereitschaft 1994)]

[STENTZEL (Lernachwierigkeiten 1986)]

[STEYeR \& EID (Mesien 1993)]

[STIENSMEIER-PELSTER \& SCHLANGEN (Hilflosigkeit 1996)] 
Nicht ausdrucken!!

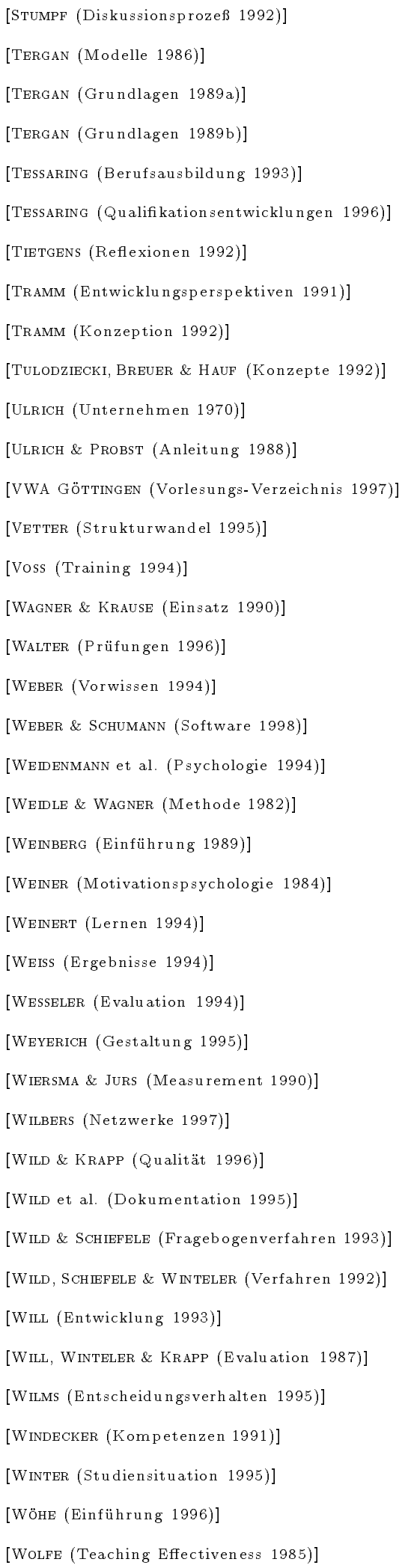


[WoLLMANN (Zauberformel 1993)]

[WoORTMANN (Qualität 1995)]

[WotTaWa (Wirkamkeit 1996)]

[Wottawa \& ThIERAU (Lehrbuch 1998)]

[WRiGHT (Erklären 1974)]

[WUNDER (Qualitätımanagement 1994)]

[WutTke (Motivation 1999)]

[Young (Education 1998)]

[ZIMMERMANN (Außbildungョge日taltung 1995)] 